\section{Report no.}

HENL-SI - 74-1
STRUCTURES AND MATERIALS RESEARCH

Department of Civil Engineering

\section{BOTICE}

PORTIONS OF THIS REPORT ARE ILLEGIBLE. It

copy to permit thed from the best available ability.

TEST RESULTS ON THE DYMATC TESTING OF EXPANSOY TYPE COMPETE AMCHRS r.

DECENBER 1974

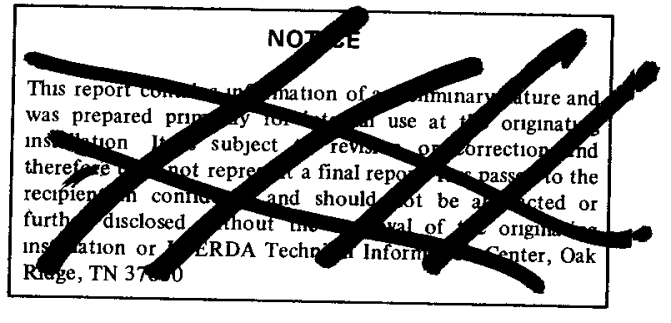

By: Basty' 'irror.

Roger Rice

Roy M. Stephen
This report was preparod as

sponsored by the United as an account of work the United States nor the United States Energy Research and Development Admunustration, nor any of subcontractors or nor any of their contractors, warranty express or their employees, makes any labblity or responsibulity for the accuracy, completeness process disclosed iny information, apparat tus, product or infinge privately owned rights
prosess dis use would not

Report lO WESTIMGHOUSE HAMORD COMPAYY

\section{MASTER}

74.

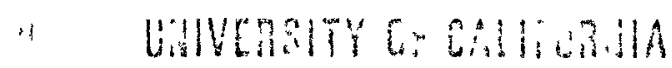

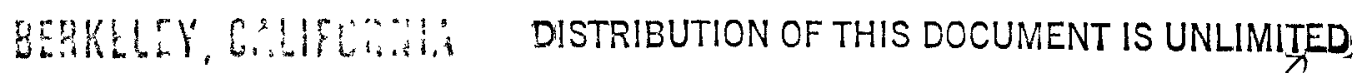

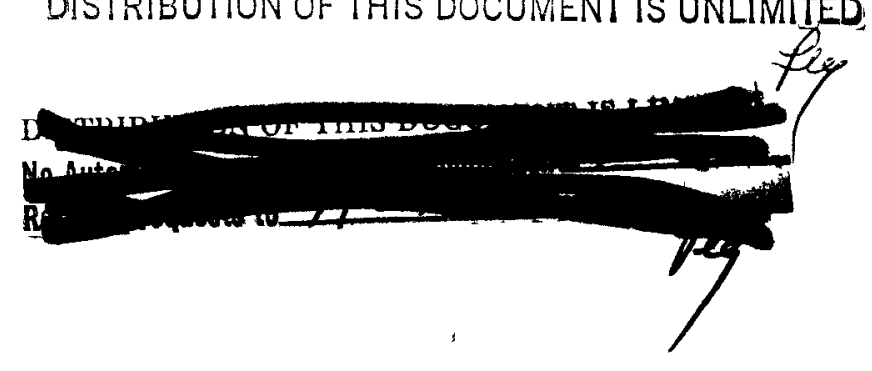




\section{DISCLAIMER}

Portions of this document may be illegible in electronic image products. Images are produced from the best available original document. 


\section{DISCLAIMER}

Portions of this document may be illegible in electronic image products. Images are produced from the best available original document. 
BERKELEY - DAVIS - MVINE - LOS ANGELES - RIVERSIDE - SAN DIECO - SAN FRANCISCO

COLLEGE OF ENGINEERING DEPARTMENT OF CIVIL ENGINEERING DIVISION OF STRUCTURAL ENGINEERING AND STRUCTURAL MECHANICS
BERKELEY, CALIFORNIA 94720

December 11, 1974

Westinghouse Hanford Company

P. O. Box 1970

Richland,WA. 99352

Attention: Mr. G. C. Peterson IN4/337

Gentlemen:

Transmitted herein is a report entitled, "Test Results on the Dynamic Testing of Expansion Type Concrete Anchors."

This test program was conducted at the University of California, Berkeley, under an Industrial Service Program, PROJECT S-69835.

I trust that you will find the report and results satisfactory.

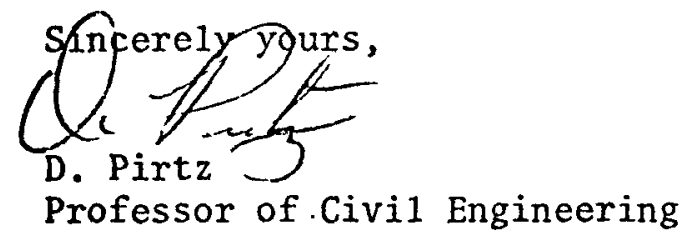

RMS : jm

cc:

K. . . M M

K. W. McMilliam 
WESTINGHOUSE HANFORD COMPANY

RICHLAND, WASHINGTON

TEST RESULTS ON THE DYNAMIC TESTING OF EXPANSION

TYPE CONCRETE ANCHORS

By

Barry Barron

Roger Rice

Roy M. Stephen

\section{OBJECTIVE}

The purpose of these tests were to determine the structural response, yield and ultimate capacity of various types of commercially available expansion type anchors when subjected to dynamic loadings similar to those encountered in: (a) steady-state machine vibrations, and (b) earthquakes. The specimens were subjected to tension, shear, and combined shear-tension loads.

DESCRIPTION OF TEST SPECIMENS

The anchors were set in precast concrete blocks measuring 15 in. by 15 in. 12 in., with $\# 2$ spiral reinforcement. Figures 1,2 and 3 show the tension, shear and combined specimens. The concrete used was of three types:

$$
\begin{aligned}
& \text { C - FFTF Regular Concrete } 4000 \text { psi mix. } \\
& \text { M - FFTF Magnetite Concrete } 5000 \text { psi mix. } \\
& \text { SS - FFTF Steel Shot Concrete } 5000 \text { psi mix. }
\end{aligned}
$$

In all cases a $11 / 4-i n$. threaded bolt with a 4 in. by 4 in. steel plate was cast into the concrete such that the threads projected from the specimen.

Shell, sleeve, and stud-type anchors in sizes of $5 / 8,3 / 4$, and 1 -inch diameter, as shown in Figure 4, were tested. Table I lists the size, type, manufacturer, and code for each of the anchors tested. All anchors were Installed according to the manufacturer's specifications and aligned with respect to the upper cast-in bolt. In some cases eccentricities were noted, 
however, these were generally less than $1 / 4$ inch. Embedment depths varled from specimen to specimen as shown on the $X-Y$ p.lots in the Appendix of this report.

For each test specimen a standard $6 \times 12$ in.-control cylinder was cast from the same batch of concrete to determine the concrete strength on the day of testing. The control cylinders and specimens were cured in moist sawdust for 14 days.

TESTING EQUIPMENT

Testing was conducted using the 500 kip MTS Dynamic Test Frame in Davis Hall, University of California, Berkeley.

\section{TEST ARRANGEMENT}

The specimens were installed in the test frame as shown in Figure 5. Two linear transformers (LVDT's) were mounted under the specimen, as noted in the figure, on opposite sides of the expansion anchor to measure the relative movement between the anchor and the test specimen. Two Honeywell $X-Y$ recorders were used in the testing. One, to measure load versus overall (or ram) displacement, and the other to measure load versus the average of the LVDT's displacement. A Tektronix 56A storage oscilloscope was used to monitor the MTS load range values during dynamic testing. This test equipment along with the MTS control console and function generator is shown in Figure 6.

\section{TEST PROCEDURE AND LOADING SEQUENCE}

For the static tests the specimens were installed and the load applied at a rate of approximately 5 kips per minute until failure occurred.

For the dynamic tests the specimens were subjected to an initial load of 20 percent of the average static test values ( $\left.S^{\prime}\right)$, cycled for 2000 cycles 
each at 5, 10, and 15 cycles per second. Displacement readings were made at each interval. At the end of the 6000 cycles the frequency was maintained at 10 cycles per second and the load was increased by 10 percent of $S^{\prime}$ every 600 . cycles up to 50 percent of $\mathrm{S}^{\prime}$. At this point (7800 accumulated cycles) the load was raised to 60 percent $S^{\prime}$ and the specimen cycled unt 11100,000 cycles. At 100,000 cycles the load was increased 2000 pounds every 2000 cycles until failure occurred. Table II outlines the loading sequence used. In many cases, due either to the high loads or large displacements, it was not possible to maintain frequencies above 5 cycles per second. In this case the maximum possible frequency was used and was noted on the $X-Y$ plots.

For the long-term fatigue tests the specimens were loaded to 20 percent of S' for 2,000,000 cycles at 10 cycles per second (frequencies varied on certain specimens as noted above, see $X-Y$ plots for actual frequencies). In the case where the specimen survived $2,000,000$ cycles, the $10 a d$ was then increased by 2000 pounds every 2000 cycles until failure occurred.

The minimum load for all dynamic and fatigue tests varied between 500 and 1200 pounds.

\section{RESULTS}

The results are tabulated in Table III according to size, anchor type, and type of specimen for easy reference. The table lists from left to right: specimen number, type of concrete, strength of concrete on test date, type of anchor (see Table I), type of test performed, type of specimen, yield load, displacement at yield load, number of cycles at yield load, ultimate load, total number of cycles, and a brief comment on the mode of failure. For dynamic and fatigue tests the yield and ultimate loads are listed as the same. 
Table IV is a bar graph showing the ultimate loads of all the specimens tested, grouped according to size and anchor type. Figure 7 shows the typical failure mode for each type of specimen tested. The $\mathrm{X}-\mathrm{Y}$ plots are attached as Appendix 1 and are grouped by specimen number. Appendix 2 is a tabulation of the data by bolt type and Appendix 3 is a tabulation of the data by test type. 
TABLE 1. TYPES OF - ANCHOR BOLTS TESTED

\begin{tabular}{|c|c|c|}
\hline Anchor Type/Size & Code & Manufacturer \\
\hline $\begin{array}{l}\text { Hilti Shell Type } \\
5 / 8^{\prime \prime} \text { stud, } 27 / 32^{\prime \prime} \text { shell }\end{array}$ & $I A-5$ & \multirow{5}{*}{$\begin{array}{l}\text { Hilti Fastening Systems } \\
\text { Stamford, Connecticut }\end{array}$} \\
\hline $\begin{array}{l}\text { Hilti Shell Type } \\
3 / 4^{\prime \prime} \text { stud, 1" shel1 }\end{array}$ & LA-6 & \\
\hline $\begin{array}{l}\text { Hilti Stud Type } \\
5 / 8 " \text { dia. stud }\end{array}$ & $\mathrm{SH}-5$ & \\
\hline $\begin{array}{l}\text { Hilti Kwik-bolt Stud } \\
3 / 4 " \text { dia. stud }\end{array}$ & SH-6 & \\
\hline $\begin{array}{l}\text { Hilti Kwik-bolt Stud } \\
1^{\prime \prime} \text { dia. stud }\end{array}$ & $\mathrm{SH}-8$ & \\
\hline $\begin{array}{l}\text { Molly Parabolt Stud } \\
5 / 8^{\prime \prime} \text { dia. stud }\end{array}$ & $\mathrm{SB}-5$ & \multirow{3}{*}{$\begin{array}{l}\text { USM Corporation } \\
\text { Shelton, Connecticut }\end{array}$} \\
\hline $\begin{array}{l}\text { Mo11y Parabolt Stud } \\
3 / 4^{\prime \prime} \text { dia. stud }\end{array}$ & SB-6 & \\
\hline $\begin{array}{l}\text { Molly Parabolt Stud } \\
1 " \text { dia. stud }\end{array}$ & $\mathrm{SB}-8$ & \\
\hline $\begin{array}{l}\text { Phillips Red-head Sleeve } \\
\text { 1/2" dia. stud, 5/8" dia. } \\
\text { sleeve }\end{array}$ & LB-5 & \multirow{2}{*}{$\begin{array}{l}\text { Phillips Drill Company Inc. } \\
\text { Michigan City, Indiana }\end{array}$} \\
\hline $\begin{array}{l}\text { Phillips Red Head Sleeve } \\
5 / 8^{\prime \prime} \text { dia. stud, } 3 / 4^{\prime \prime} \text { dia. } \\
\text { sleeve }\end{array}$ & LB-6 & \\
\hline $\begin{array}{l}\text { Raw1 } \text { Stud Anchor } \\
5 / 8 " \text { dia. stud }\end{array}$ & $\mathrm{SA}-5$ & \multirow{3}{*}{$\begin{array}{l}\text { The Rawlplug Company } \\
\text { New Rochelle, New York }\end{array}$} \\
\hline $\begin{array}{l}\text { Rawl Stud Anchor } \\
3 / 4^{\prime \prime} \text { dia. stud }\end{array}$ & $S A-6$ & \\
\hline $\begin{array}{l}\text { Raw1 Stud Anchor } \\
1^{\prime \prime} \text { dia. stud }\end{array}$ & $S A-8$ & \\
\hline $\begin{array}{l}\text { Wej-it Stud Anchor } \\
5 / 8^{\prime \prime} \text { dia. stud }\end{array}$ & $\mathrm{SC}-5$ & \multirow{3}{*}{$\begin{array}{l}\text { Wej-it Corporation } \\
\text { Broomfield, Colorado }\end{array}$} \\
\hline $\begin{array}{l}\text { Weij-it Stud Anchor } \\
3 / 4^{\prime \prime} \text { dia. stud }\end{array}$ & $\mathrm{SC}-6$ & \\
\hline $\begin{array}{l}\text { Weif-it Stud Anchor } \\
1^{\prime \prime} \text { dia. stud }\end{array}$ & $\mathrm{SC}-8$ & \\
\hline
\end{tabular}


TABLE 2. DYNAMIC LOAD SEQUENCE

\begin{tabular}{|c|c|c|c|}
\hline $\begin{array}{c}\text { Test } \\
\text { Period }\end{array}$ & $\begin{array}{c}\text { Max. } \\
\text { Load }\end{array}$ & $\begin{array}{c}\text { Cycle/ } \\
\text { Second }\end{array}$ & $\begin{array}{c}\text { Total } \\
\text { Cycles }\end{array}$ \\
\hline 1 & $.2 S^{\prime}$ & 5 & 2000 \\
2 & $.2 S^{\prime}$ & 10 & 4000 \\
3 & $.2 S^{\prime}$ & 15 & 6000 \\
4 & $.3 S^{\prime}$ & 10 & 6600 \\
5 & $.4 S^{\prime}$ & 10 & 7200 \\
6 & $.5 S^{\prime}$ & 10 & 7800 \\
7 & $.6 S^{\prime}$ & 10 & 100,000 \\
8 & $.6 S^{\prime}+2000 \#$ & 10 & 102,000 \\
9 & $.6 S^{\prime}+4000$ * & 10 & 104,000 \\
1 & $.6 S^{\prime}+(i-7) 2000 \#$ & 10 & $100,000+(i-7) 2000$ \\
\hline
\end{tabular}


TABLE 3. RESULTS OF ANCHOR TEST PROCRAM

TAELE OF DATA SORTED GY BOLT DIAMETER AND TEST TYPE IN THAT ORDEP.

5/8 INCH BOLTS TESTED IN TENSION

\begin{tabular}{|c|c|c|c|c|c|c|c|c|c|c|c|c|}
\hline SPEC & CONC & $F \cdot C$ & BOLT & \multicolumn{2}{|c|}{ TEST } & $P 1$ & 01 & CYCLE & $P U$ & DU & CYCLE & FAILURE \\
\hline NUMB & TYPE & KSI & TYPE & \multicolumn{2}{|c|}{ TYPE } & KIP & IN & $K C$ & $K \backslash \rho$ & $I N$ & $K C$ & MODE \\
\hline $\begin{array}{l}11 \\
112\end{array}$ & $\begin{array}{l}\text { REG } \\
\text { REG }\end{array}$ & $\begin{array}{l}5.60 \\
7.49\end{array}$ & $\begin{array}{l}L B-5 \\
L A-5\end{array}$ & $\begin{array}{l}\text { STAT } \\
\text { DYN }\end{array}$ & $\begin{array}{l}T \\
T\end{array}$ & $\begin{array}{l}12 \cdot 0 \\
6 \cdot 6\end{array}$ & $\begin{array}{l}.25 \\
.08\end{array}$ & -- & $\begin{array}{l}13 \cdot 0 \\
10 \cdot 0\end{array}$ & $\begin{array}{l}.40 \\
.22\end{array}$ & $\overline{102}$ & $\begin{array}{l}\text { BOLT FAILED } \\
\text { CONC FAILES }\end{array}$ \\
\hline 100 & REG & $6 \cdot 20$ & $L 4-5$ & DYN & $T$ & $7 \cdot 8$ & .06 & $7 \cdot 8$ & $10 \cdot 6$ & .14 & 101 & CONC FAILEO \\
\hline 14 & REG & 4.50 & $\angle E-5$ & DYN & $T$ & $11 \cdot 0$ & .20 & $10 \cdot 2$ & $11 \cdot 0$ & .45 & $11 \cdot 7$ & BOLT FAILED \\
\hline 202 & $R \in G$ & $4 \cdot 40$ & $L B-5$ & DYN & $T$ & $6 \cdot 8$ & $\cdot 15$ & $22 \cdot 0$ & $6 \cdot 8$ & .20 & 71 & BOLT FAILED \\
\hline 201 & REG & 0.10 & $L B-5$ & FAT & $T$ & $2 \cdot 6$ & .25 & $3 \cdot 0$ & $2 \cdot 6$ & .35 & 504 & BOLT FAILED \\
\hline $\begin{array}{l}205 \\
105\end{array}$ & $\begin{array}{l}\text { REG } \\
\text { REG }\end{array}$ & $\begin{array}{l}5 \cdot 50 \\
6 \cdot 54\end{array}$ & $\begin{array}{l}L B-5 \\
S B-5\end{array}$ & $\begin{array}{l}\text { FAT } \\
\text { STAT }\end{array}$ & $\begin{array}{l}T \\
T\end{array}$ & $\begin{array}{l}2 \cdot 6 \\
8 \cdot 0\end{array}$ & $\begin{array}{l}.02 \\
.20\end{array}$ & $\begin{array}{l}2000 \\
--\end{array}$ & $\begin{array}{l}2 \cdot 6 \\
14 \cdot 4\end{array}$ & $\begin{array}{l}.02 \\
.40\end{array}$ & 2000 & $\begin{array}{l}\text { BOLT FAILED } \\
\text { DULLOUT }\end{array}$ \\
\hline $\begin{array}{l}203 \\
89\end{array}$ & $\begin{array}{l}\text { REG } \\
R E G\end{array}$ & $\begin{array}{l}6.20 \\
6.86\end{array}$ & $\begin{array}{l}S B-5 \\
S C-5\end{array}$ & $\begin{array}{l}\text { STAT } \\
\text { DYN }\end{array}$ & $\begin{array}{l}T \\
T\end{array}$ & $\begin{array}{l}11 \cdot 6 \\
5 \cdot 5\end{array}$ & $\begin{array}{l}.35 \\
.07\end{array}$ & -- & $\begin{array}{l}11 \cdot 6 \\
10 \cdot 7\end{array}$ & $\begin{array}{l}.80 \\
.20\end{array}$ & $-\overline{102}$ & $\begin{array}{l}\text { PULLOUT } \\
\text { PULLOUT }\end{array}$ \\
\hline 85 & REG & 6.55 & $S C-5$ & DYN & $T$ & $b \cdot 5$ & .05 & $7 \cdot 8$ & 12.6 & .20 & 101 & PULLOUT \\
\hline 37 & REG & 5.50 & $S A-5$ & DYN & $T$ & $8 \cdot 6$ & $\cdot 22$ & $7 \cdot 8$ & 18.5 & .60 & 110 & PULLOUT \\
\hline 114 & REG & 6.86 & $S A-5$ & DYN & $T$ & $8 \cdot 6$ & .50 & $7 \cdot 8$ & $12 \cdot 6$ & 1.0 & 102 & CONC FAILED \\
\hline 5 & REG & 6.30 & $S B-5$ & DYN & $T$ & 7.0 & .45 & $7 \cdot 8$ & 15.0 & .58 & 112 & PULLOUT \\
\hline 1 & REG & 6.60 & $S B-5$ & DYN & $T$ & $7 \cdot 0$ & .07 & $7 \cdot 8$ & $12 \cdot 0$ & .45 & 102 & PULLOUT \\
\hline
\end{tabular}


TABLE 3. CONTINUED

TASLE OF UATA SORTED GY BOLT DIAMETER AND TEST TYPE IN THAT ORDER (CONT•)

5/8 INCH BOLTS TESTED IN SHEAR

$\begin{array}{lllllllllll}\text { SPEC } & \text { CONC } & \text { FIC } & \text { GOLT } & \text { TEST } & \text { PI } & \text { DI } & \text { CYCLE } & \text { PU } & \text { DU CYCLE } & \text { FAILURE } \\ \text { NUMB } & \text { TYPE } & \text { KSI } & \text {-TYPE } & \text { TYPE } & \text { KIP } & \text { IN } & \text { KC } & \text { KIP } & \text { IN KC K } & \text { MODE }\end{array}$

\begin{tabular}{|c|c|c|c|c|c|c|c|c|c|c|c|c|c|}
\hline 210 & REG & 4.90 & LB-5 & STAT & v & 19.0 & .45 & -- & 20.0 & .70 & $-\infty$ & BOLT & SHEADED \\
\hline 16 & REG & 5.00 & LB-5 & STAT & $v$ & 17.0 & .20 & -- & 24.0 & .70 & -- & BOL T & SHEADEL \\
\hline$C 2-12$ & REG & 6.87 & LB்-5 & STAT & v & 16.0 & $\cdot 30$ & -- & $17 \cdot 2$ & .40 & -- & BOLT & SHE ARED \\
\hline$C 2-14$ & $R E G$ & 6.22 & $L A-5$ & DYN & $v$ & $9 \cdot 6$ & .15 & $7 \cdot 8$ & $9 \cdot 6$ & . 30 & $51 \cdot 0$ & EOLT & $\triangle H E A R E U$ \\
\hline$c 2-15$ & REG & 6.52 & $L A-5$ & DYN & v & 9.6 & .15 & $7 \cdot 8$ & 9.6 & .25 & 39.0 & BOLT & SHEARED \\
\hline 207 & REG & $4 \cdot 50$ & L3-5 & DYN & v & 14.4 & .20 & 7.8 & 14.4 & .40 & 31.6 & BOLT & $S H E A R E D$ \\
\hline 12 & REG & $5 \cdot 50$ & $L B-5$ & DYN & $v$ & $14 \cdot 4$ & .35 & $7 \cdot 8$ & $14 \cdot 4$ & .65 & $32 \cdot 0$ & SOLT & SHEARED \\
\hline 206 & REG & $5 \cdot 80$ & LB-5 & FAT & v & $4 \cdot 6$ & .07 & .60 & $26 \cdot 5$ & & 2000 & BOL T & SHEADED \\
\hline 72 & REG & $7 \cdot 17$ & LB-5 & FAT & $v$ & $4 \cdot 6$ & .05 & $7 \cdot 8$ & 26.6 & 1.0 & 2000 & BOLT & SHEADEC \\
\hline 208 & REG & 6.20 & SE-5 & STAT & v & 28.0 & .15 & -- & 33.0 & .45 & -- & BOL T & SHEARED \\
\hline$c 2-13$ & REG & 5.90 & $S B-5$ & STAT & $v$ & 16.0 & .10 & -- & 21.0 & .17 & $-\infty$ & BOLT & SHEAREZ \\
\hline 7 & REG & 6.40 & $S B-5$ & DYN & v & $20 \cdot 0$ & .10 & $7 \cdot 8$ & $20 \cdot 0$ & .20 & $9 \cdot 2$ & BOLT & SHEARED \\
\hline 8 & REG & --- & $S B-5$ & DYN & v & 18.0 & $\cdot 22$ & $7 \cdot 8$ & 18.0 & -- & 10.2 & BOL T & SHEEAREC \\
\hline$c 2-10$ & REG & 6.40 & $s c-5$ & OYN & $v$ & $14 \cdot 4$ & $\cdot 15$ & $7 \cdot 8$ & $14 \cdot 4$ & .23 & 33.9 & BOLT & SHEARE \\
\hline$C 2-11$ & $R E G G$ & 6.64 & $s c-5$ & DYN & $v$ & 14.4 & .25 & $7 \cdot 8$ & 14.4 & .75 & 23.0 & BOLT & SHEARED \\
\hline 209 & REG & $7 \cdot 16$ & $S A-5$ & DYN & v & 19.2 & .17 & $7 \cdot 8$ & $19 \cdot 2$ & .45 & 17.9 & BOLT & SHEAREL \\
\hline C2-9 & REG & 6.77 & $S A-5$ & DYN & $v$ & 19.2 & . 30 & $7.8:$ & $19 \cdot 2$ & .35 & $8 \cdot 0$ & BOL T & SHEAREL \\
\hline
\end{tabular}


TABLL OF UATA SORTED GY BOLT DIAMETER AND TEST TYPE IN THAT ORDER (CONT•) $5 / 8$ INCH BOLTS TESTED IN COMBINED TENSION AND SHEAR

\begin{tabular}{|c|c|c|c|c|c|c|c|c|c|c|c|c|}
\hline SPEC & CONC & $\mathrm{F}, \mathrm{C}$ & . SOLT & \multicolumn{2}{|c|}{ TEST } & $P_{1}$ & D 1 & CYCLE & $P U$ & DU & CYCLE & FAILUPE \\
\hline NUMS & TYPE & KSI & - TYPE & \multicolumn{2}{|c|}{ TYPË } & $K I P$ & IN & $K C$ & KIP & IN & $K C$ & MODE \\
\hline 39 & REG & $6 \cdot 11$ & $L A-5$ & STAT & C & $4 \cdot 0$ & .10 & $-\infty$ & $11 \cdot 2$ & .95 & -- & BOLT FAILED \\
\hline 21 & REG & $5 \cdot 80$ & LBS 5 & STAT & C & $6 \cdot 4$ & $\cdot 15$ & -- & $11 \cdot 8$ & 1.0 & -- & PULLOUT \\
\hline 46 & REG & 5.30 & LBS-5 & STAT & C & 8.0 & .30 & -- & 11.4 & $1 \cdot 1$ & - & PULLOUT \\
\hline 43 & REG & $5 \cdot 70$ & La-5 & DYN & C & 7.0 & .05 & $7 \cdot 2$ & $7 \cdot 0$ & .46 & 41 & PULLOUT \\
\hline 33 & REG & 5.00 & $L B-5$ & DYN & $c$ & $7 \cdot 2$ & .15 & 7.8 & $7 \cdot 2$ & .40 & $16 \cdot 5$ & BOLT SHEAREC \\
\hline 44 & REG & 6.61 & $L A-5$ & DYN & $C$ & $6 \cdot 6$ & .07 & $7 \cdot 8$ & $6 \cdot 6$ & .07 & $7 \cdot 8$ & PULLOUT \\
\hline 34 & REG & 5.92 & $L A-5$ & DYN & C & $6 \cdot 6$ & .07 & $7 \cdot 8$ & $6 \cdot 6$ & .07 & $7 \cdot 8$ & PULLOUT \\
\hline 35 & REG & 4.90 & SE -5 & STAT & C & 11.6 & .07 & - & $11 \cdot 6$ & .10 & -- & CONC FAILEO \\
\hline 25 & REG & -- & $S B-5$ & STAT & $c$ & $6 \cdot 8$ & 12 & -- & $10 \cdot 6$ & .43 & -- & CONC FAILED \\
\hline 10 & REG & 5.80 & $S E-5$ & DYN & $c$ & $6 \cdot 6$ & .07 & $7 \cdot 8$ & $6 \cdot 6$ & $-\infty$ & 17.9 & EOLT SHEAREC \\
\hline 45 & REG & $6 \cdot 10$ & $S B-5$ & DYN & $C$ & $6 \cdot 6$ & .10 & $7 \cdot 8$ & $7 \cdot 6$ & .35 & 102 & BOLT SHEAREC \\
\hline 18 & REG & $6 \cdot 70$ & $s c-5$ & DYN & $C$ & 6.6 & $\cdot 27$ & $7 \cdot 8$ & $6 \cdot 6$ & $-\infty$ & 28.0 & DULLOUT \\
\hline 4 & REG & $6 \cdot 20$ & $s c-5$ & DYN & C & $6 \cdot 6$ & .05 & $7 \cdot 8$ & $6 \cdot 6$ & .07 & $15 \cdot 6$ & PULLOUT \\
\hline 24 & REG & 6.50 & $S A-5$ & DYN & C & 6.6 & .09 & $7 \cdot 8$ & $6 \cdot 5$ &.$: 0$ & $22 \cdot 0$ & PU!LLUT' \\
\hline 36 & REG & $6 \cdot 30$ & $S A-5$ & DYN & $c$ & $6 \cdot 6$ & .15 & $7 \cdot 8$ & $6 \cdot 6$ & .45 & $37 \cdot 0$ & PULLOUT \\
\hline
\end{tabular}


TABLE 3. .CONTINUED

- AgLE OF LATA SORTED EY BOLT DIAMETER AND. TEST TYPE IN.THAT ORDER (CONT.)

$3 / 4$ INCH BOLTS TESTED IN TENSION

SPEC CONC FIC .. BOLT TEST PI DI CYCLE PU DU CYCLE FAILURE NUMB TYPE KSI TYPE TYPE KIP IN KC KIP IN KC MODE

\begin{tabular}{|c|c|c|c|c|c|c|c|c|c|c|c|c|}
\hline 106 & REG & $7 \cdot 16$ & LB-G & STAT & $T$ & $14 \cdot 8$ & $\cdot 78$ & $-\infty$ & 15.4 & .98 & -- & PULLOUT \\
\hline 99 & REG & 0.91 & $L B-G$ & STAT & $T$ & -- & -- & & 9.4 & -- & $-\infty$ & PULLOUT \\
\hline 87 & REG & 5.51 & LB-6 & DYN & $\mathrm{T}$ & $7 \cdot 8$ & $\cdot 15$ & 7.8 & 15.6 & 1.01 & 103 & PULLOUT \\
\hline 86 & REG & $7 \cdot 24$ & LB-G & DYN & $T$ & $7 \cdot 8$ & - 80 & $7 \cdot 8$ & $9 \cdot 7$ & .96 & $9 \cdot 7$ & PULLOUT \\
\hline 13 & REG & 6.08 & LEB-6 & FAT & $T$ & $2 \cdot 6$ & .02 & $1 \cdot 0$ & $4 \cdot 2$ & .03 & 2000 & EOLT FAILED \\
\hline 94 & REG & $6 \cdot 38$ & $L B-6$ & FAT & $T$ & $2 \cdot 6$ & .05 & 1.0 & $2 \cdot 6$ & $\cdot 15$ & 535 & BOLT FAILED \\
\hline 121 & MAG & 6.17 & L $3-6$ & STAT & $T$ & - & - & & $16 \cdot 6$ & -- & -- & DULLOUT \\
\hline 128 & MAG & 6.20 & LS-6 & STAT & $T$ & 3.7 & .01 & -- & 5.6 & -- & -- & PULLOUT \\
\hline 127 & MAG & 6.17 & LB-6 & DYN & $T$ & 7.0 & $1 \cdot 0$ & 7.8 & 13.0 & $1 \cdot 26$ & 107.8 & CONC, FAILED \\
\hline 130 & MAG & $6 \cdot 15$ & LB-6 & DYN & $T$ & 7.0 & .46 & 7.8 & 12.5 & .76 & 102 & CONC FAILED \\
\hline 132 & ss & 6.27 & LB-6 & STAT & $T$ & $2 \cdot 8$ & .02 & -- & 4.0 & -- & -- & PULLOUT \\
\hline 134 & SS & $6 \cdot 29$ & LB-6 & STAT & $T$ & $4 \cdot 8$ & $\cdot 38$ & -- & $7 \cdot 2$ & 1.05 & $\therefore-$ & PULLOUT \\
\hline 137 & SS & $6 \cdot 20$ & LB-S & DYN & $T$ & $3 \cdot 4$ & - 38 & 7.8 & $6 \cdot 4$ & .97 & 110 & PULLOUT \\
\hline 135 & $S S$ & 5.24 & LB-6 & DYN & $T$ & - & $-\infty$ & - & $2 \cdot 2$ & .83 & $6 \cdot 8$ & PULLOUT \\
\hline 110 & REG & 7.49 & $58-6$ & STAT & $\mathrm{T}$ & 5.0 & .10 & $-\infty$ & 17.3 & .50 & -- & PULLOUT \\
\hline 109 & REG & 7.44 & $S A-6$ & STAT & $T$ & 6.0 & $\cdot 20$ & -- & $22 \cdot 0$ & .50 & -- & DULLOUT \\
\hline 115 & REG & 6.86 & $S A-6$ & DYN & $T$ & 10.0 & $\cdot 25$ & $7 \cdot 8$ & $18 \cdot 2$ & .45 & 105 & PULLOUT \\
\hline 120 & REG & $7 \cdot 12$ & SA-6 & DYN & 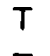 & $10 \cdot 0$ & $\cdot 18$ & 7.8 & $21 \cdot 8$ & .45 & 108 & CONC FAILED \\
\hline $\begin{array}{l}204 \\
73\end{array}$ & $\begin{array}{l}\text { REG } \\
\text { REG }\end{array}$ & - & $S A-6$ & $\begin{array}{l}F A T \\
F A T\end{array}$ & $T$ & $4 \cdot 0$ & .01 & -- & $4 \cdot 0$ & .03 & 386 & BOLT SHEARES \\
\hline 123 & MAG & 6.93 & SE-G & STAT & $T$ & $8 \cdot 0$ & .04 & -- & $14 \cdot 2$ & .15 & -- & CONC FAILED \\
\hline $1: 5$ & MAG & 7.05 & $S A-6$ & STAT & $T$ & 8.0 & .05 & -- & $25 \cdot 5$ & .42 & -- & CONC FAILED \\
\hline $1<9$ & MAG & 6.78 & $S B-6$ & DYN & $T$ & 13.6 & $\cdot 10$ & $7 \cdot 8$ & 15.7 & .42 & 100 & BOLT FAILED \\
\hline 122 & MAG & 7.07 & $5 A-6$ & DYN & $T$ & $13 \cdot 6$ & $\cdot 18$ & $7 \cdot 8$ & 13.6 & .26 & 94 & FOL T FAILED \\
\hline
\end{tabular}


TABLE 3. CONTINUED

TABLE OF DATA SORTED BY BOLT DIAMETER AND TEST TYPE IN THAT ORDER (CONT •) 3/4 INCH BOLTS TESTED IN TENSION CONT.

SPEC CONC FIC BOLT TESTT PI DI CYCLE PU DU CYCLE FAILURE NUMS TYPE KSI "TYPE TYPE KIP IN KC KIP IN KC MODE

$\begin{array}{lllllllllllll}139 & \text { SS } & 6.50 & \text { SA-6 } & \text { STAT } & T & 12.0 & .30 & -- & 15.6 & .60 & -- & \text { PULLOUT } \\ 138 & \text { SS } & 7.38 & \text { SB-6 } & \text { STAT } & T & 6.0 & .40 & -- & 7.6 & .60 & -- & \text { PULLOUT } \\ 131 & \text { SS } & 7.33 & \text { SA-6 } & \text { DYN } & T & 9.6 & .21 & 7.8 & 13.6 & .30 & 101 & \text { PULLOUT } \\ 140 & \text { SS } & 7.58 & \text { SA-6 } & \text { DYN } & T & 8.4 & .27 & 7.8 & 14.4 & .40 & 102 & \text { PULLOUT } \\ 133 & \text { SS } & 7.61 & \text { SB-6 } & \text { FAT } & T & 3.2 & .05 & -- & 3.2 & .70 & 1440 & \text { BOLT FAILED }\end{array}$


TABLE 3. CONTINUED

TAZLE OF UATA SORTEd BY bOLT DIAMETER AND TEST TYPE IN THAT ORDER (CONT.)

3/4 INCH BOLTS TESTED IN SHEAR

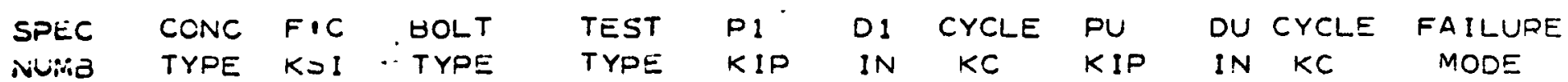

\begin{tabular}{|c|c|c|c|c|c|c|c|c|c|c|c|c|}
\hline 77 & REG & 6.78 & LB-6 & STAT & $\mathrm{v}$ & 21.5 & .30 & -- & $29 \cdot 2$ & .25 & -- & BOLT FAILED \\
\hline 76 & REG & 6.67 & LB-6 & STAT & v & $28 \cdot 0$ & .40 & -- & $31 \cdot 6$ & .65 & -- & BOLT FAILES \\
\hline 75 & REG & $7 \cdot 35$ & LB-6 & DYN & $v$ & 17.8 & .27 & $7 \cdot 8$ & 17.8 & .80 & $27 \cdot 5$ & BOLT FAILE= \\
\hline 70 & REG & $6 \cdot 54$ & LB-6 & DYN & $v$ & $17 \cdot 8$ & $1 \cdot 10$ & $7 \cdot 8$ & 17.8 & -- & $19 \cdot 8$ & BOLT FAILES \\
\hline 63 & MAG & 6.54 & LB-6 & STAT & $v$ & 23.0 & - 34 & -- & $32 \cdot 4$ & -- & $-\infty$ & BOLT FAILEO \\
\hline 65 & MAG & 6.57 & LB-6 & STAT & $v$ & $25 \cdot 0$ & .40 & -- & $28 \cdot 2$ & .90 & -- & BOLT FAILED \\
\hline 66 & MAG & 6.48 & LB-G & DYN & $v$ & 17.4 & .25 & $7 \cdot 8$ & $17 \cdot 4$ & .55 & $39 \cdot 6$ & $F A I L E D$ \\
\hline 64 & MAG & $6 \cdot 70$ & LB-6 & DYNV & $v$ & 17.4 & .27 & 7.8 & $17 \cdot 4$ & $1 \cdot 25$ & 26.9 & BOLT FAILE? \\
\hline 9 & ss & 6.40 & LB- 6 & STAT & v & 18.8 & $\cdot 17$ & -- & $22 \cdot 0$ & 1.20 & -- & D'ルLLCUT \\
\hline 63 & SS & 6.96 & LB-6 & STAT & $v$ & $26 \cdot 0$ & .40 & -- & 28.0 & .60 & -- & PULLOUT \\
\hline 70 & SS & 5.16 & LB-6 & DYN & v & 10.8 & $\cdot 22$ & 7.8 & 10.0 & -- & 34 & PIJLLOUT \\
\hline 71 & SS & 6.43 & LS-6 & DYN & v & $16 \cdot 8$ & 1.10 & 7.8 & 10.9 & -- & 7.8 & PULLOUT \\
\hline$c 2-1$ & REG & 5.87 & $S H-6$ & STAT & $v$ & $30 \cdot 1$ & $\cdot 23$ & -- & $32 \cdot 8$ & $\cdot 30$ & - & BOLT SHEAFE: \\
\hline$c 2-2$ & REG & 6.10 & $S A-6$ & STAT & $v$ & 30.3 & .15 & -- & 34.0 & $\cdot 20$ & - & SHEACE: \\
\hline$c 2-3$ & REG & 6.38 & $58-6$ & DYN & $v$ & $20 \cdot 1$ & .13 & $7 \cdot 8$ & 23.4 & .25 & 101 & SHEARE: \\
\hline$c 2-4$ & REG & 6.68 & $S A-6$ & DYN & $v$ & $20 \cdot 1$ & $\cdot 20$ & $7 \cdot 8$ & $20 \cdot 1$ & .25 & 18.7 & SHEAREI \\
\hline iAic -2 & MAG & 5.81 & $S A-6$ & STAT & $v$ & 38.0 & $\cdot 25$ & -- & 42.0 & .33 & $-\infty$ & CONC FAILED \\
\hline$N i c-1$ & MAG & 6.08 & $S H-S$ & STAT & $v$ & 34.0 & $\cdot 30$ & -- & 37.8 & .70 & -- & BOLT FAILED \\
\hline$M 2-4$ & MAG & 5.87 & $\mathrm{SH}-6$ & DYN & v & $24 \cdot 0$ & $\cdot 30$ & $7 \cdot 8$ & 24.0 & .90 & $8 \cdot 1$ & BOLT FAILED \\
\hline iviz -3 & MAG & 5.49 & $53-6$ & DYN & v & $24 \cdot 0$ & $\cdot 20$ & $7 \cdot 8$ & $24 \cdot 0$ & .25 & $8 \cdot 1$ & BOLT FAILEO \\
\hline $52-1$ & SS & 7.30 & $S A-6$ & STAT & $v$ & $24 \cdot 0$ & $\cdot 20$ & -- & $32 \cdot 0$ & .30 & -- & CONC FAILEO \\
\hline $\begin{array}{l}52-2 \\
52-3\end{array}$ & $\begin{array}{l}\text { SS } \\
\text { SS }\end{array}$ & $\begin{array}{l}6.84 \\
7.44\end{array}$ & $\begin{array}{l}S H-6 \\
S B-6\end{array}$ & $\begin{array}{l}\text { STAT } \\
\text { DYN }\end{array}$ & $\begin{array}{l}v \\
v\end{array}$ & $\begin{array}{l}20.0 \\
18.4\end{array}$ & $\begin{array}{l}.15 \\
.20\end{array}$ & $-\overline{7.8}$ & 26.0 & .22 & $-\infty, 0$ & CONC FAILED \\
\hline $\begin{array}{l}52-4 \\
52-5\end{array}$ & $\begin{array}{l}\text { SS } \\
\text { SS }\end{array}$ & $\begin{array}{l}7.10 \\
7.23\end{array}$ & $\begin{array}{l}S A-G \\
S A-6\end{array}$ & $\begin{array}{l}\text { DYN } \\
\text { FAT }\end{array}$ & $\begin{array}{l}v \\
v\end{array}$ & $\begin{array}{l}18.0 \\
5.8\end{array}$ & .10 & 7.8 & 26.3 & .19 & $\begin{array}{l}103 \\
1500\end{array}$ & $\begin{array}{l}\text { CONC FM ILE? } \\
\text { FAILEO STAT }\end{array}$ \\
\hline
\end{tabular}


TABLE 3. CONTINUED

TAELE OF DATA SORTED BY BOLT DIAMETER AND TEST TYPE IN.THAT ORDER (CONT.) 3/4 INCH BOLTS TESTED IN COMBINED TENSION AND SHEAR $\begin{array}{lllllllllll}\text { SHEC } & \text { CONC FIC } & \text { SOLT } & \text { TEST } & \text { PI } & \text { DI } & \text { CYCLE } & \text { PU } & \text { DU CYCLE FAILURE } \\ \text { NUMB } & \text { TYPE KSI } & \text { TYPE } & \text { TYPE } & \text { KIP } & \text { IN } & \text { KC } & \text { KIP } & \text { IN KC K } & \text { MODE }\end{array}$

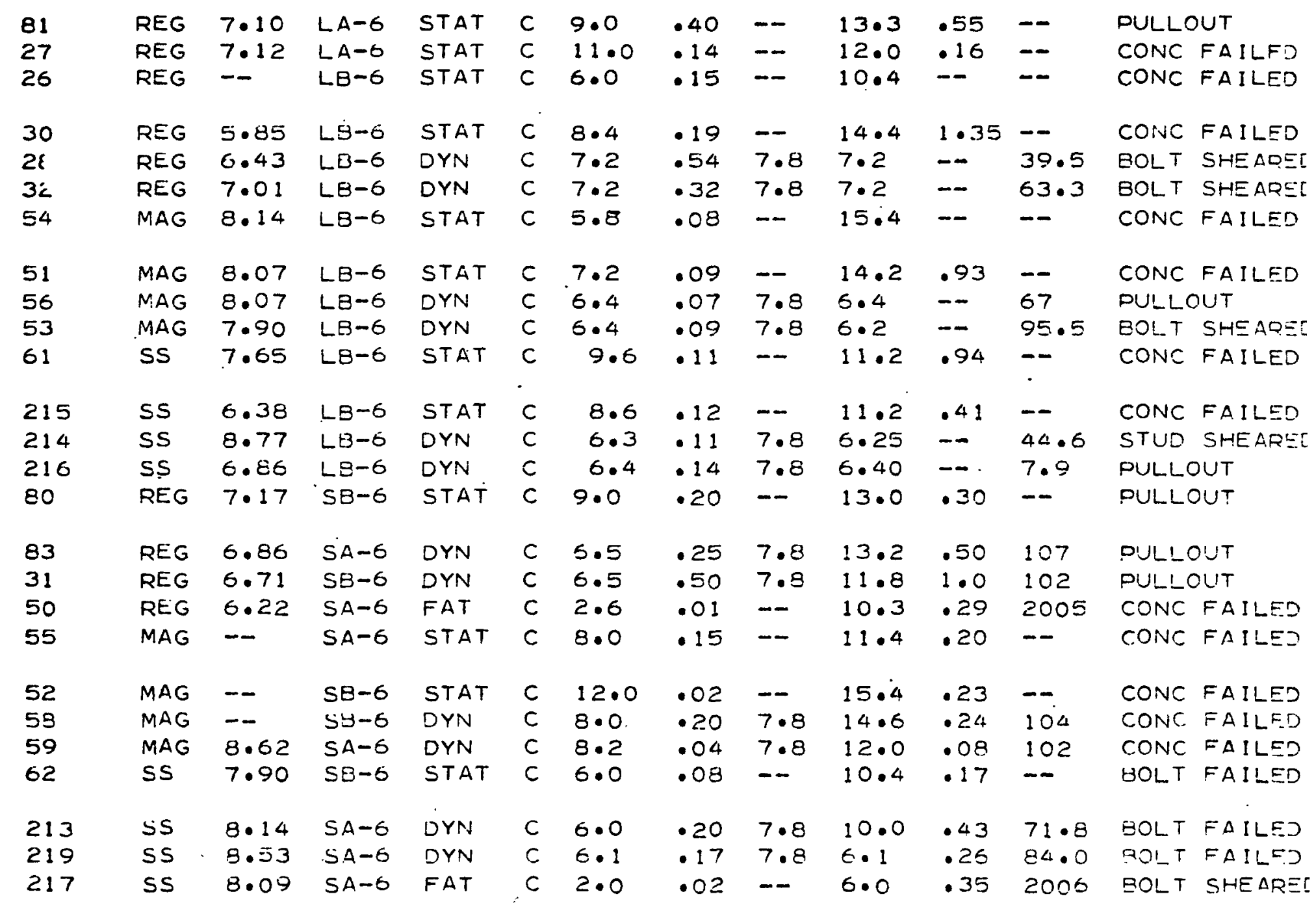


TABLE 3. CONTINUED

TAIOLE OF GATA SORTEO SY EOLT DIAMETER AND TEST TYPE IN THAT ORDER (CONT.)

\section{INCH SCLTS TESTED IN TENSION}

\begin{tabular}{|c|c|c|c|c|c|c|c|c|c|c|c|c|c|}
\hline \multirow{2}{*}{$\begin{array}{l}\text { SFEC } \\
\text { NUDiViE }\end{array}$} & \multirow{2}{*}{$\begin{array}{l}\text { CONC } \\
\text { TYPE }\end{array}$} & \multirow{2}{*}{$\begin{array}{l}F \cdot C \\
\text { KSI }\end{array}$} & \multirow{2}{*}{$\begin{array}{l}\text { BOLT } \\
\text { TYPE }\end{array}$} & \multicolumn{2}{|c|}{ TEST } & $P_{1}$ & $D 1$ & CYCLE & PU & DU & CYCLE & \multicolumn{2}{|c|}{ FAILURE } \\
\hline & & & & \multicolumn{2}{|c|}{ TYPE } & $K I P$ & IN & $K C$ & $K I P$ & IN & $K C$ & MODE & \\
\hline 80 & REG & 6.96 & $5 A-8$ & STAT & $T$ & 24.0 & .50 & -- & 26.4 & .50 & -- & CONC & FAILES \\
\hline 113 & REG & 6.13 & $S B-8$ & STAT & $T$ & 16.0 & 1.0 & -- & 22.5 & 1.20 & -- & CONC & FAILED \\
\hline 107 & REG & 7.00 & $5 B-8$ & DYN & $T$ & 15.8 & .65 & $7 \cdot 8$ & $20 \cdot 8$ & .85 & 101 & CONC & FAILEO \\
\hline 91 & REG & 5.69 & $5 A-8$ & DYN & $T$ & 16.0 & .10 & 7.8 & 26.0 & .33 & 103 & CONC & FAILEO \\
\hline
\end{tabular}

1 INCH BOLTS TESTED IN SHEAR

SPEC CONC FIC BOLT TEST PI DI CYCLE PU DU CYCLE FAILURE NUMO TYPE KSI TYPE TYPE KIP IN KC KIP IN KC MODE

$\begin{array}{lllllllllllll}C 2-5 & \text { REG } & 6.65 & \text { SA-8 } & \text { STAT } & V & 28.0 & .1 & & 34.5 & .50 & -1 & \text { CONC FAILEO } \\ C 2-6 & \text { REG } & 7.14 & \text { SA-8 } & \text { STAT } & V & 23.0 & .1 & & 35.4 & .50 & -1 & \\ C Z-7 & \text { REG } & 6.36 & \text { SA-8 } & \text { DYN } & V & 21.3 & .25 & 7.8 & 38.0 & .50 & 76 & \text { BOLT FAILED } \\ C 2-8 & \text { REG } & 6.10 & \text { SA-8 } & \text { DYN } & V & 21.8 & .10 & 7.8 & 31.0 & .25 & 106 & \text { CONC FAILED }\end{array}$

I INCH SOLTS TESTED IN COMBINED TENSION AND SHEAR

\begin{tabular}{|c|c|c|c|c|c|c|c|c|c|c|c|c|c|}
\hline \multirow{2}{*}{$\begin{array}{l}\text { SPEC } \\
\text { Nuivia }\end{array}$} & \multirow{2}{*}{$\begin{array}{l}\text { CONC } \\
\text { TYPE }\end{array}$} & \multirow{2}{*}{$\begin{array}{l}F: C \\
\text { KSI }\end{array}$} & \multirow{2}{*}{$\begin{array}{l}\text { BOLT } \\
\text { TYPE }\end{array}$} & \multicolumn{2}{|c|}{$\begin{array}{l}\text { TEST } \\
\text { TYOF }\end{array}$} & \multirow{2}{*}{$\begin{array}{l}P 1 \\
K I P\end{array}$} & \multirow{2}{*}{$\begin{array}{l}\text { DI } \\
\text { IN }\end{array}$} & \multirow{2}{*}{$\begin{array}{c}\text { CYCLE } \\
K C\end{array}$} & \multirow{2}{*}{$\begin{array}{l}P U \\
K I P\end{array}$} & \multirow{2}{*}{$\begin{array}{l}\text { DU } \\
\text { IN }\end{array}$} & \multirow{2}{*}{$\begin{array}{c}\text { CYCLE } \\
K C\end{array}$} & \multicolumn{2}{|c|}{ FAILURE } \\
\hline & & & & TYF & & & & & & & & \multicolumn{2}{|c|}{ MODE } \\
\hline 17 & REG & 5.02 & $S B-3$ & STAT & C & 10.0 & $\cdot 20$ & -- & $13 \cdot 0$ & .25 & -- & CONC & FAILED \\
\hline 23 & REG & 7.14 & $S A-8$ & STAT & c & 10.0 & .20 & -- & 12.6 & .25 & -- & CONC & FAILED \\
\hline 29 & REG & 6.52 & $S A-8$ & DYN & c & 8.0 & - 14 & 7.8 & $8 \cdot 0$ & .34 & 74 & CONC & FAILED \\
\hline 20 & REG & 6.77 & $S A-8$ & DYN & C & $8 \cdot 0$ & .36 & 7.8 & 11.6 & .57 & 102 & CONC & FAILSO \\
\hline
\end{tabular}


TABLE IV. COMPARATIVE BAR CHART FOR EXPANSION ANCHORS.

\section{EXPLANATION}

- Each bar on the chart represents one test, with the length of the bar representing the ultimate load. In the case of the shear test where two bolts are used the plotted value is taken as one half the ultimate total load. Lines without a KC (kilo cycle) notation above them are static, all others are dynamic. The KC notation refers to the number of cycles (in thousands) that the specimen sustained.

At the top of the page directly above the bar is listed the type (tension, shear, or combined) test, and the diameter of the bolt used in that test. Note at the bottom of the page the type of bolt (sleeve, shell or stud) and type of concrete (regular, magnite or steel shot) used in the. test.

In most cases the short bars ( 4 kips or less) represent long term fatigue tests, where the specimen was loaded with a low load and failed due to eccentricity in the load and or premature metal fatigue due to an imperfection in the bolt.

The small " $w$ " at the bottom of some bars represents a Wej-it standard bolt, which is slightly differently from the other manufactures stud anchors. Specimen \#'s 73 and 121 are not included in this chart due to Insufficient data; a11 other specimens are included. 


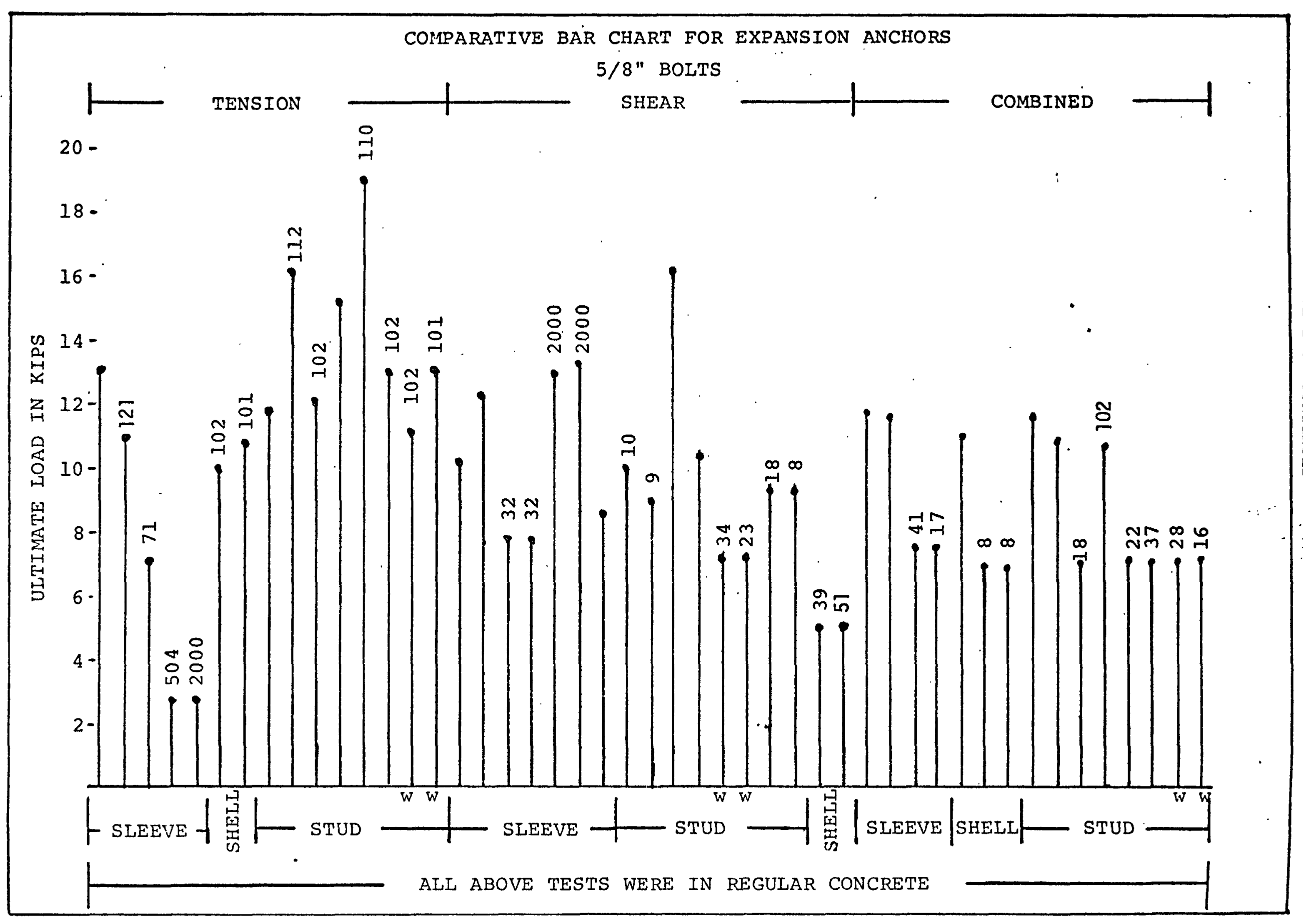




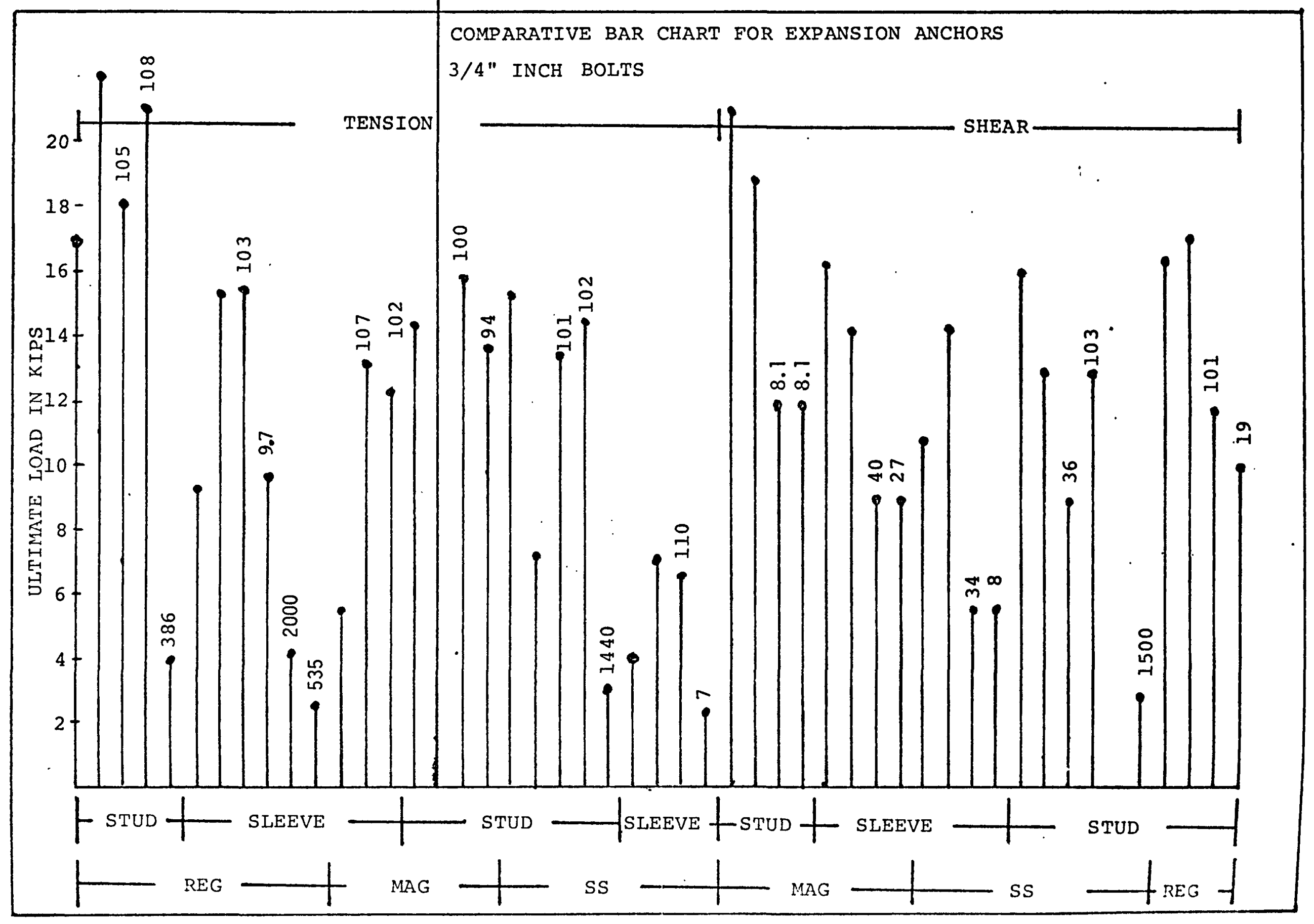




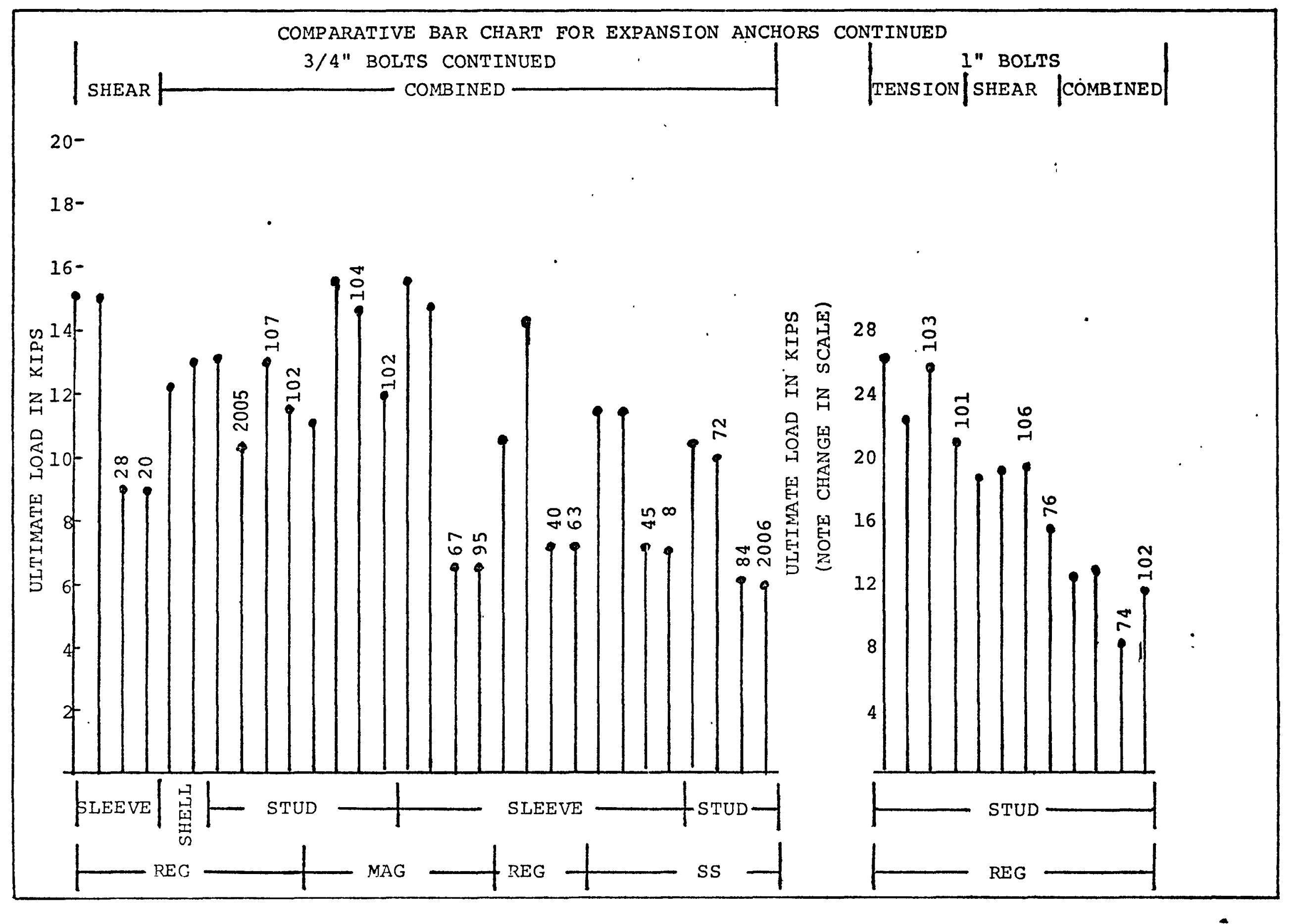




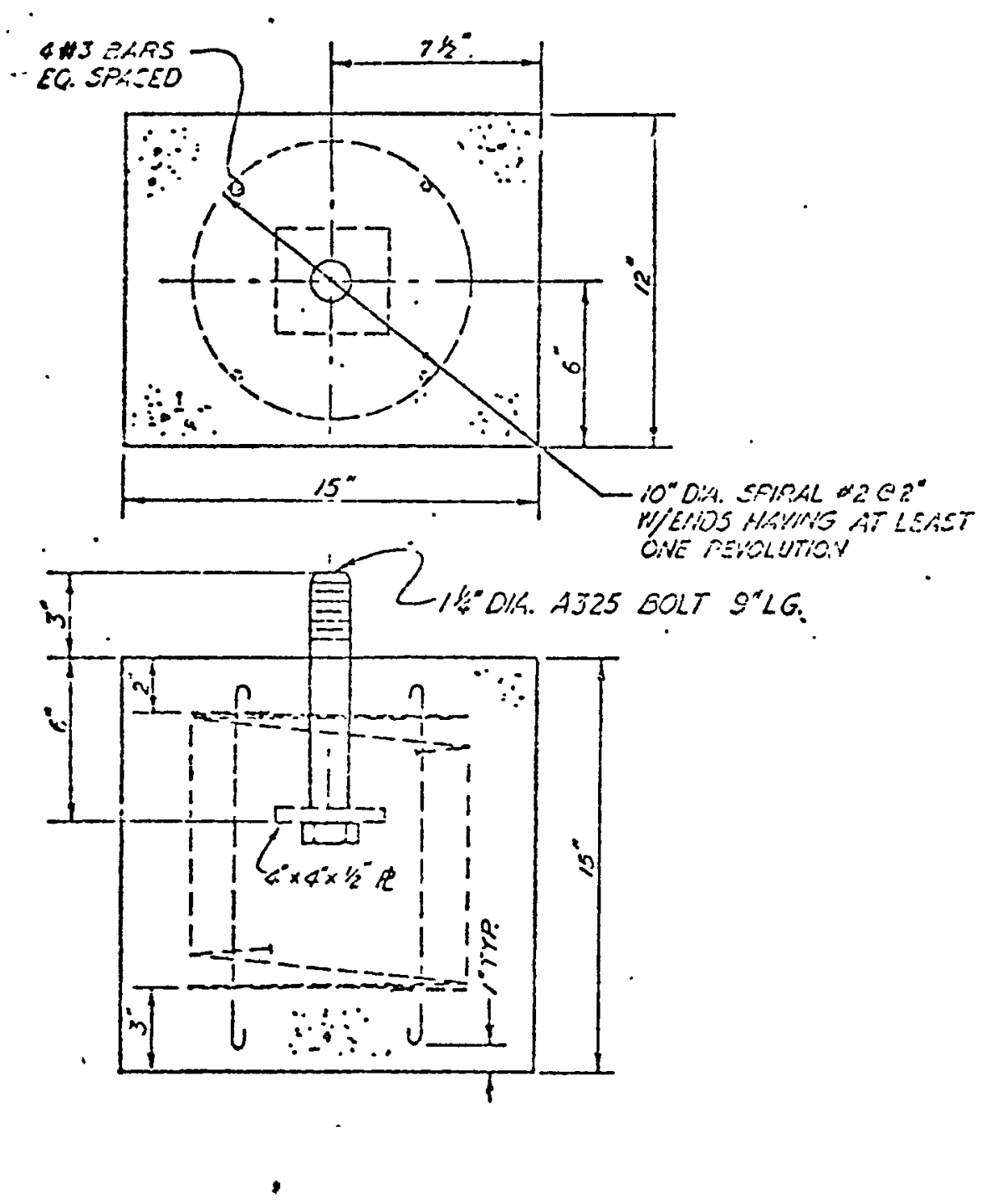

Fig. 1. TENSION TEST SPECIMEN 


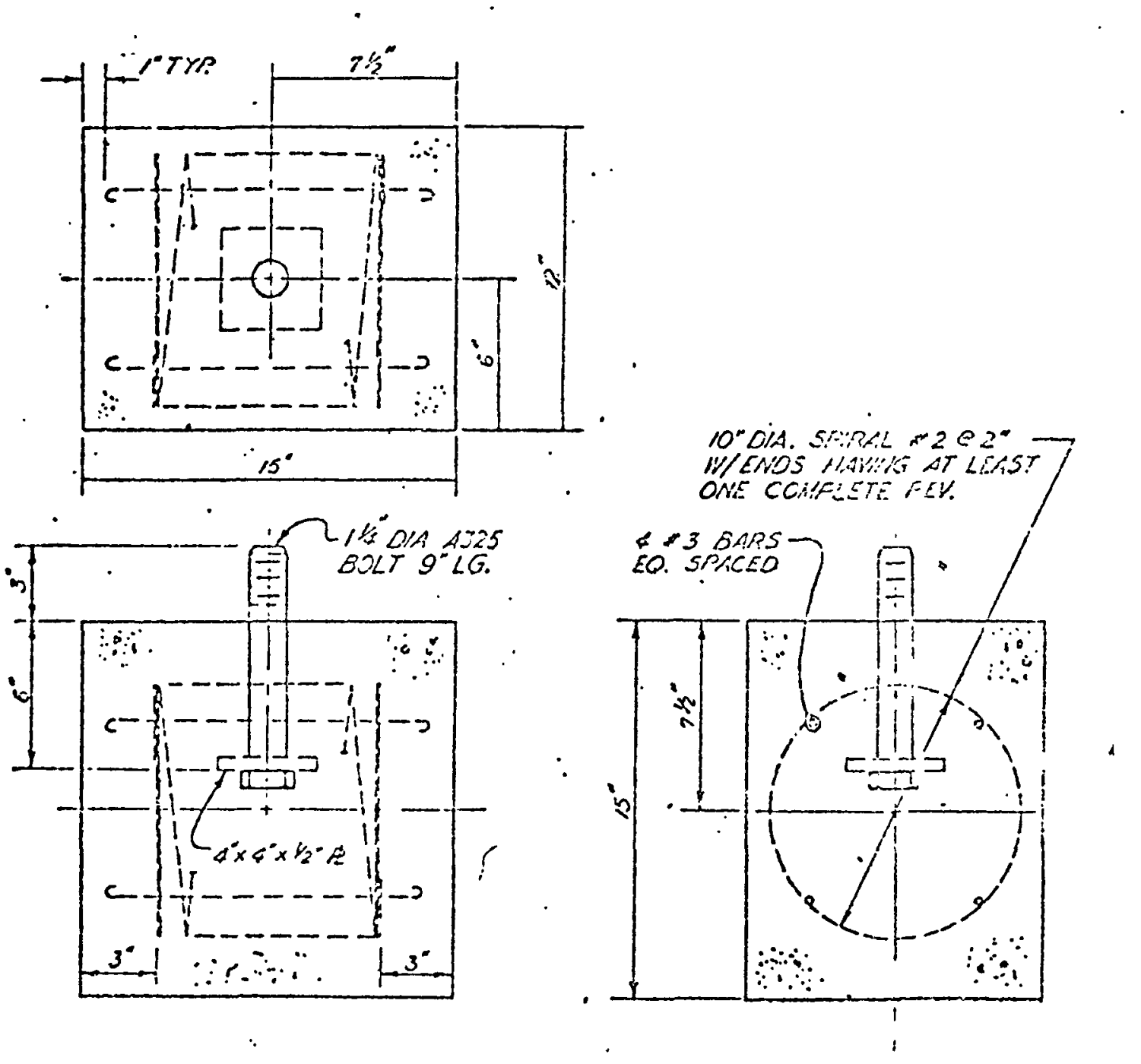

Fig. 2. SHEAR TEST SPECIMEN 


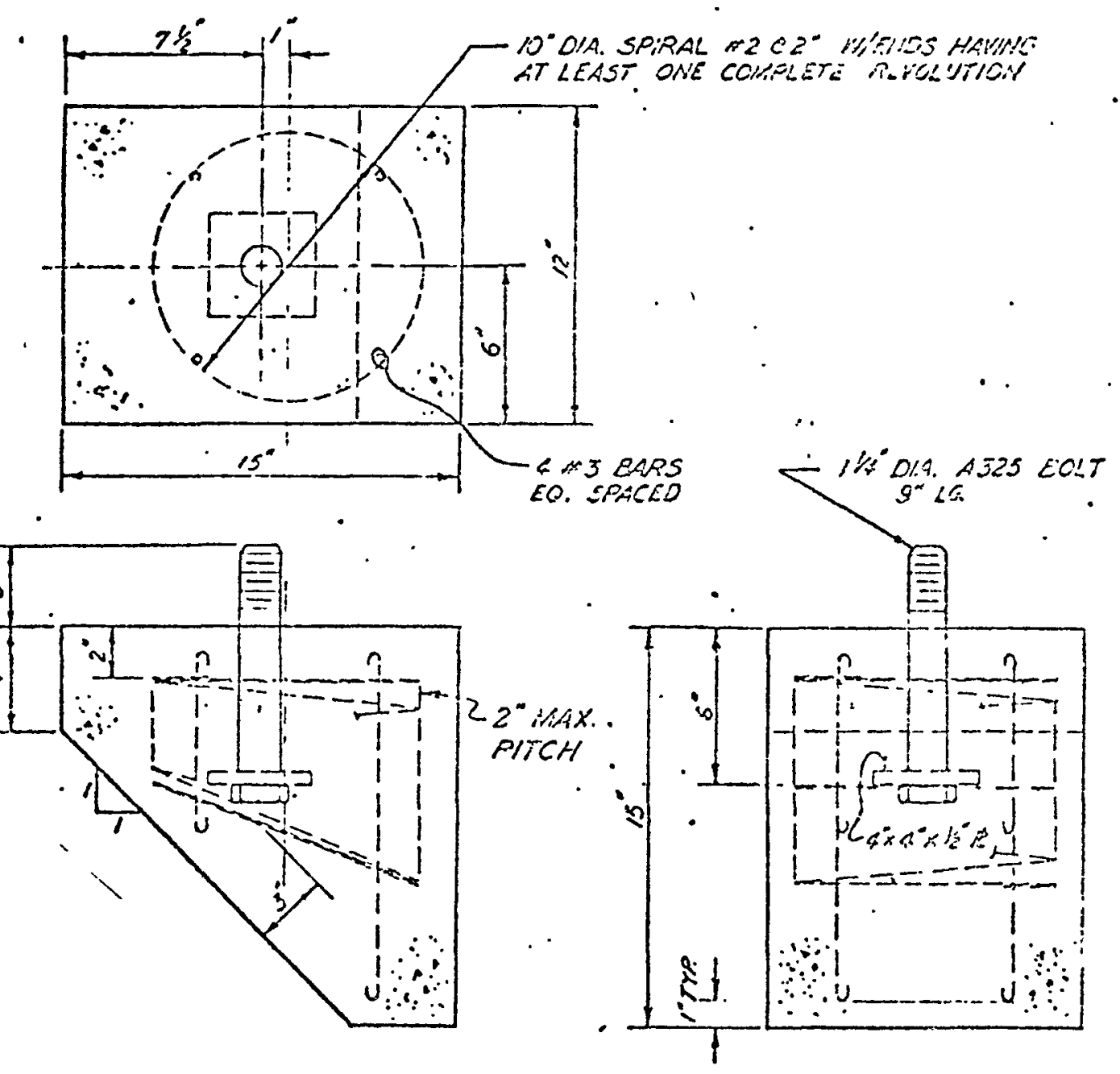

Fig. 3. COMBINED TEST SPECIMEN 

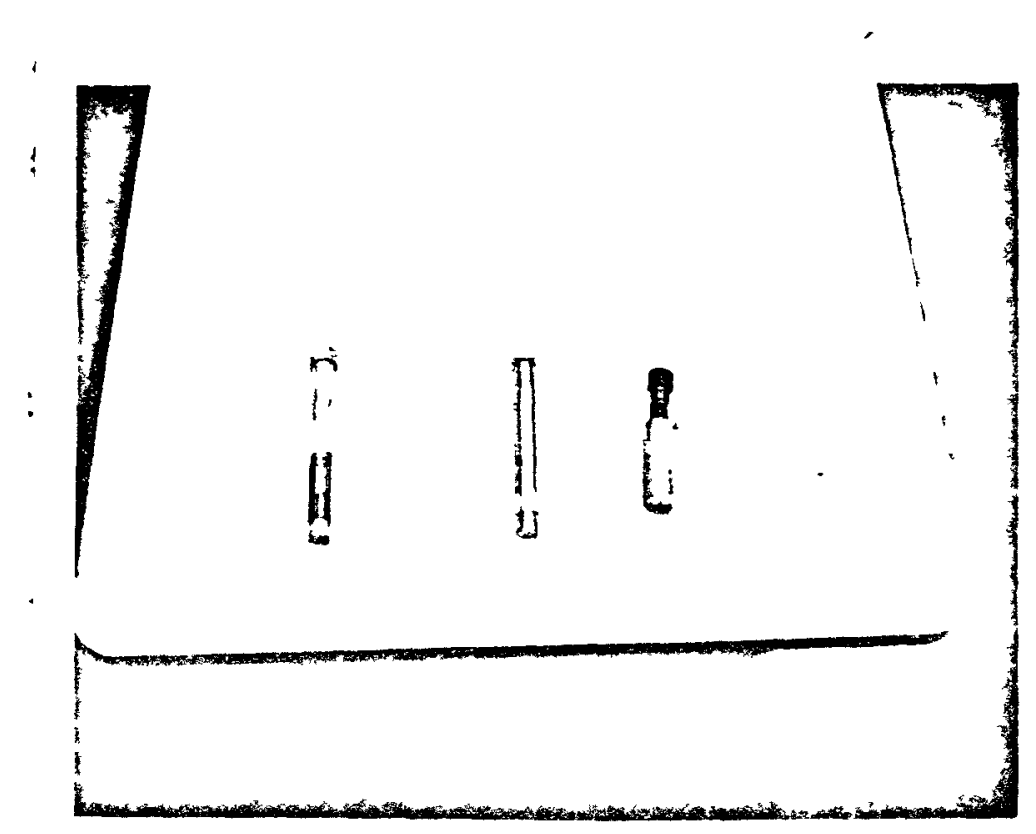

Fig. 4. TYPICAL SLEEVE, STUD AND SHELL TYPE ANCHOR 


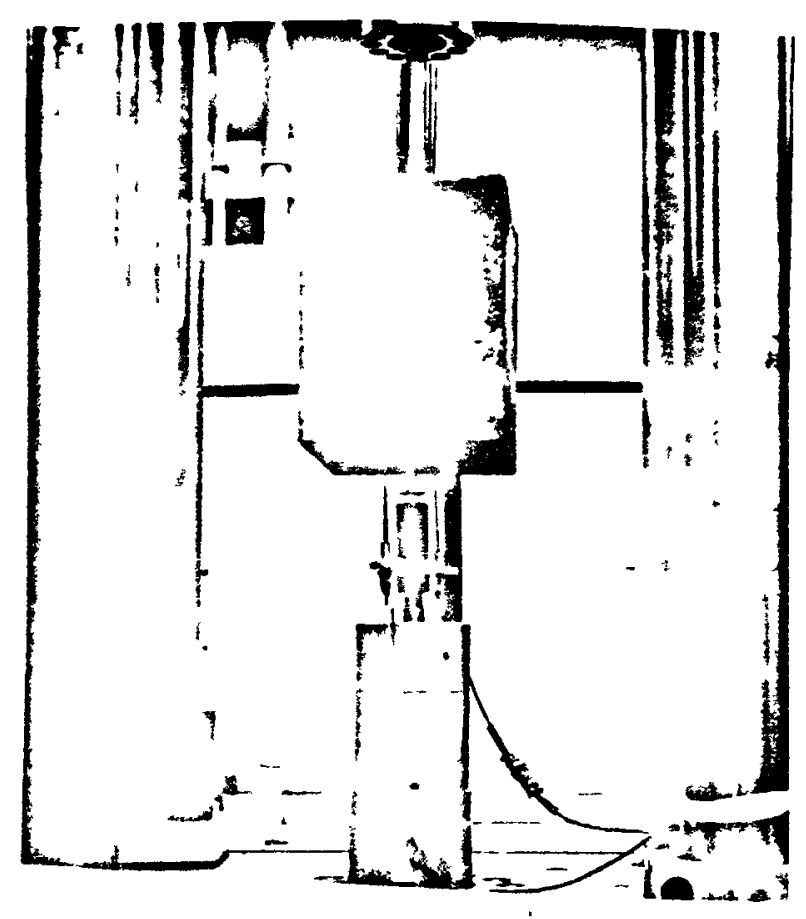

TENSION TEST SET-UP

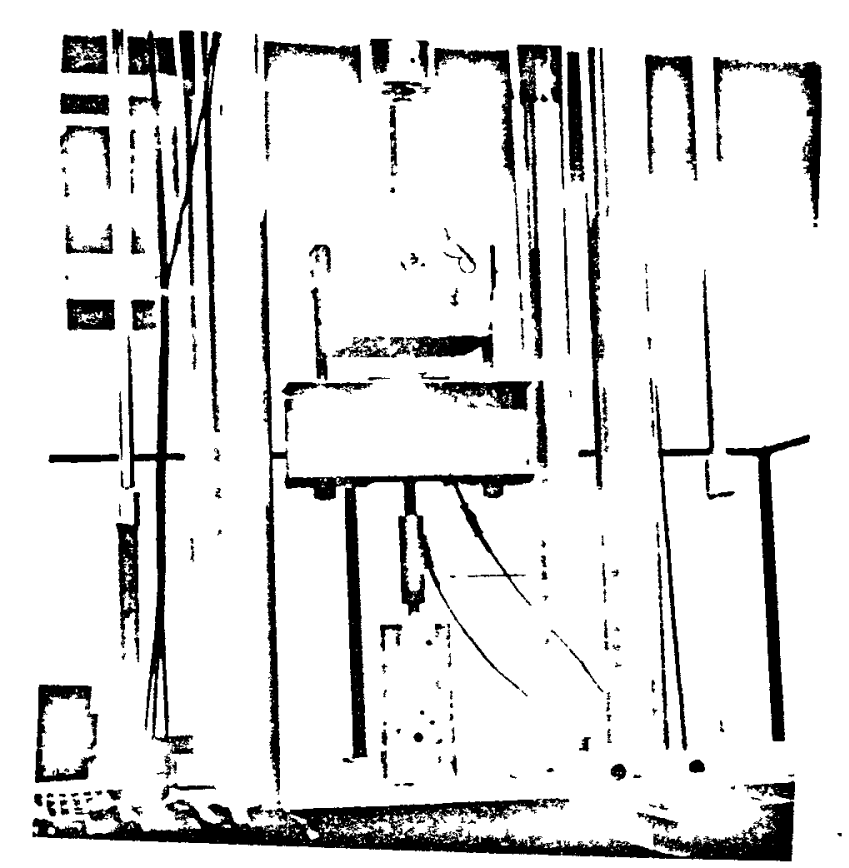

SHEAR TEST SET-UP

Fig. 5. TYPICAL TEST ARRANGEMENT 


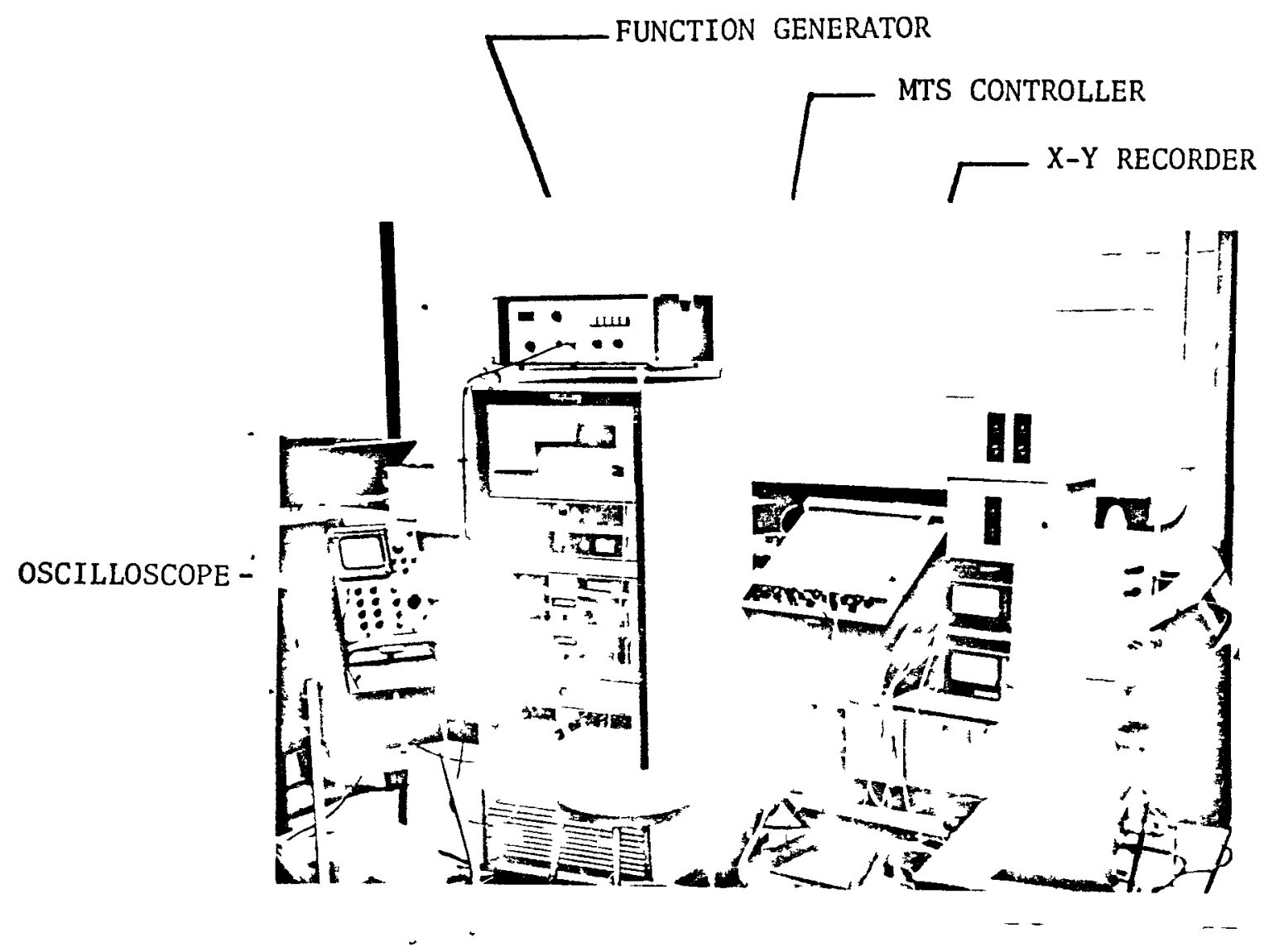

Fig. 6. ARRANGEMENT OF TEST EQUIPMENT 

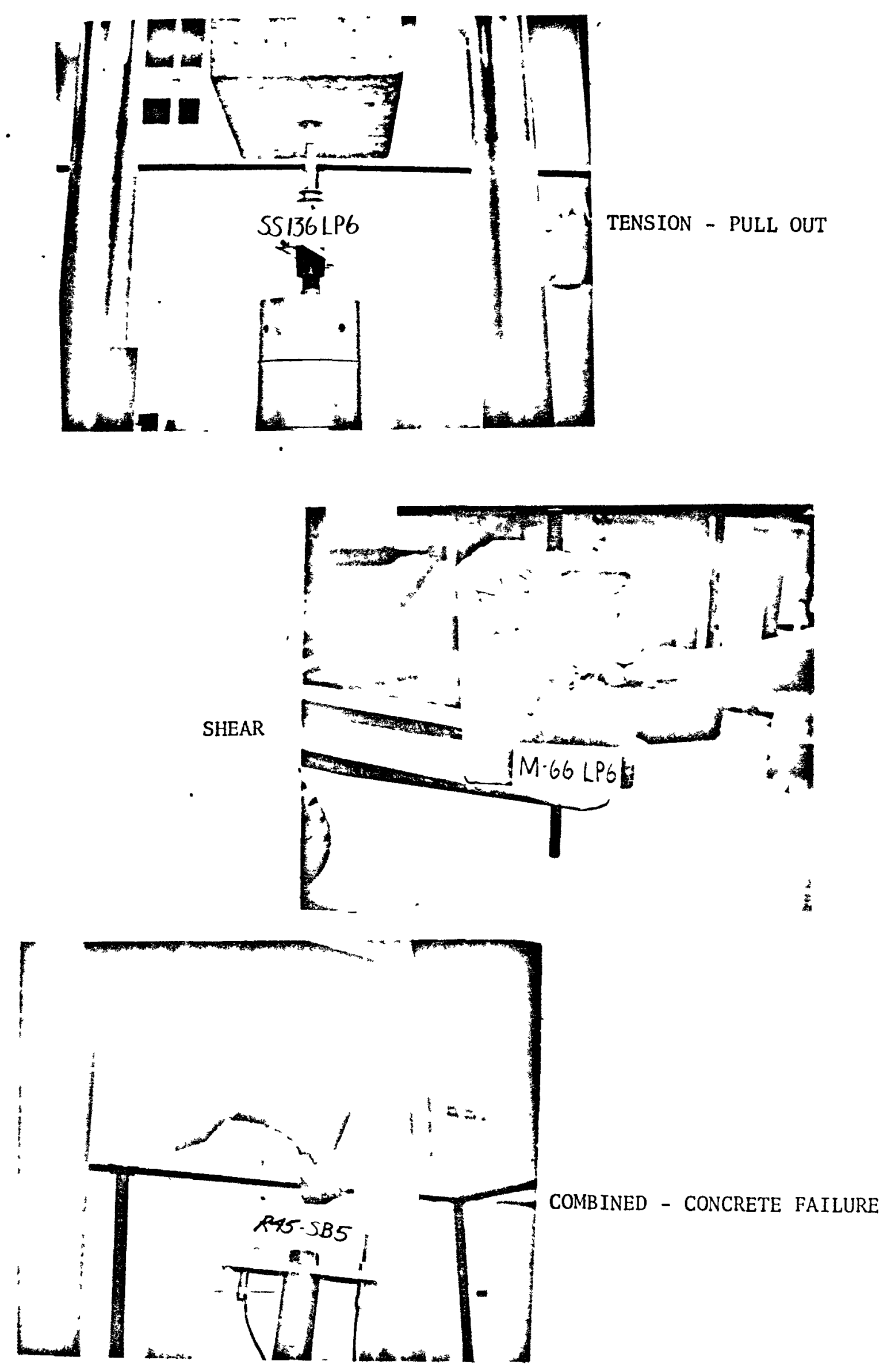

fig. 7. TYPICAL FAILURE MODES FOR EACH TYPE OF SPECIMEN 
APPENDIX 1 


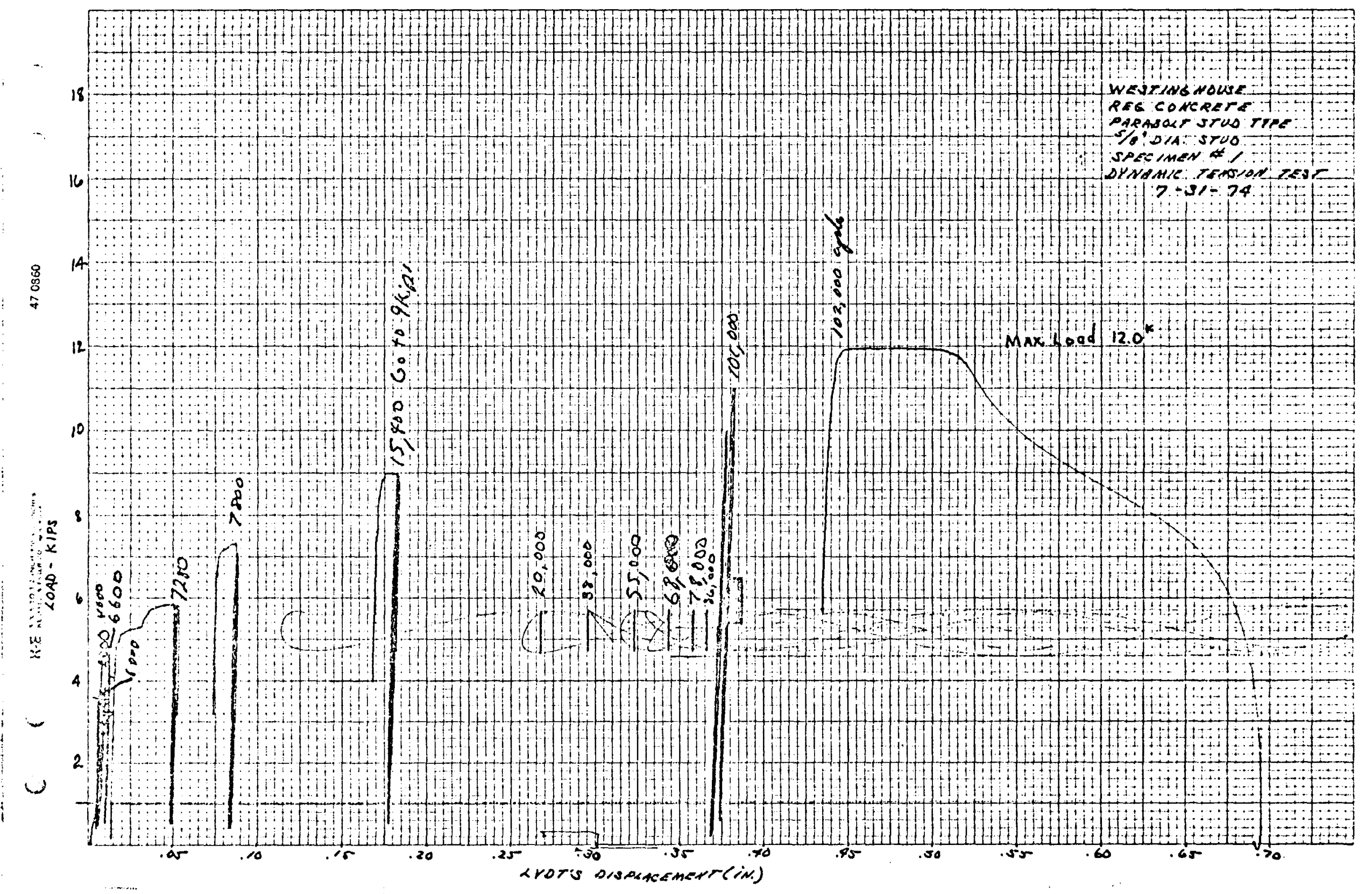




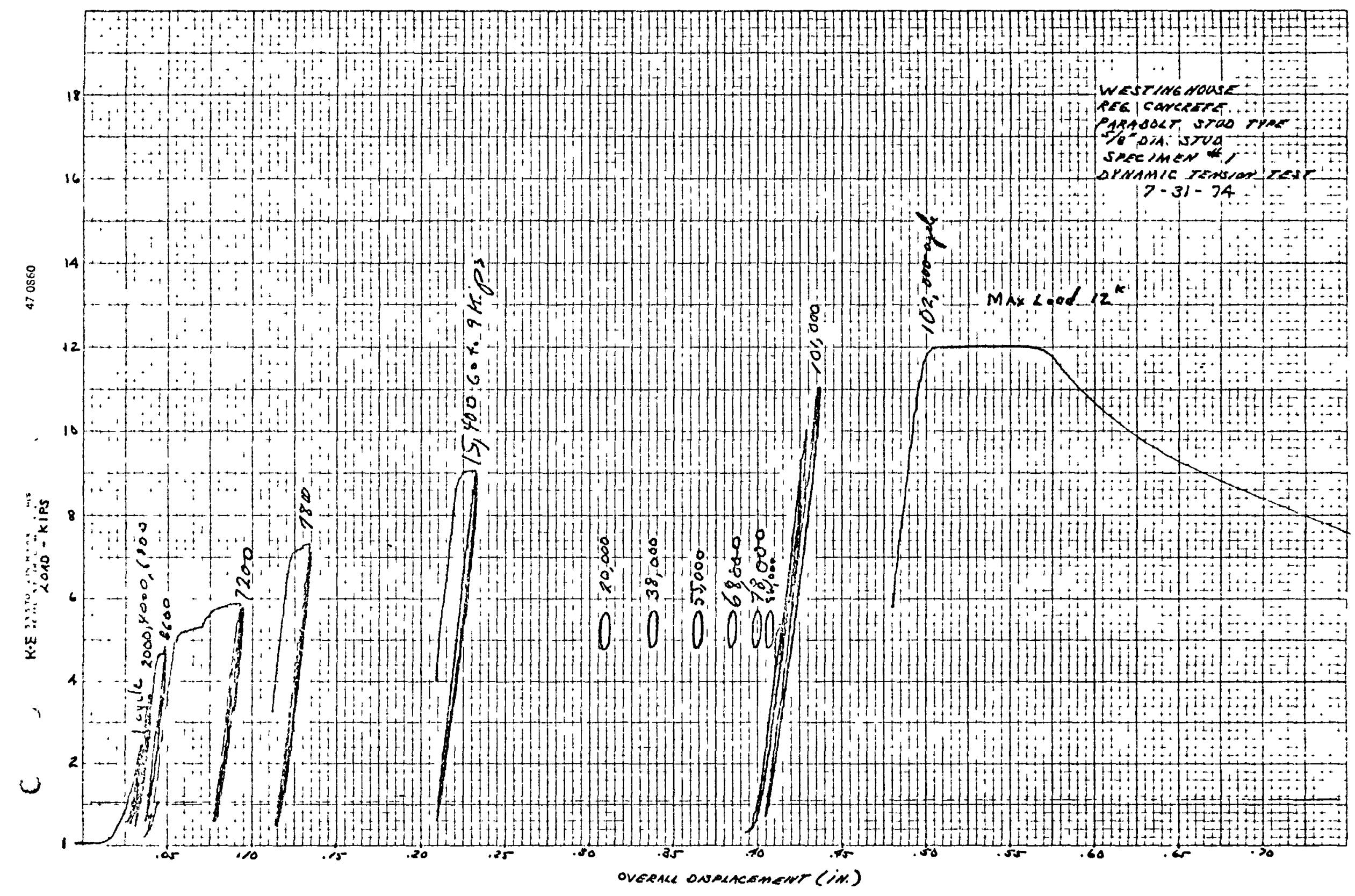




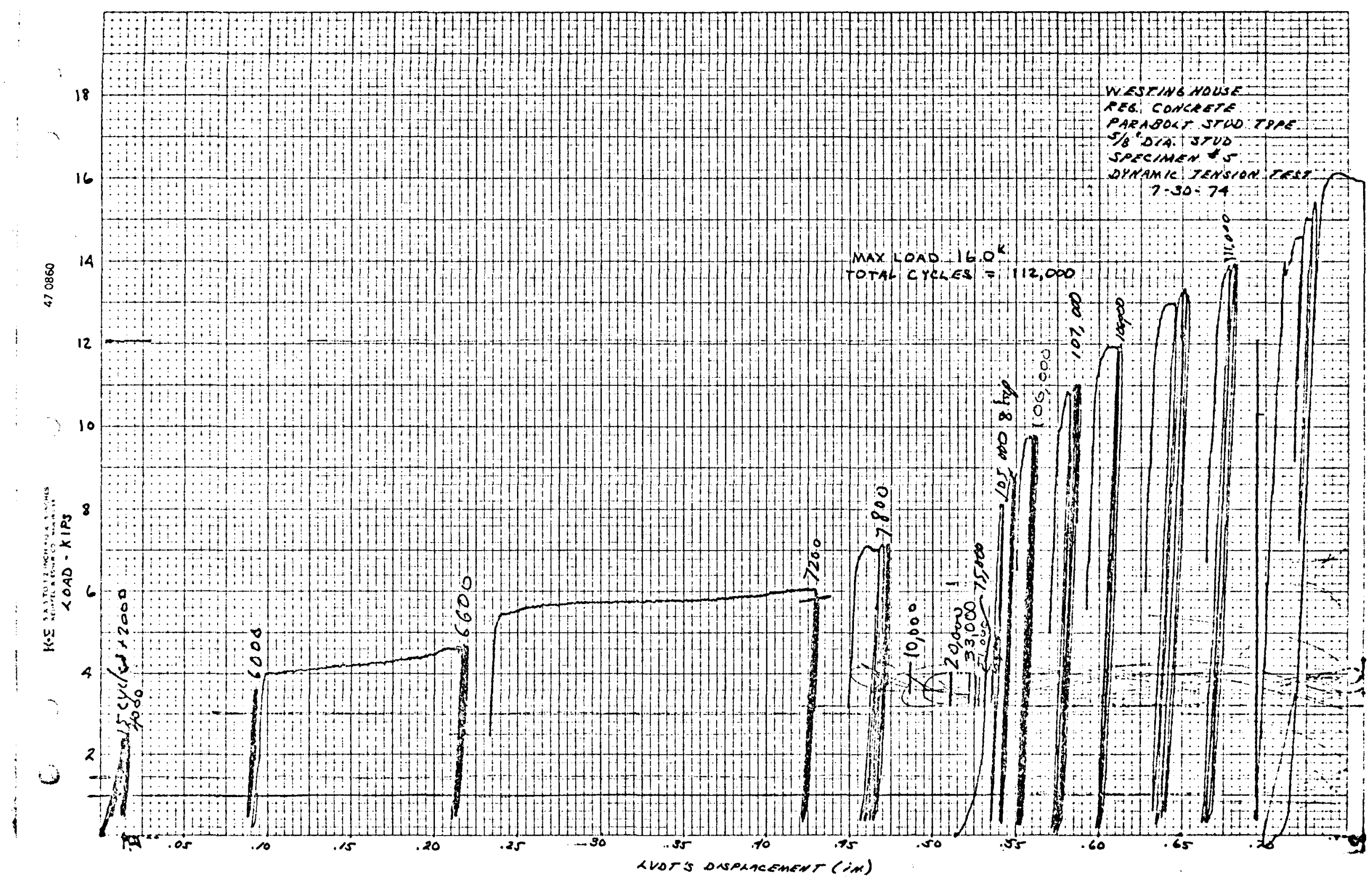




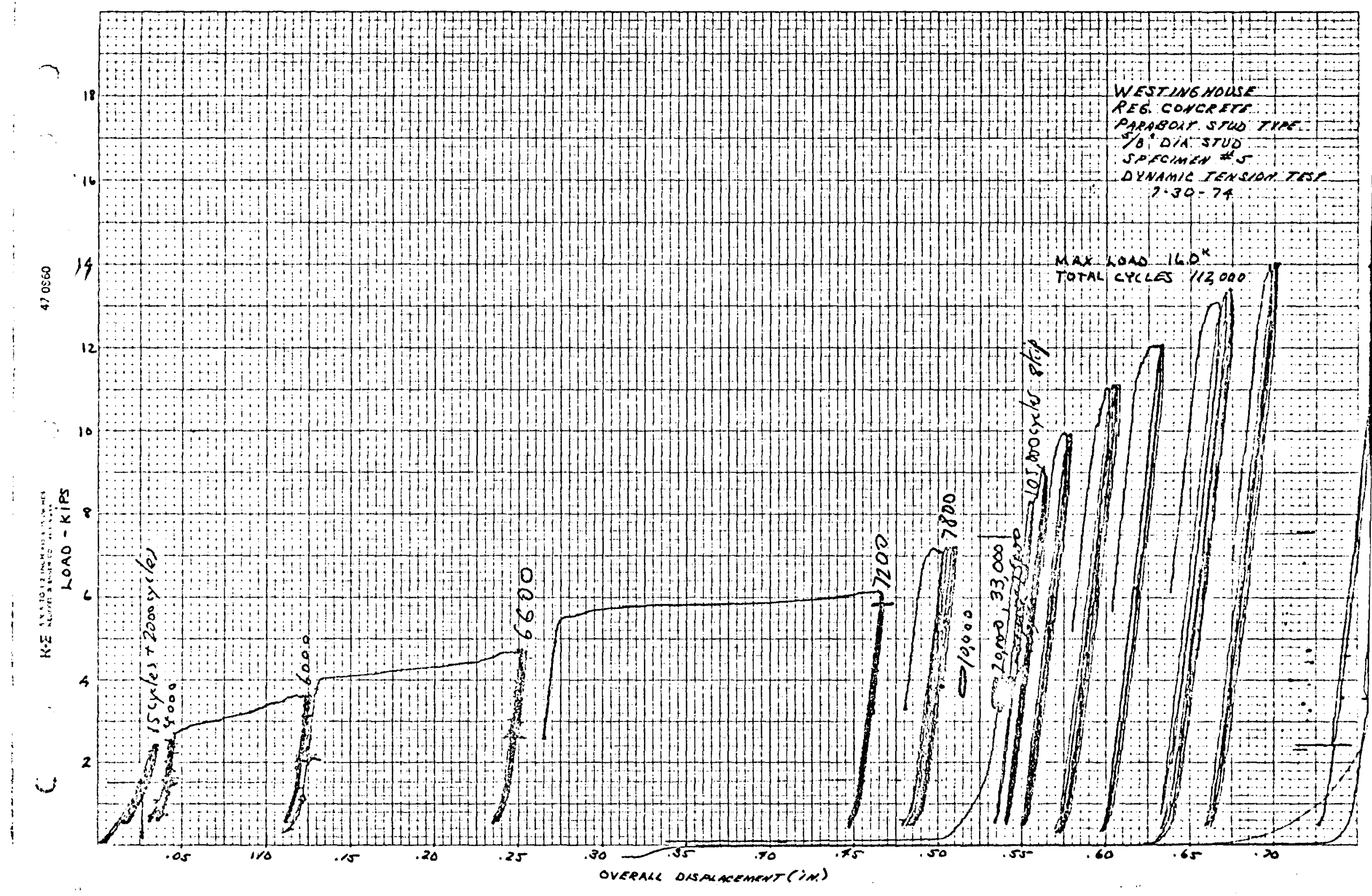




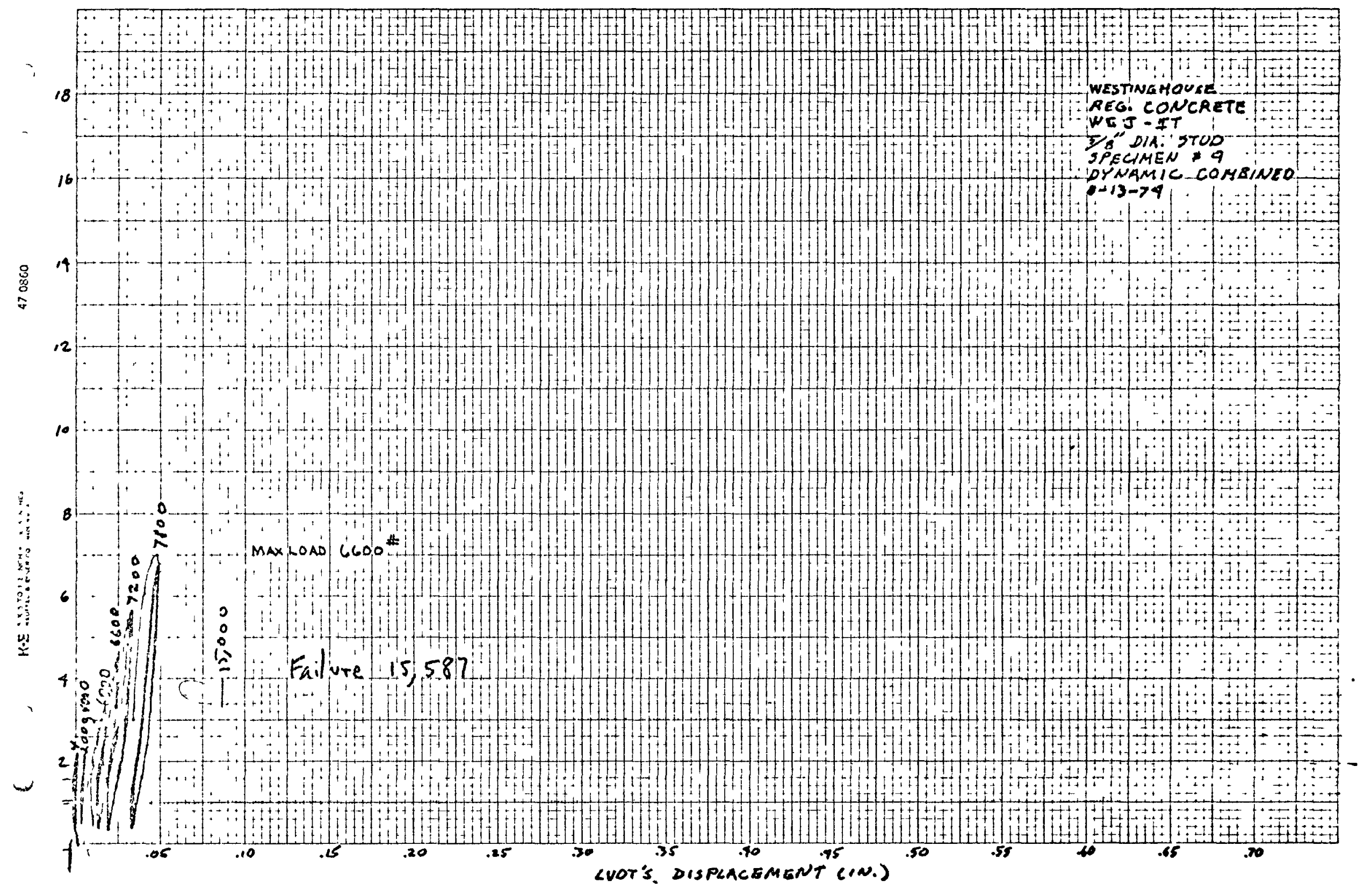




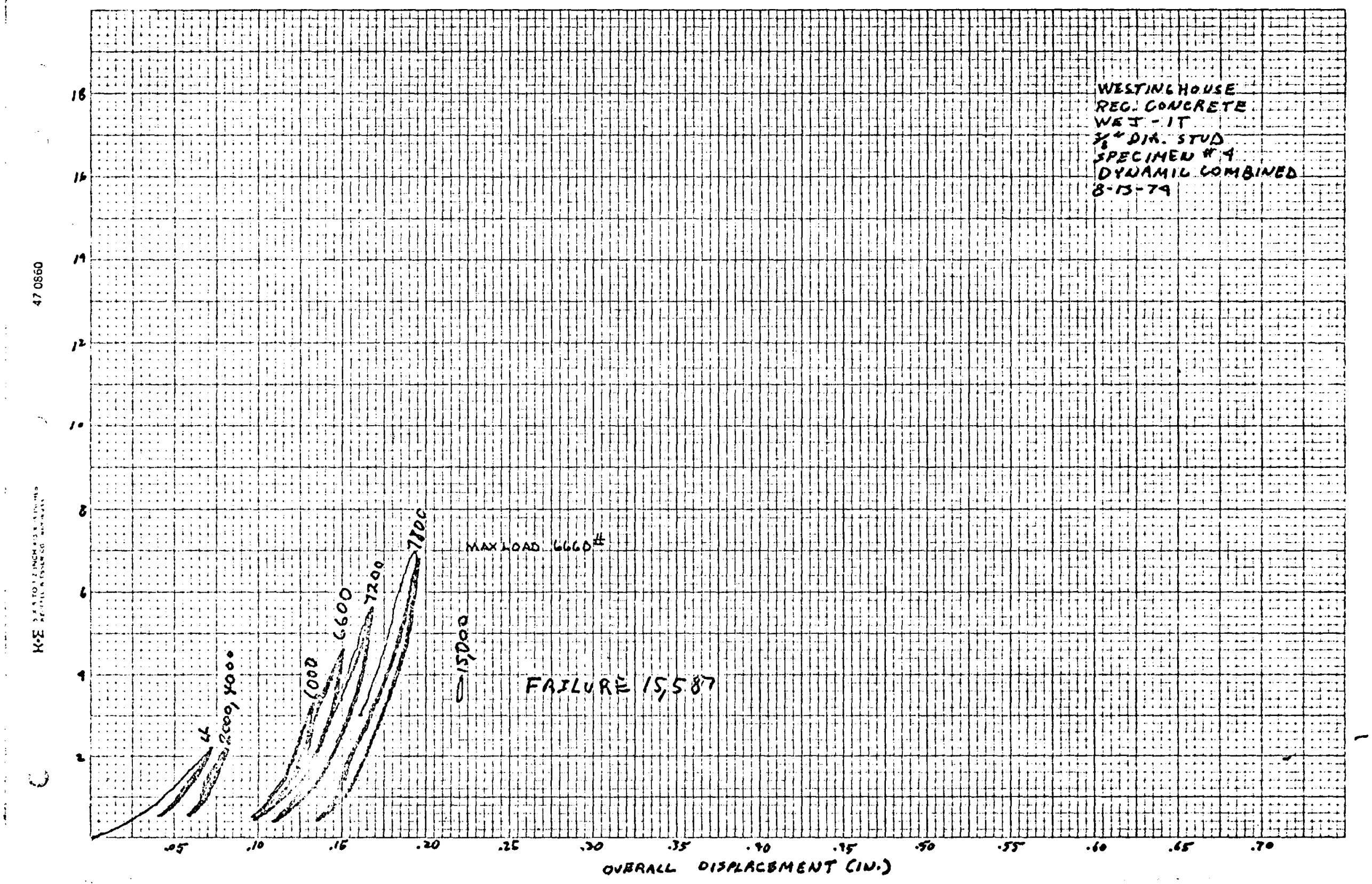




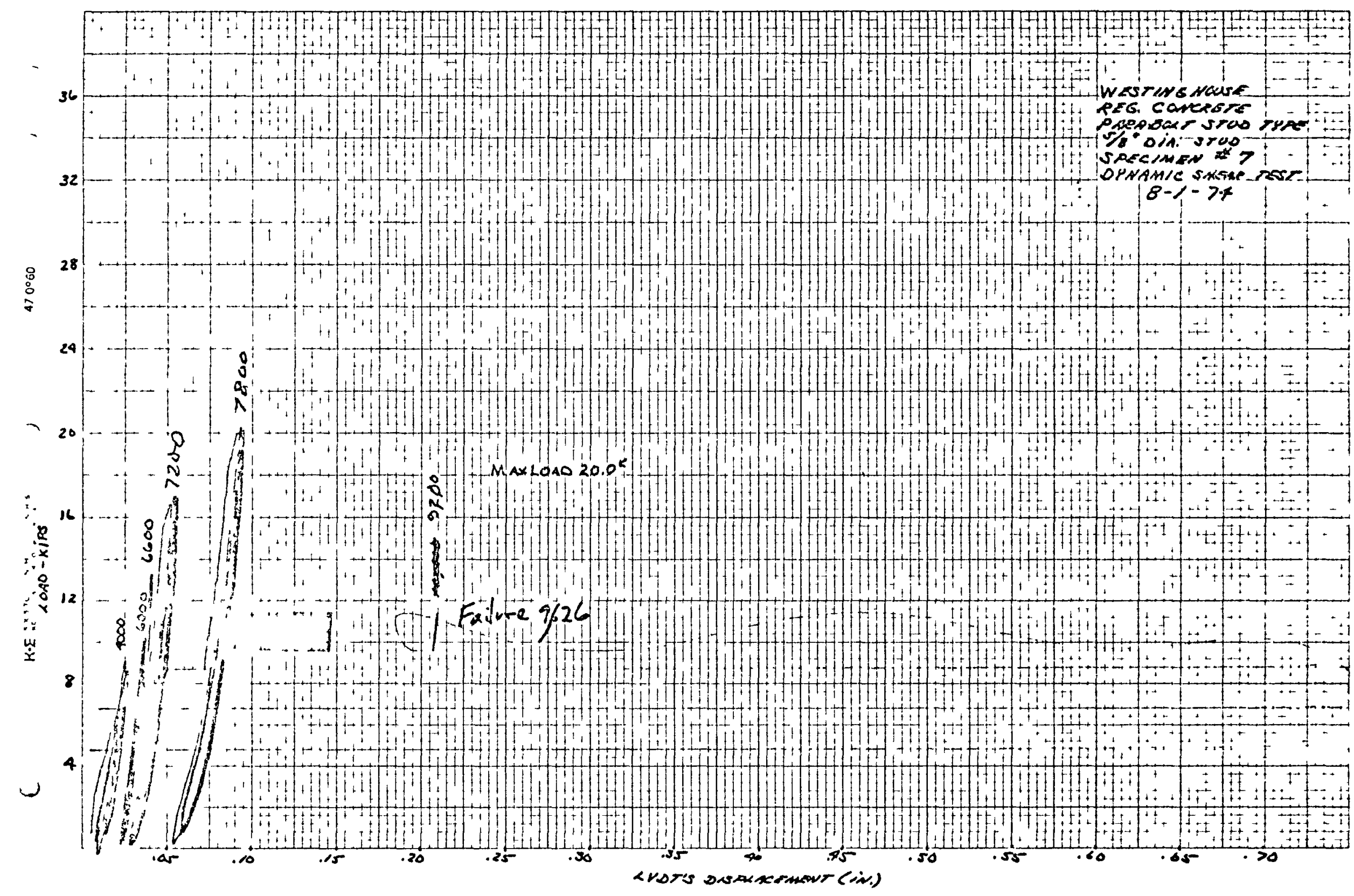




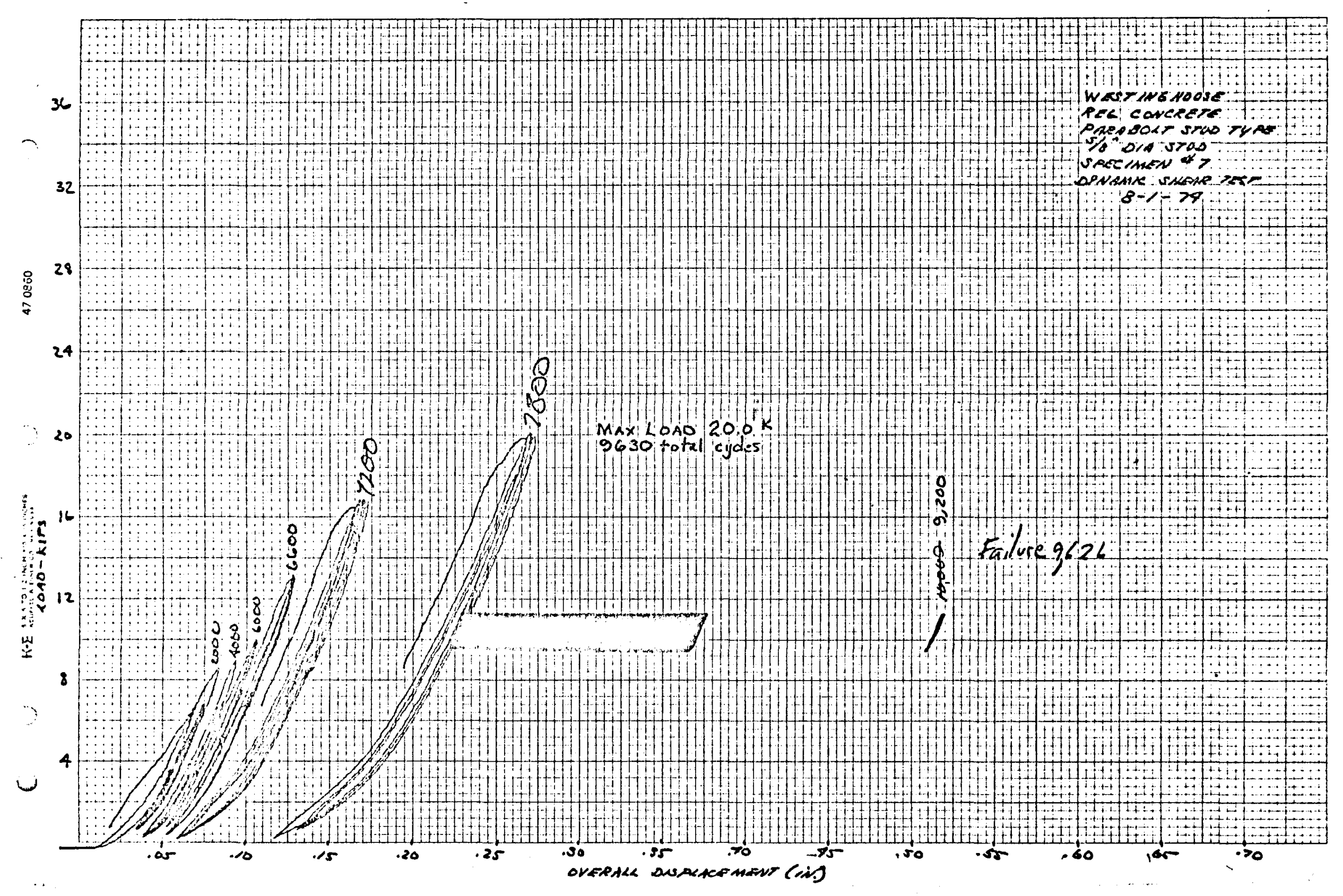




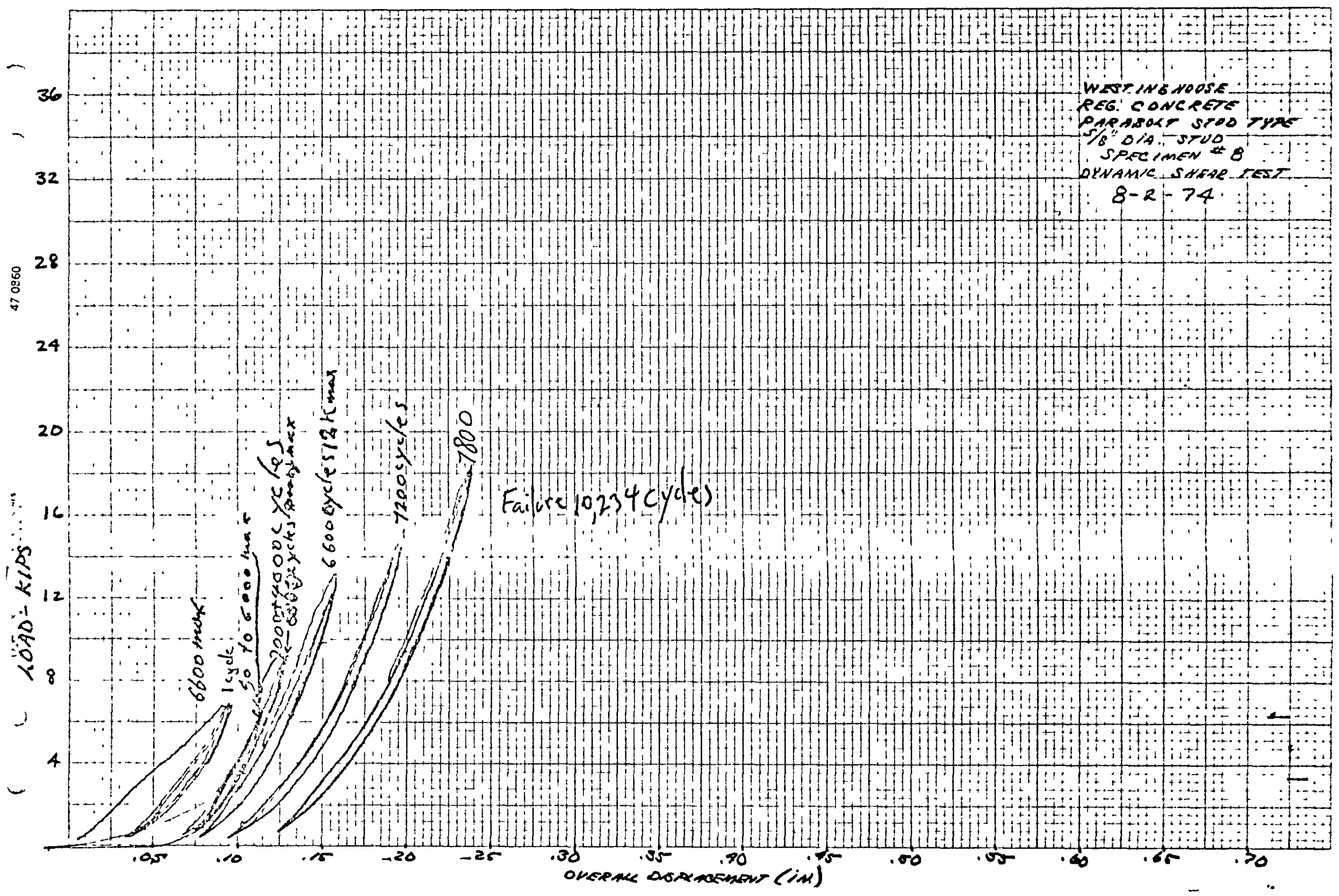




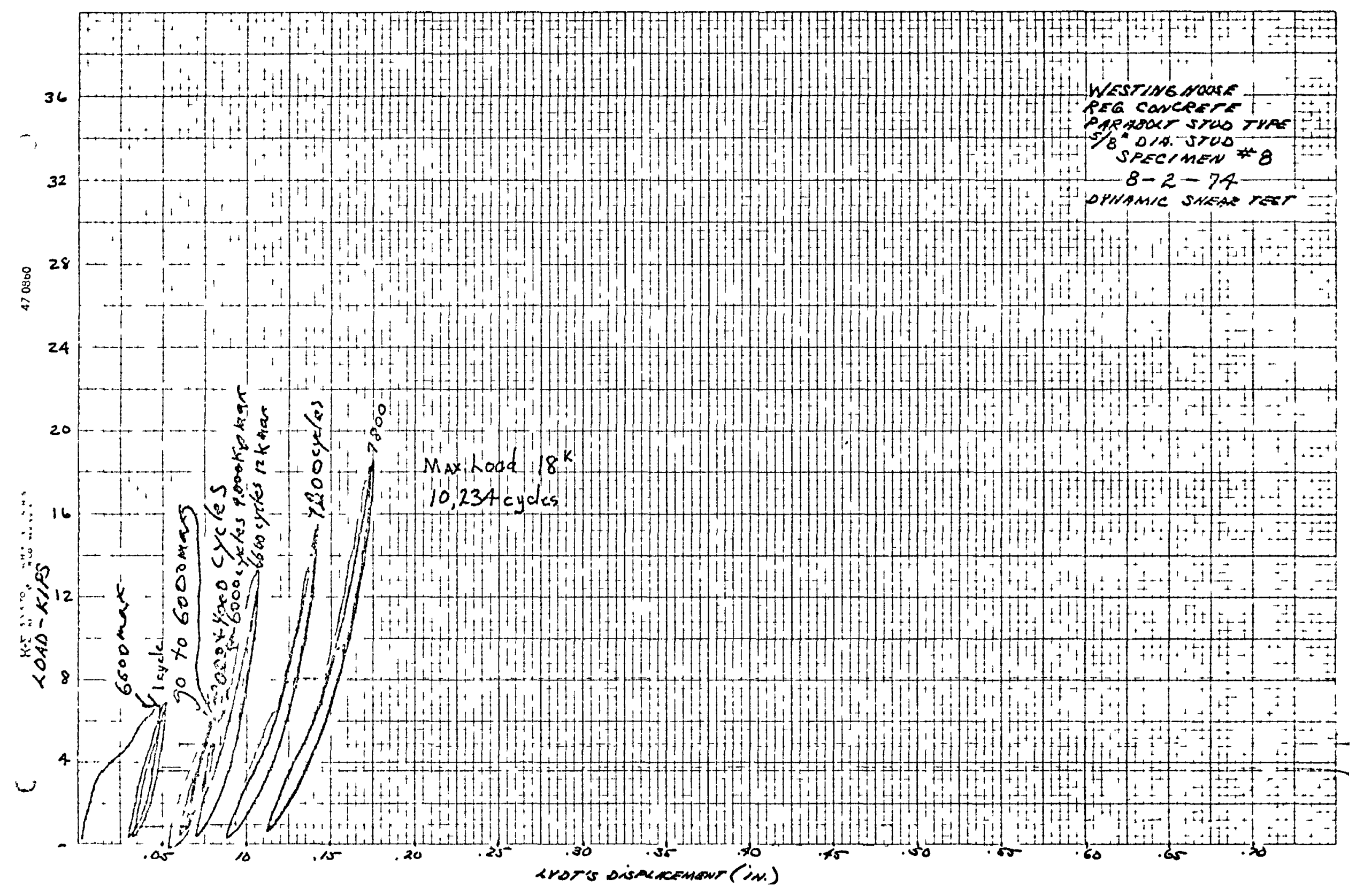




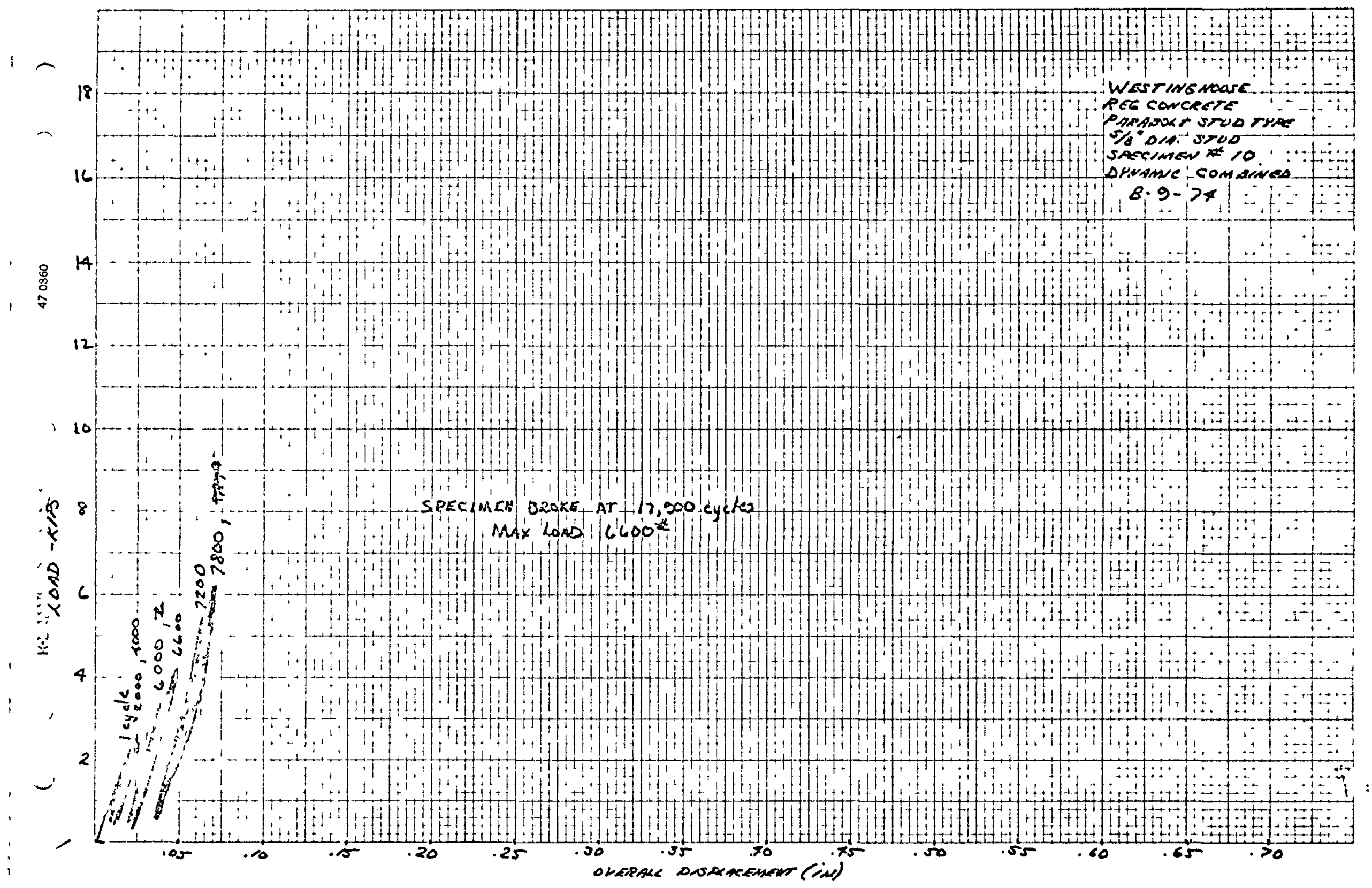




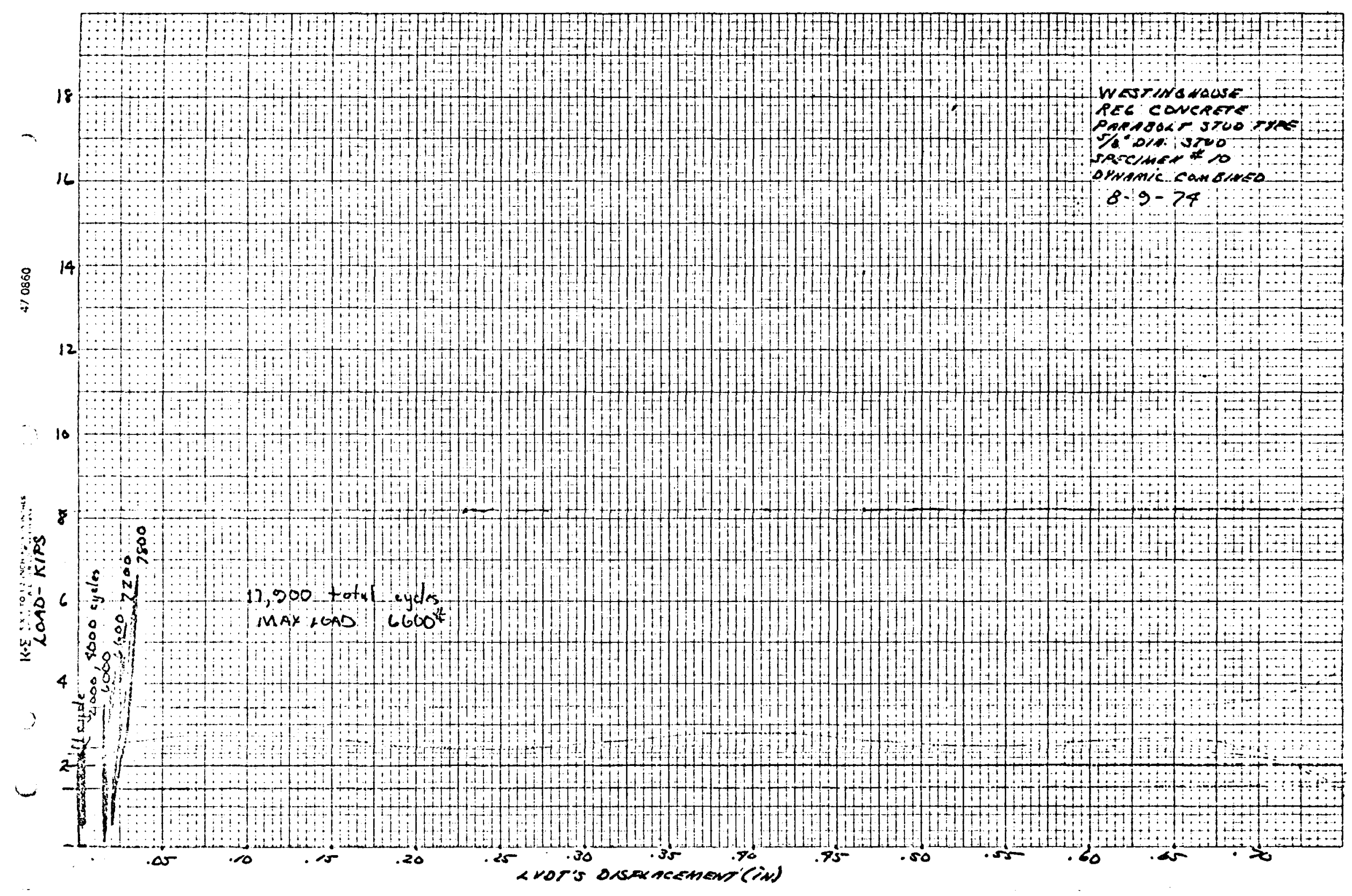




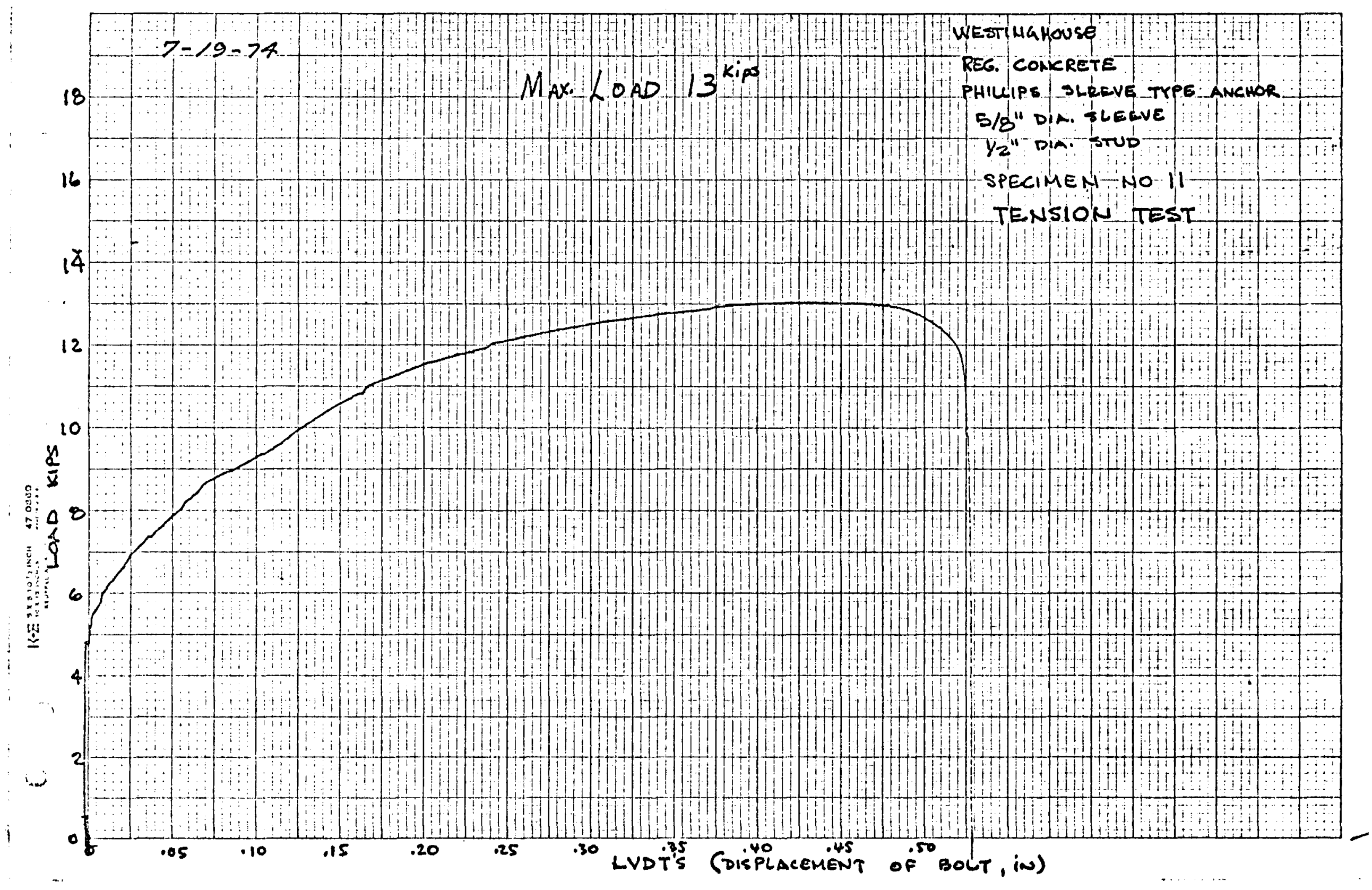




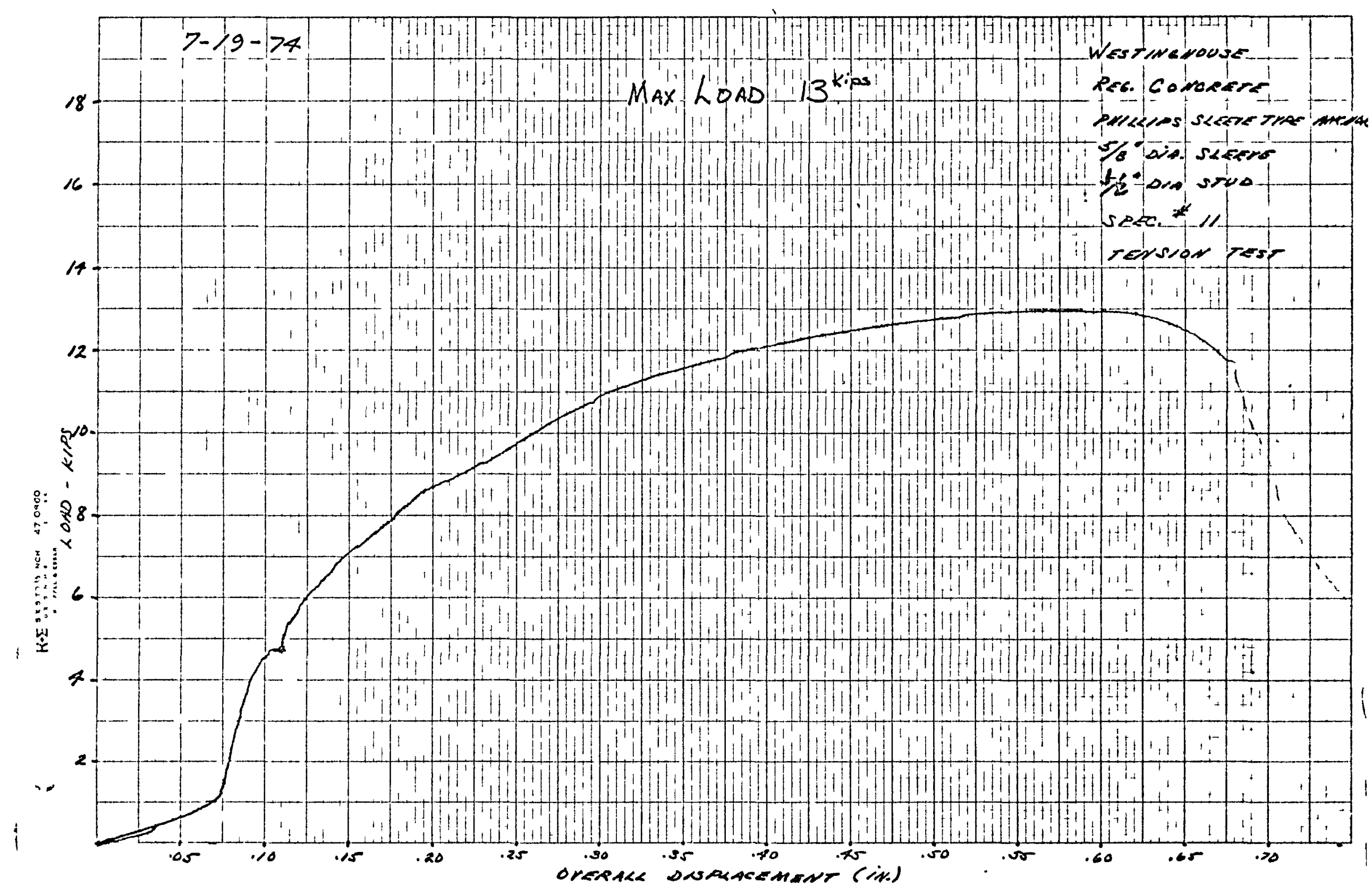




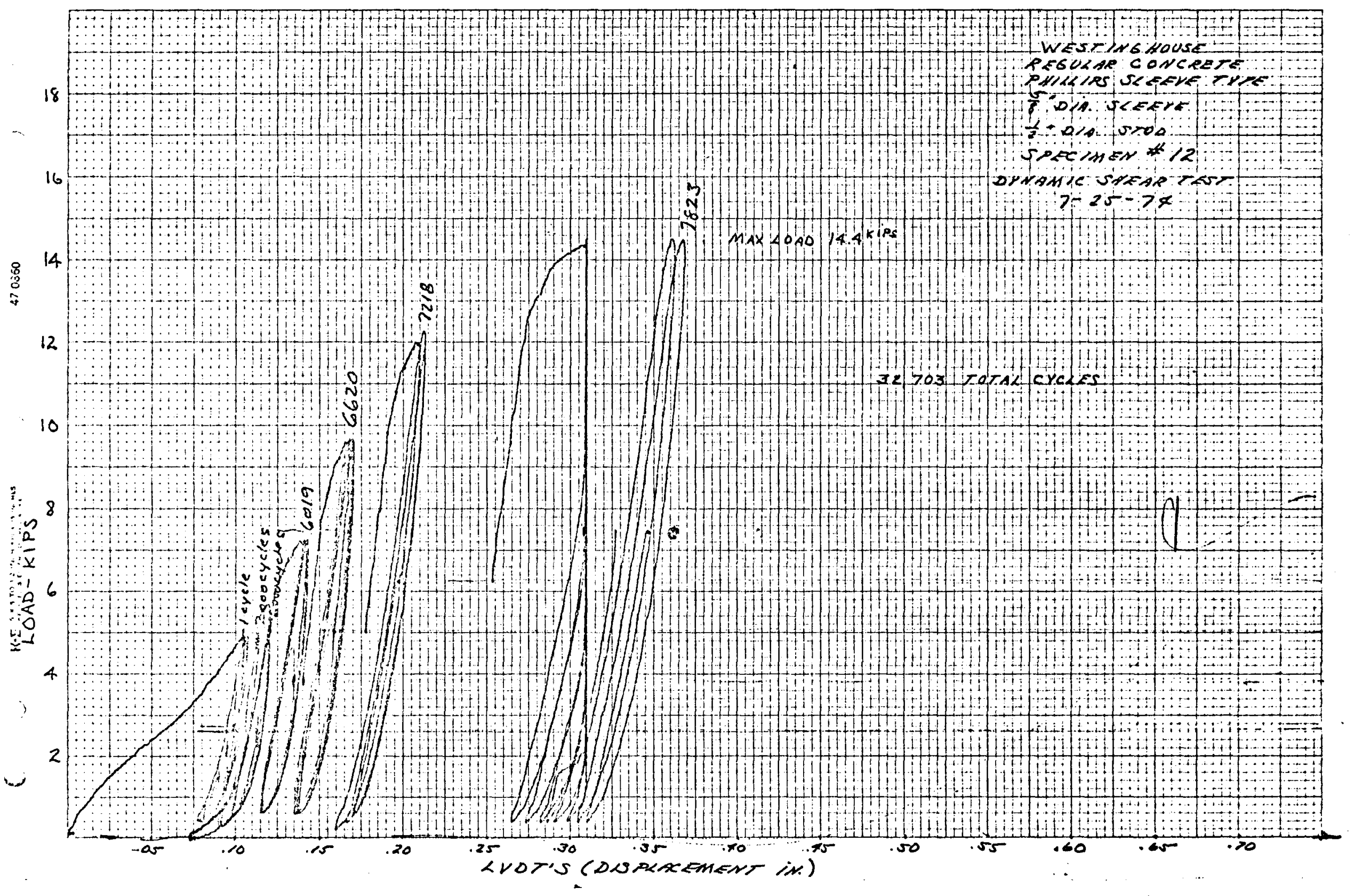




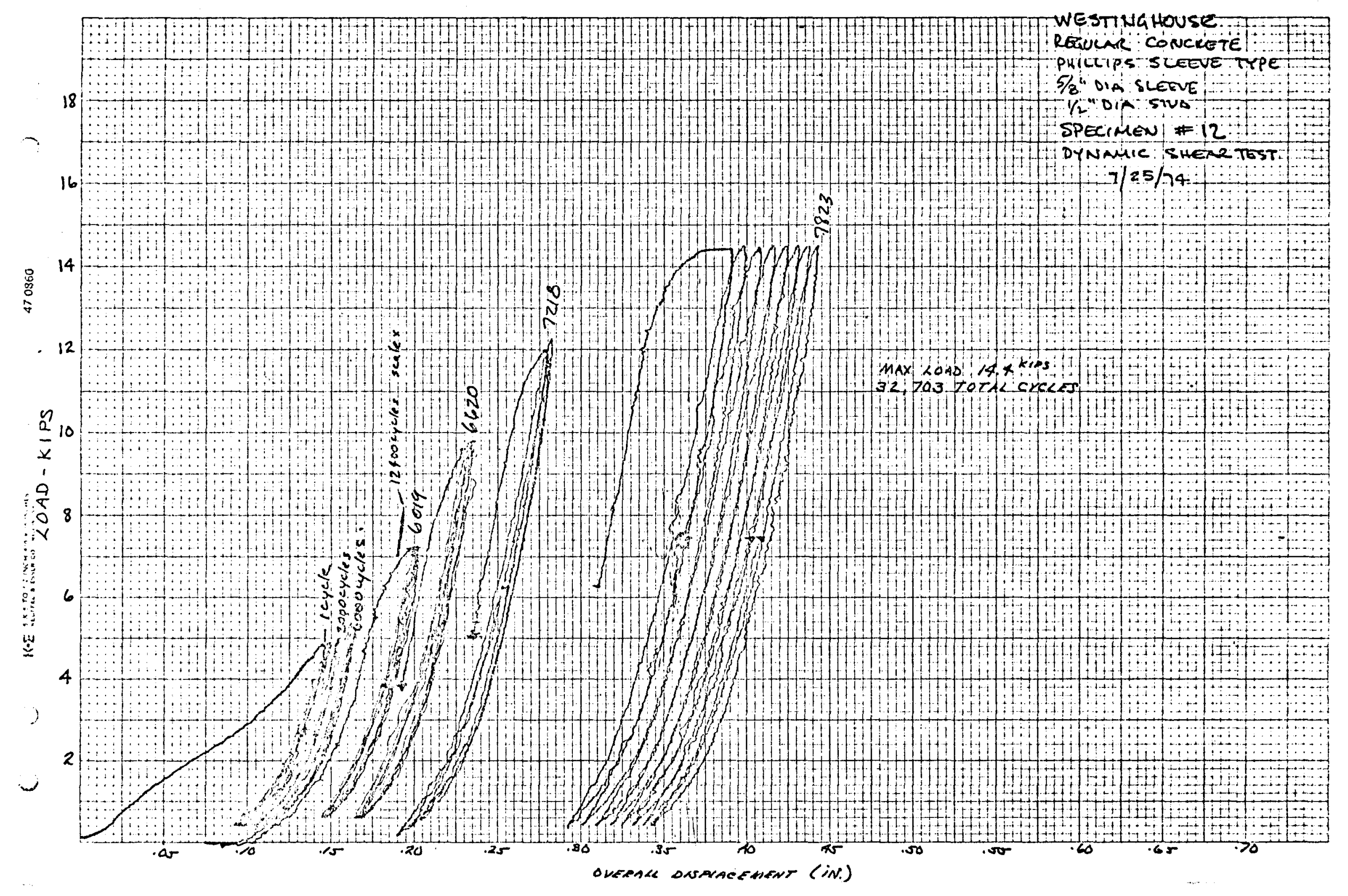




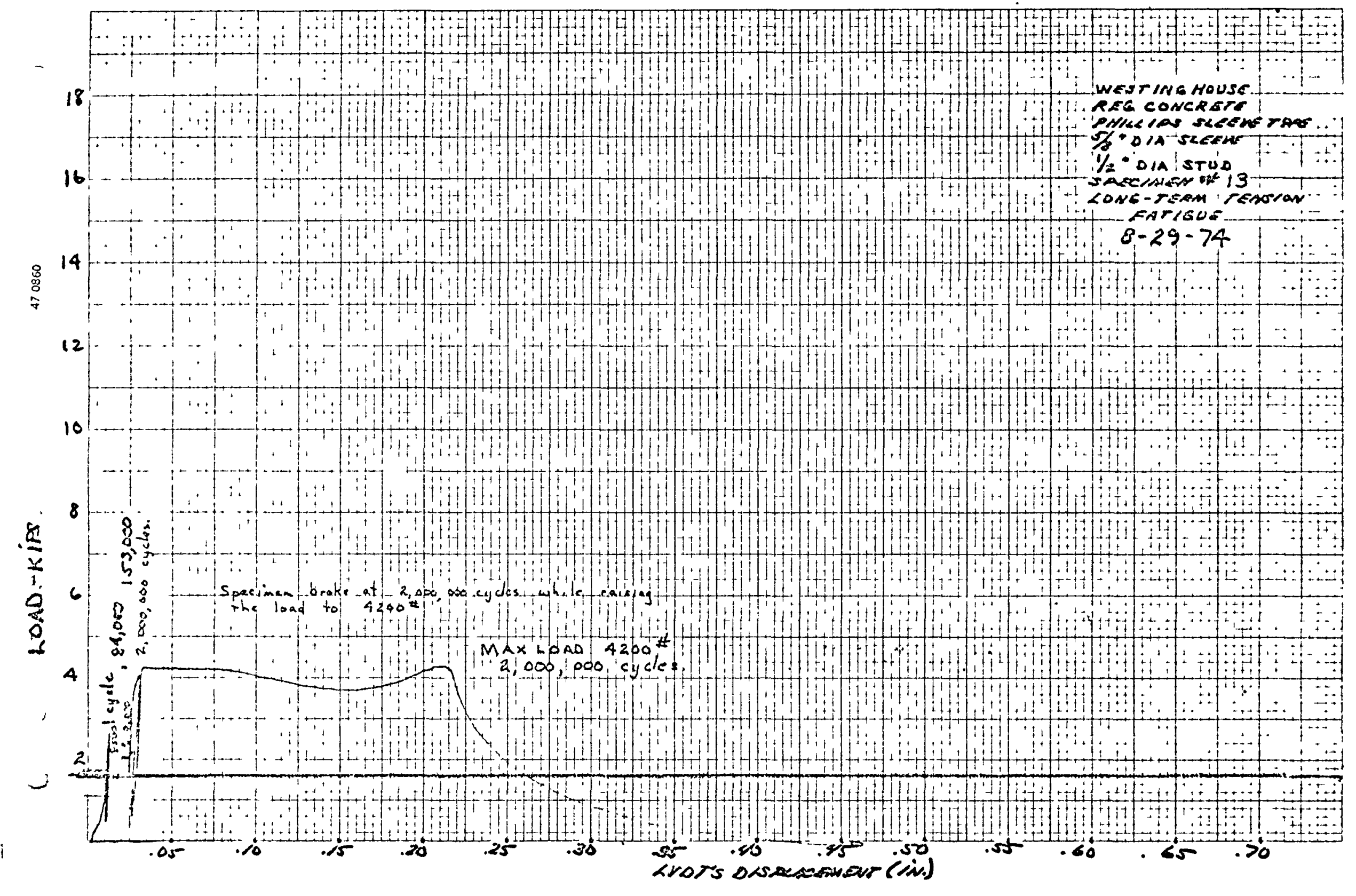




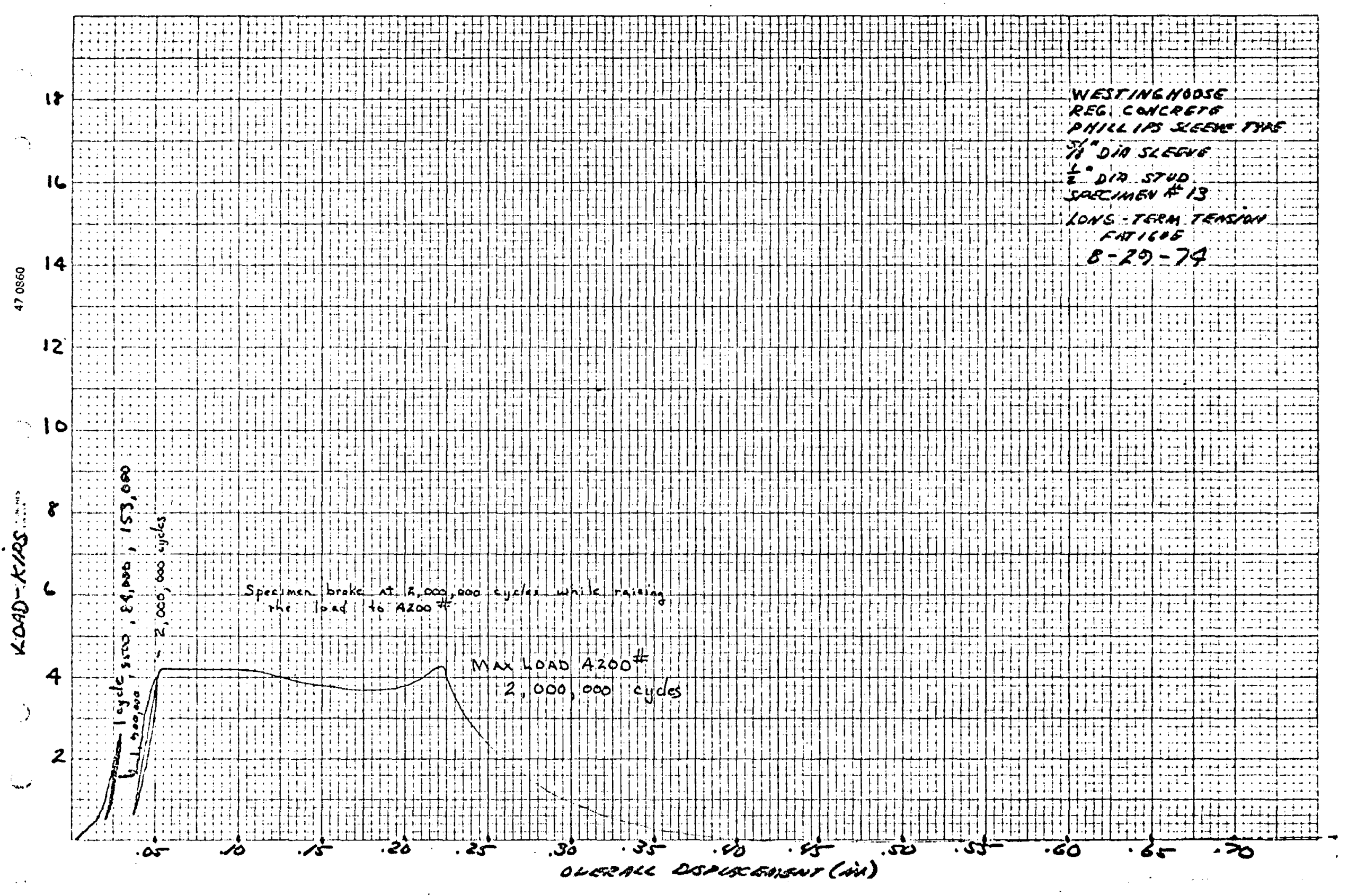




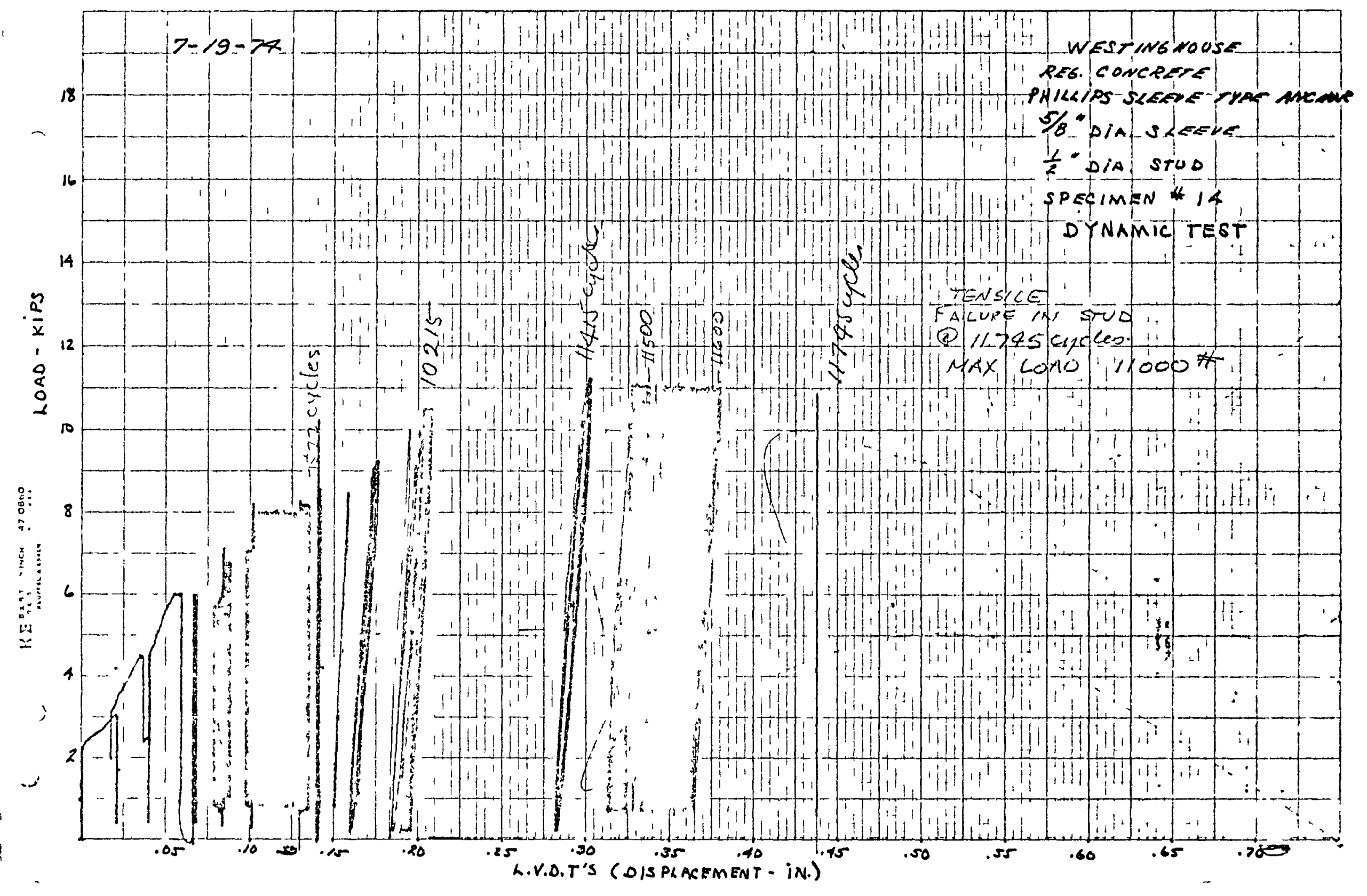




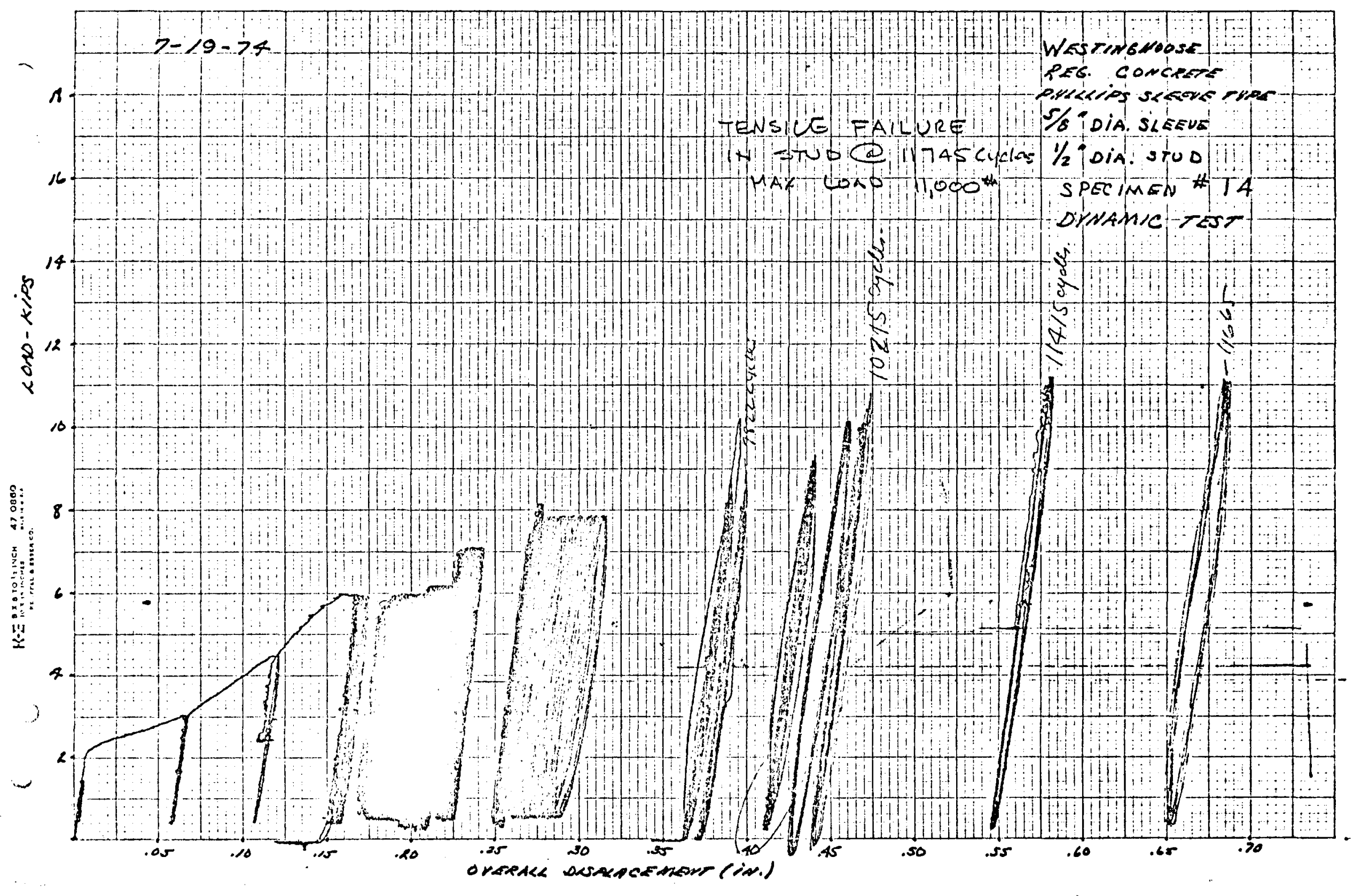




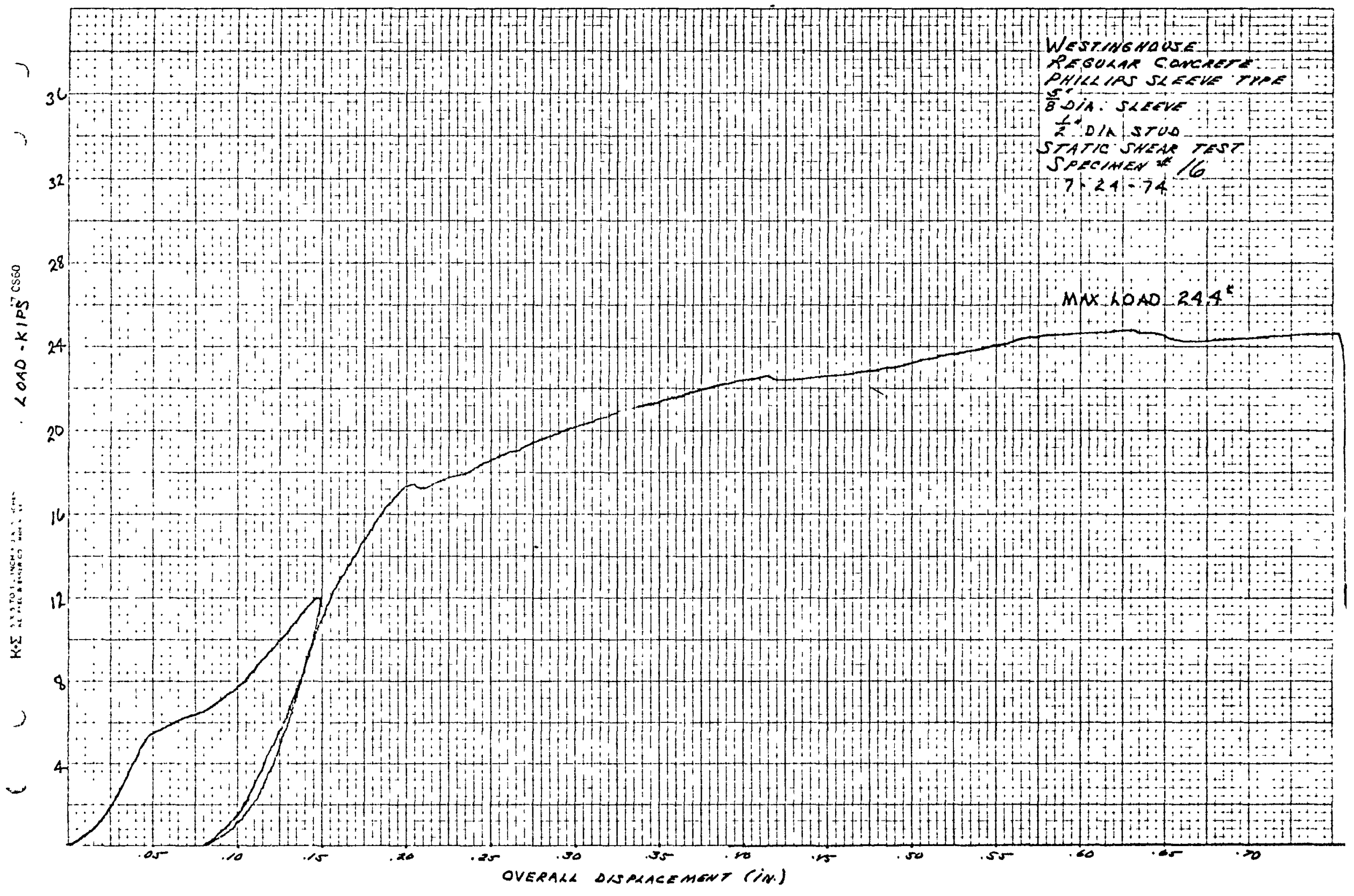




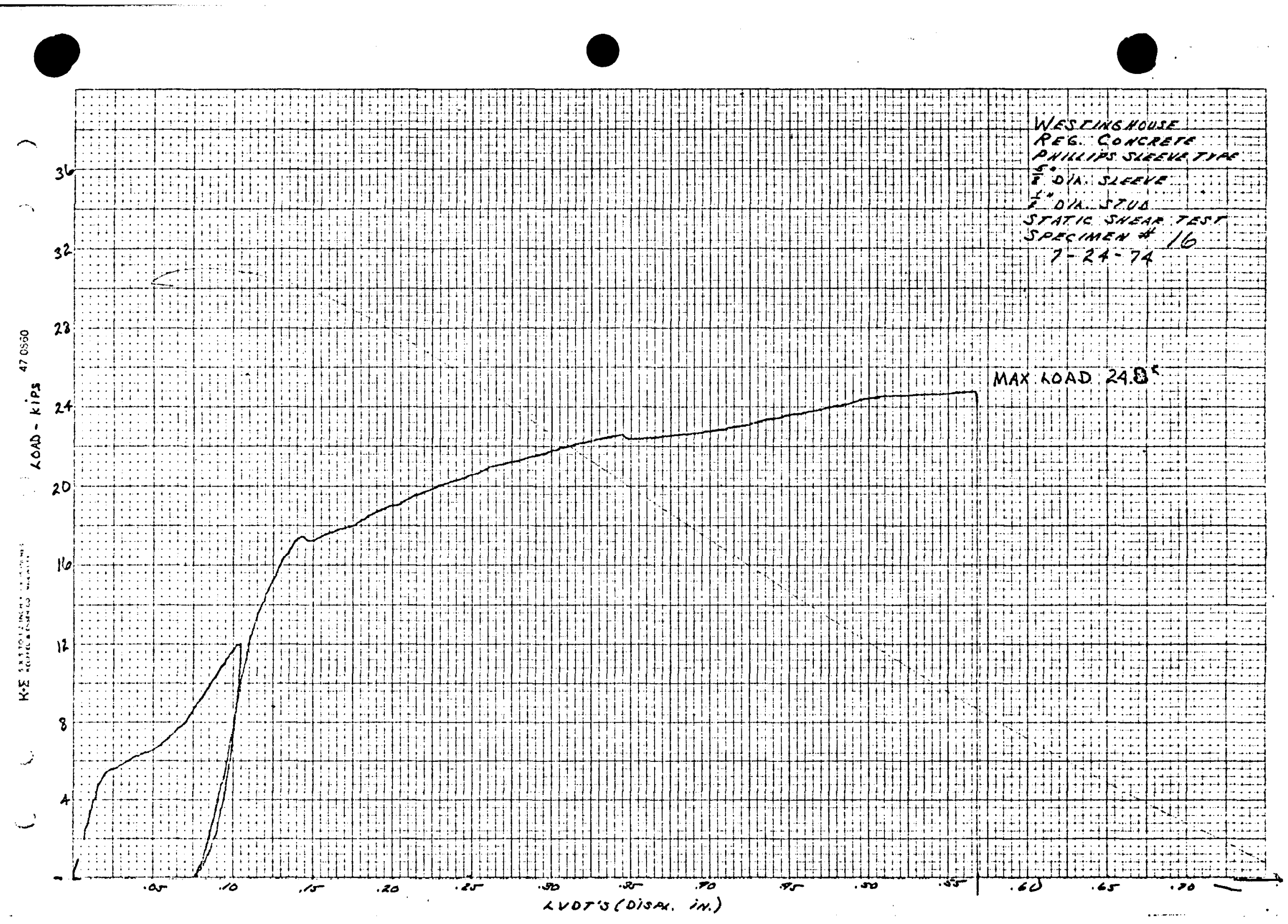




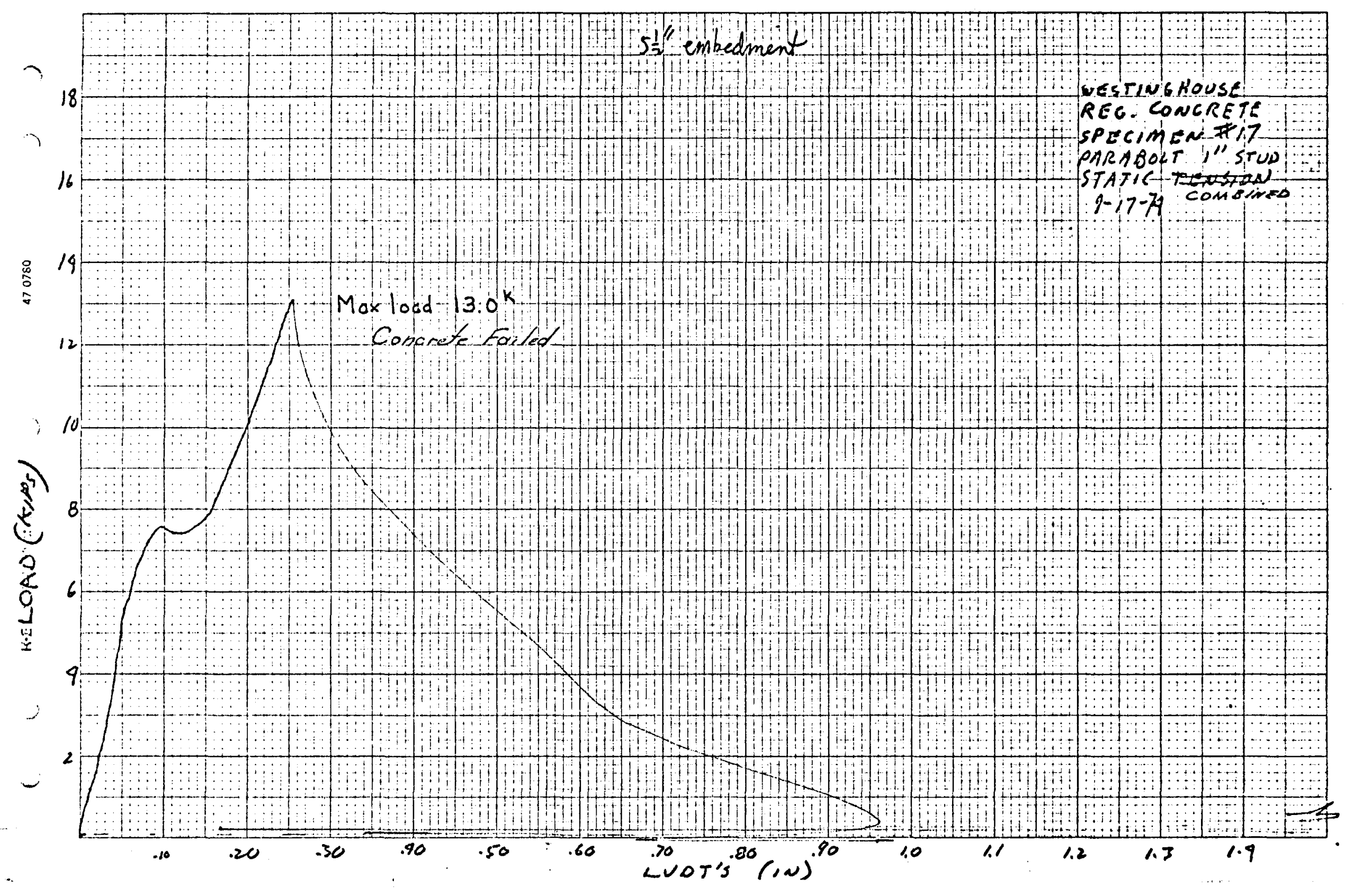




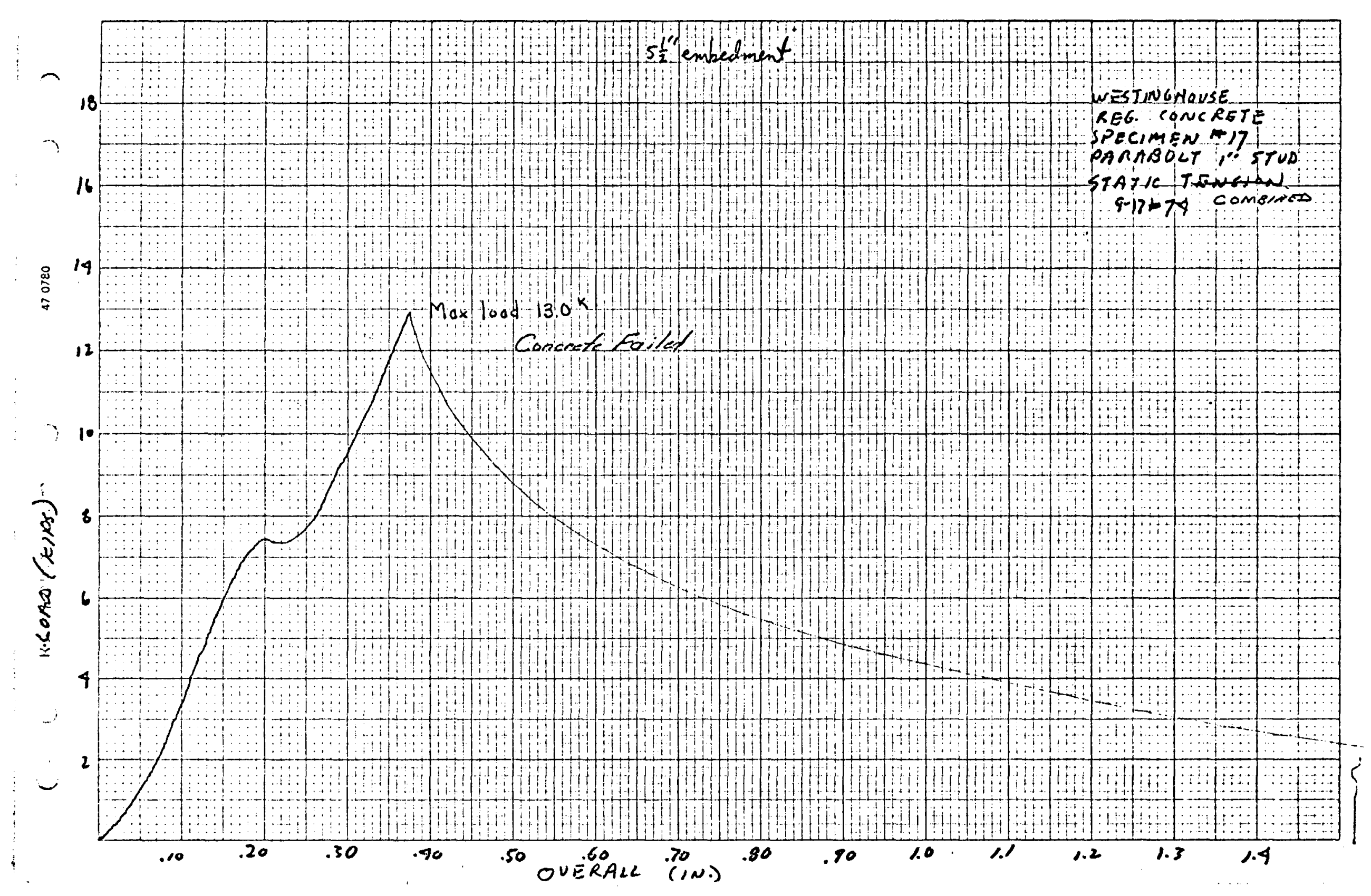




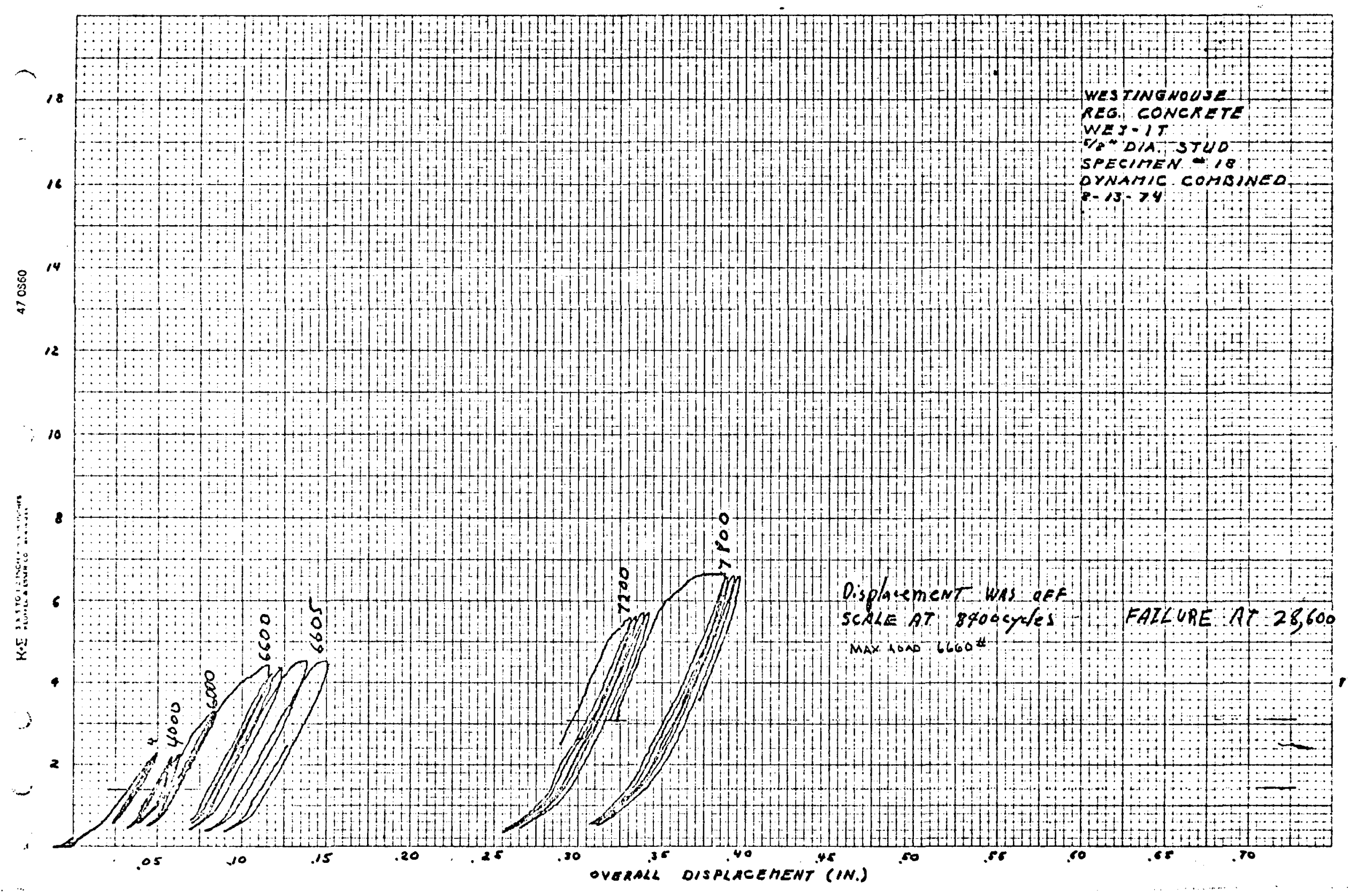




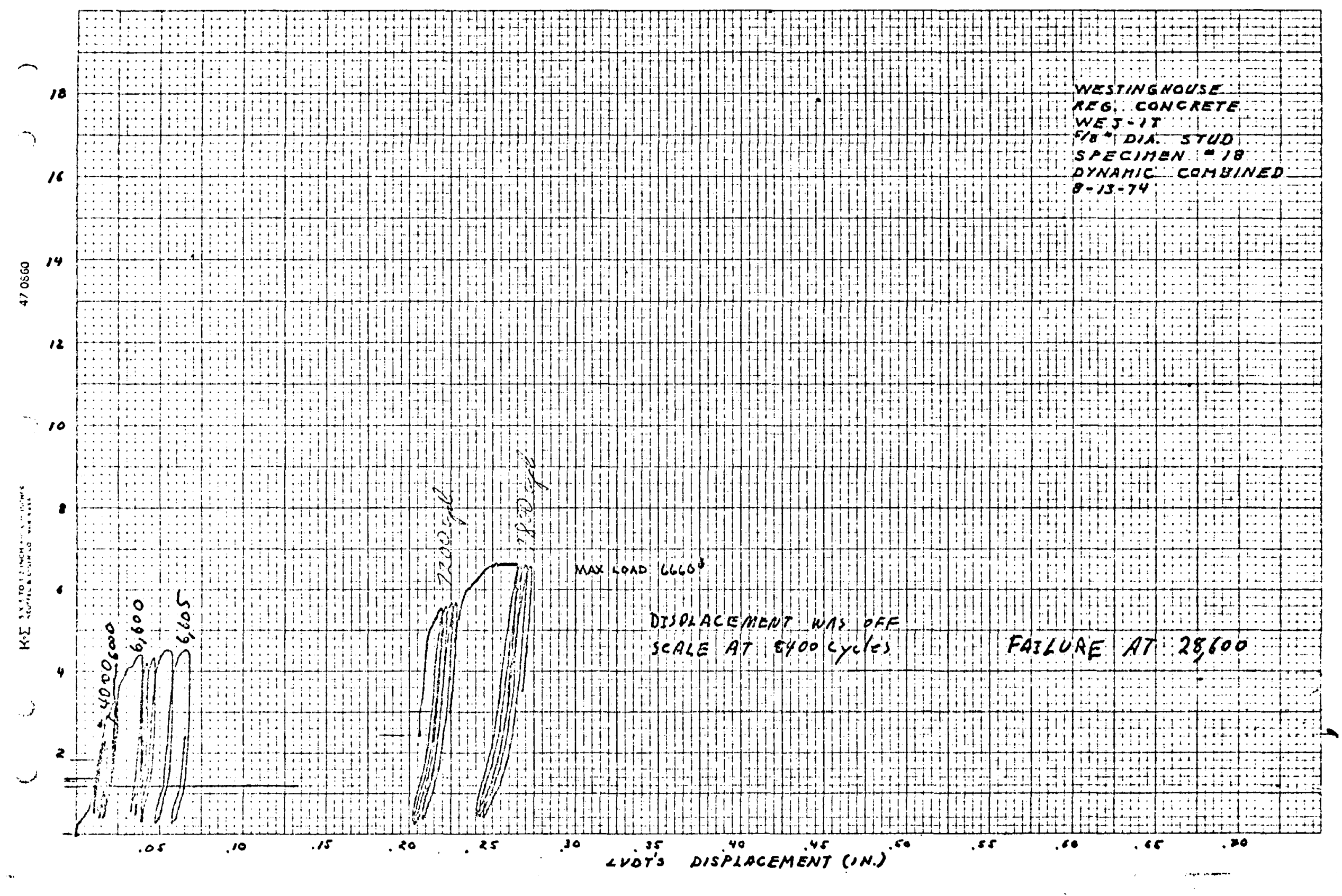




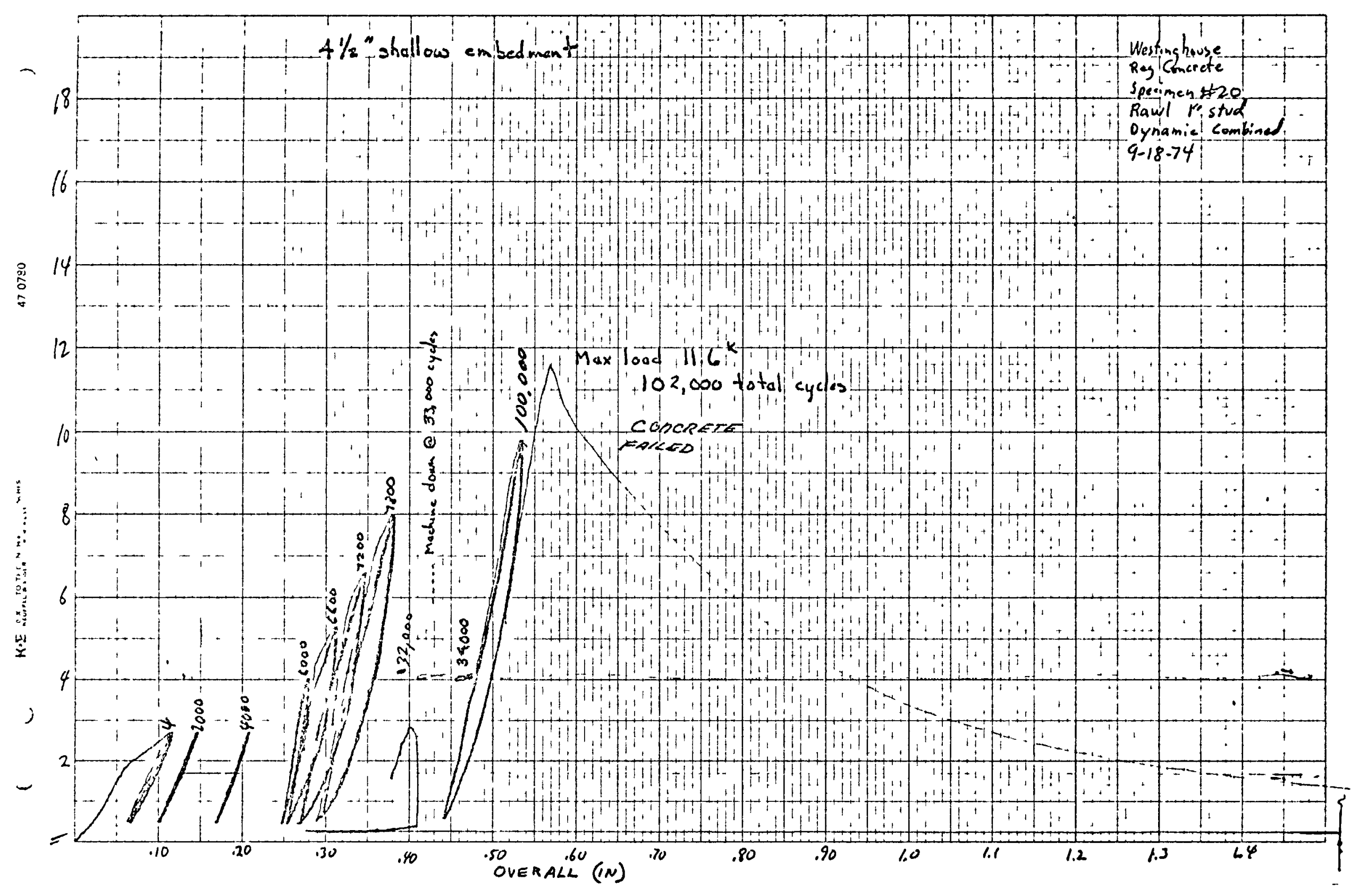




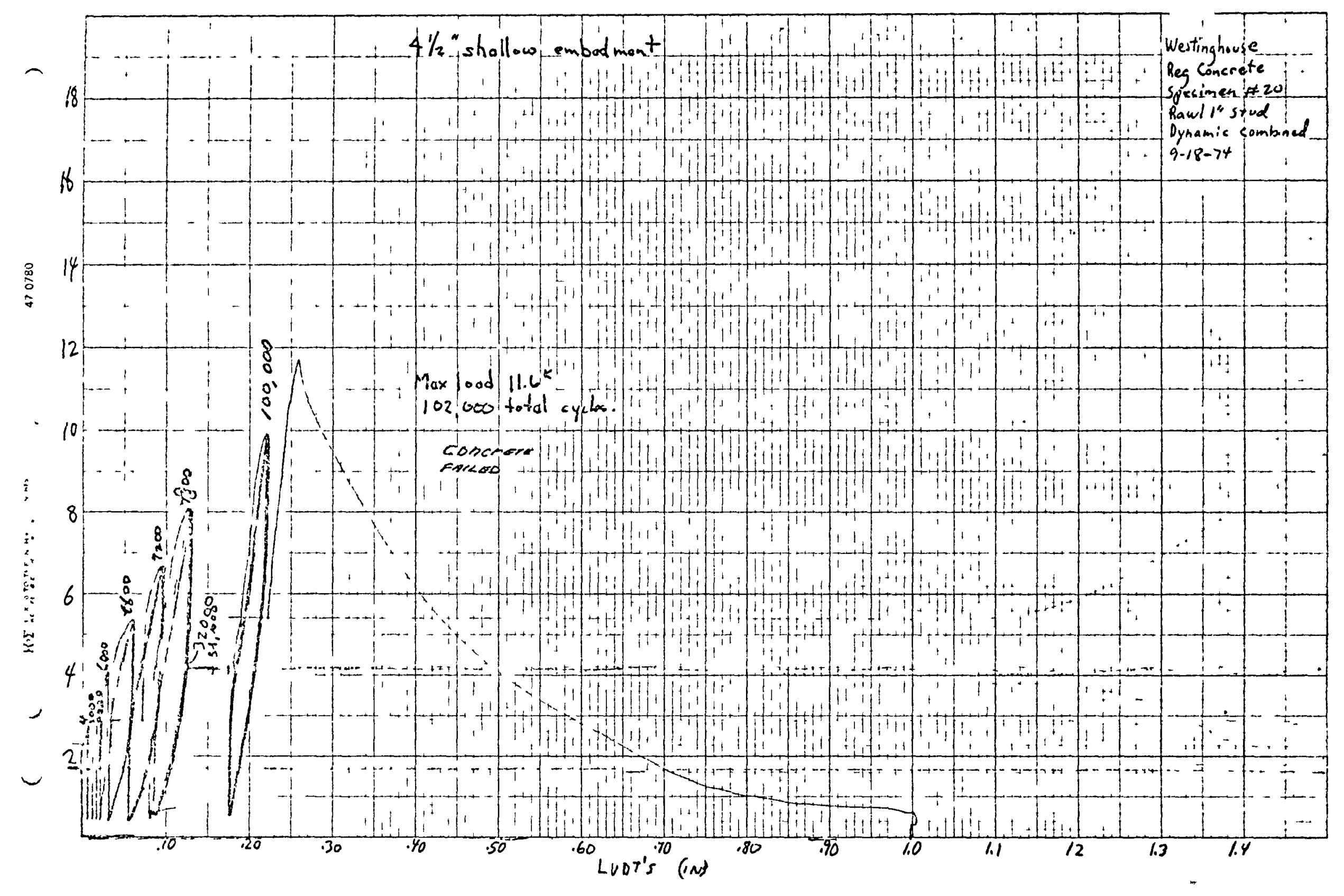




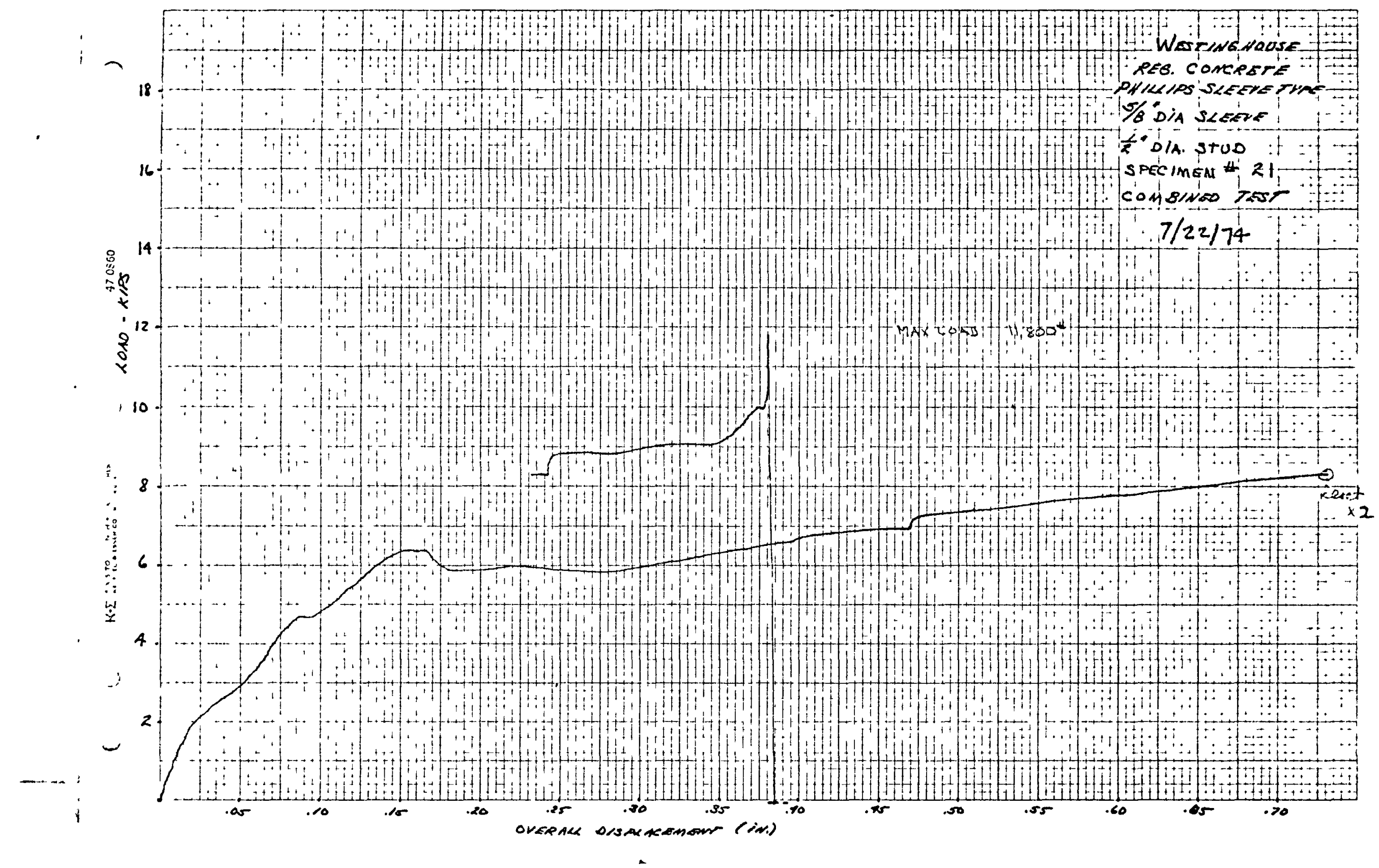




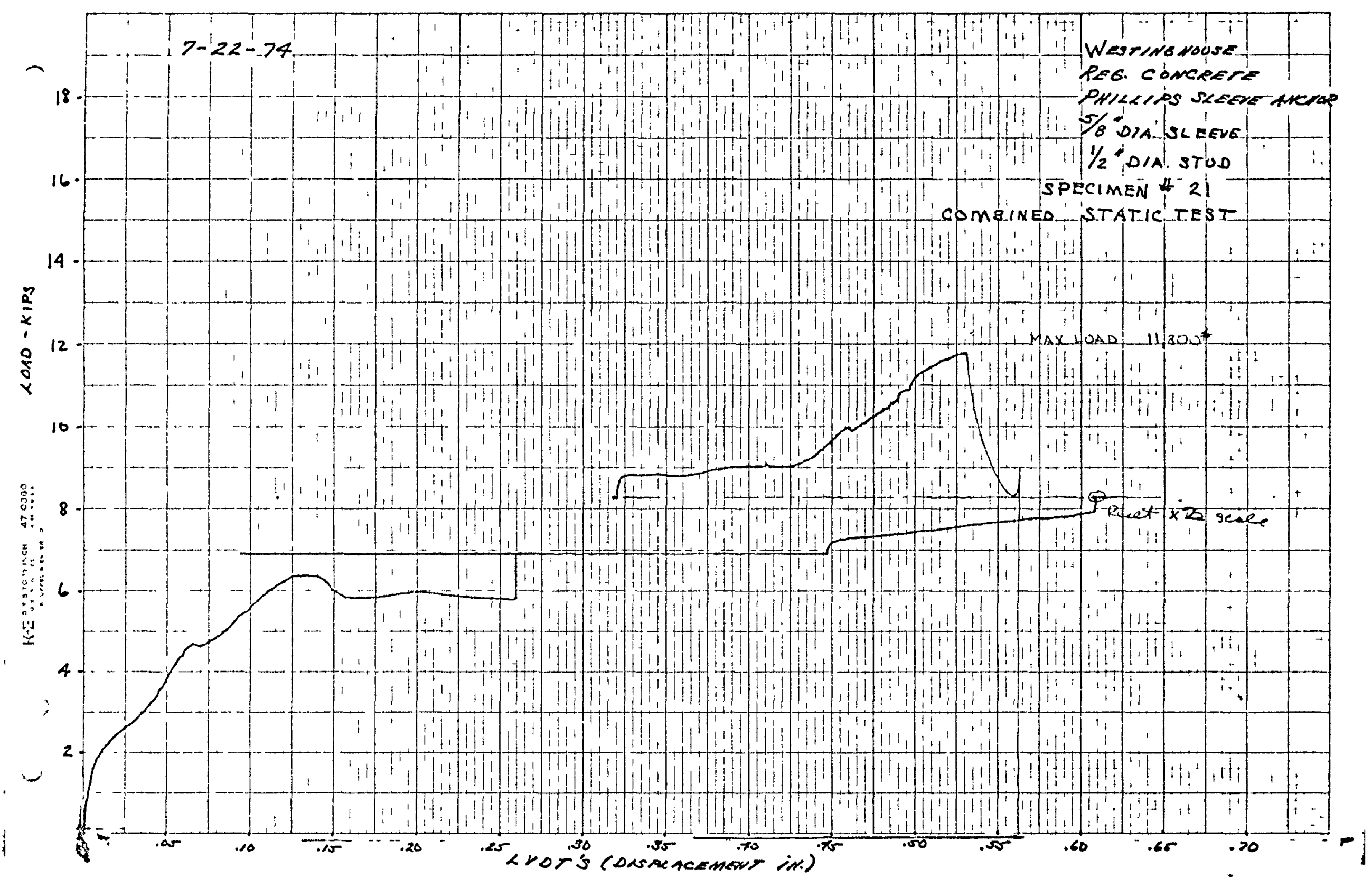




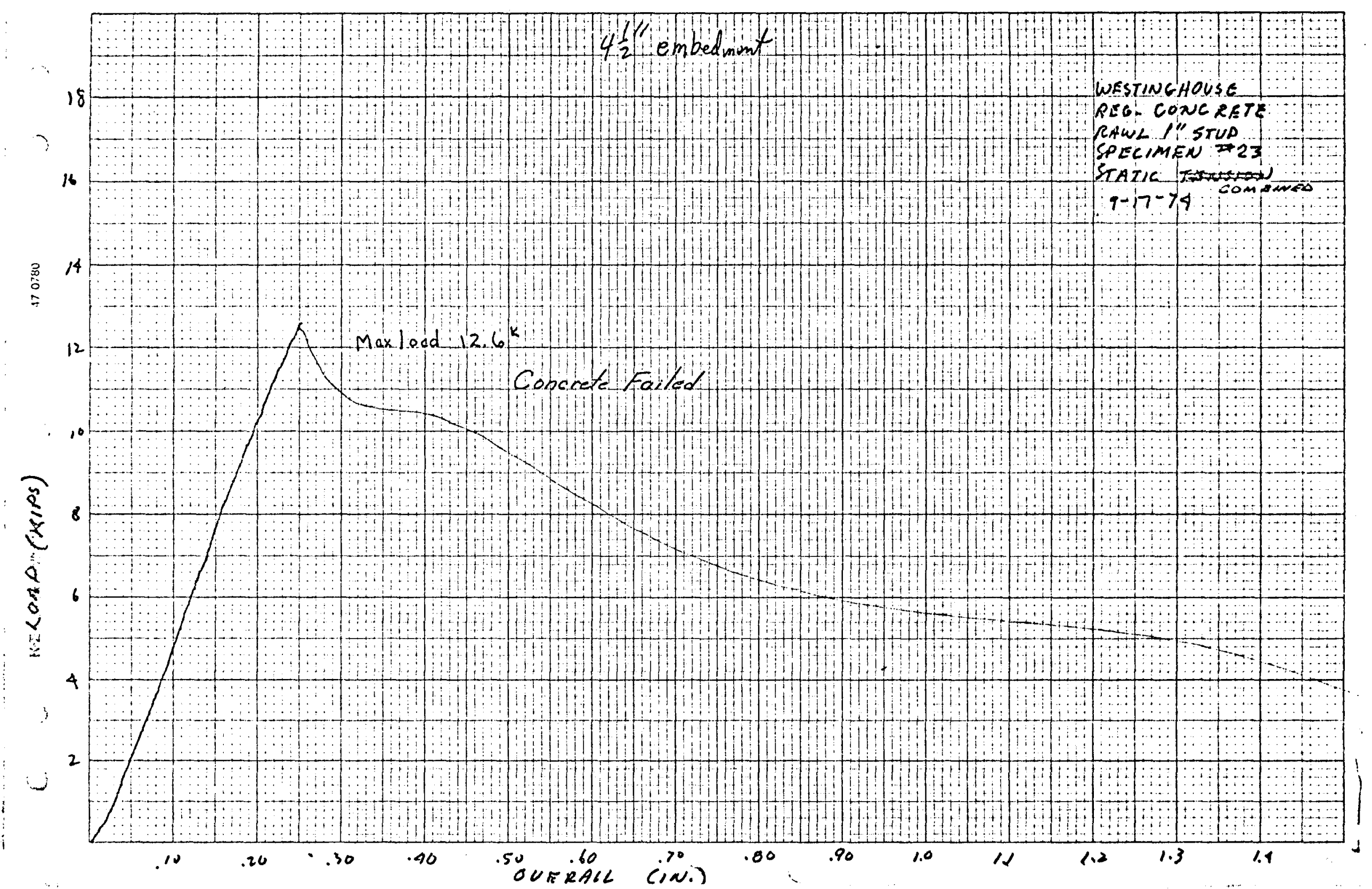




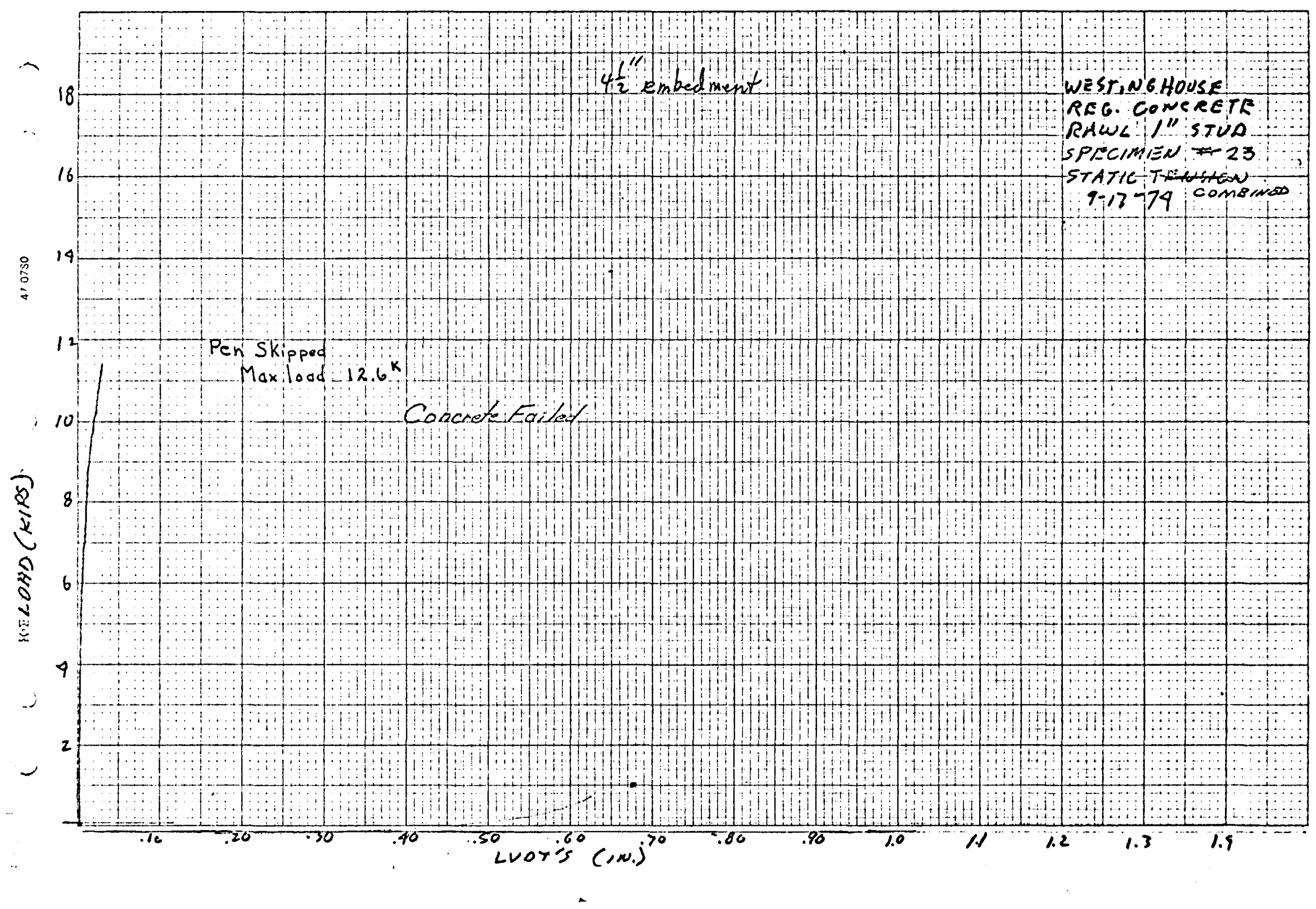




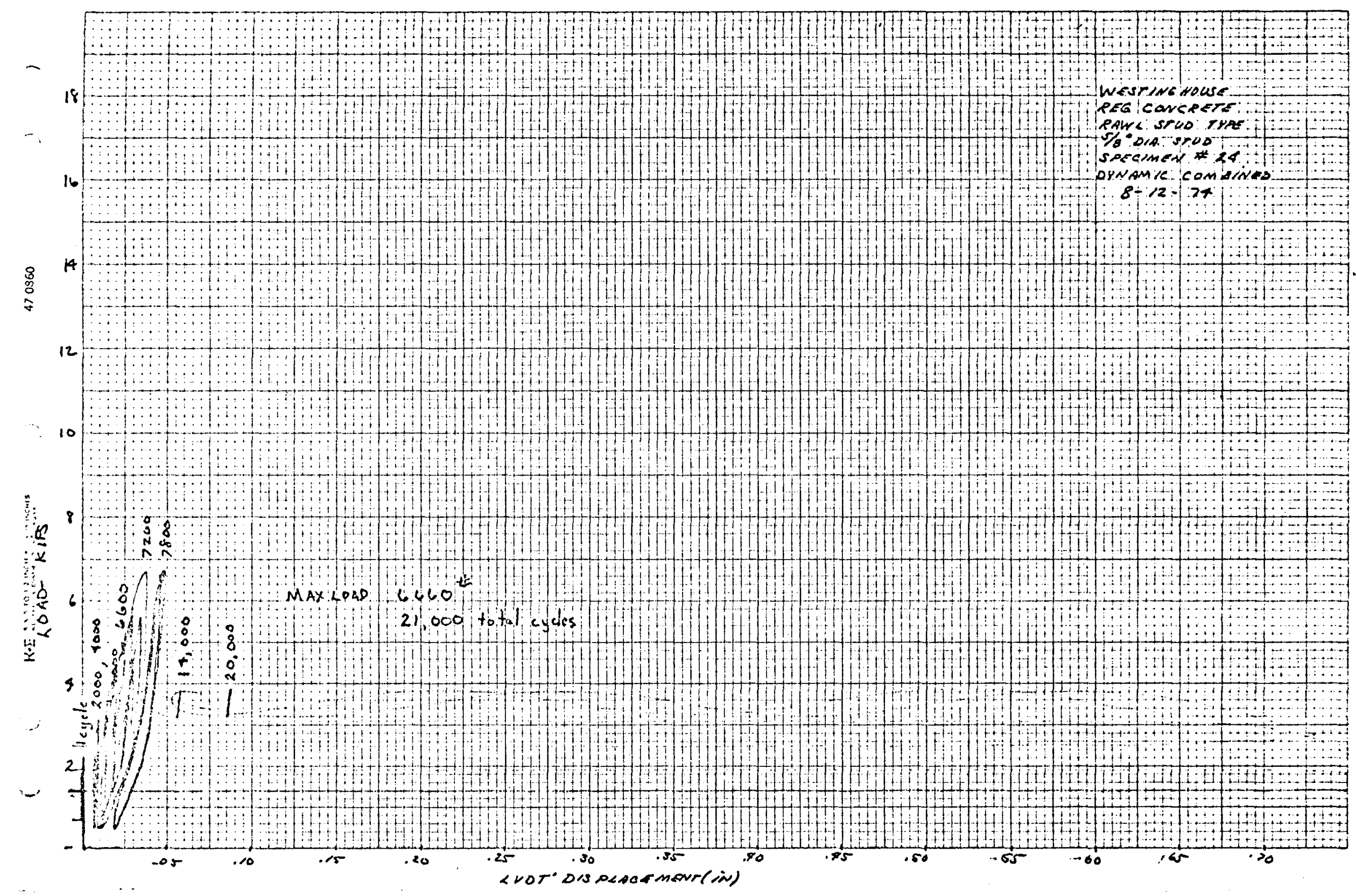




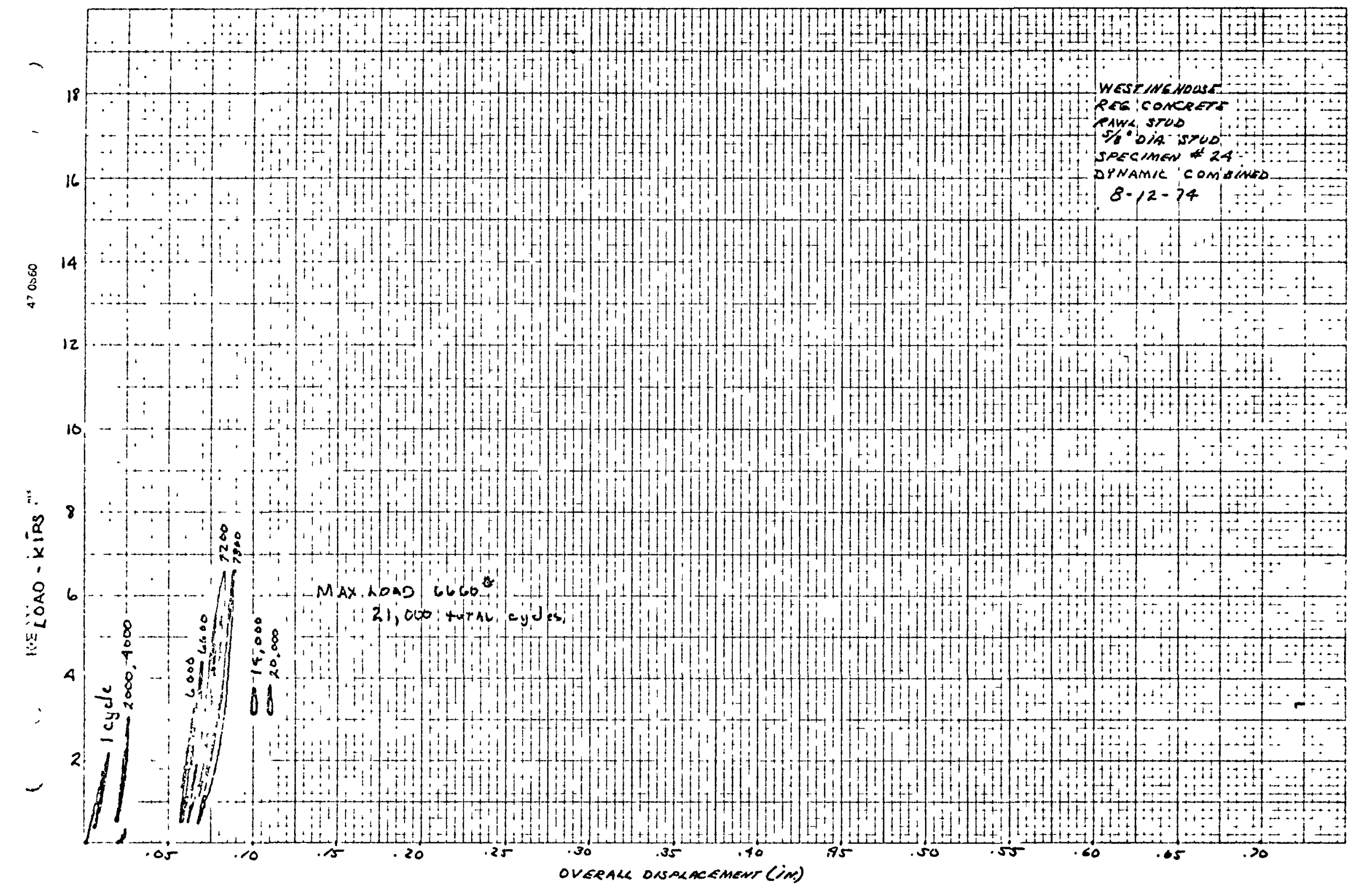




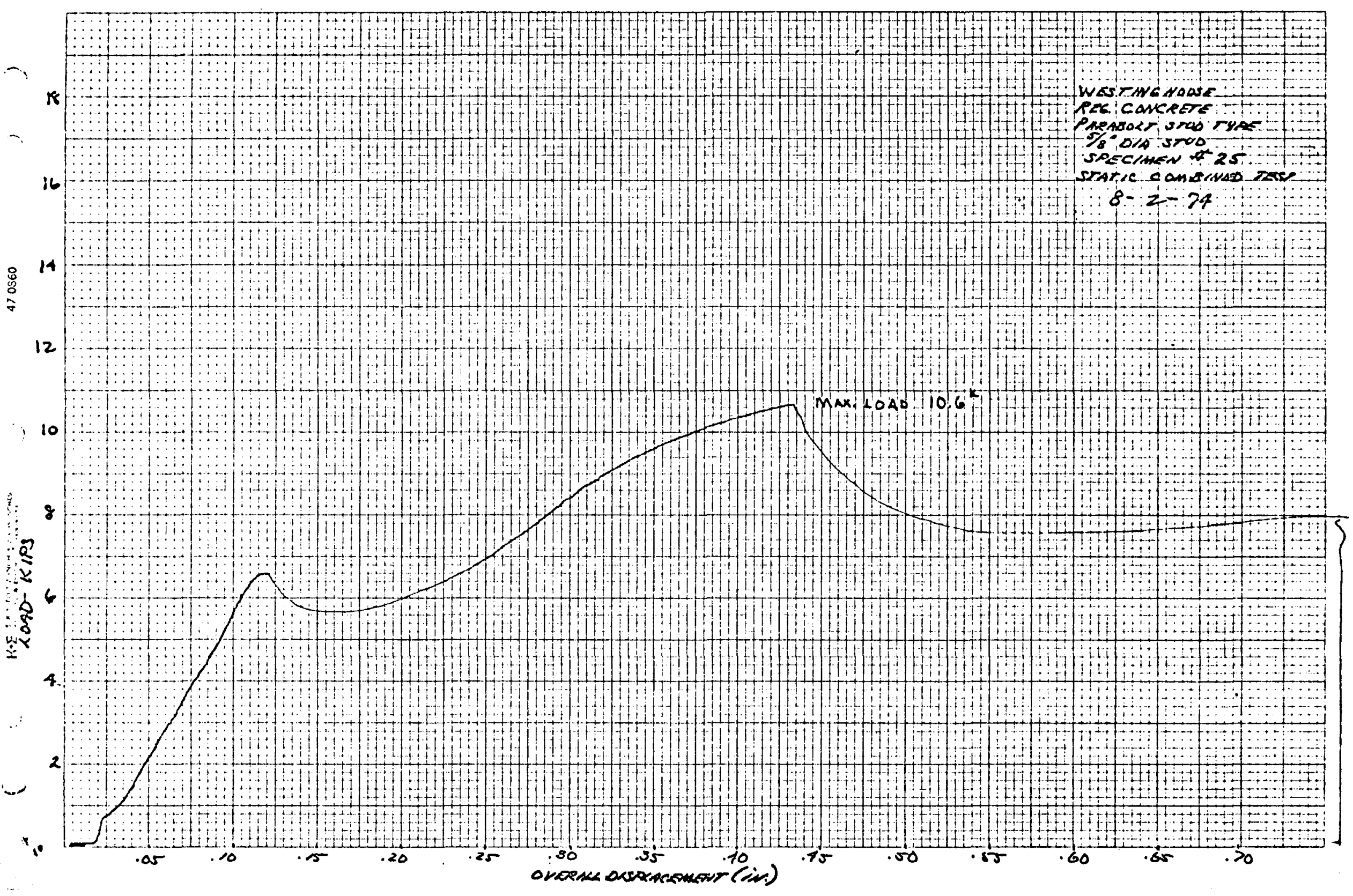




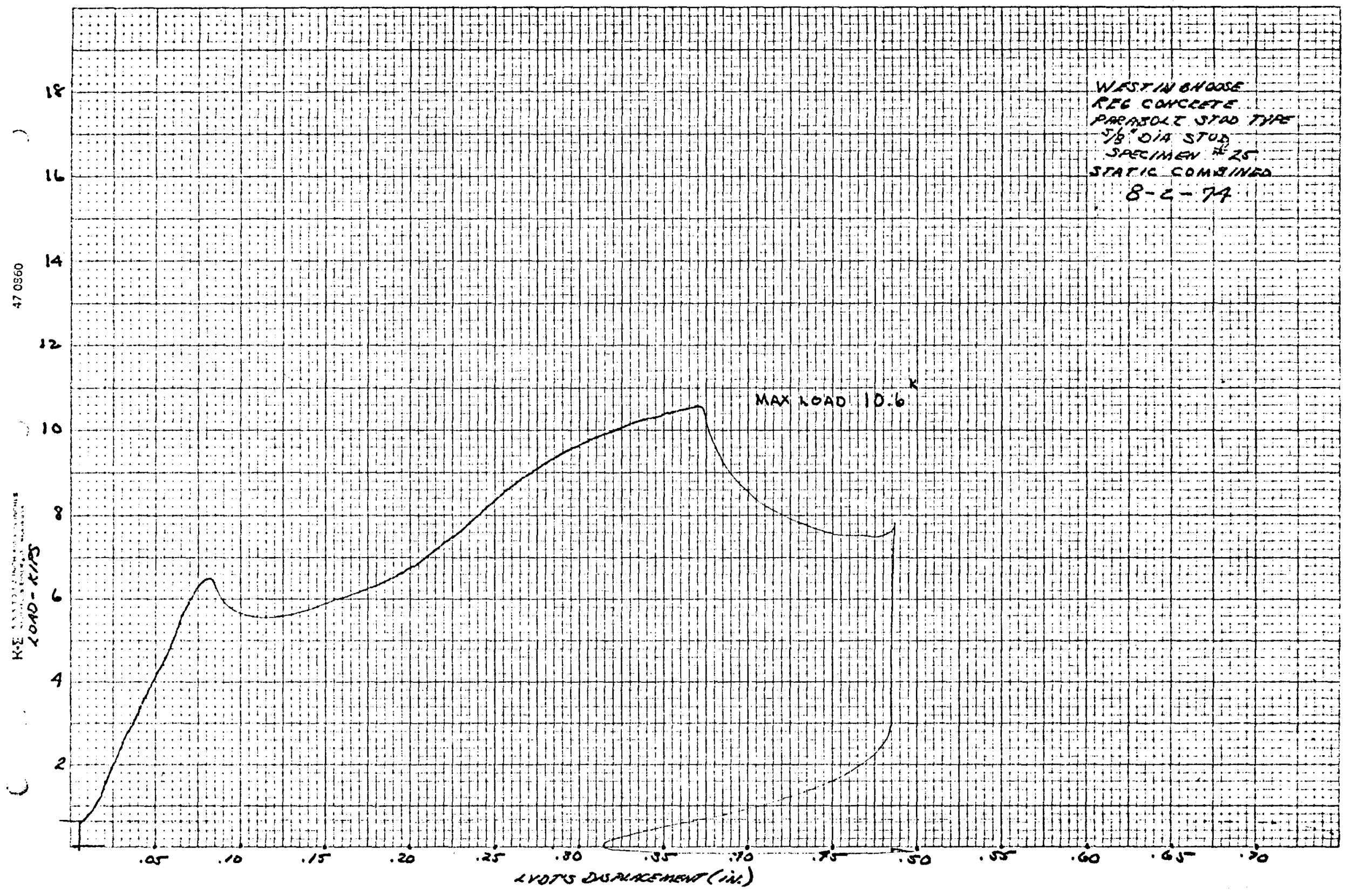




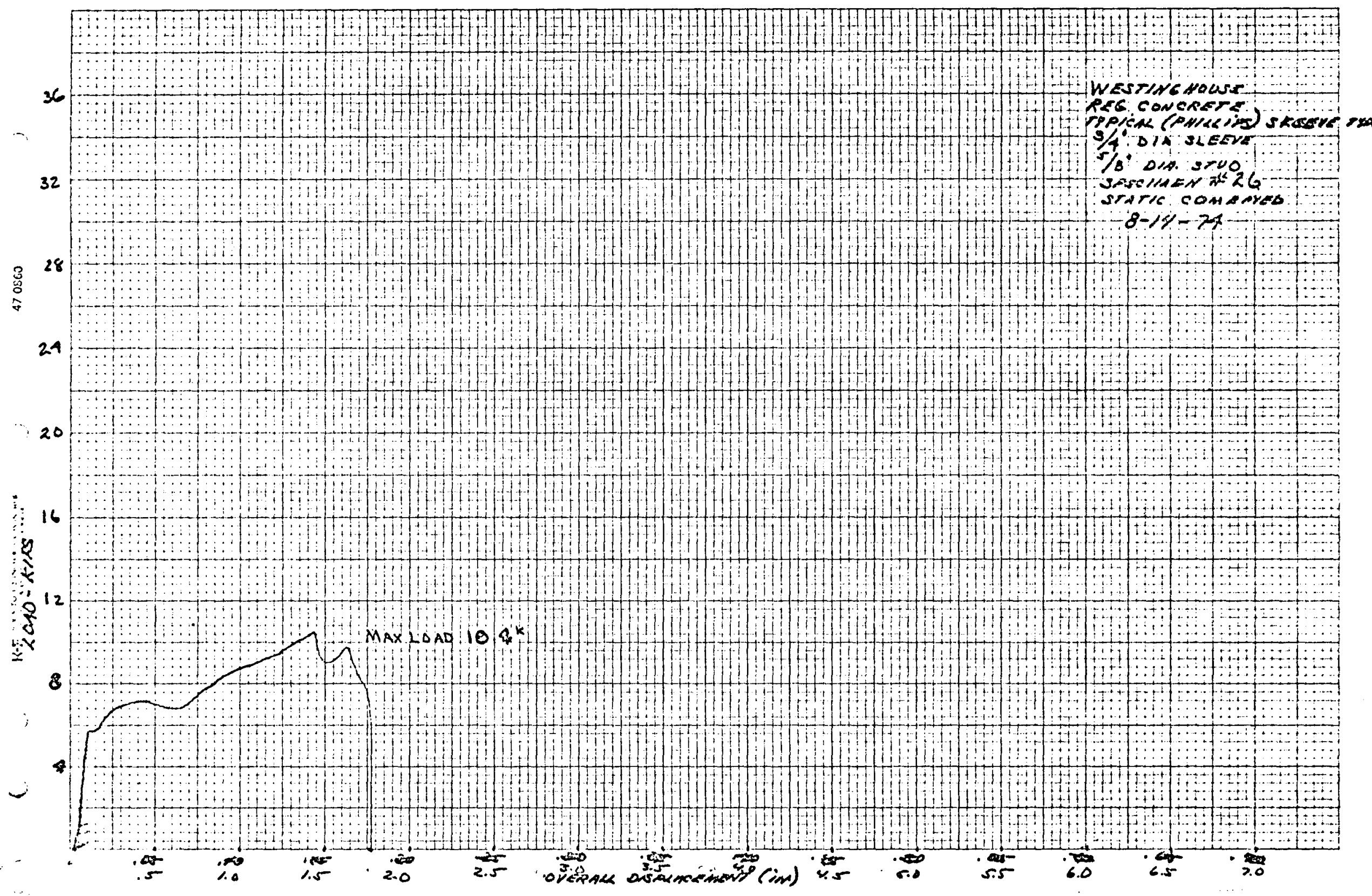




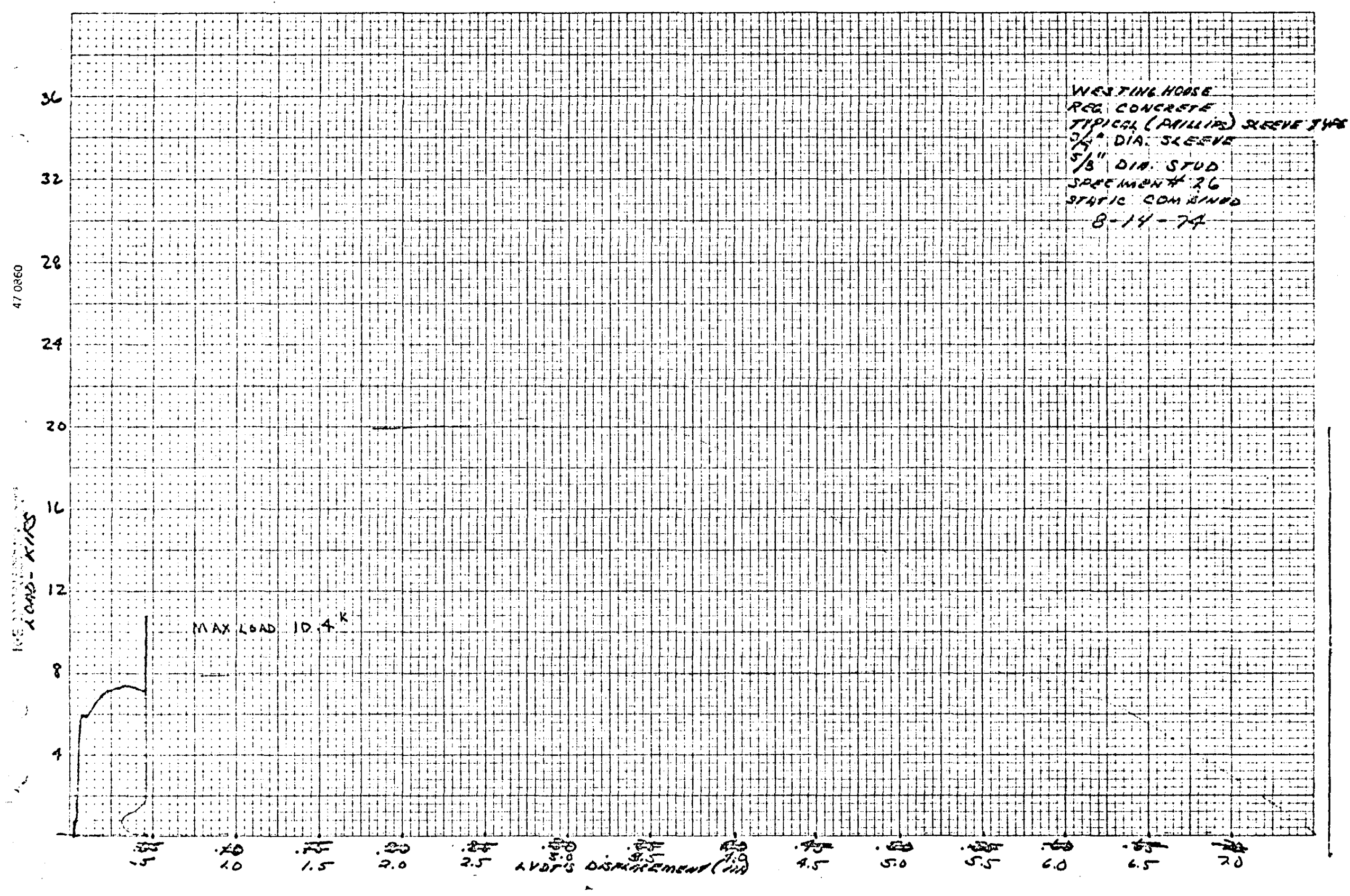




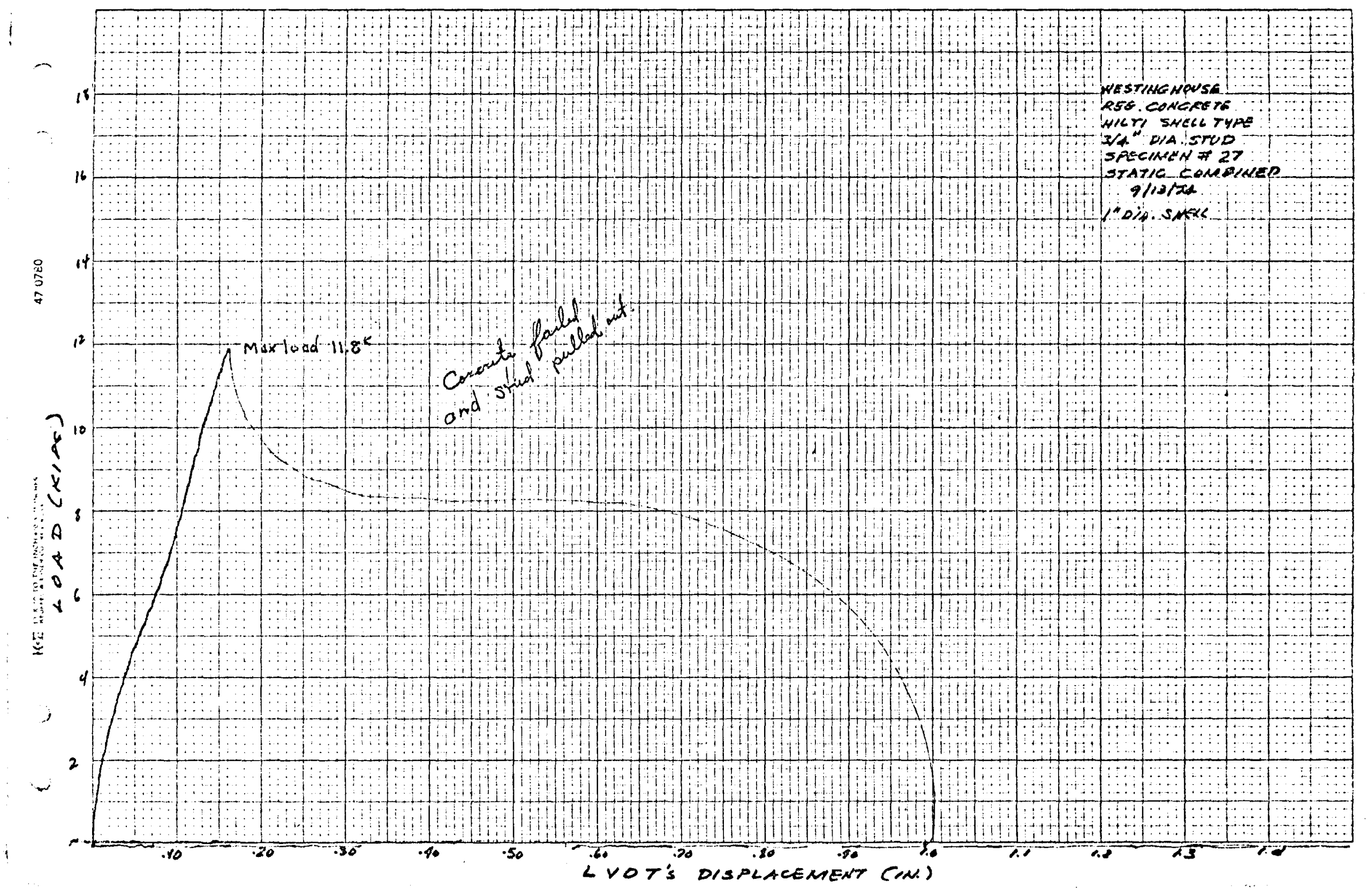




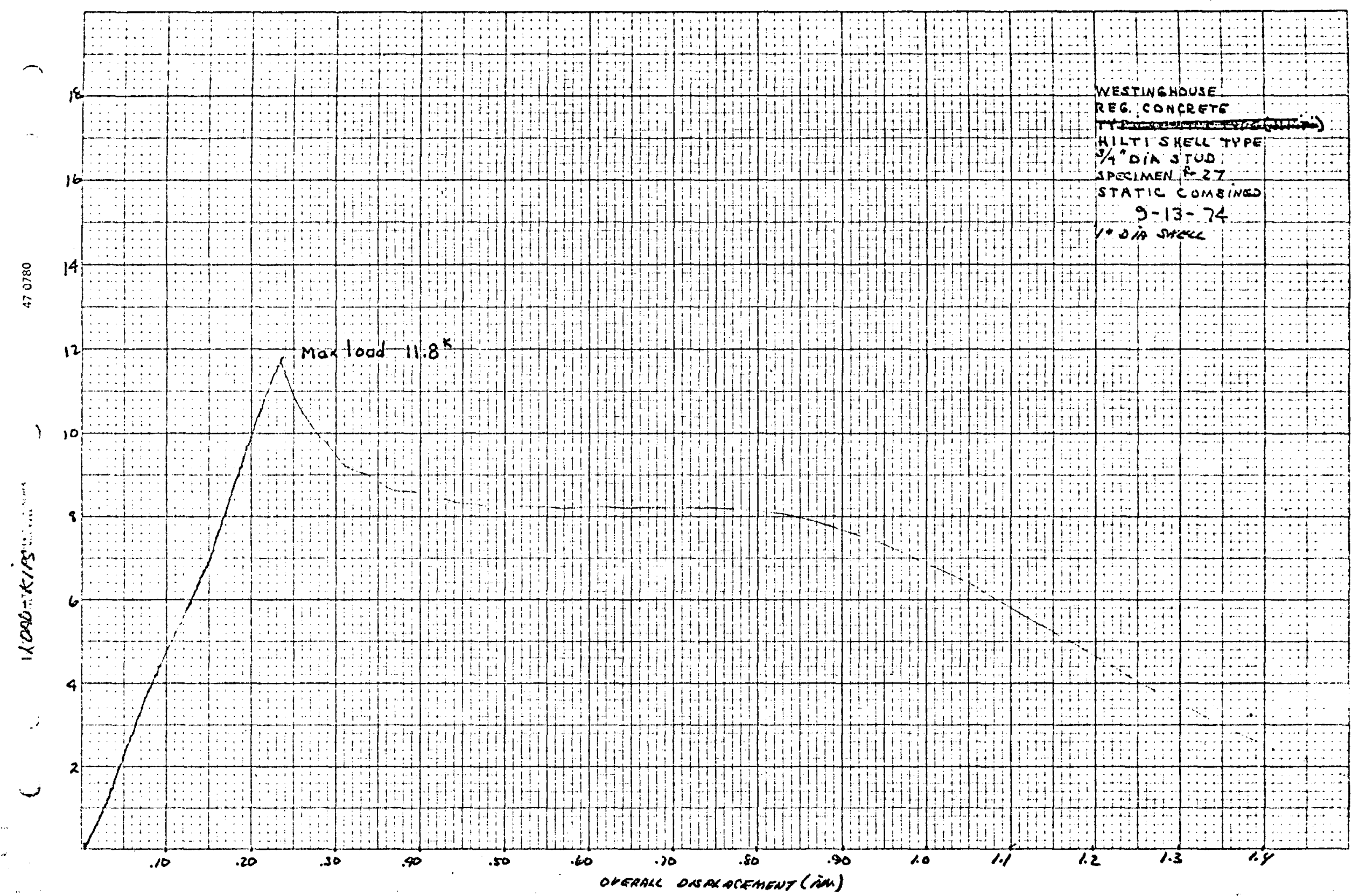




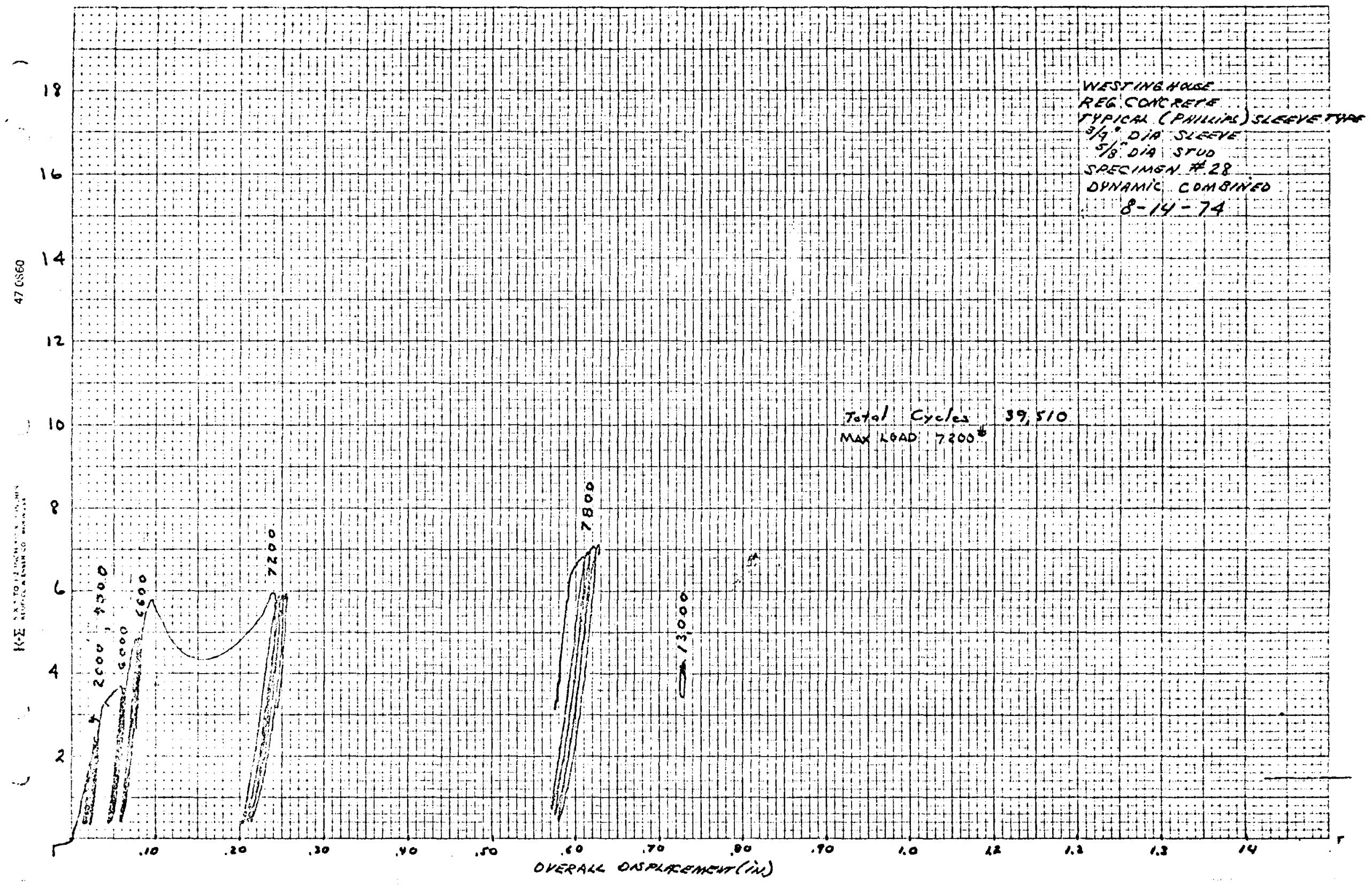




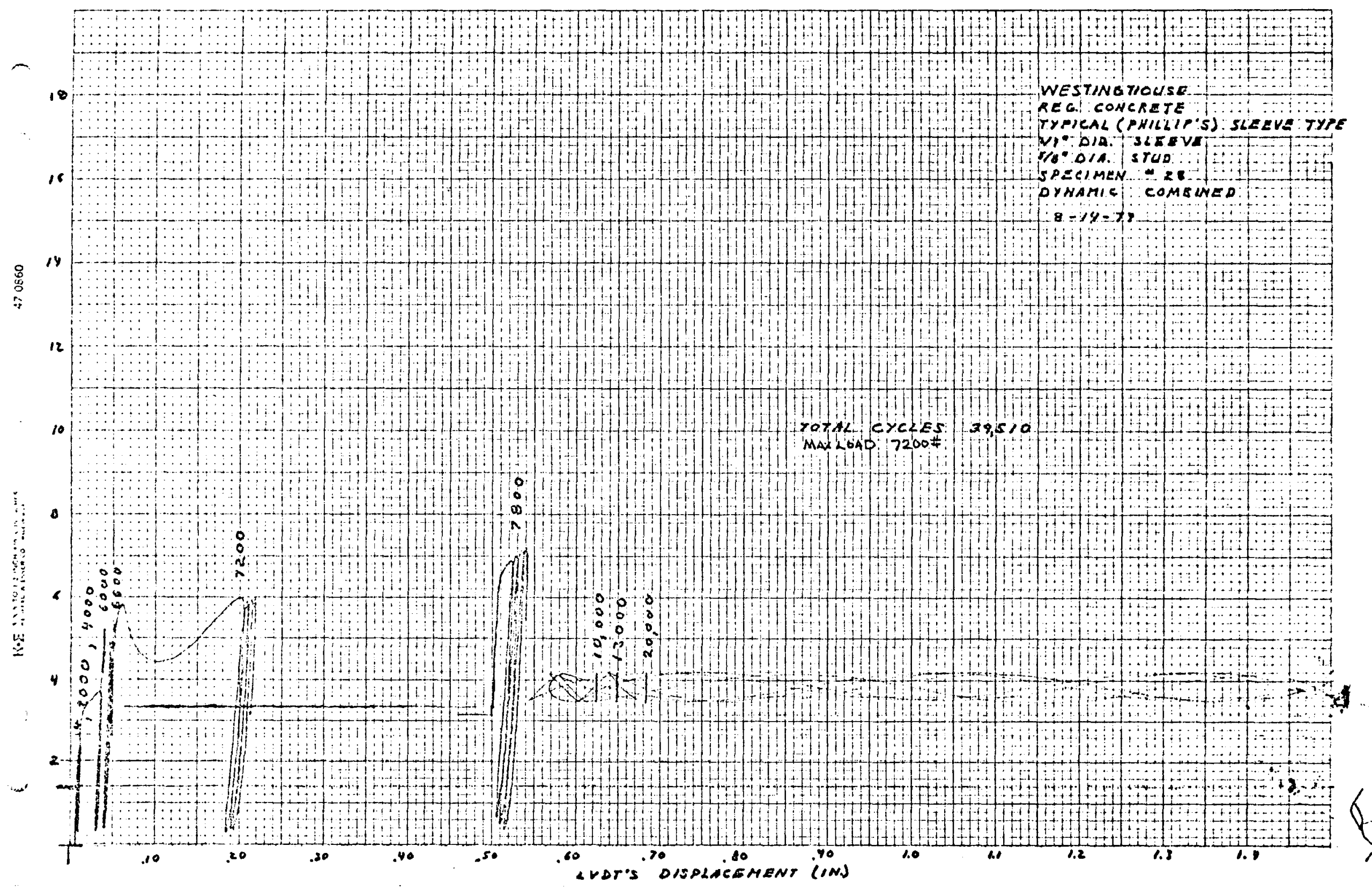




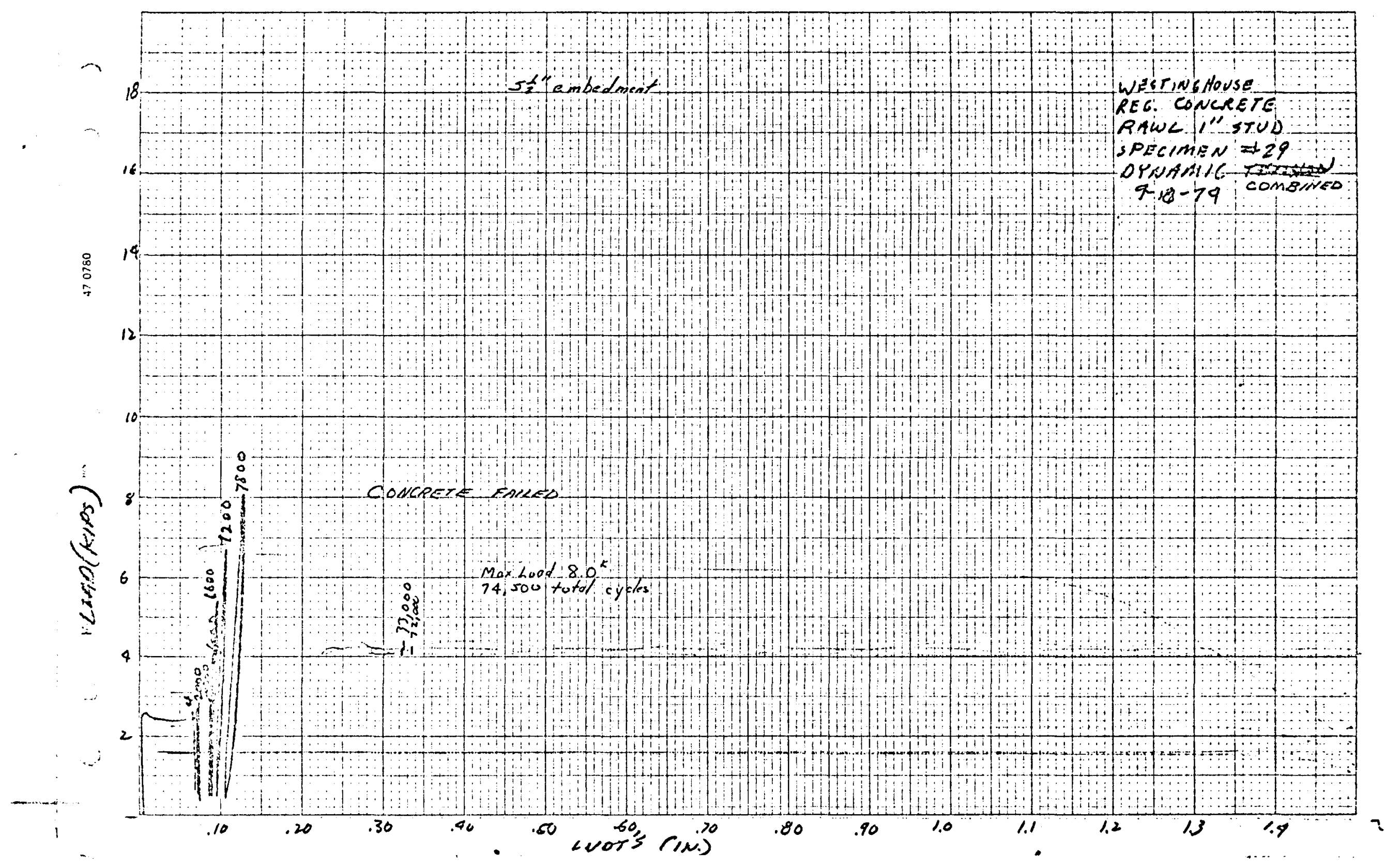




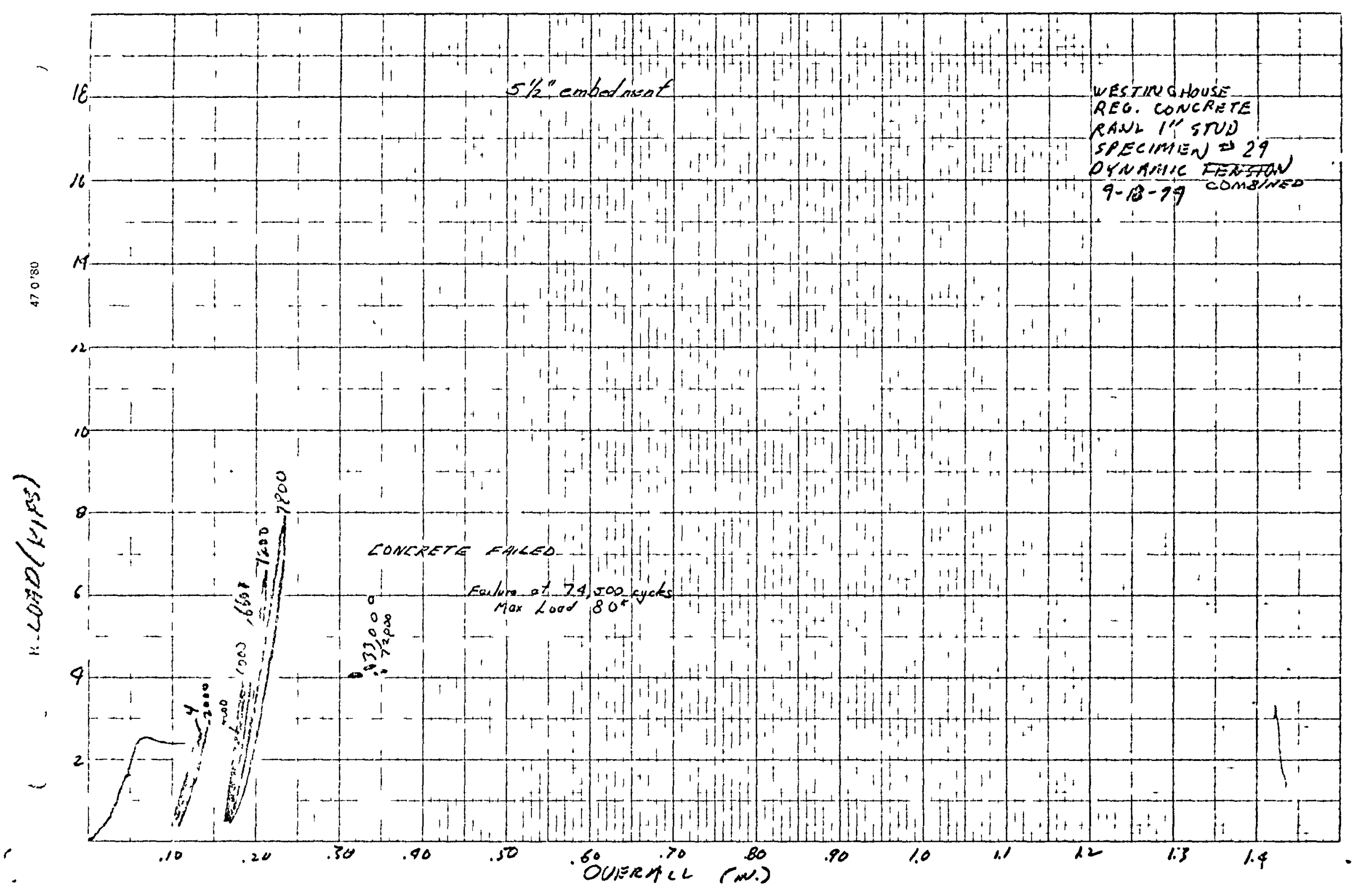




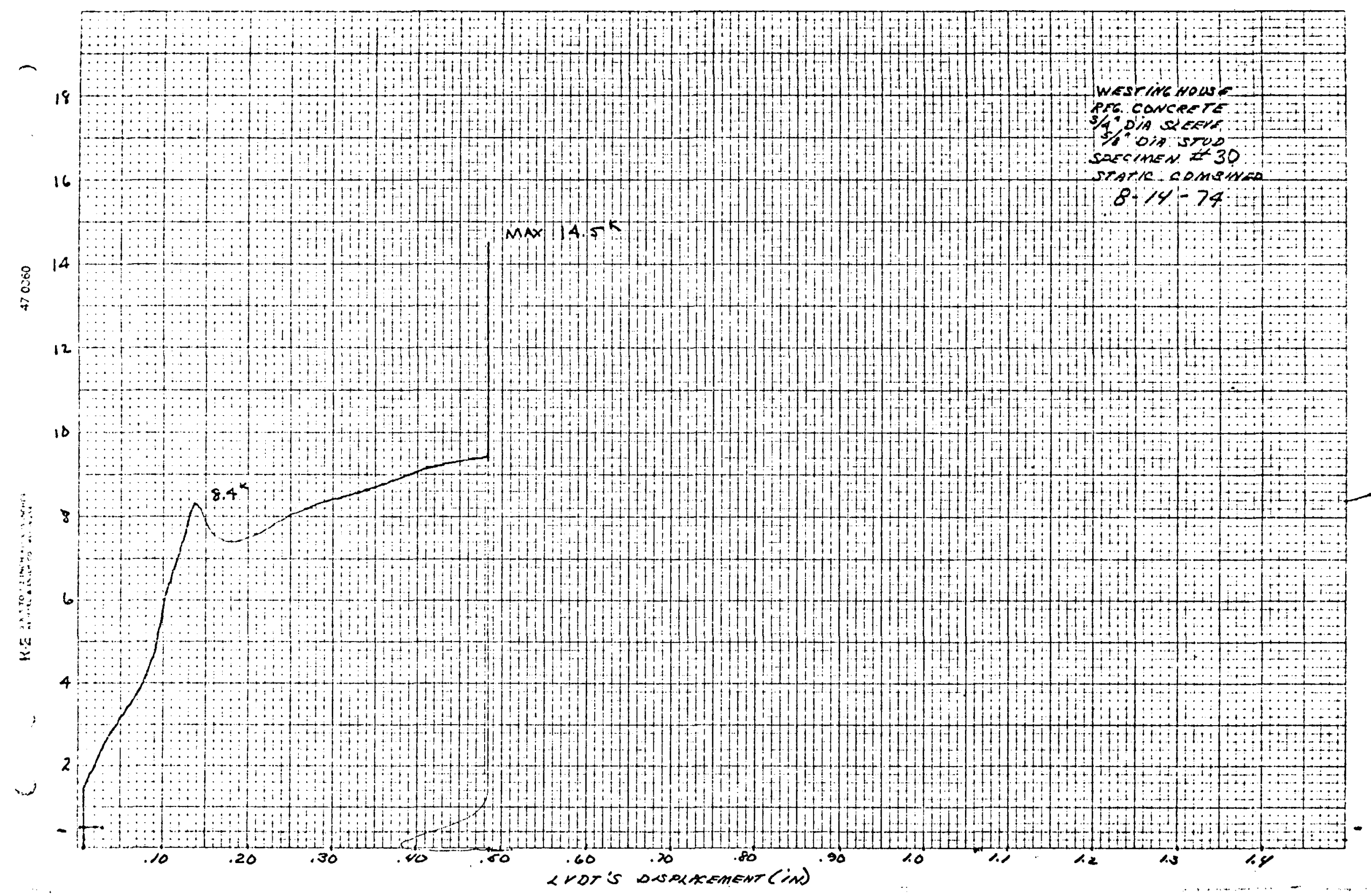




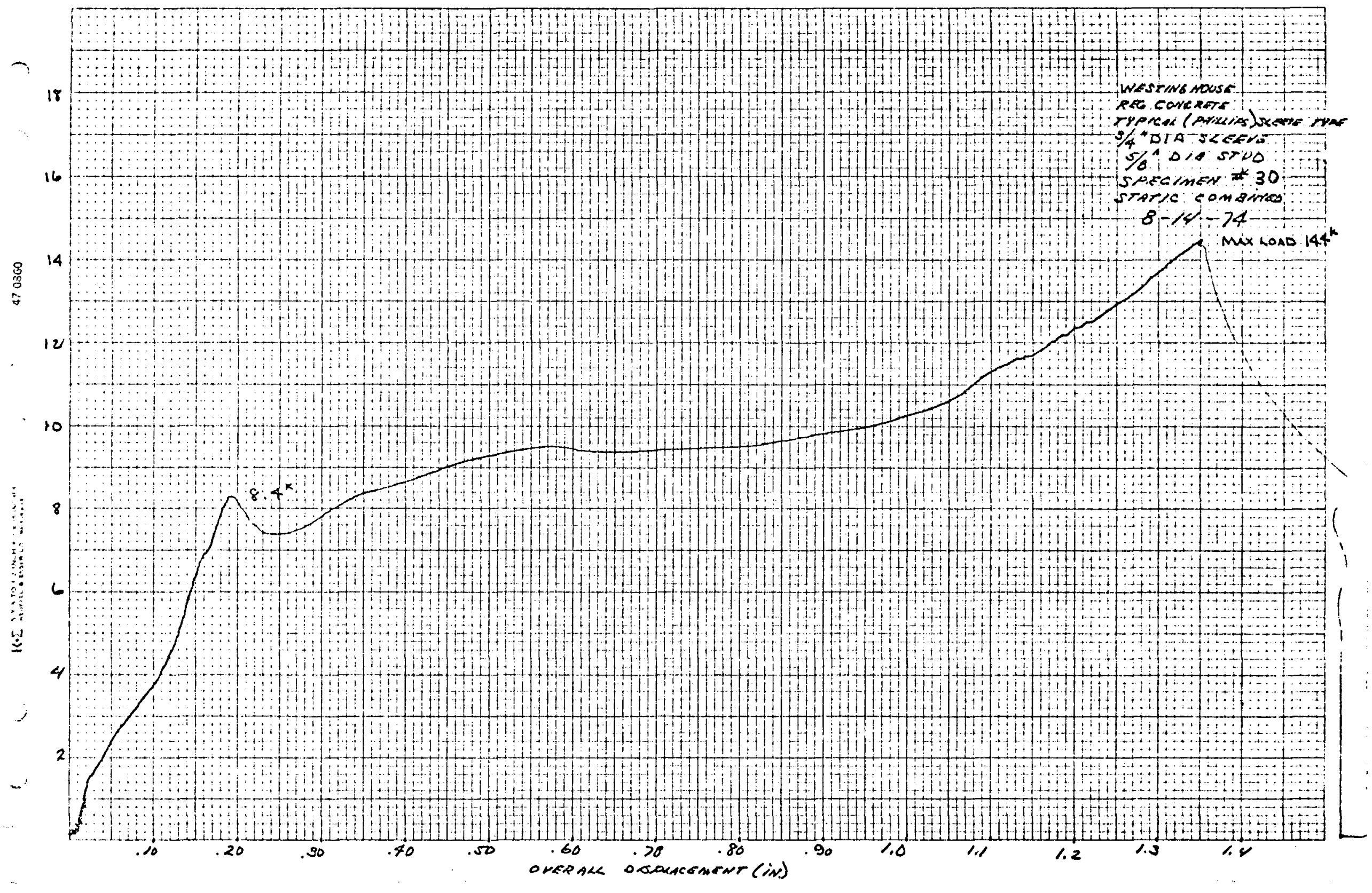




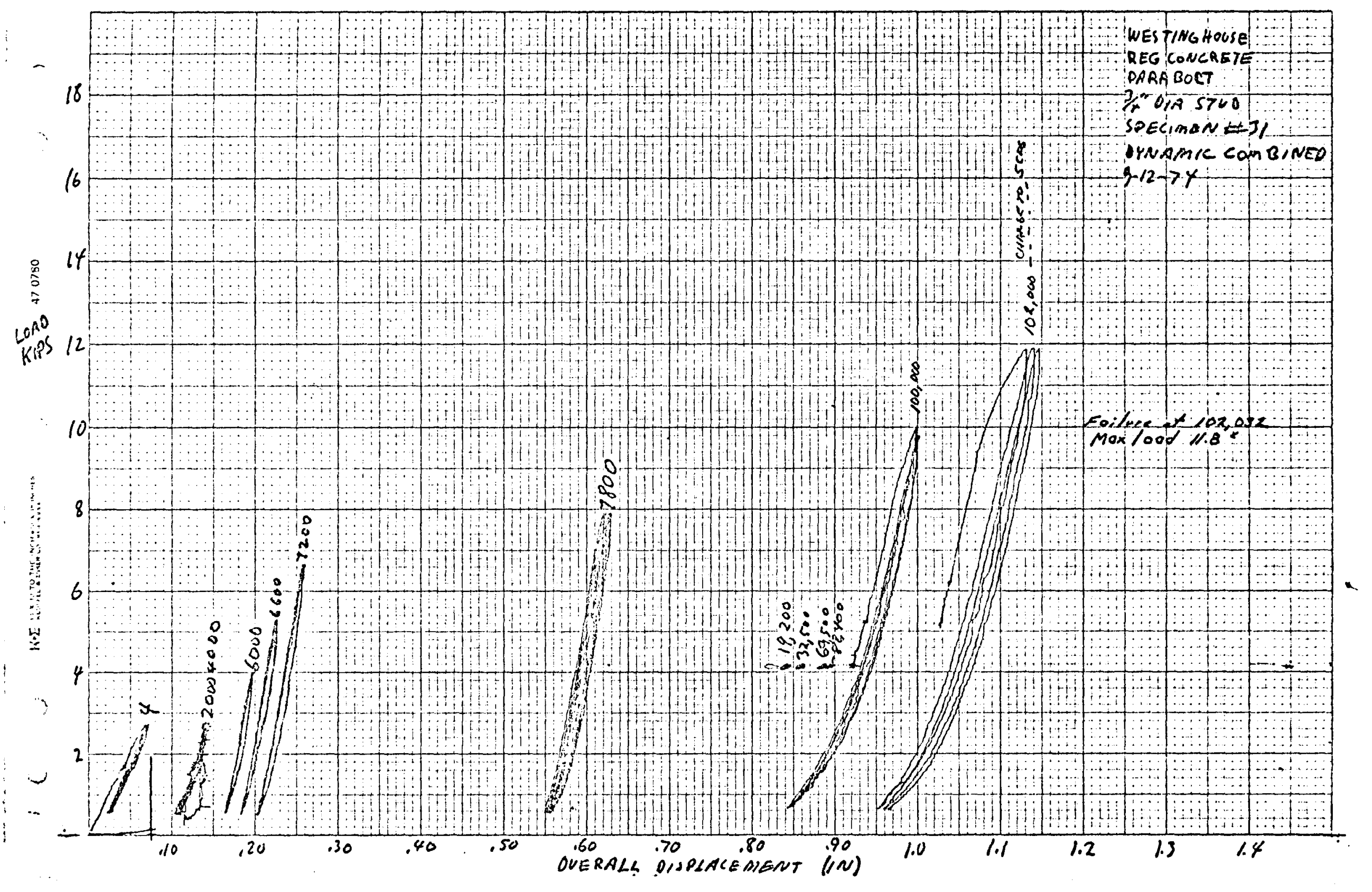




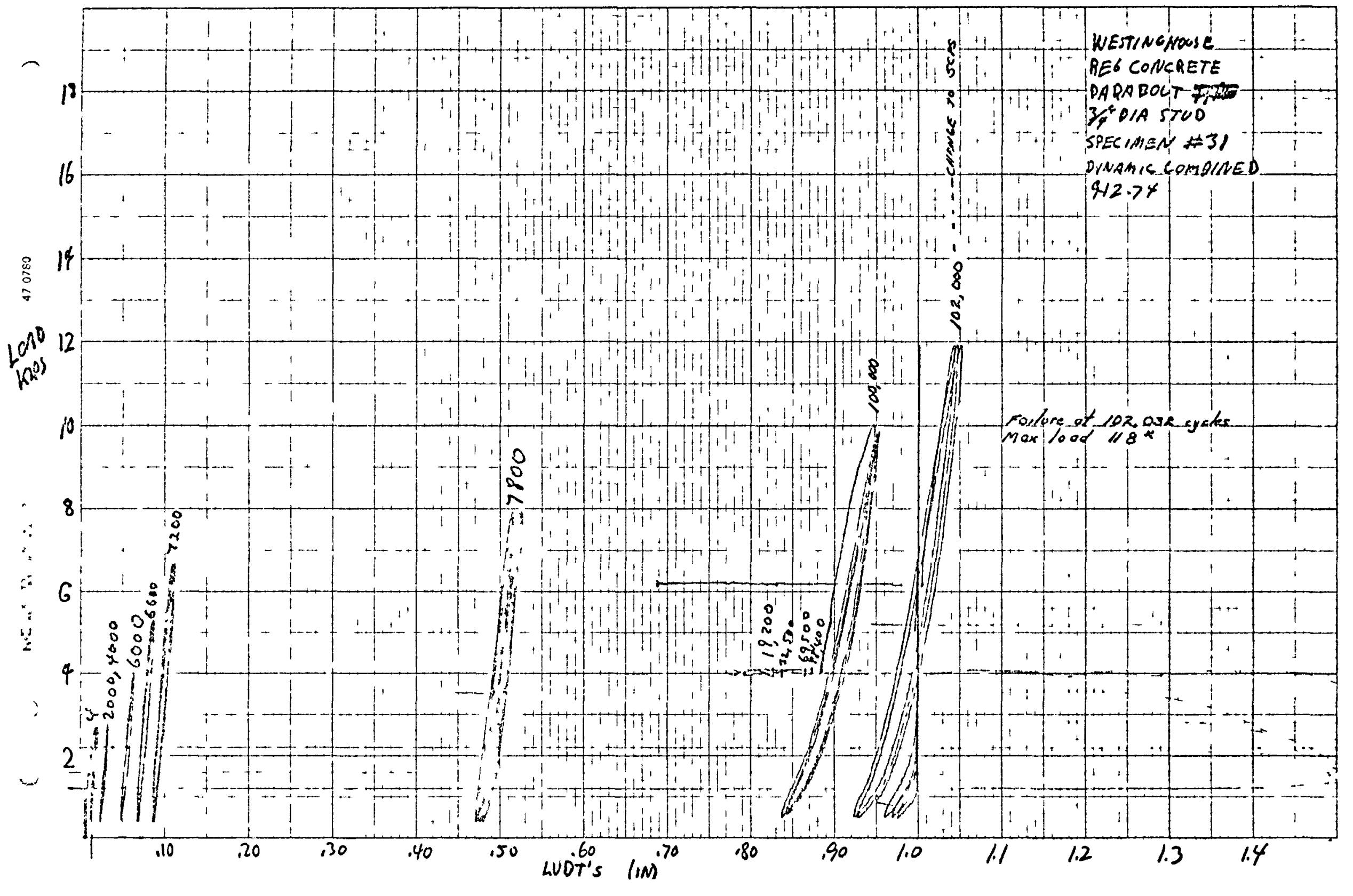




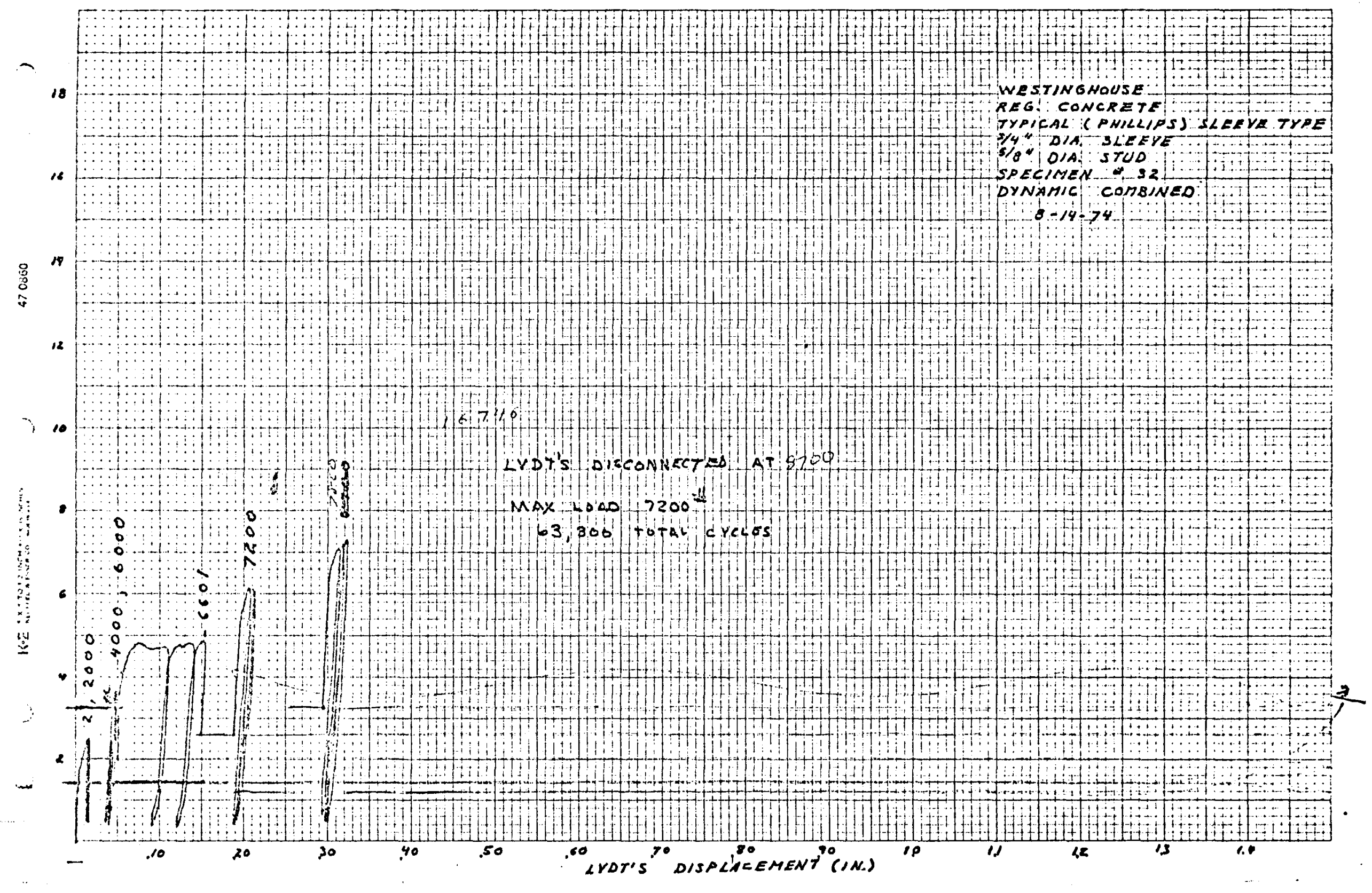




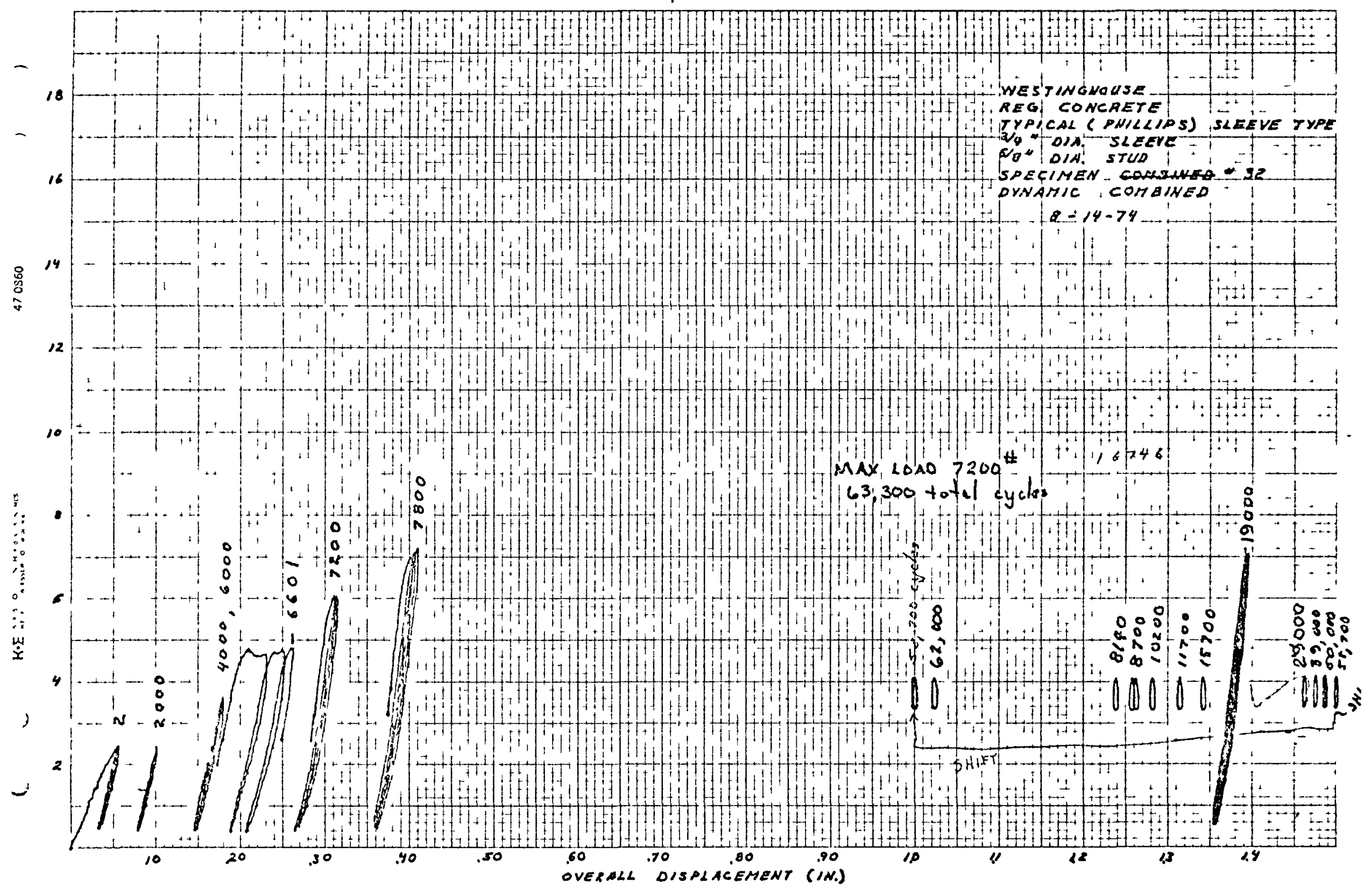




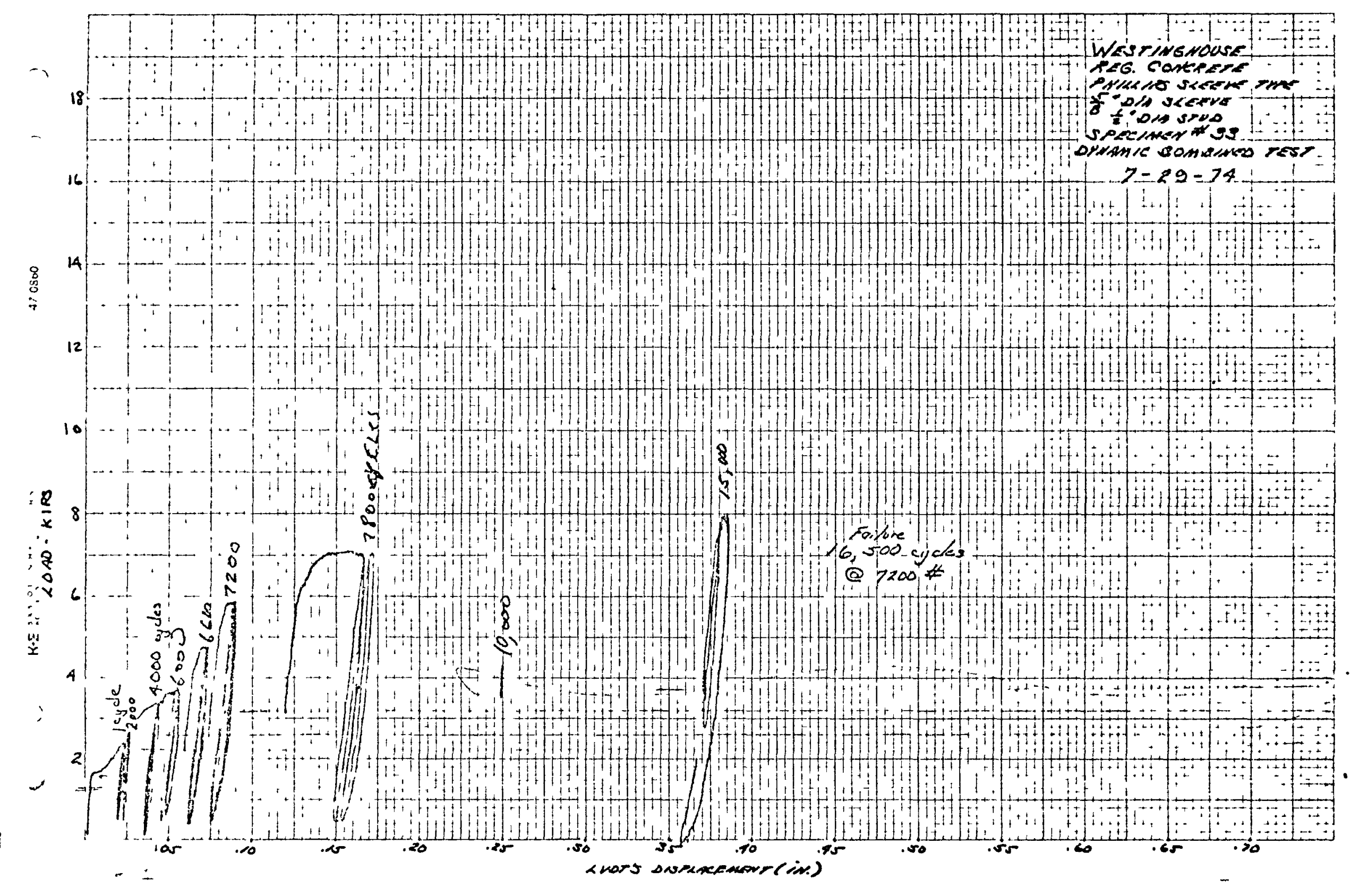




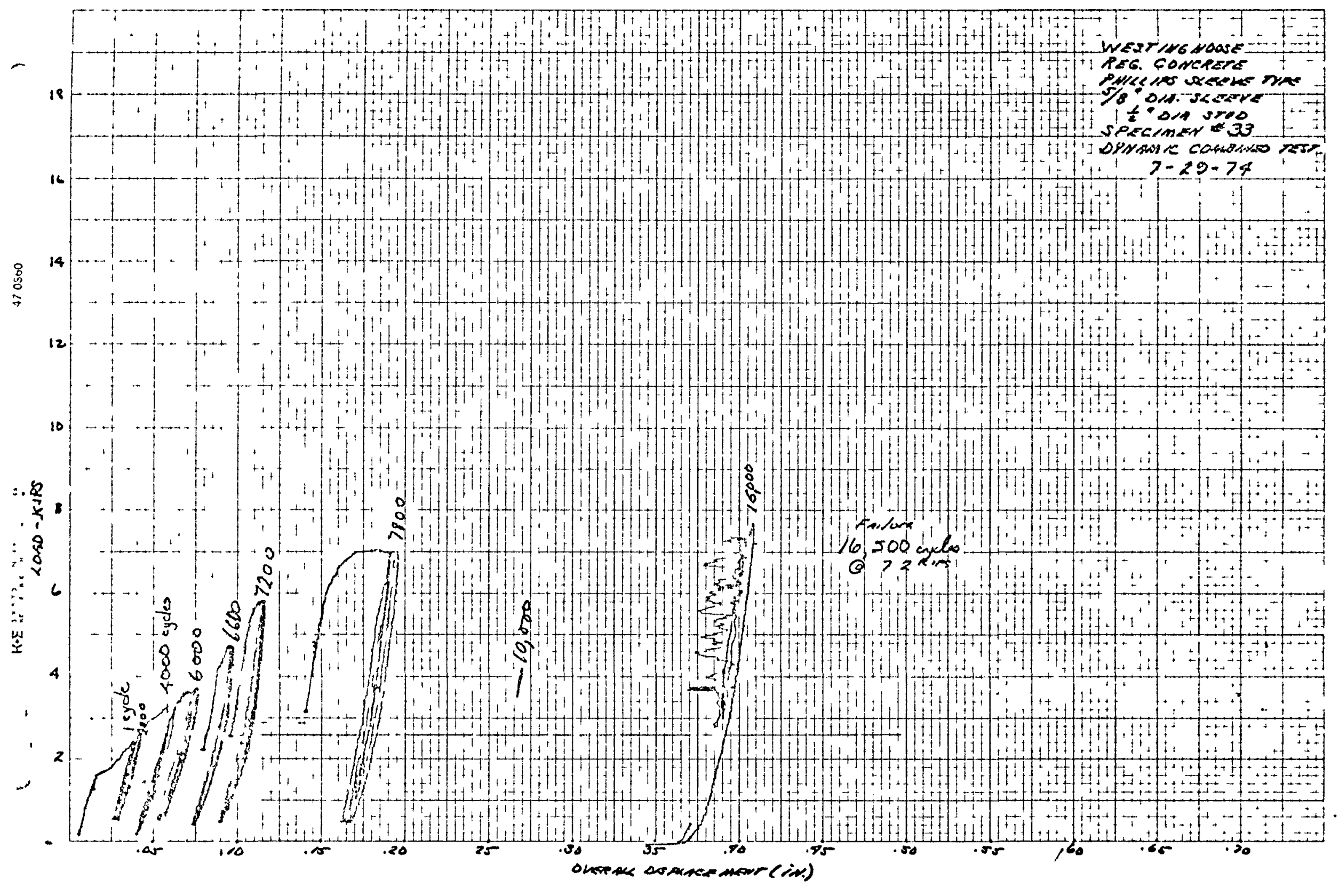




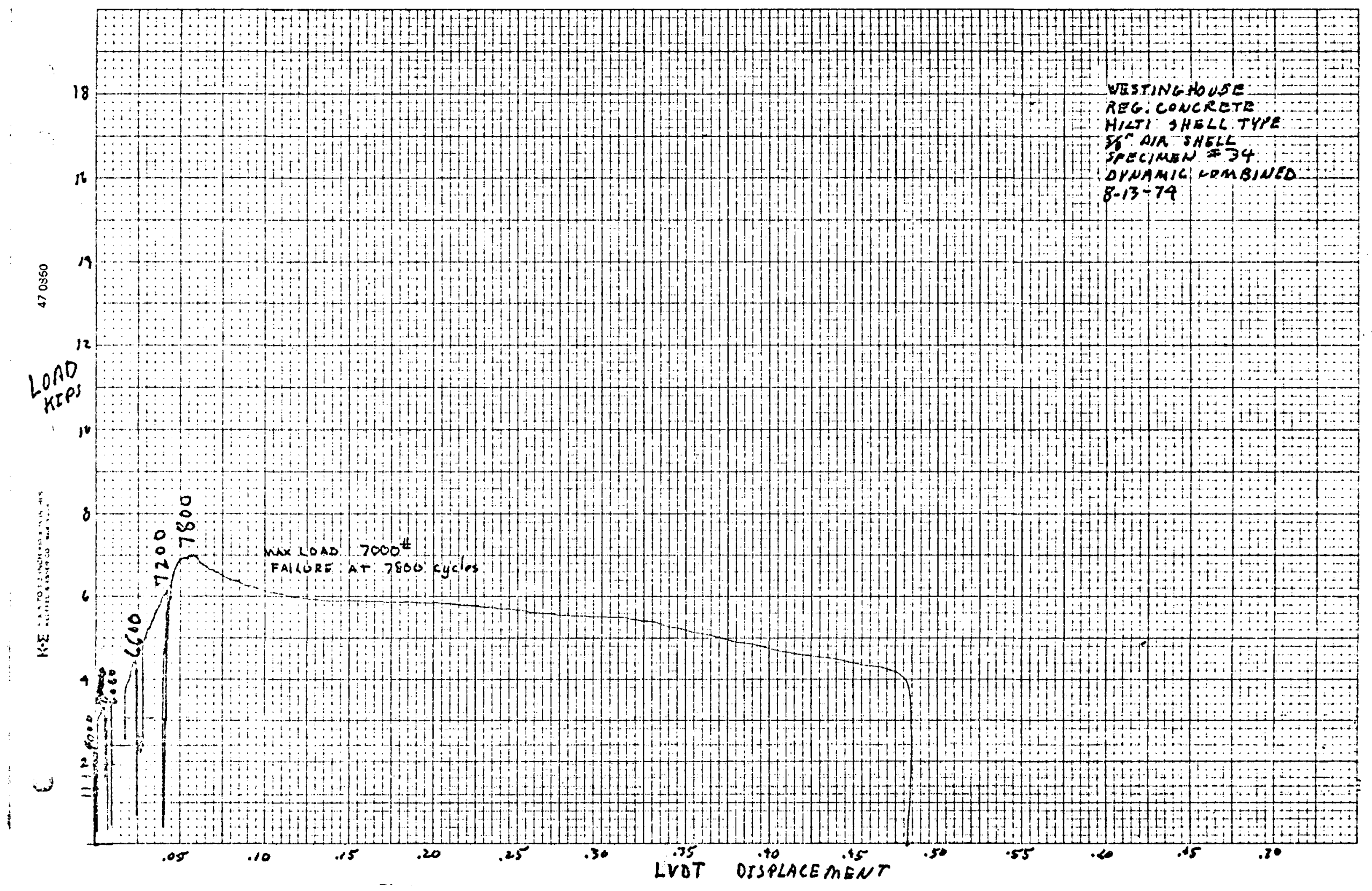




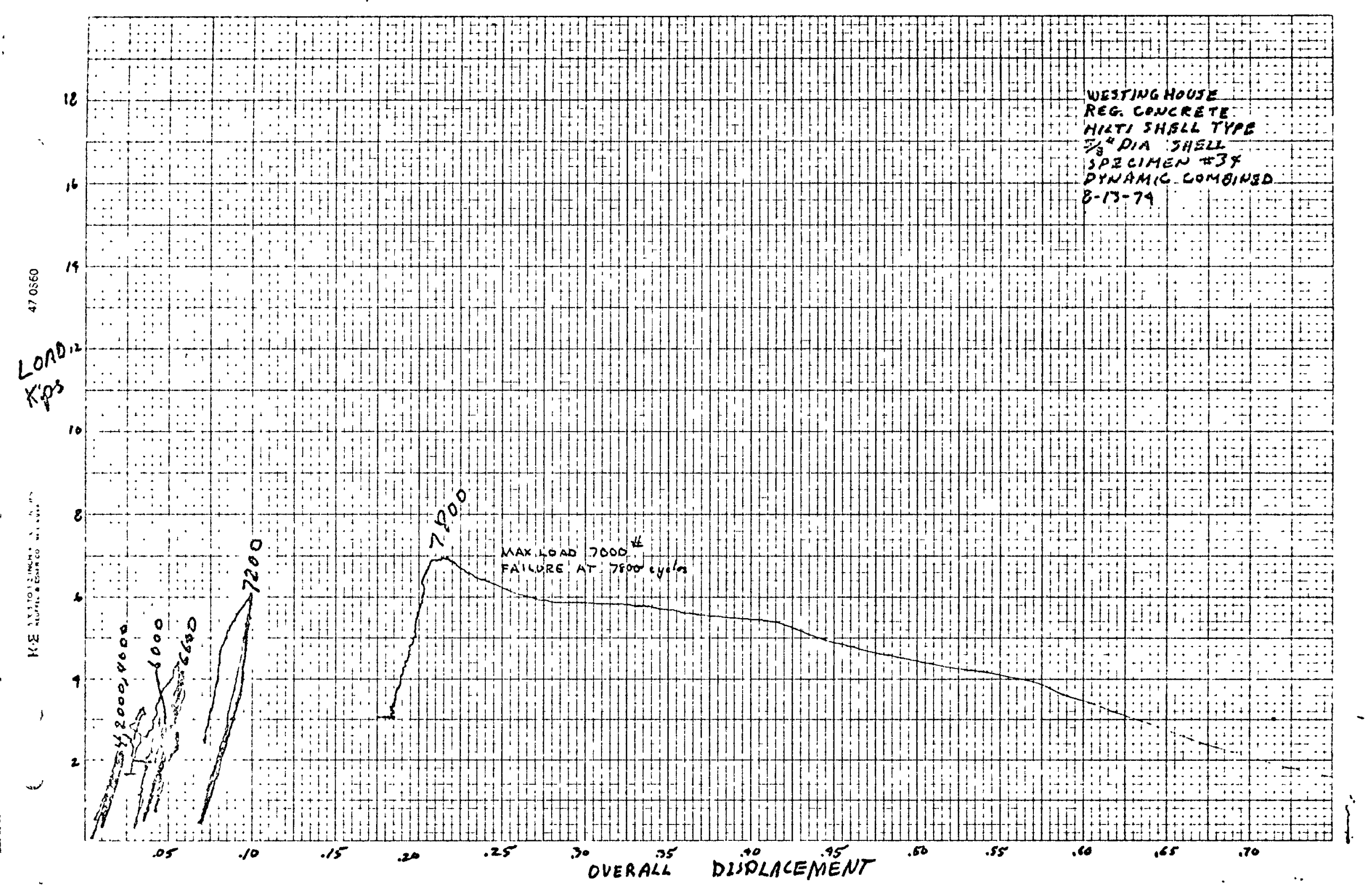




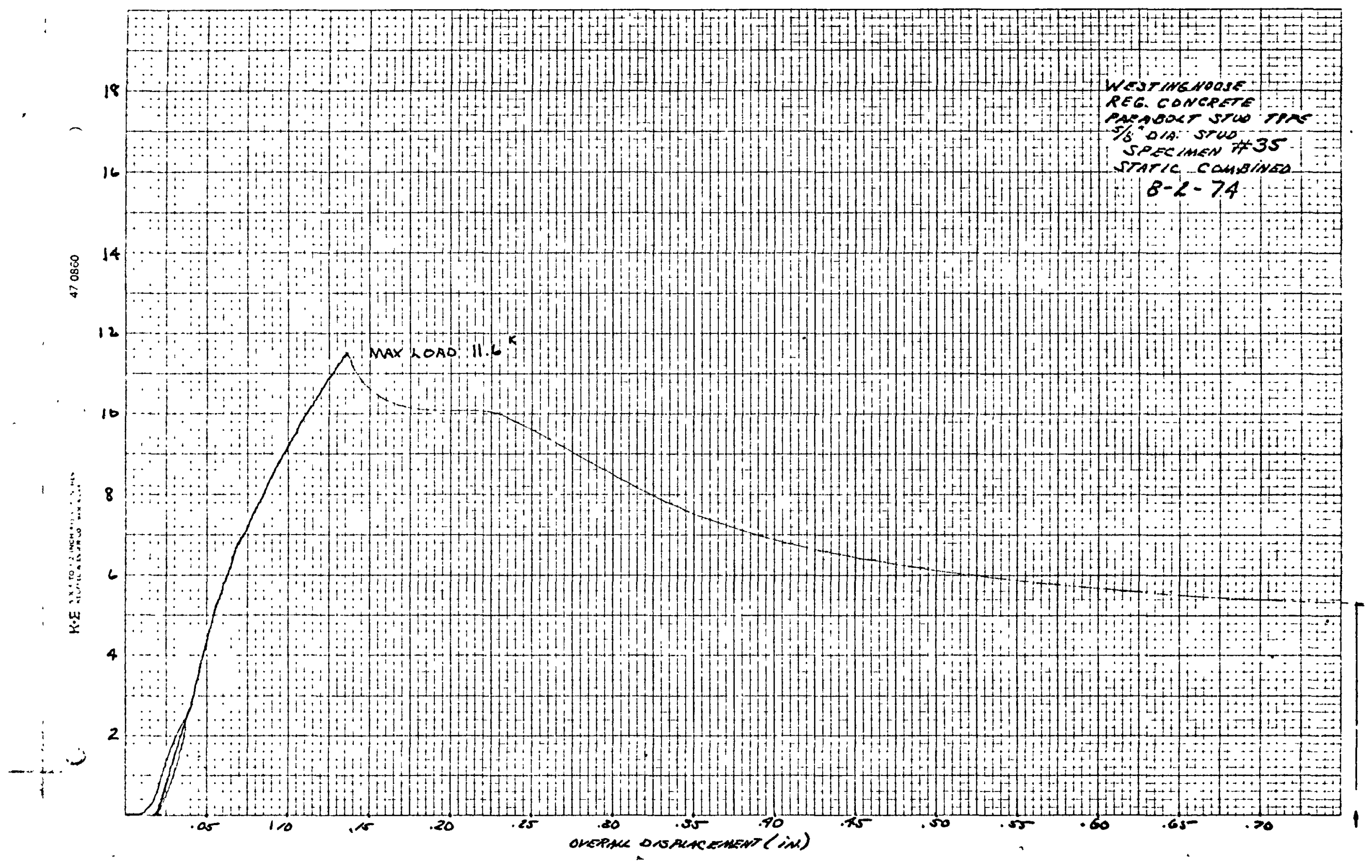




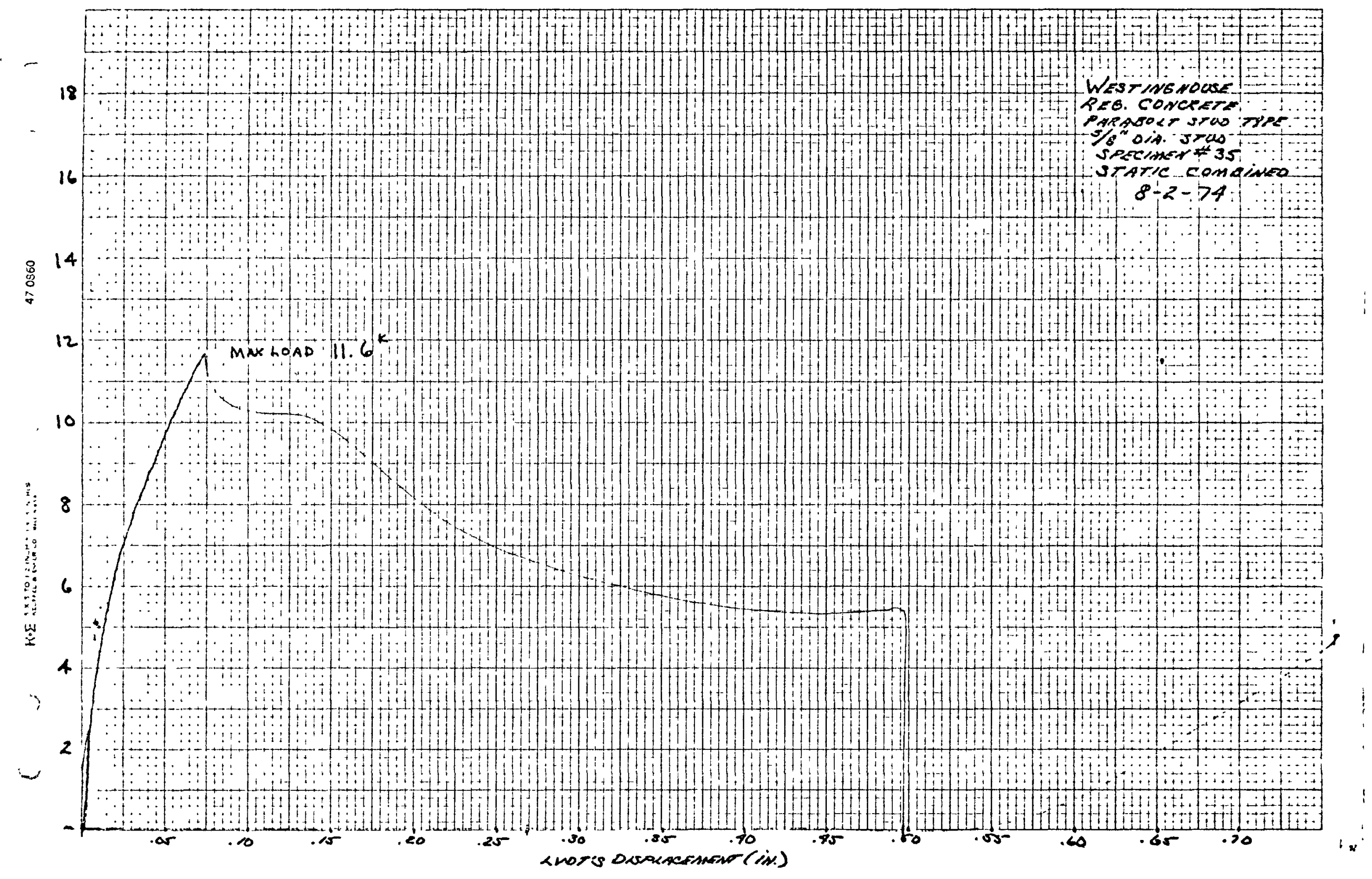




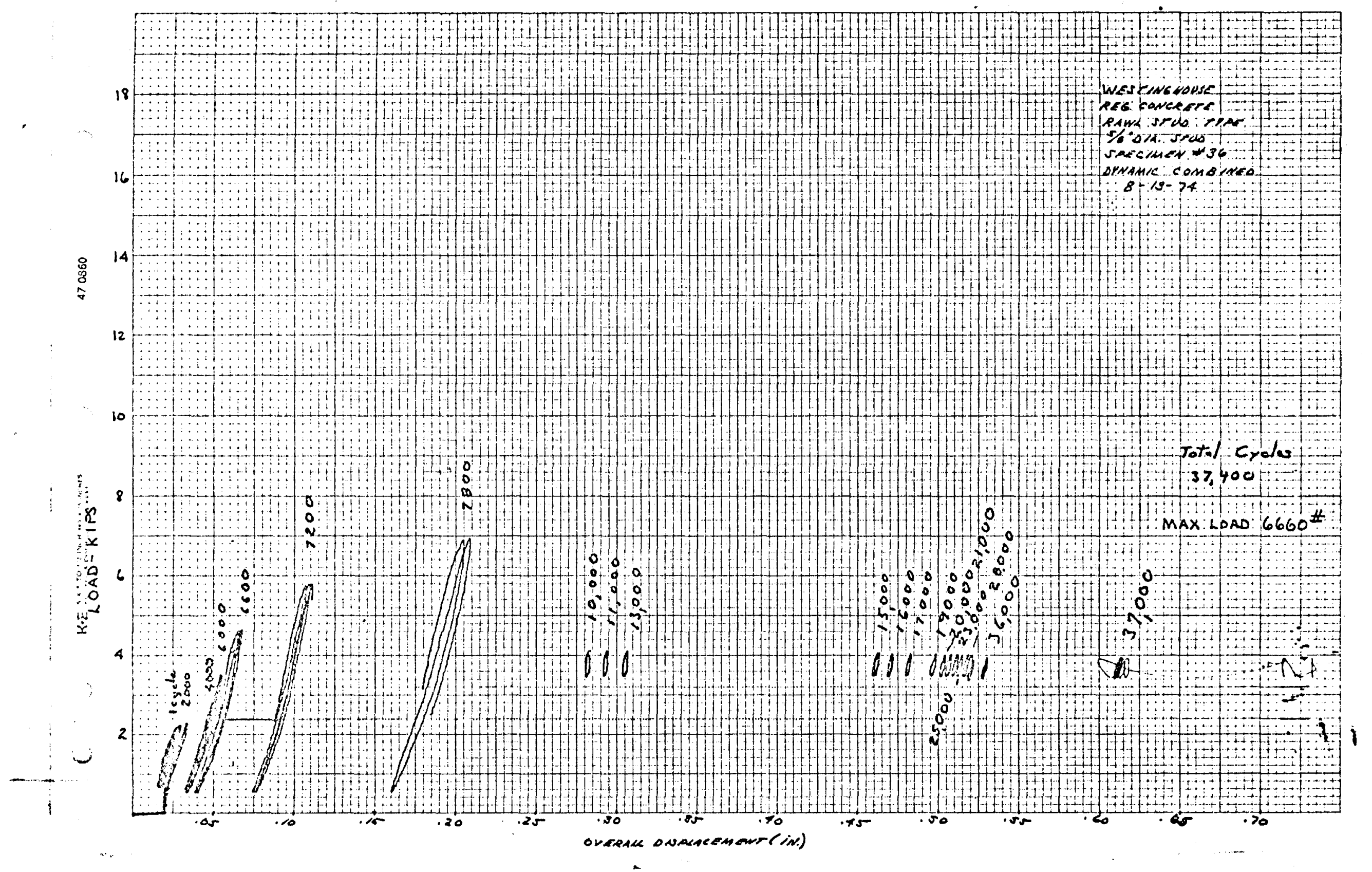




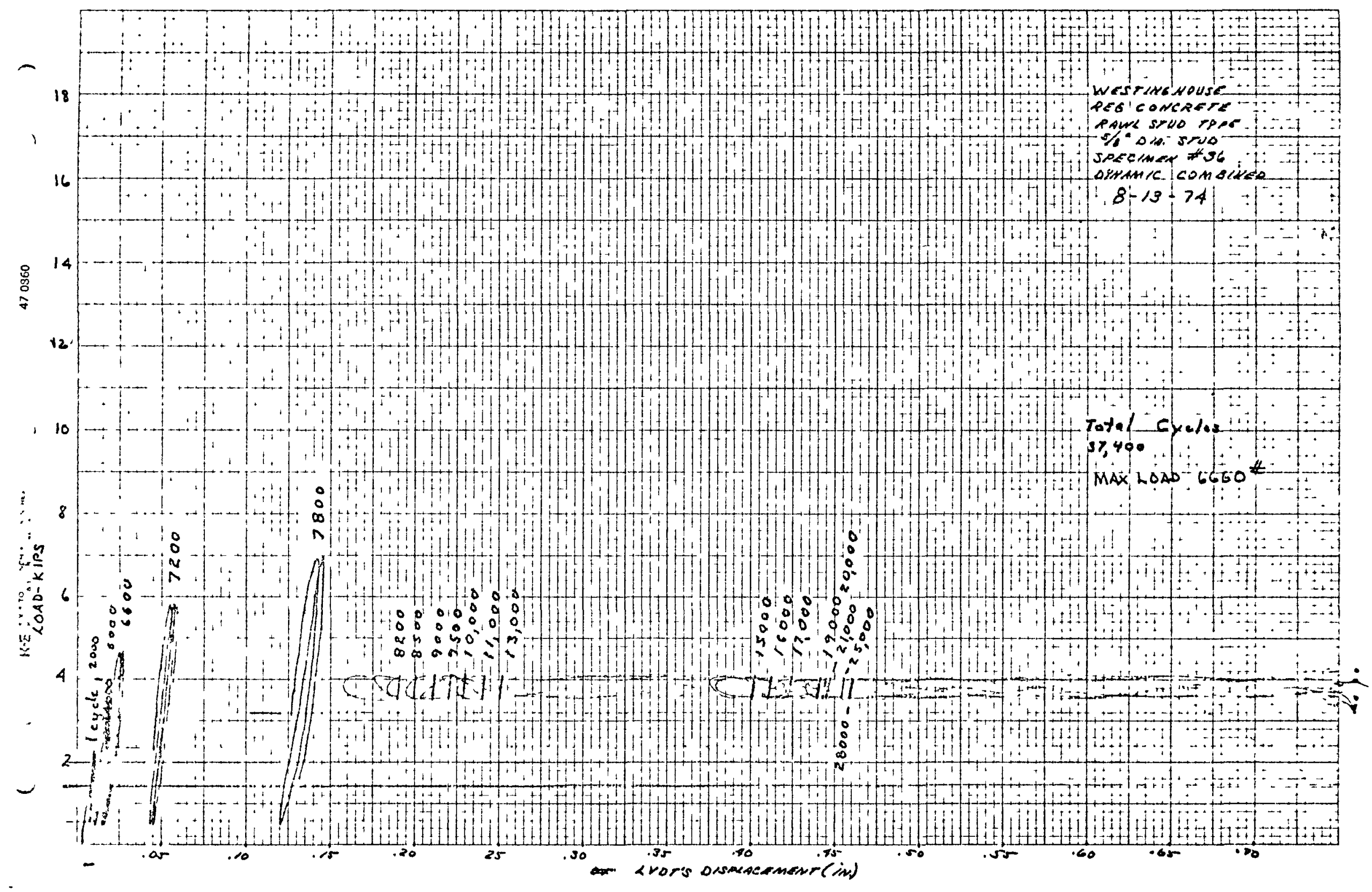




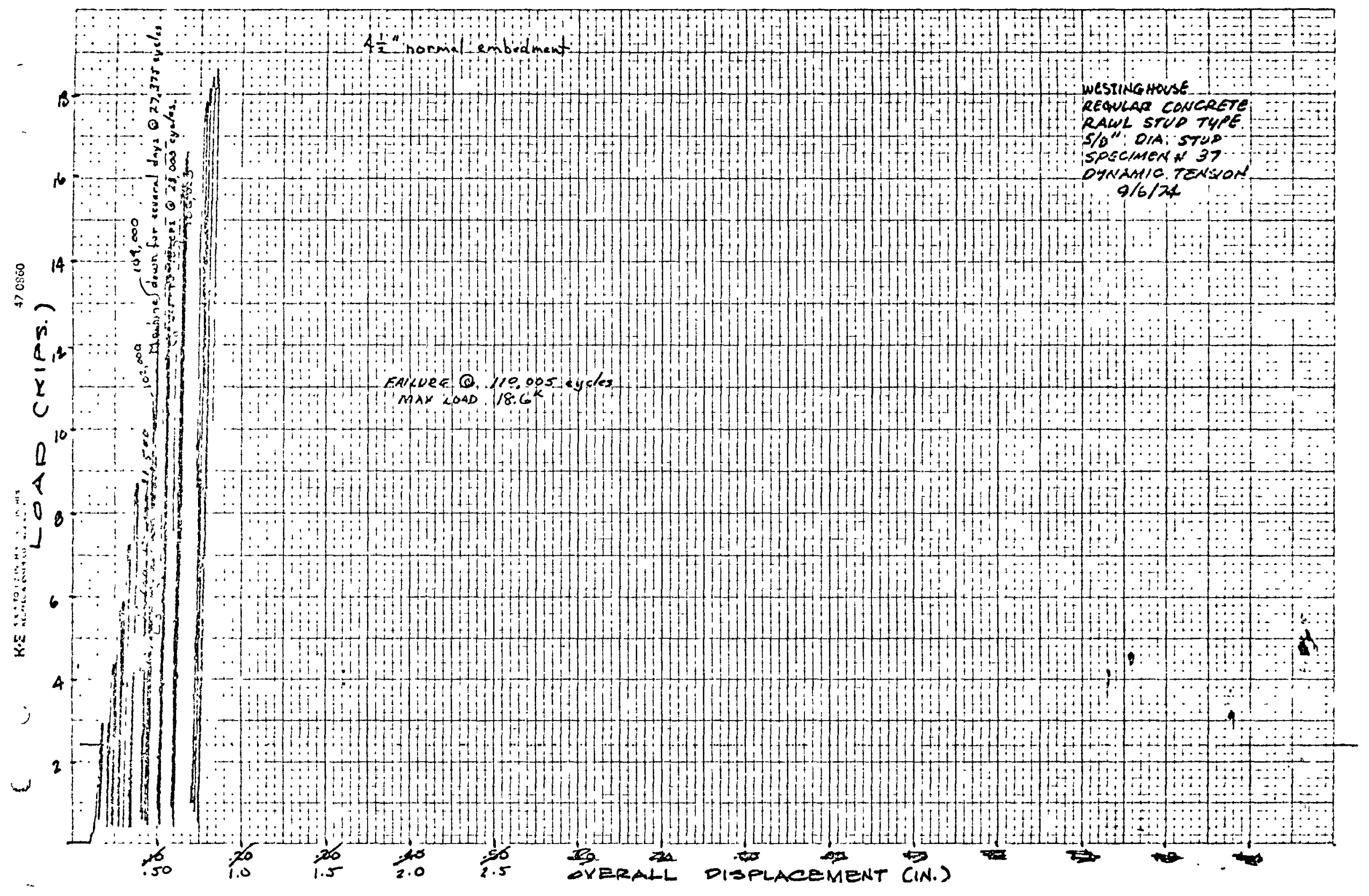




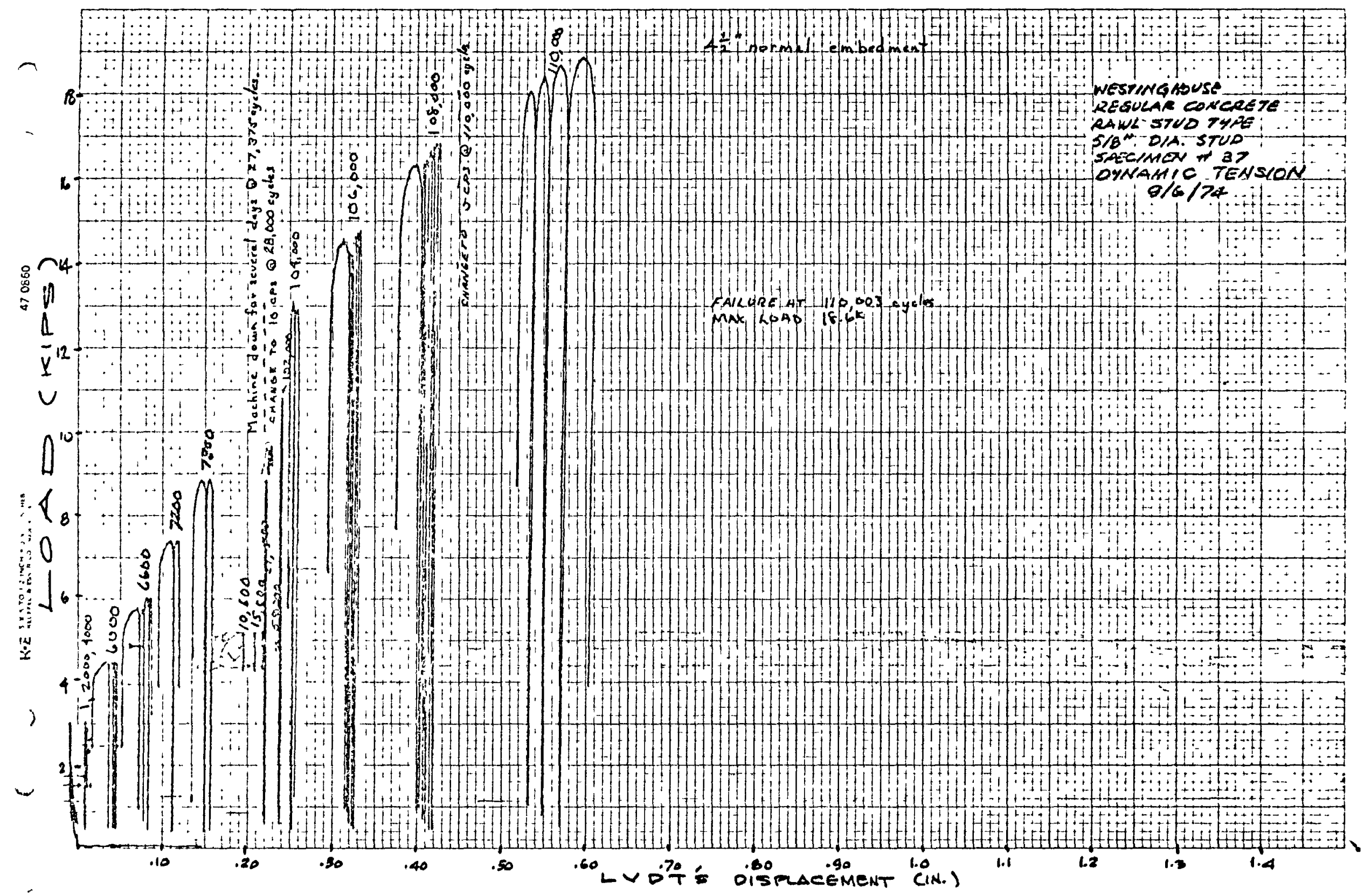




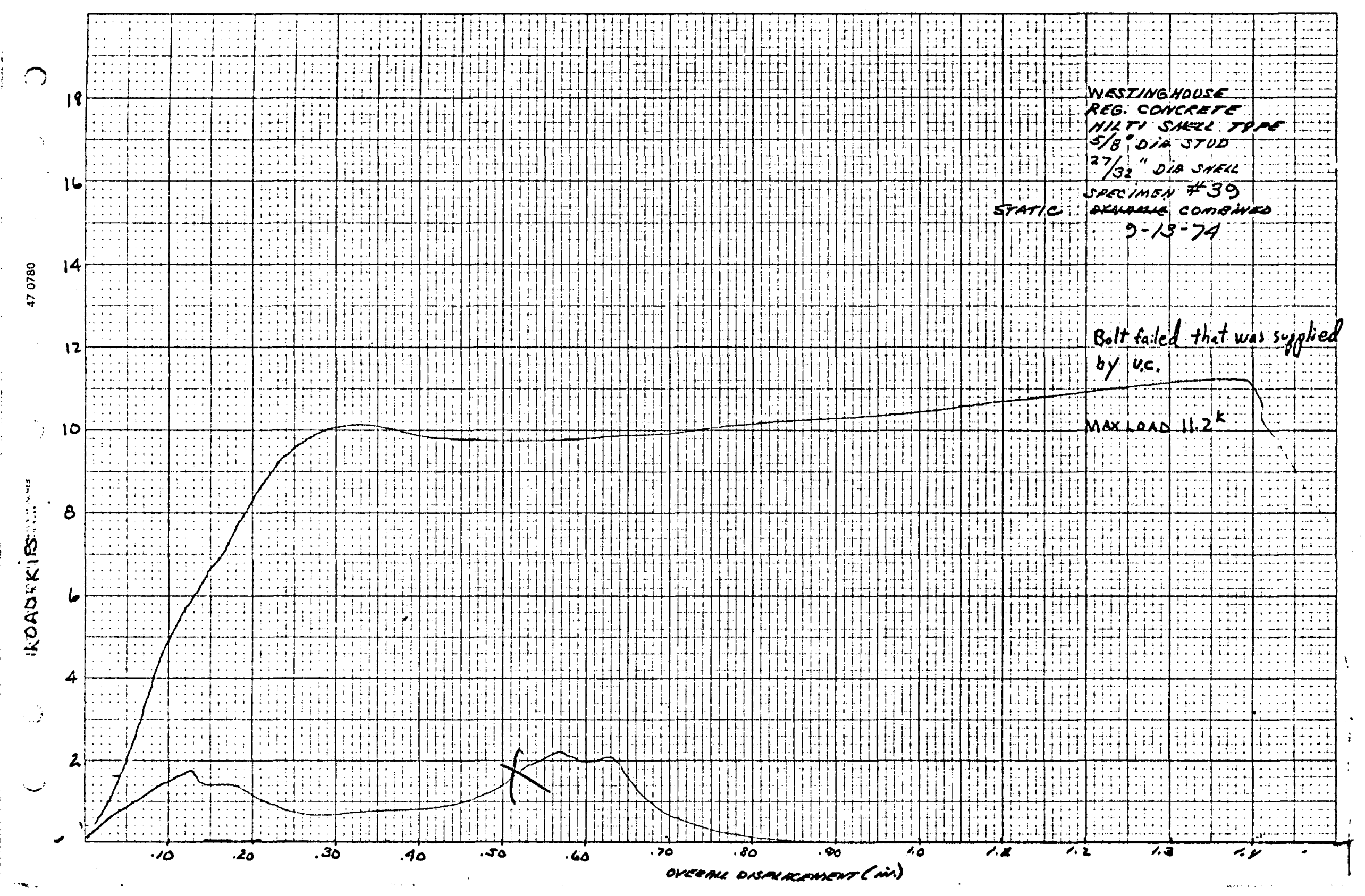




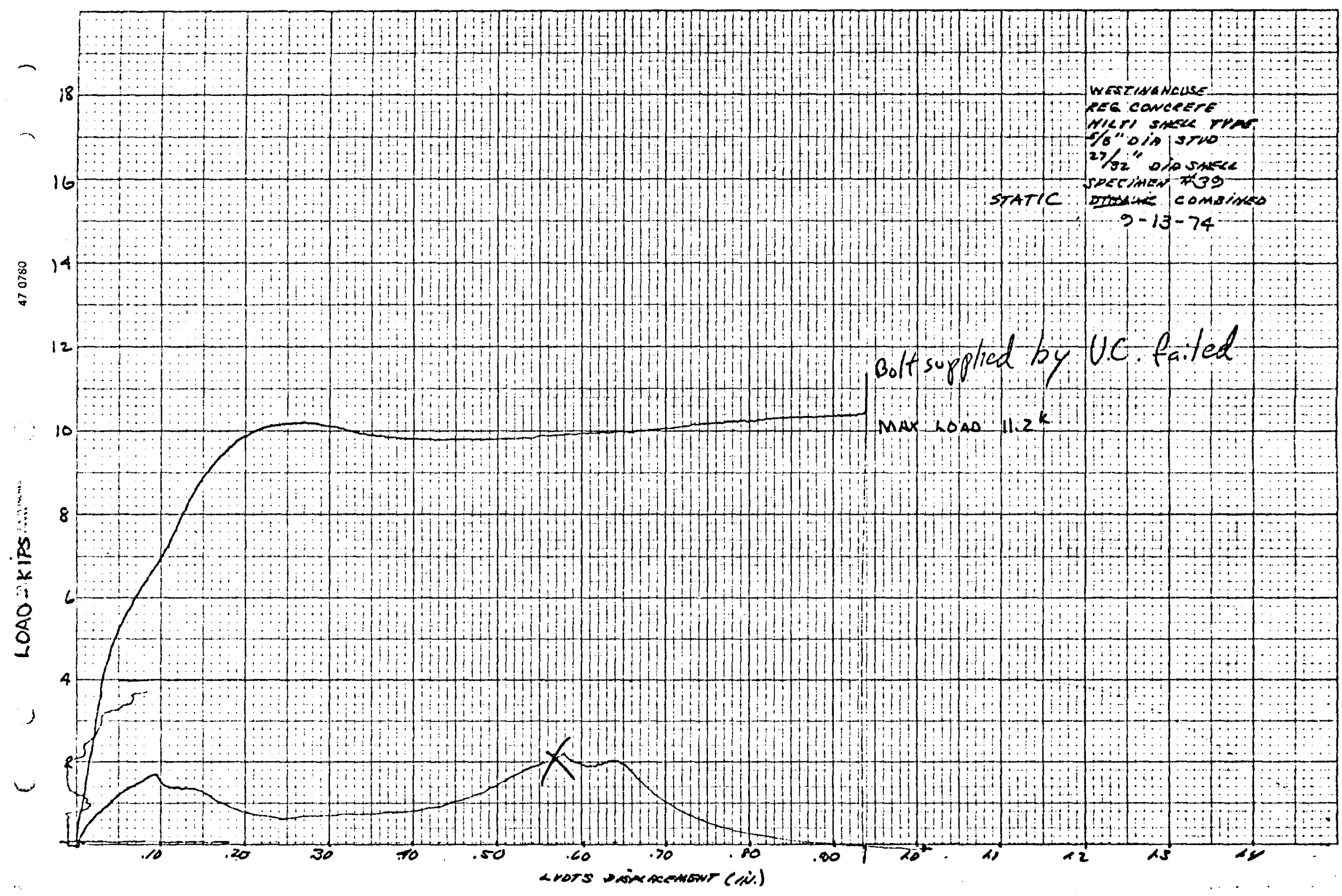




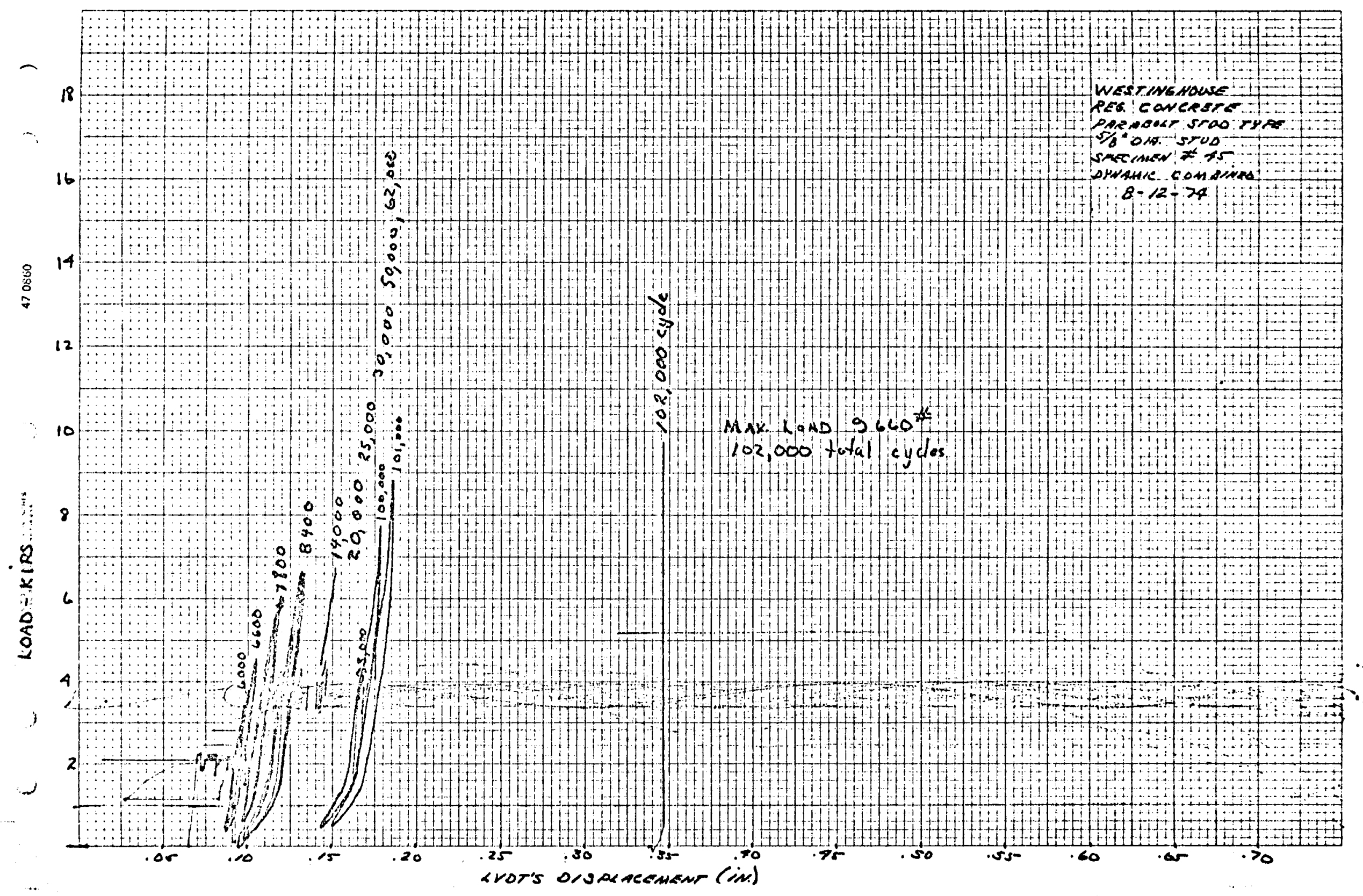




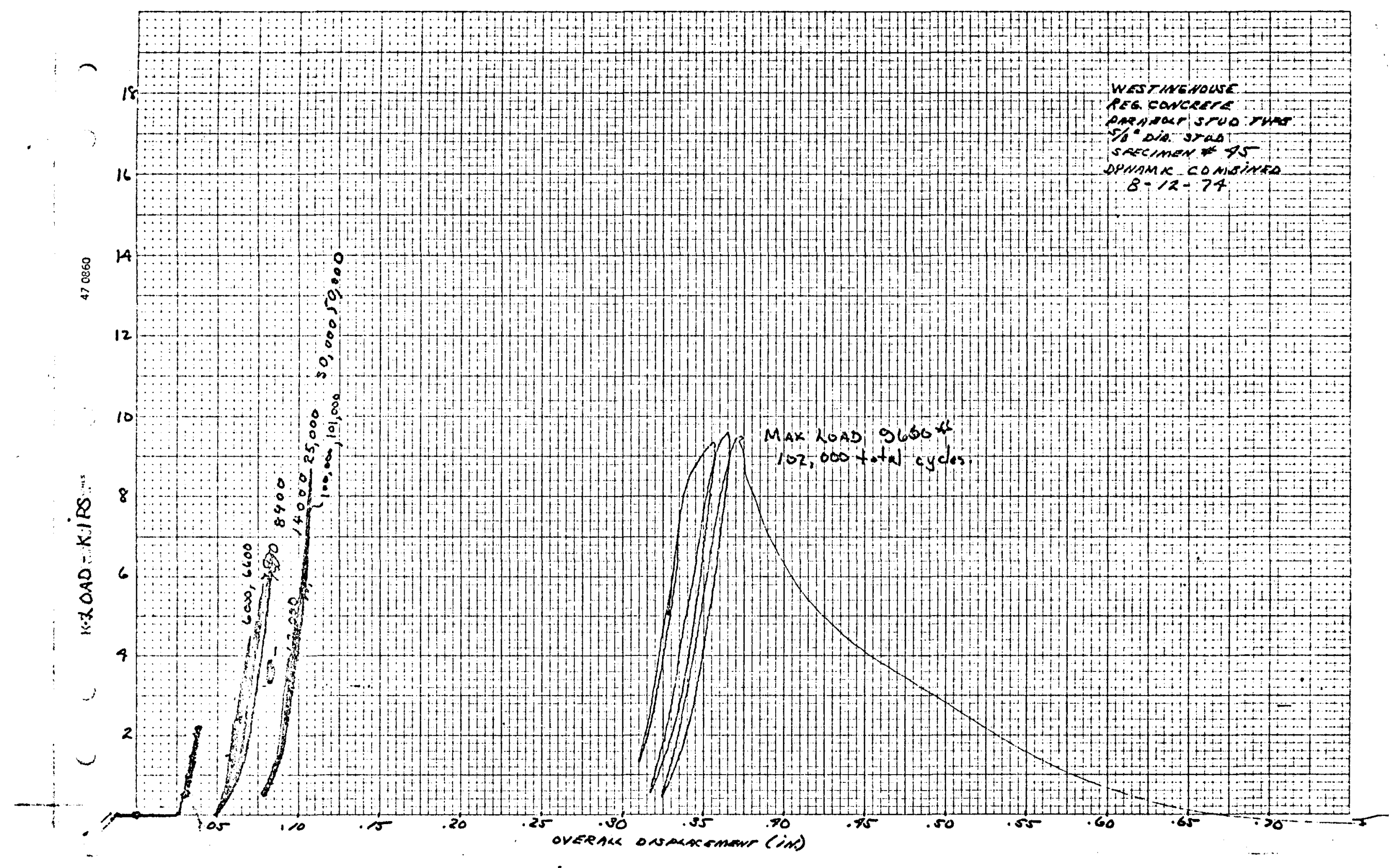




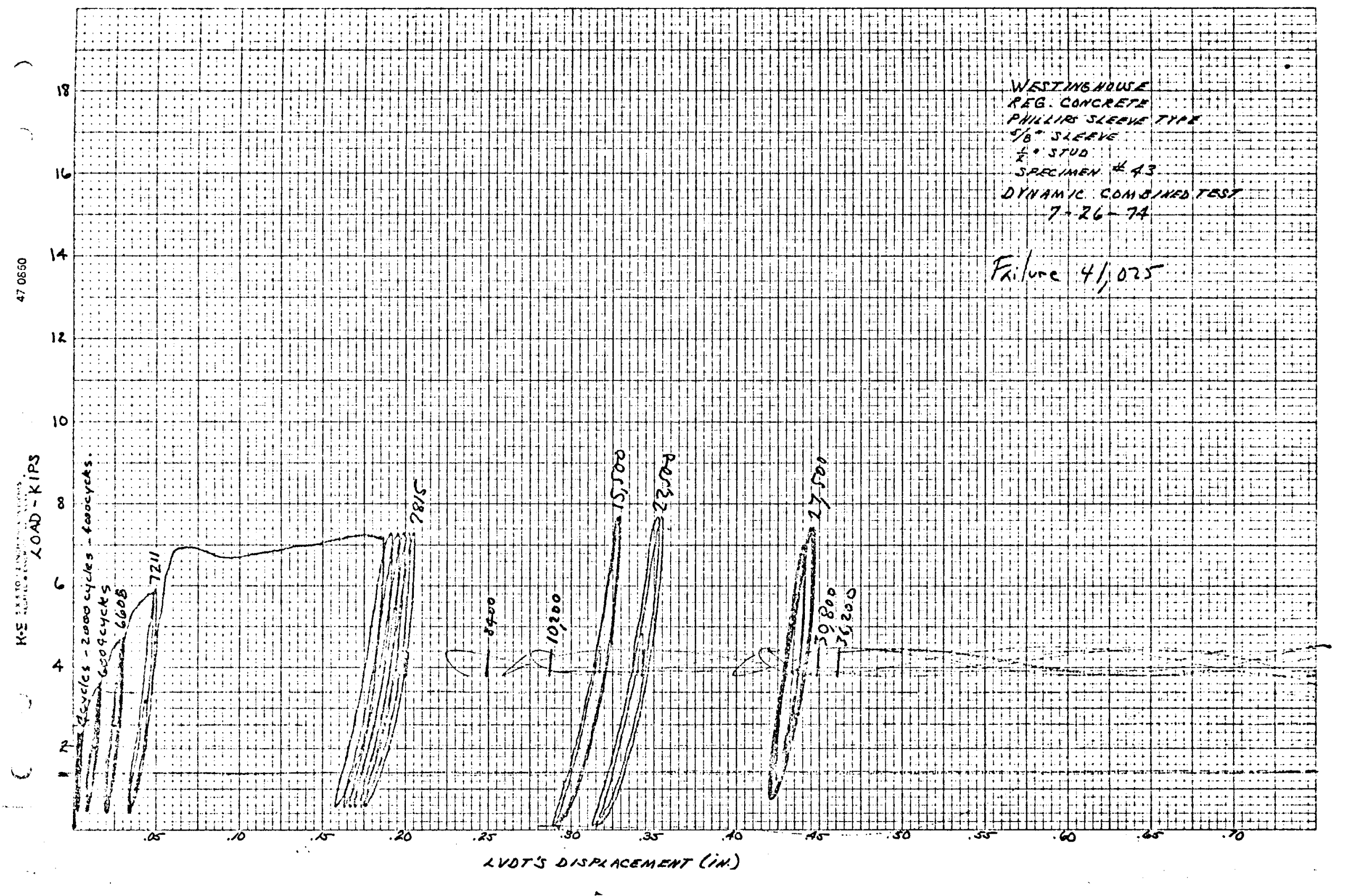




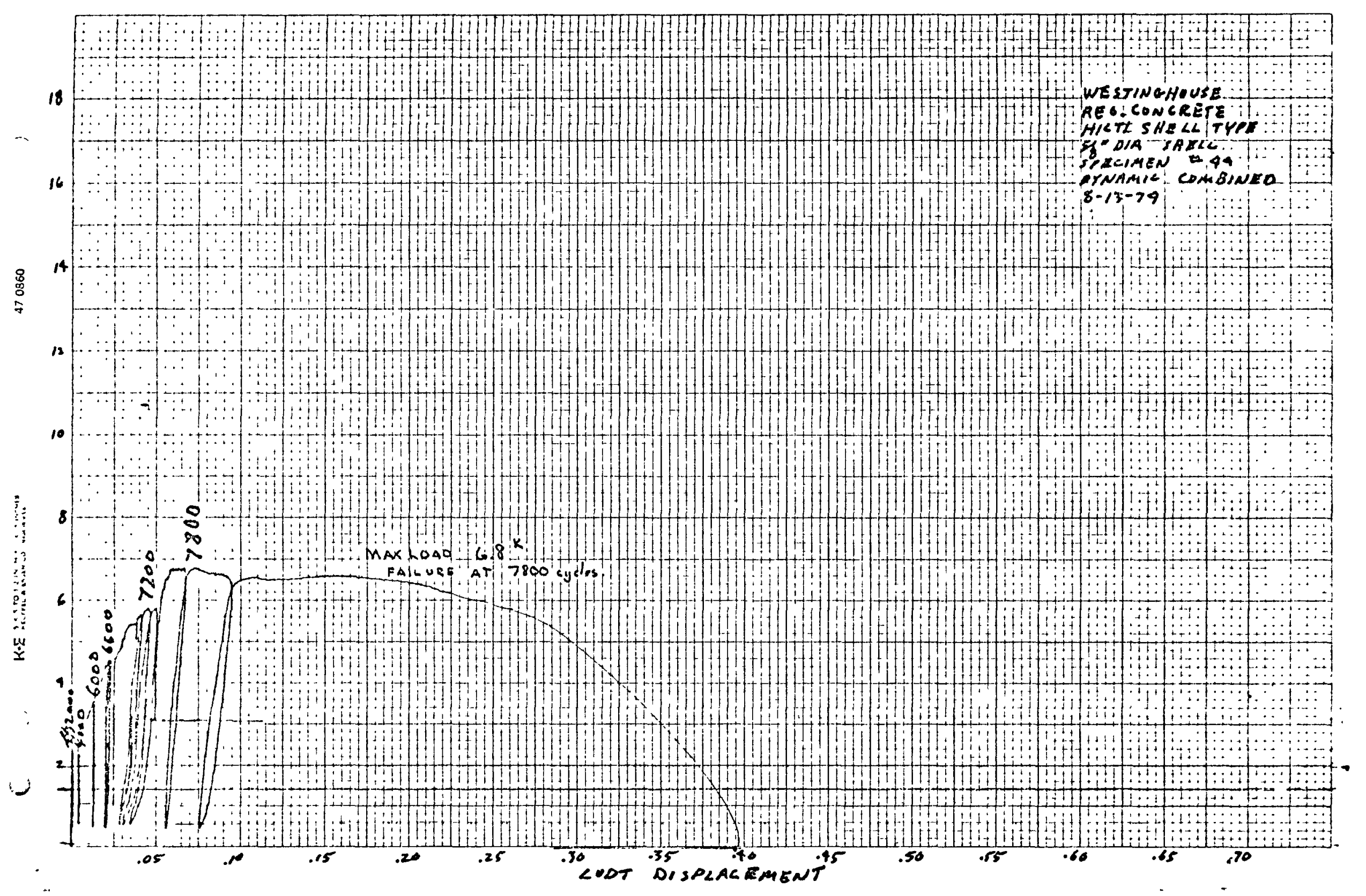




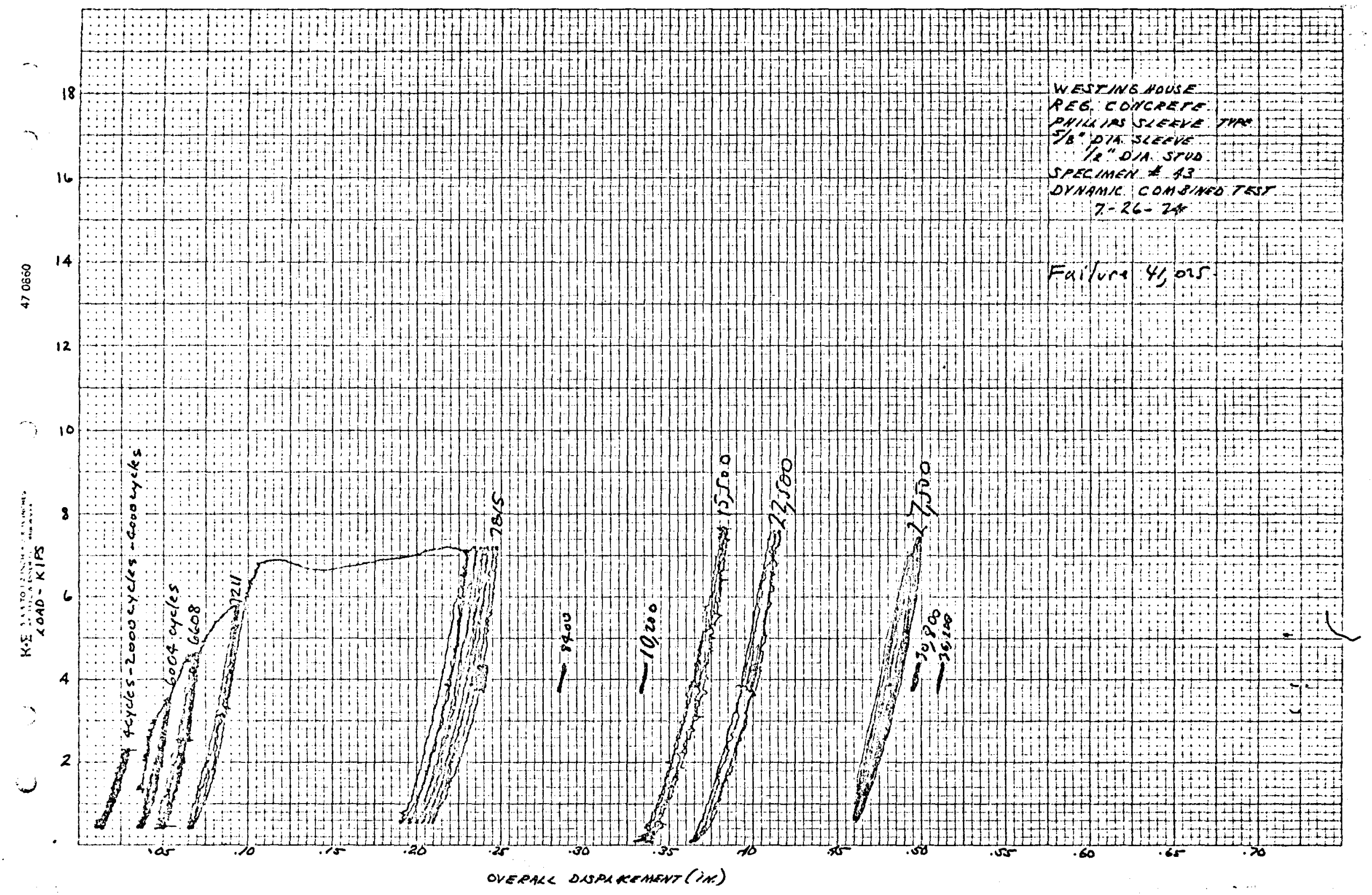




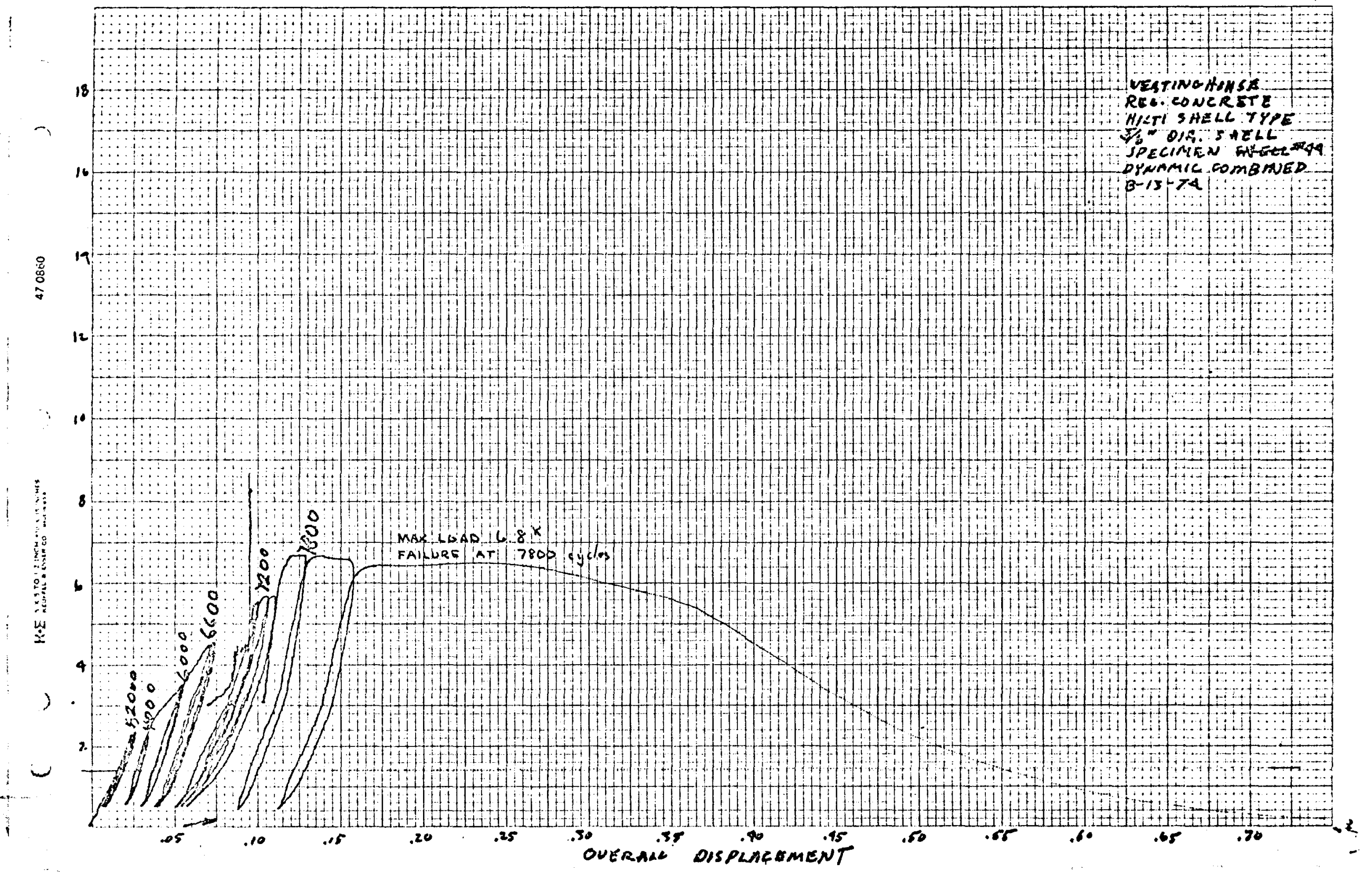




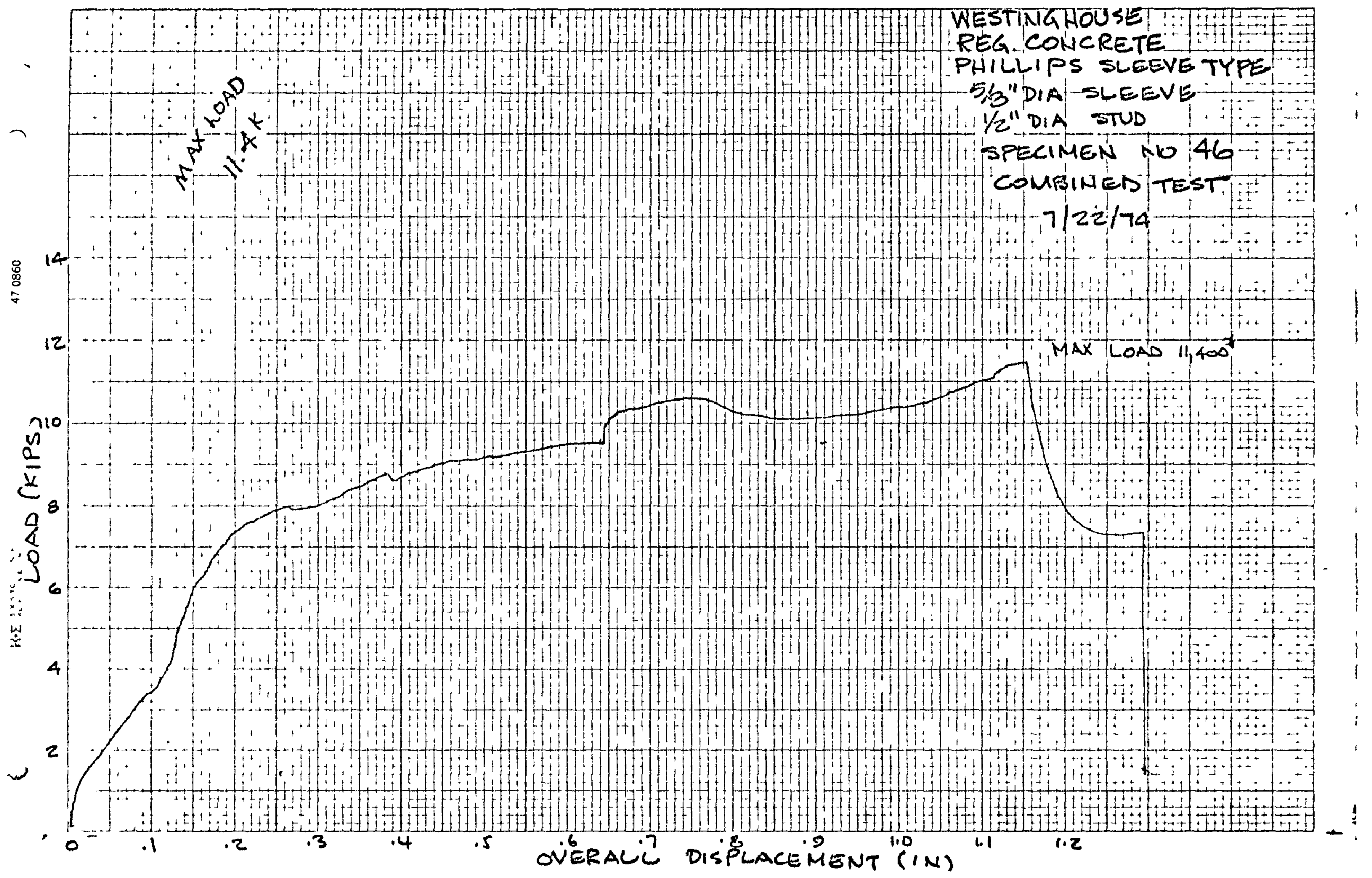




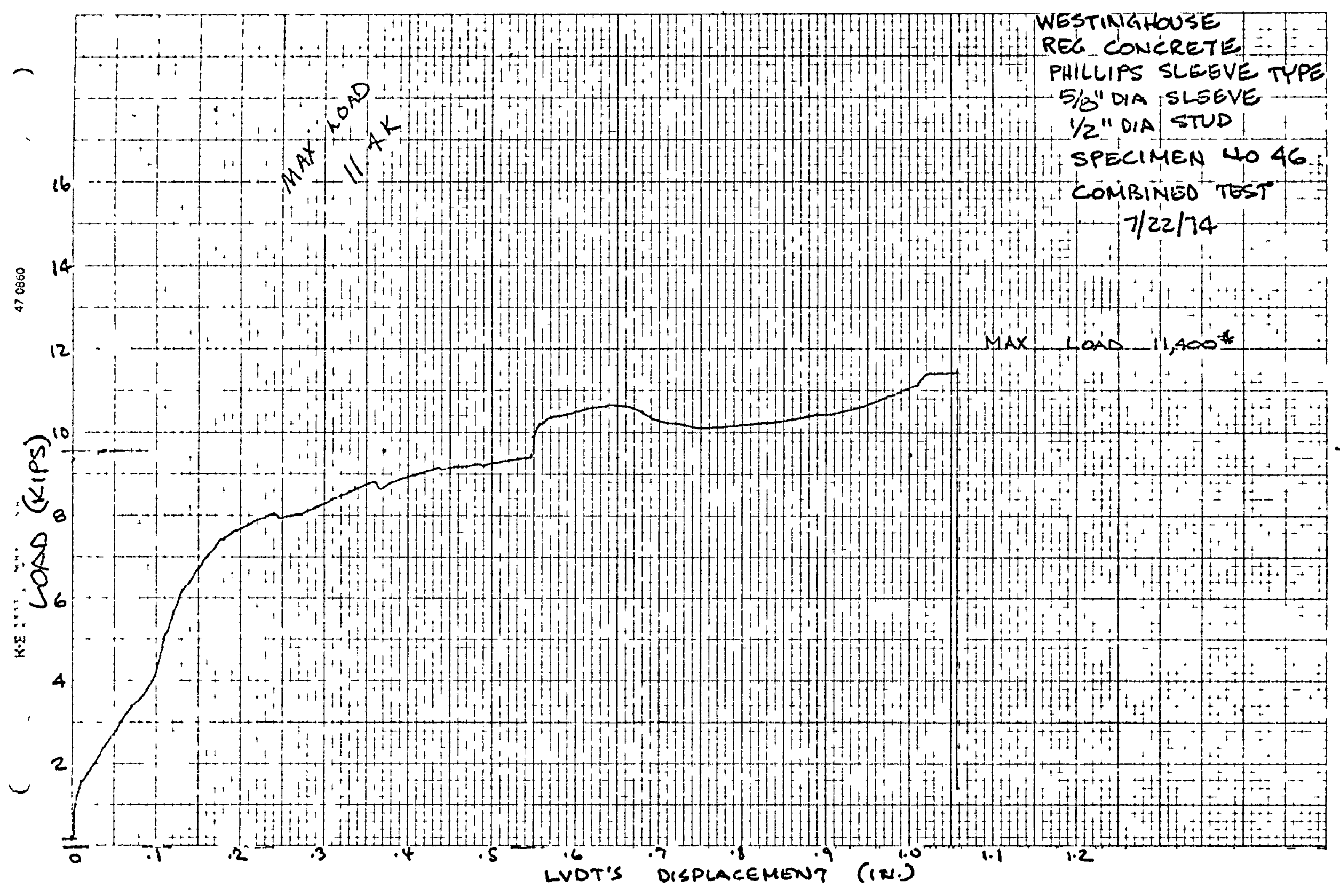




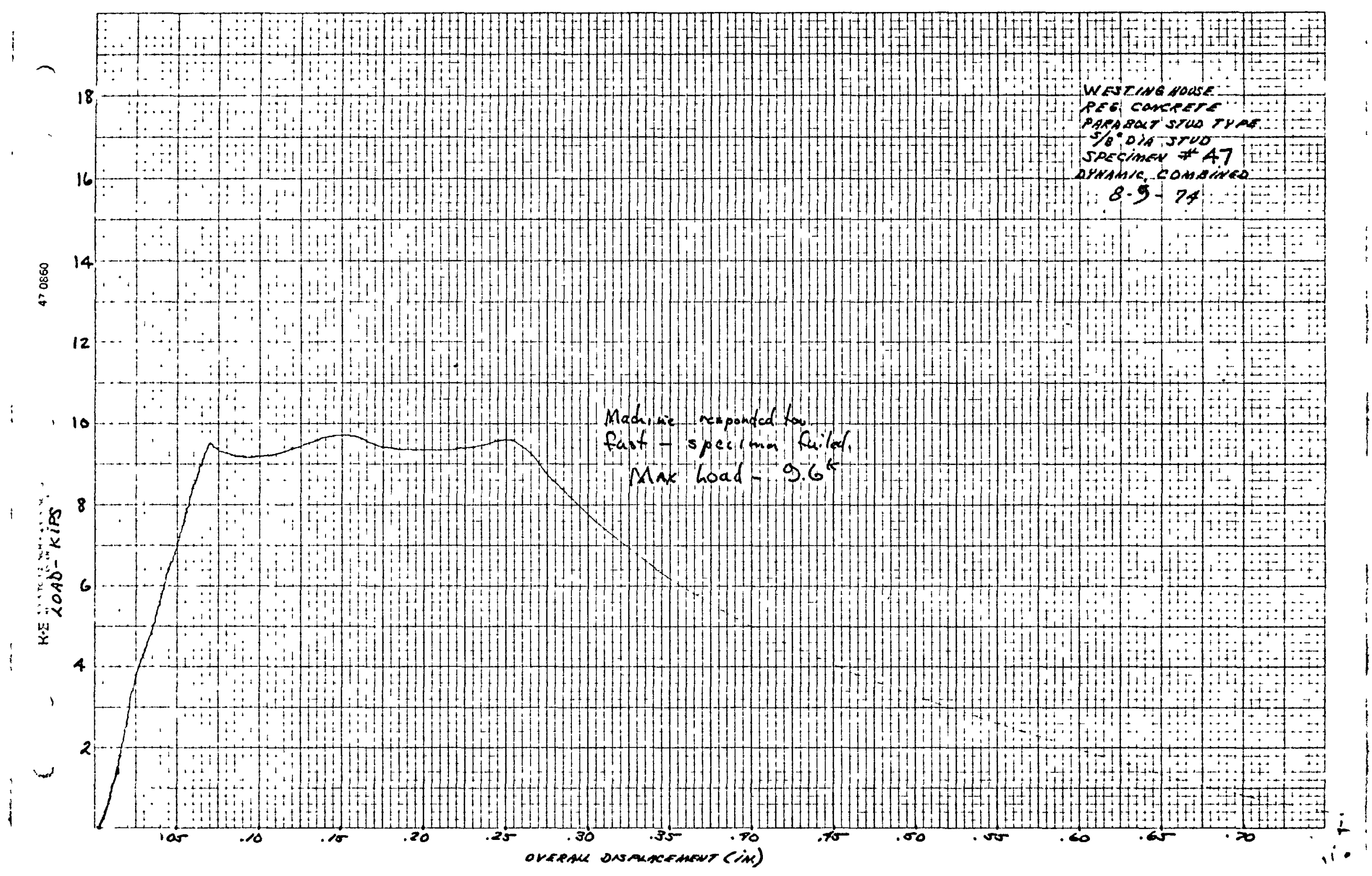




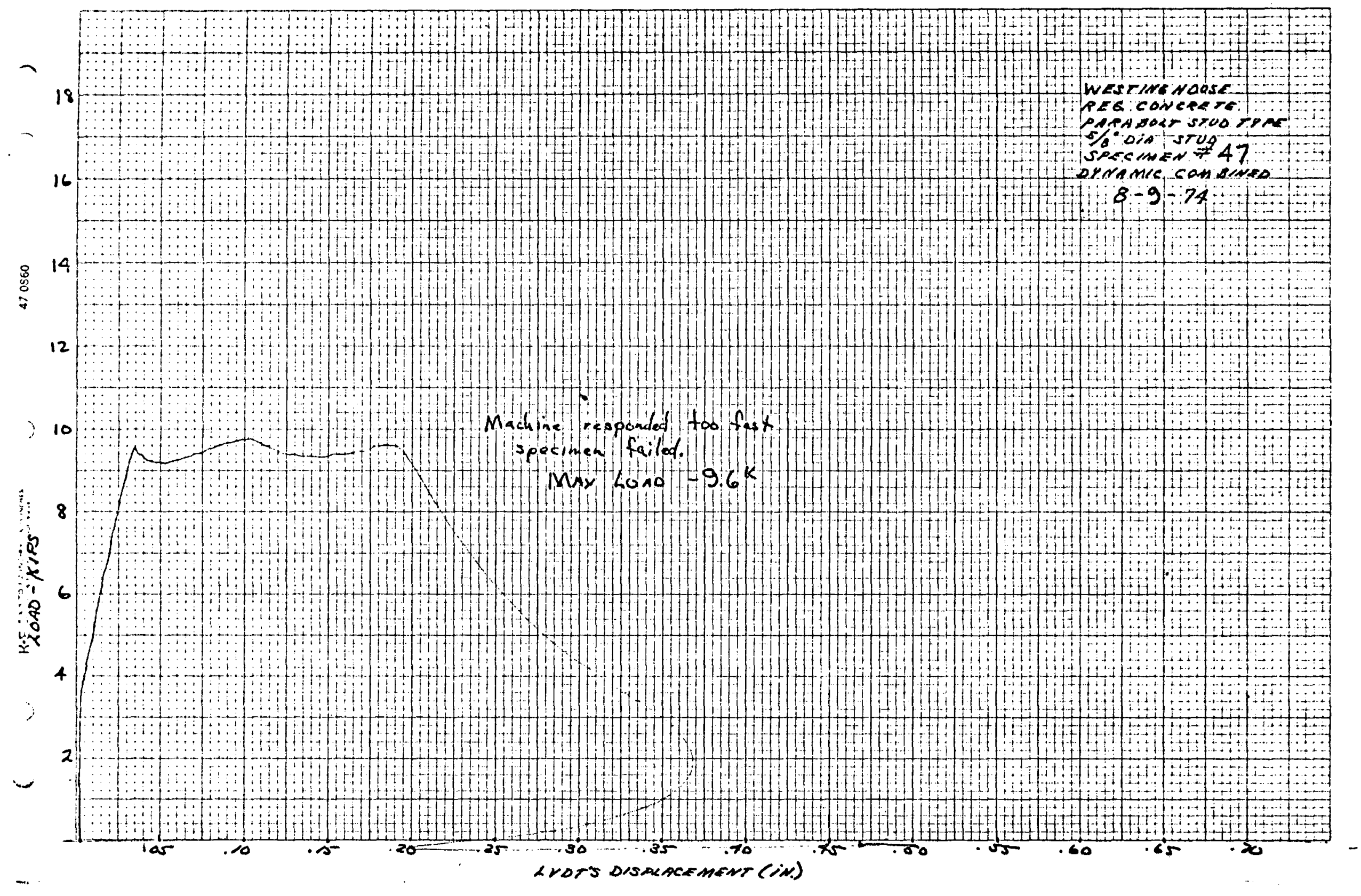




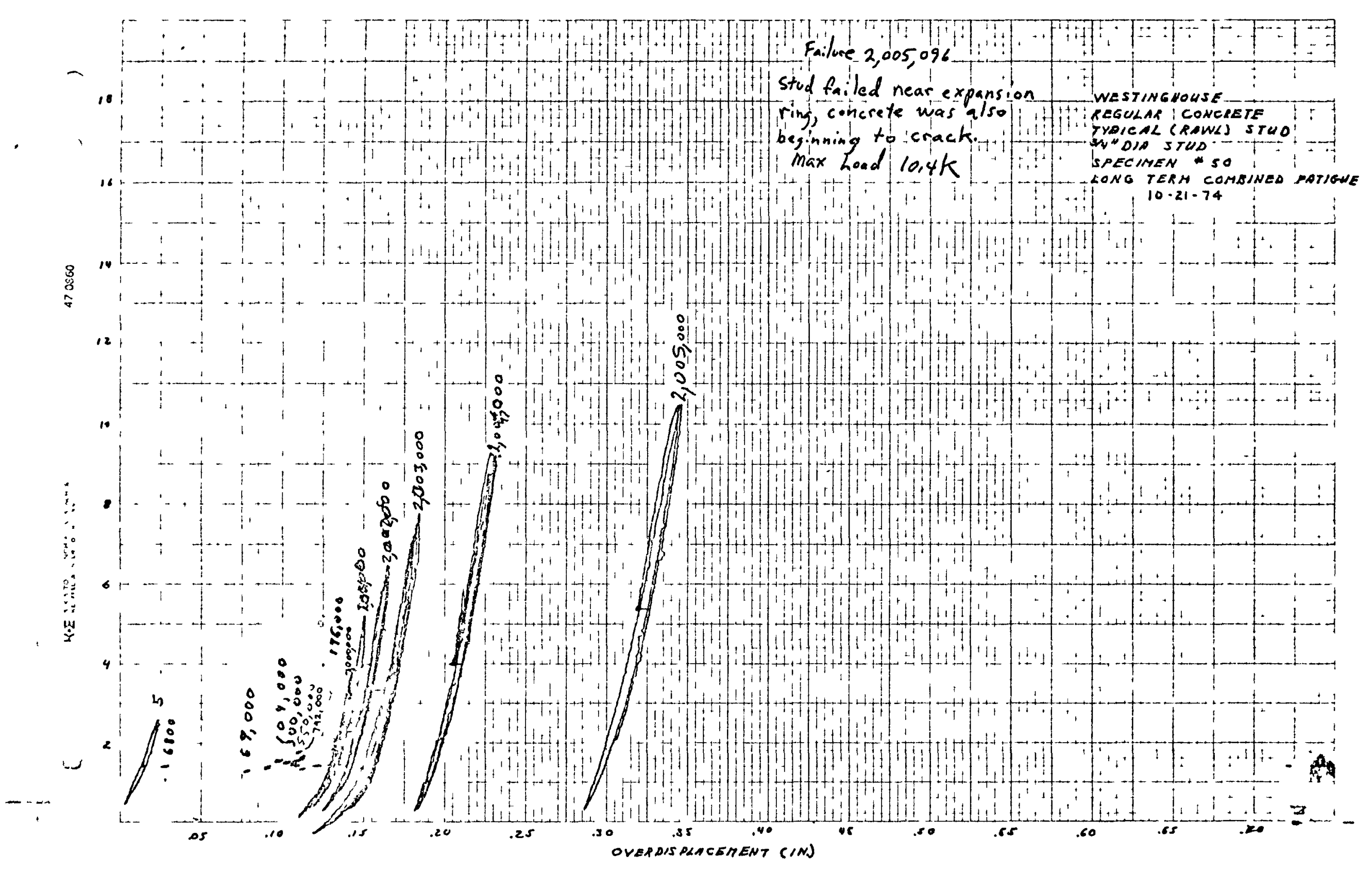




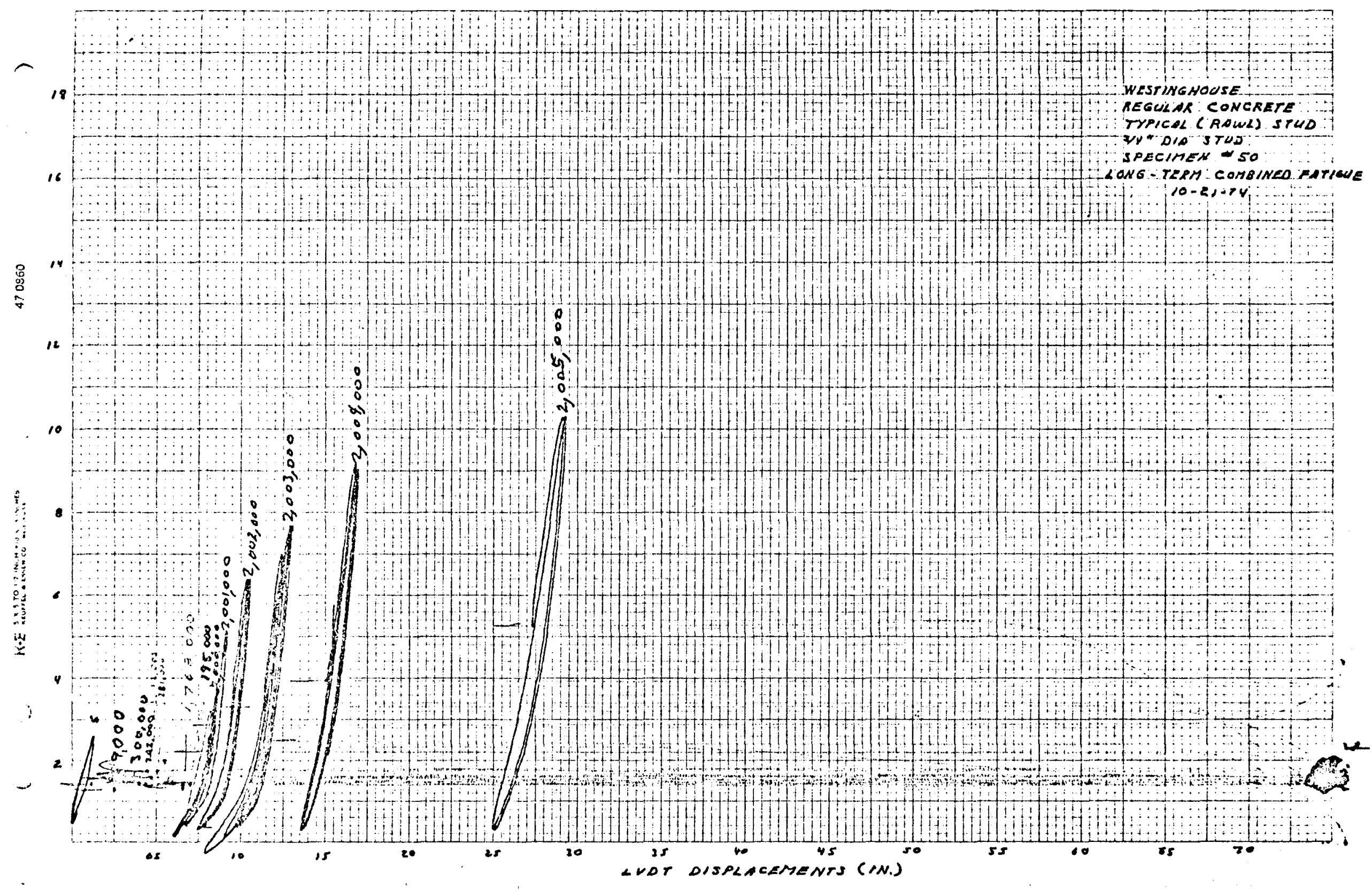




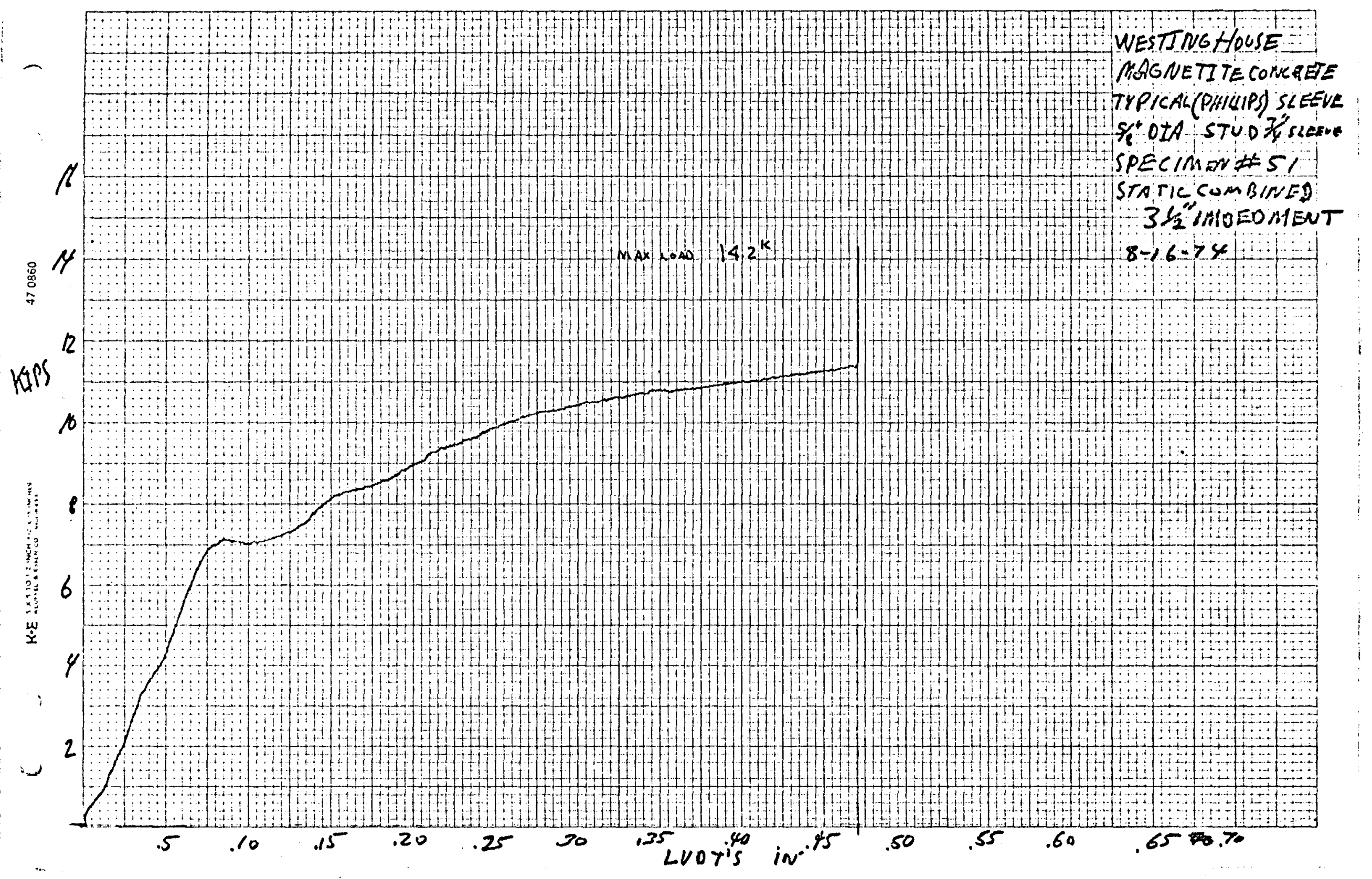




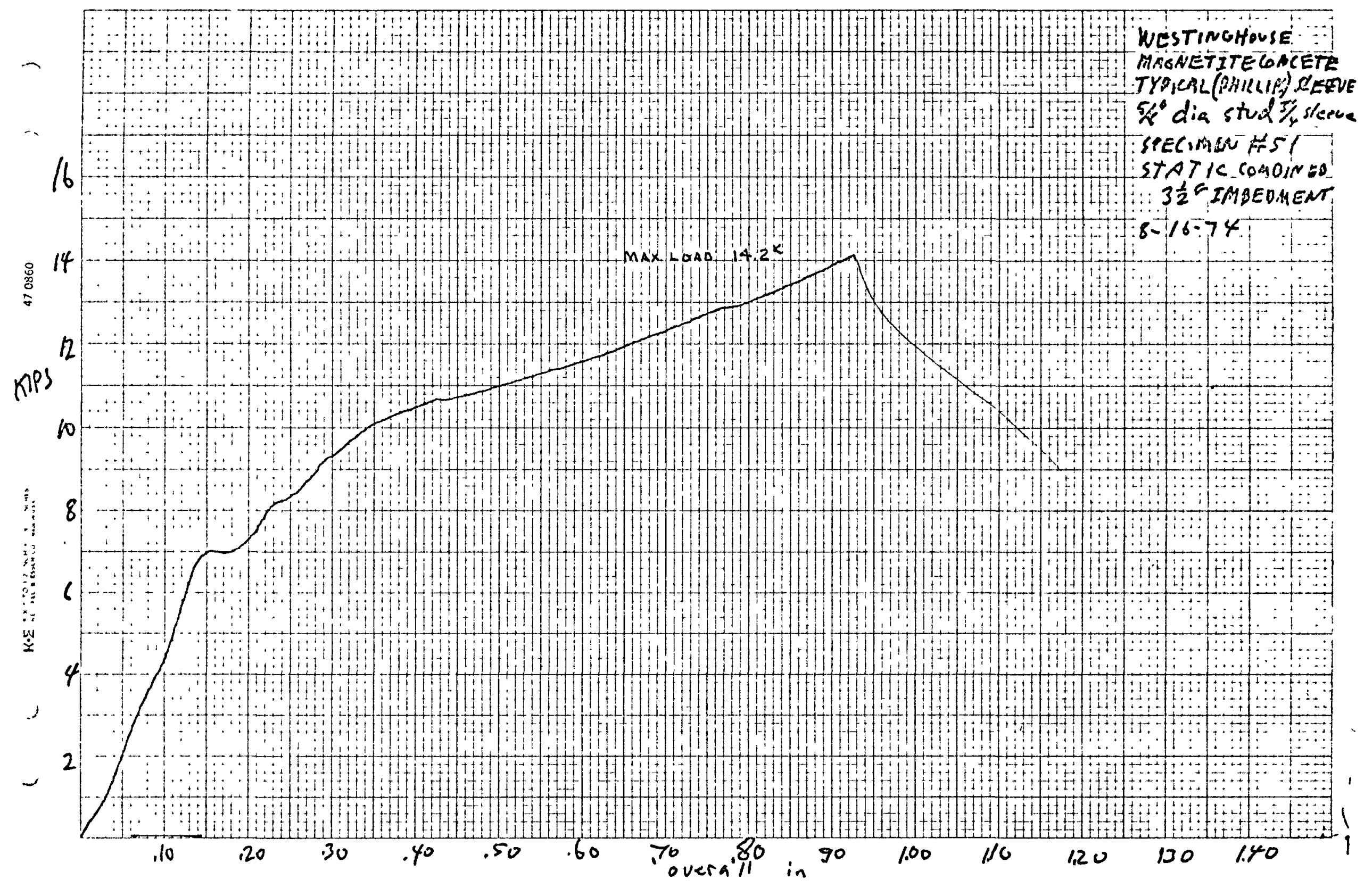




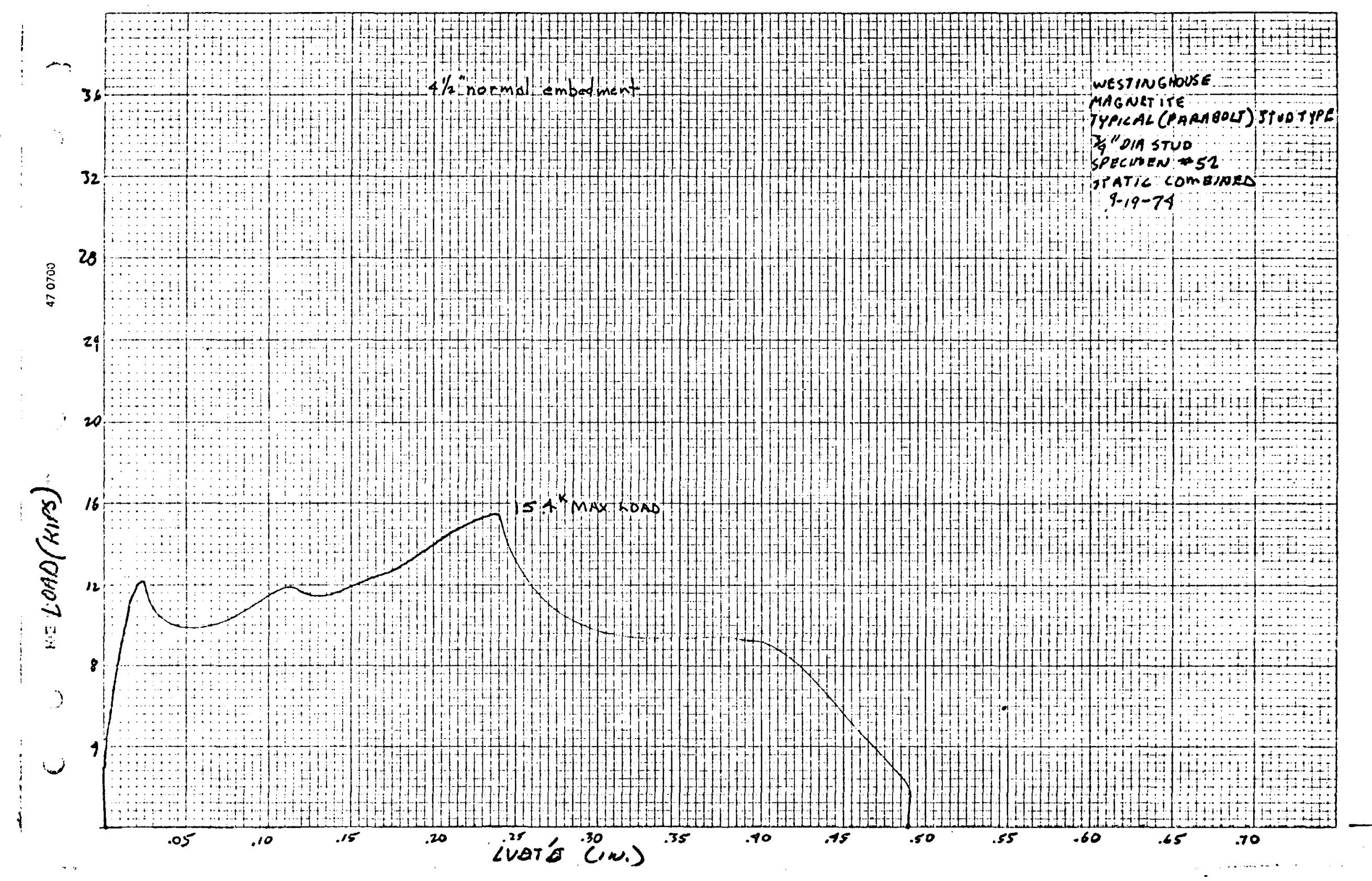




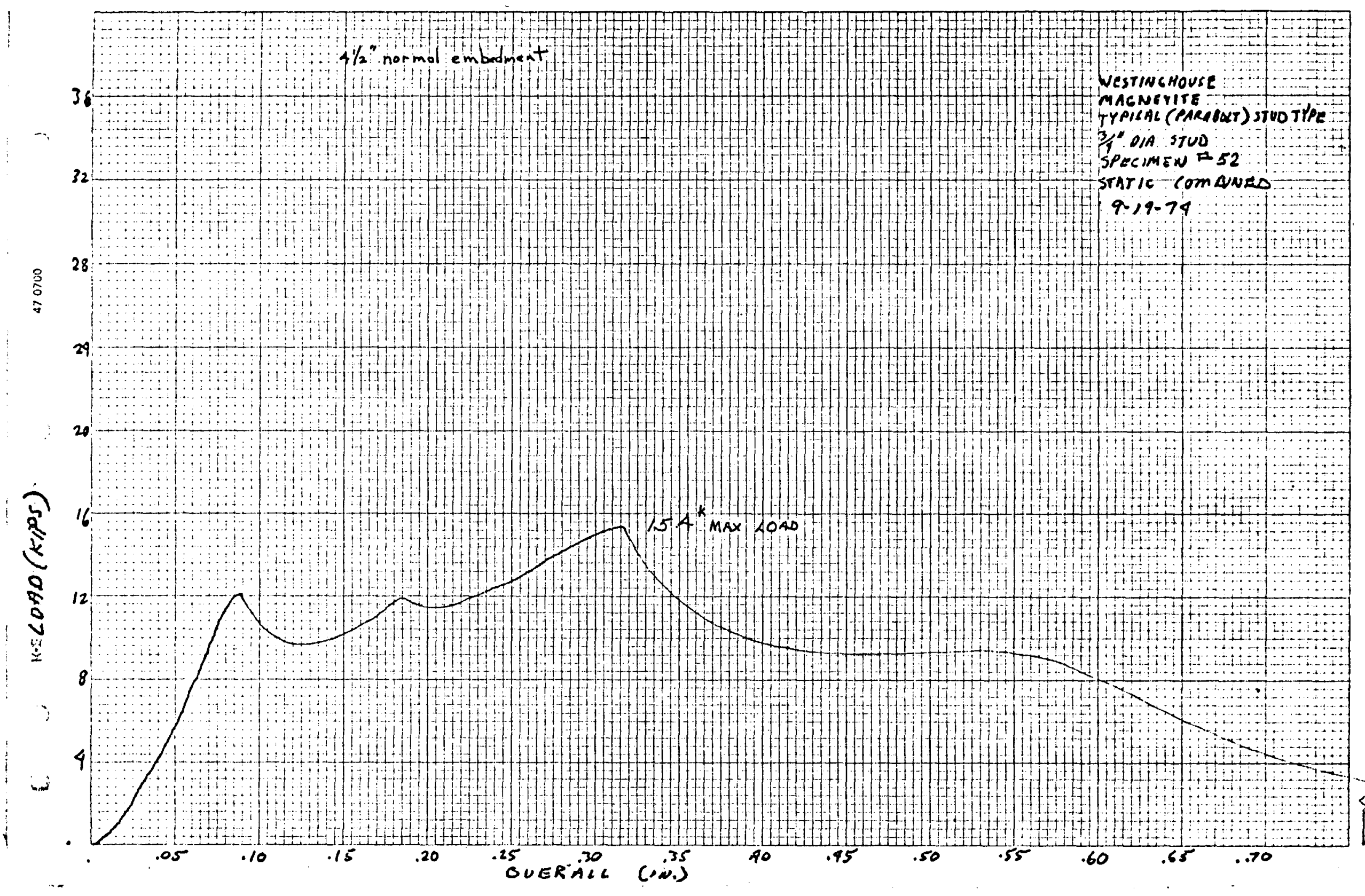




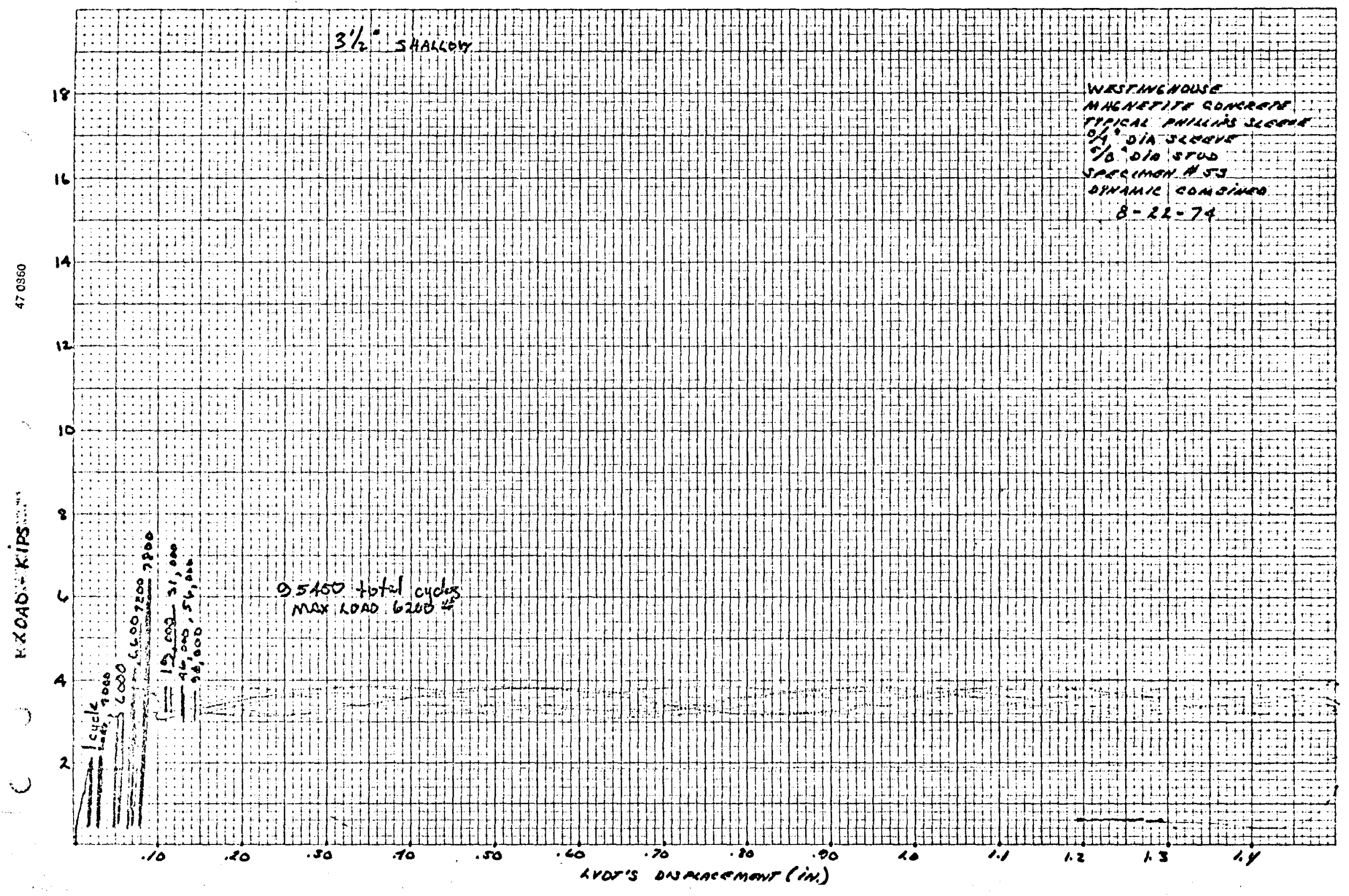




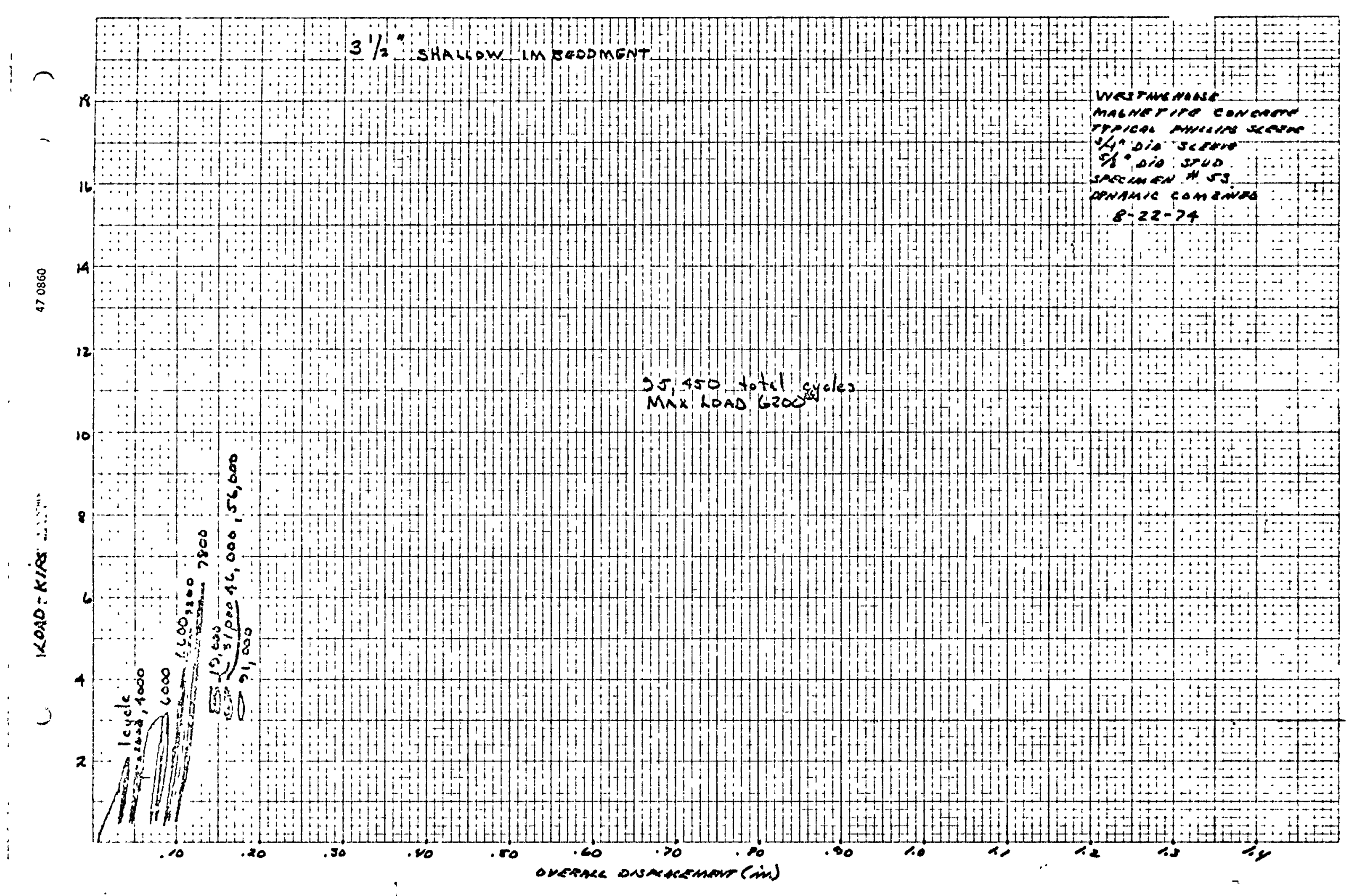




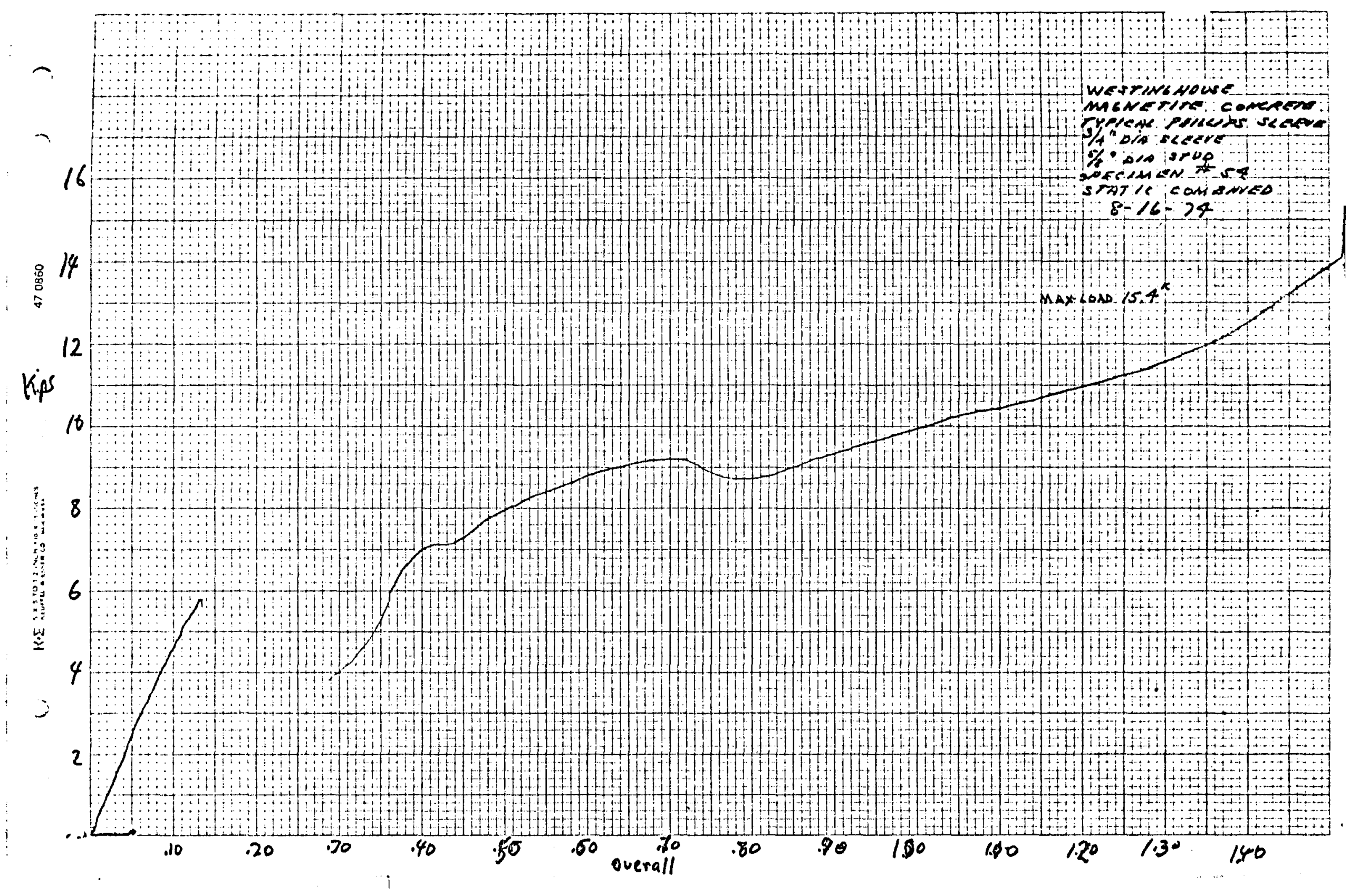




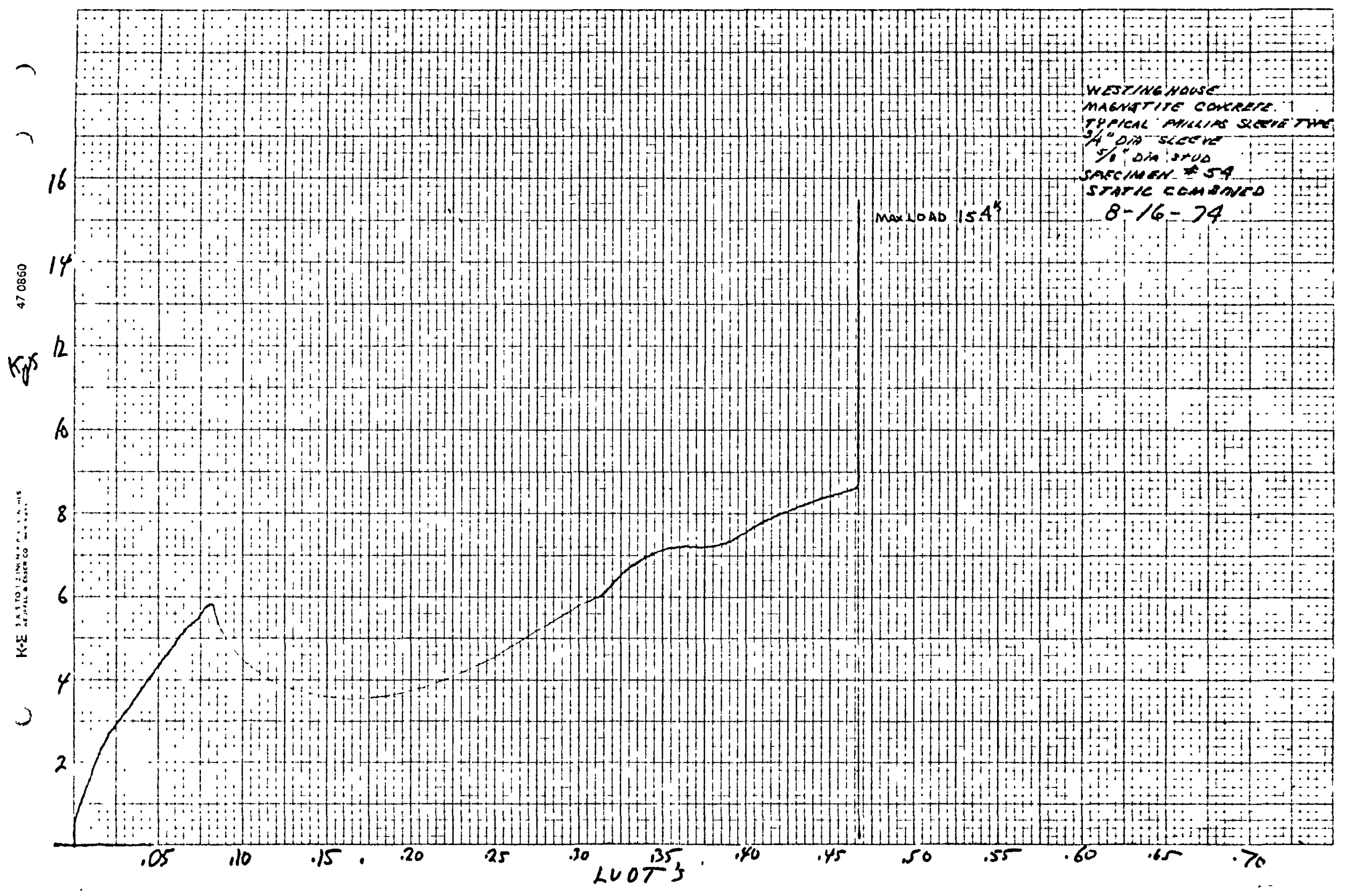




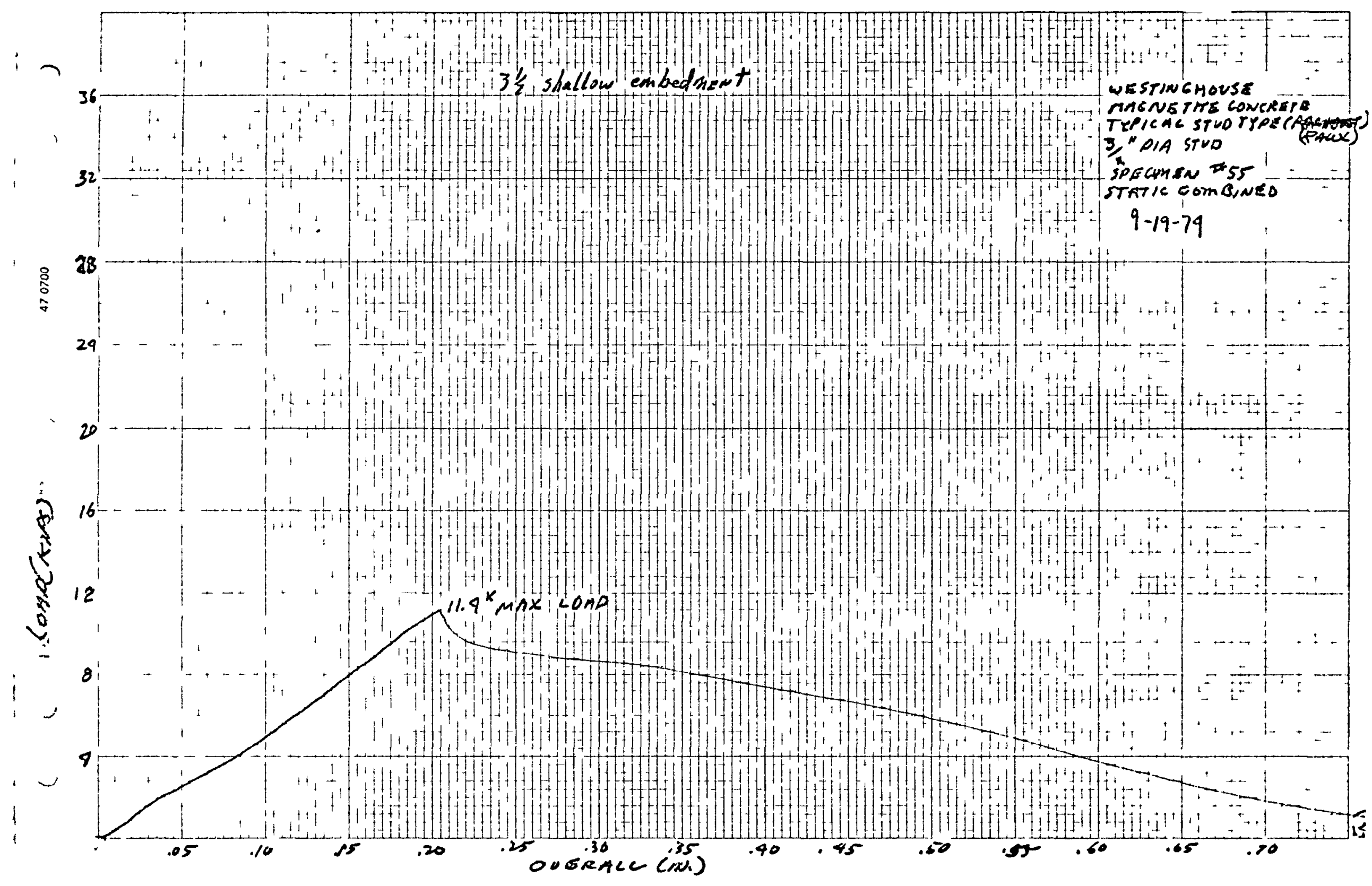




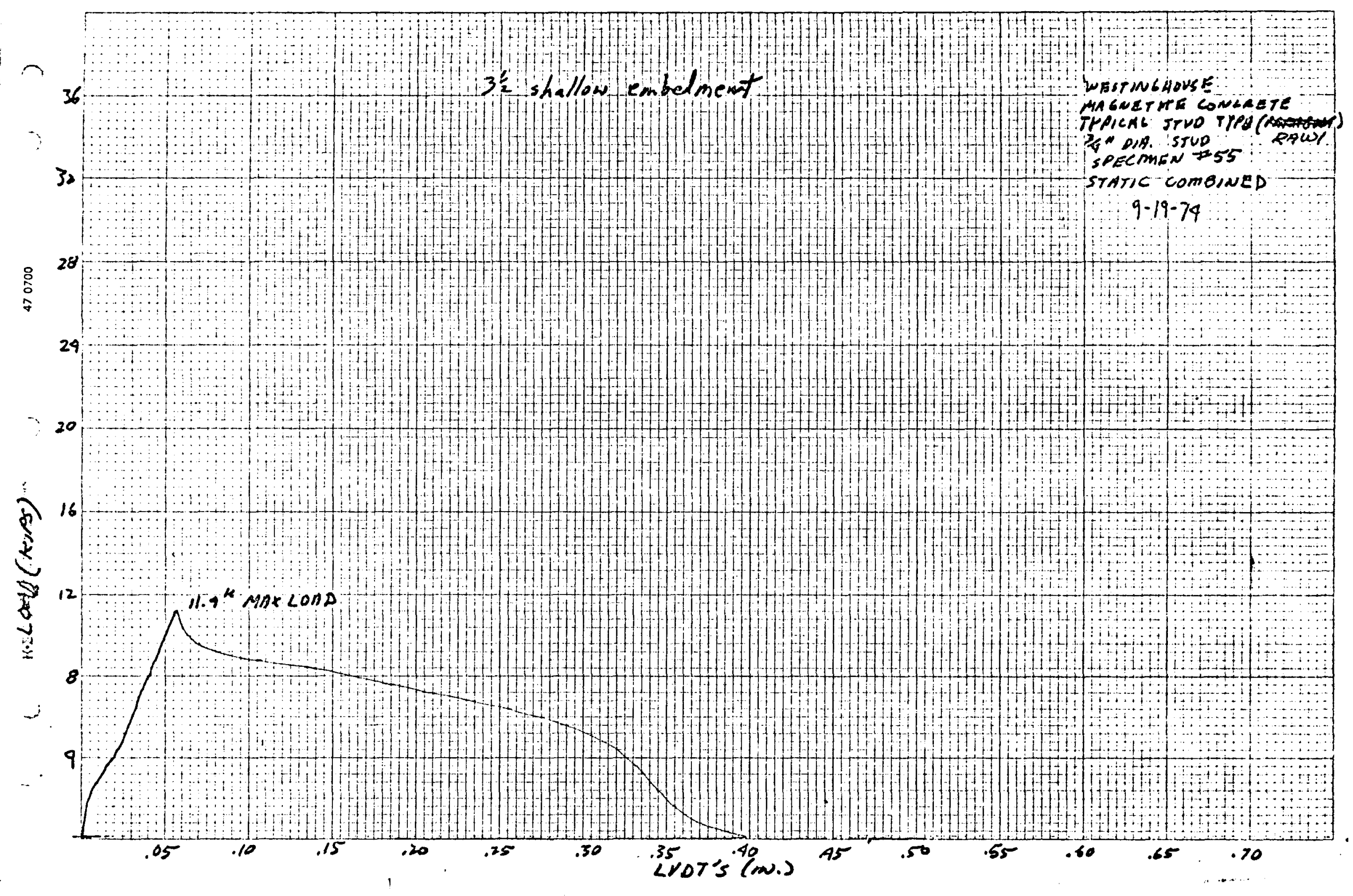




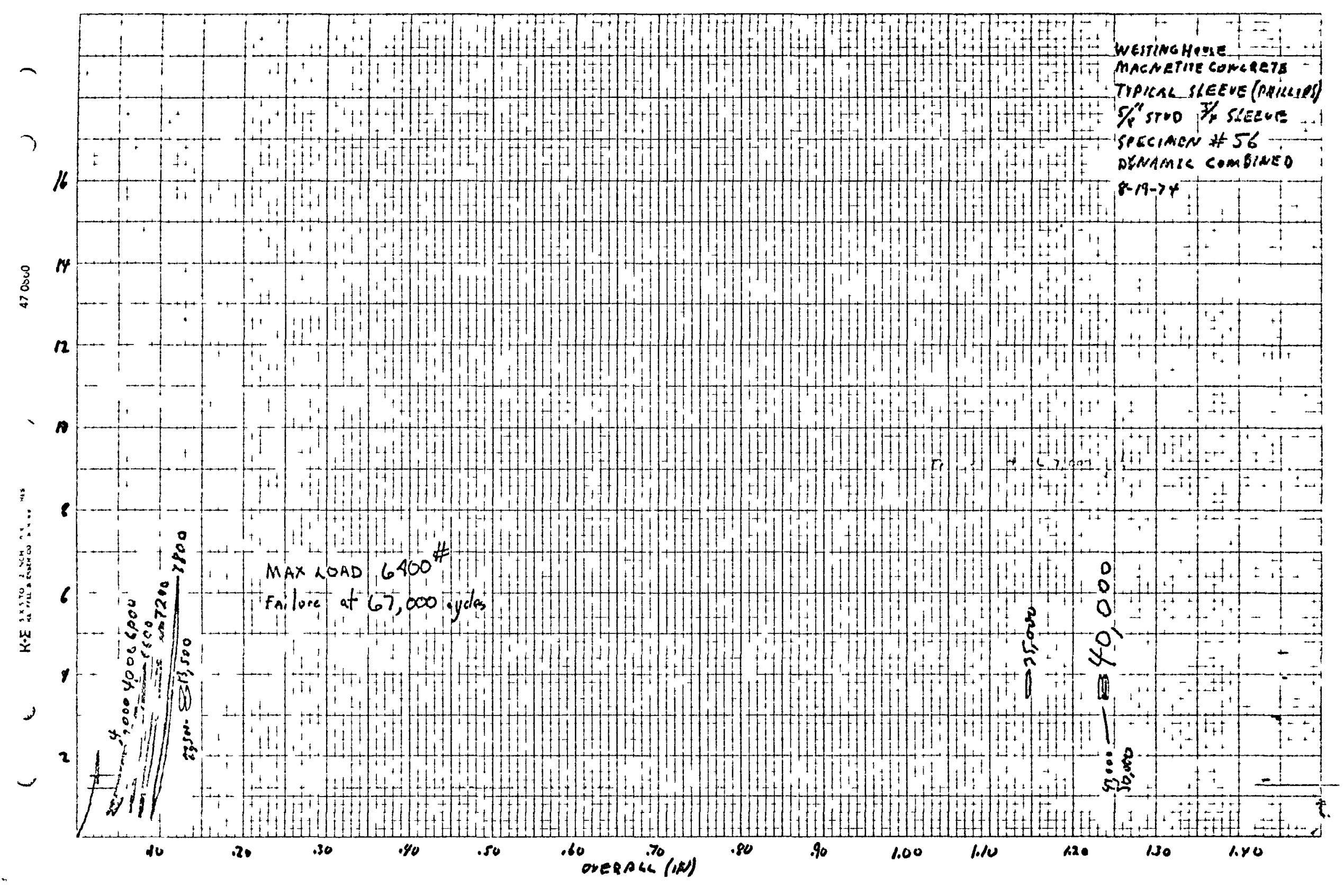




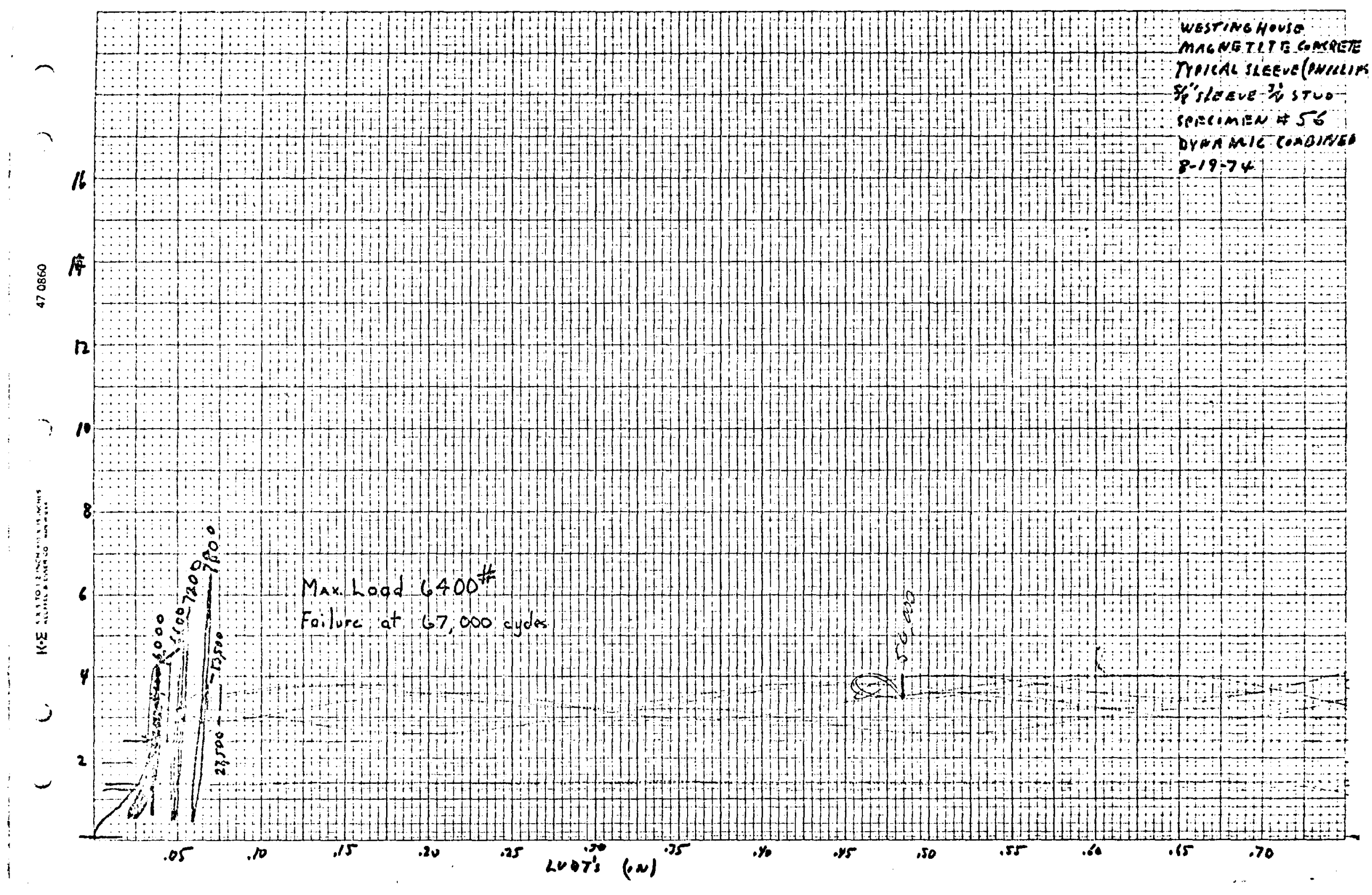




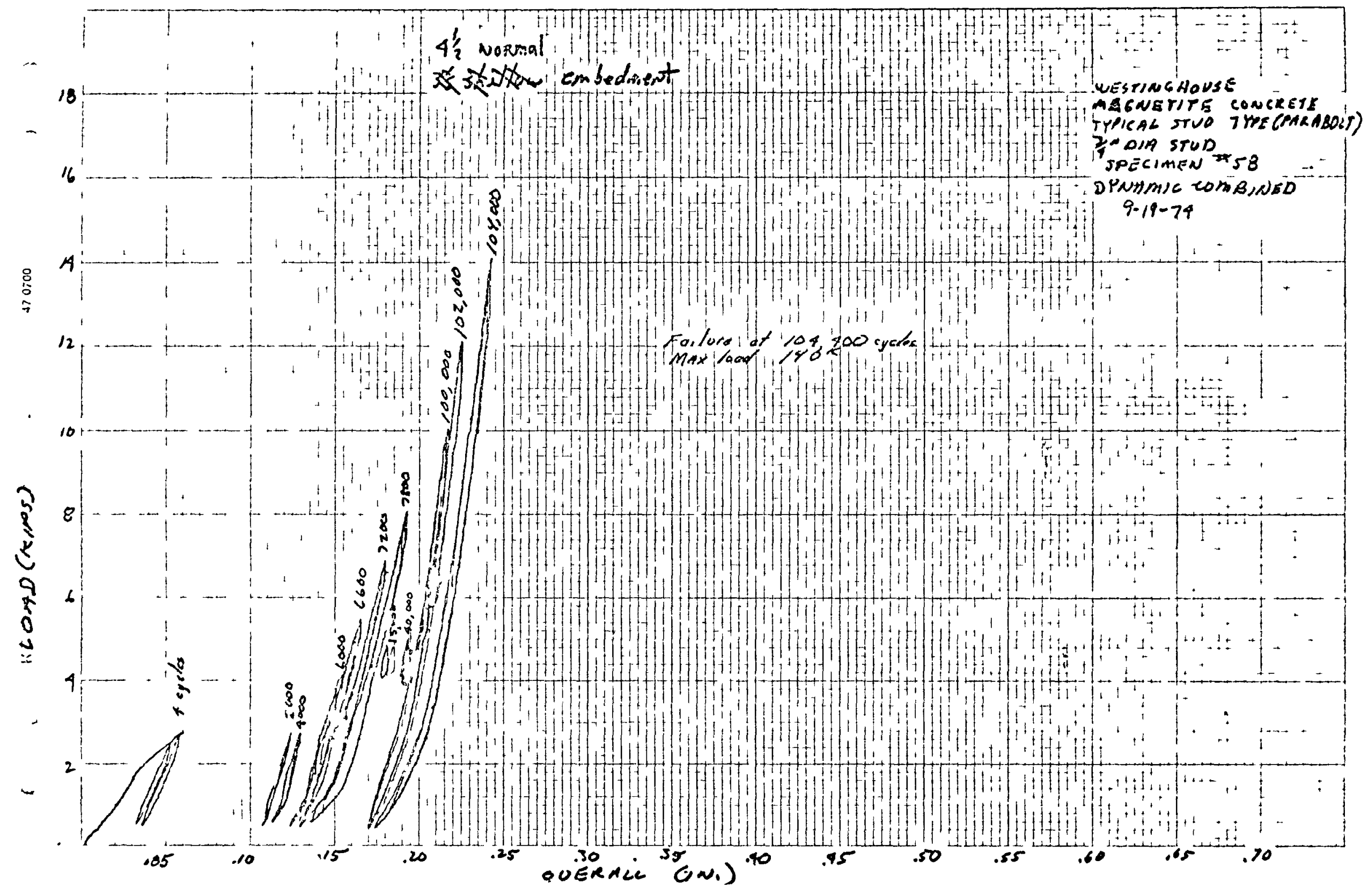




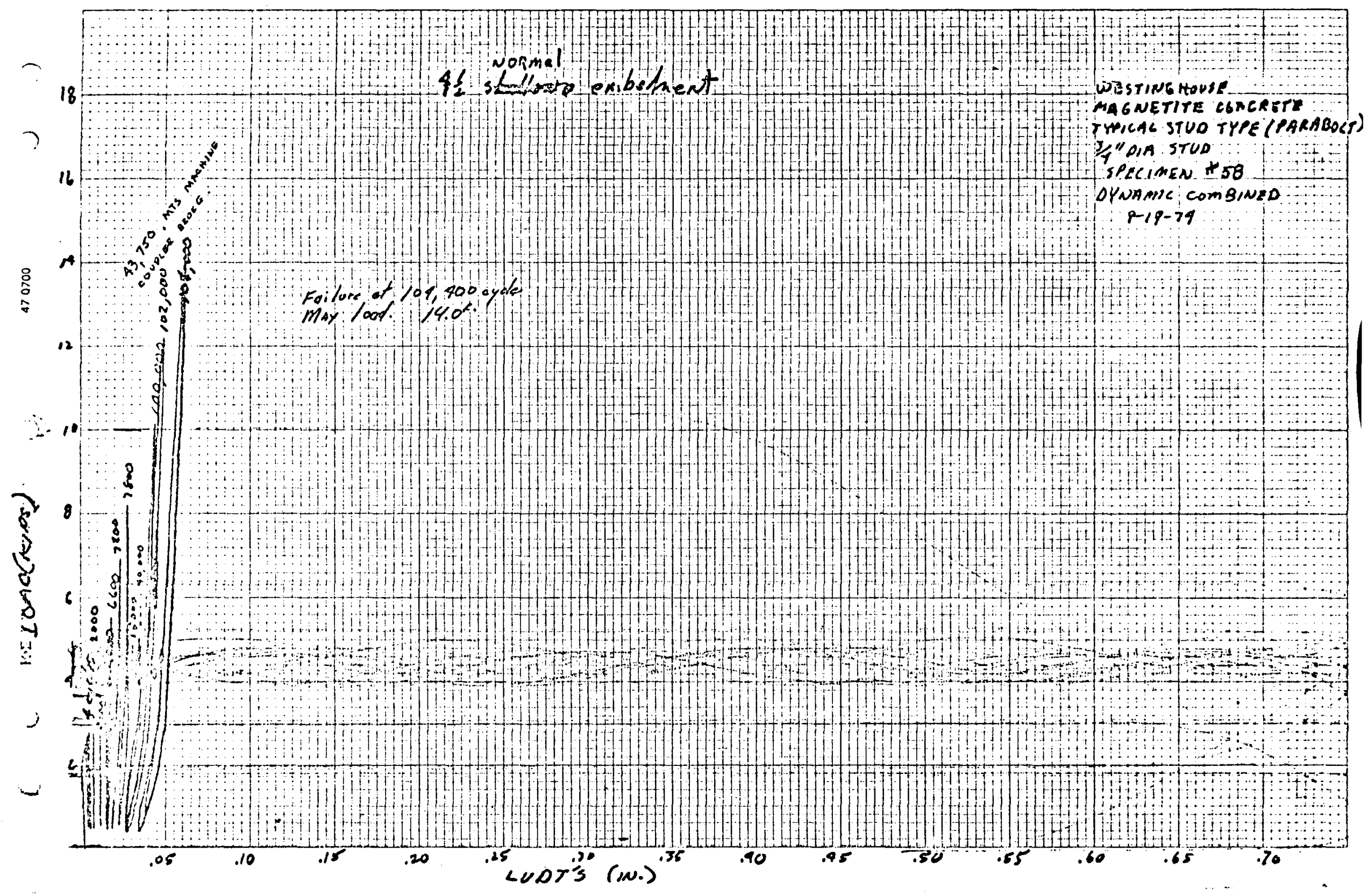




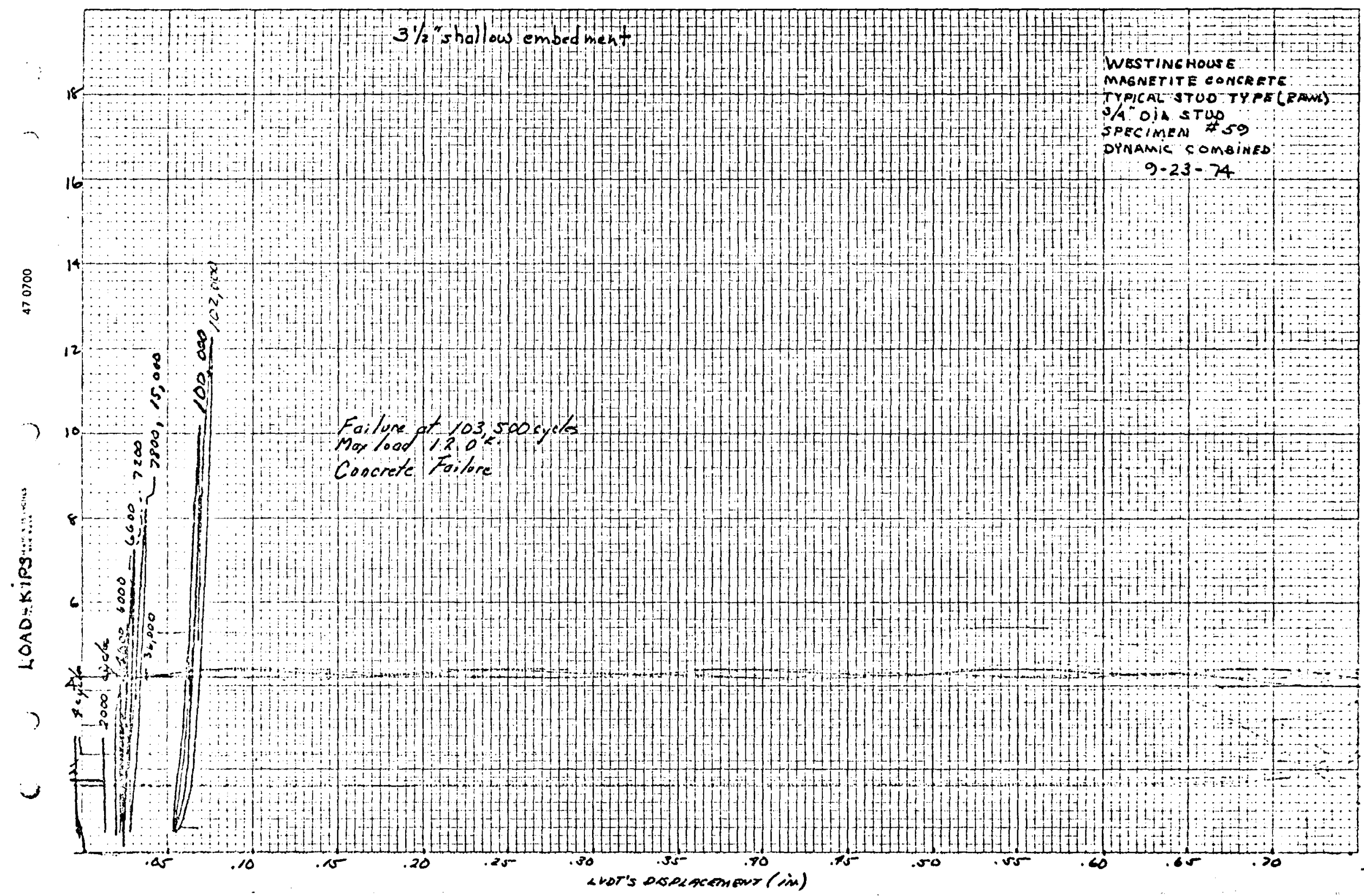




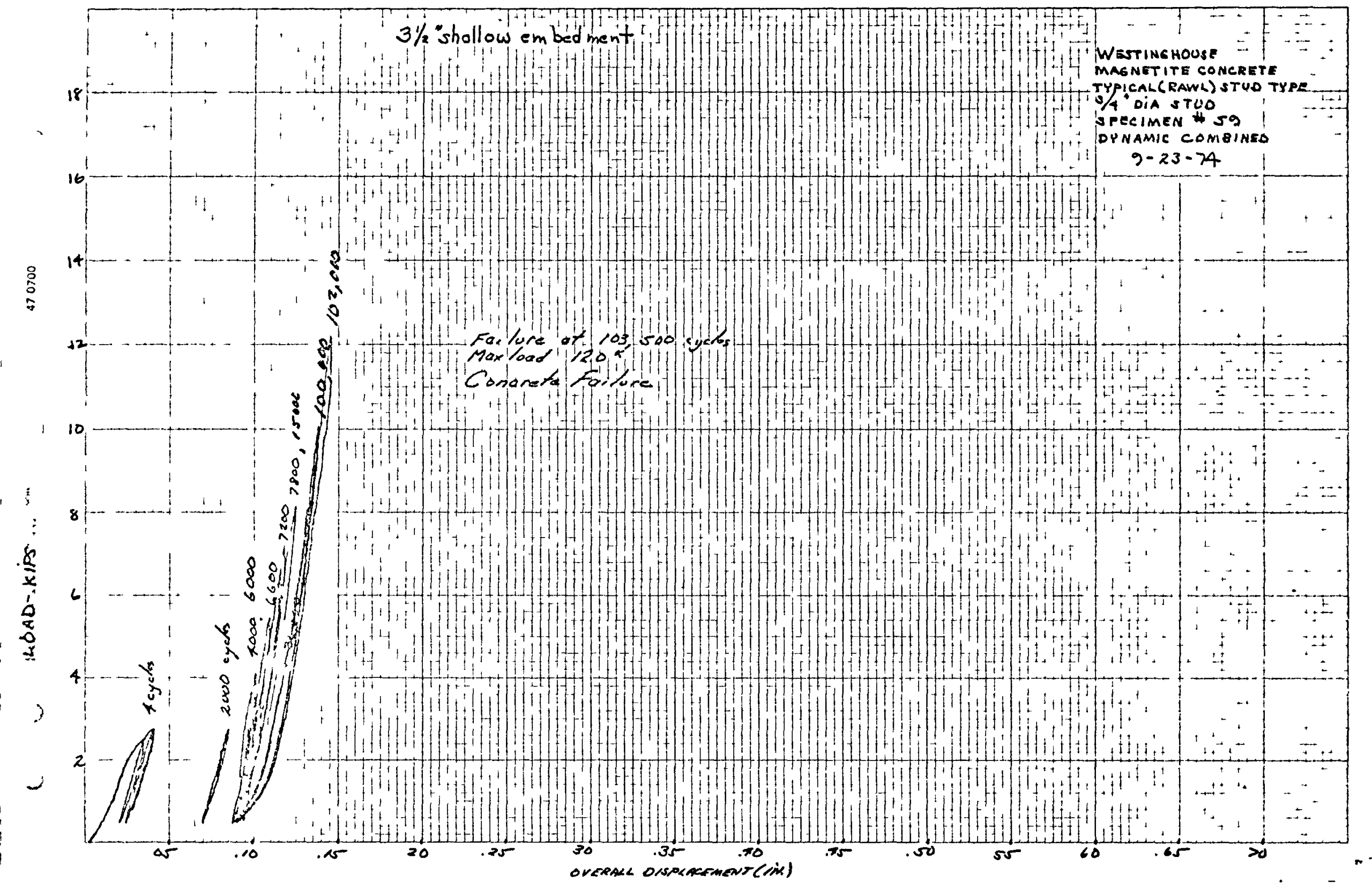




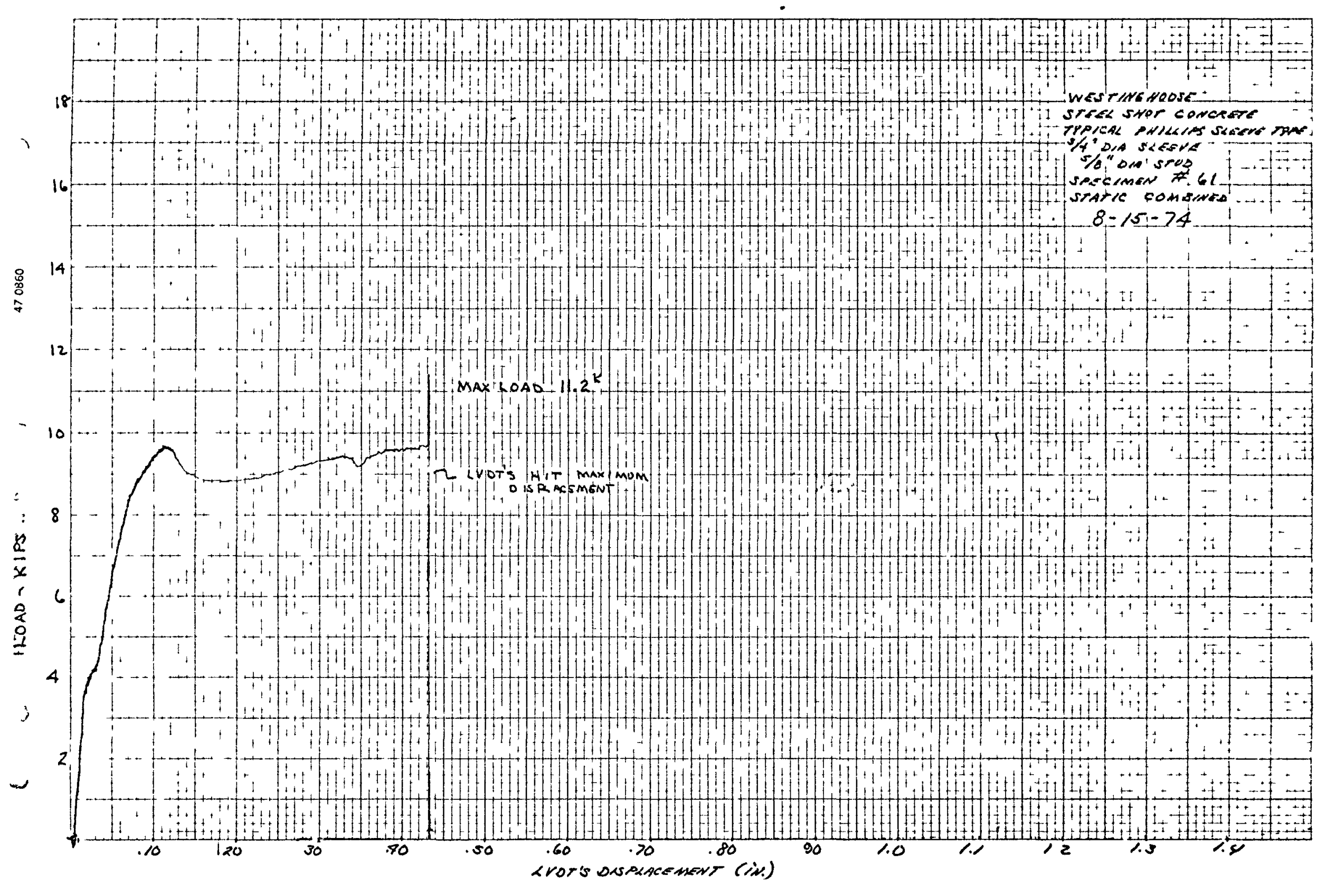




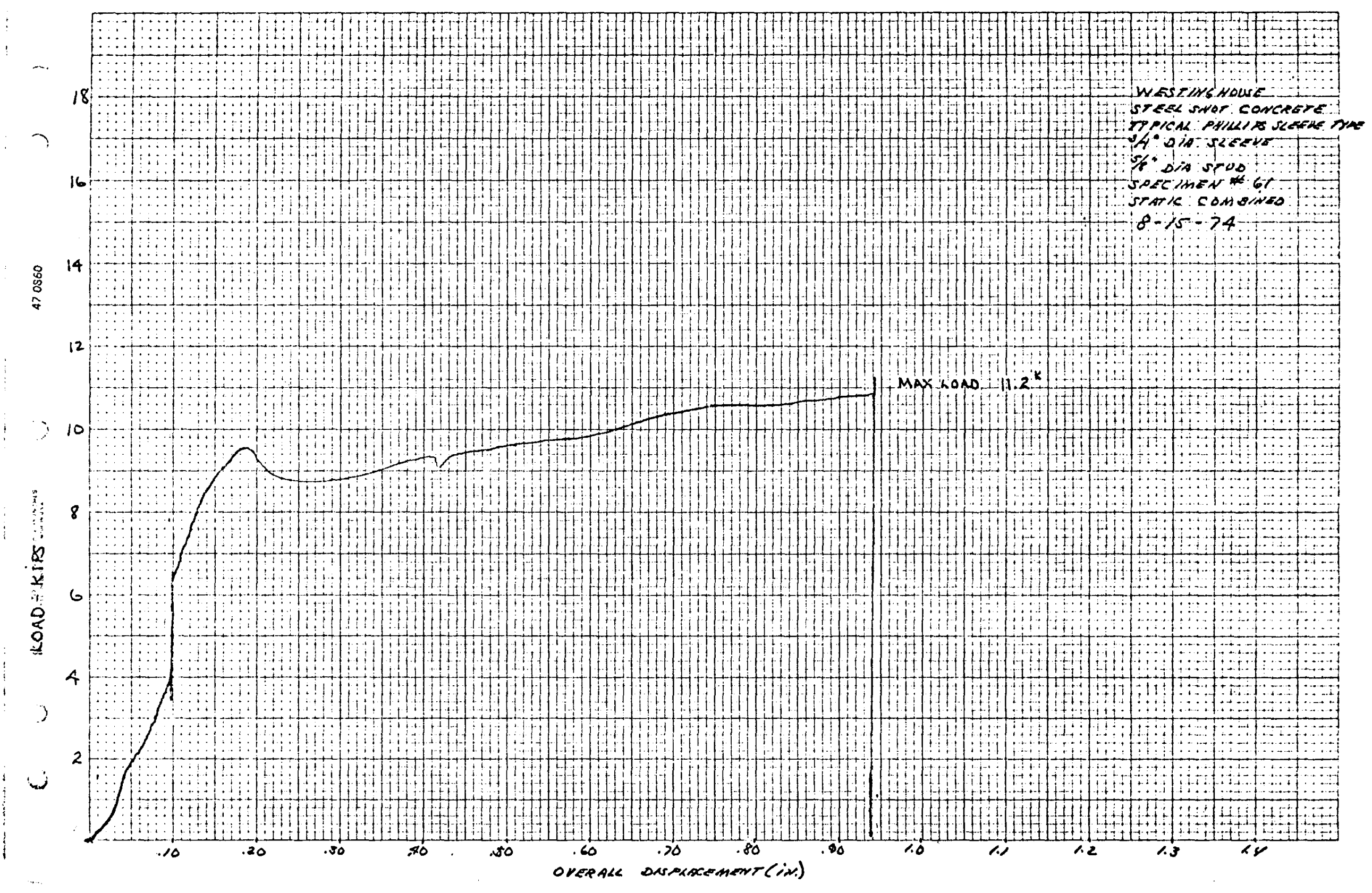




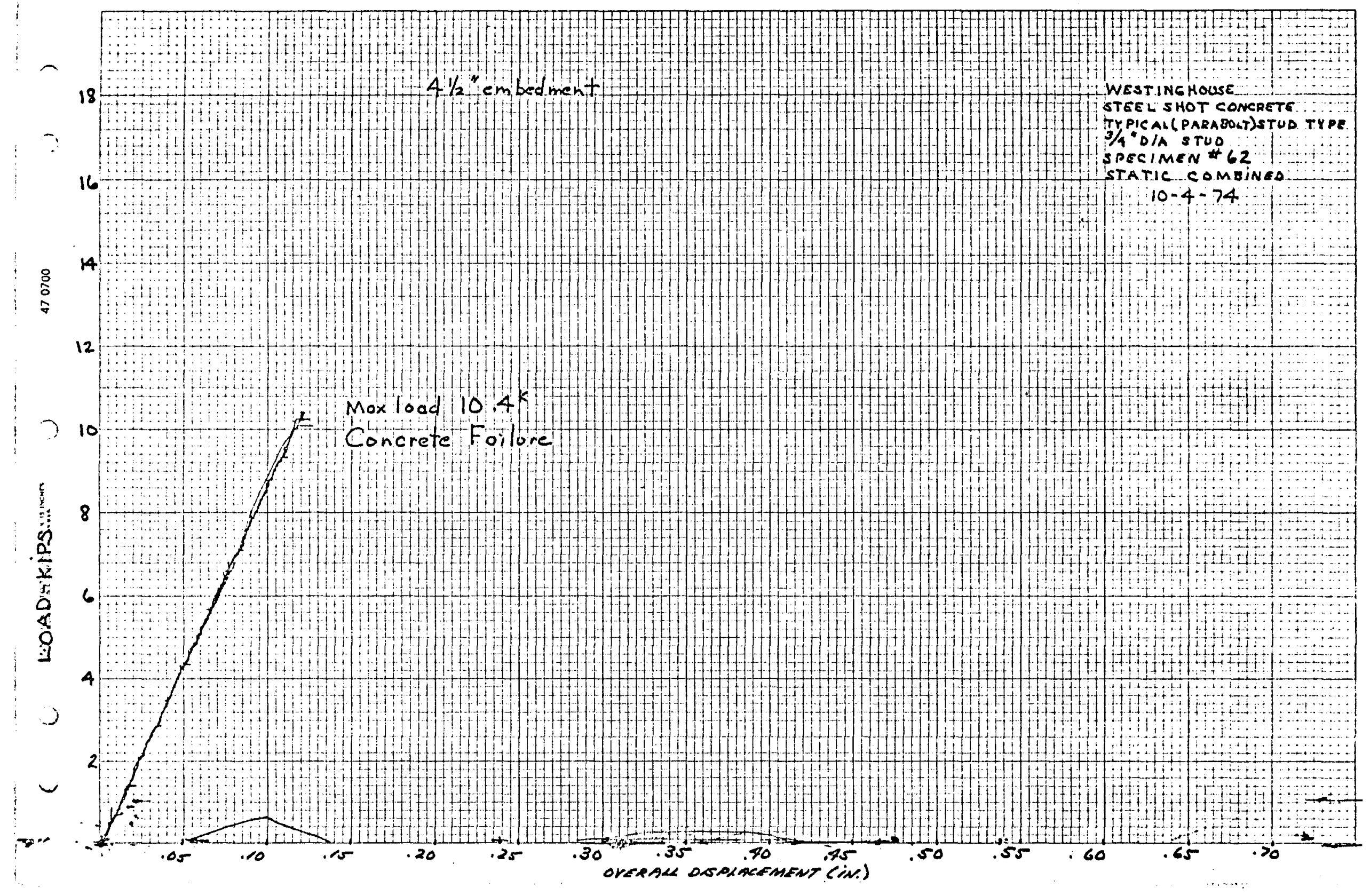




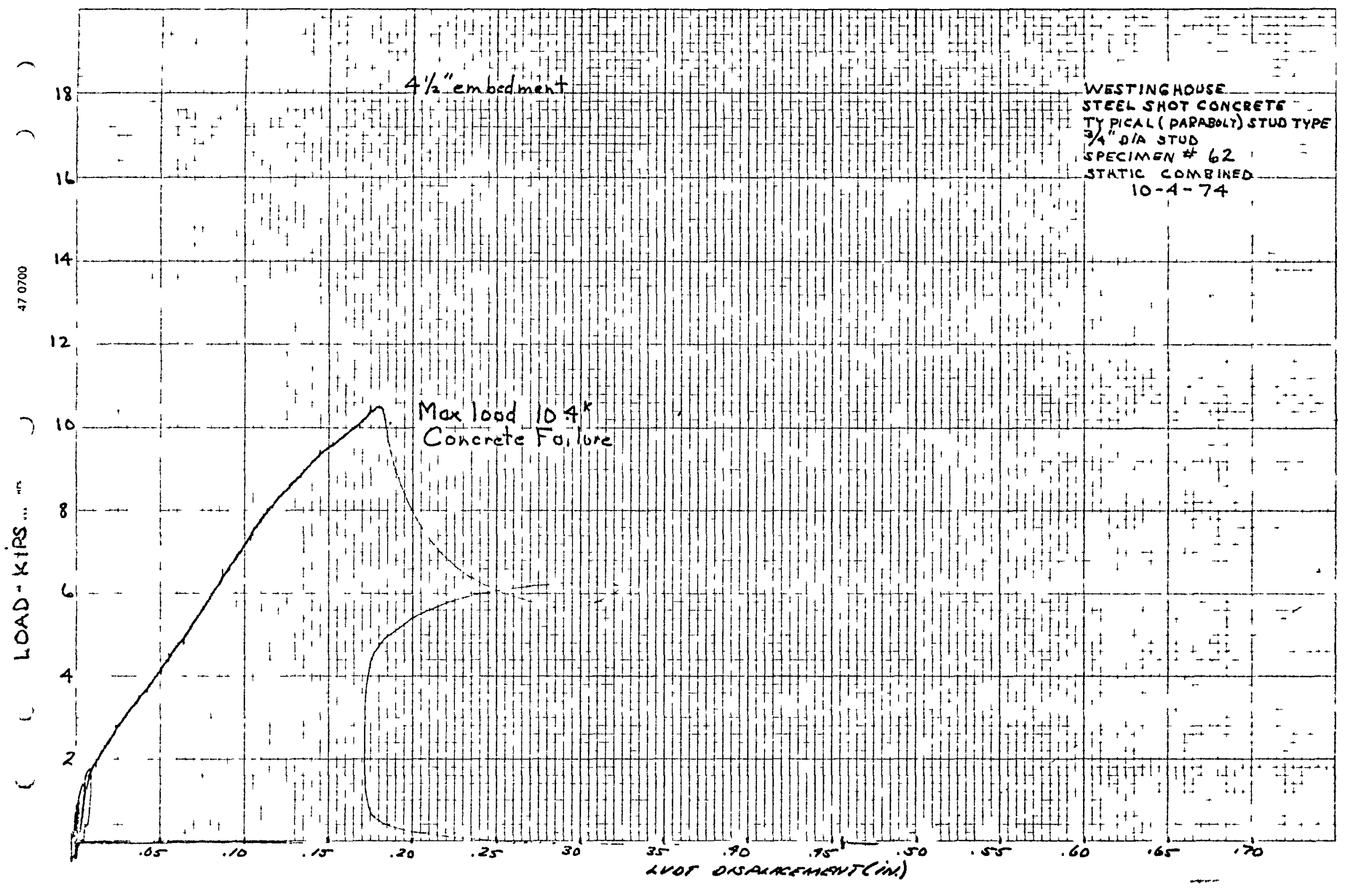




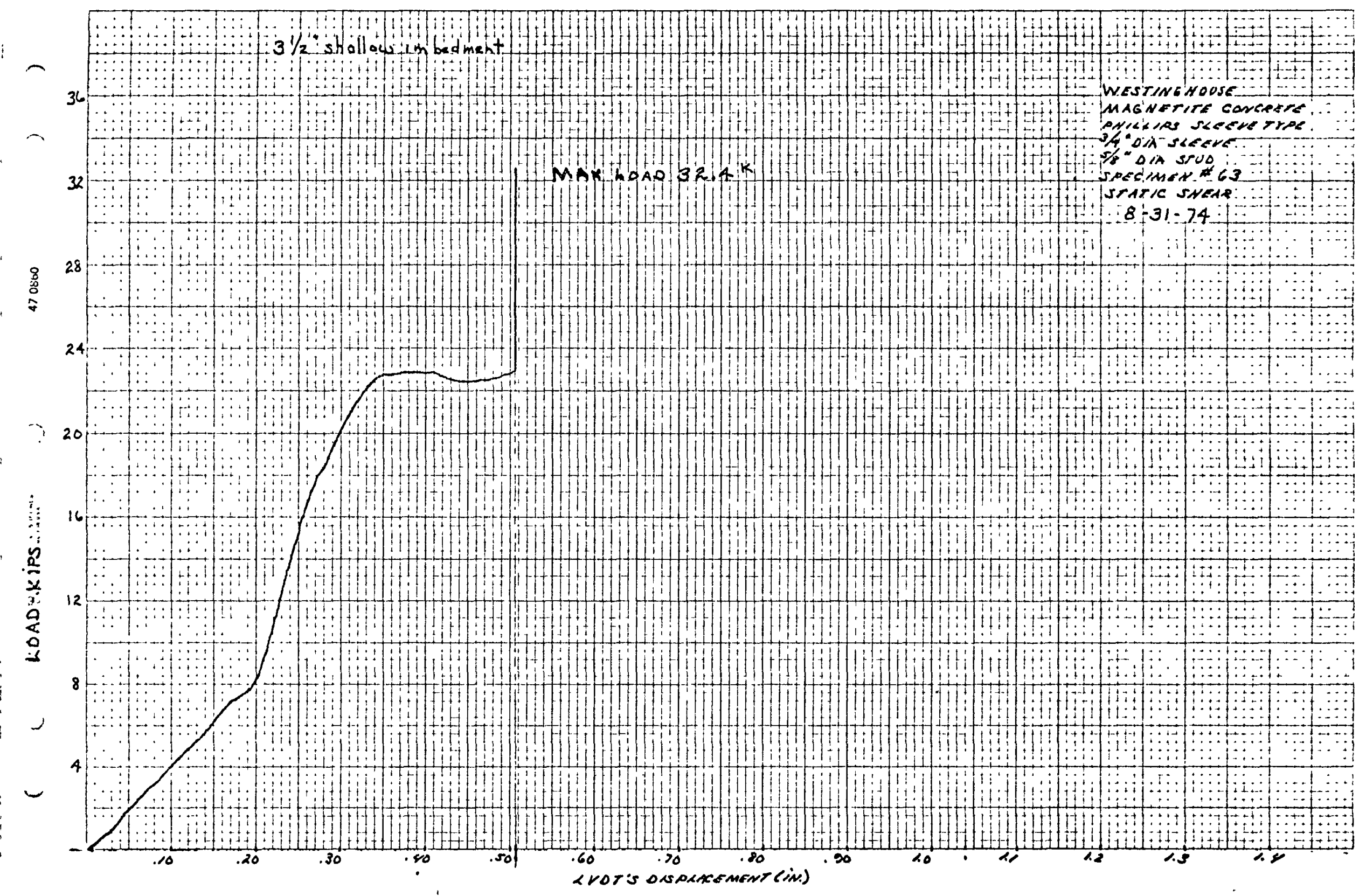




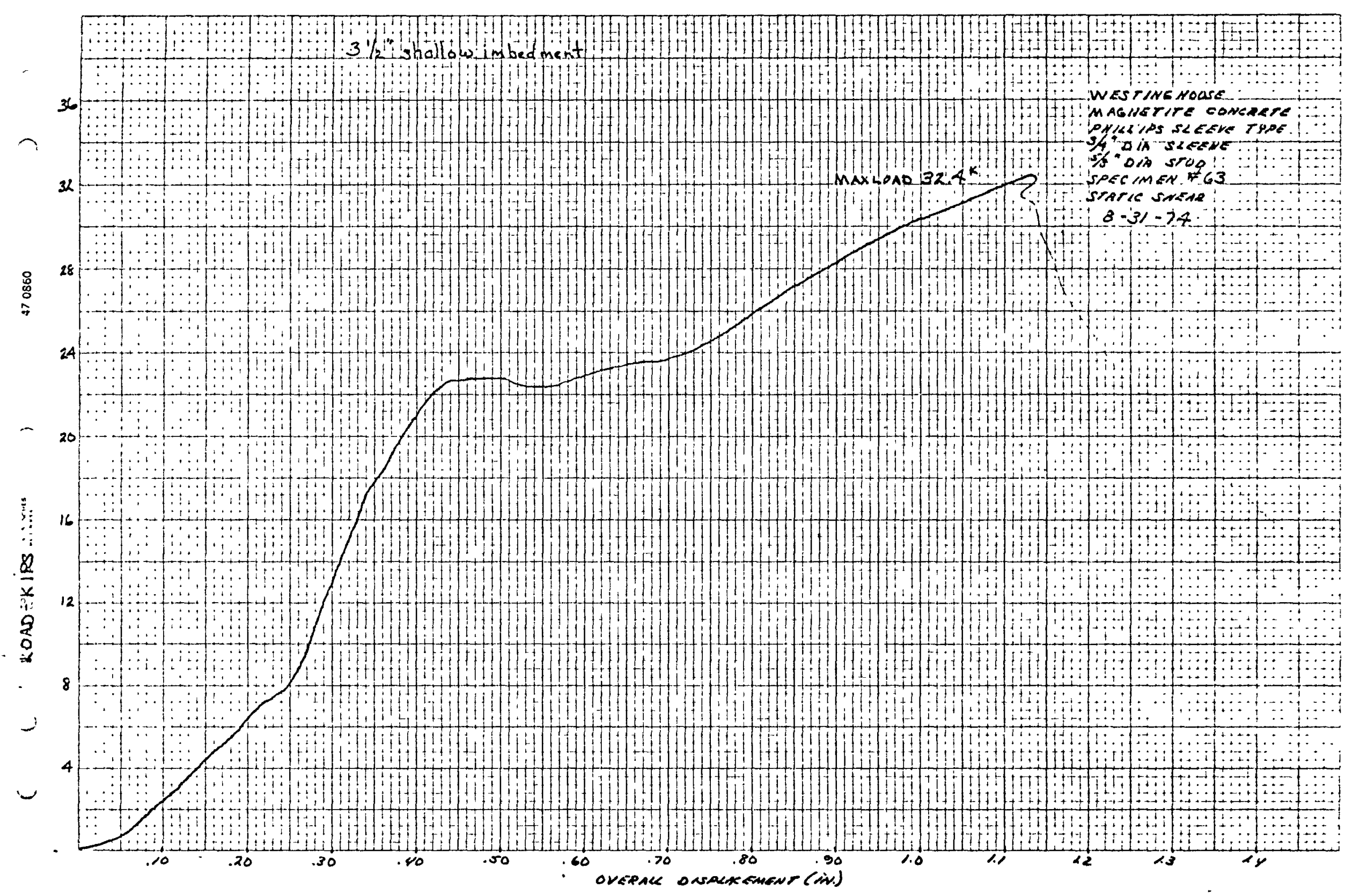




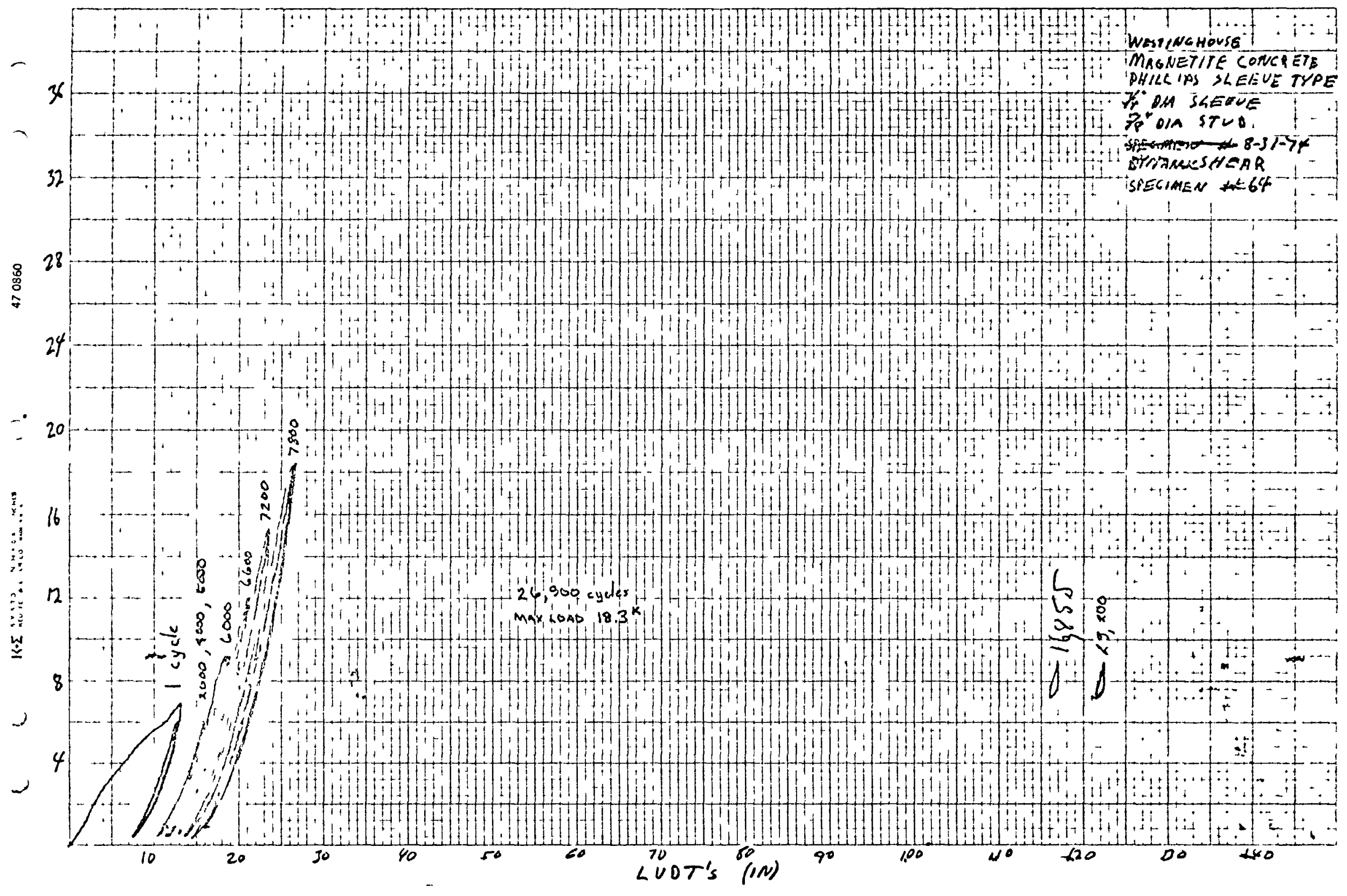




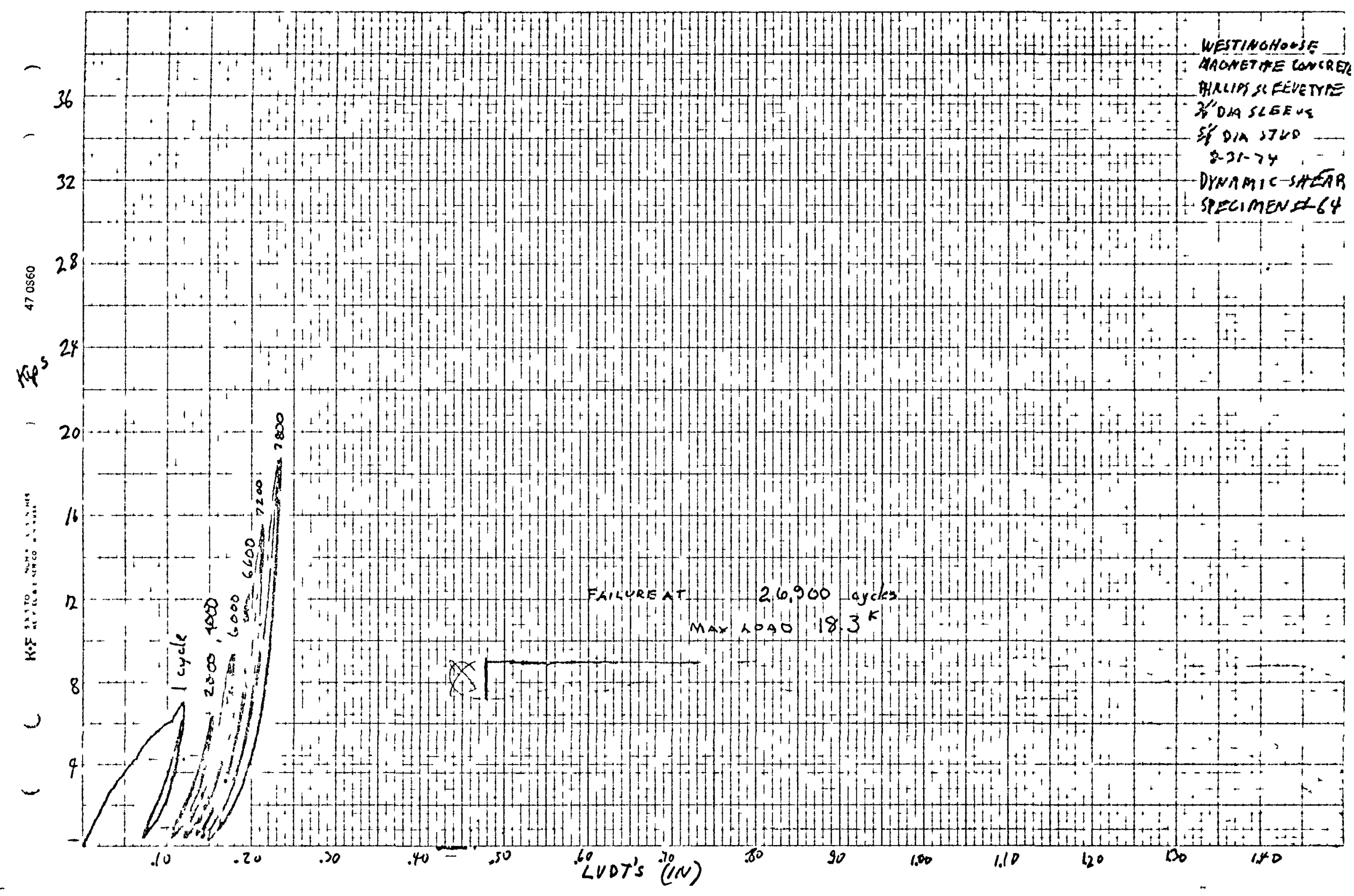




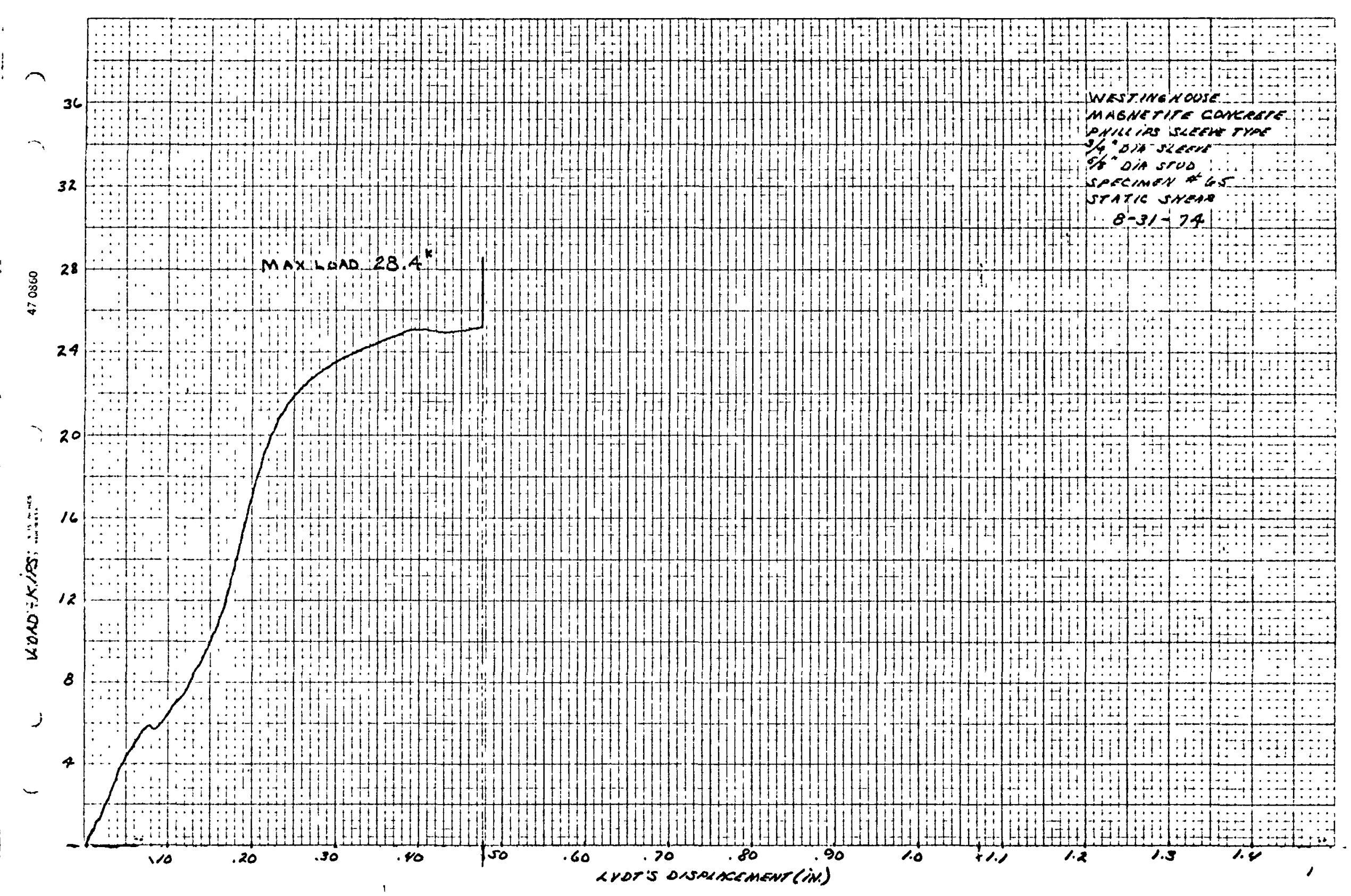




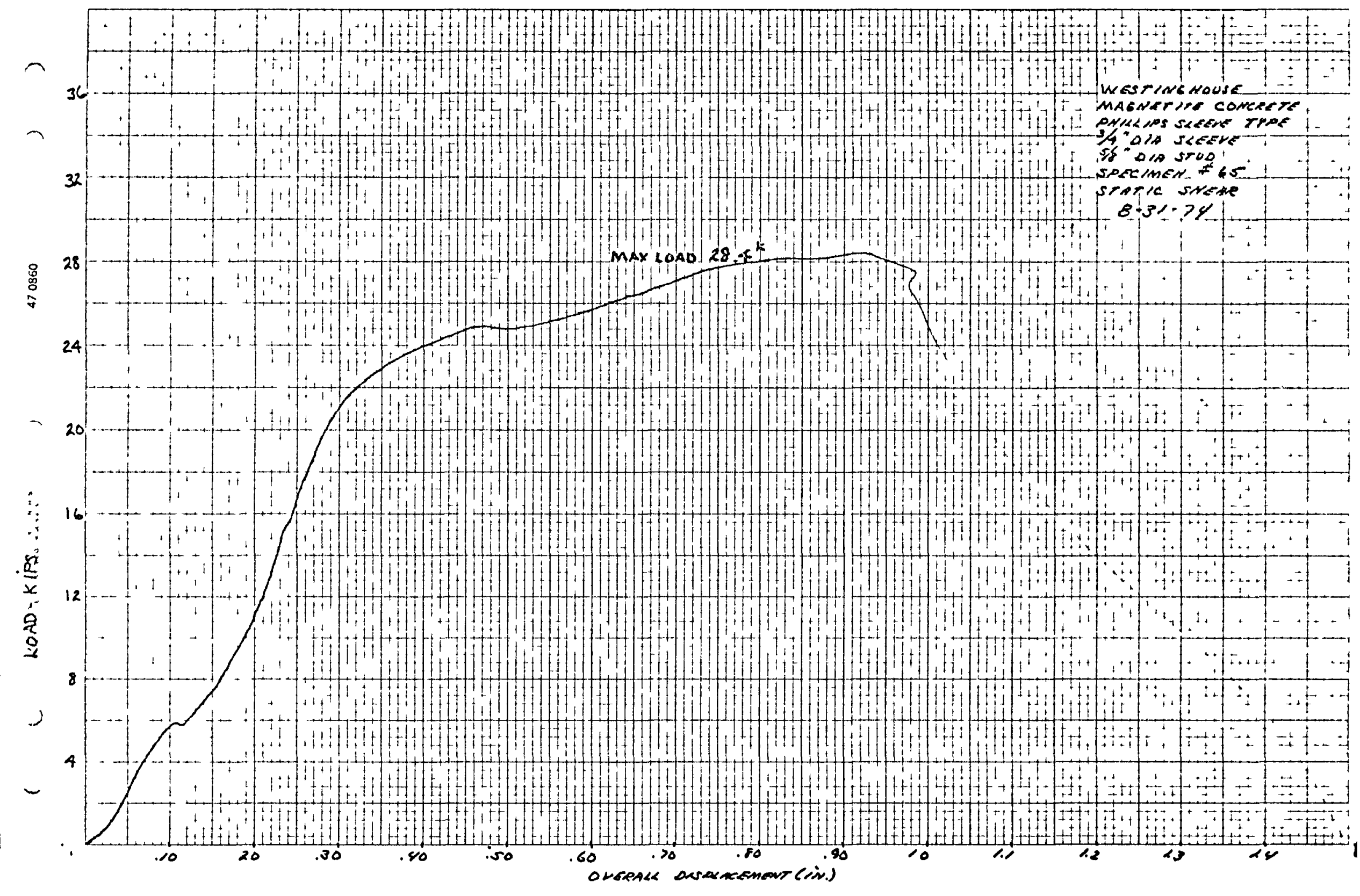




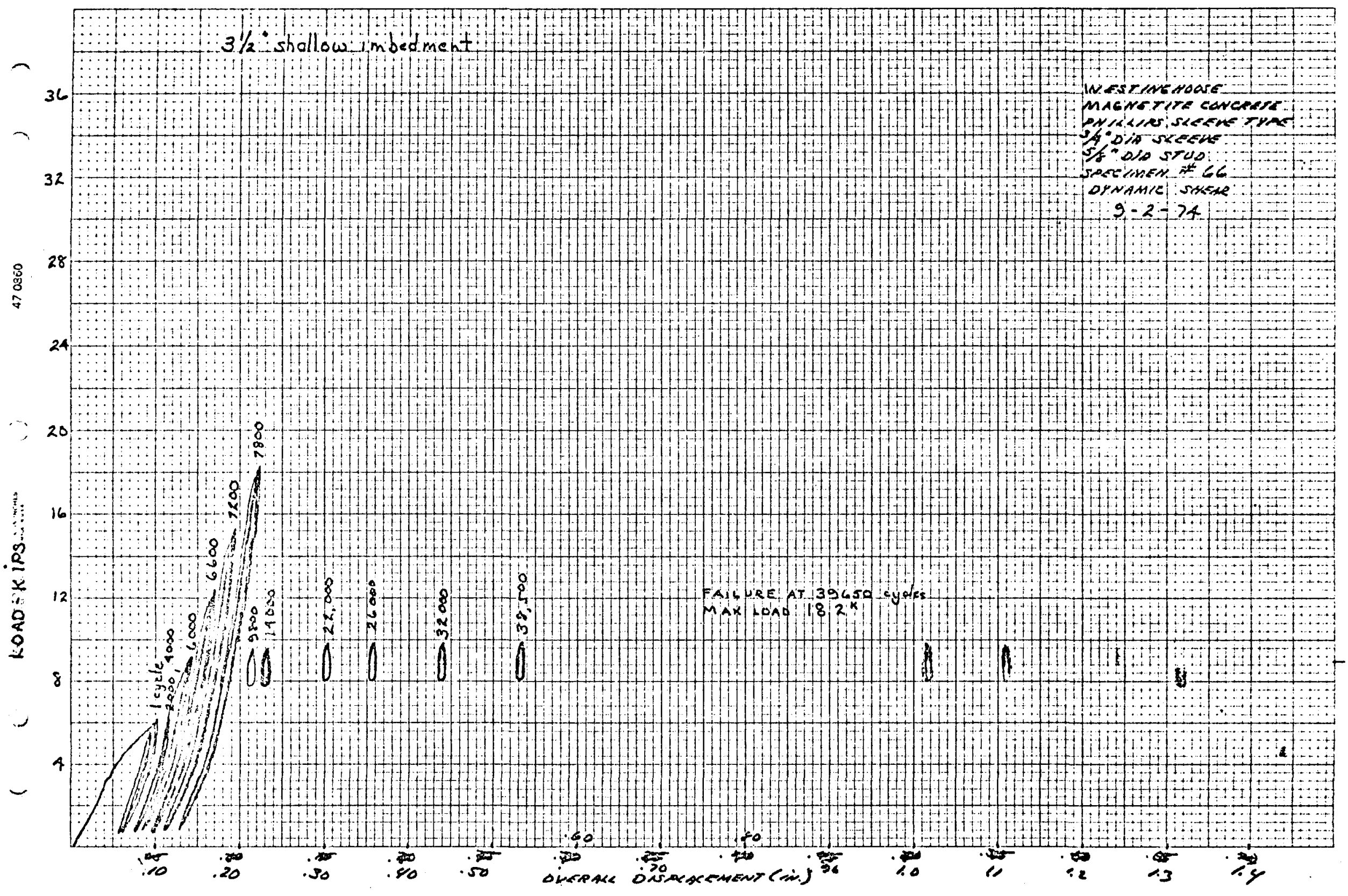




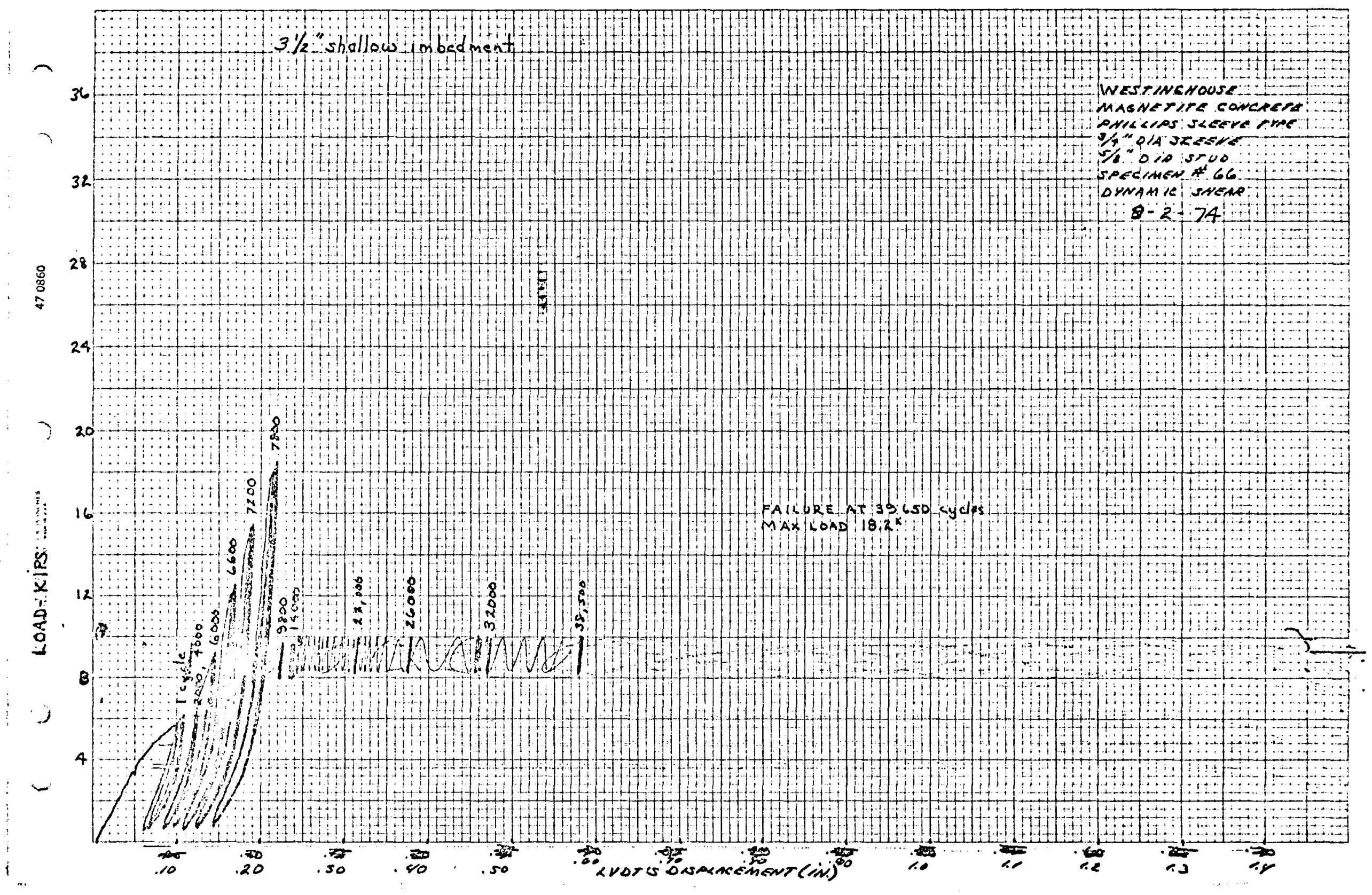




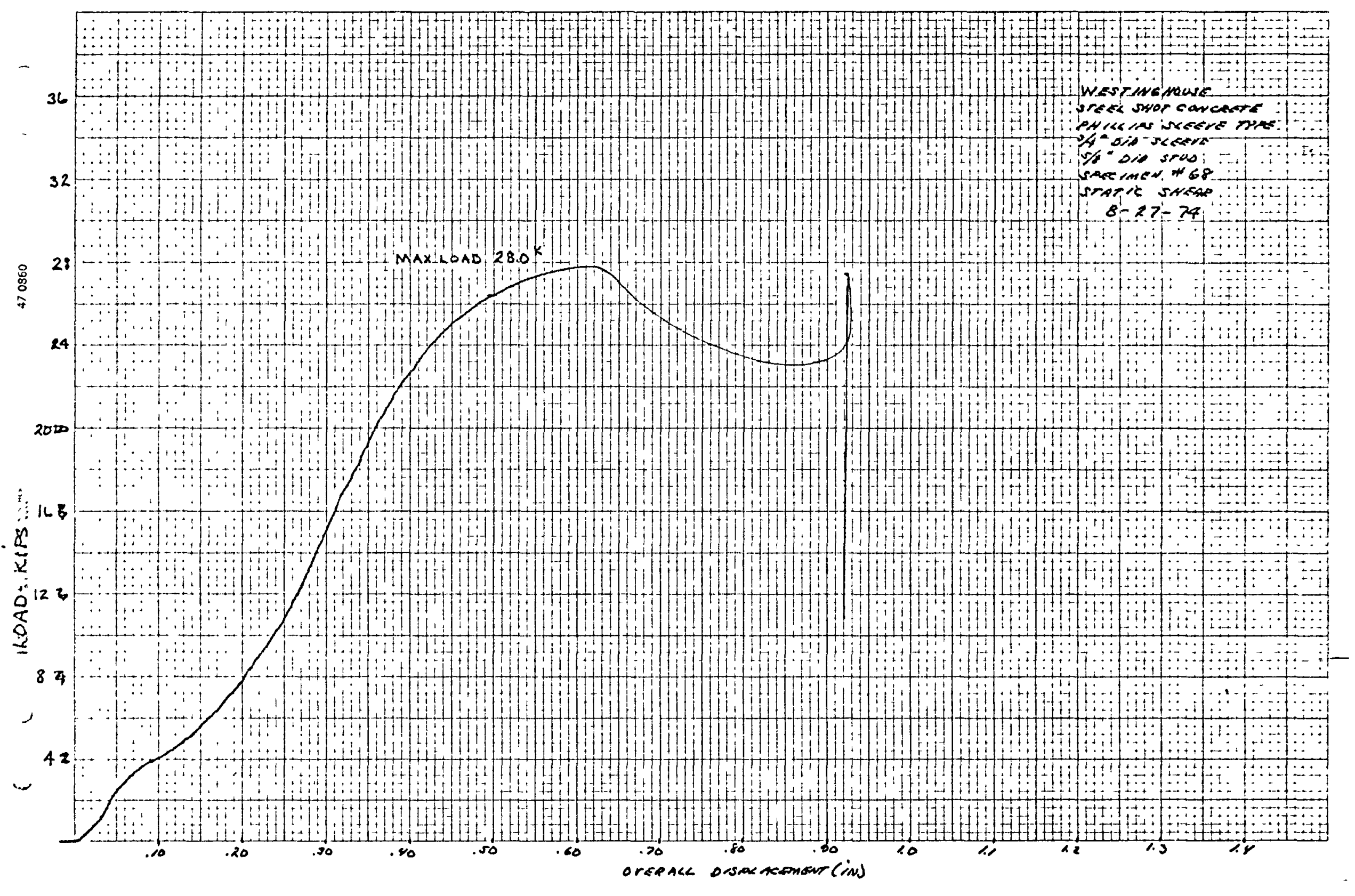




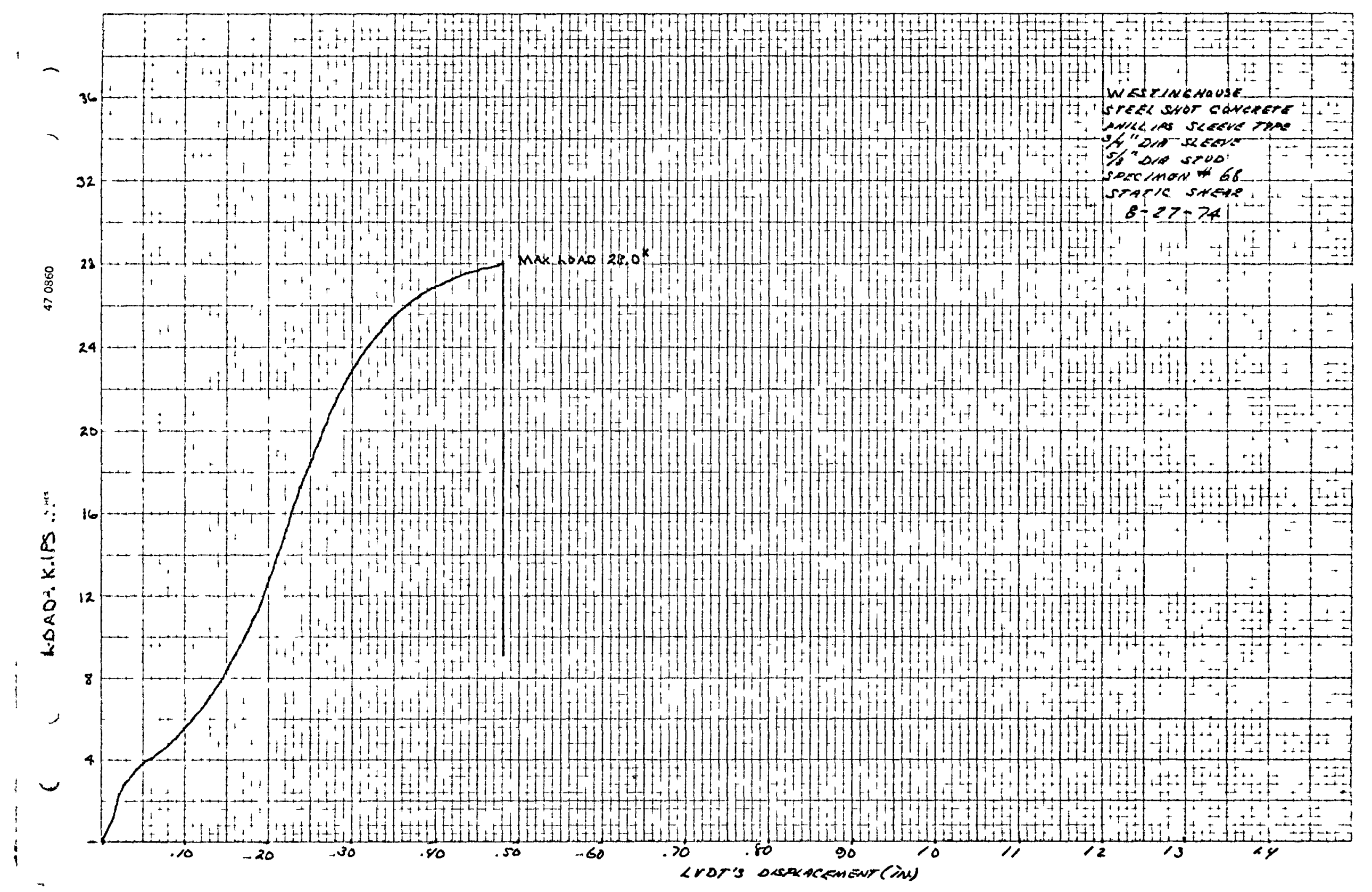




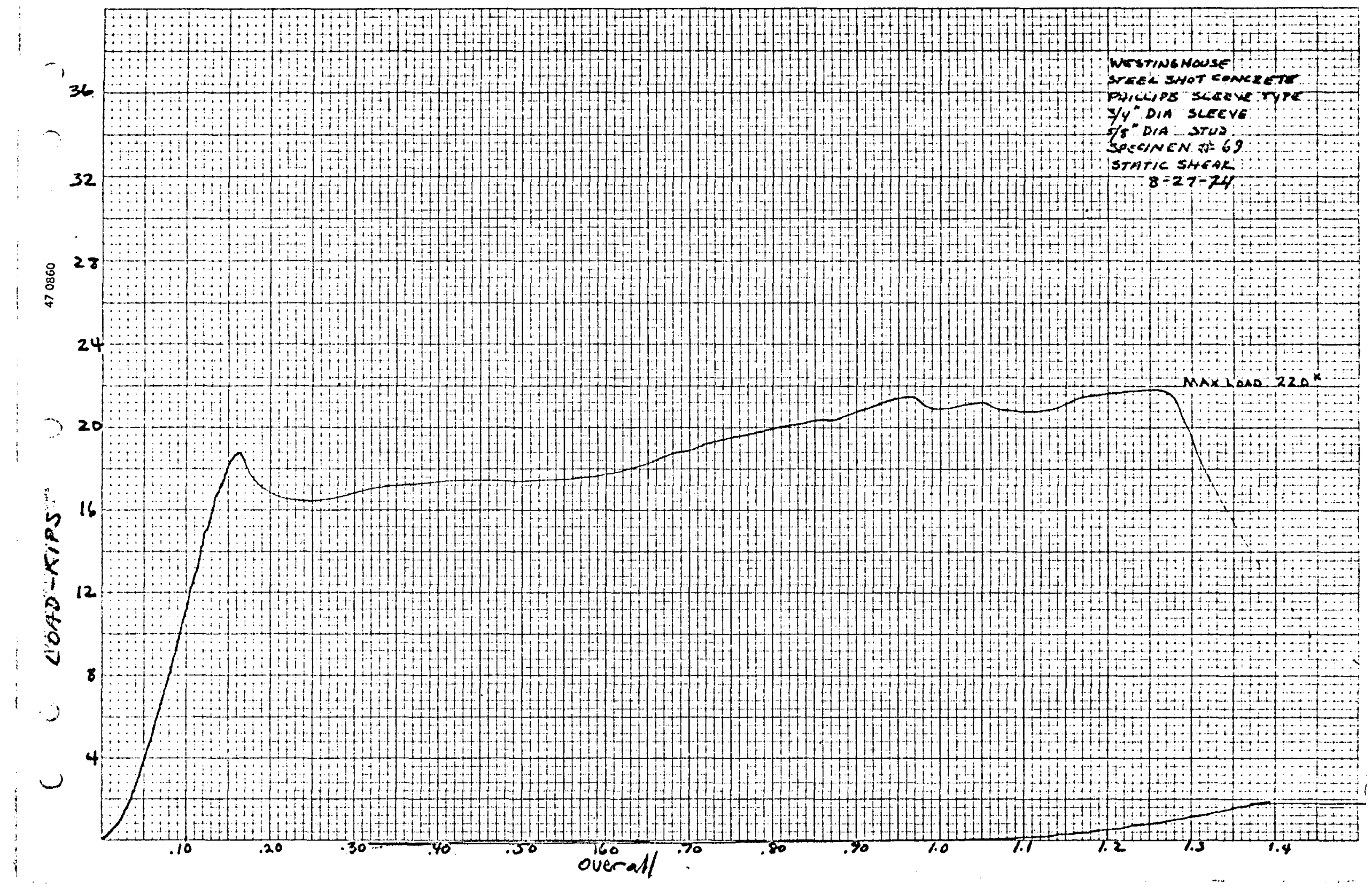




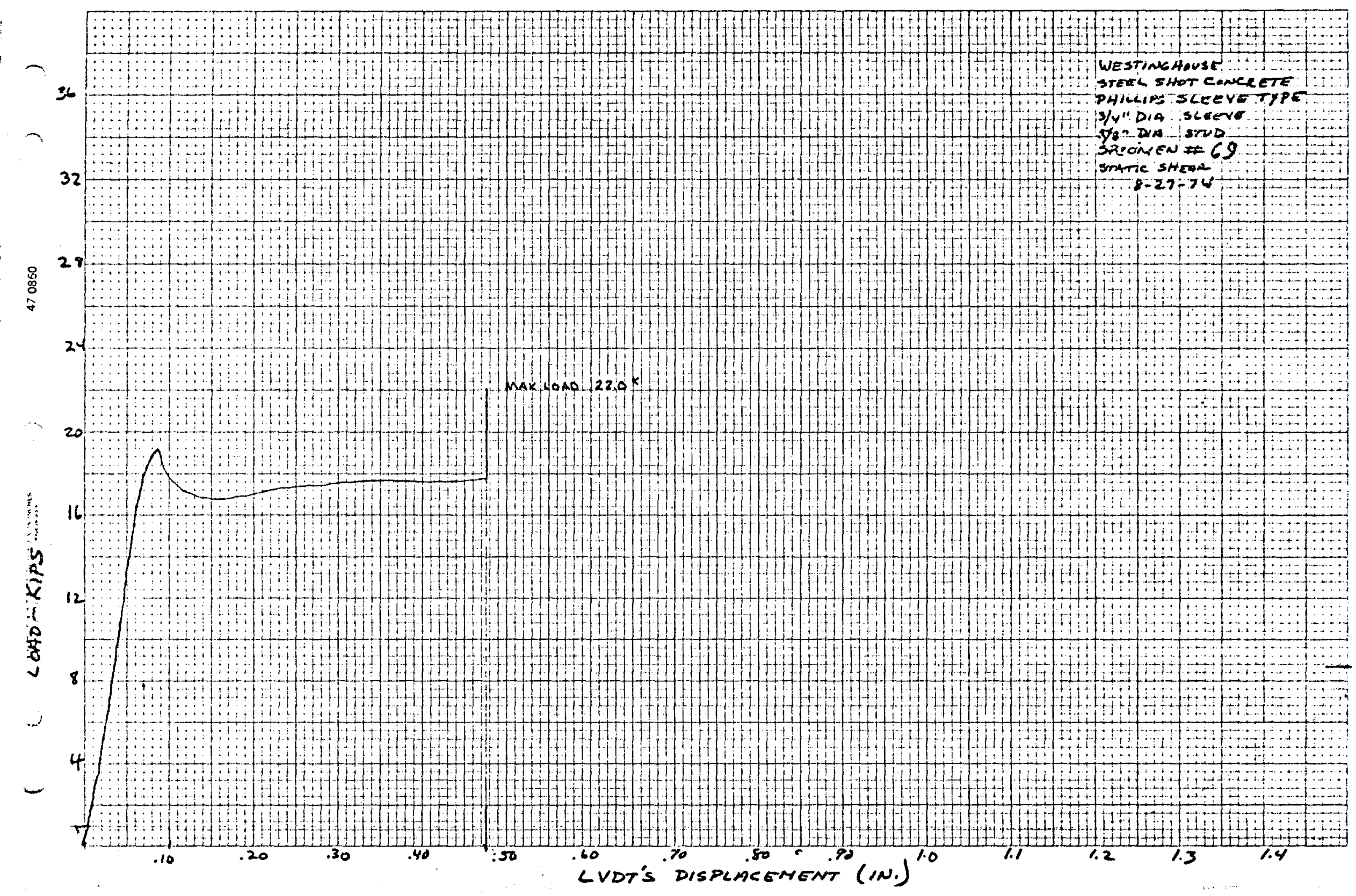




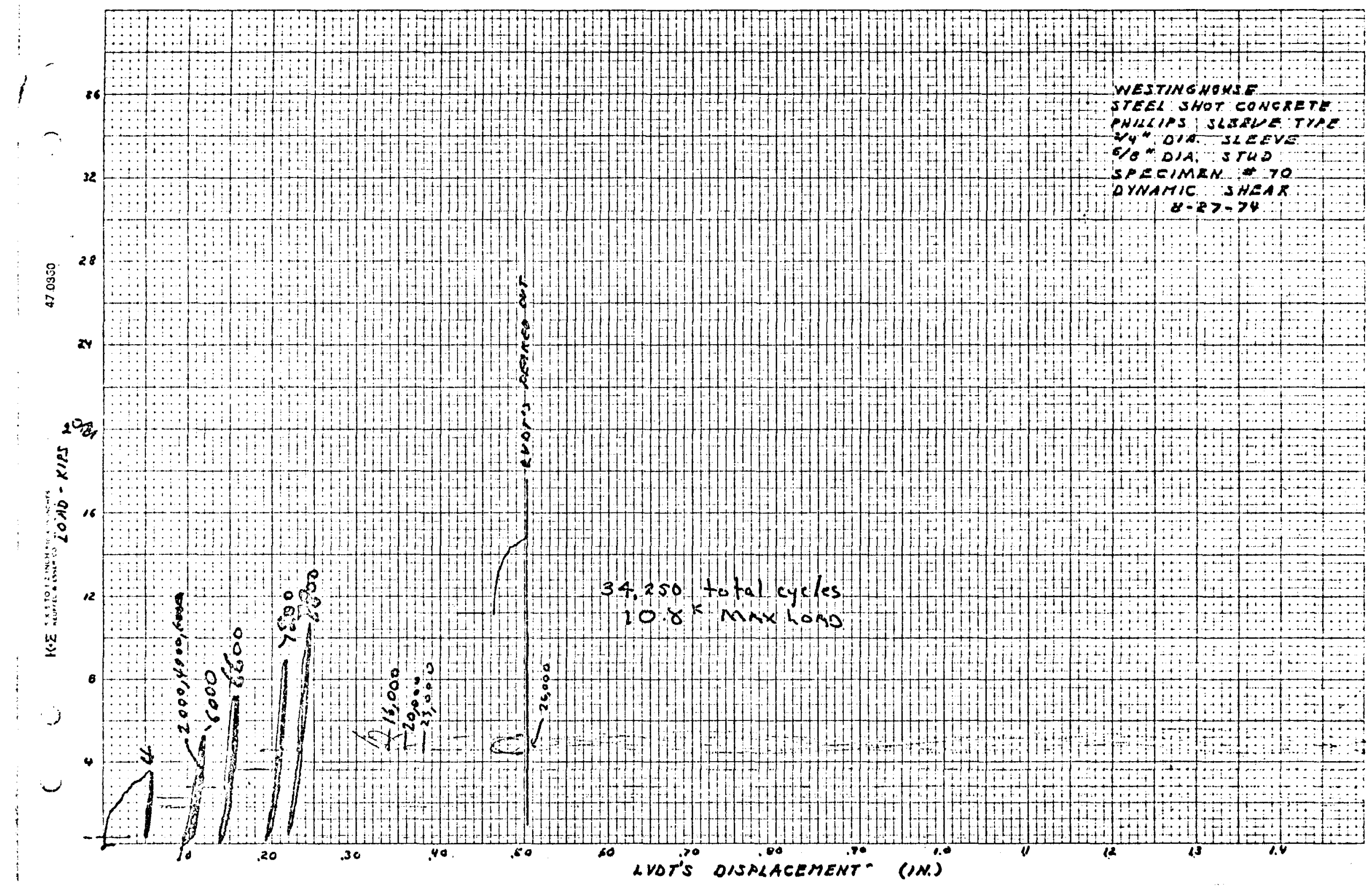




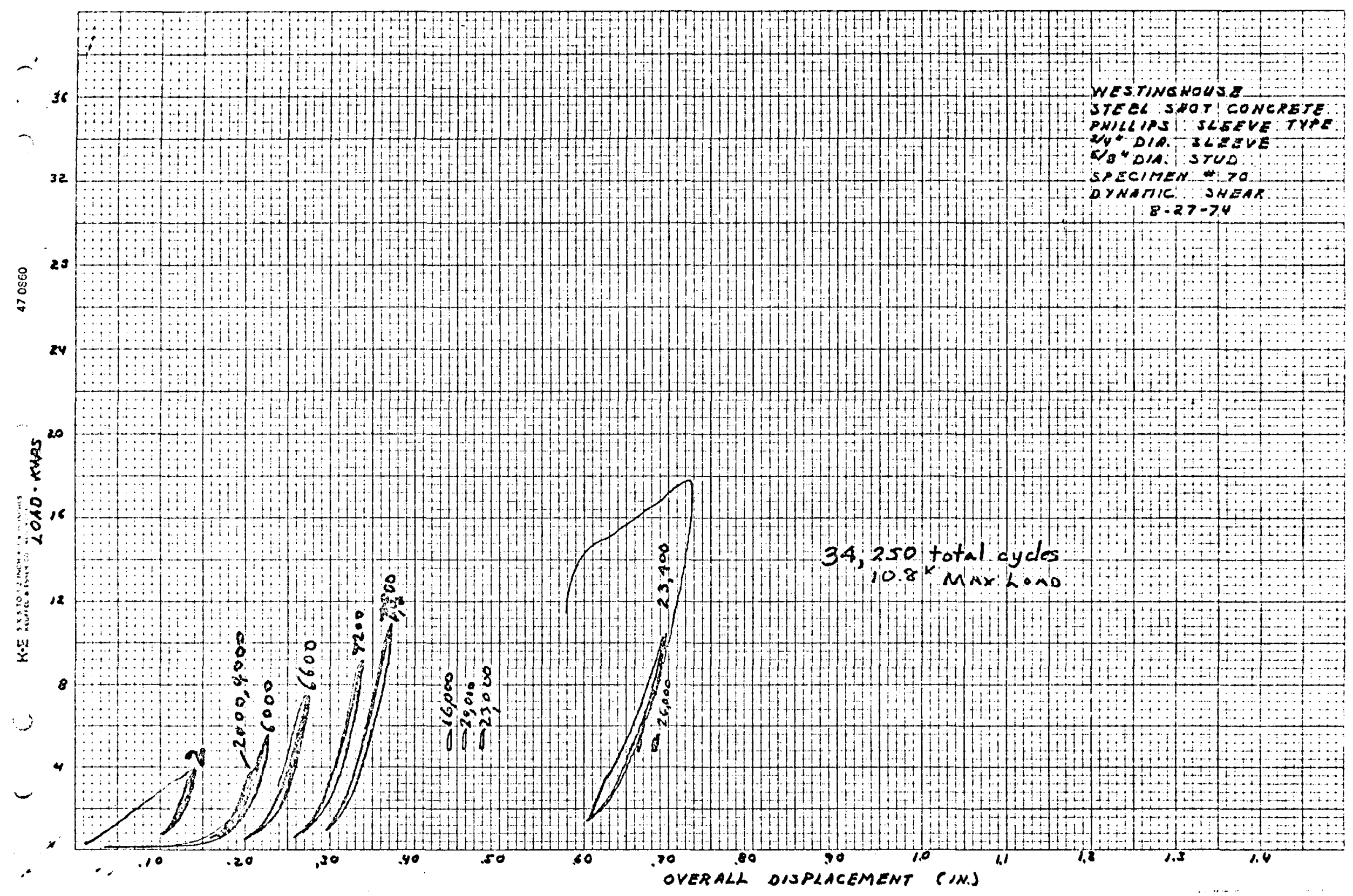




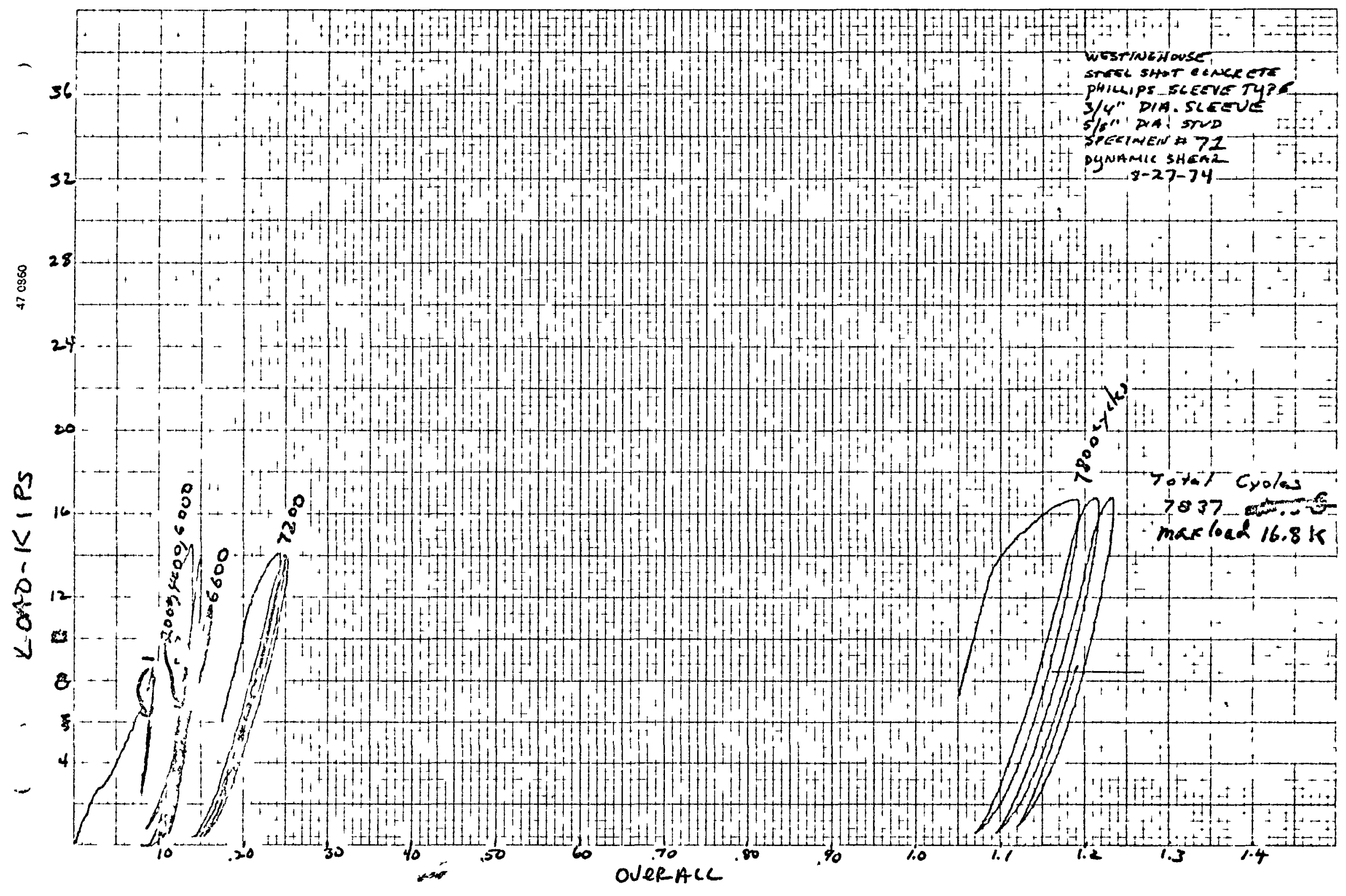




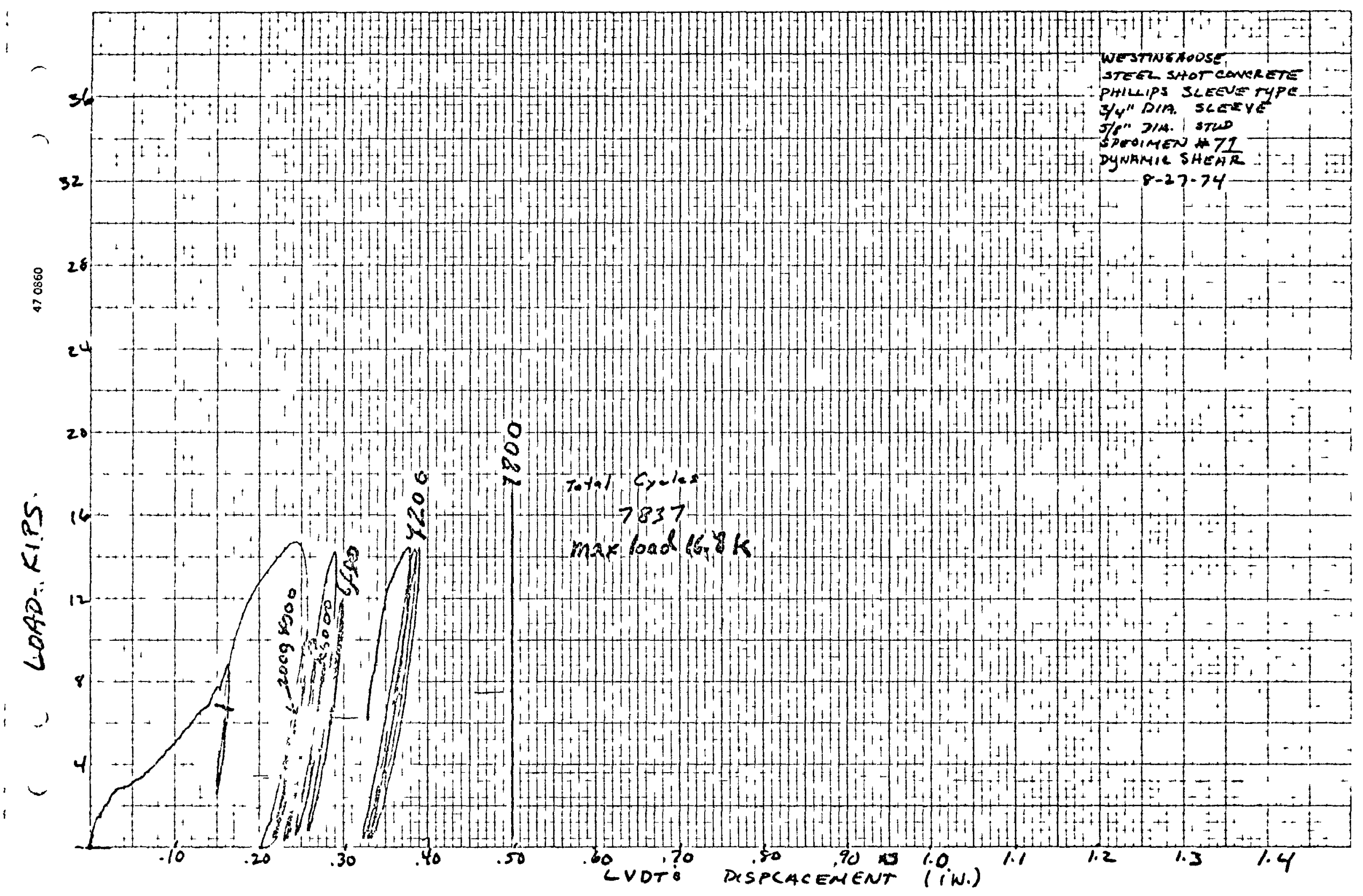




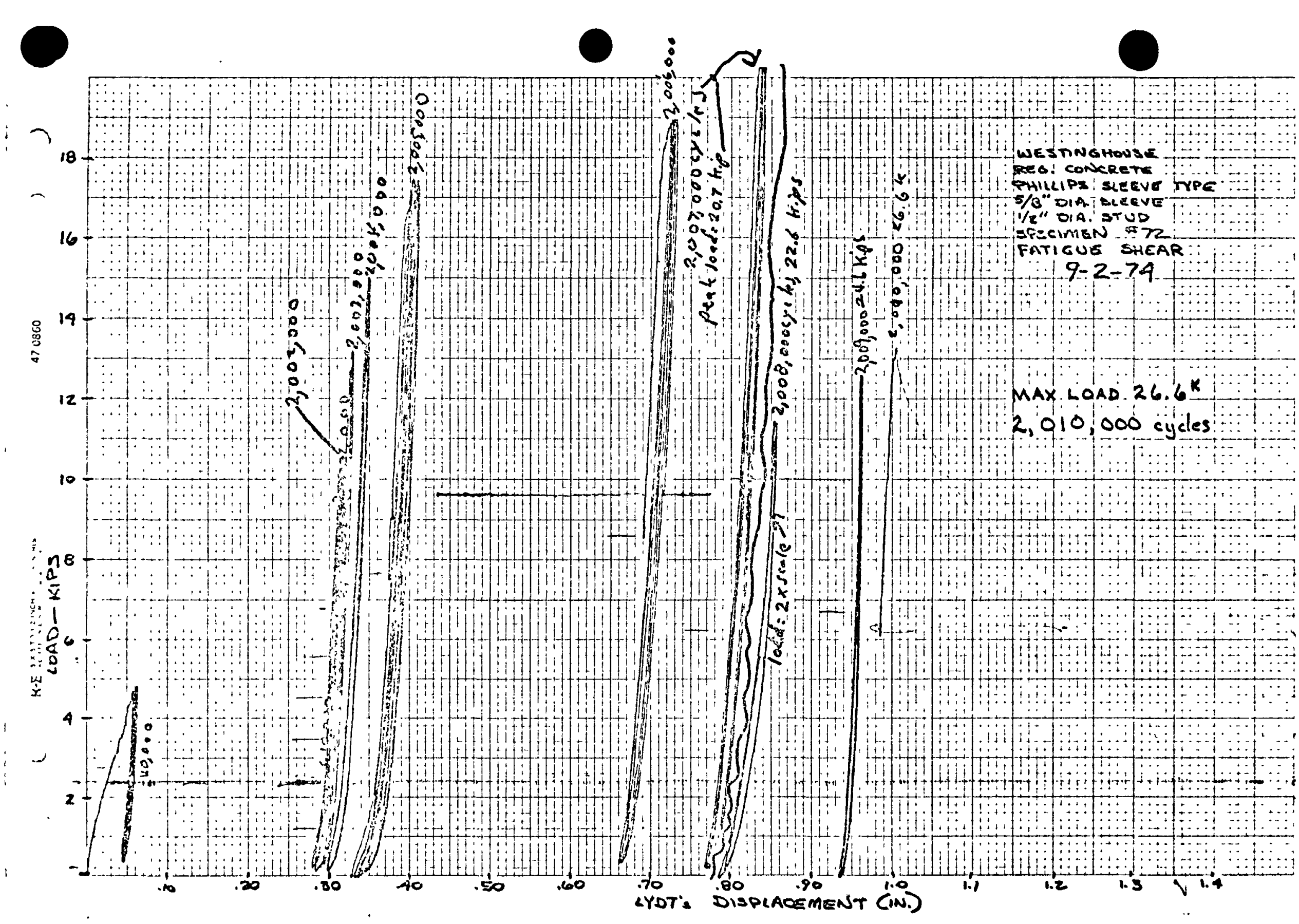




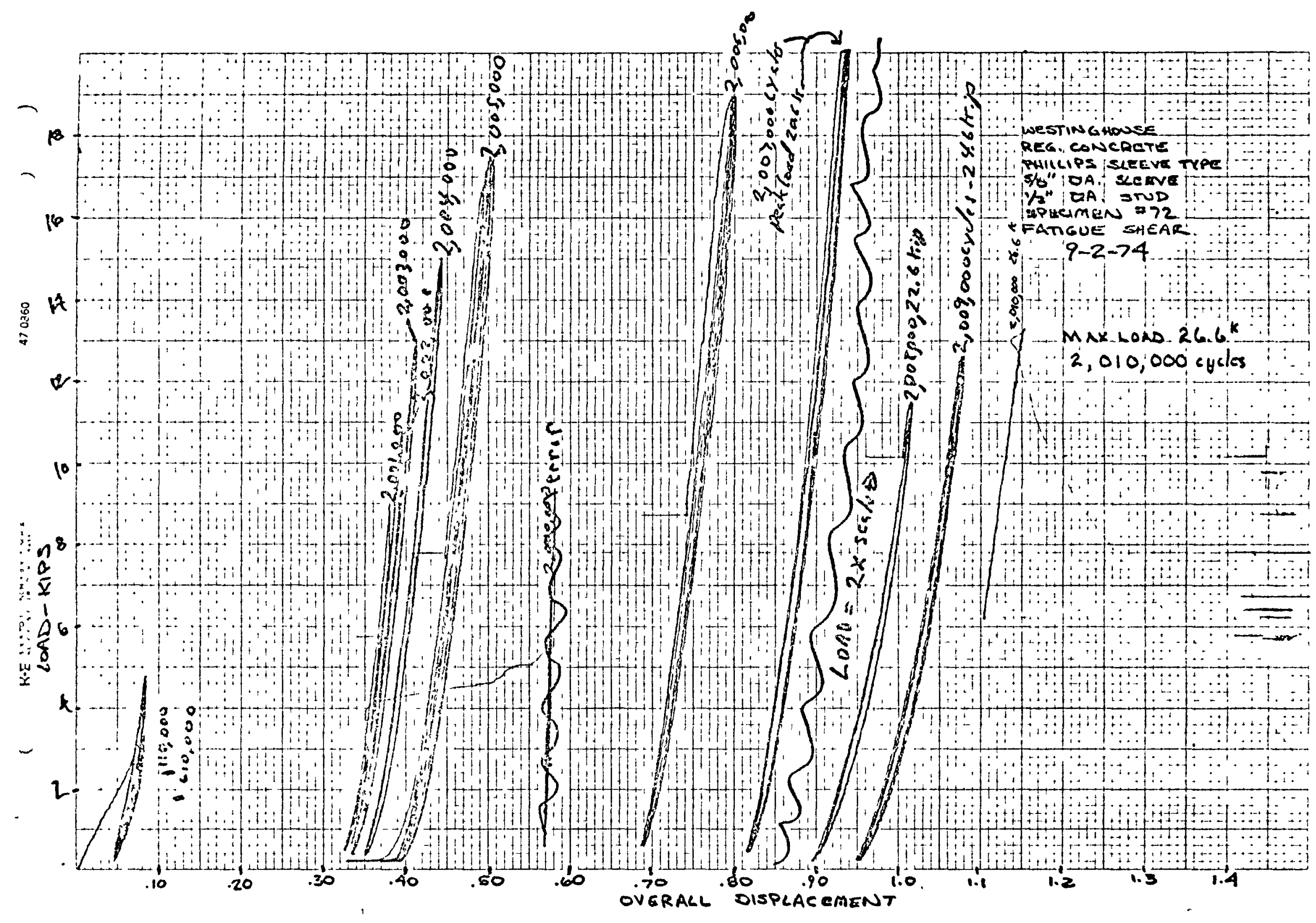




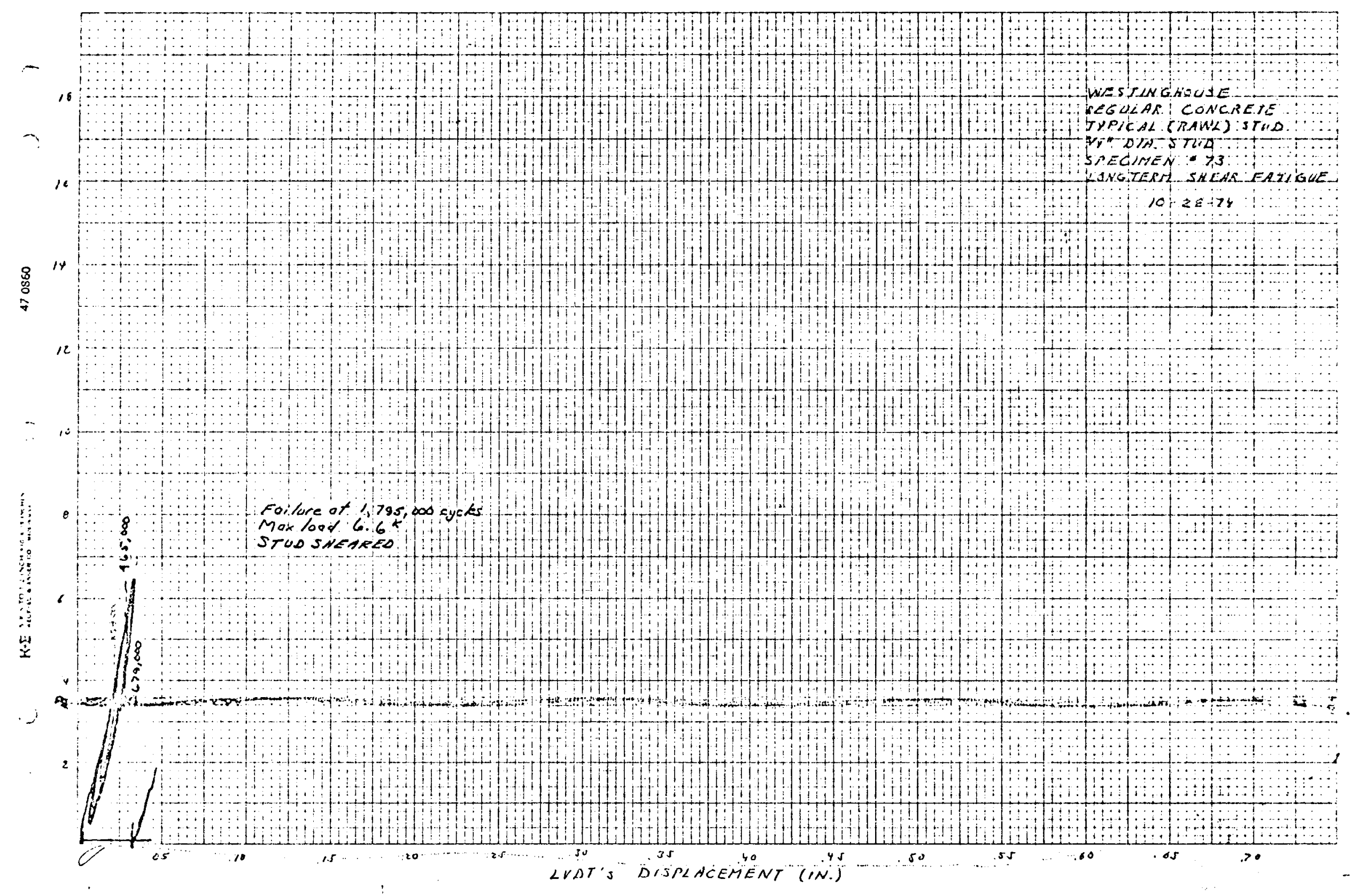




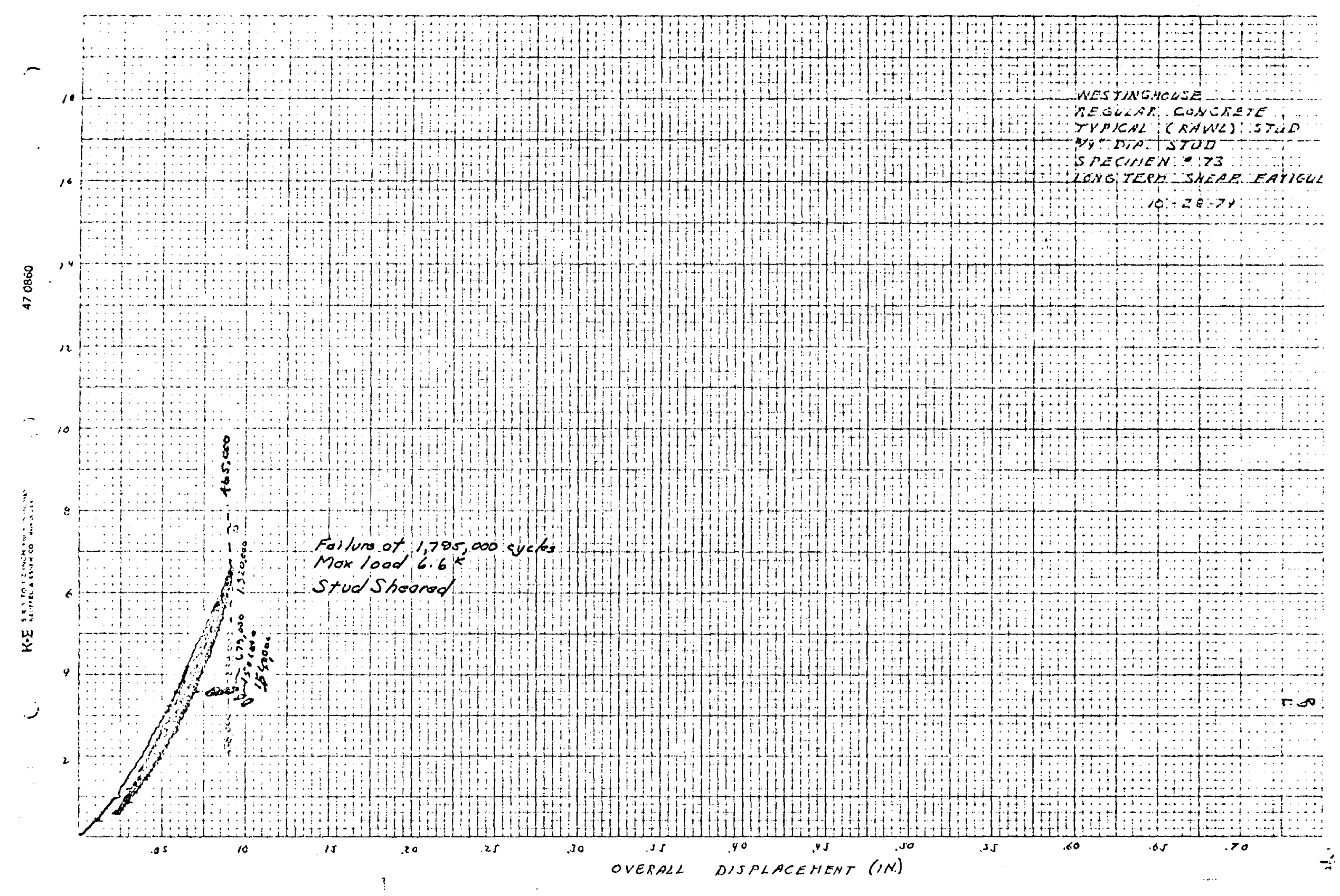




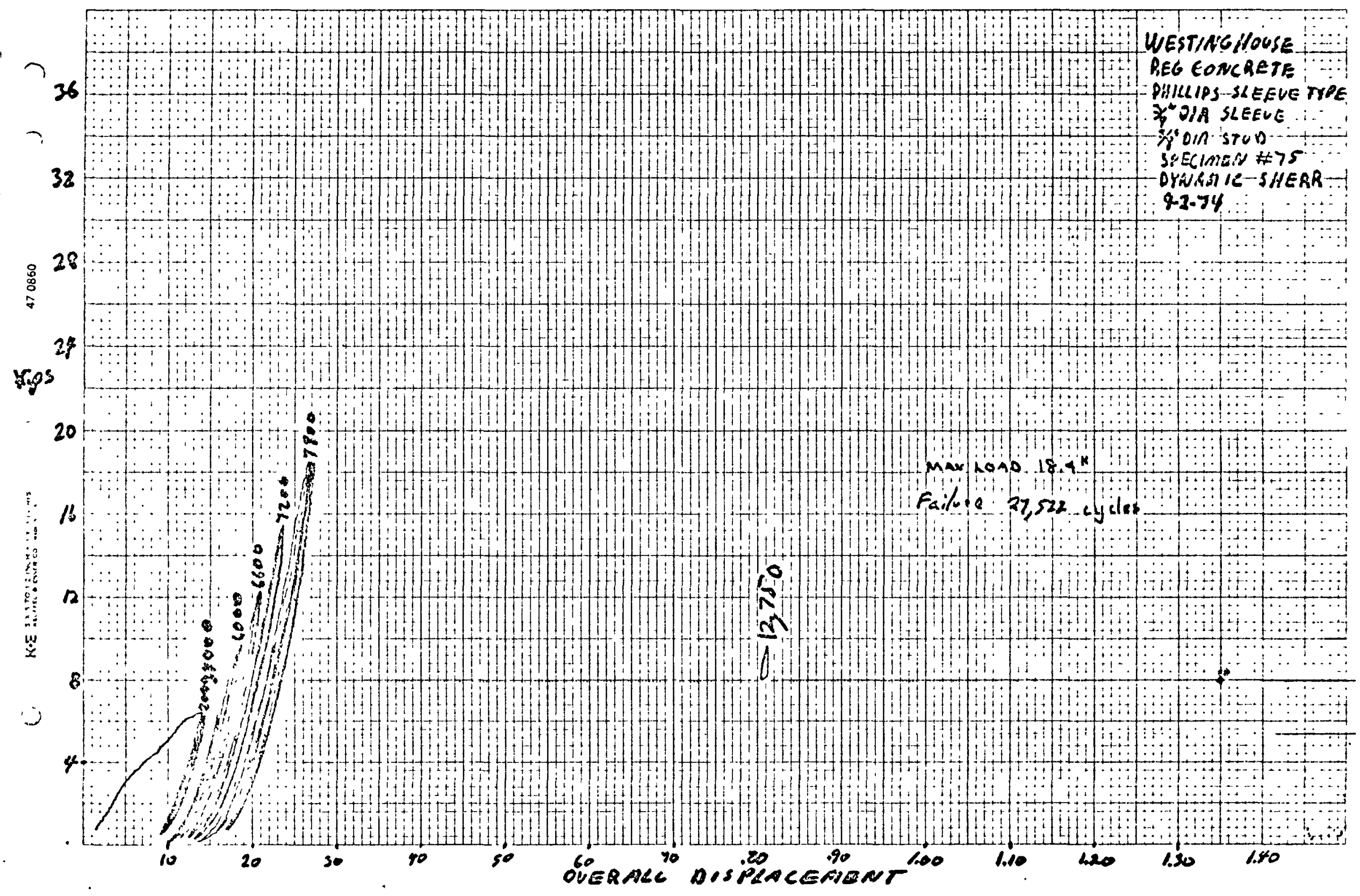




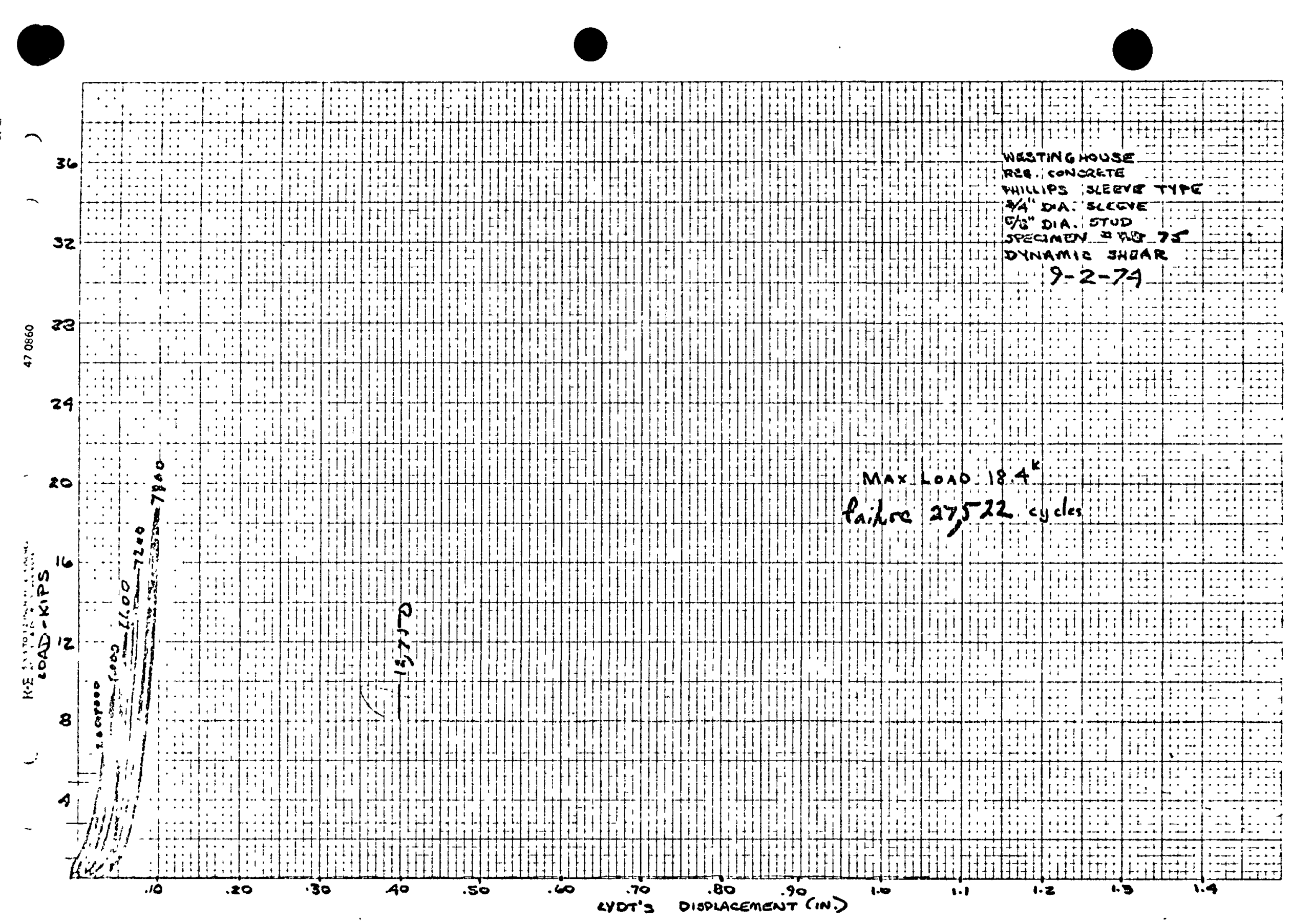




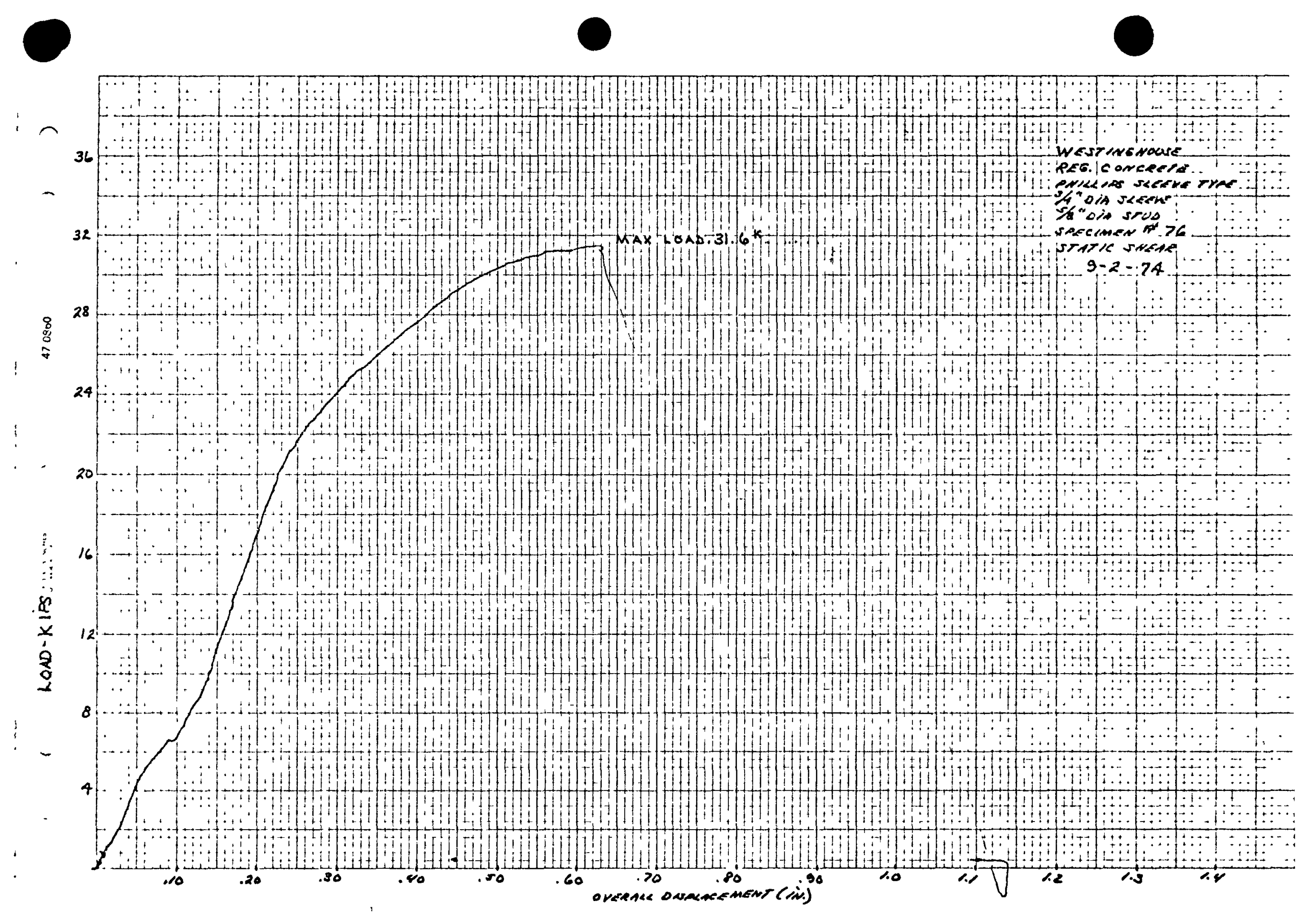




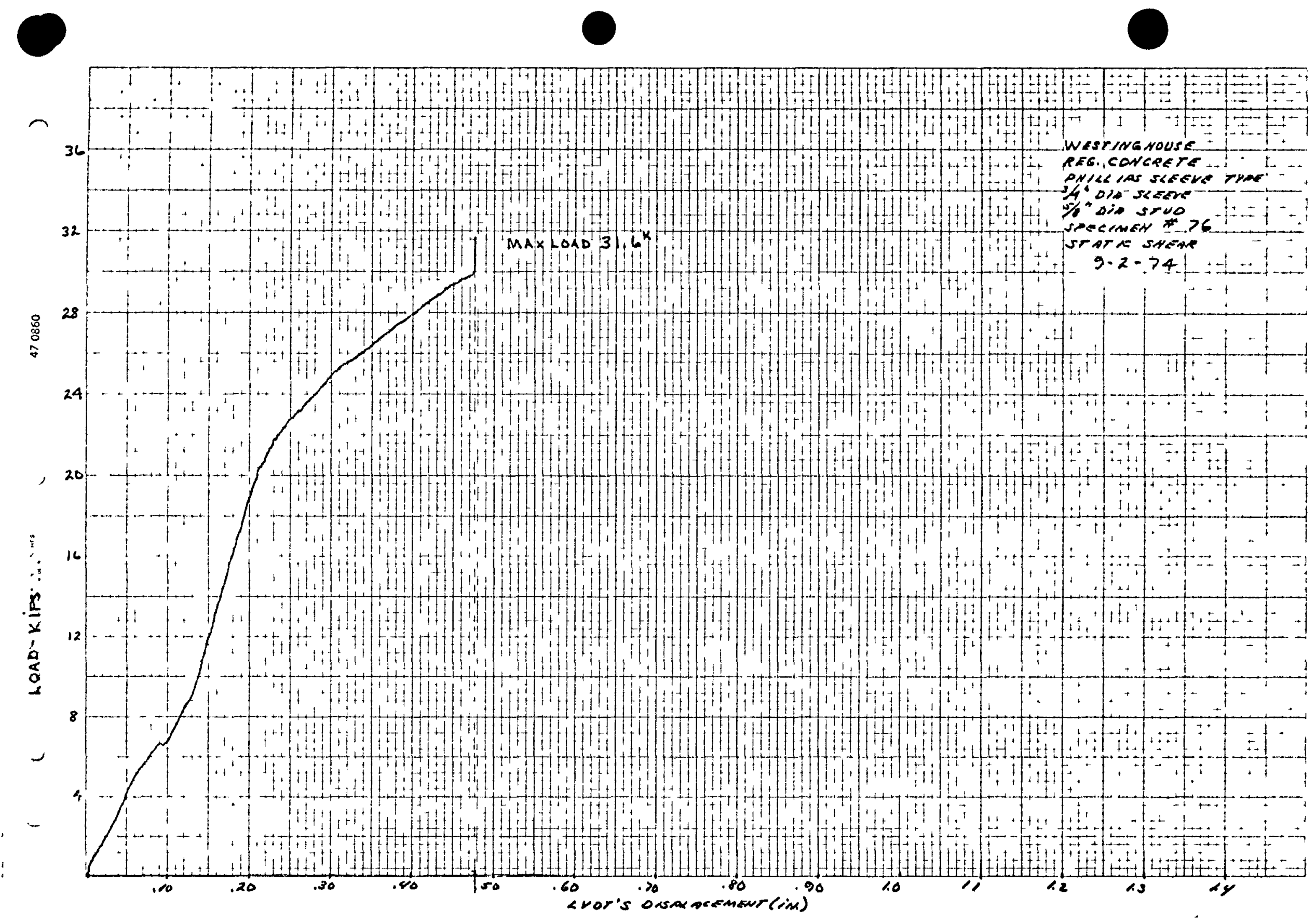




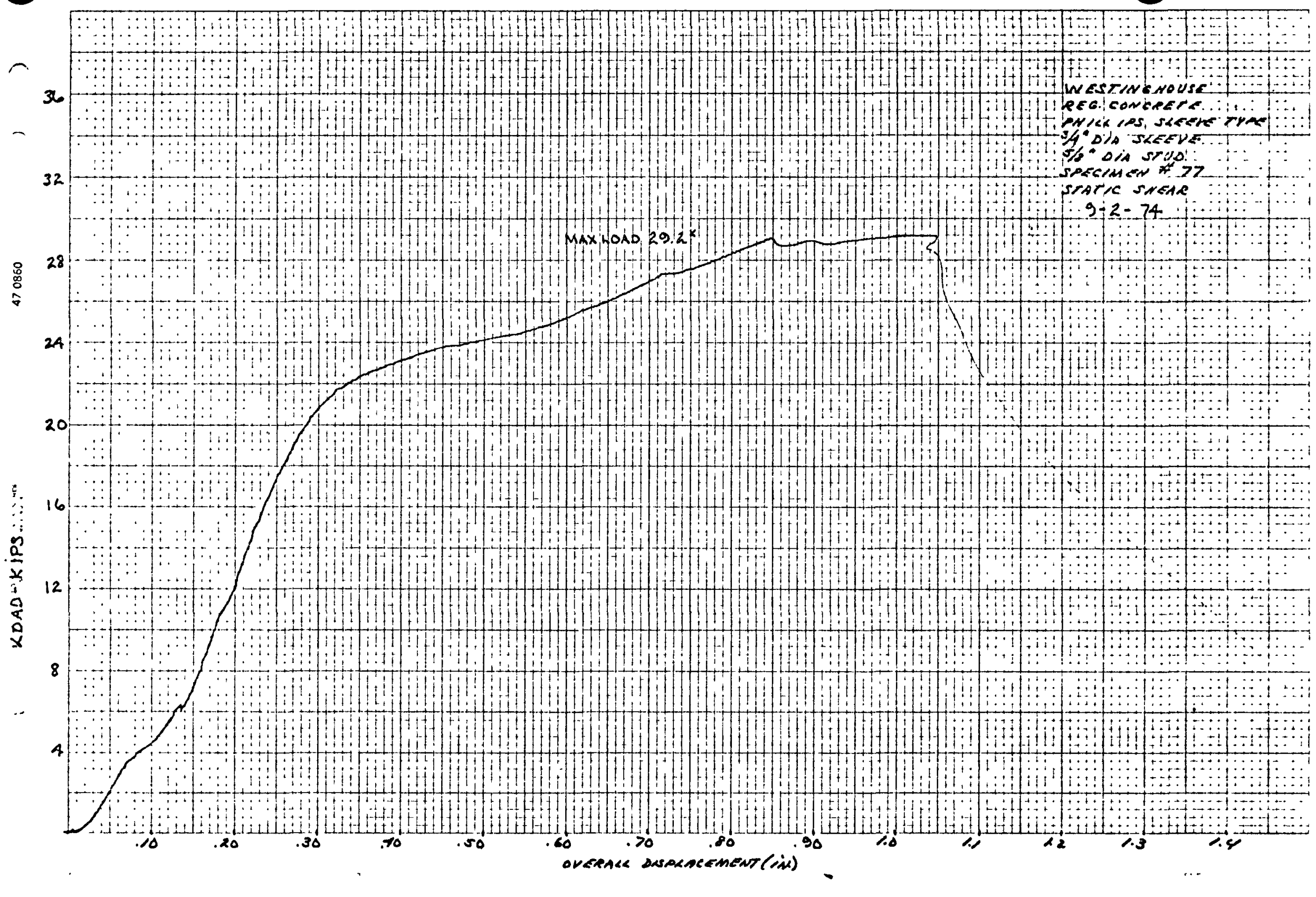




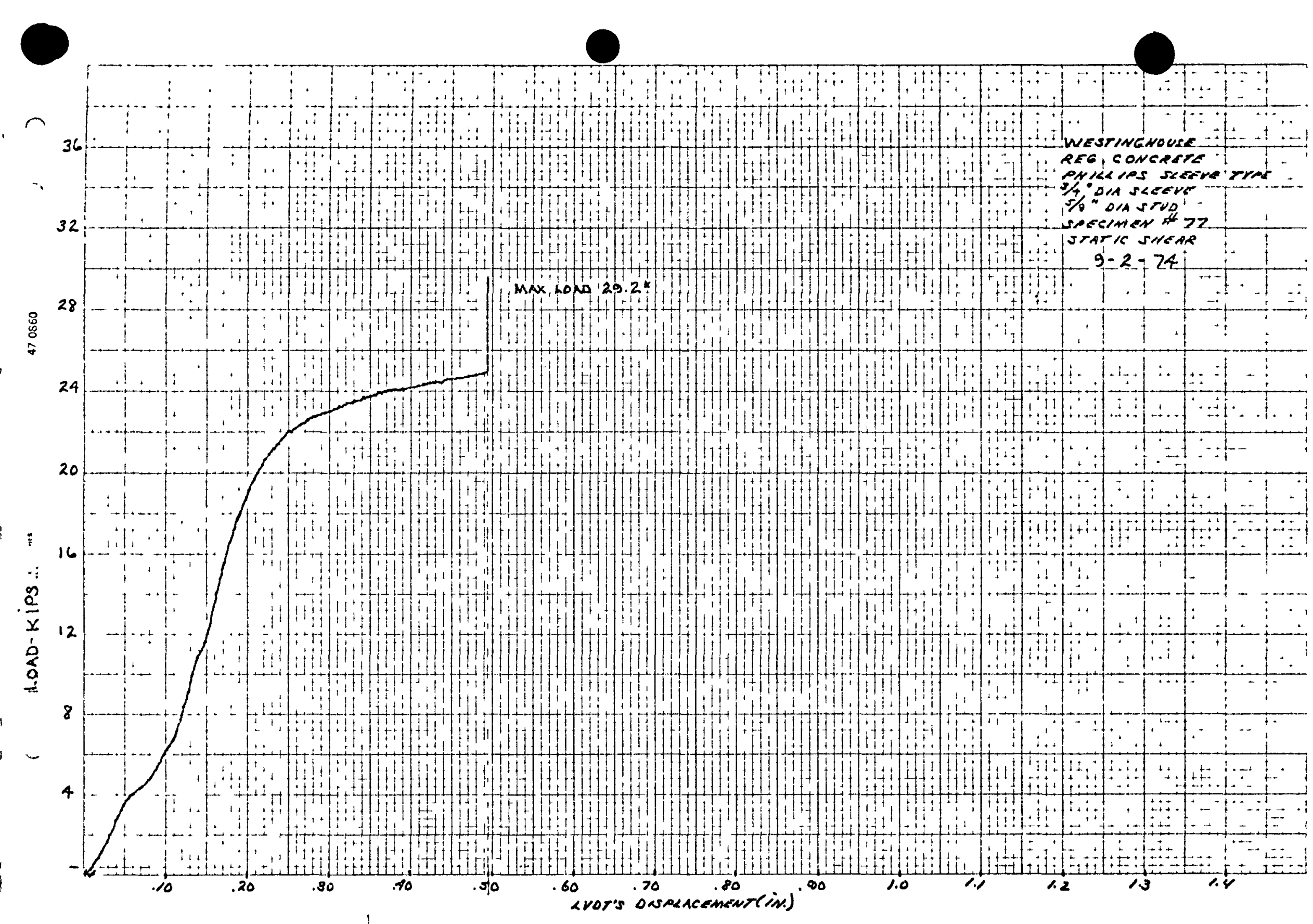




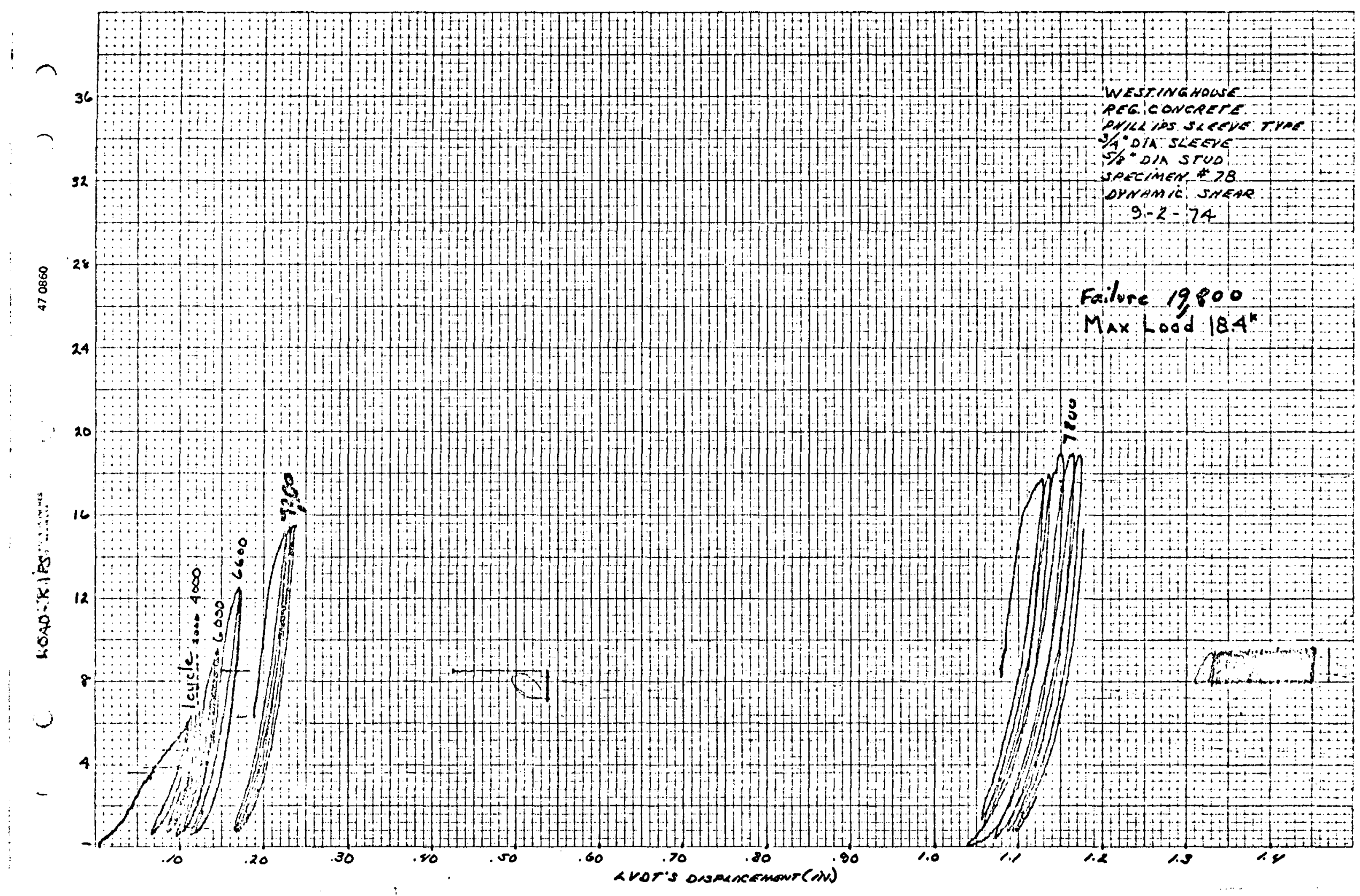




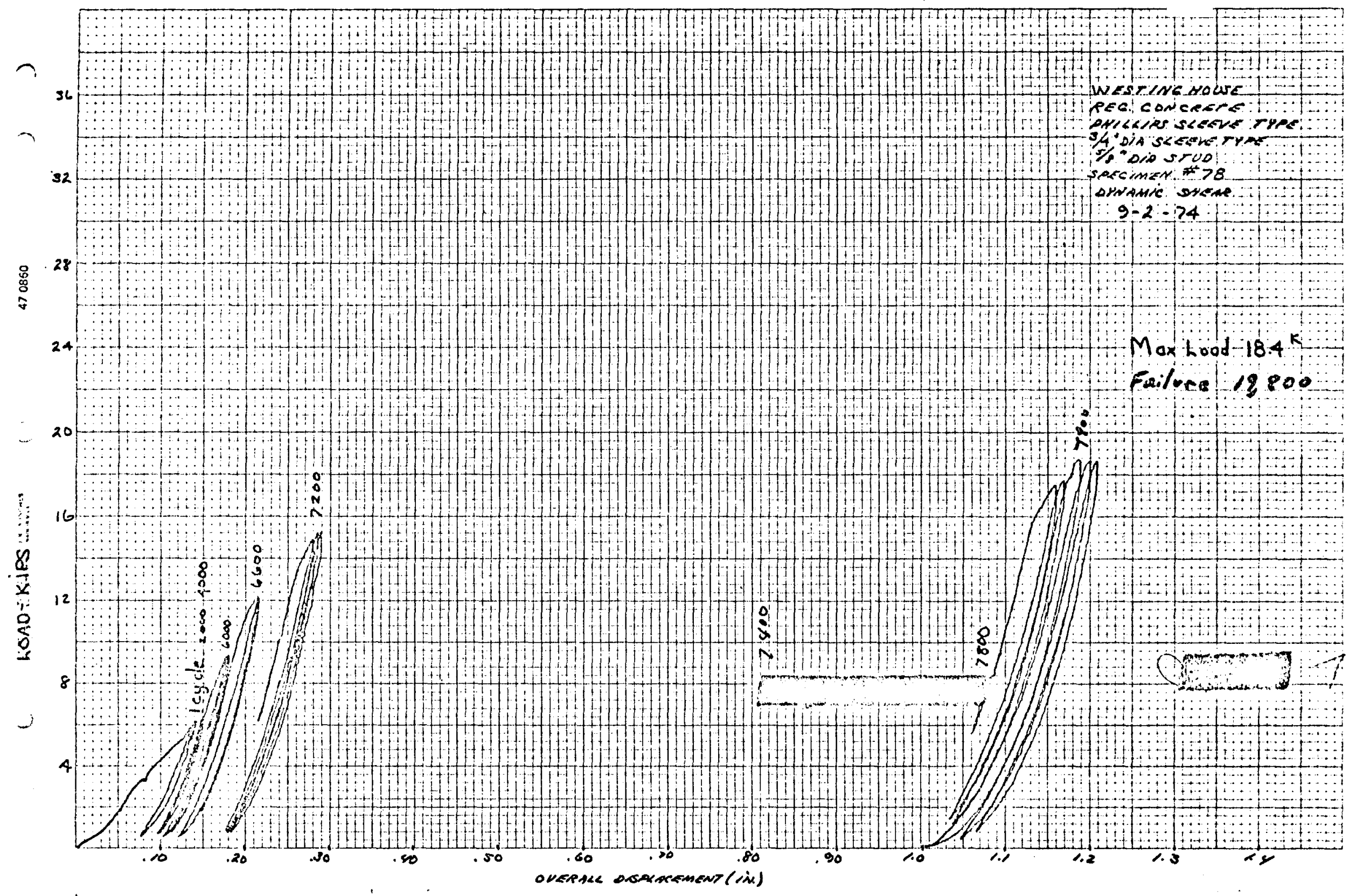




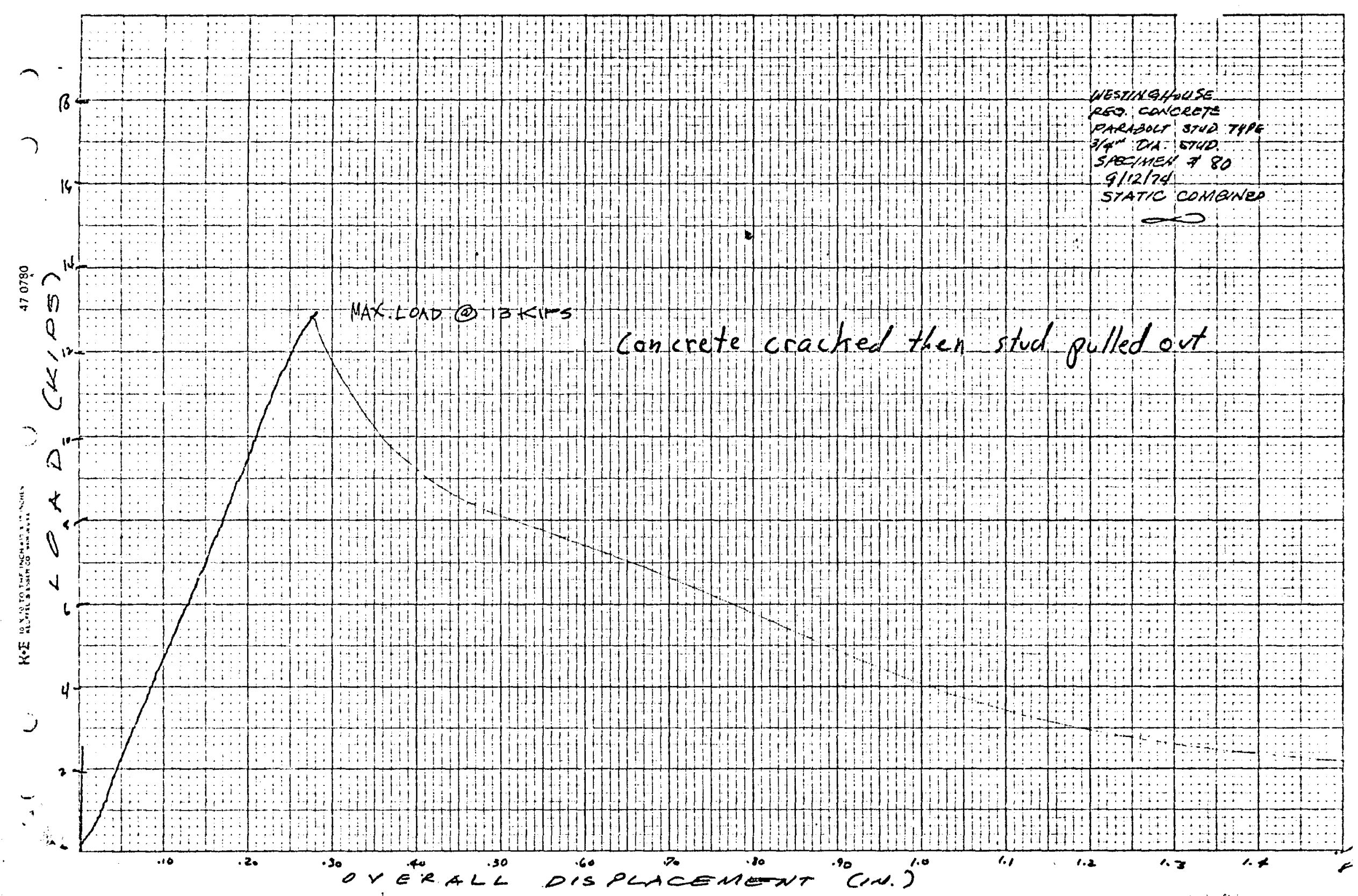




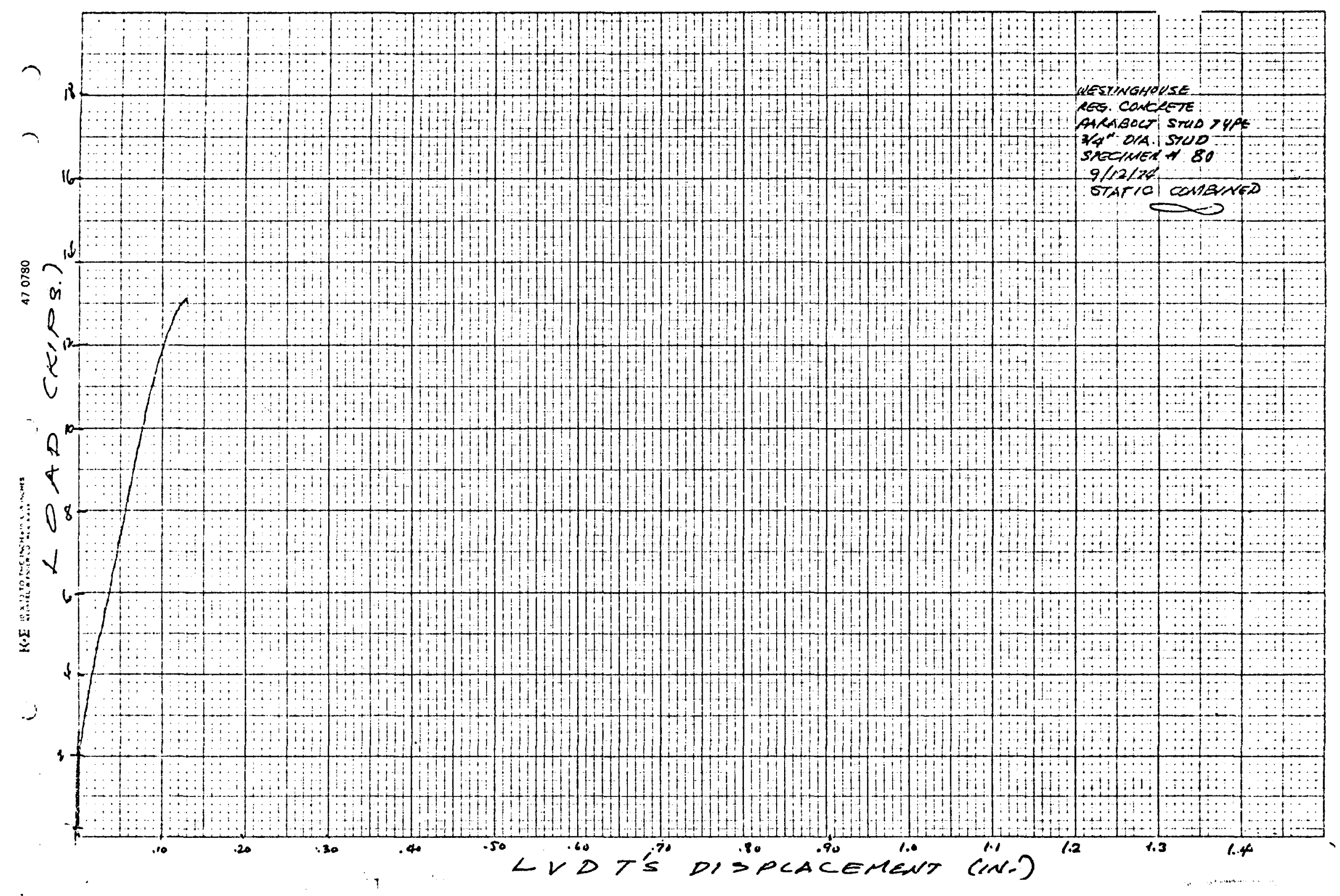




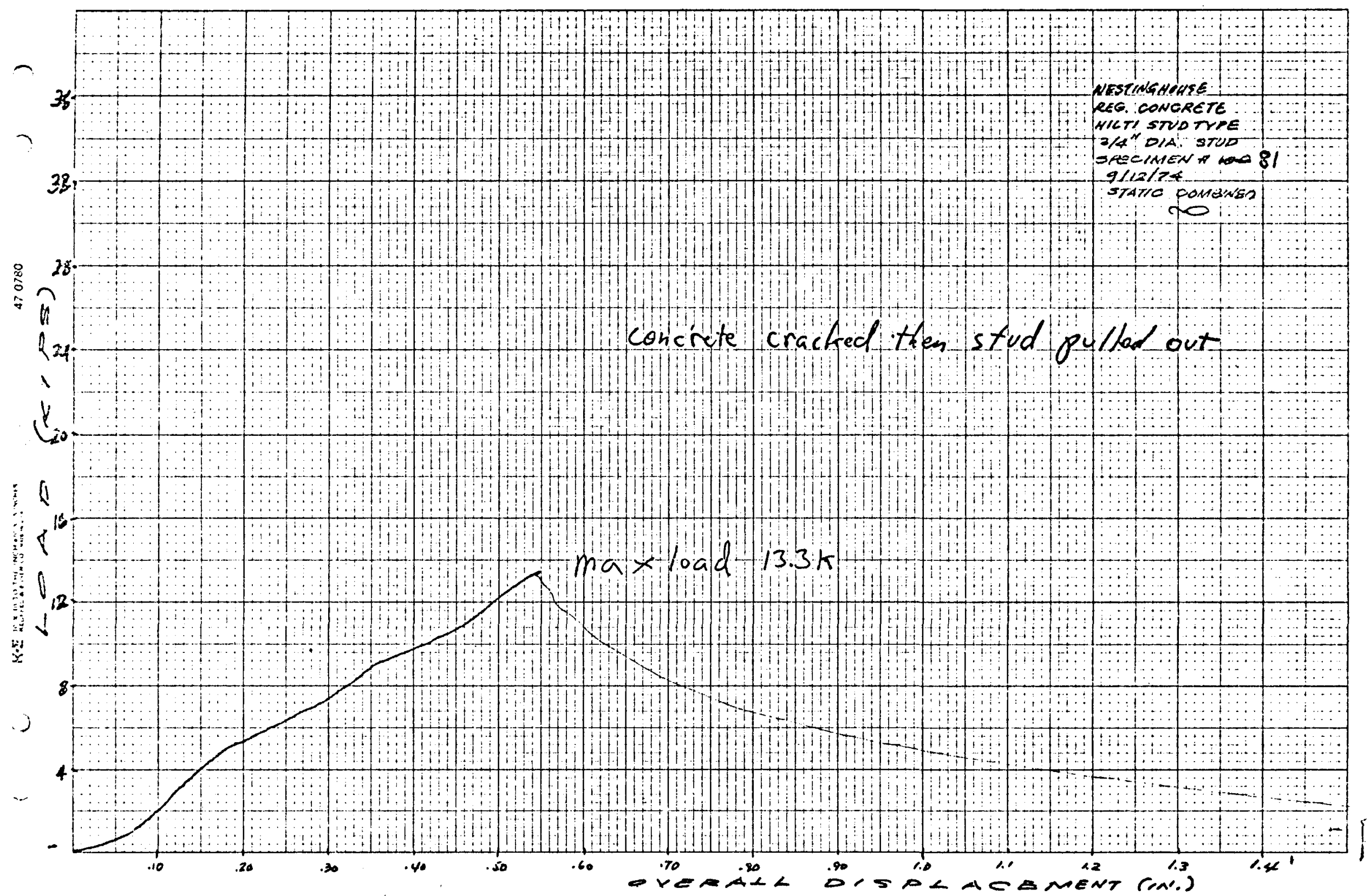




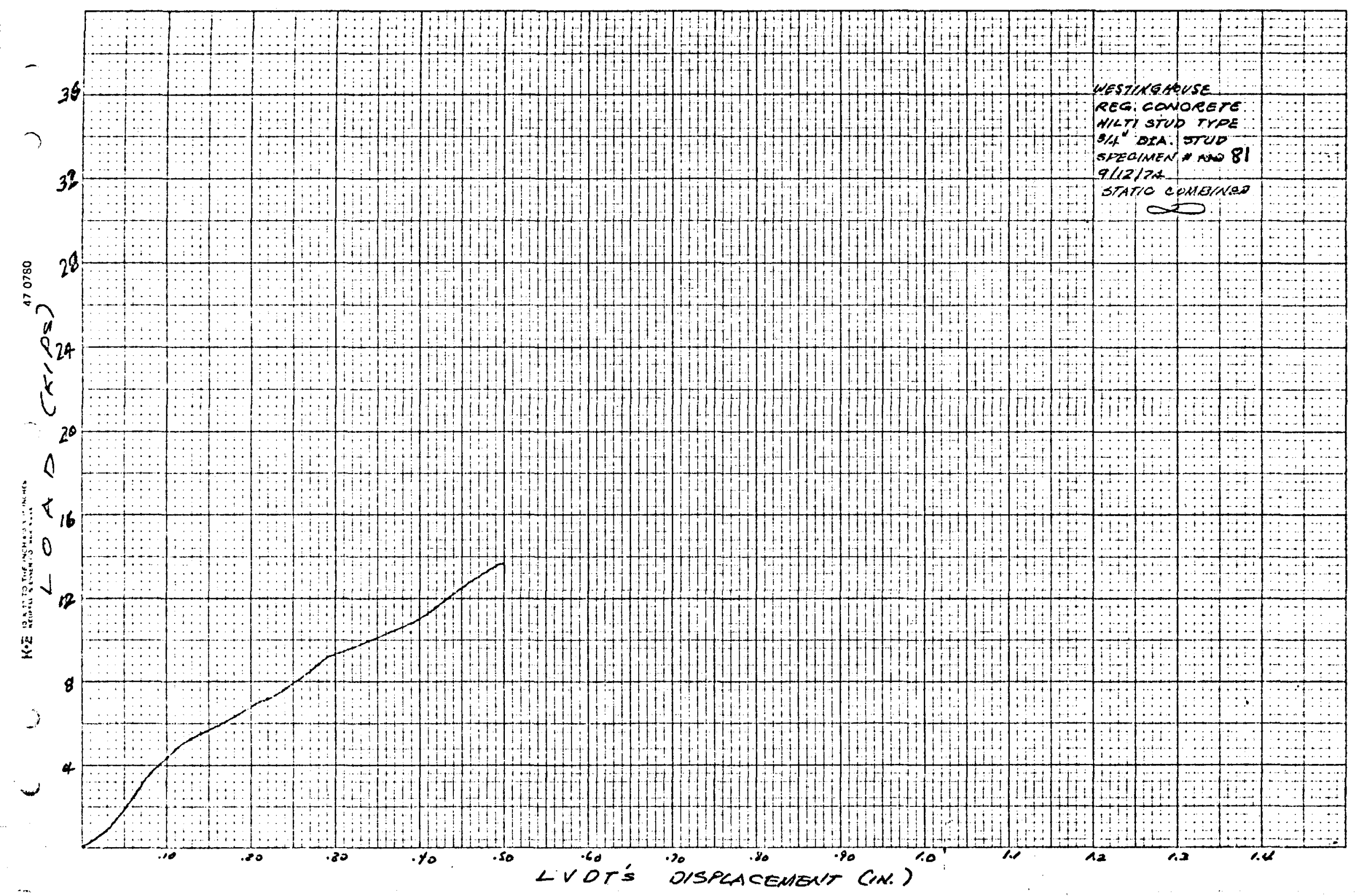




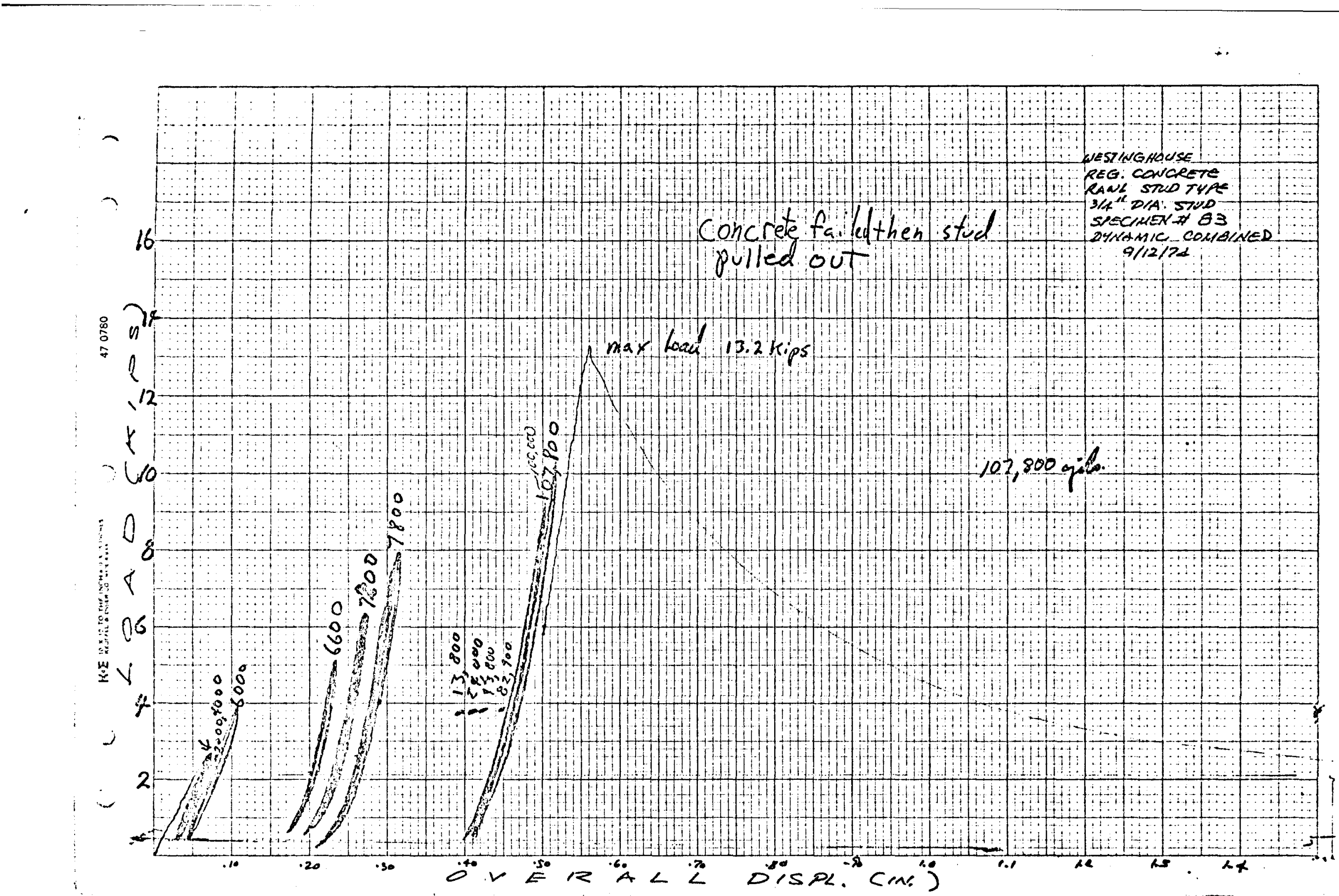




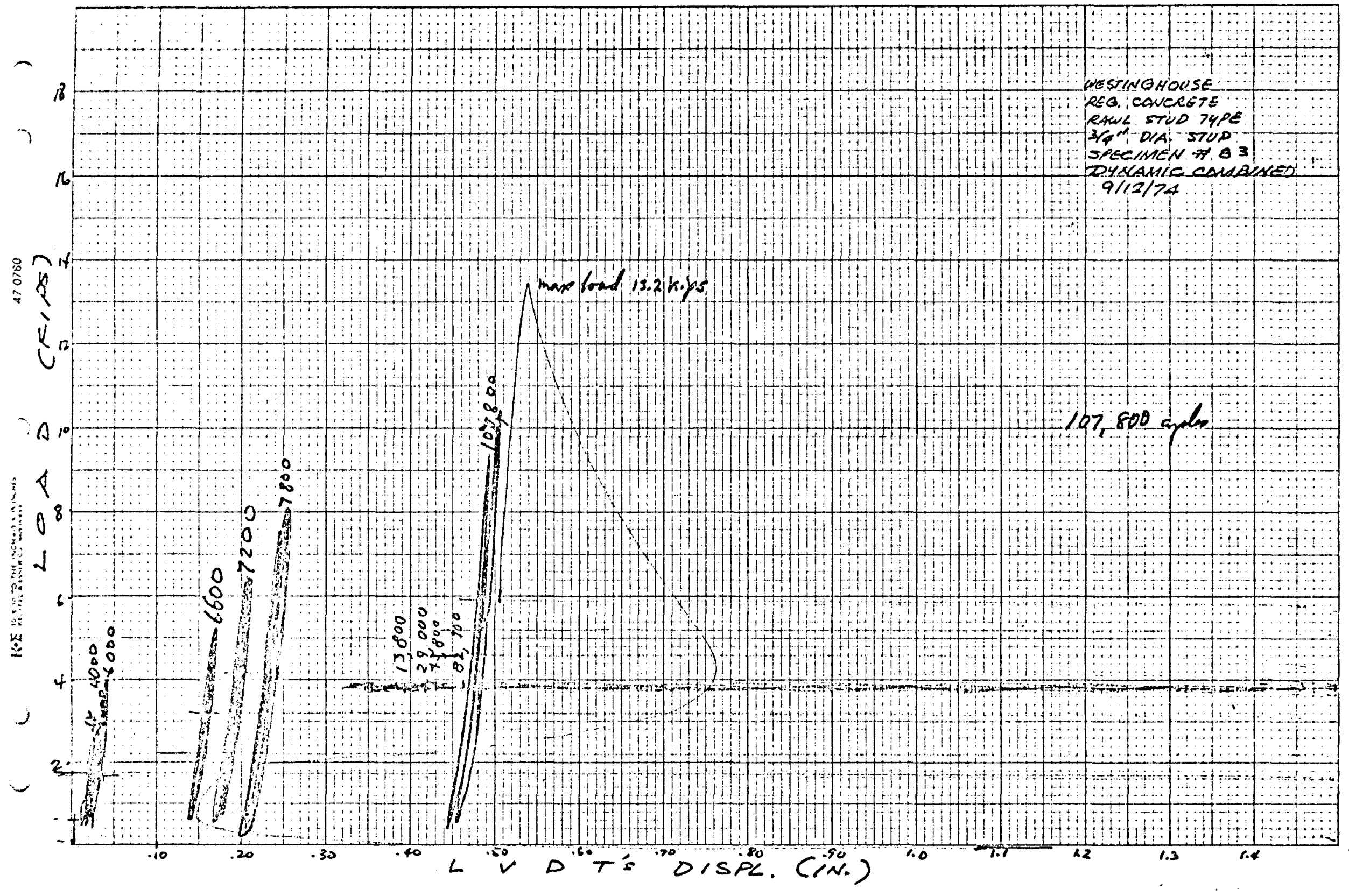




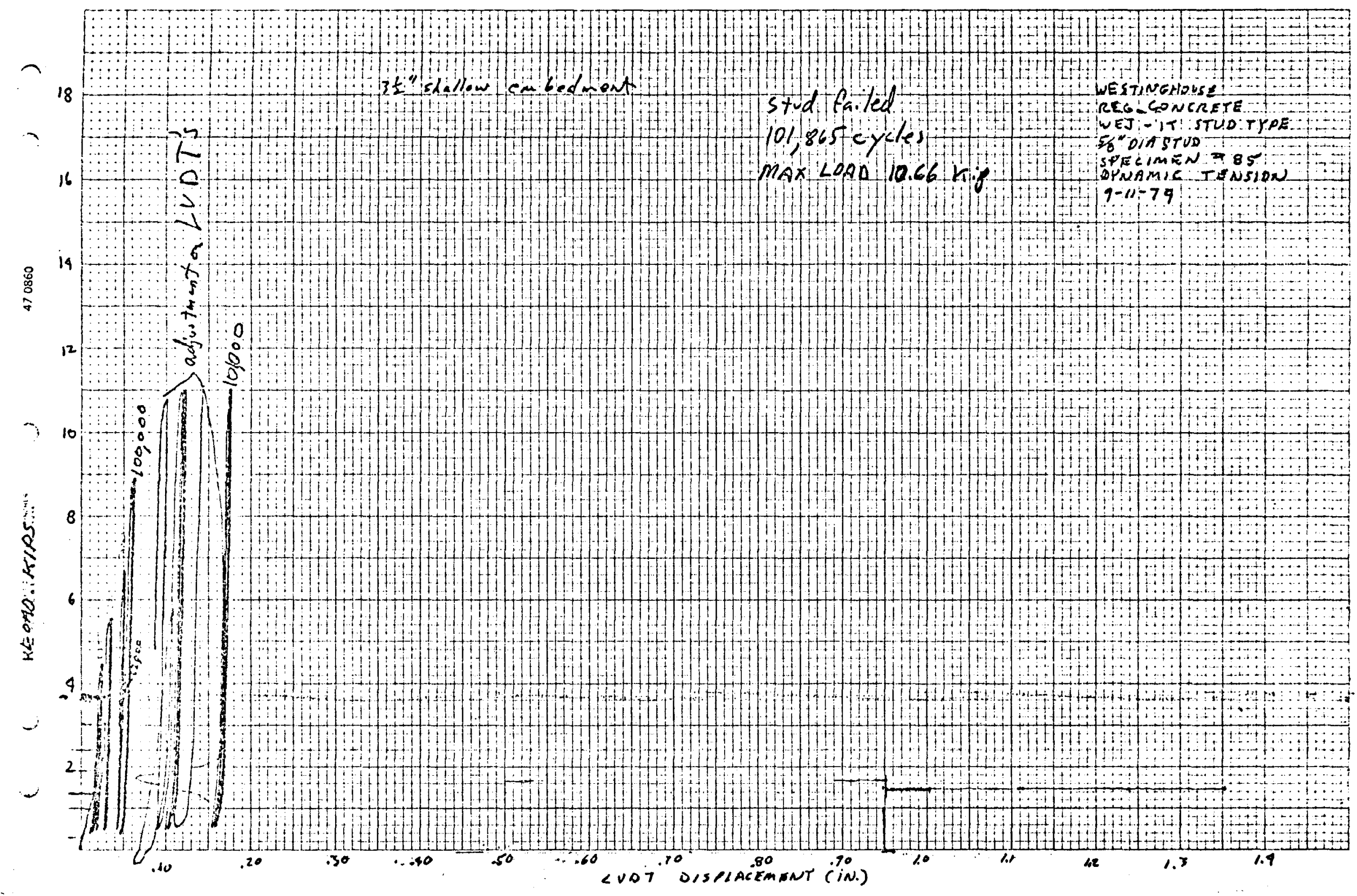




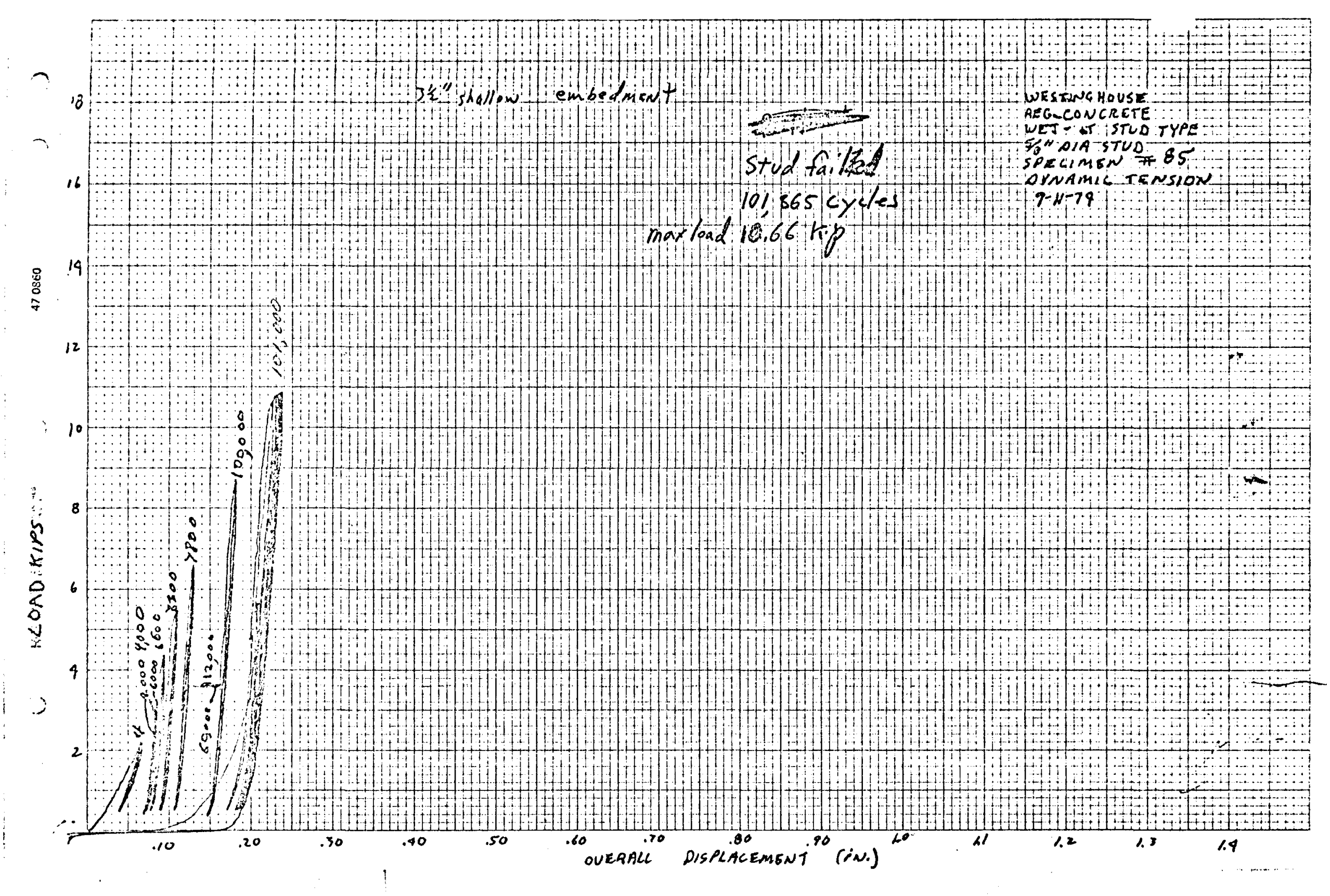




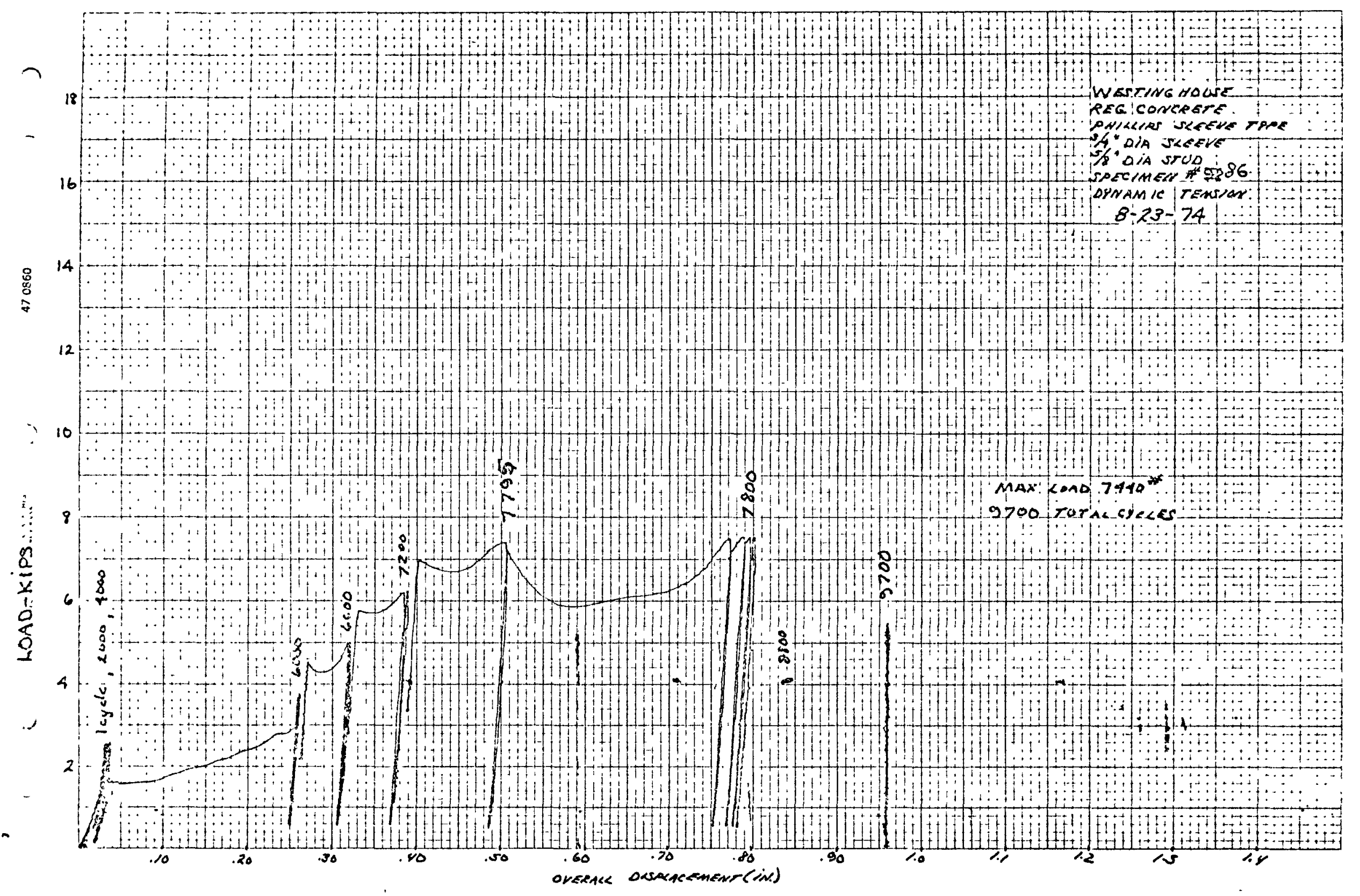




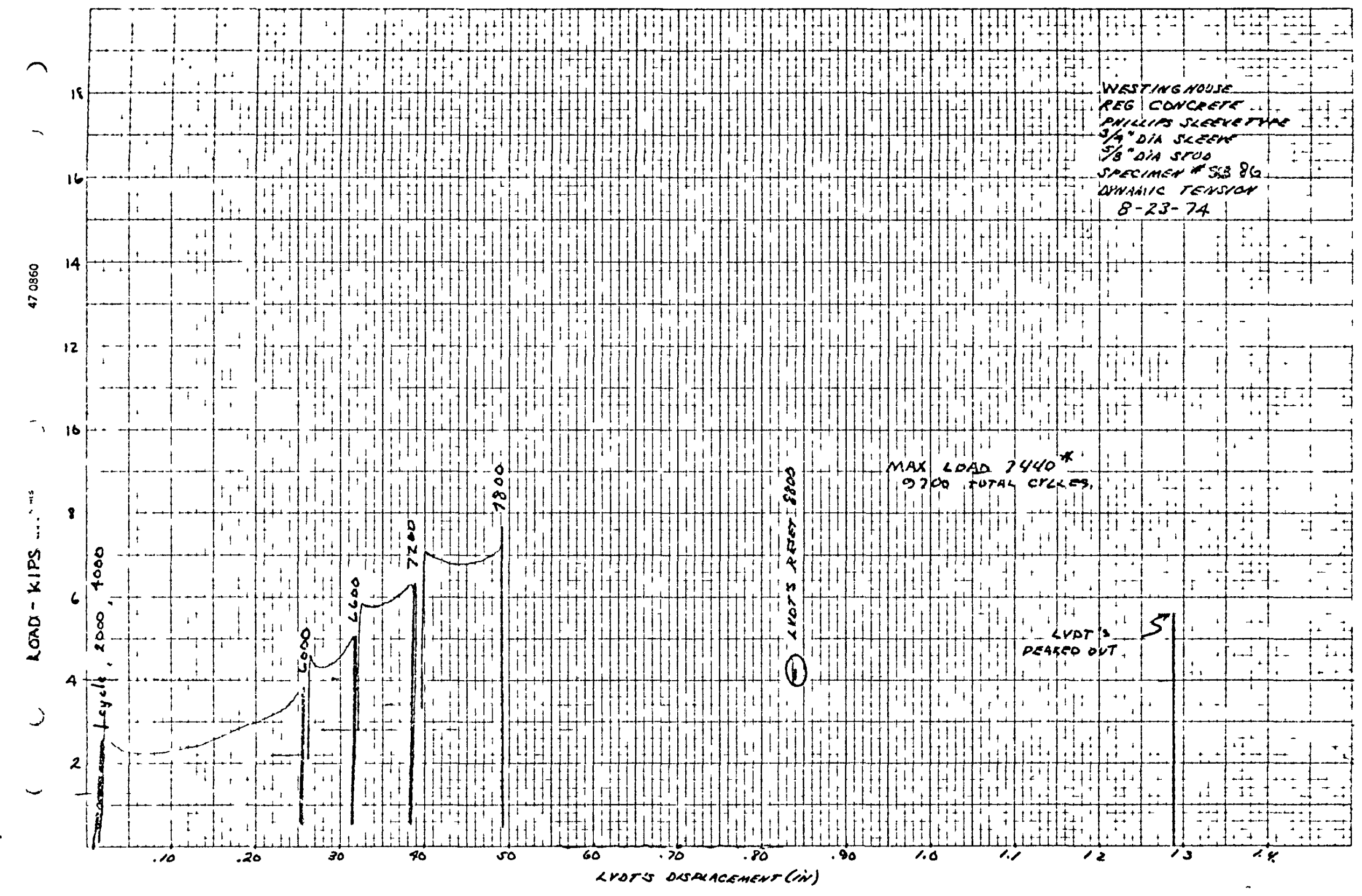




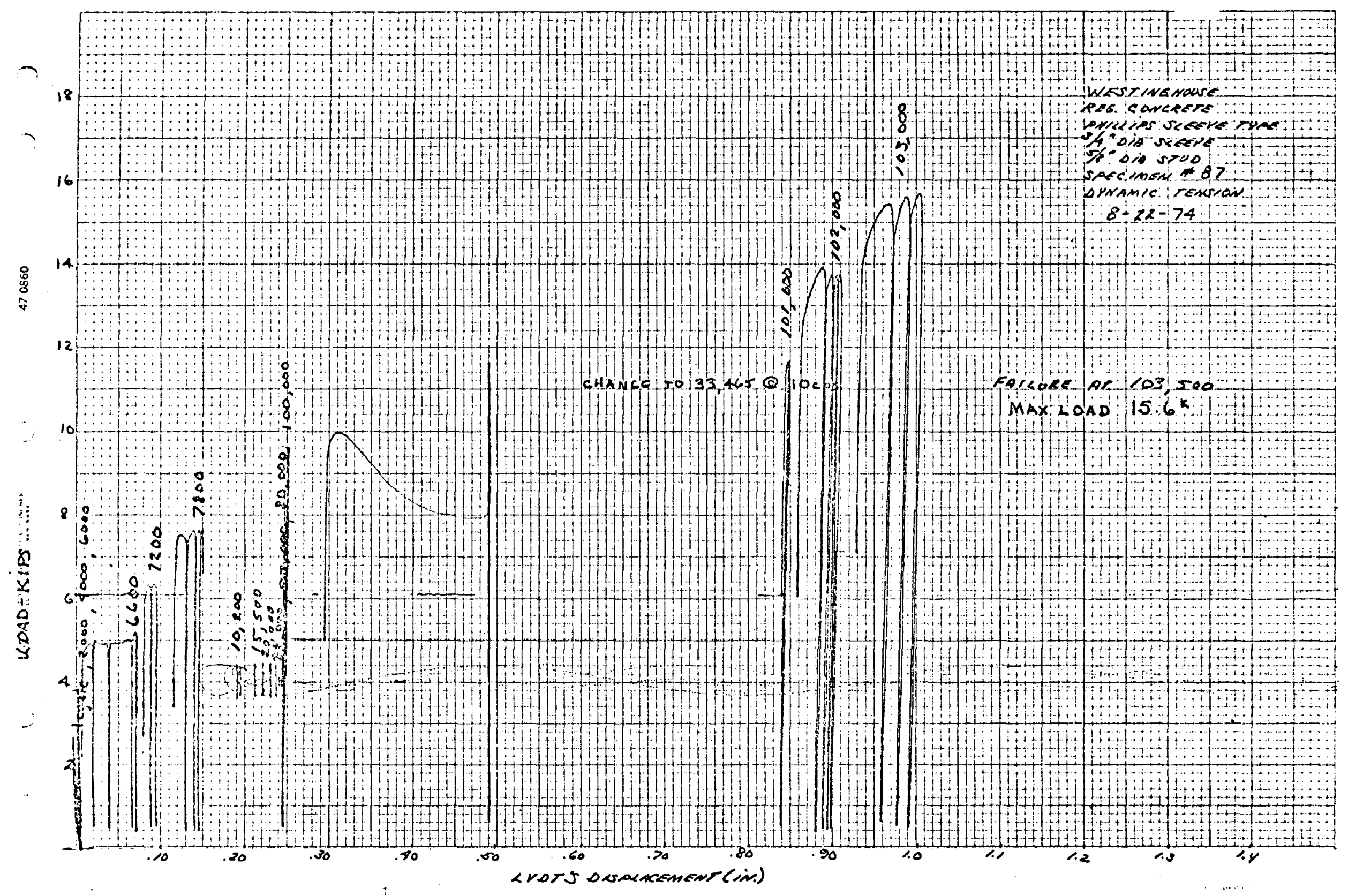




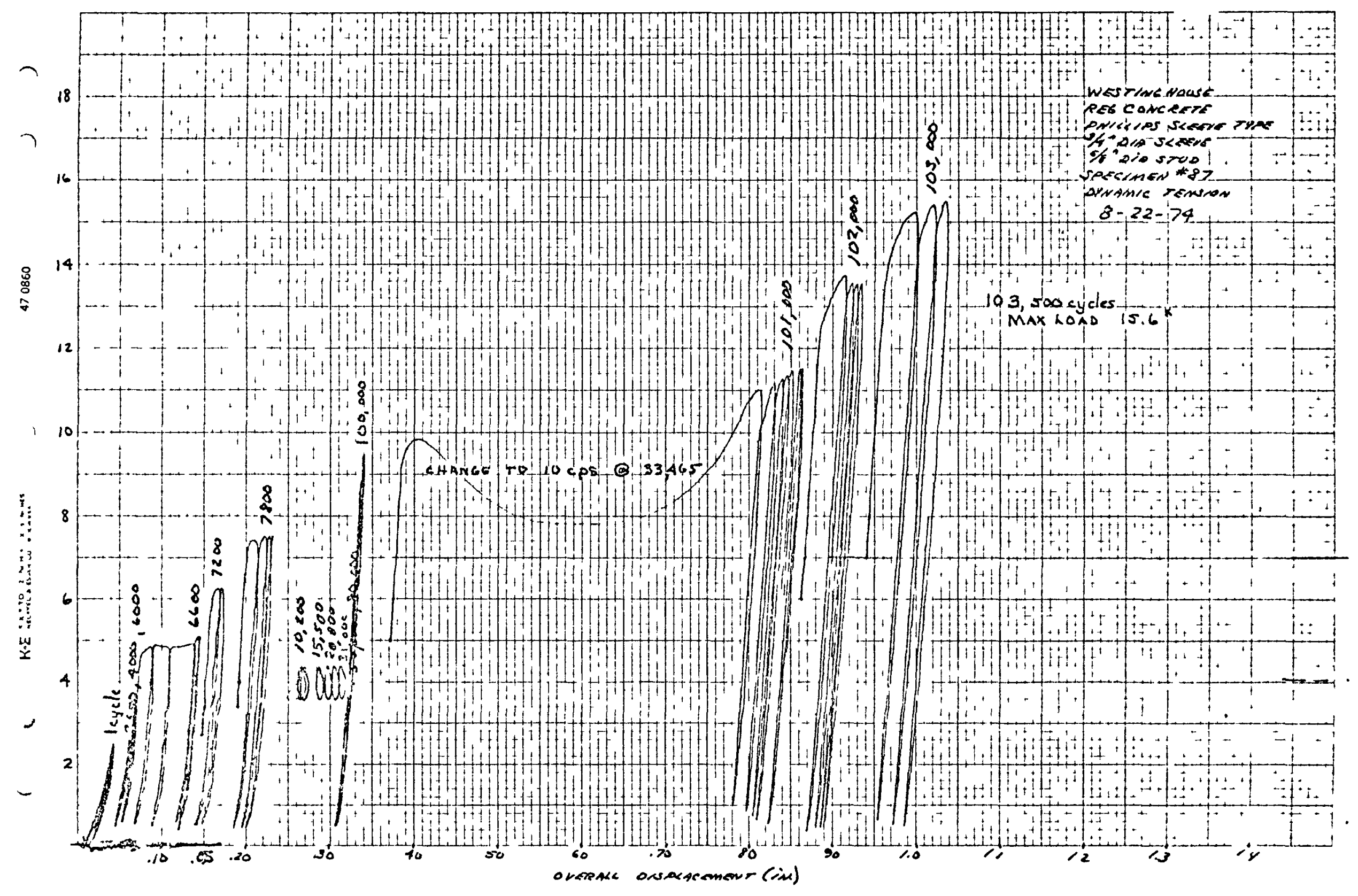




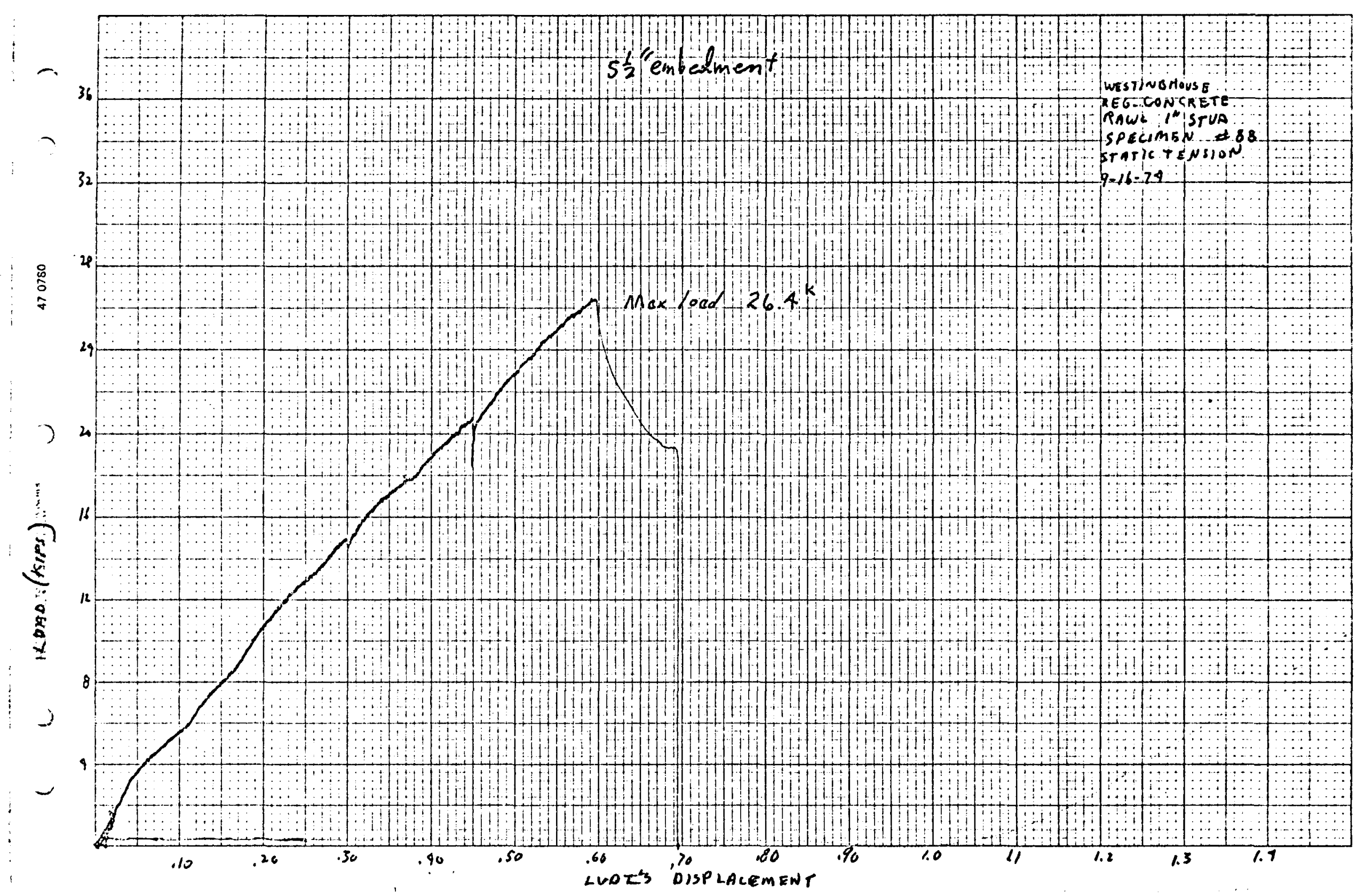




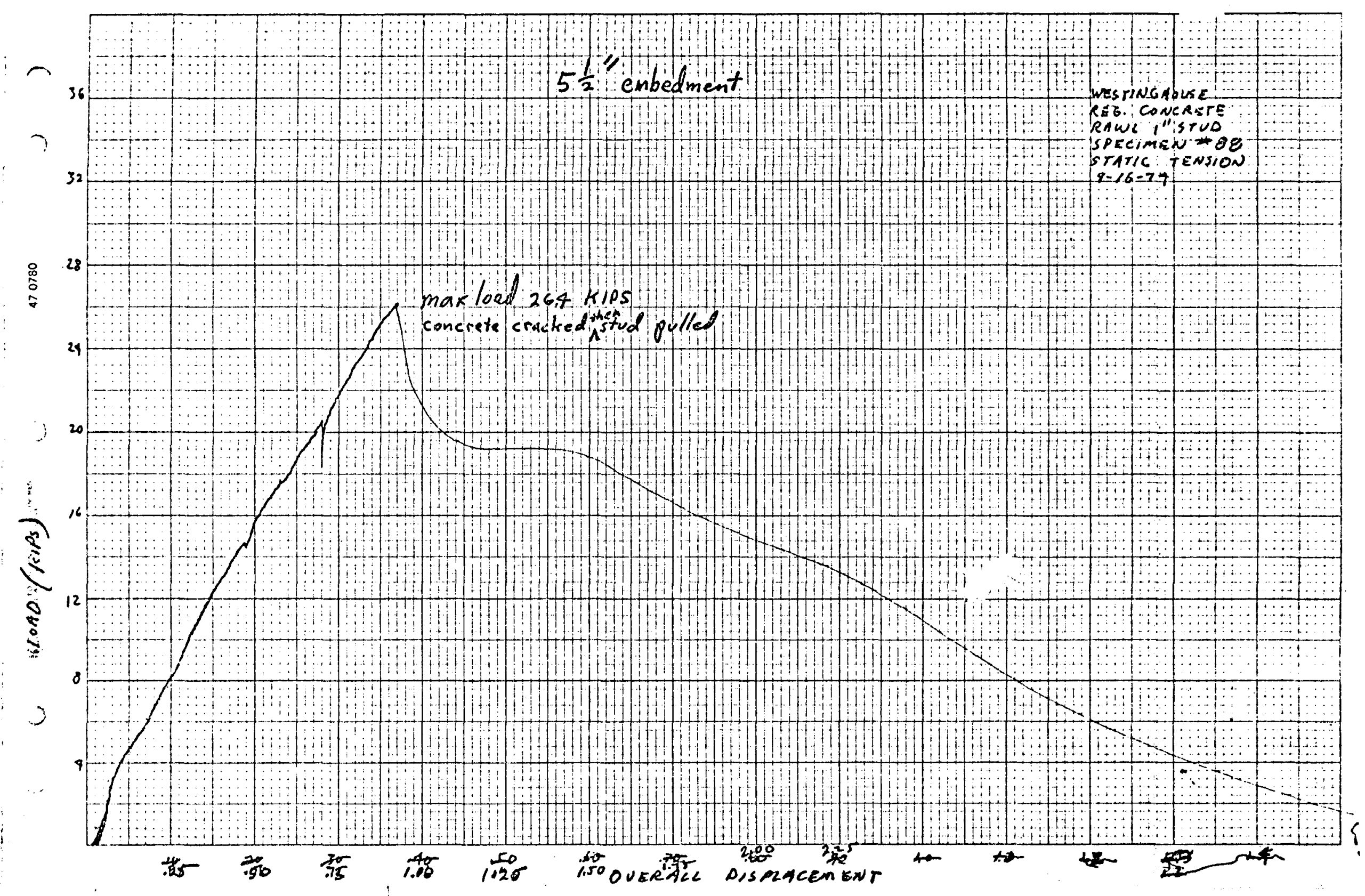




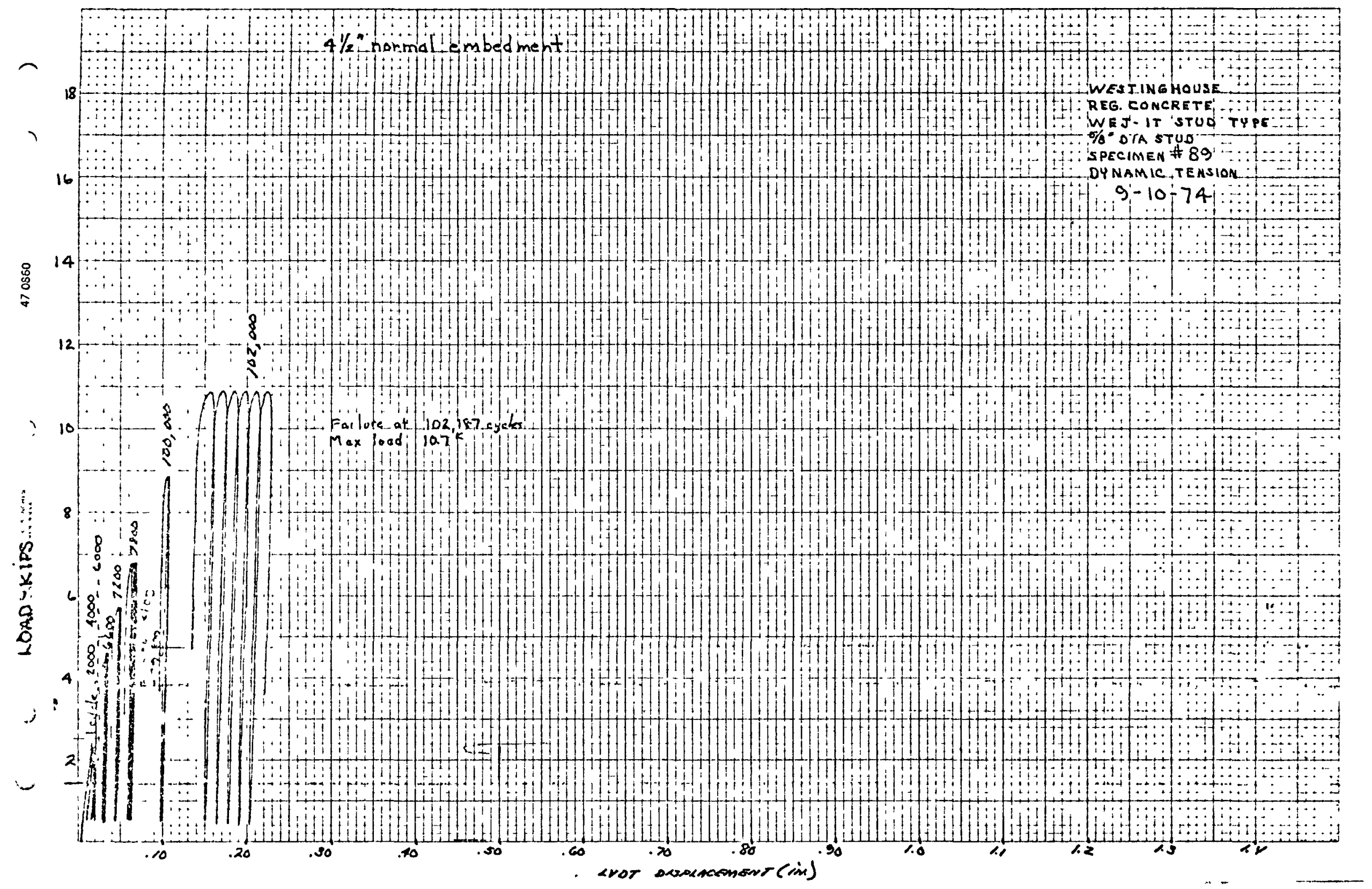




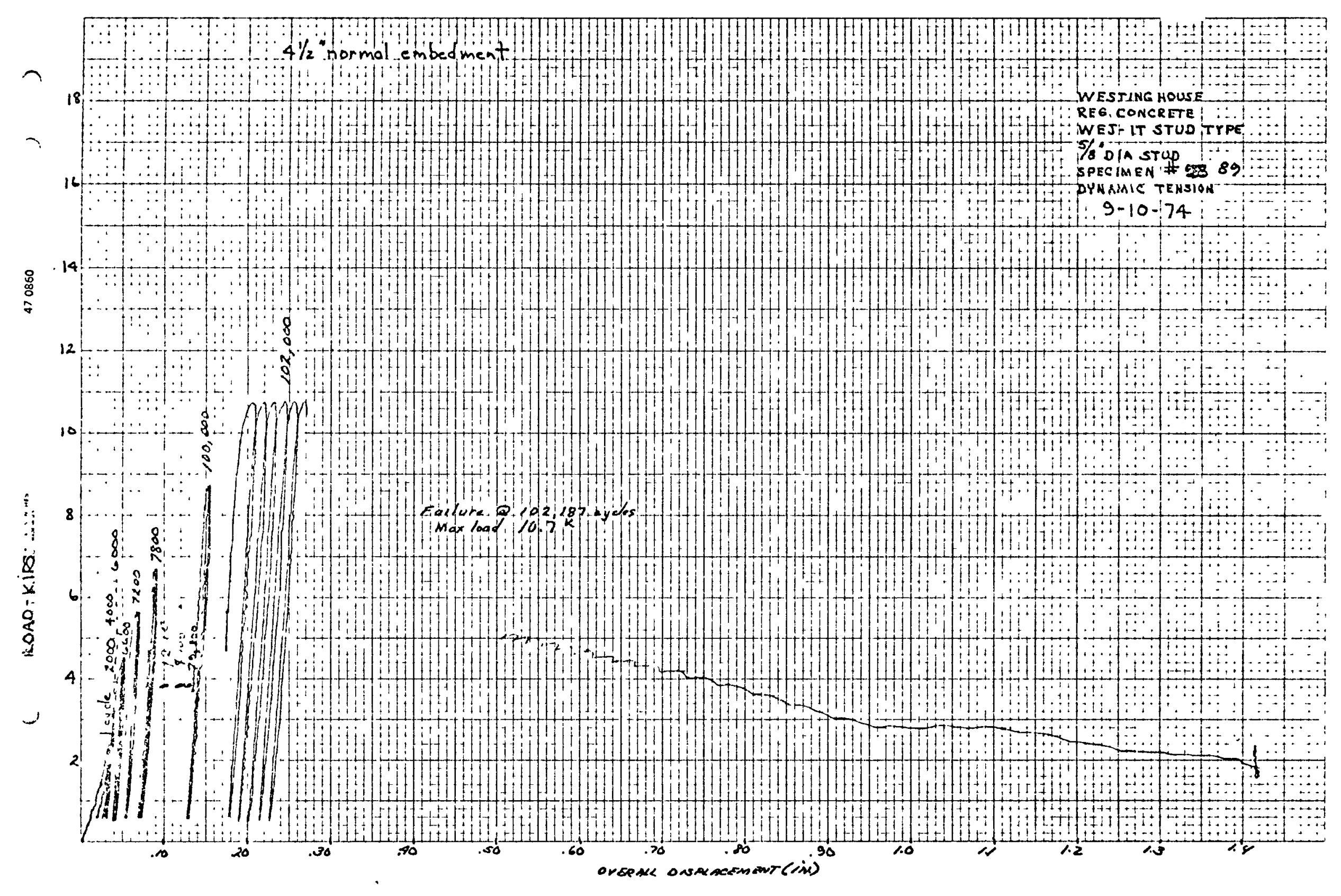




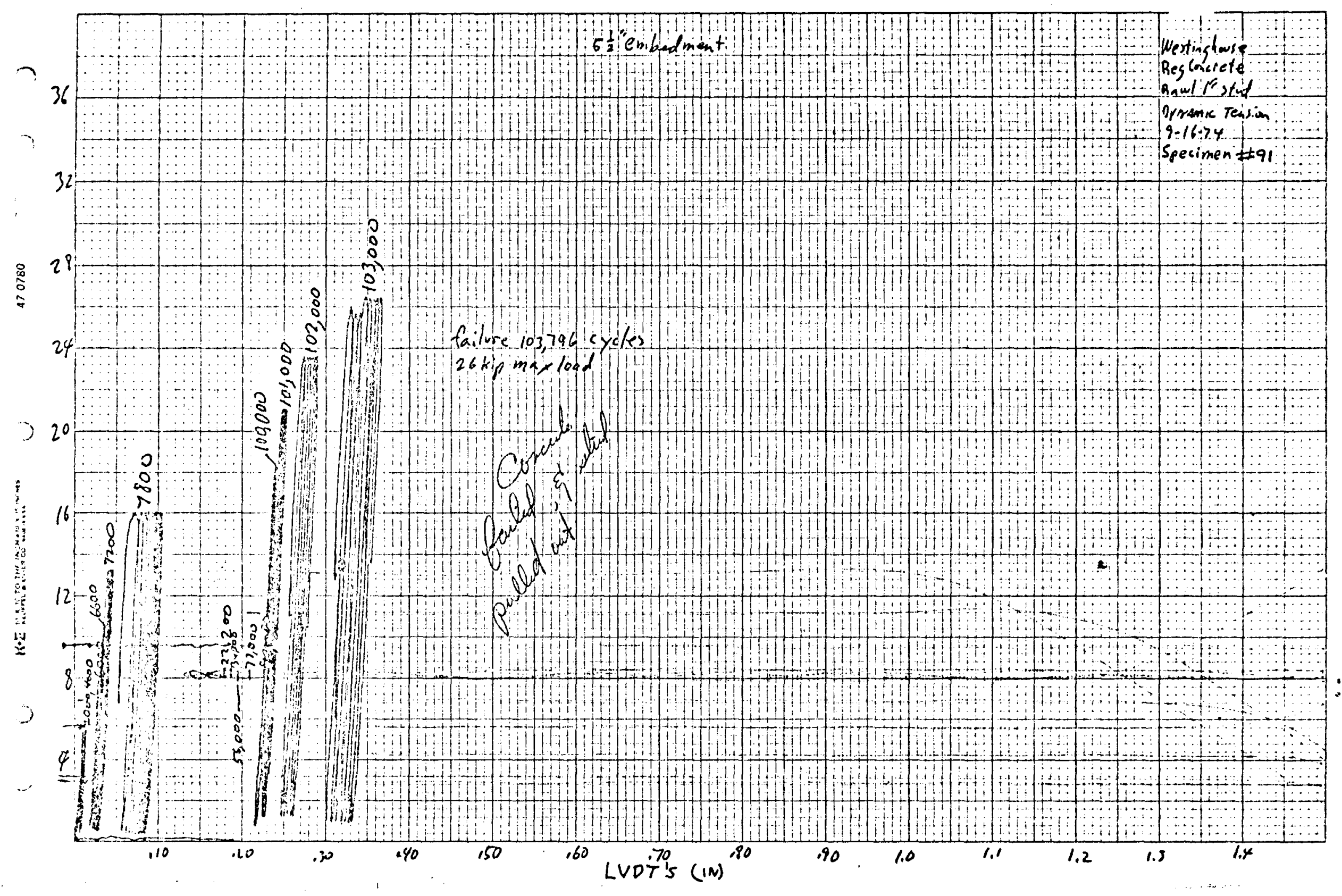




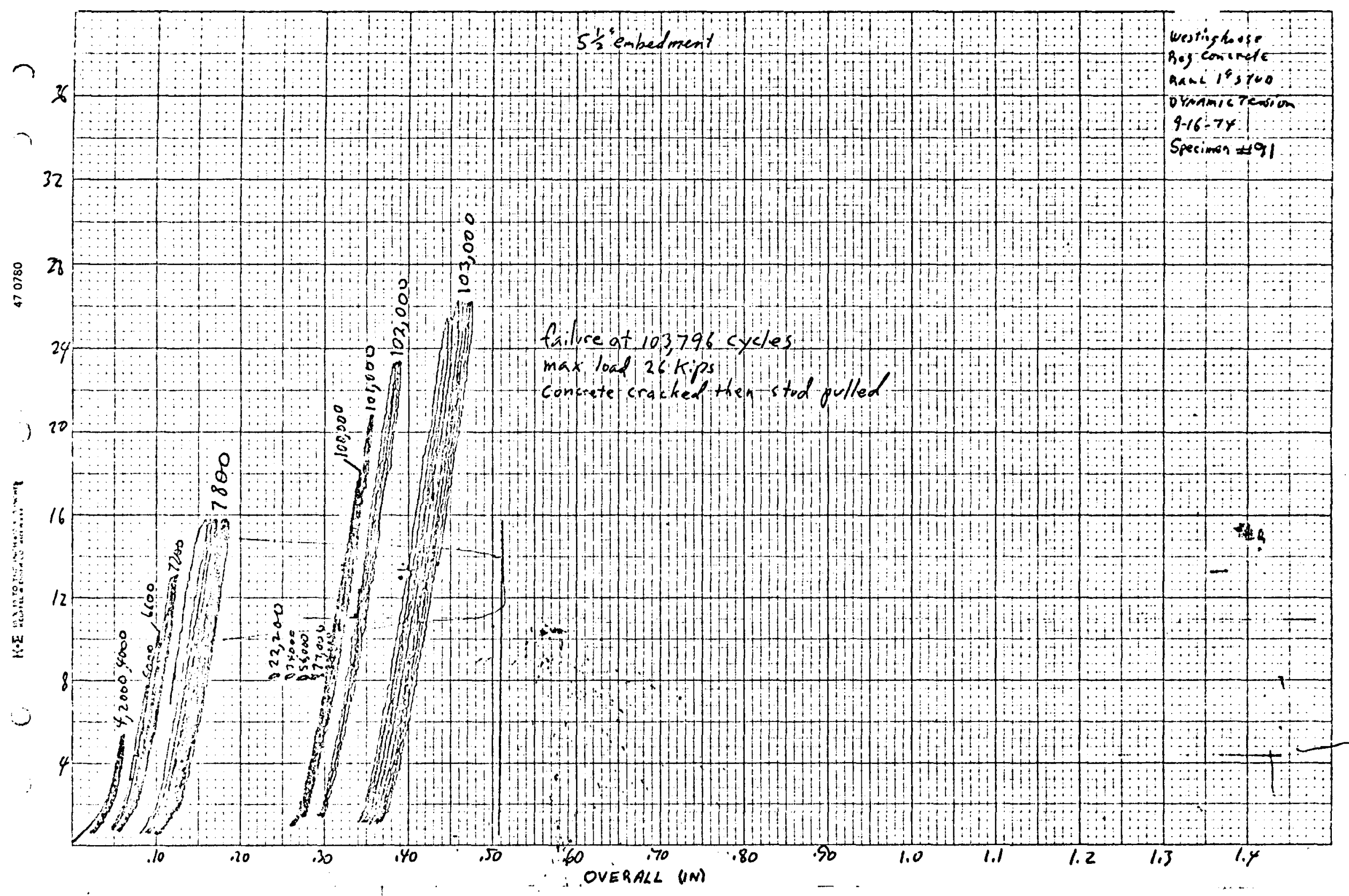




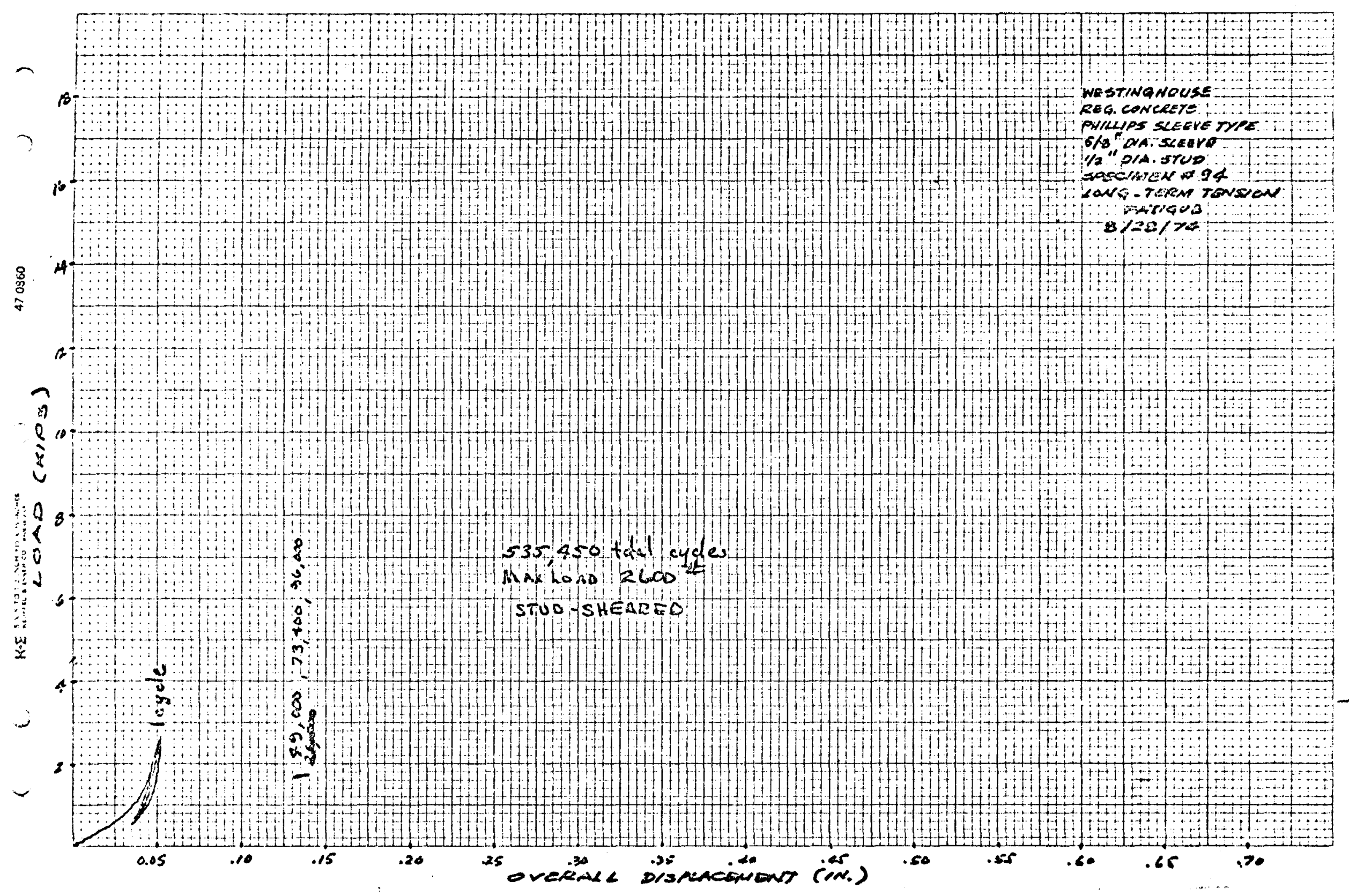




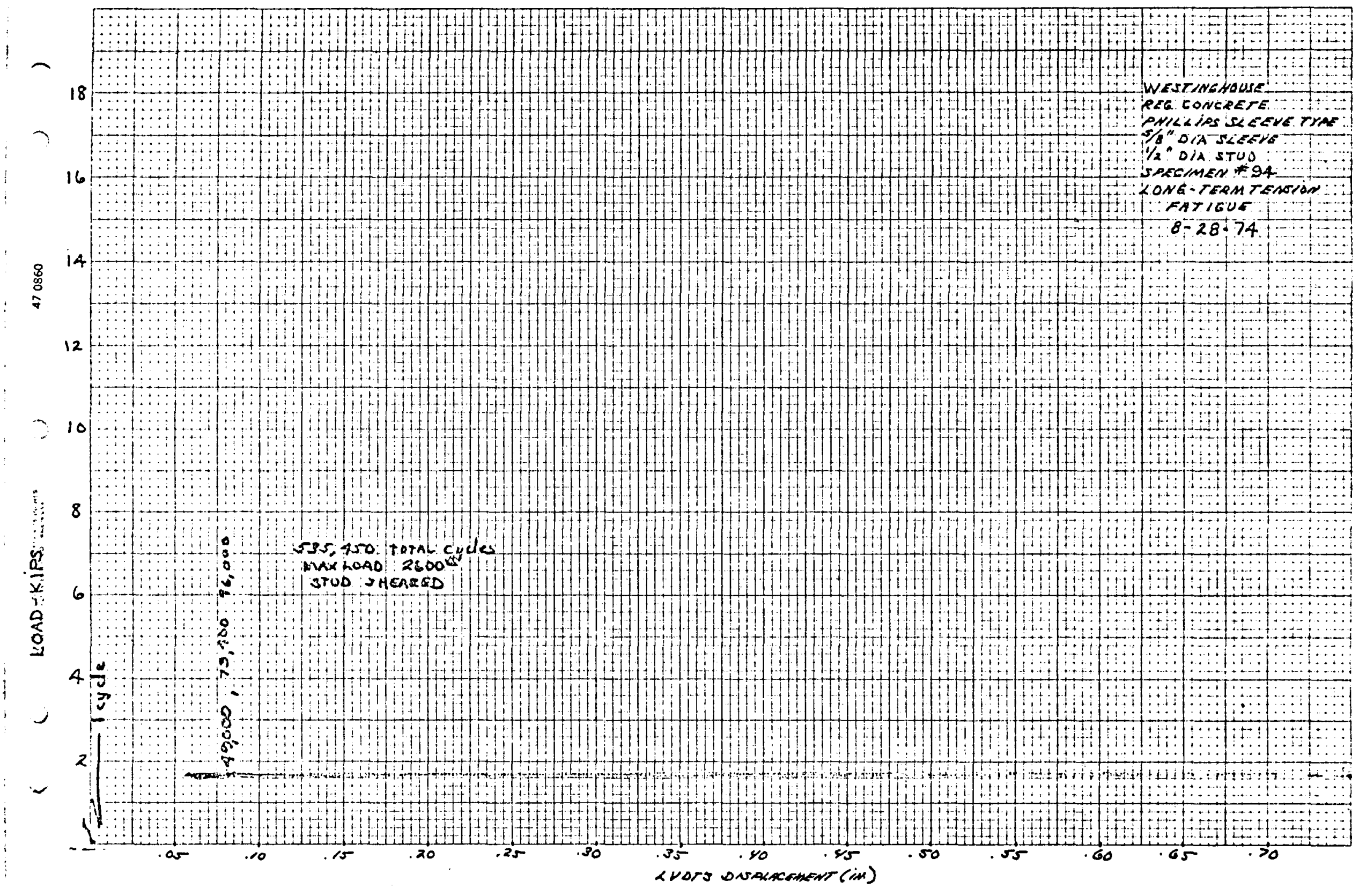




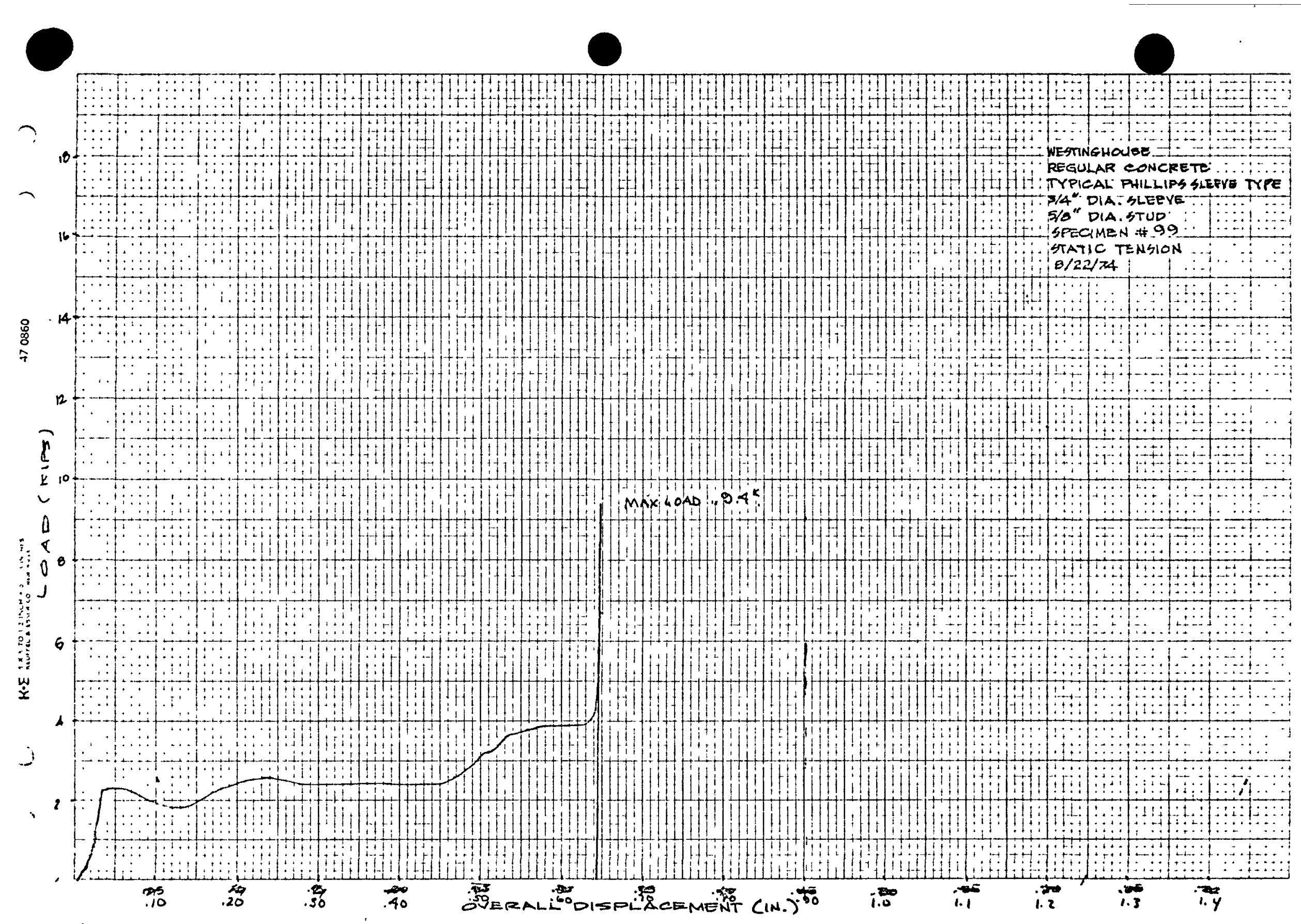




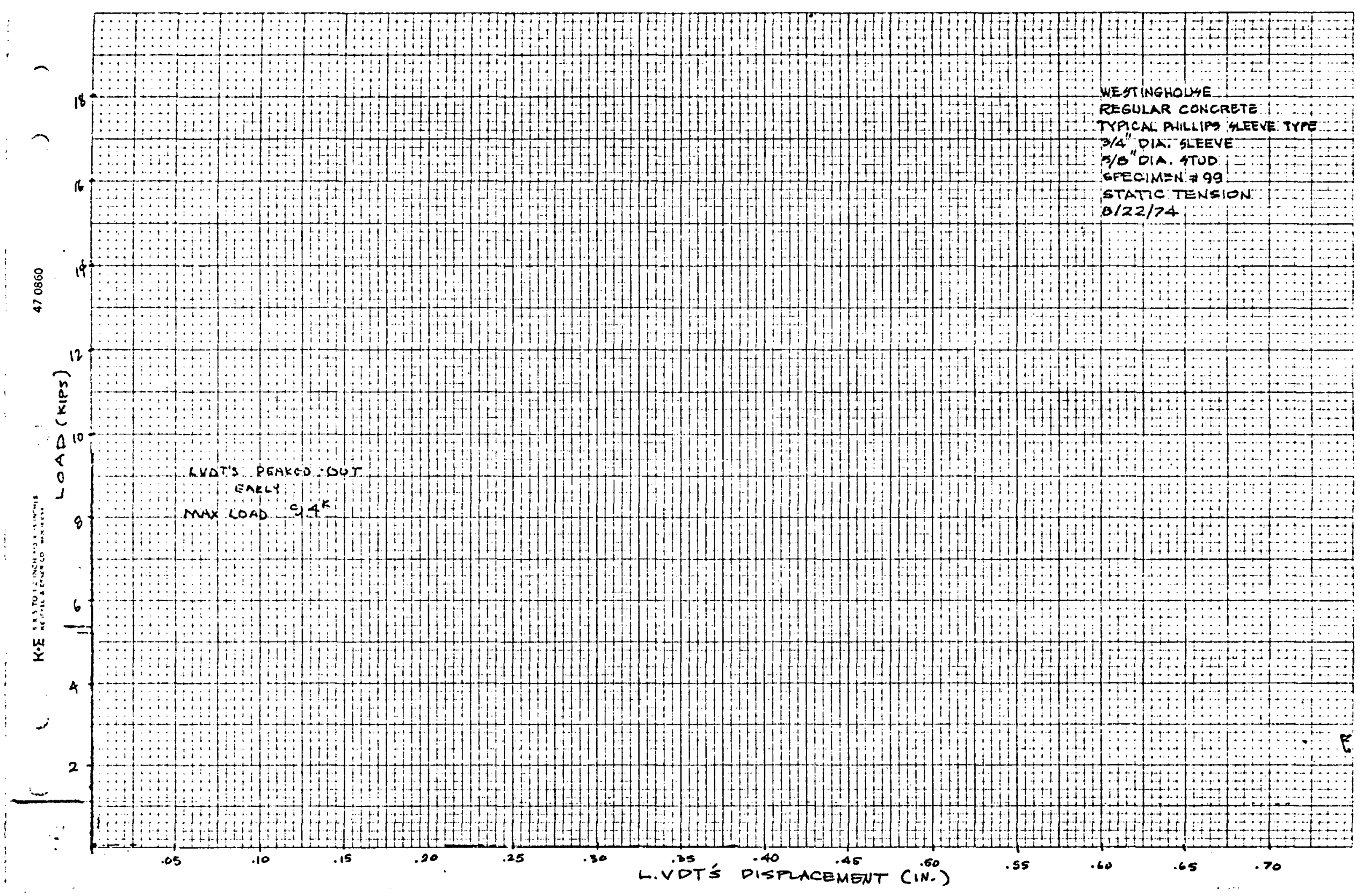




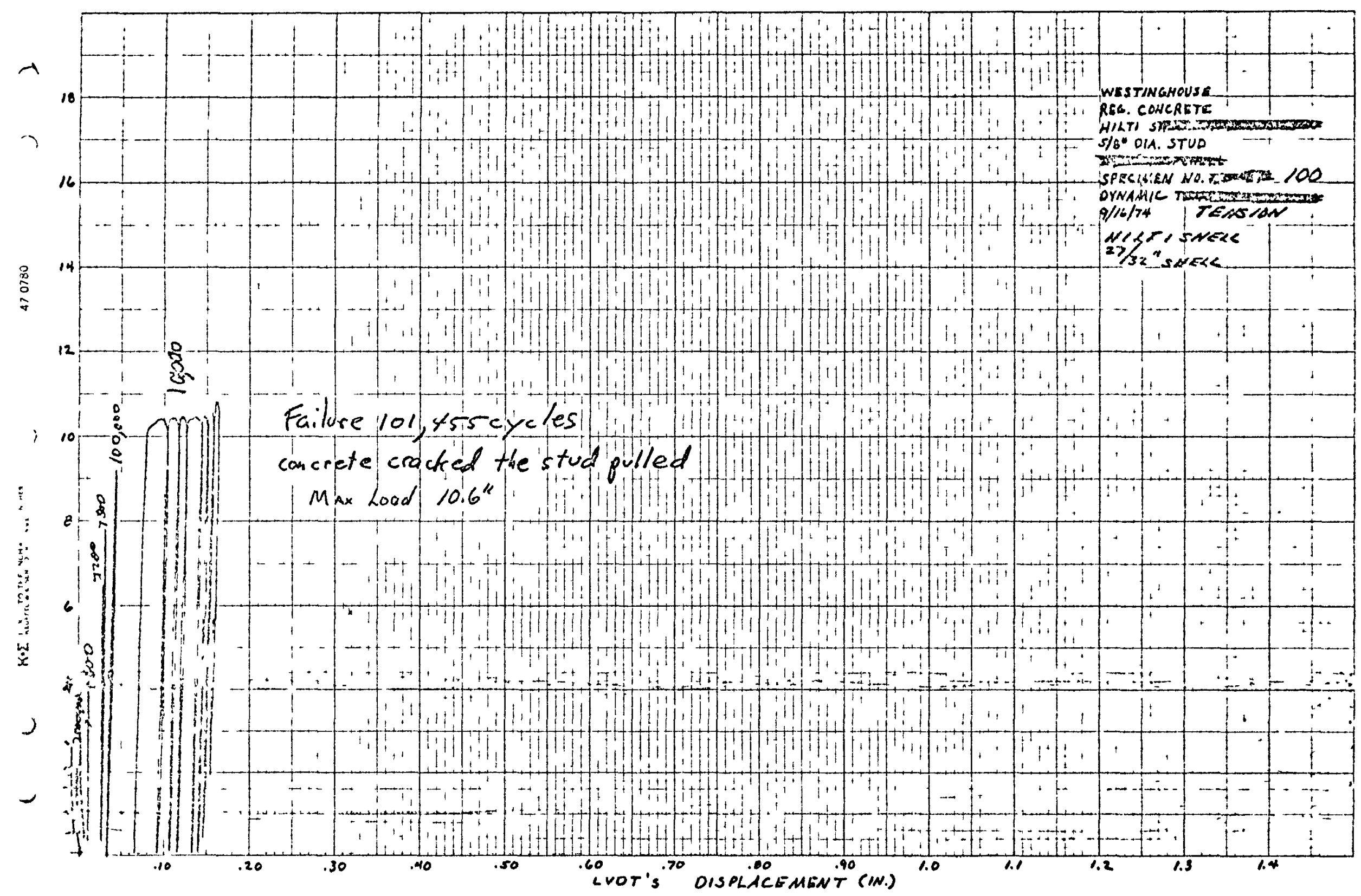




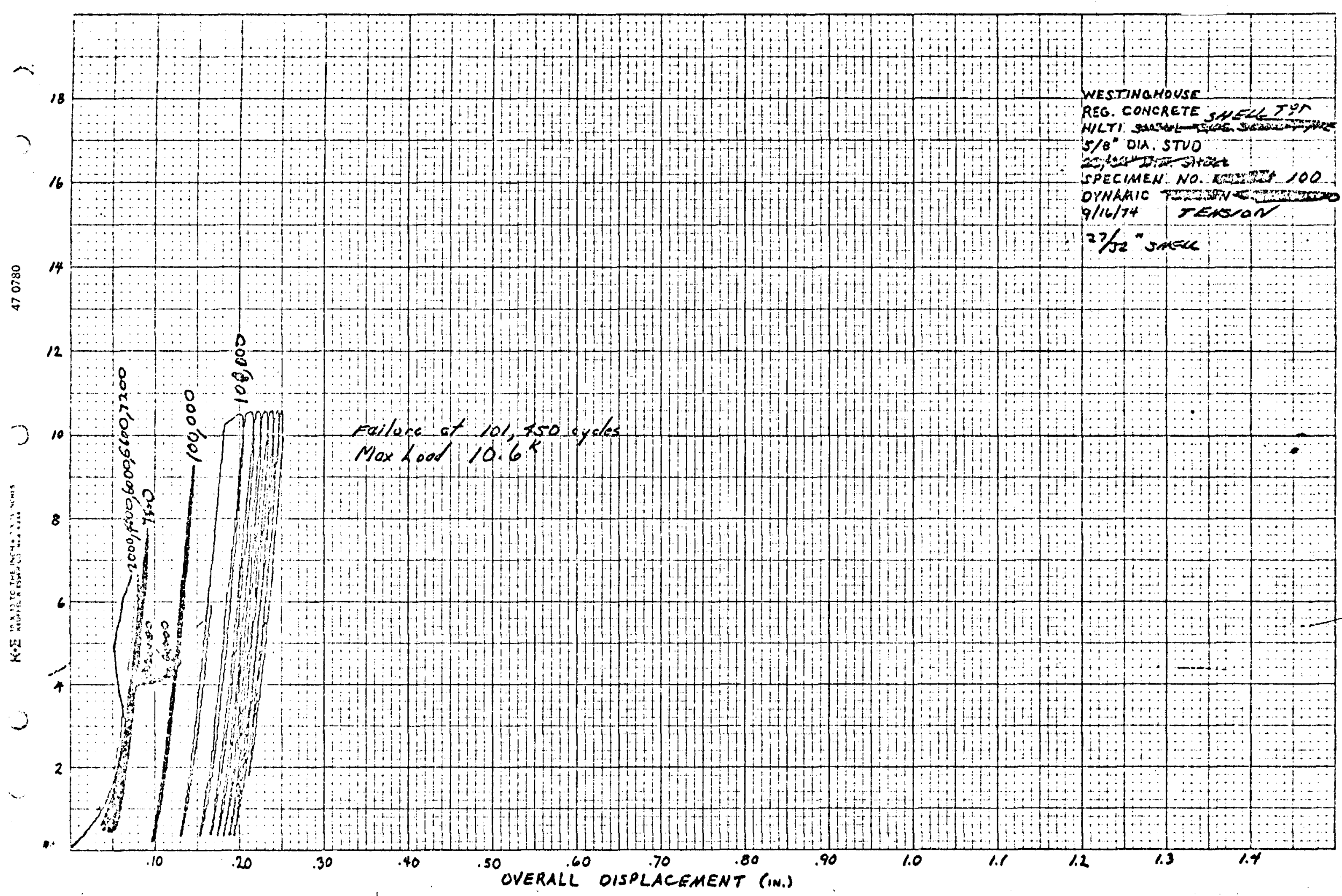




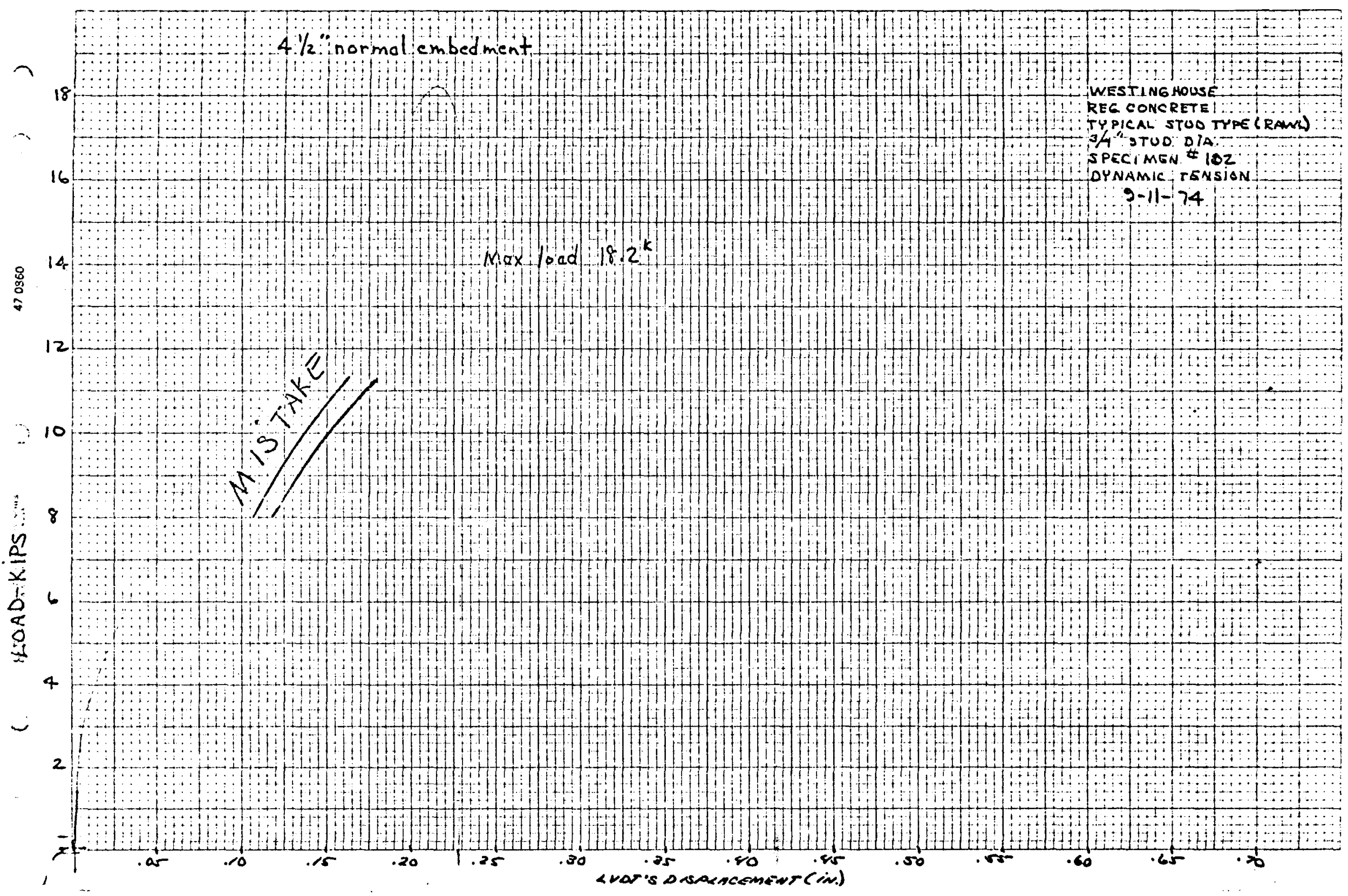




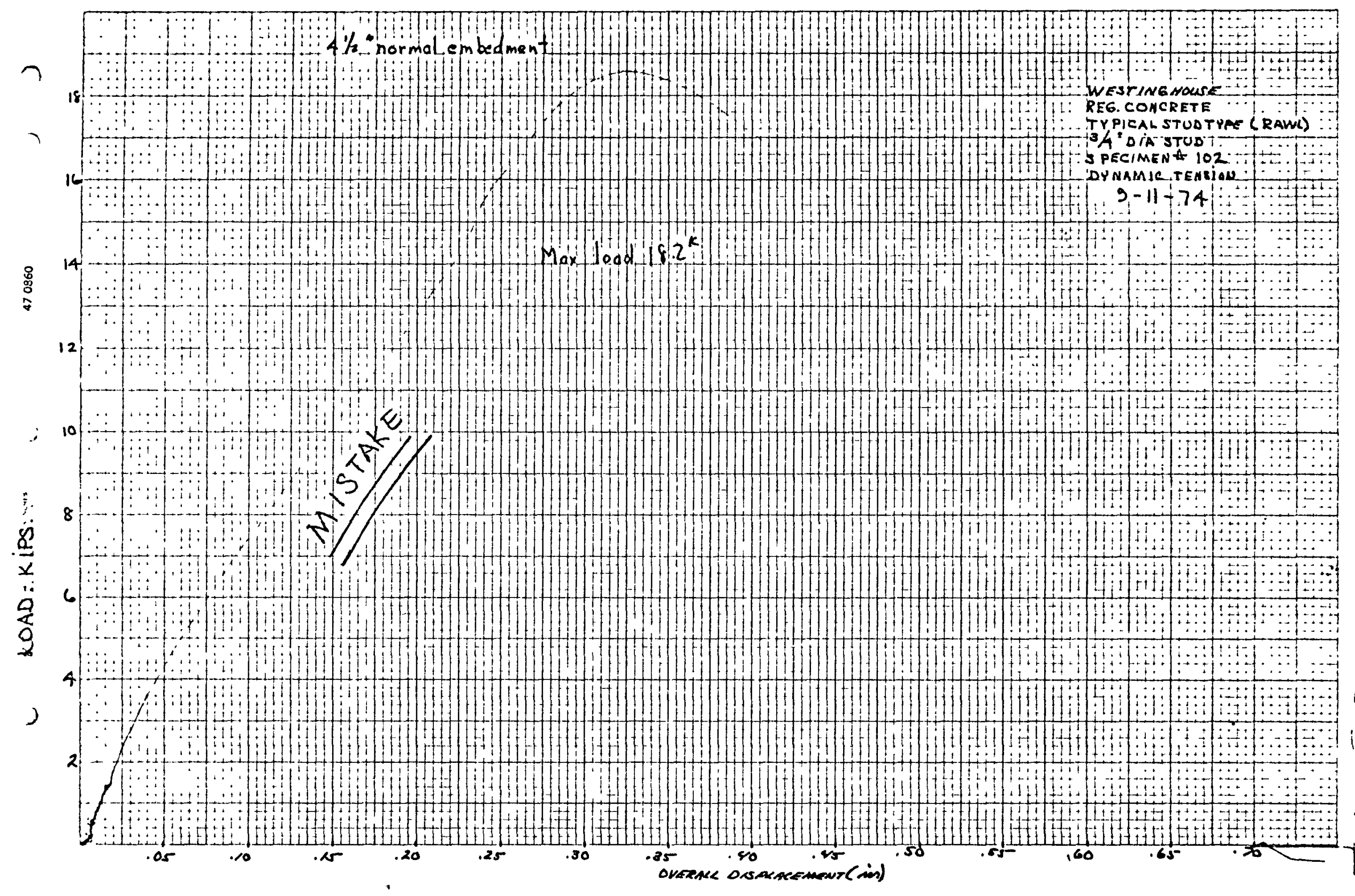




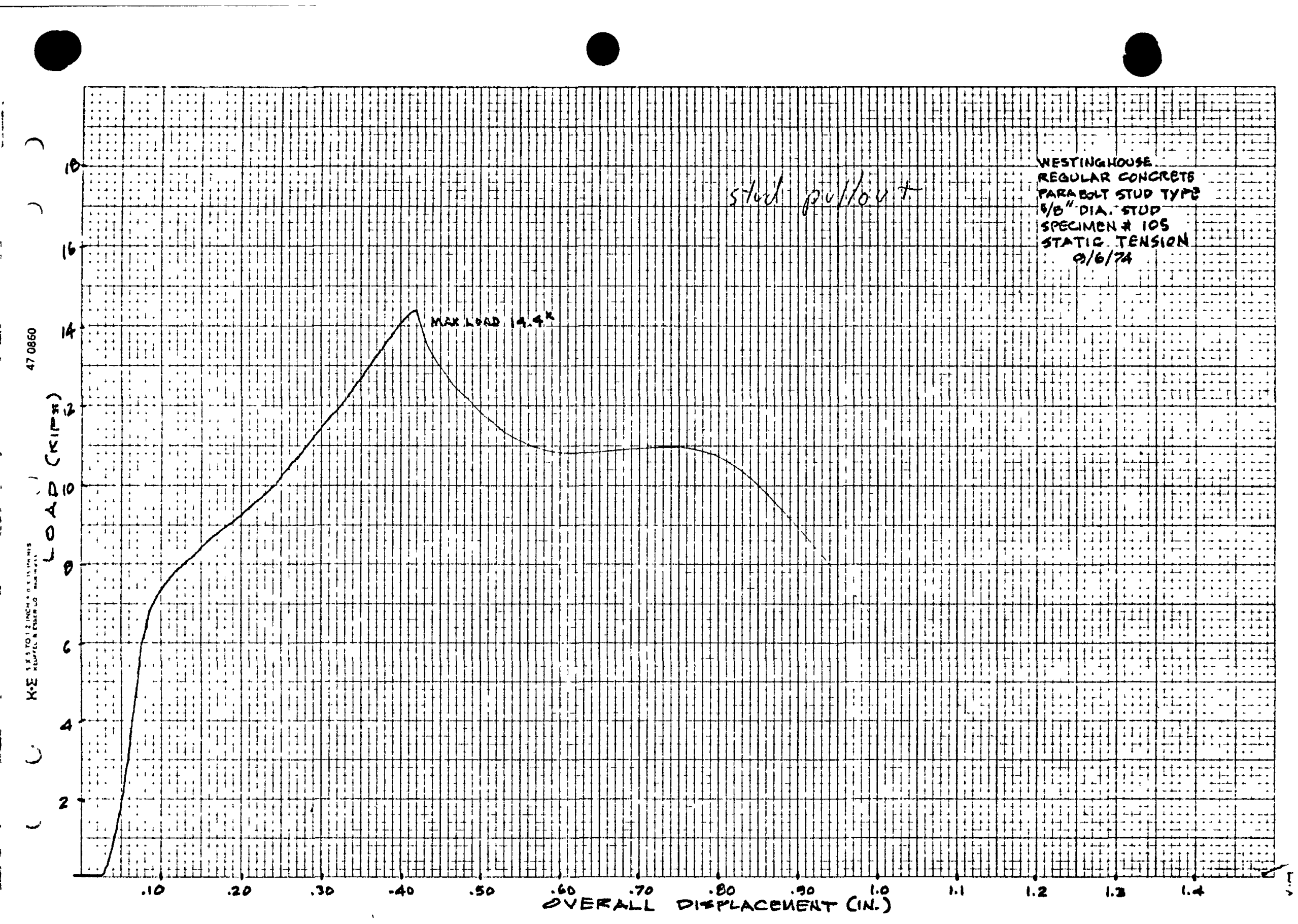




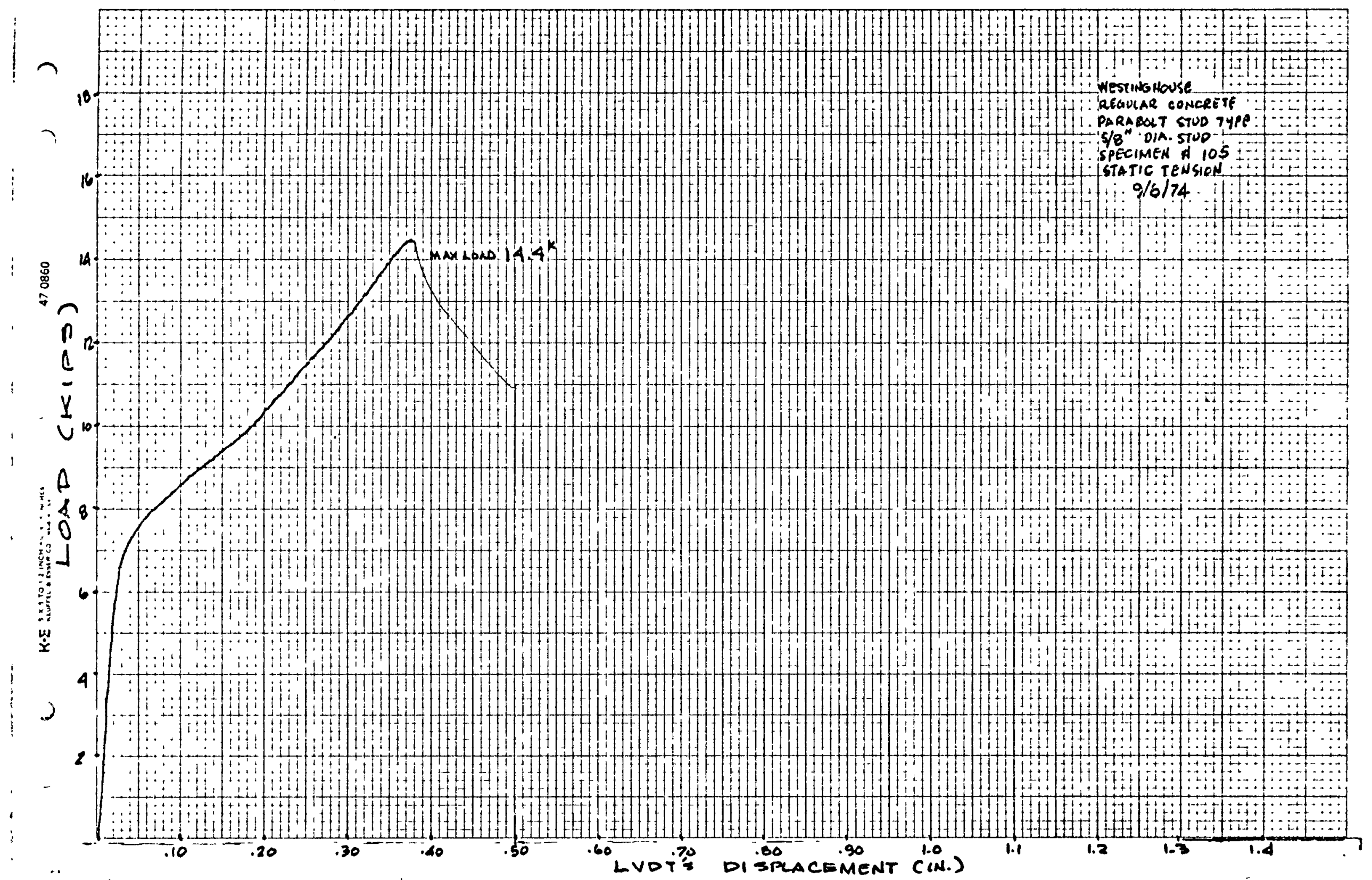




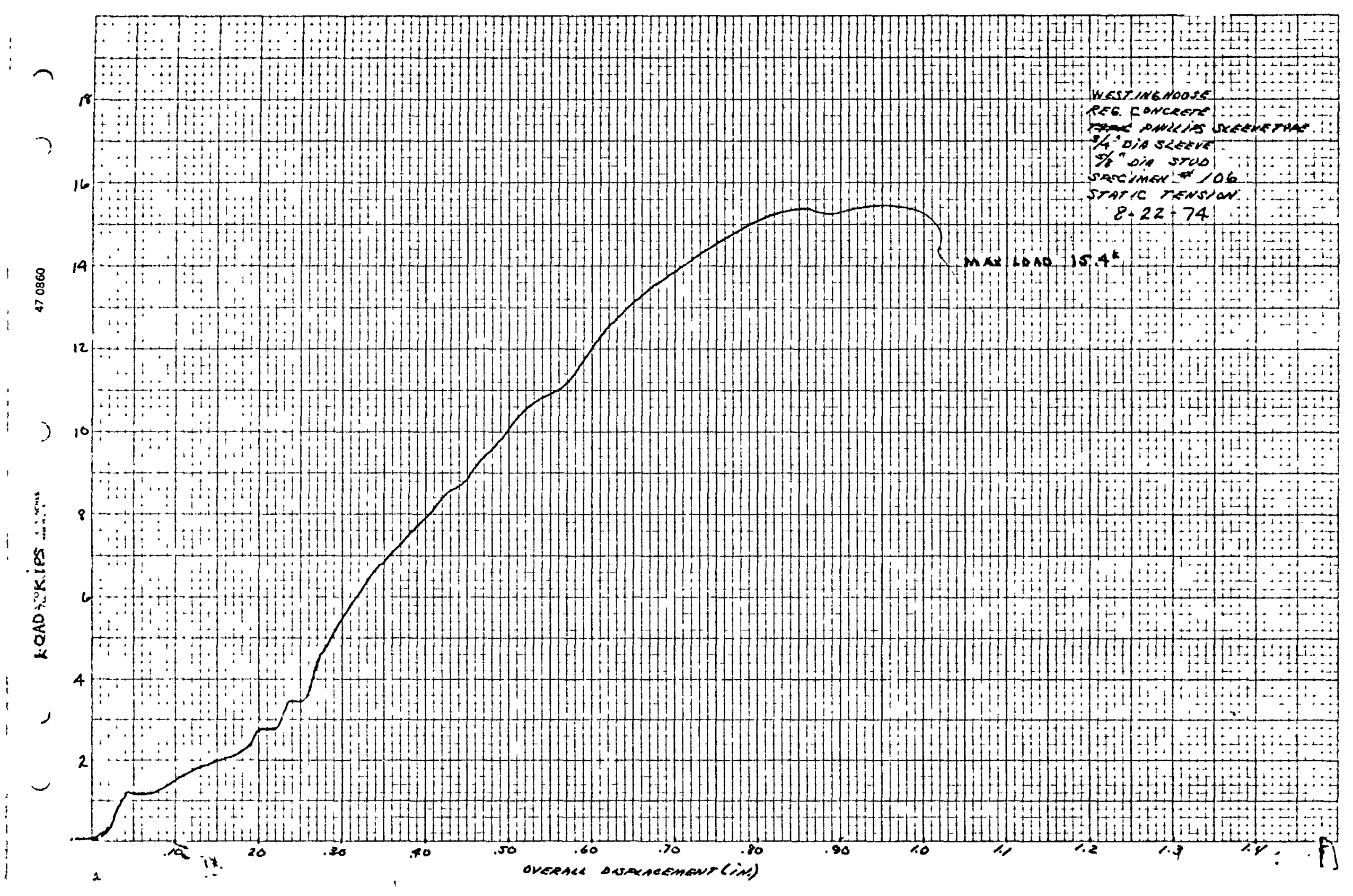




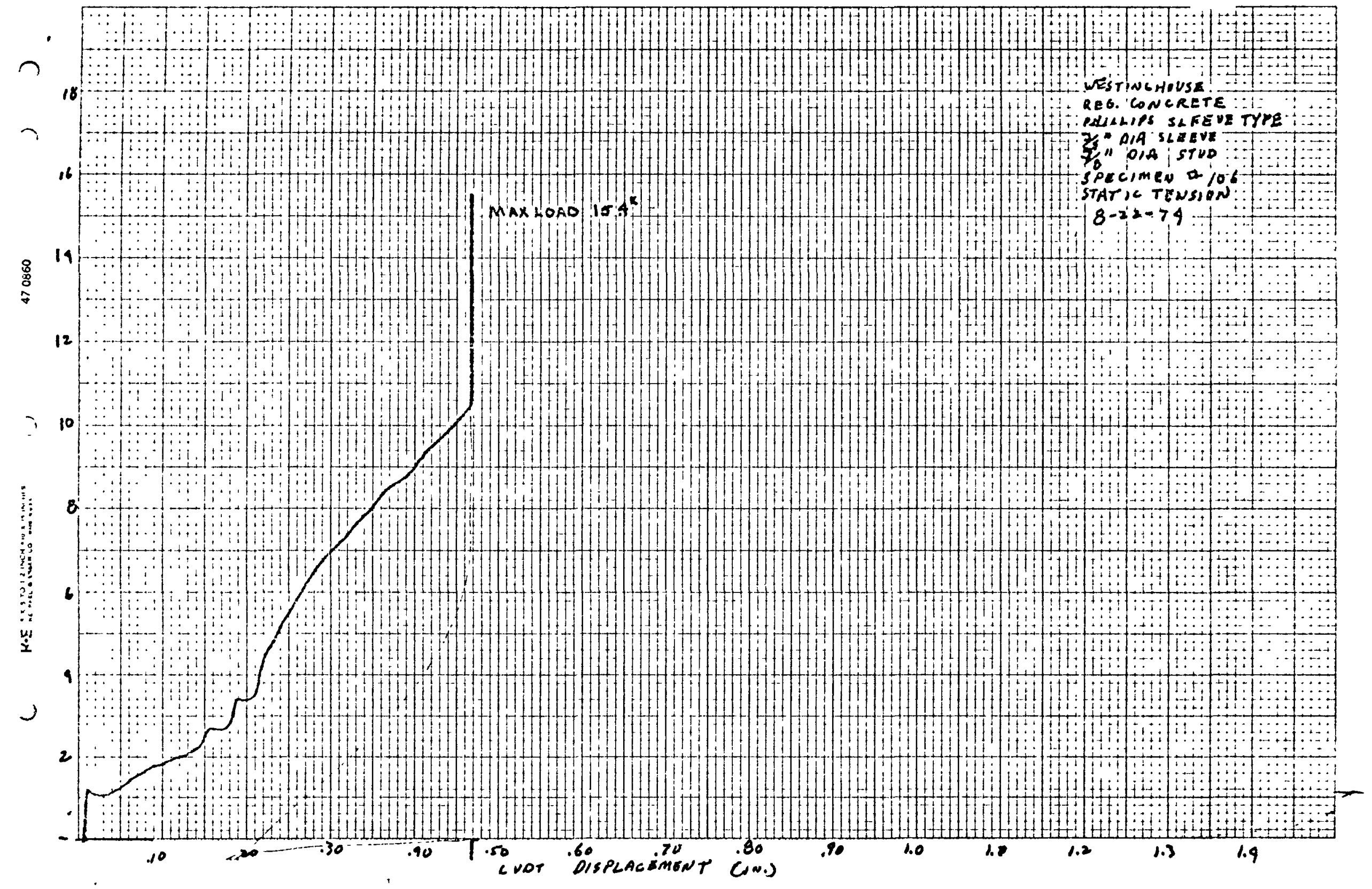




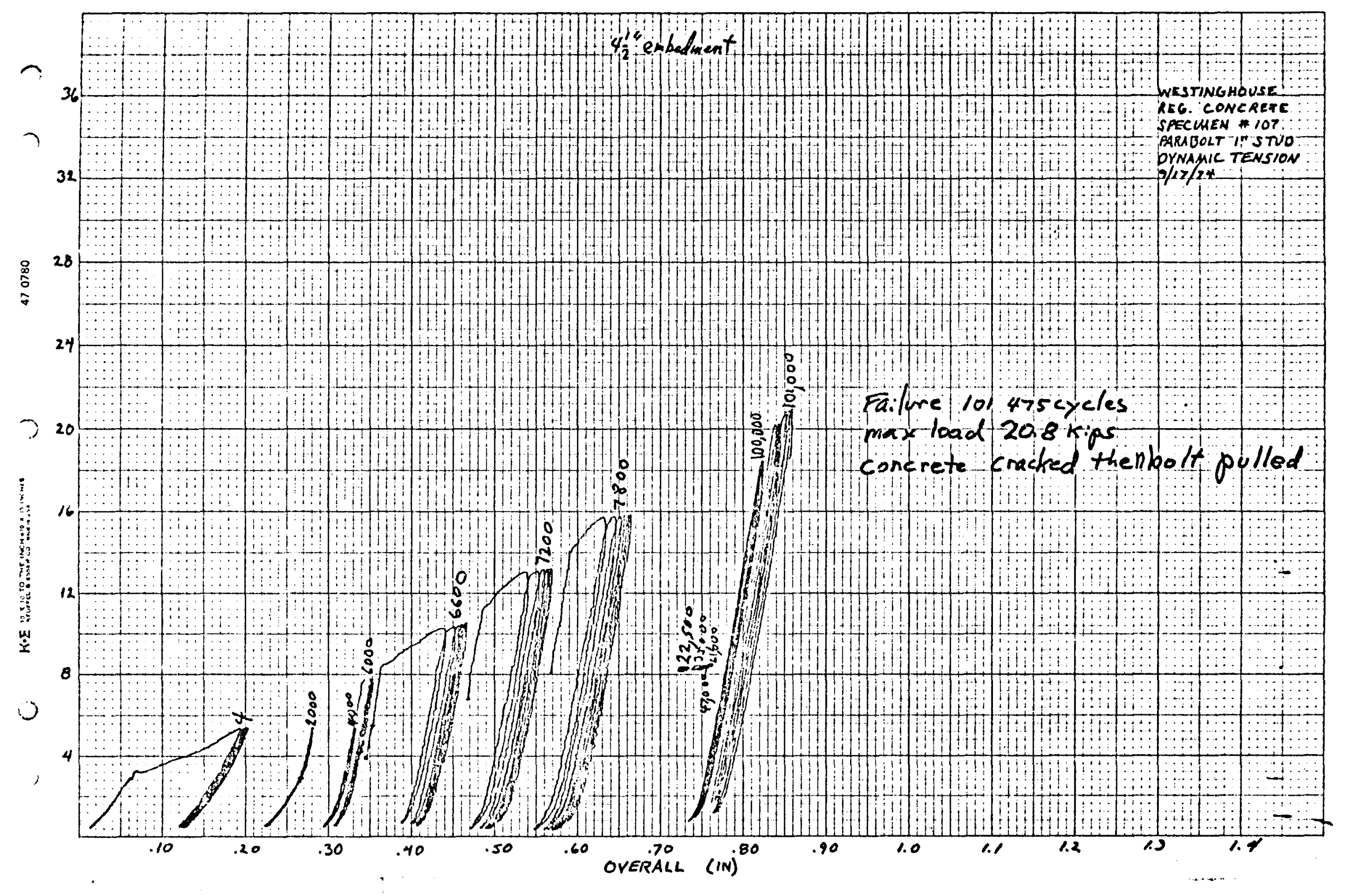




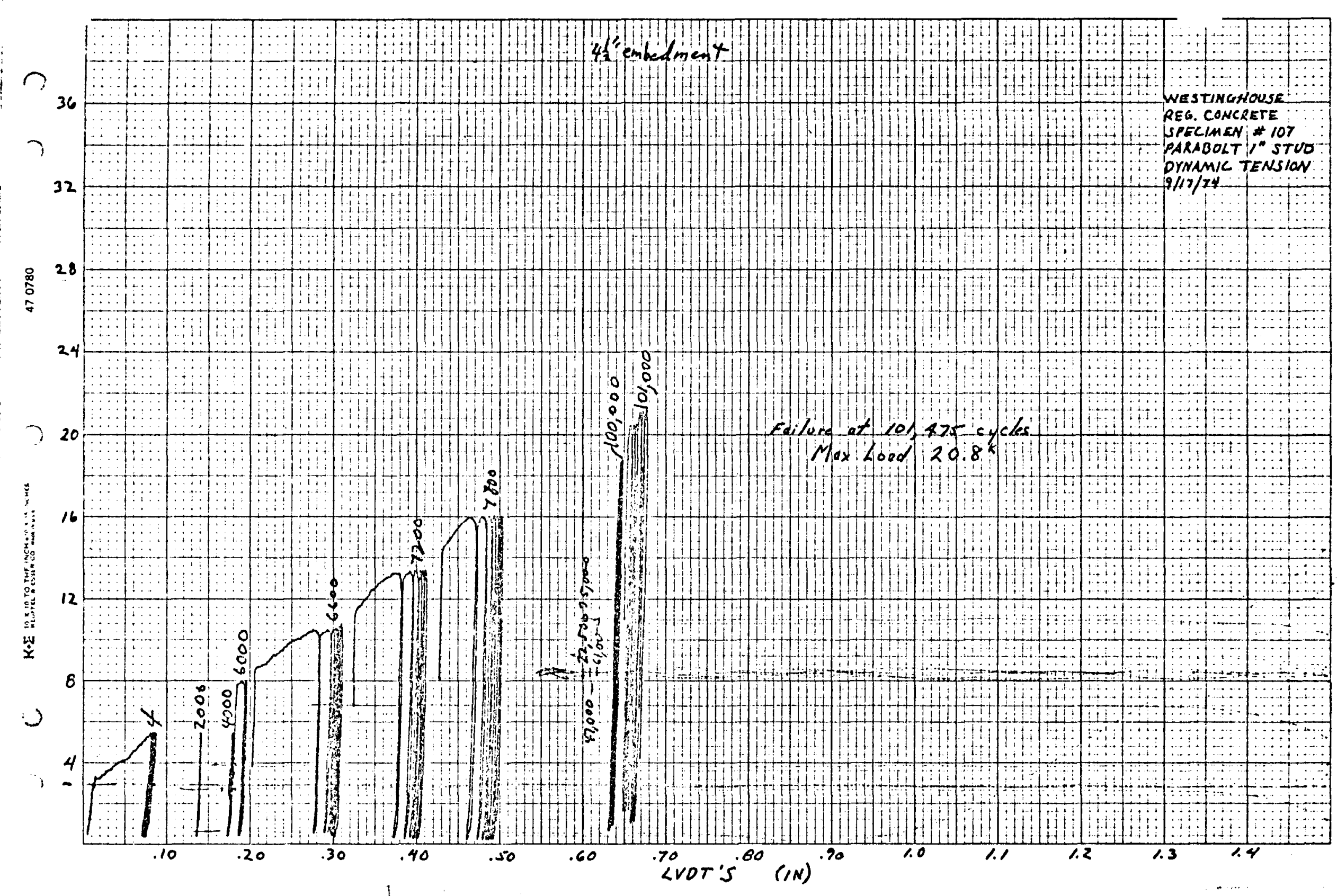




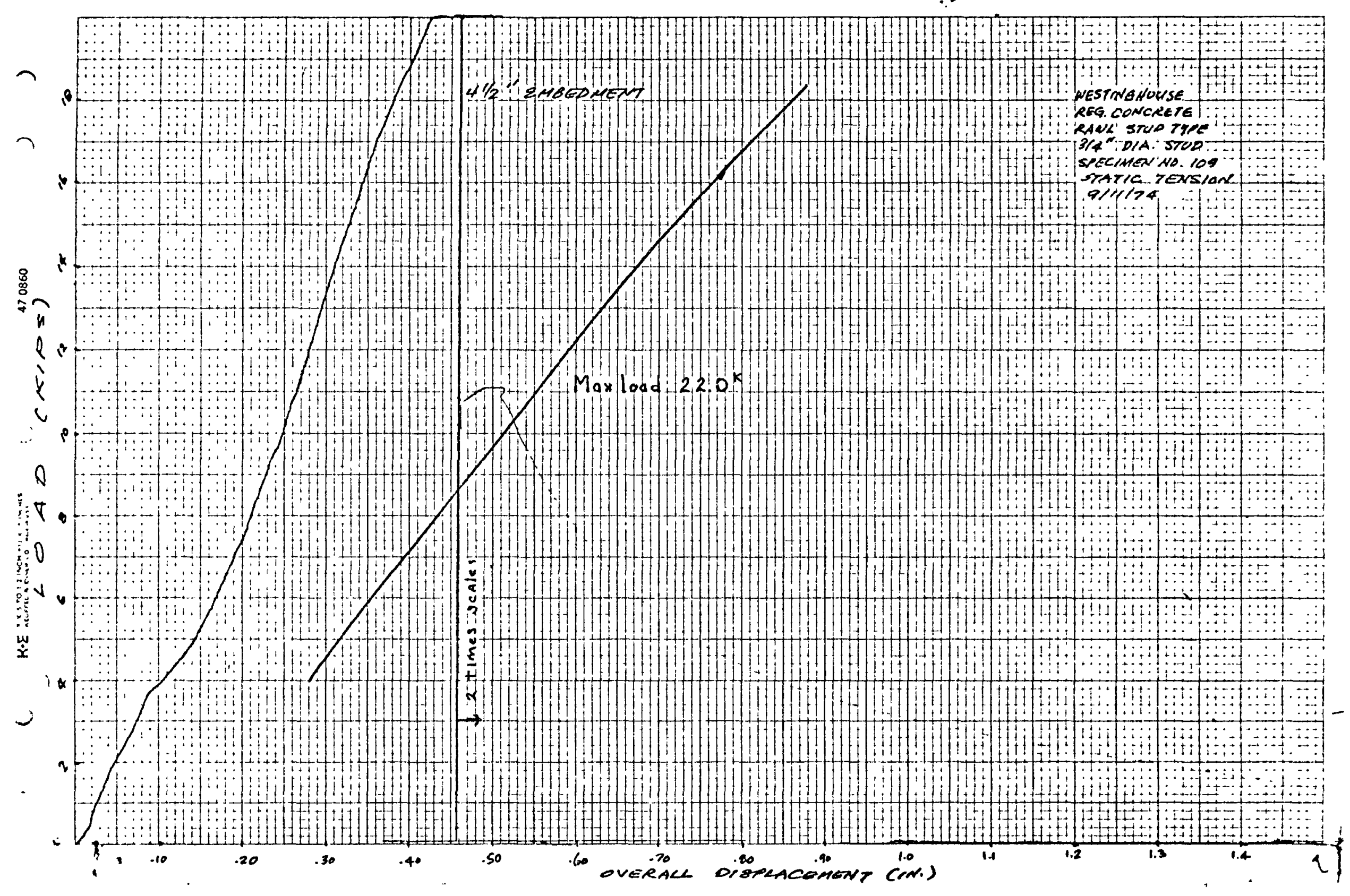




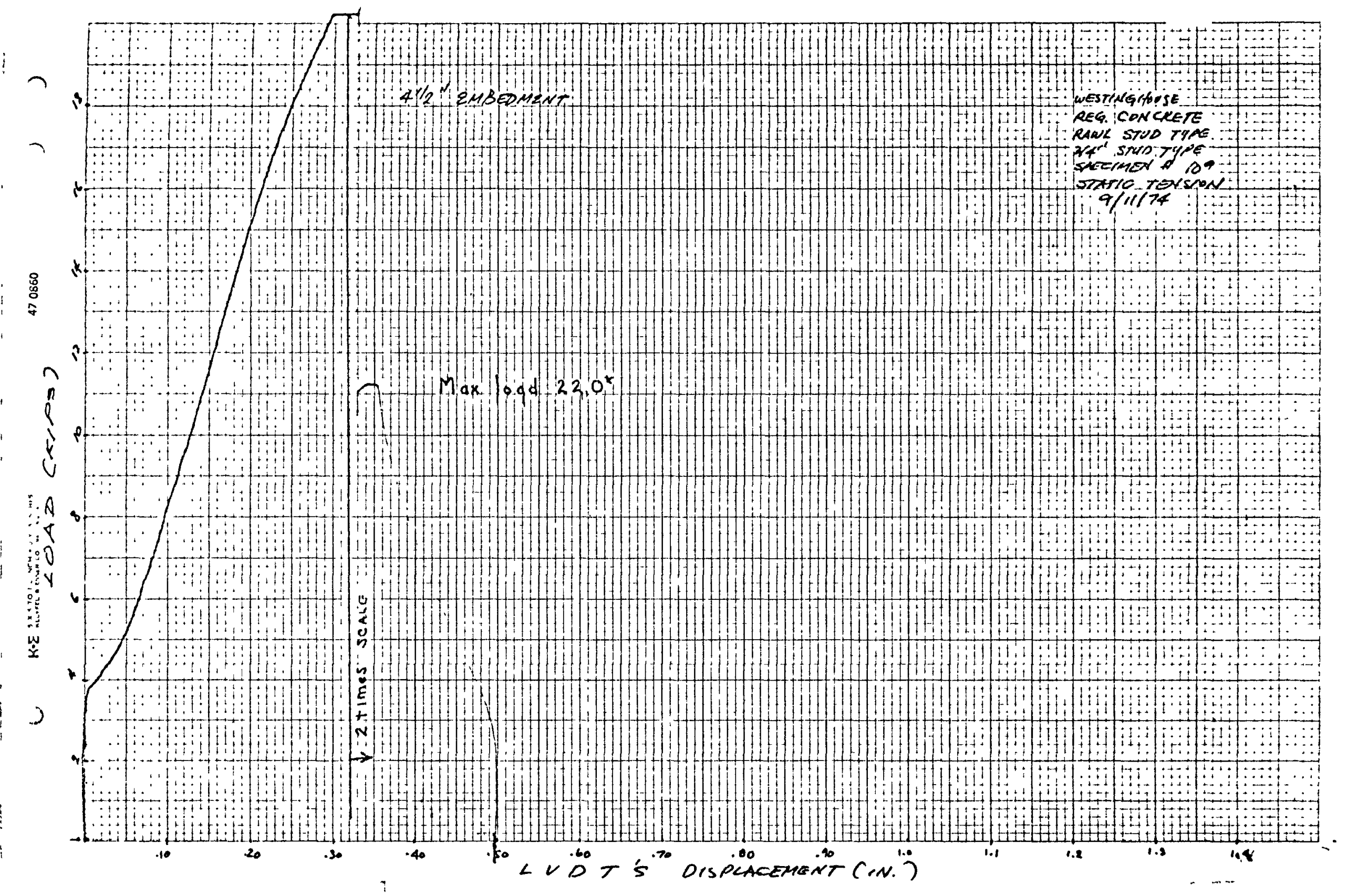




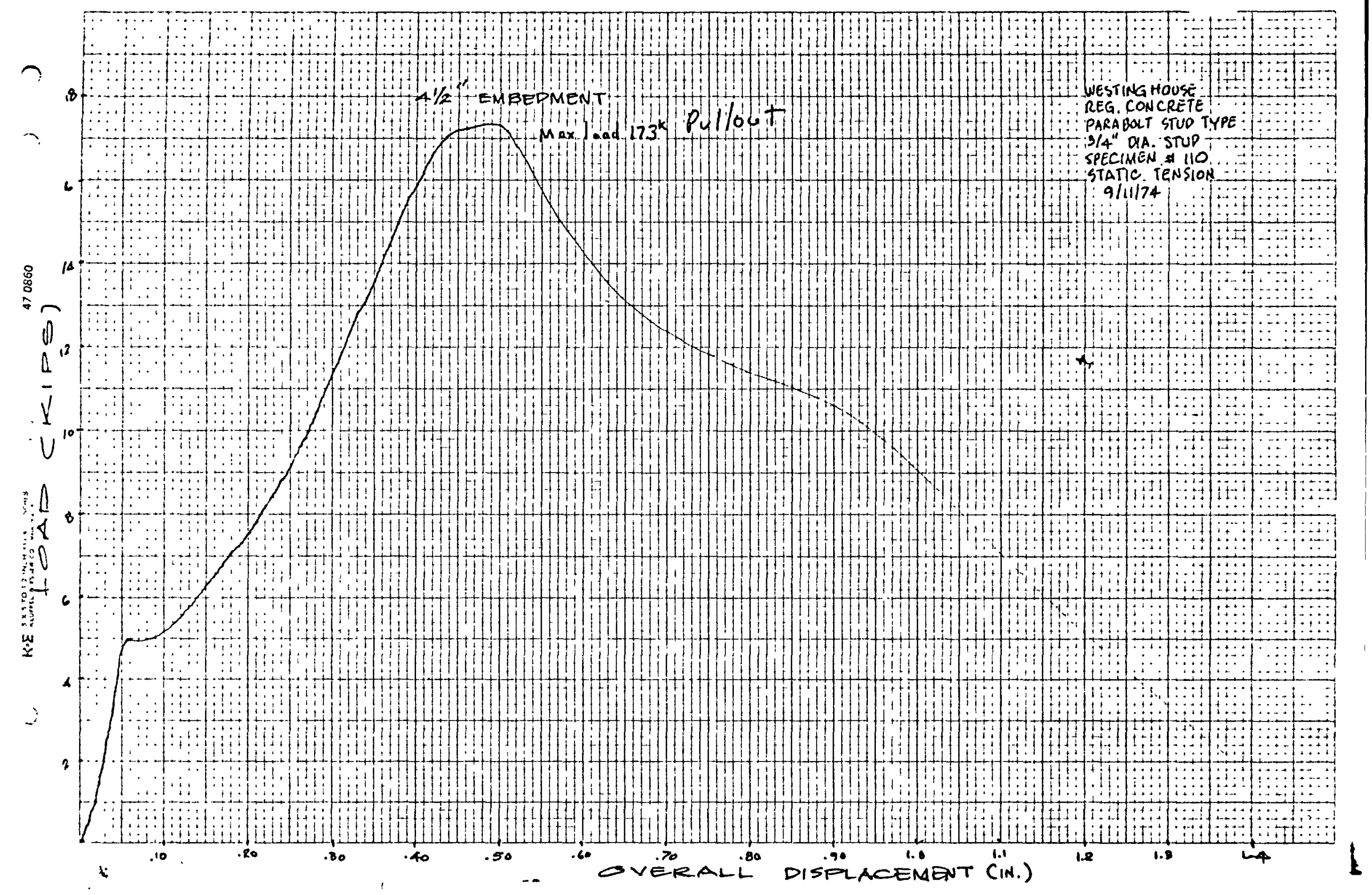




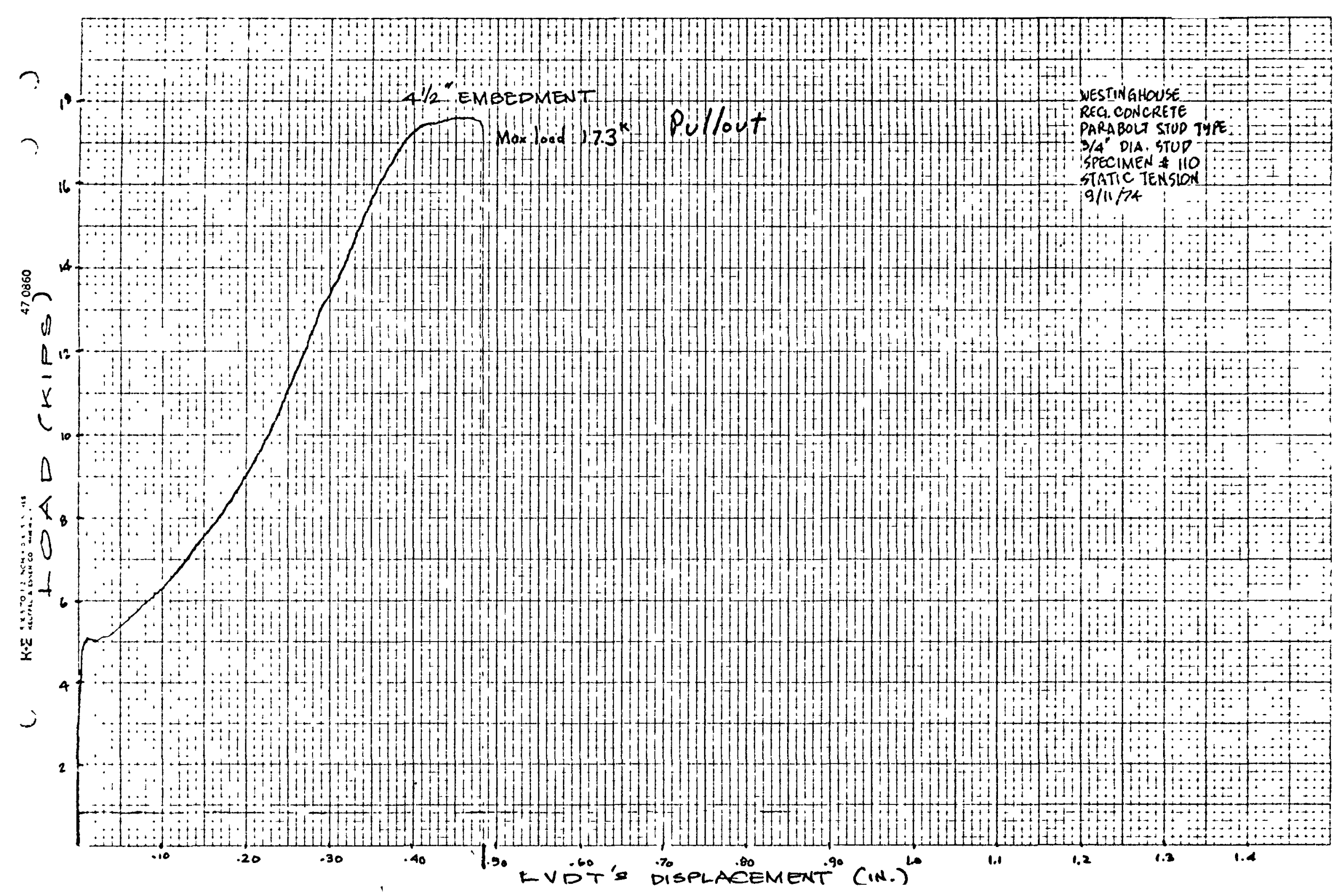




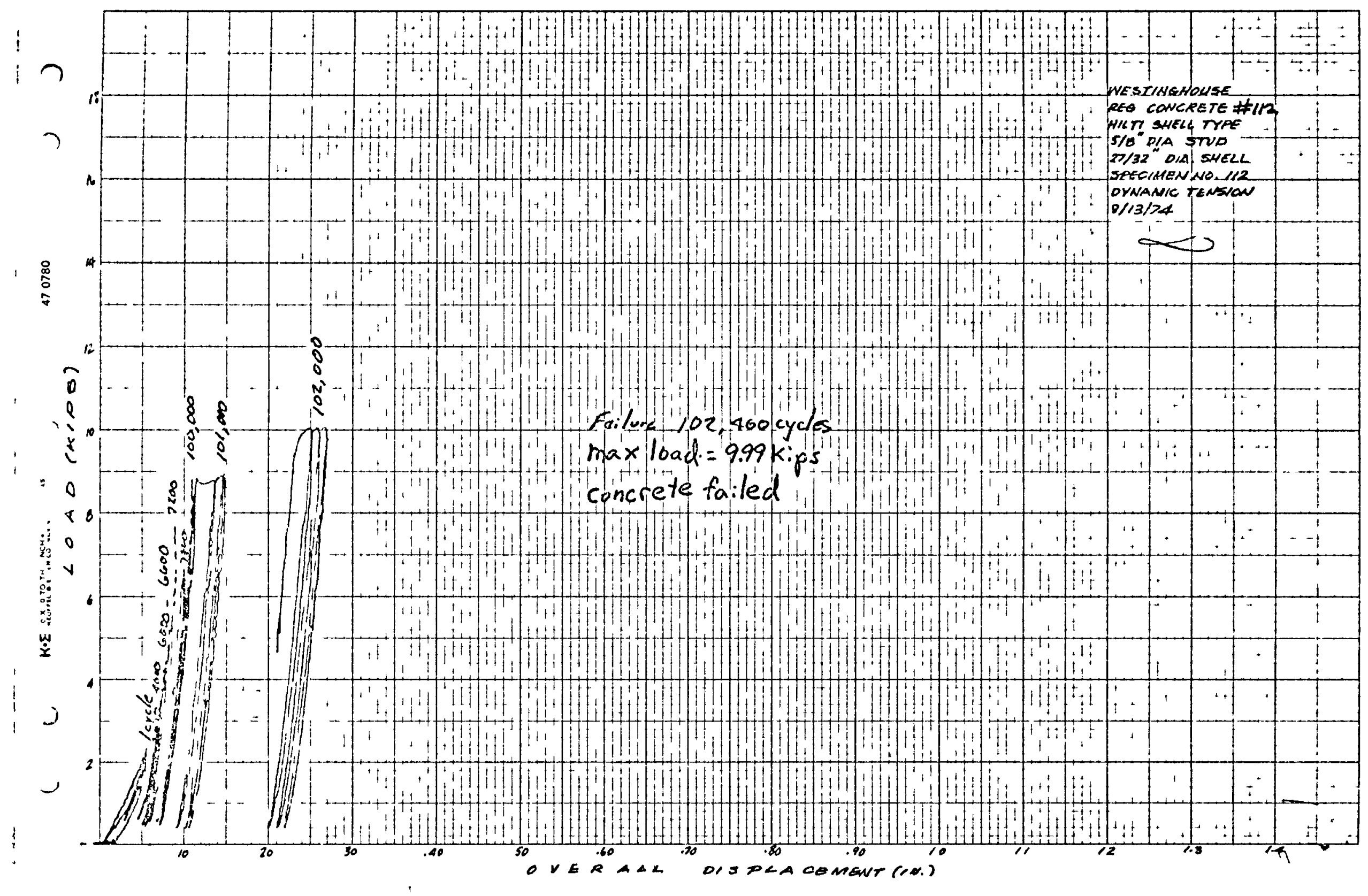




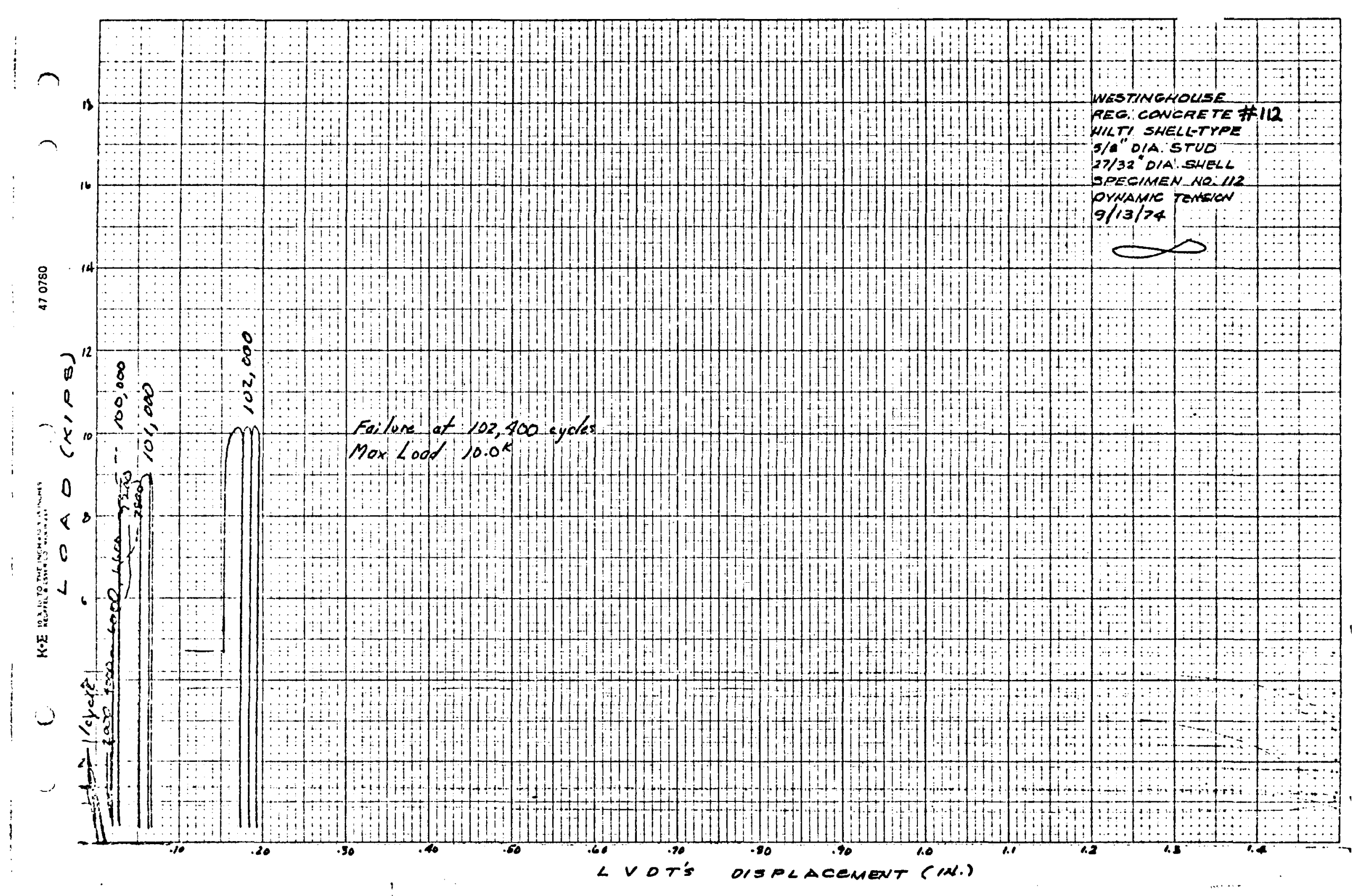




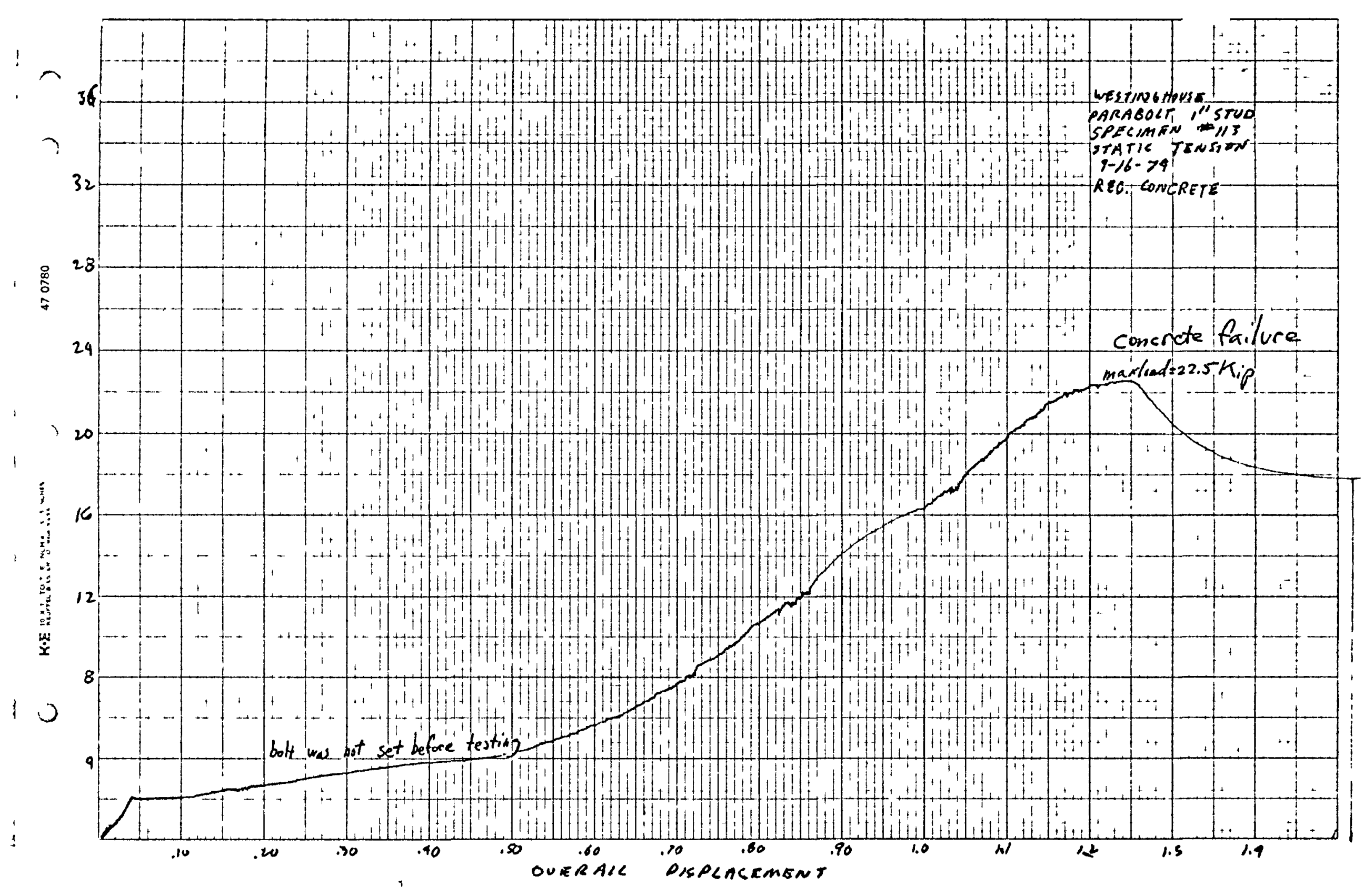




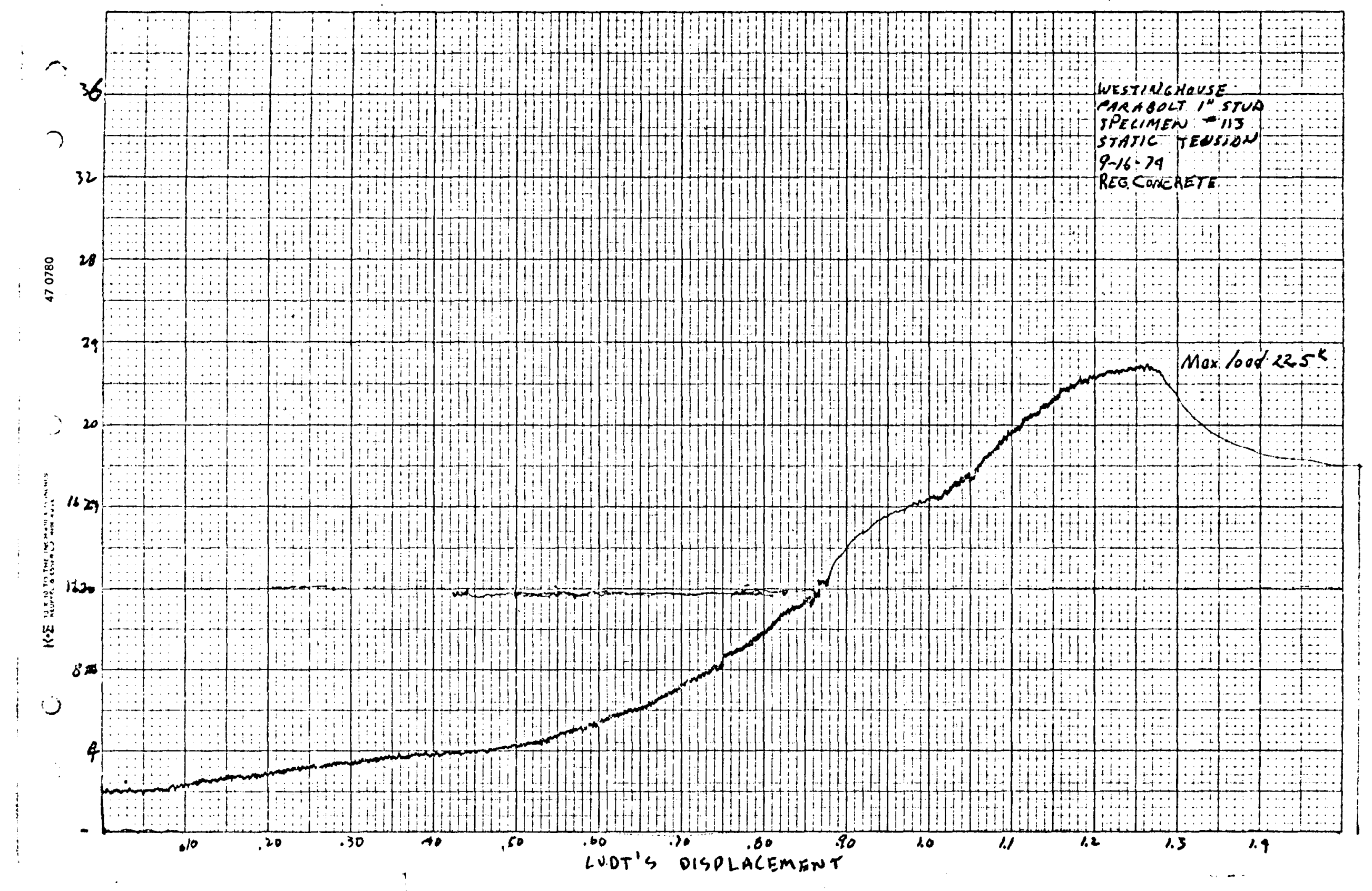




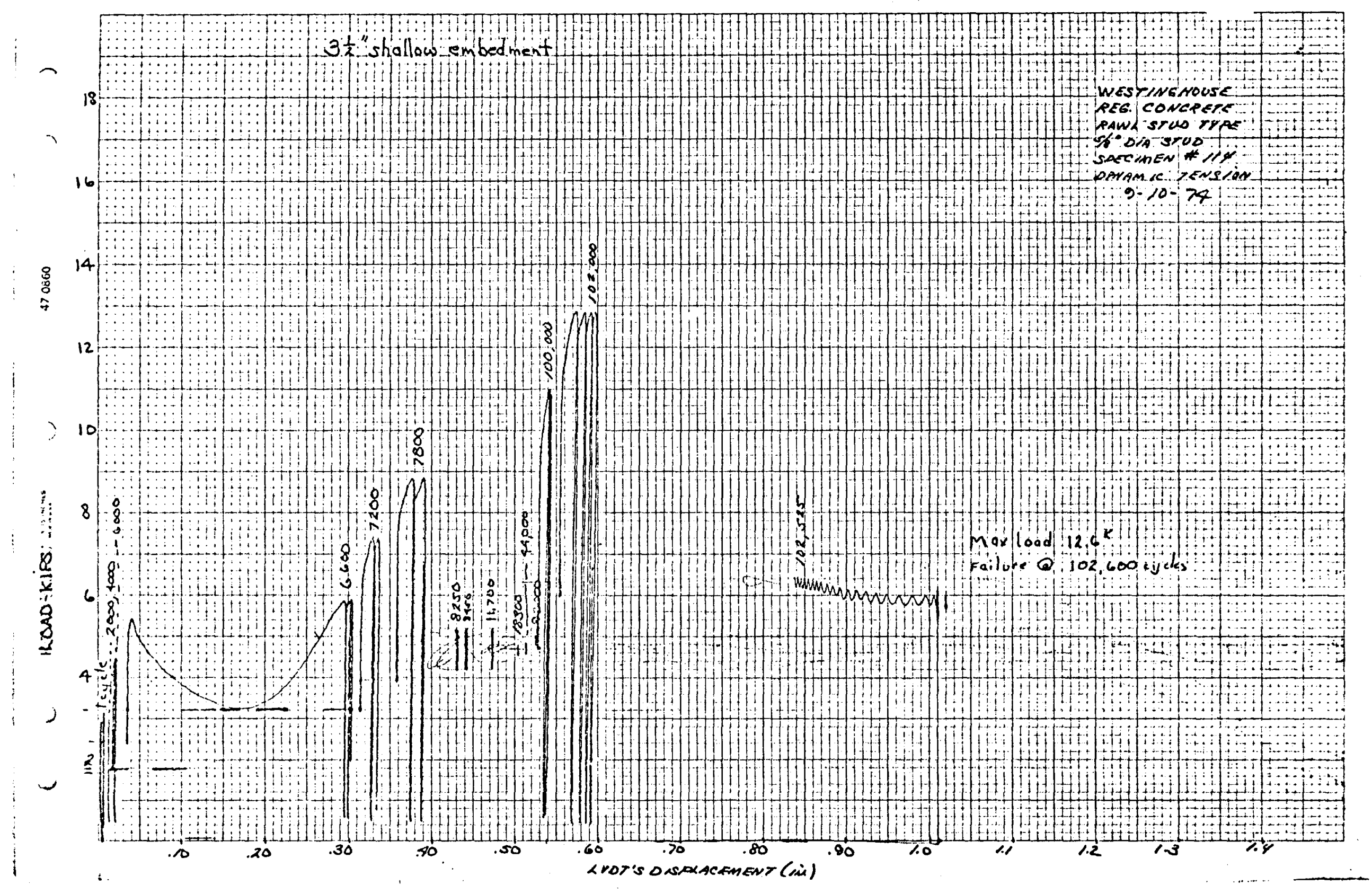




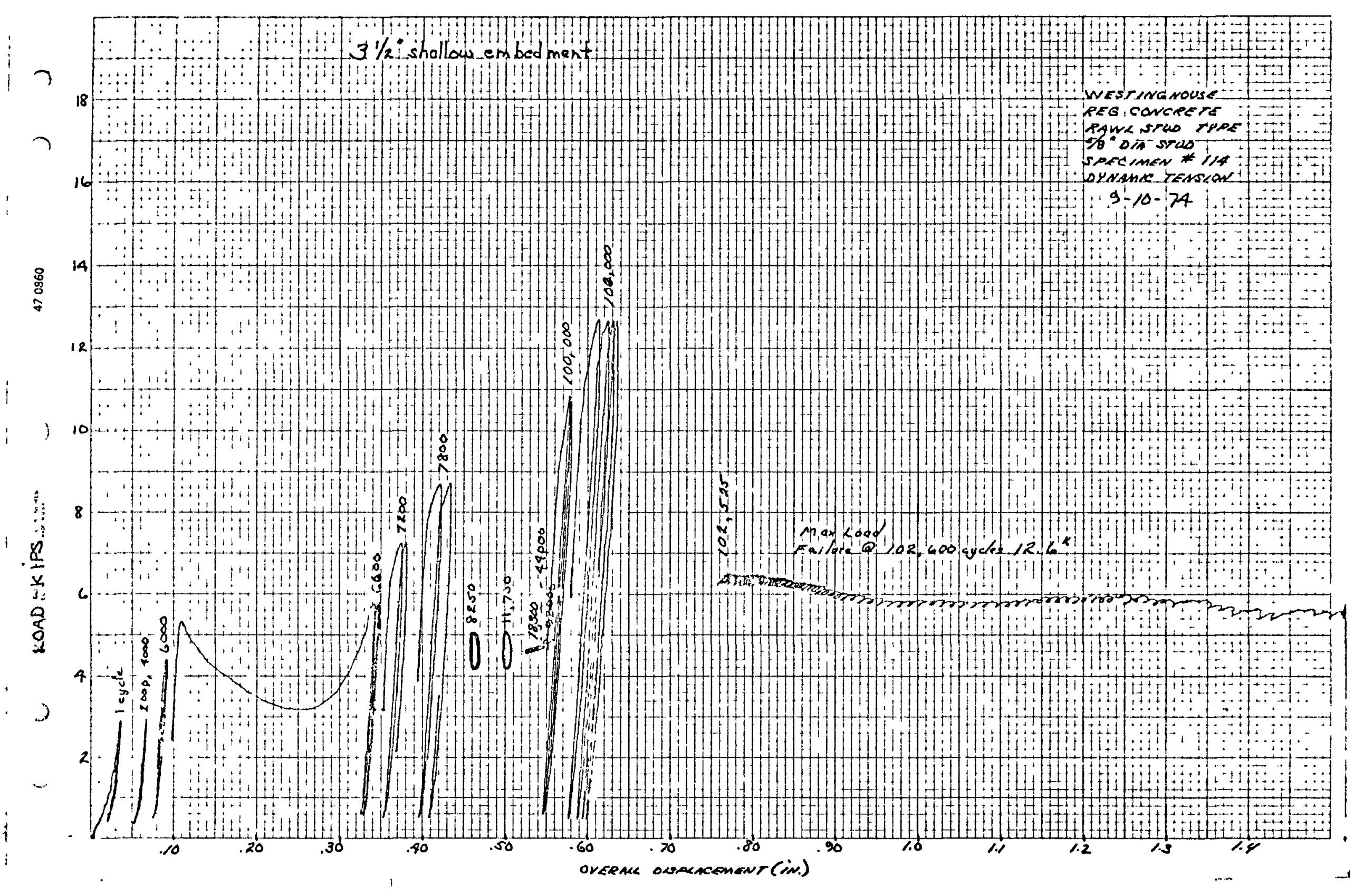




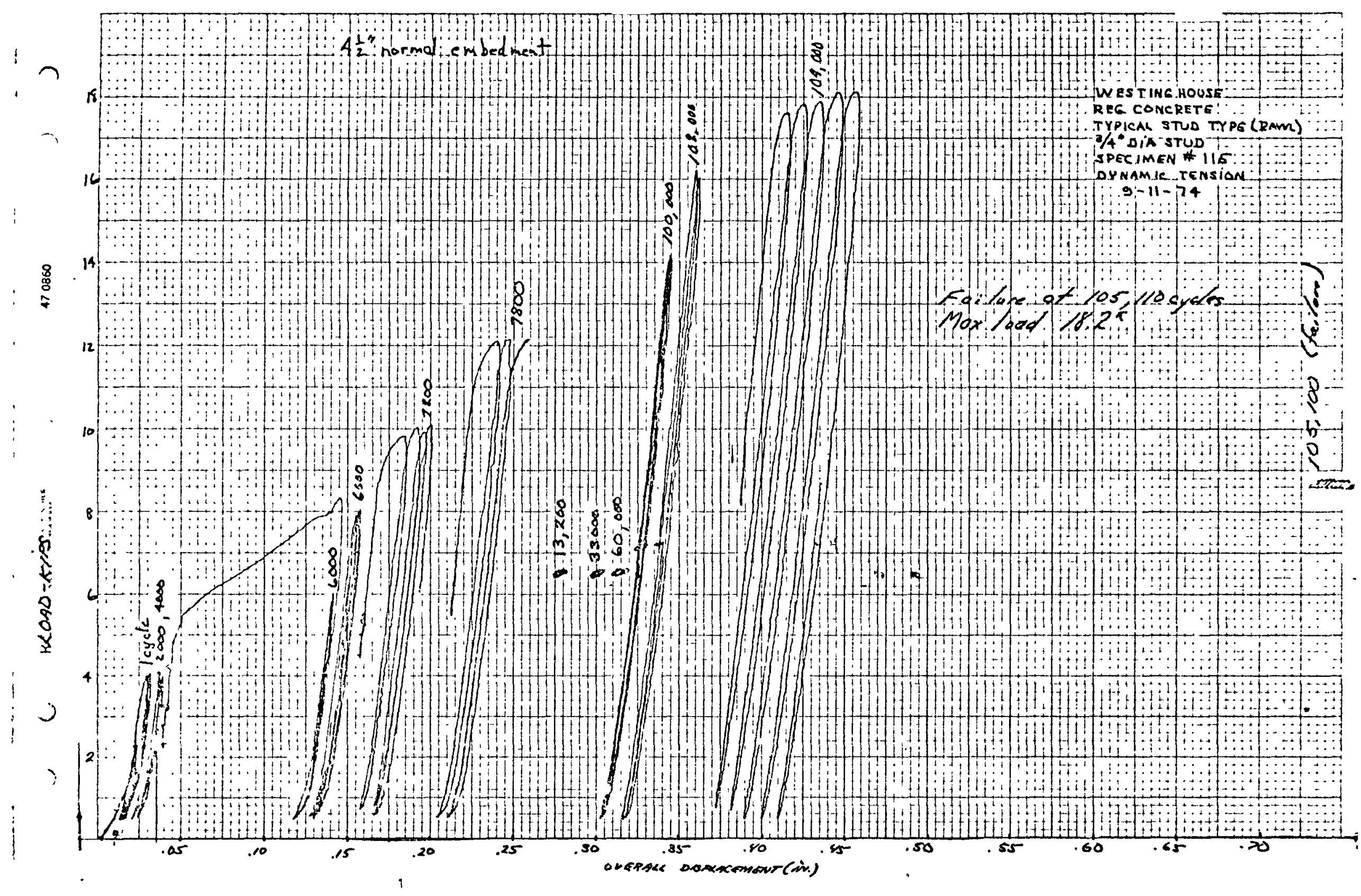




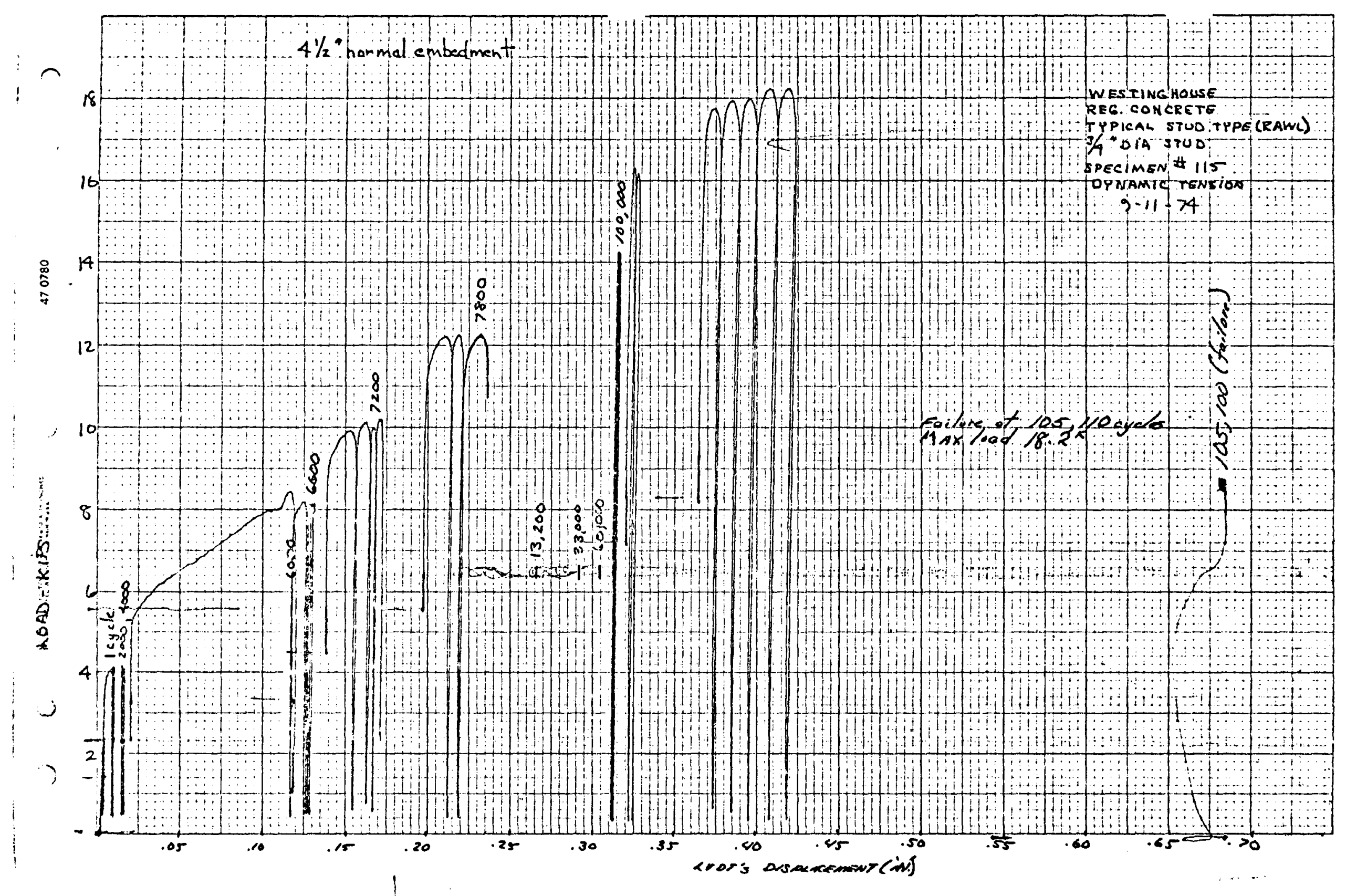




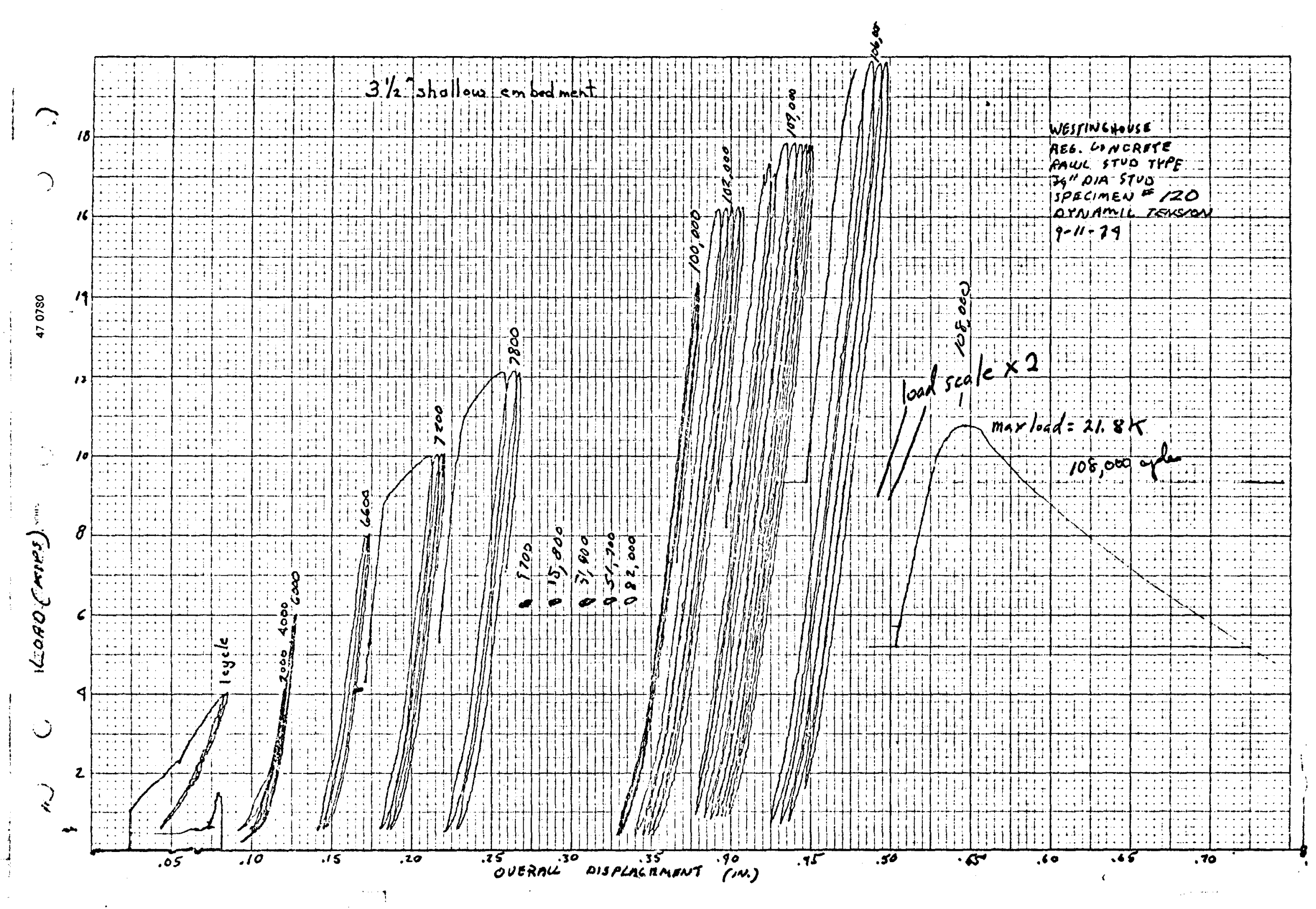




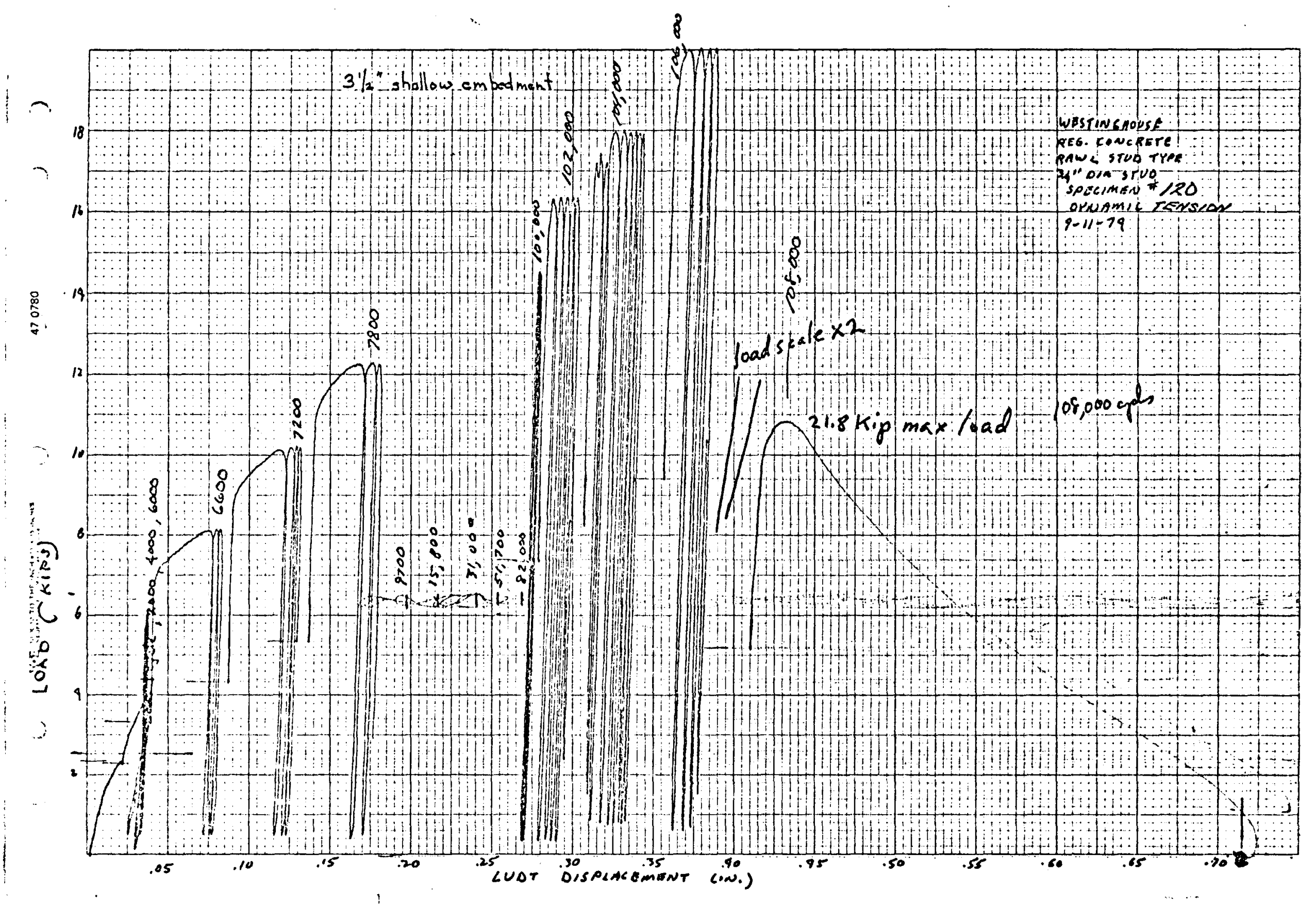




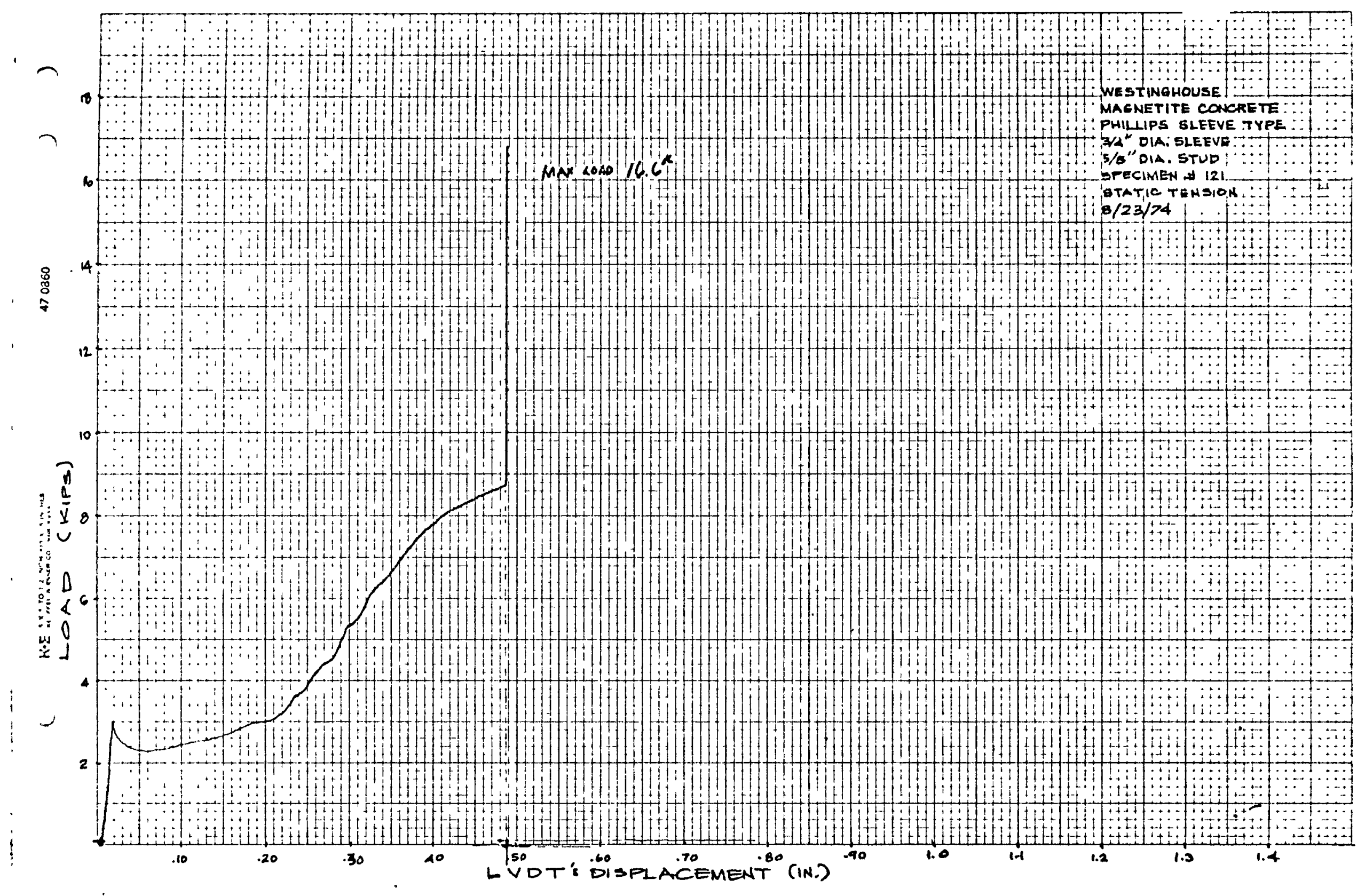




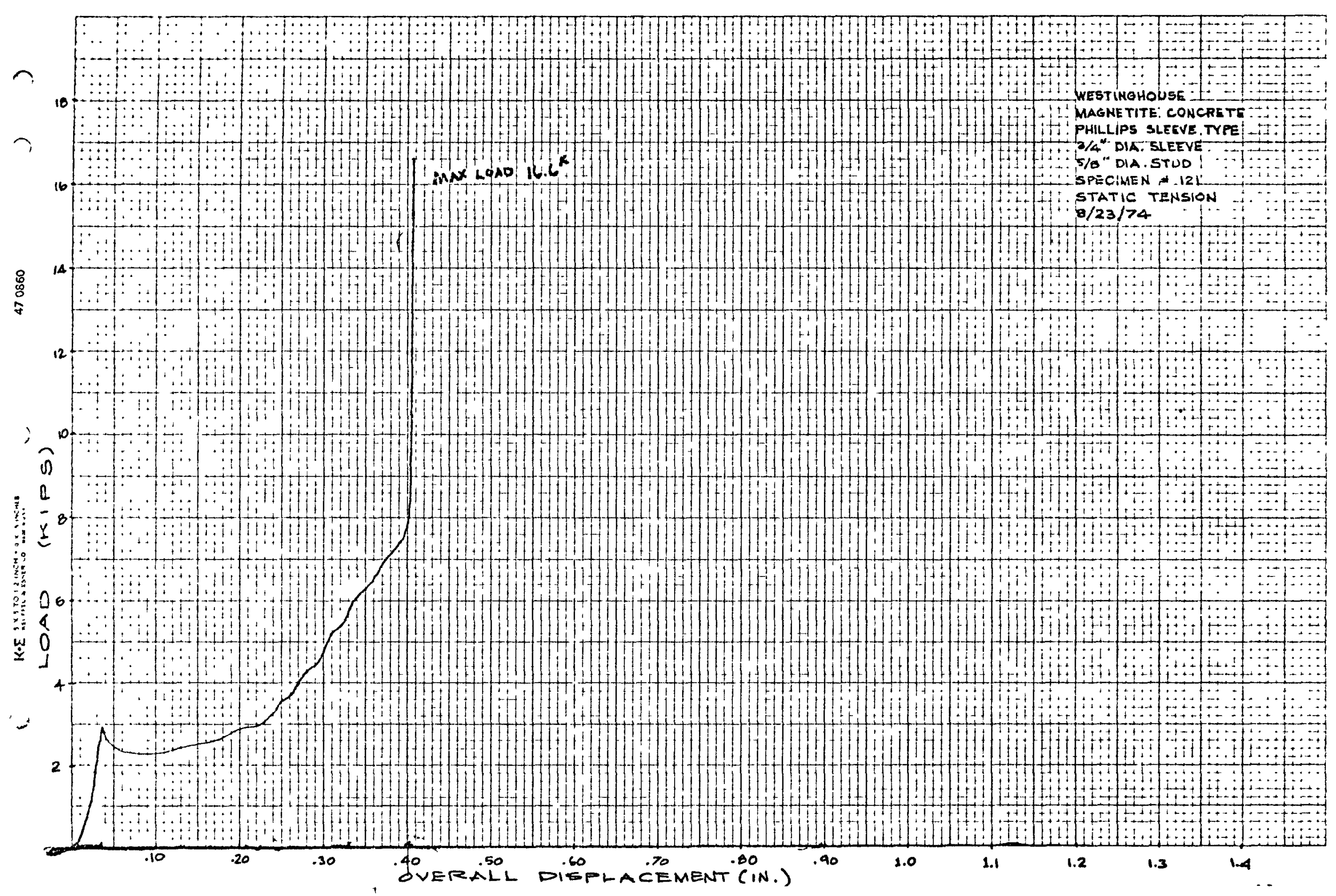




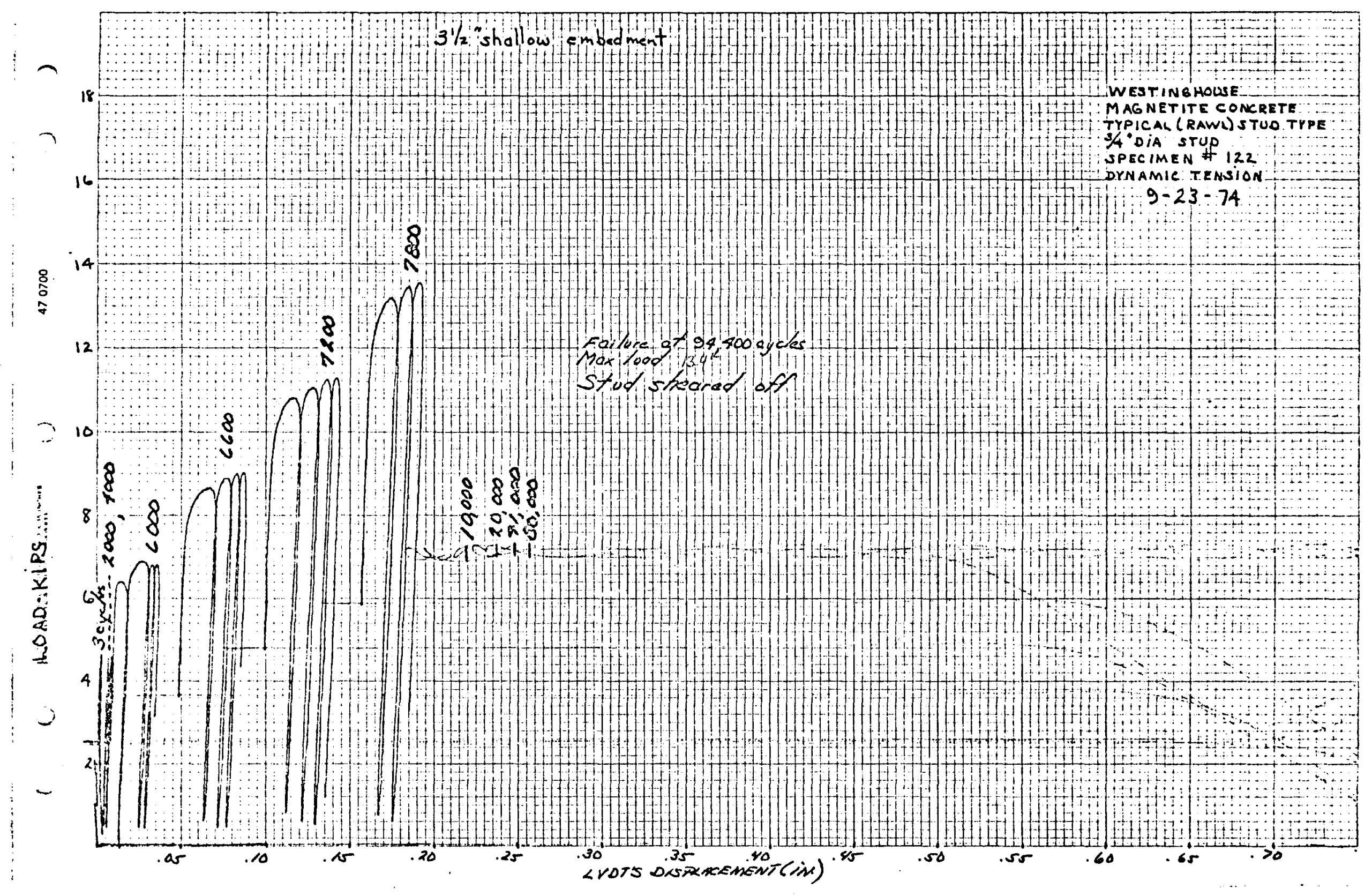




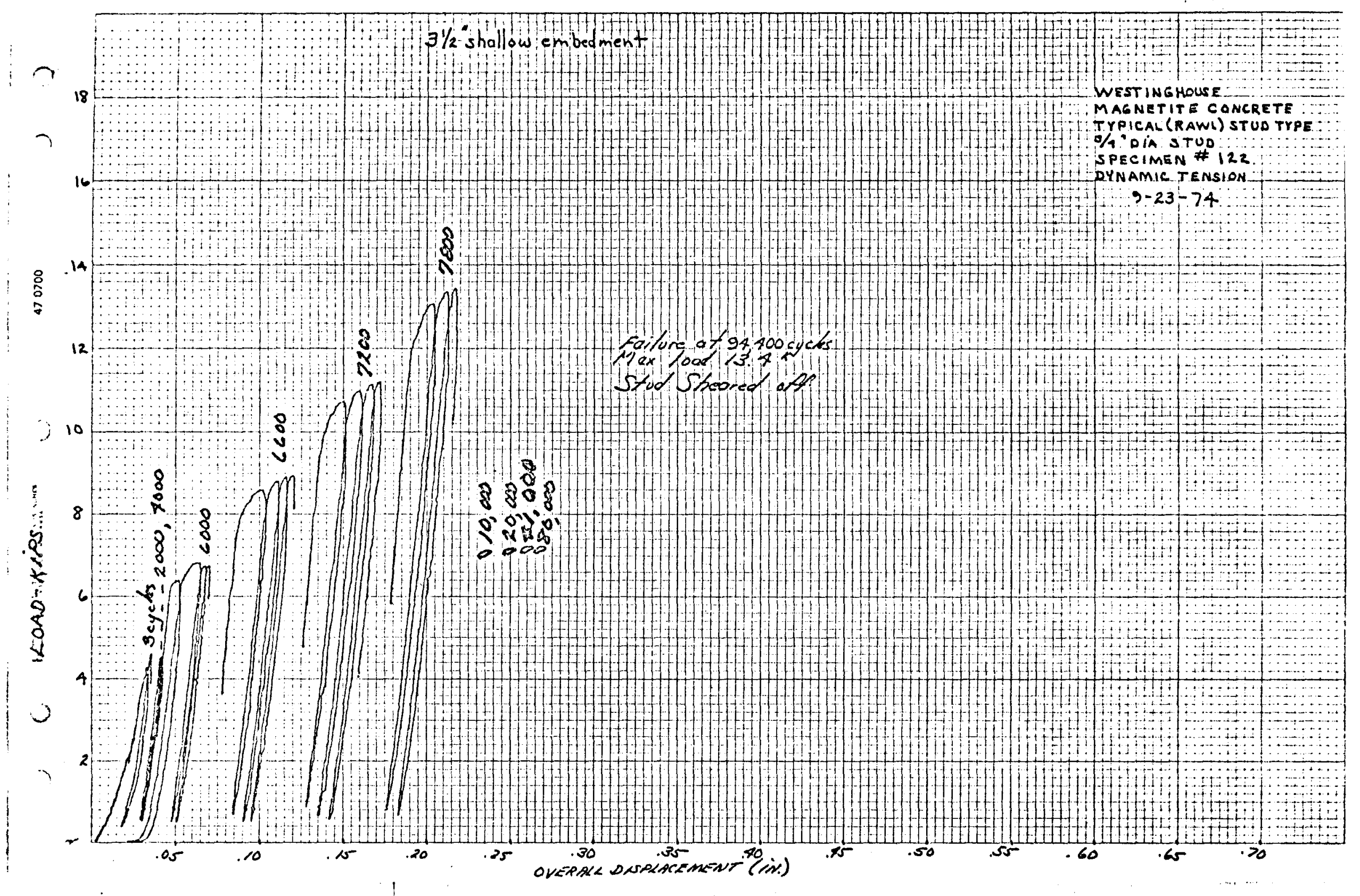




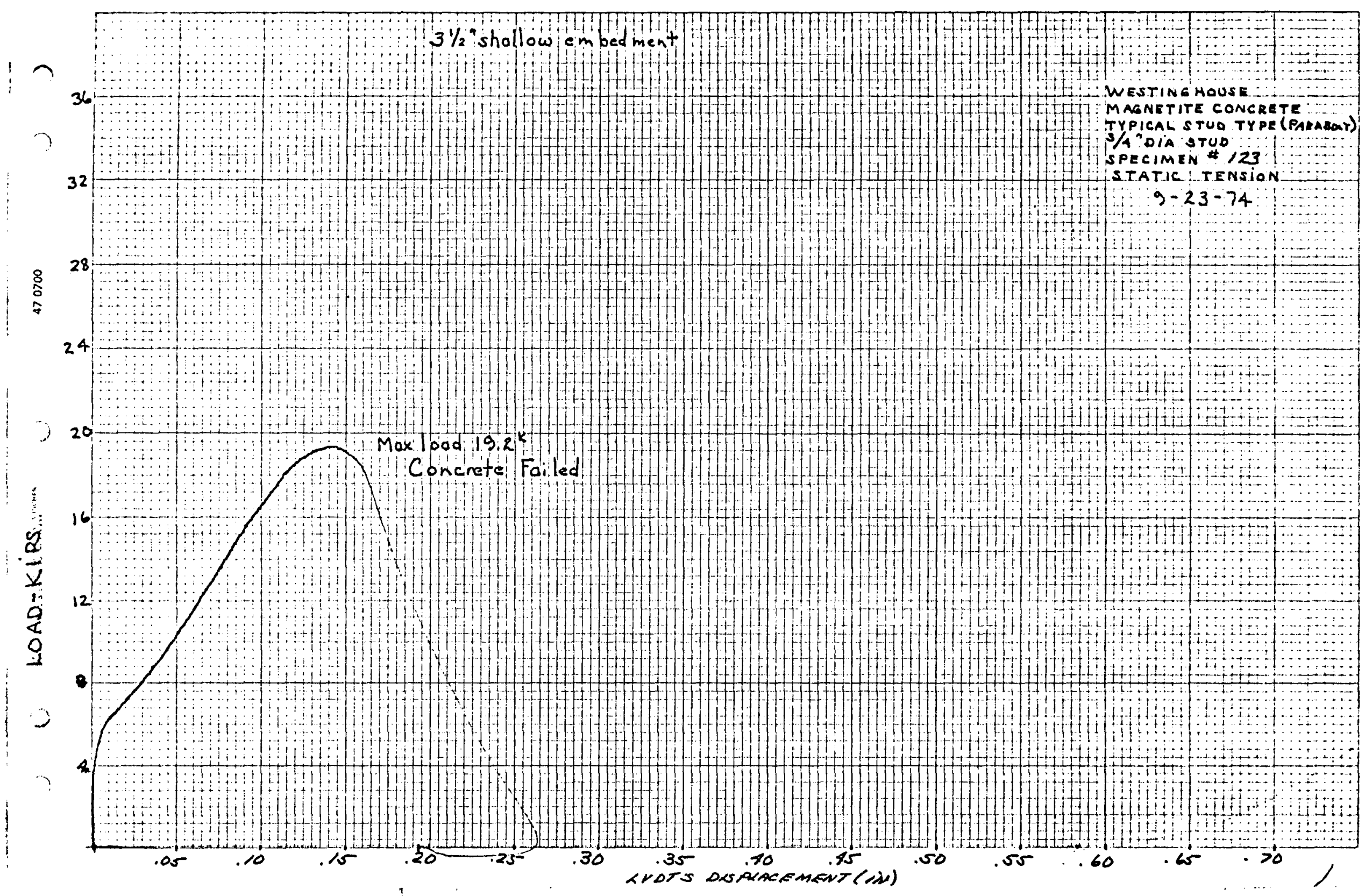




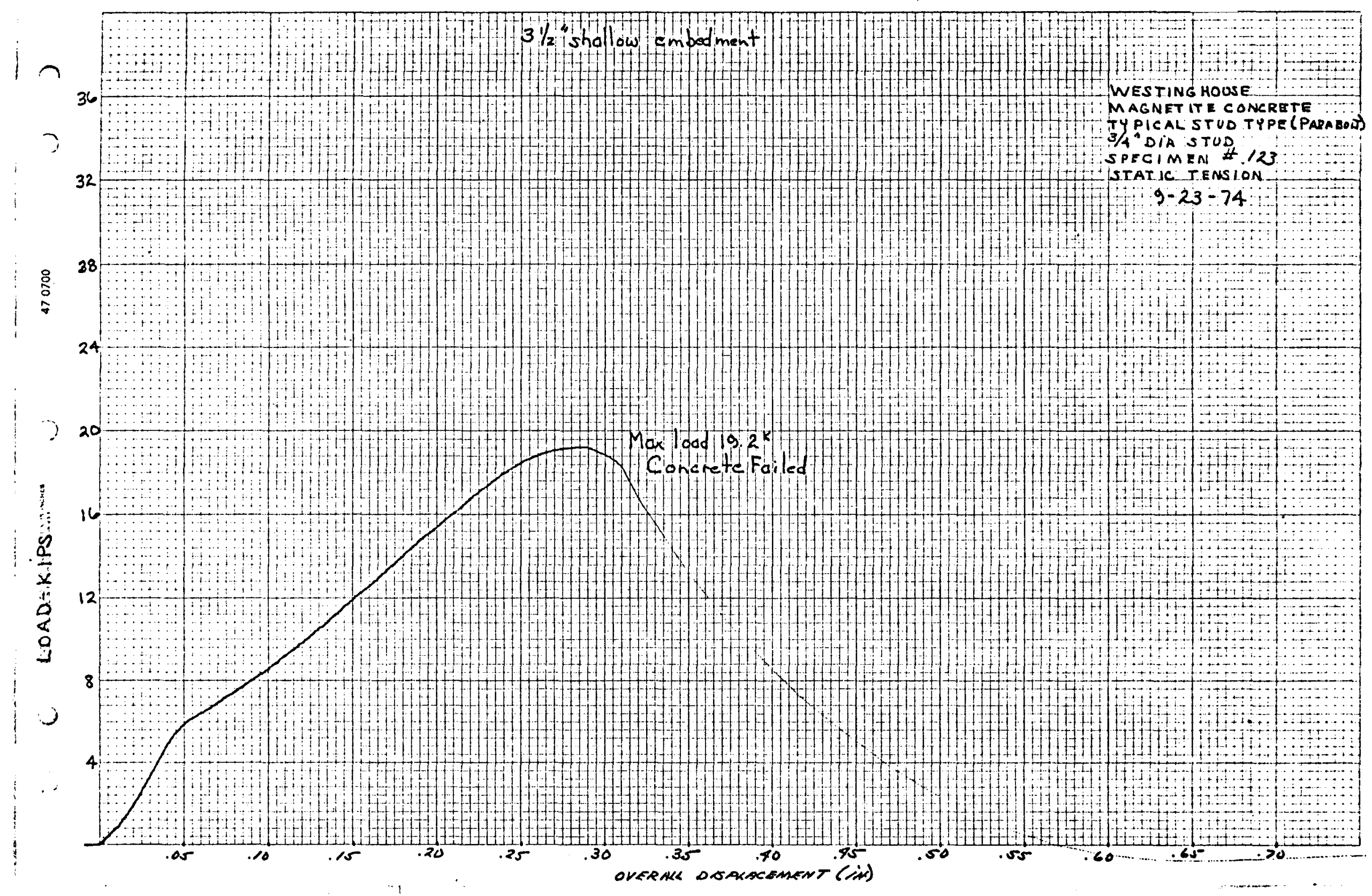




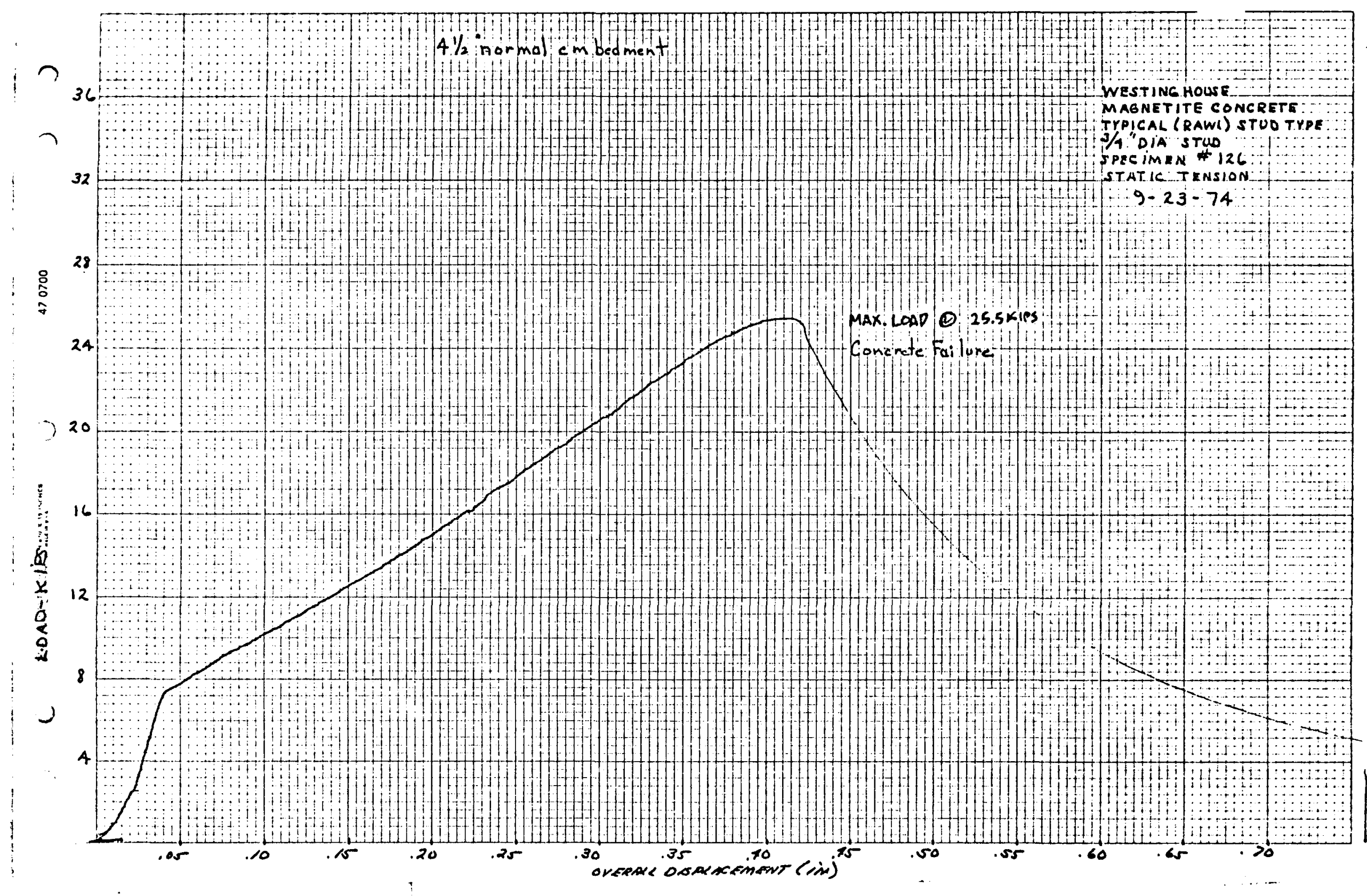




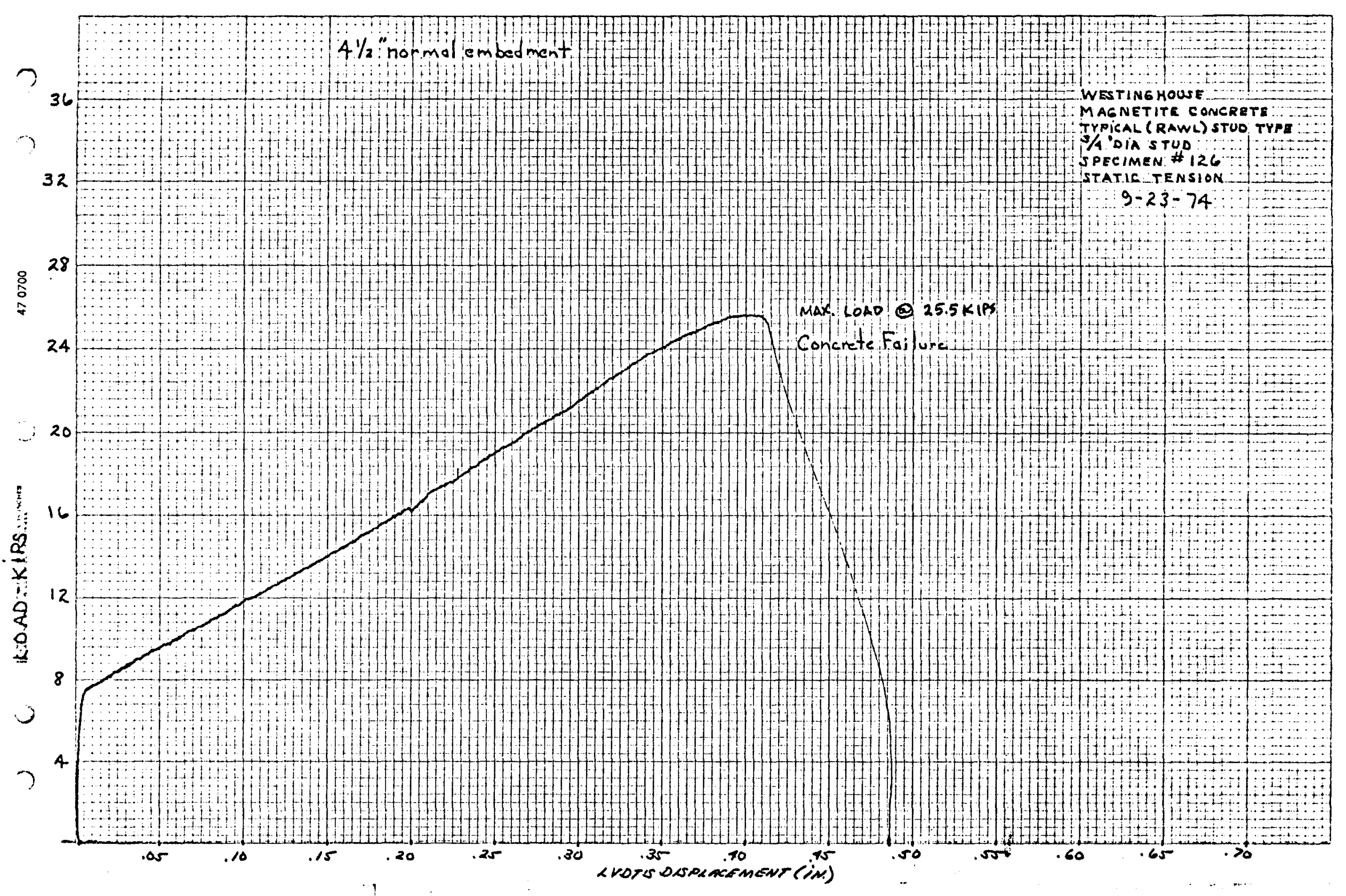




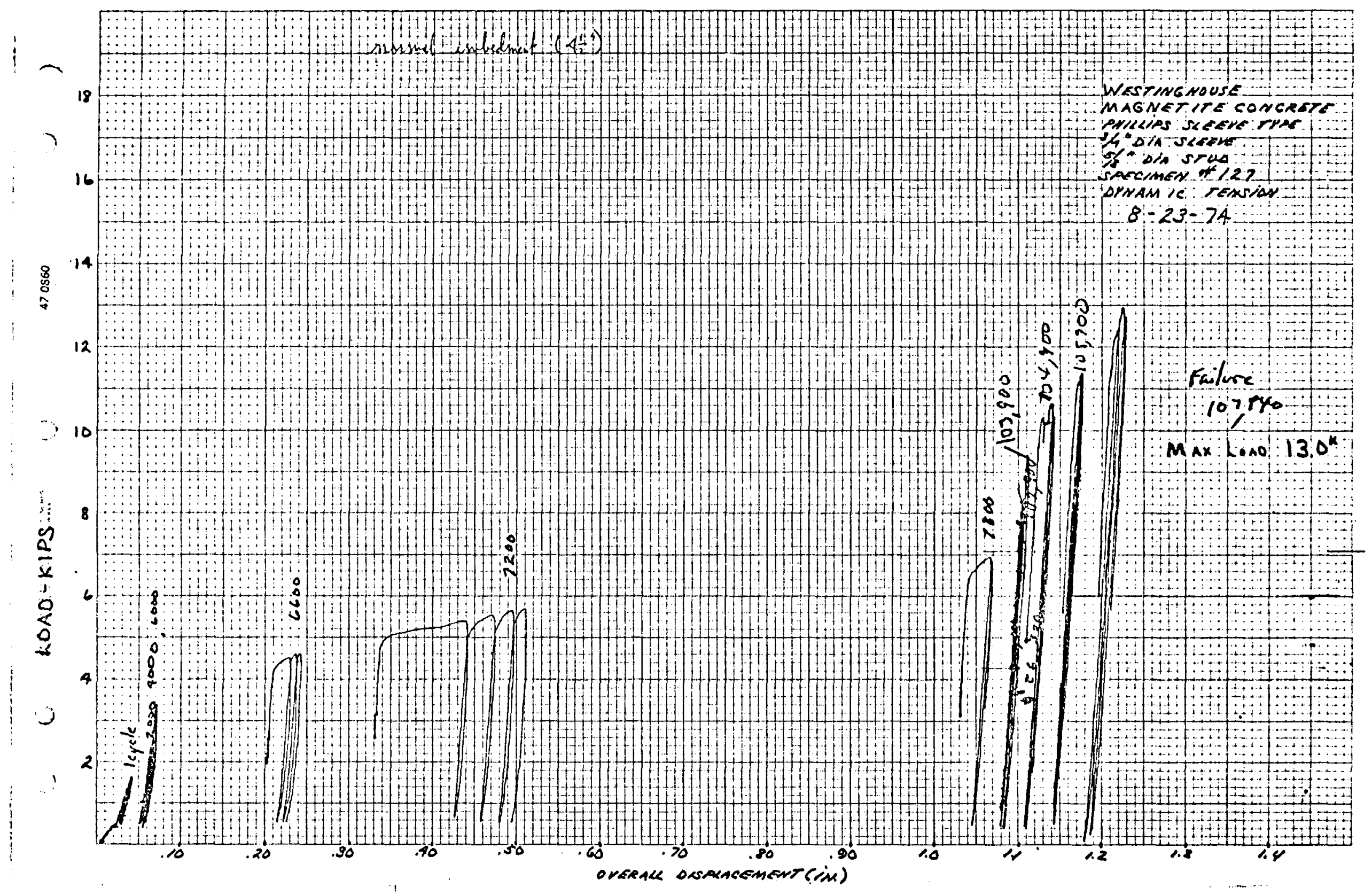




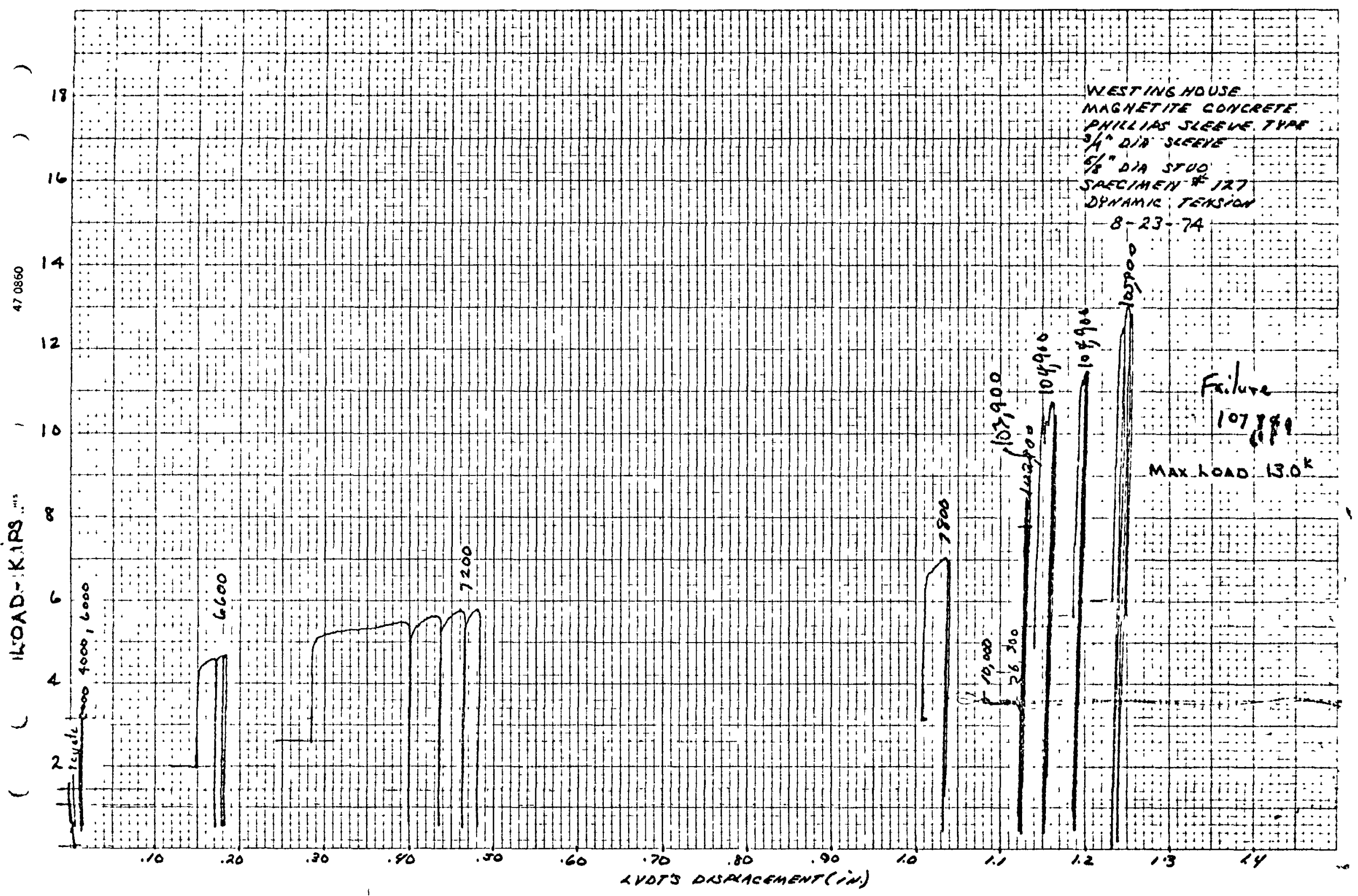




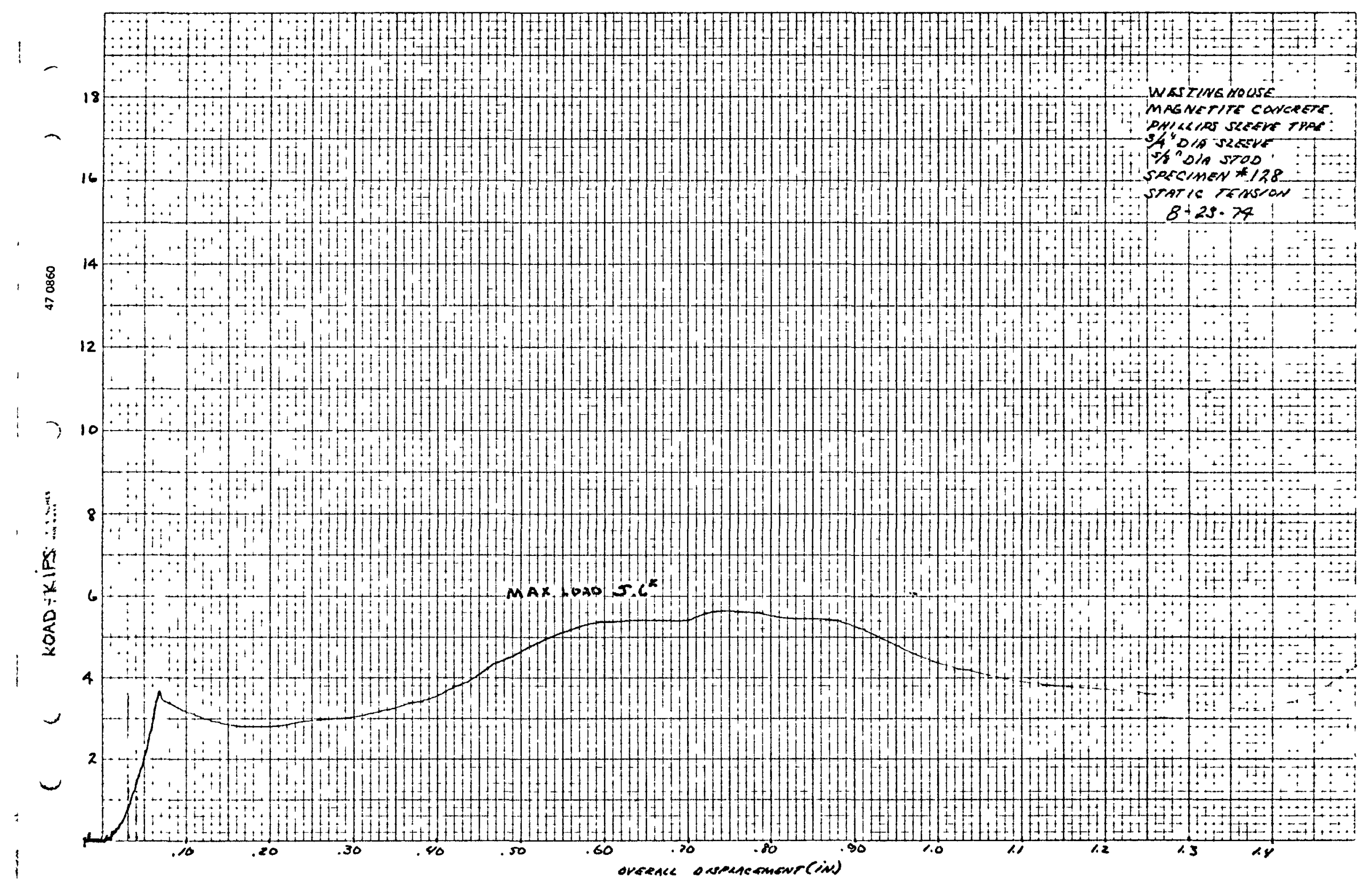




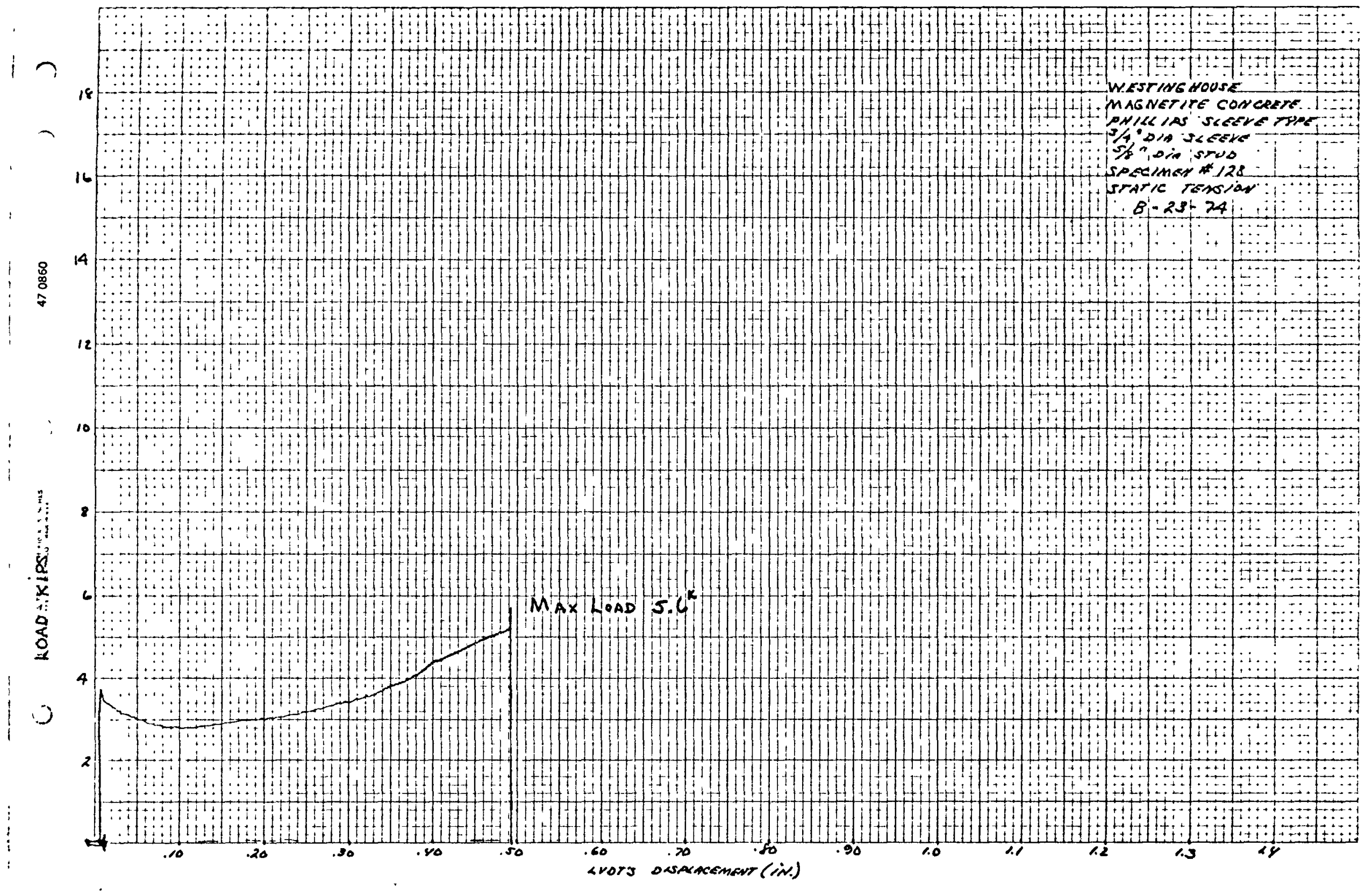




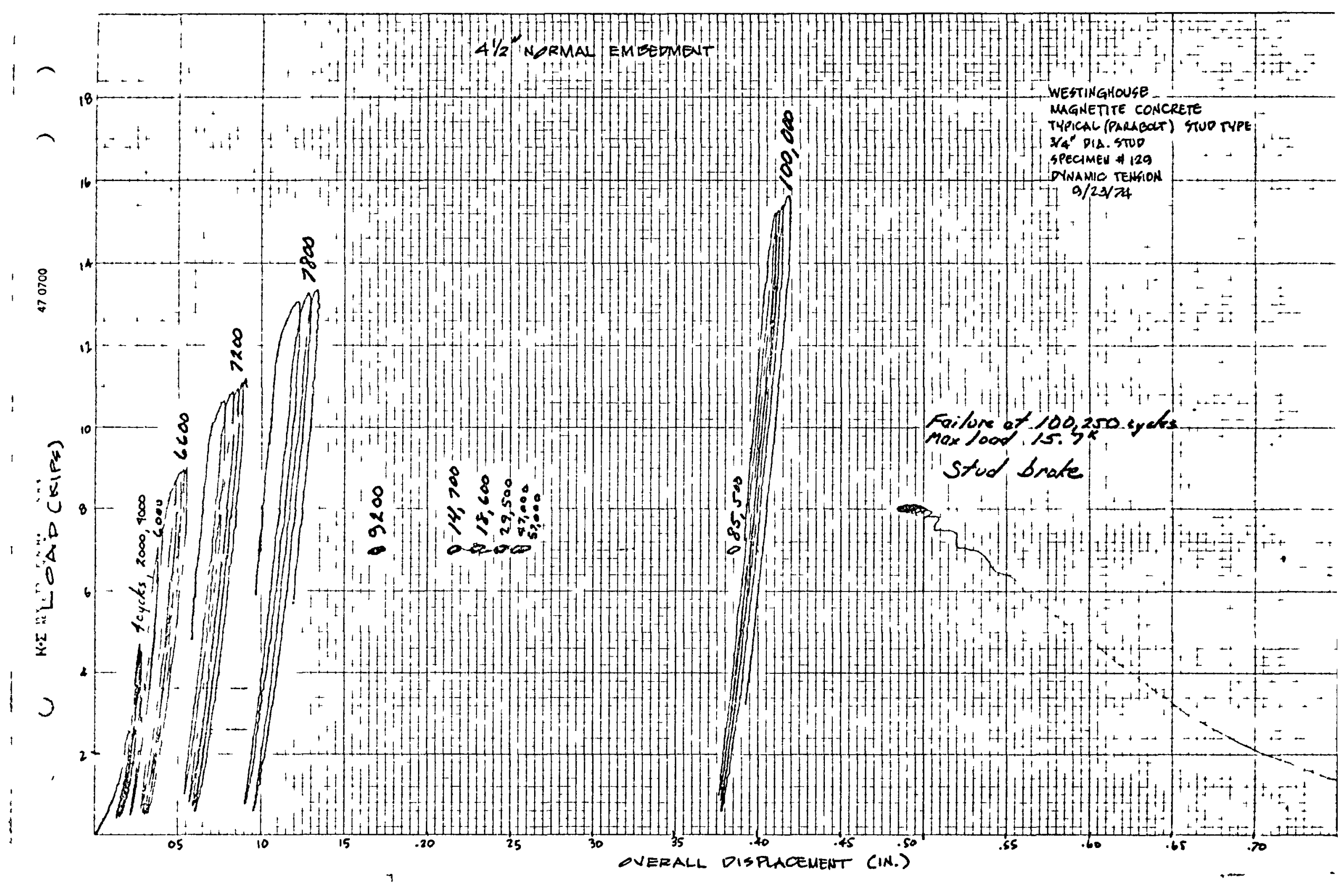




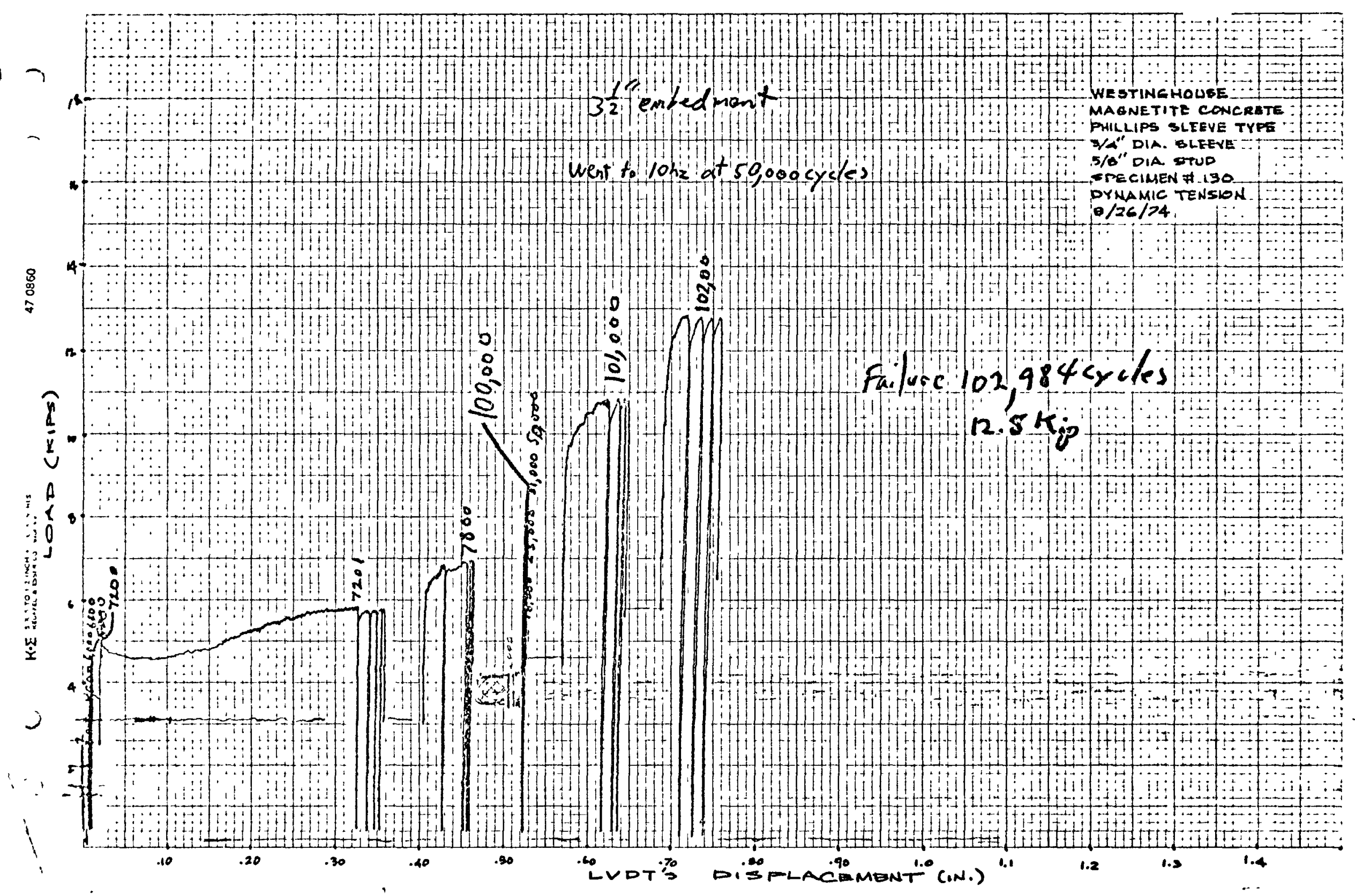




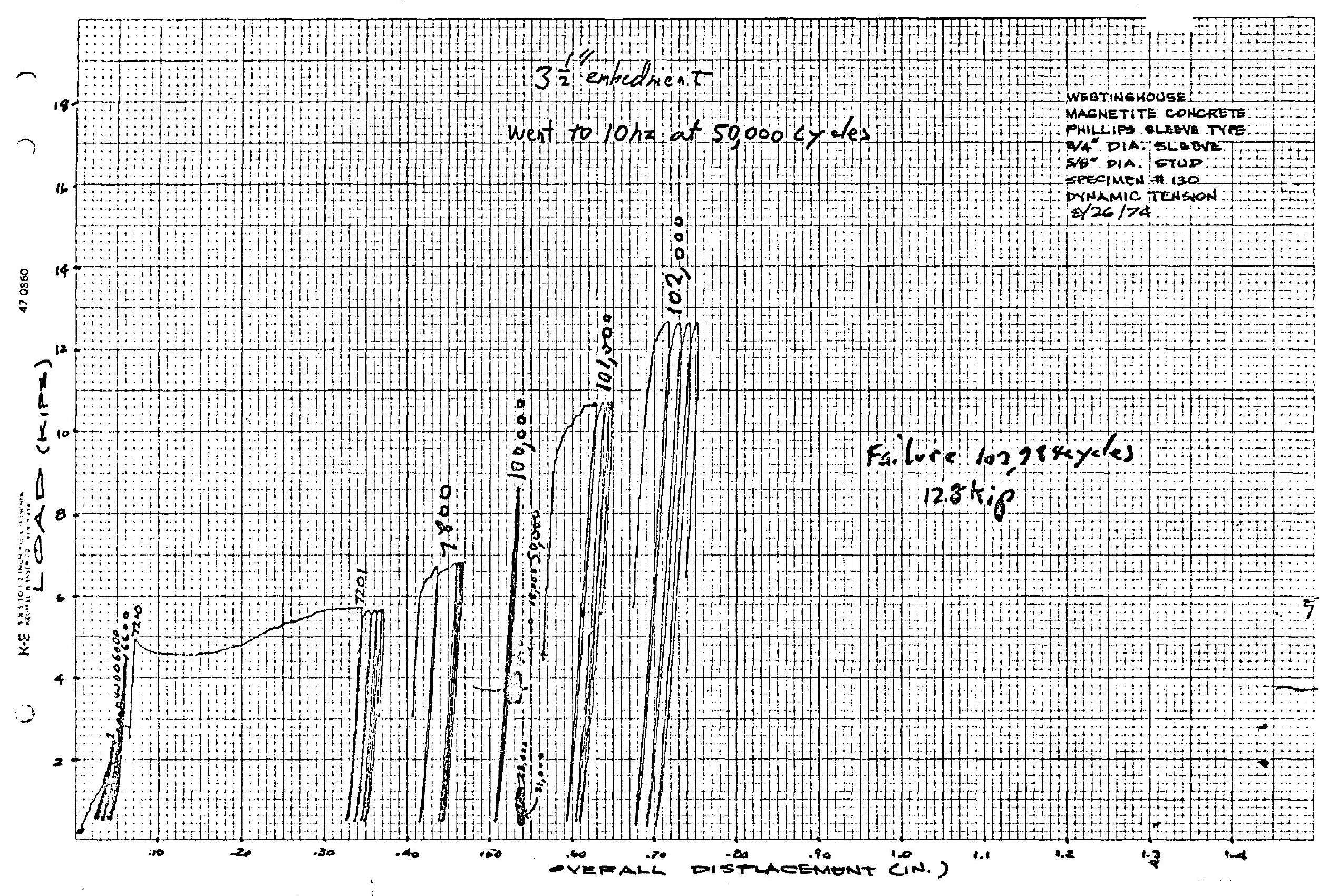




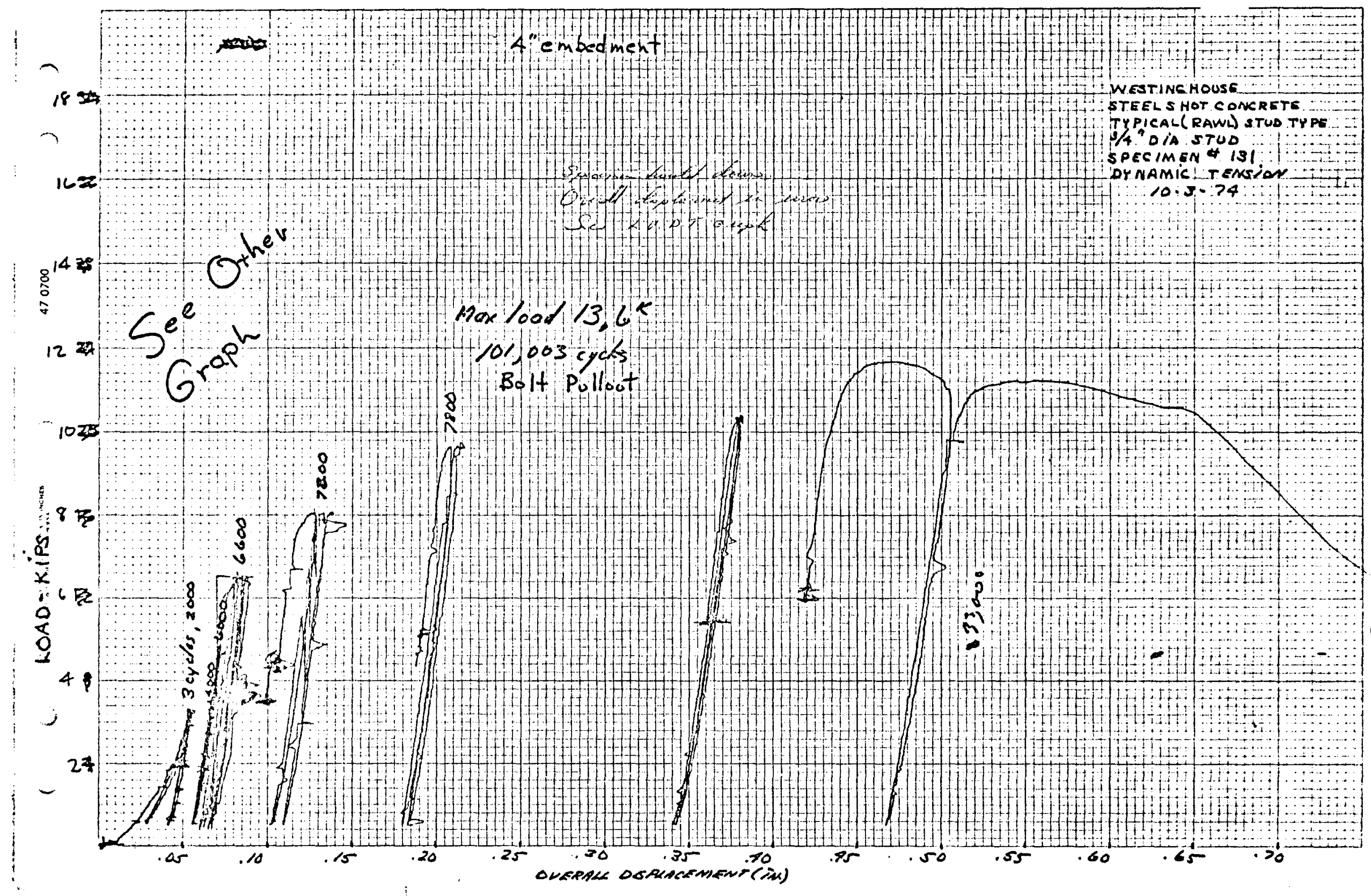




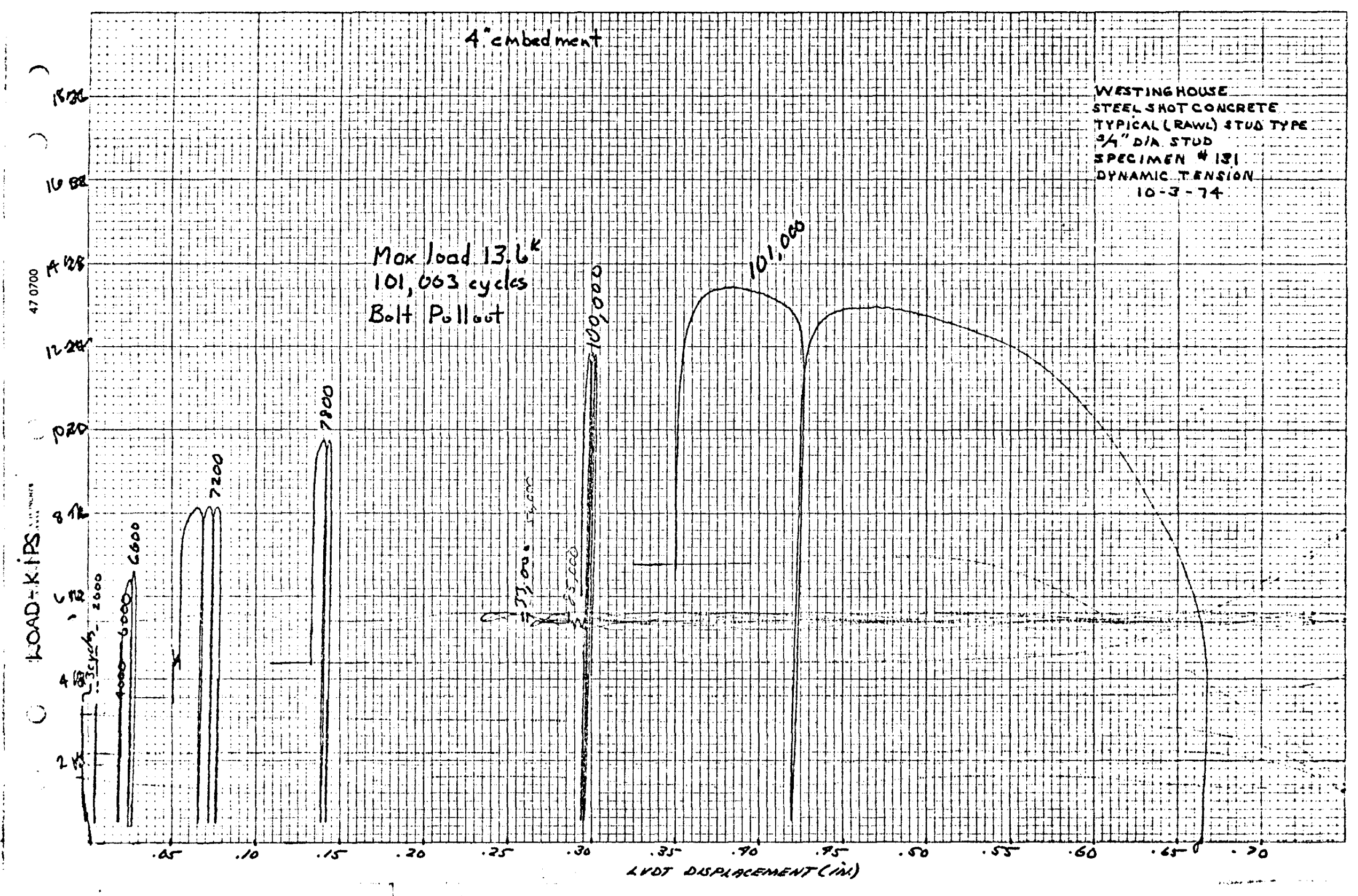




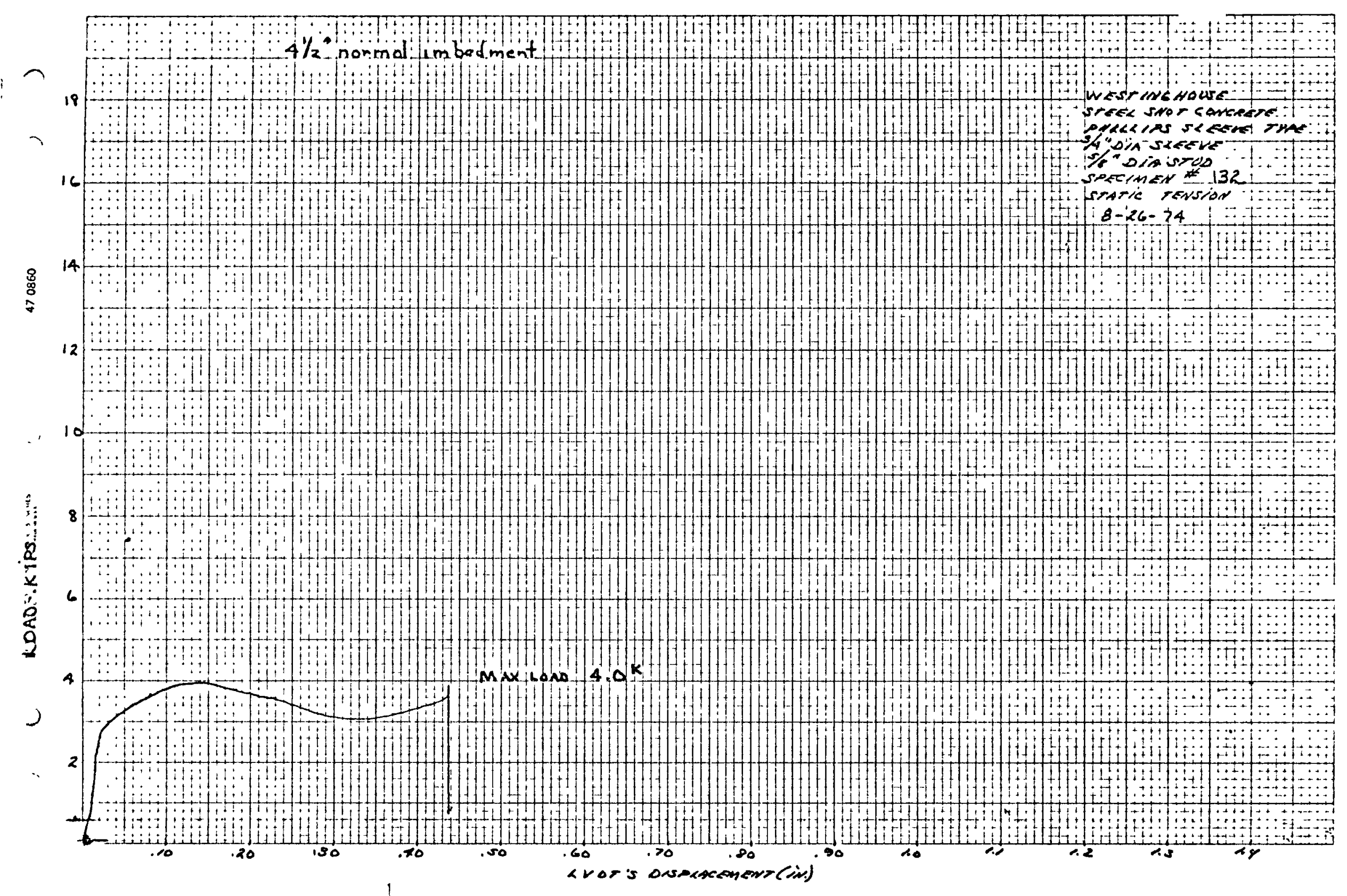




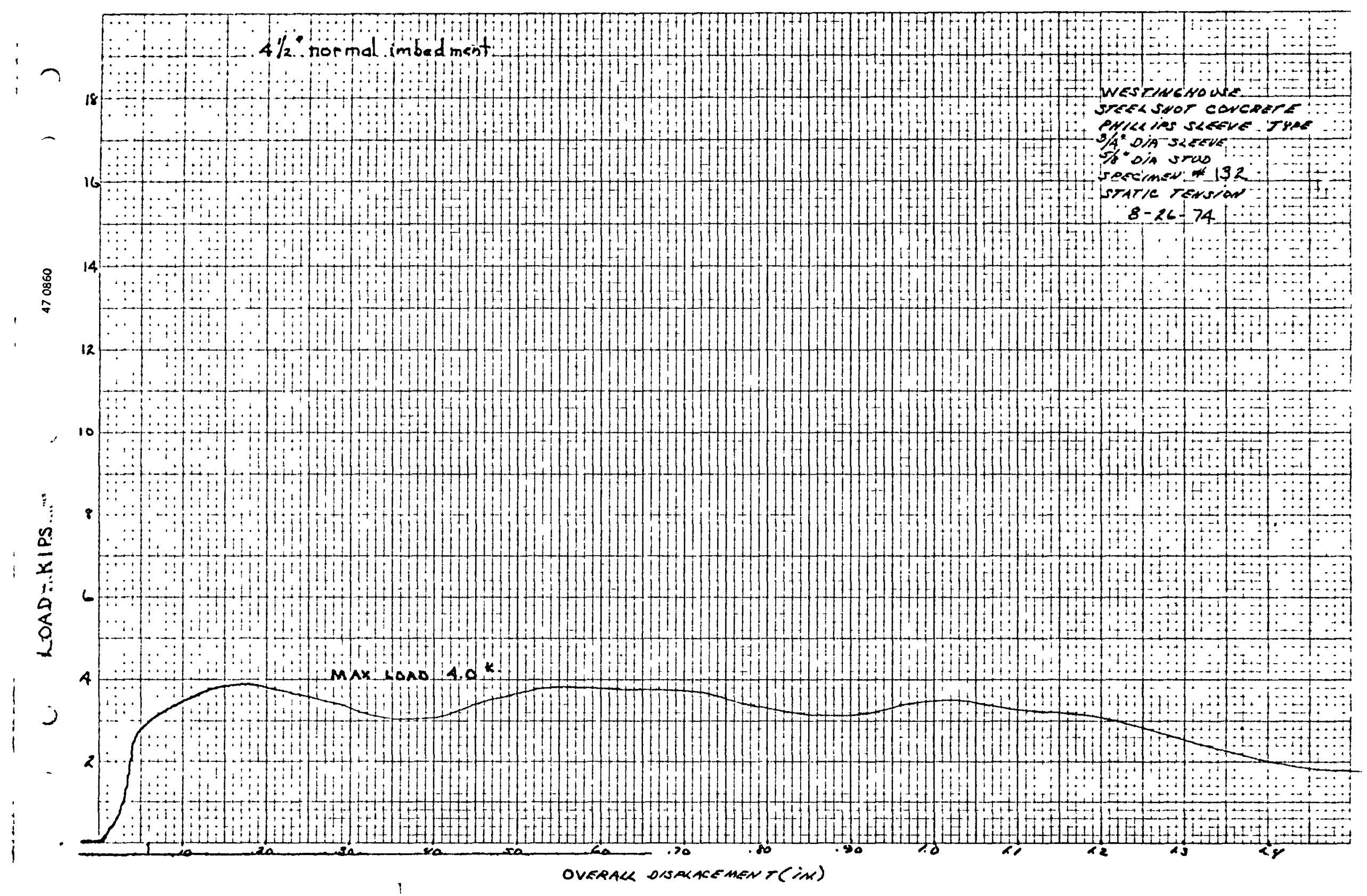




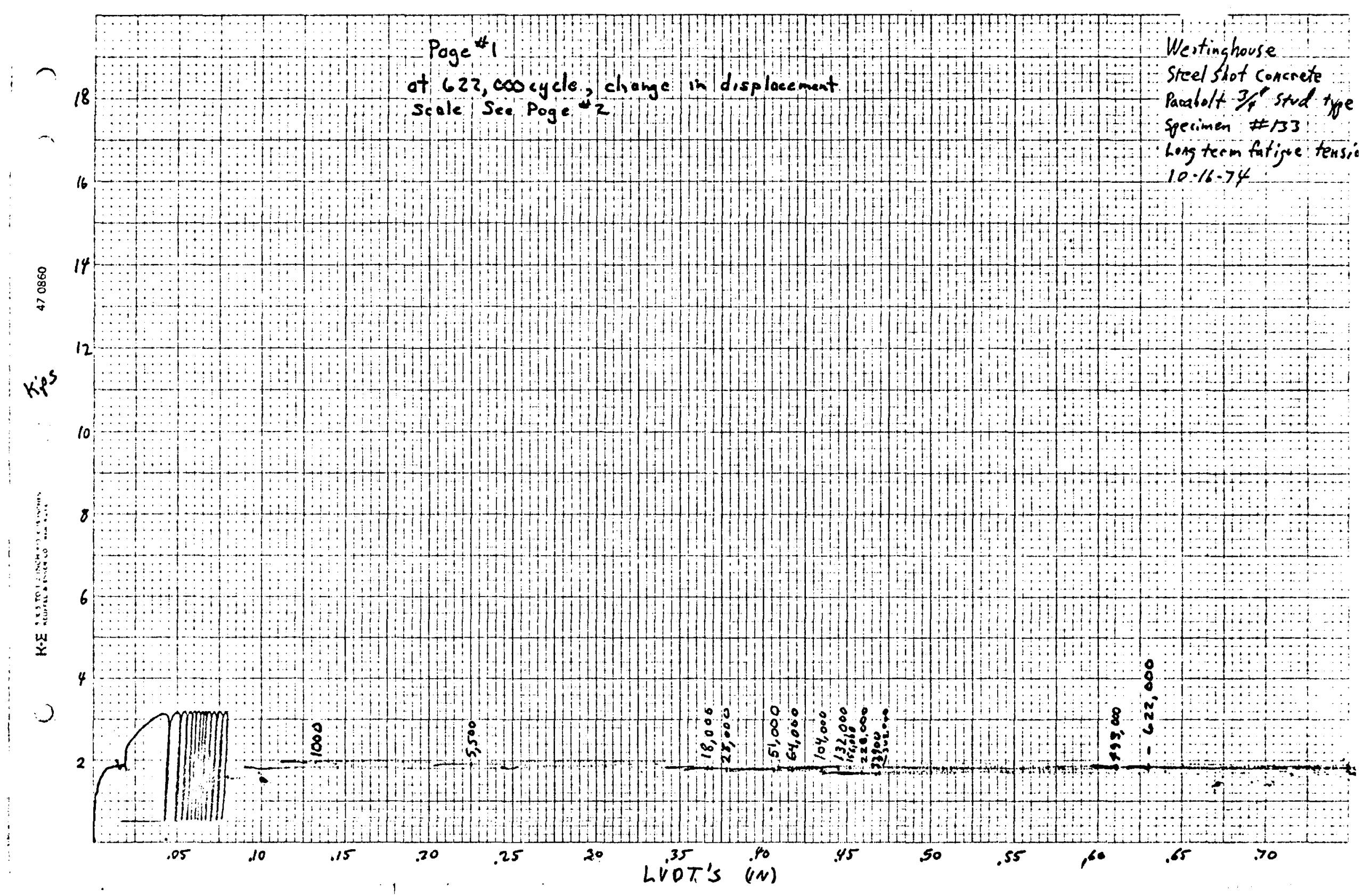




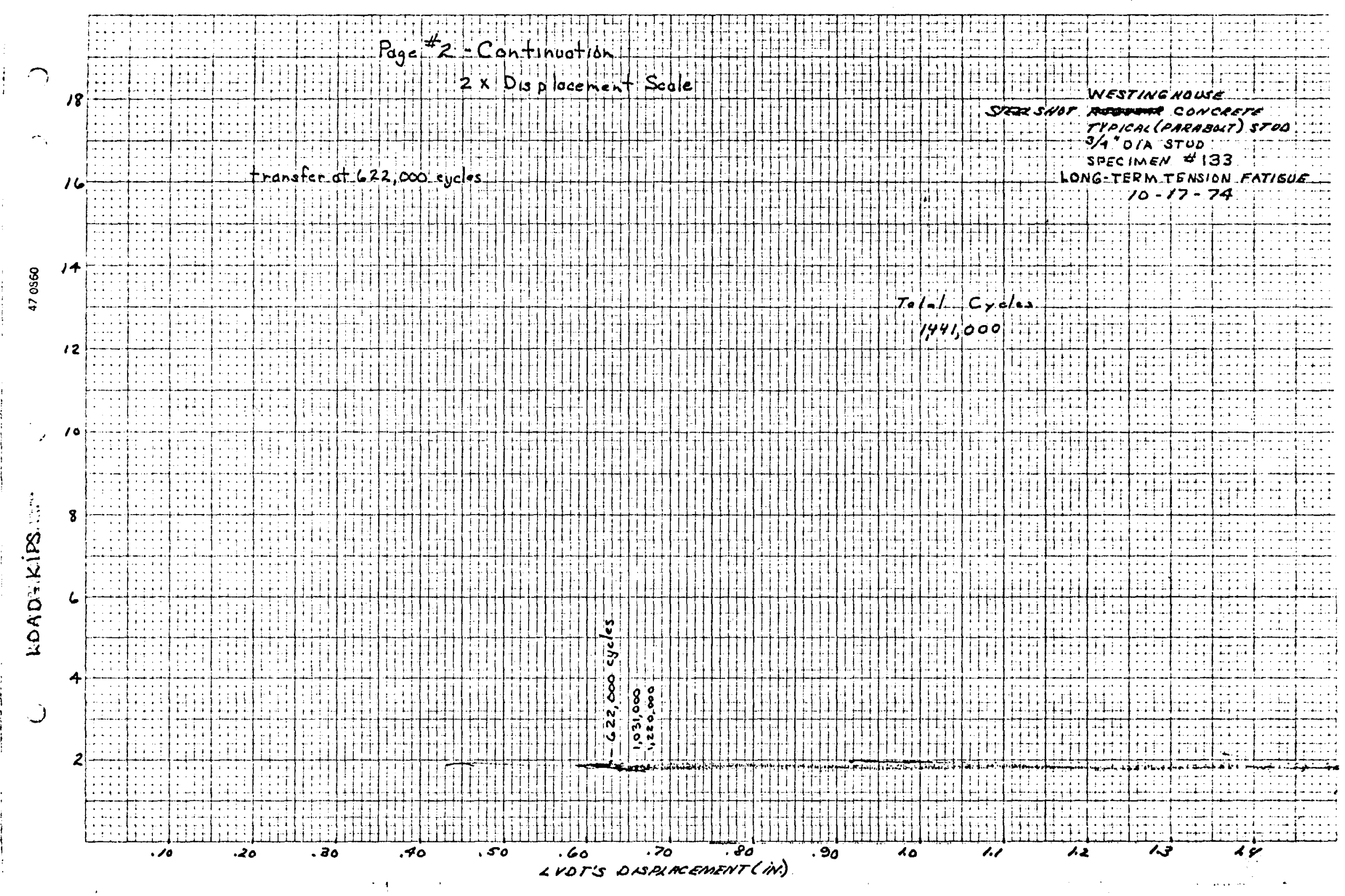




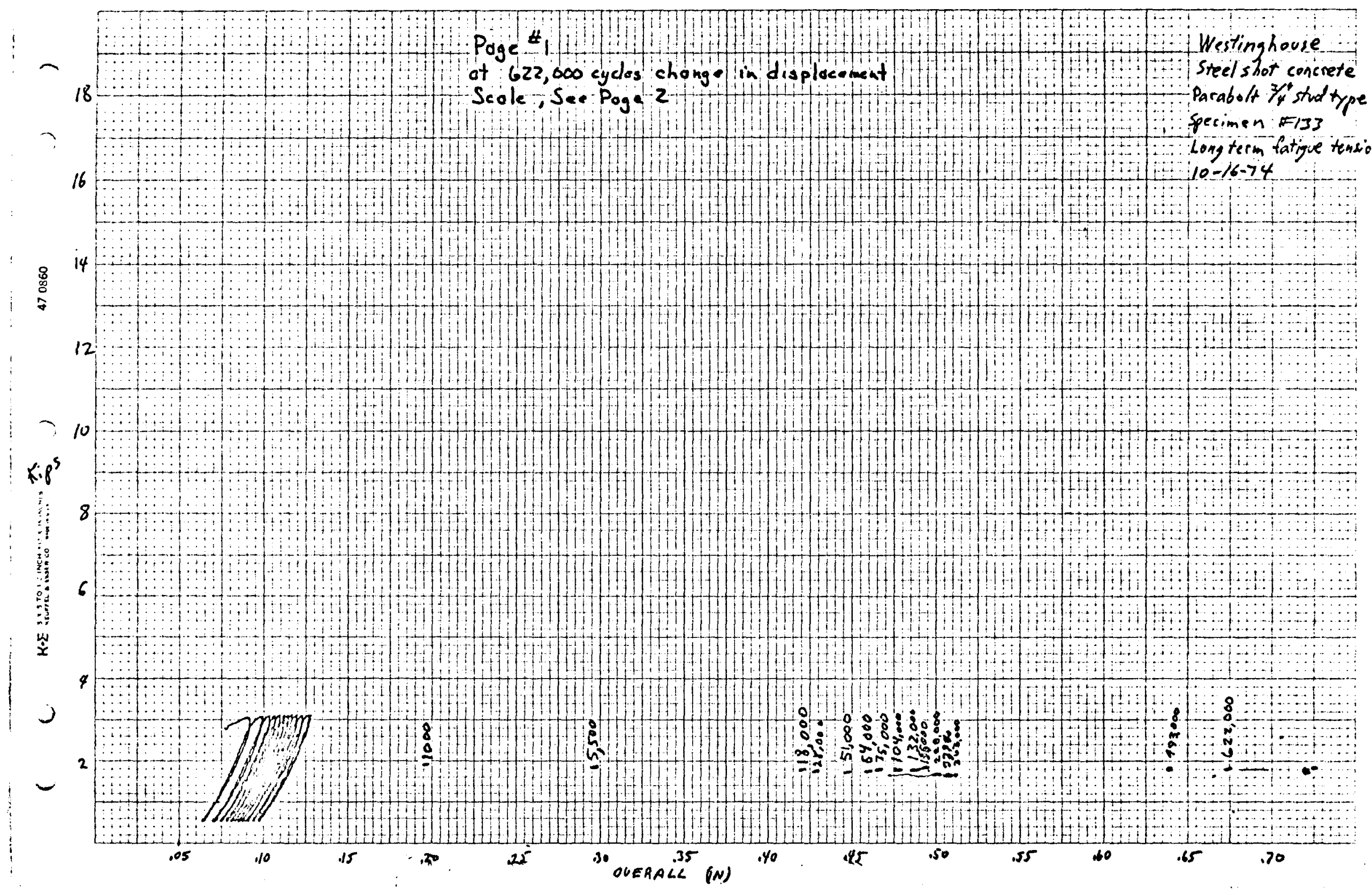




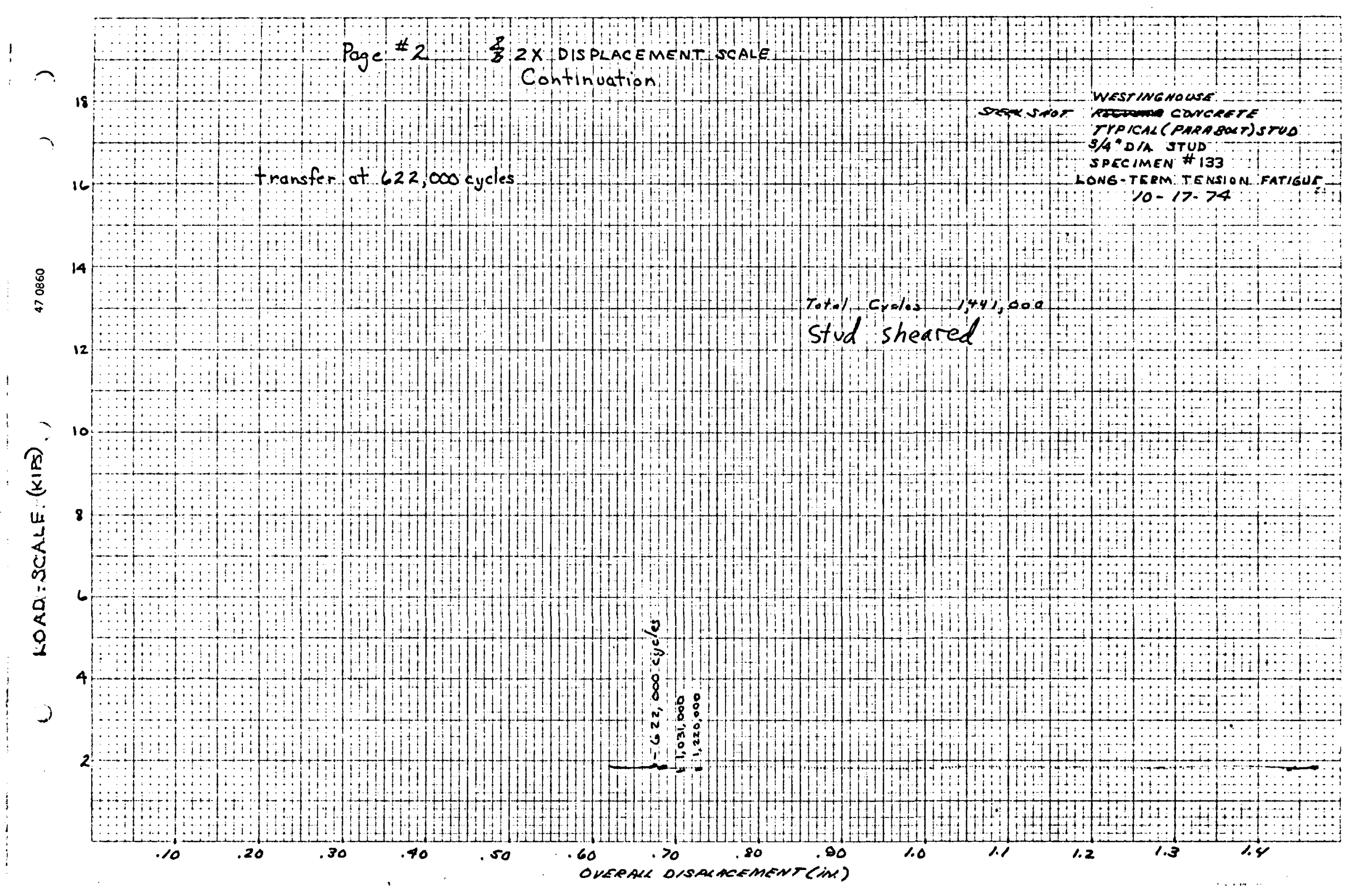




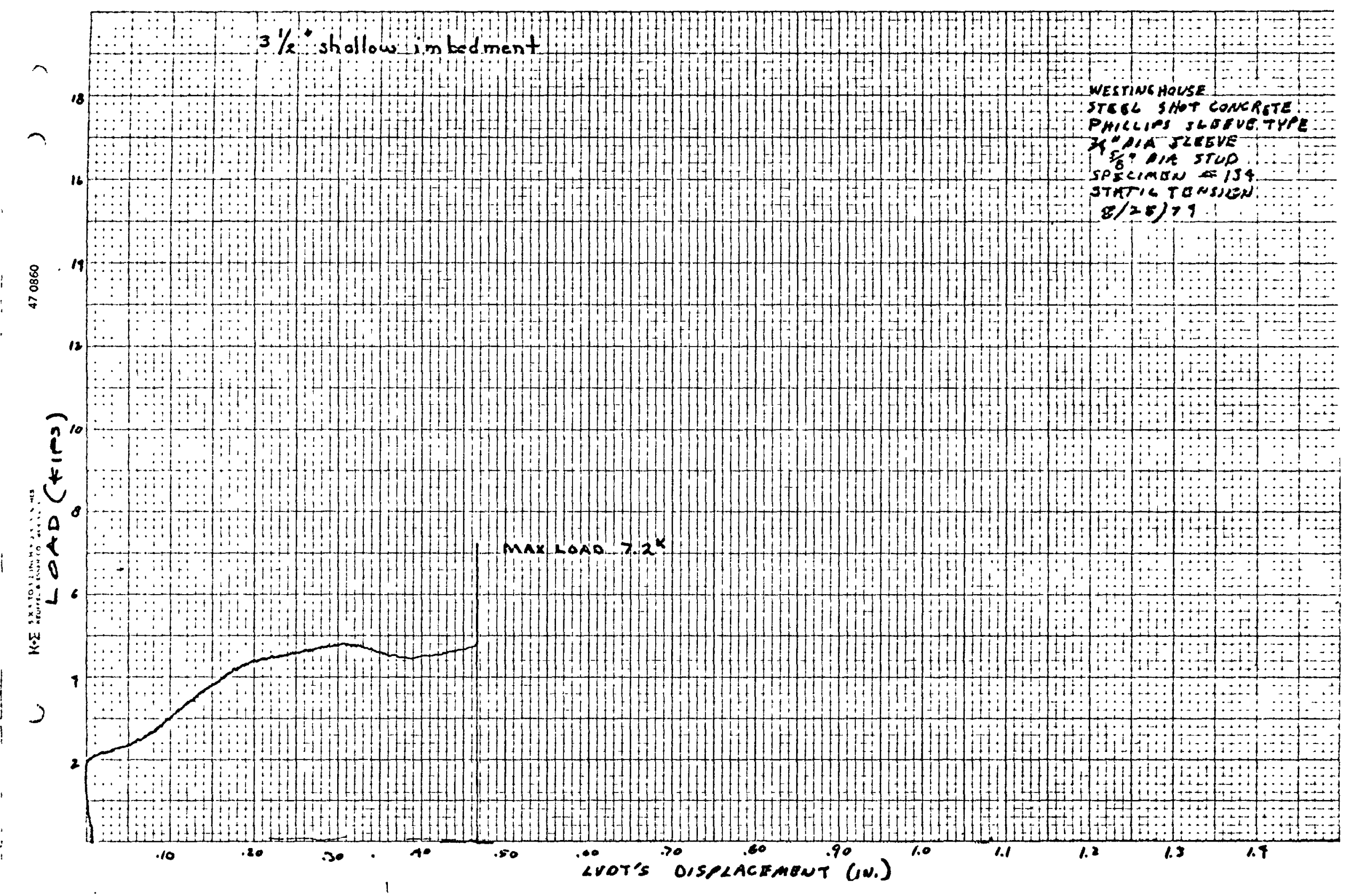




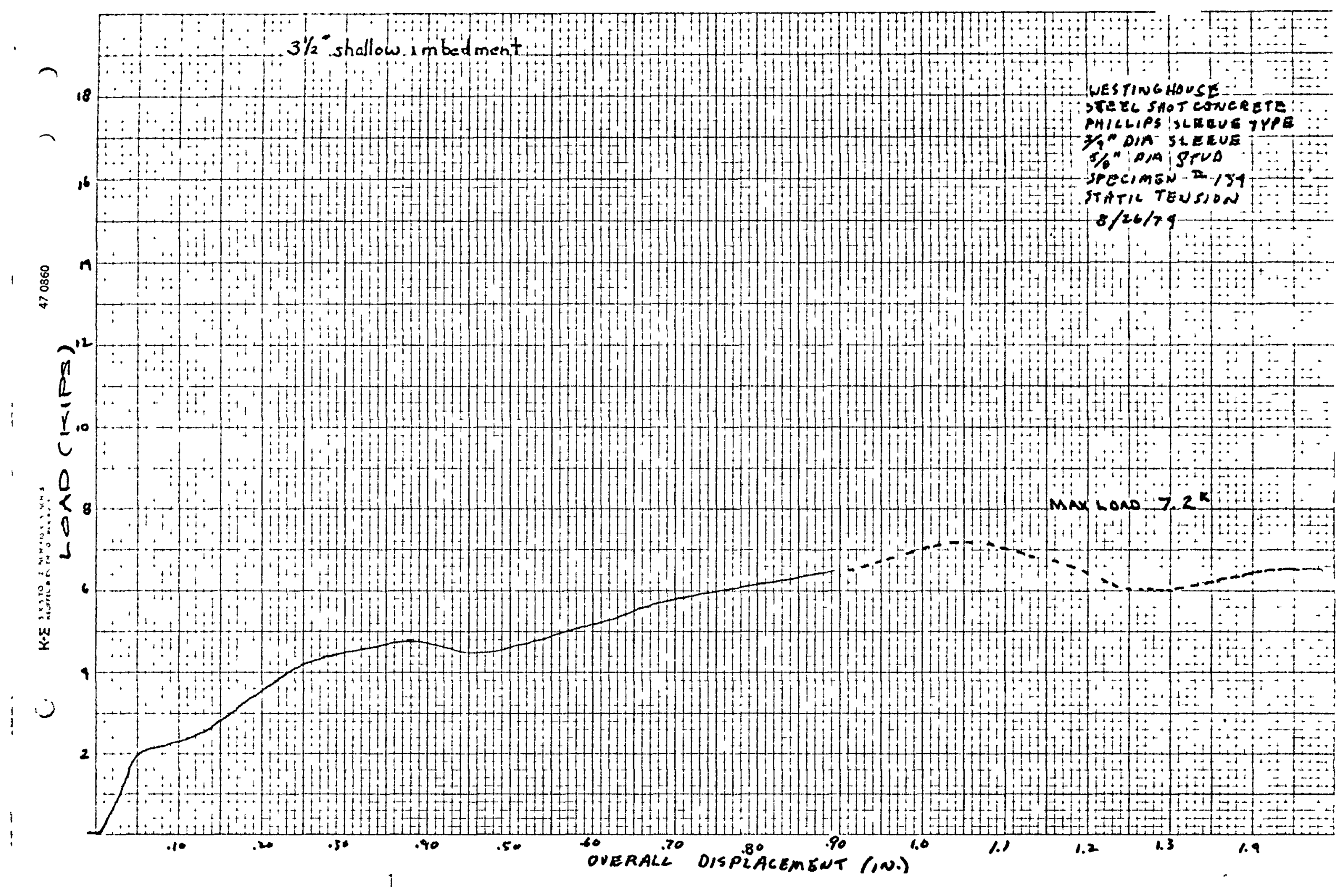




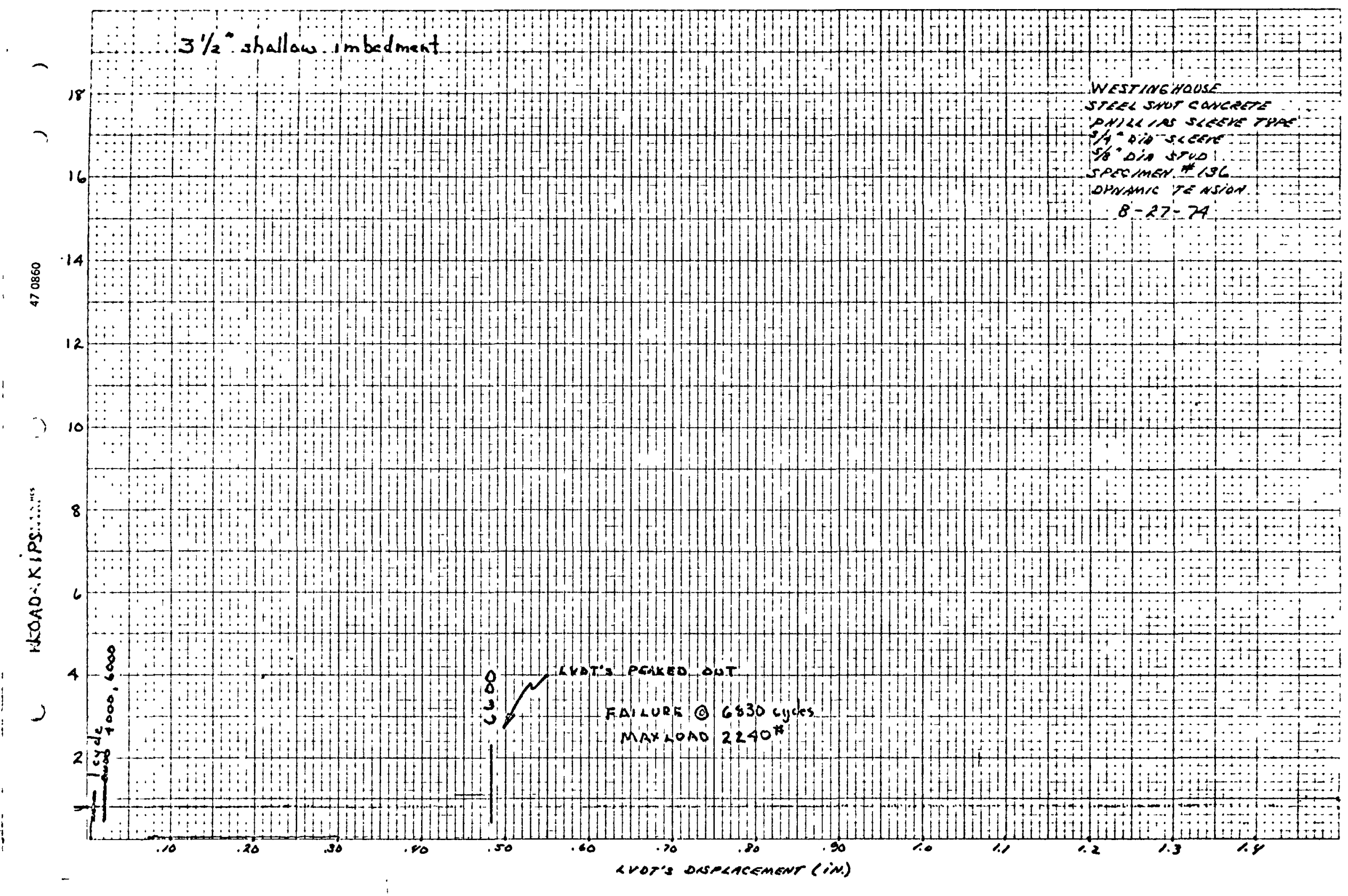




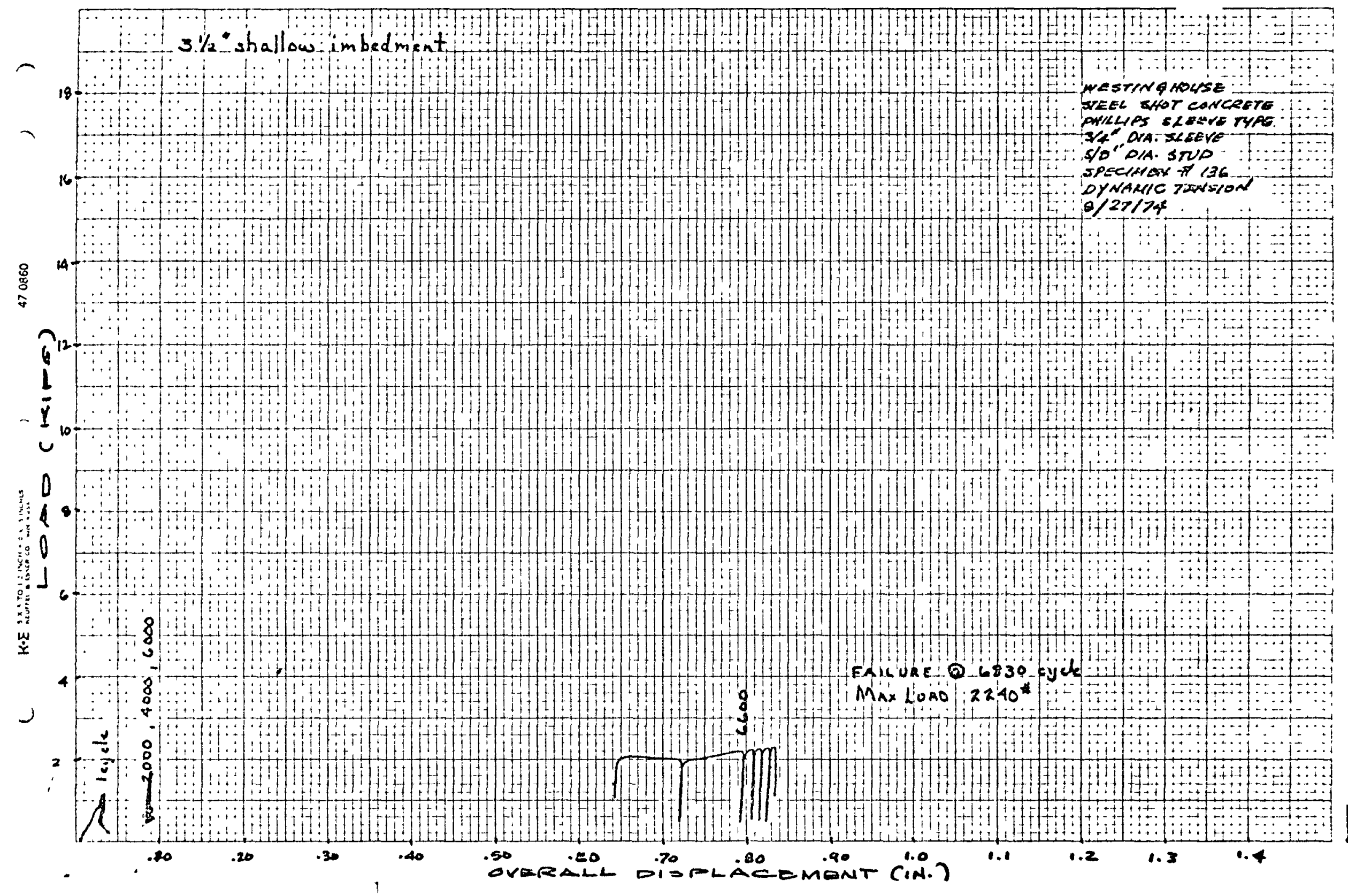




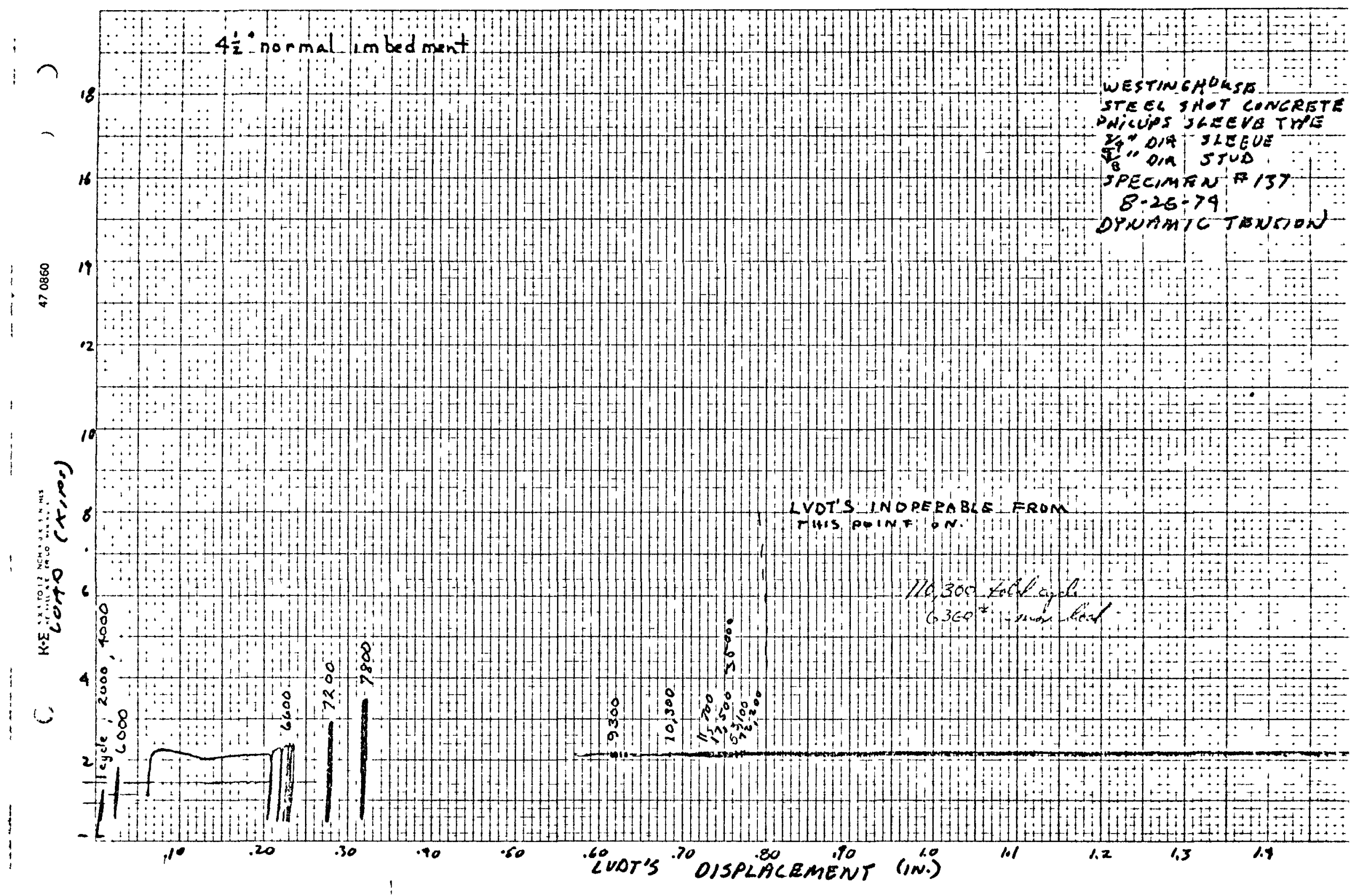




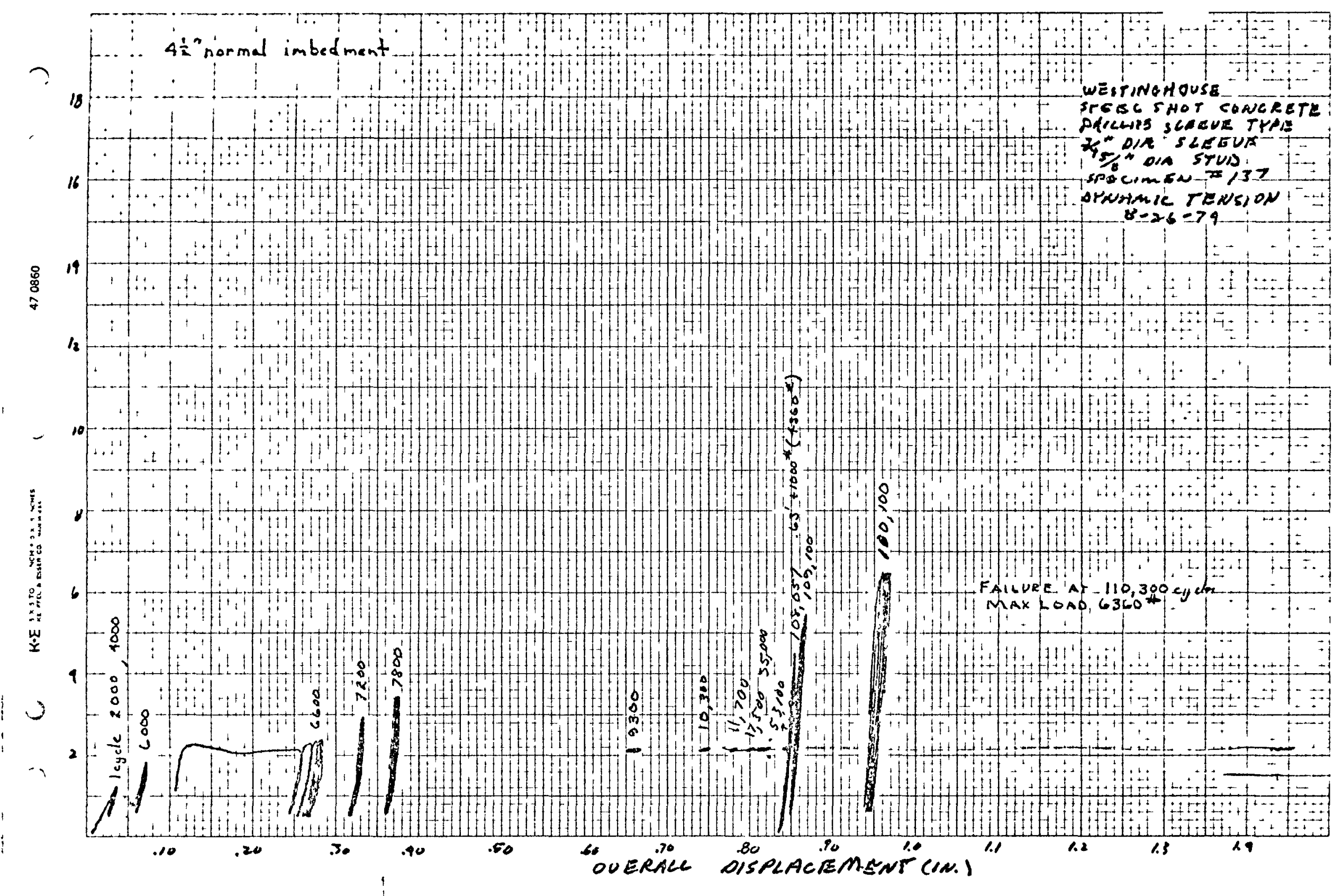




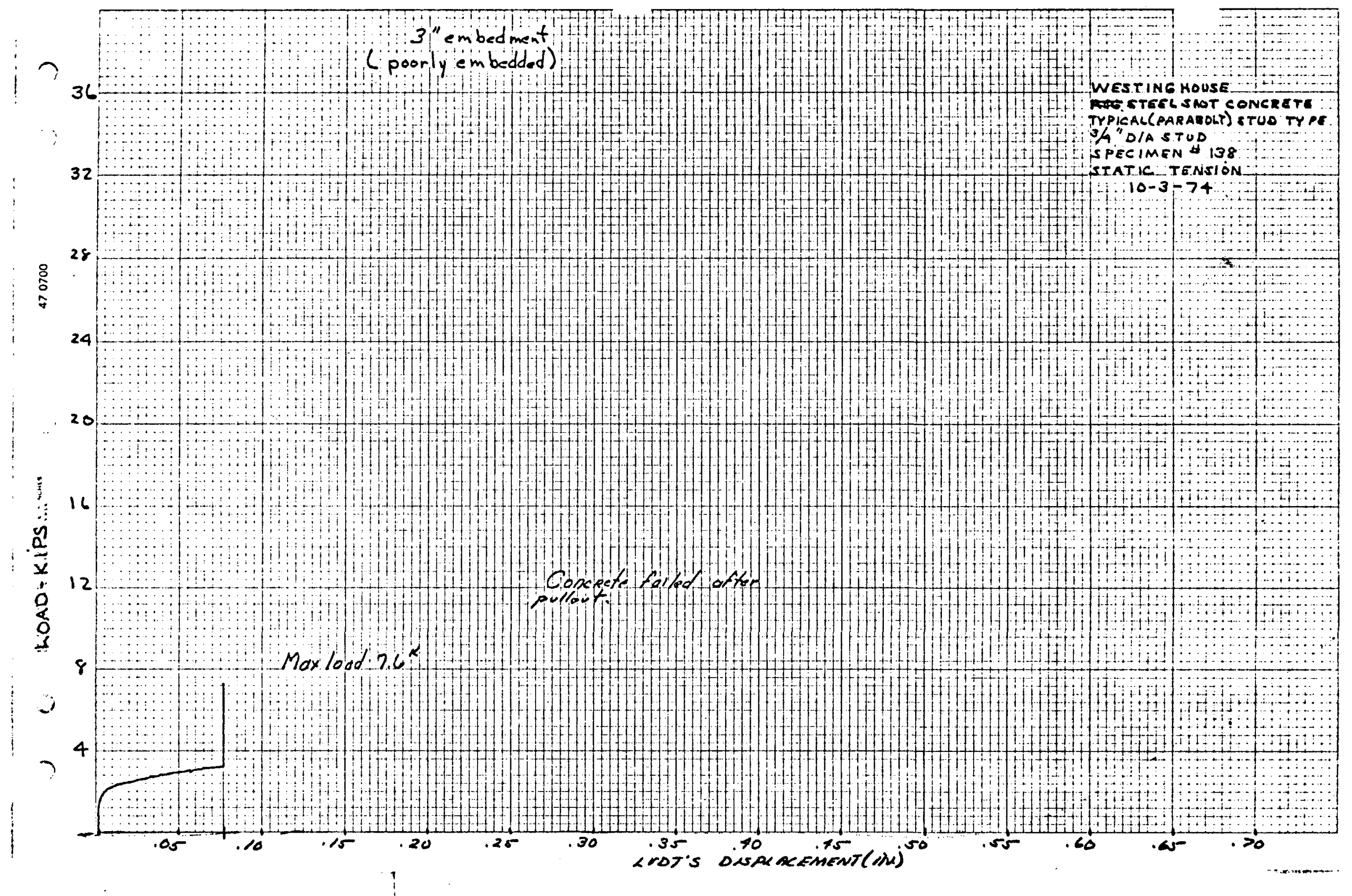




\section{-}

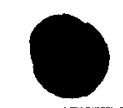

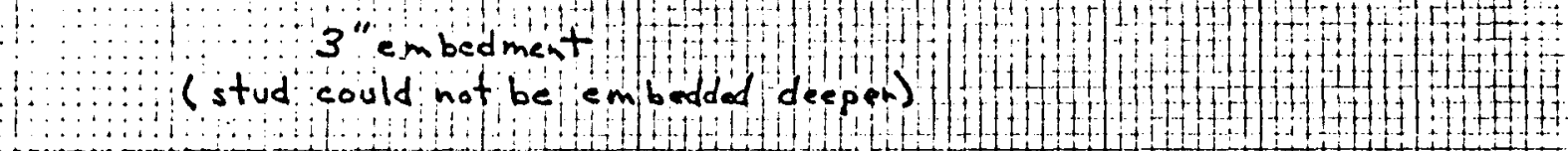

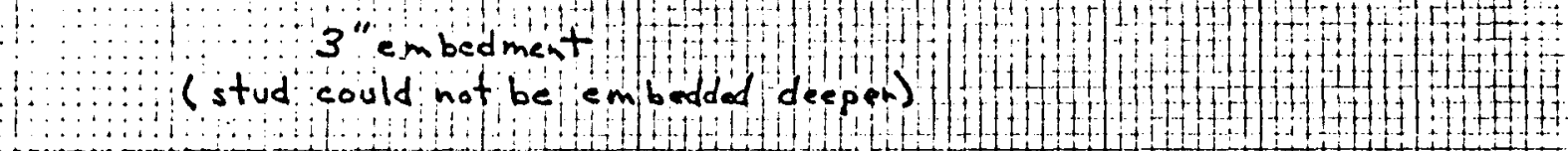

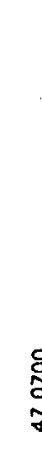

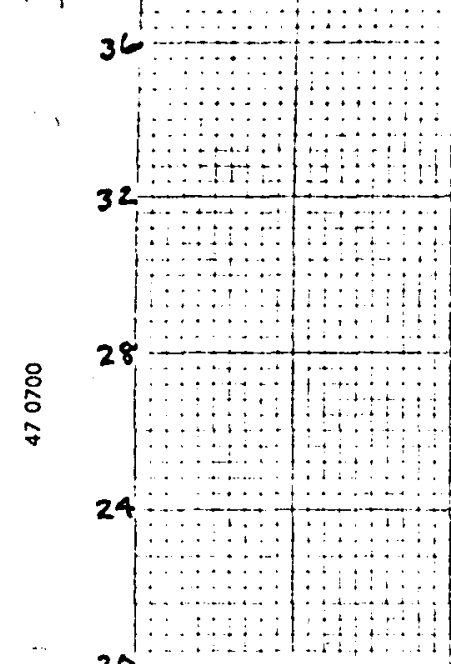

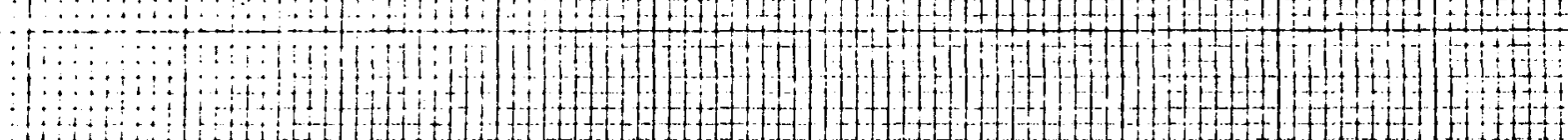

WESTINGMOUSE

STEEL SMOT CONCRETE:

TYPICAL ETUD TYPE LPABAEOC

3/4 DiA STUD

IPECIMEN \# 138

STATIC TENSION

$10-3-74$

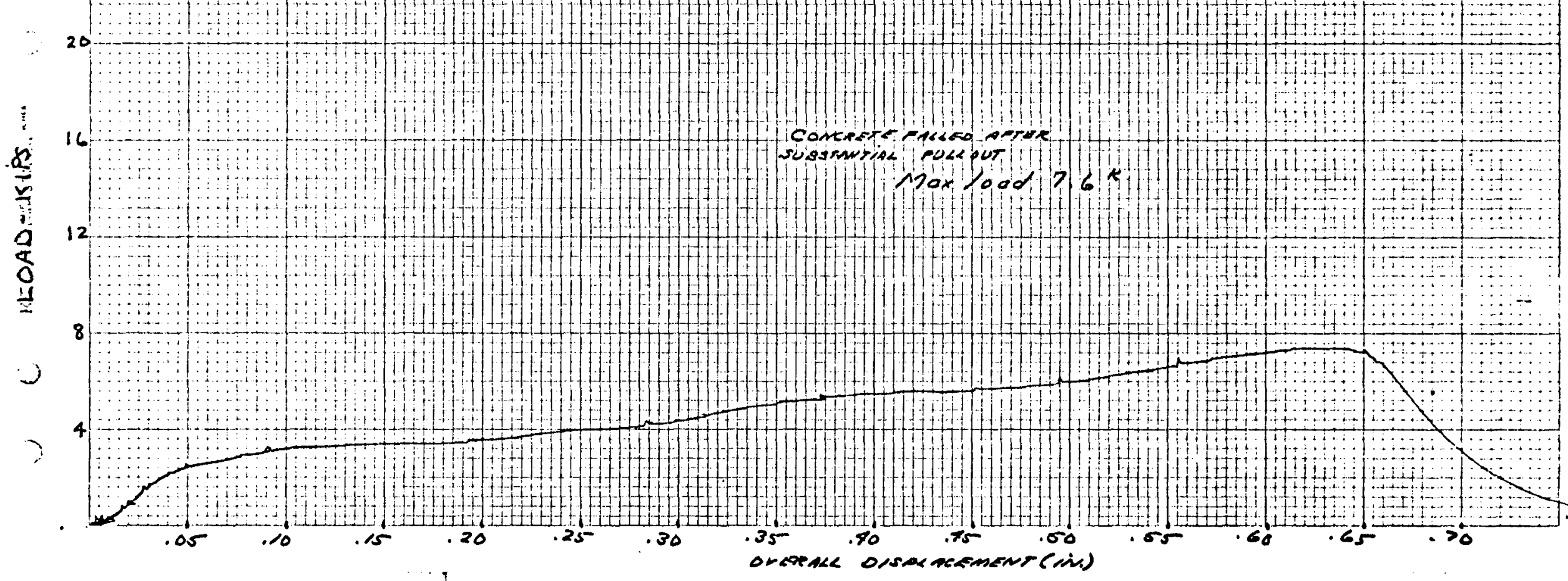




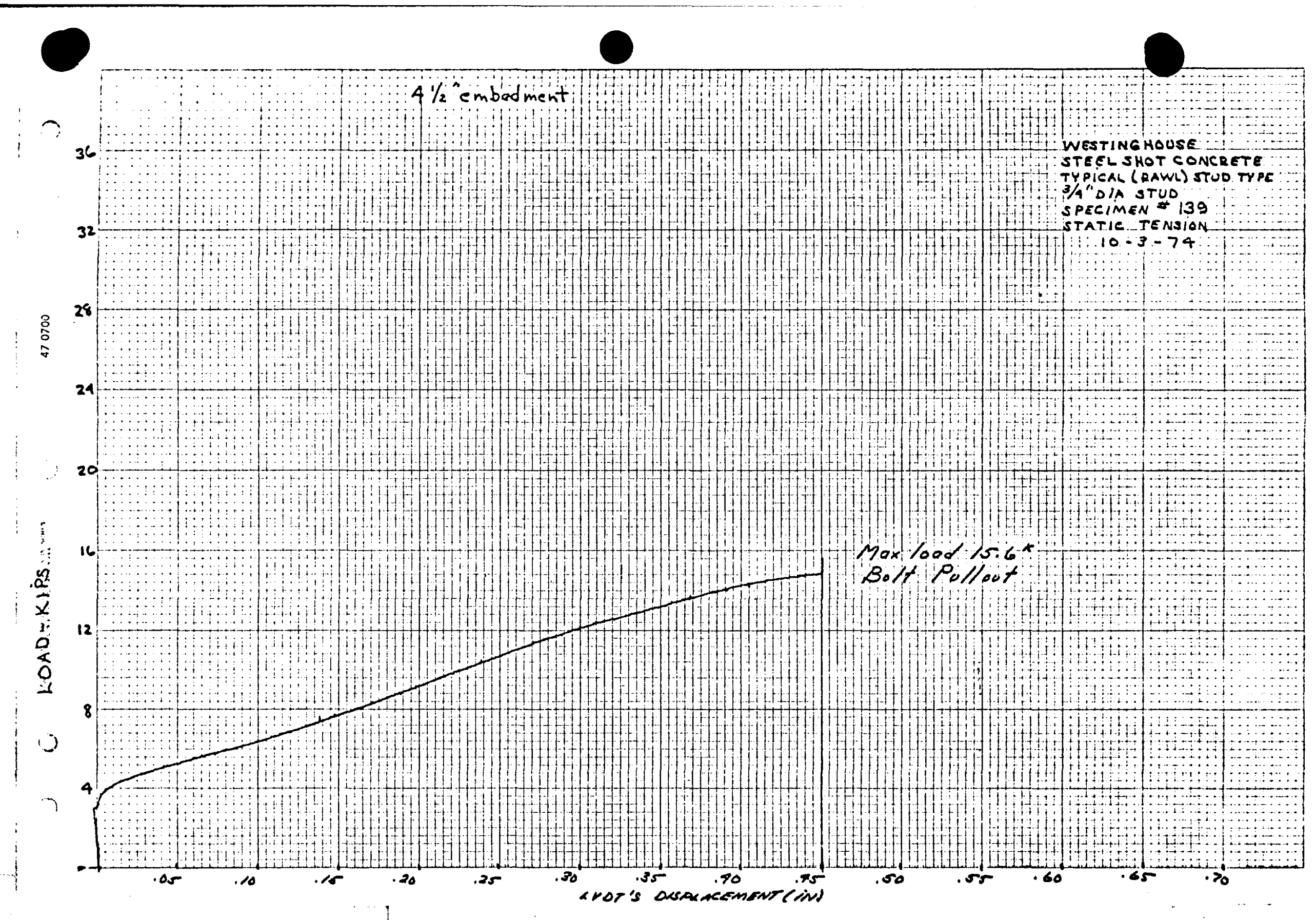




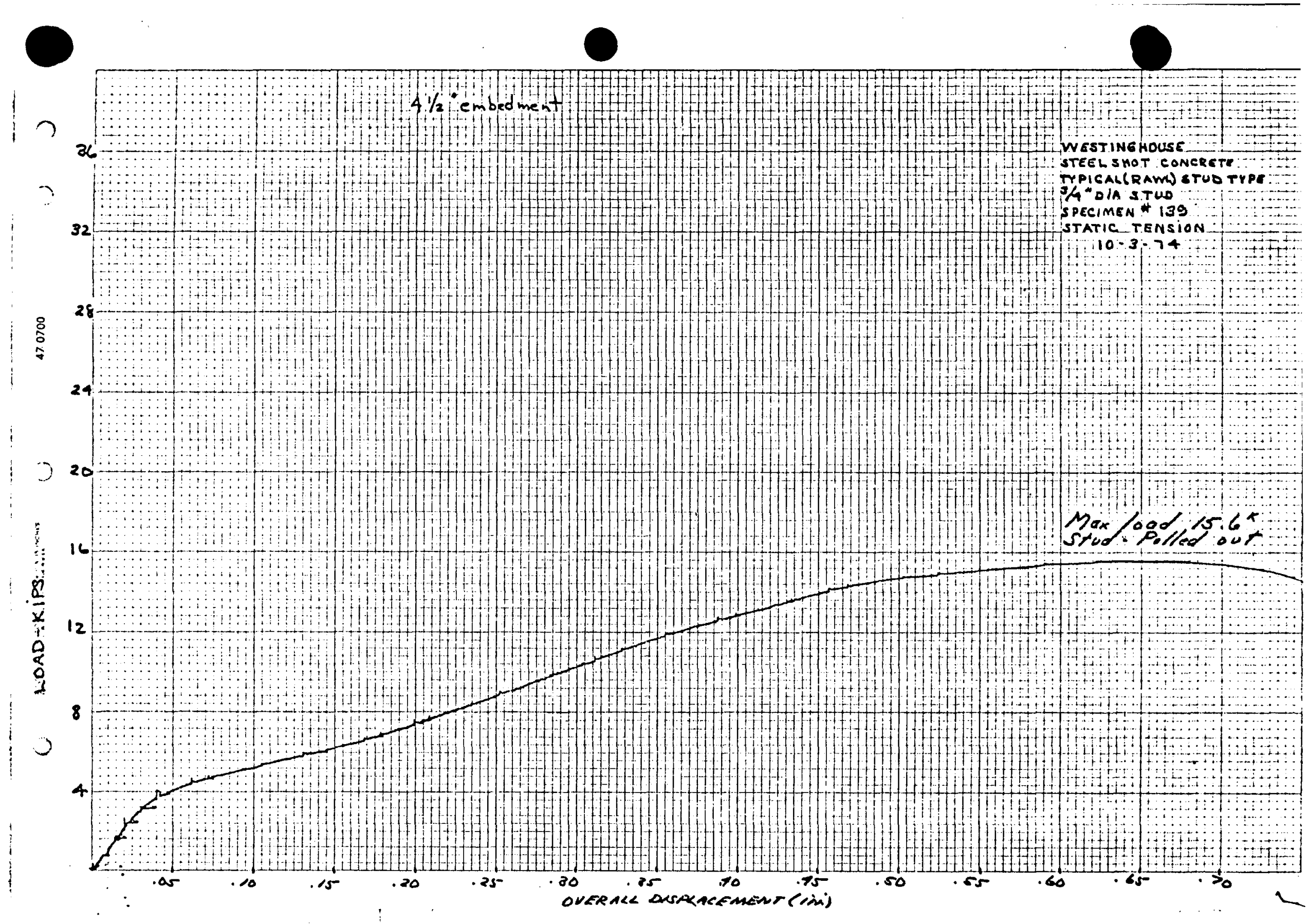




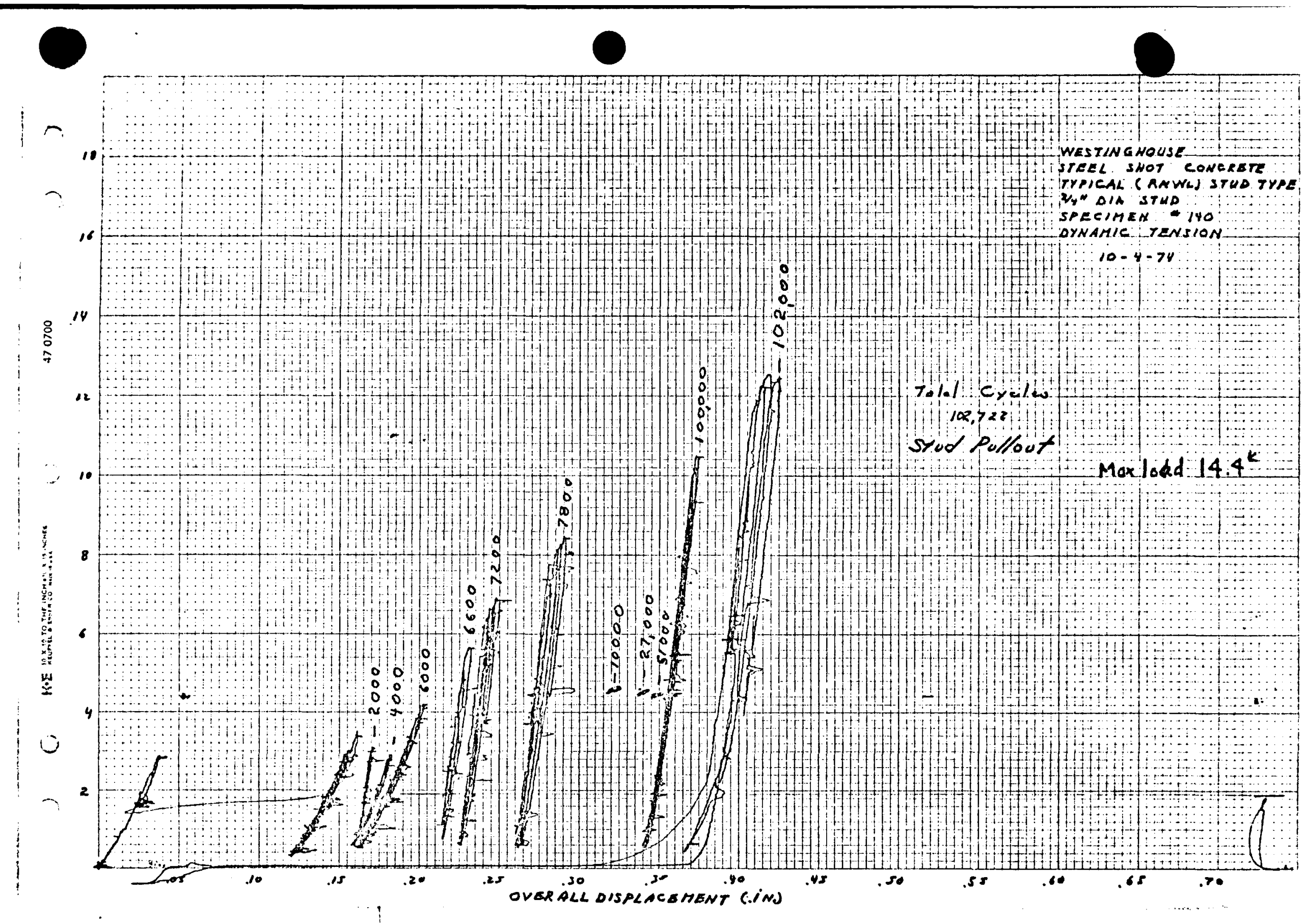




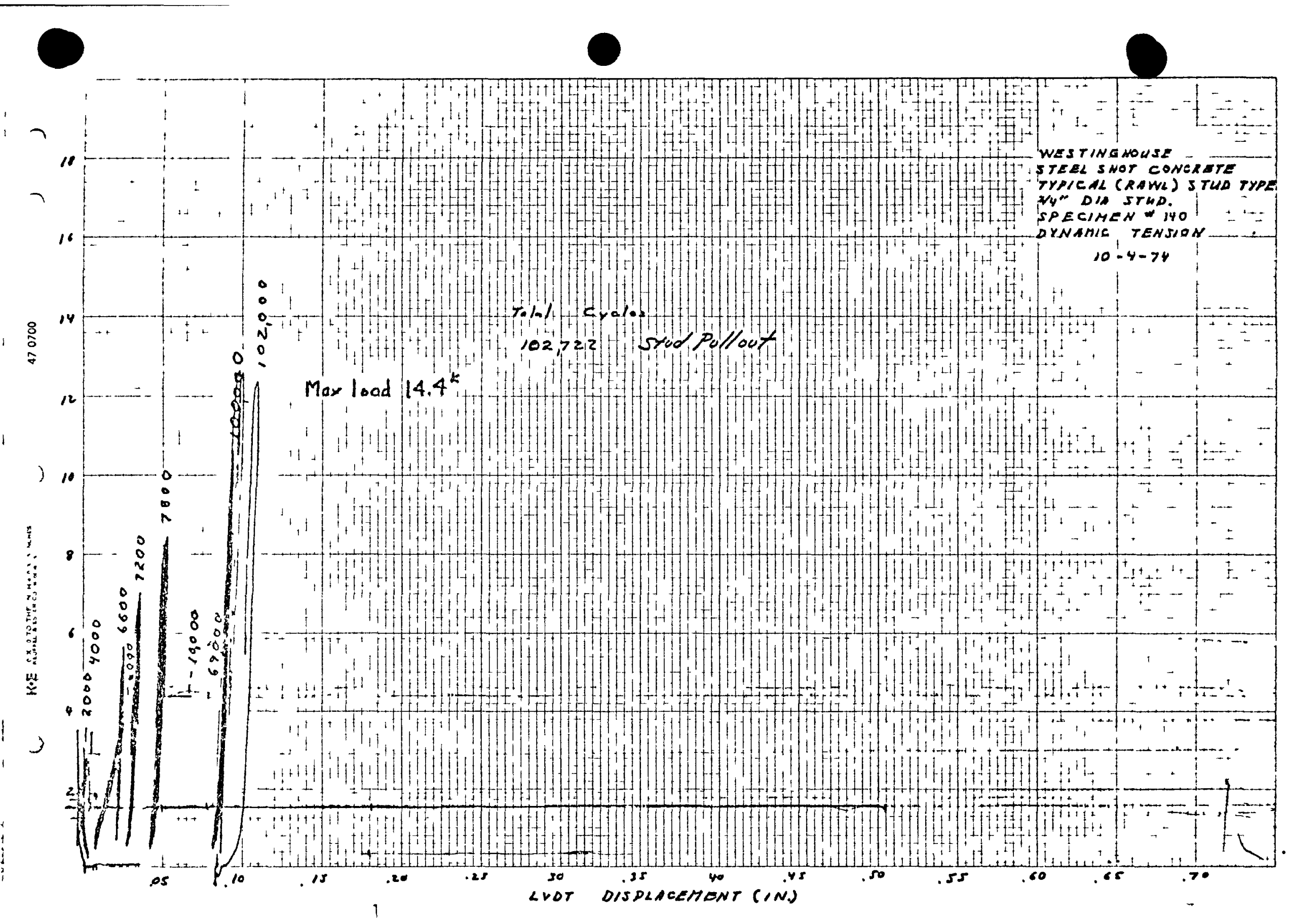




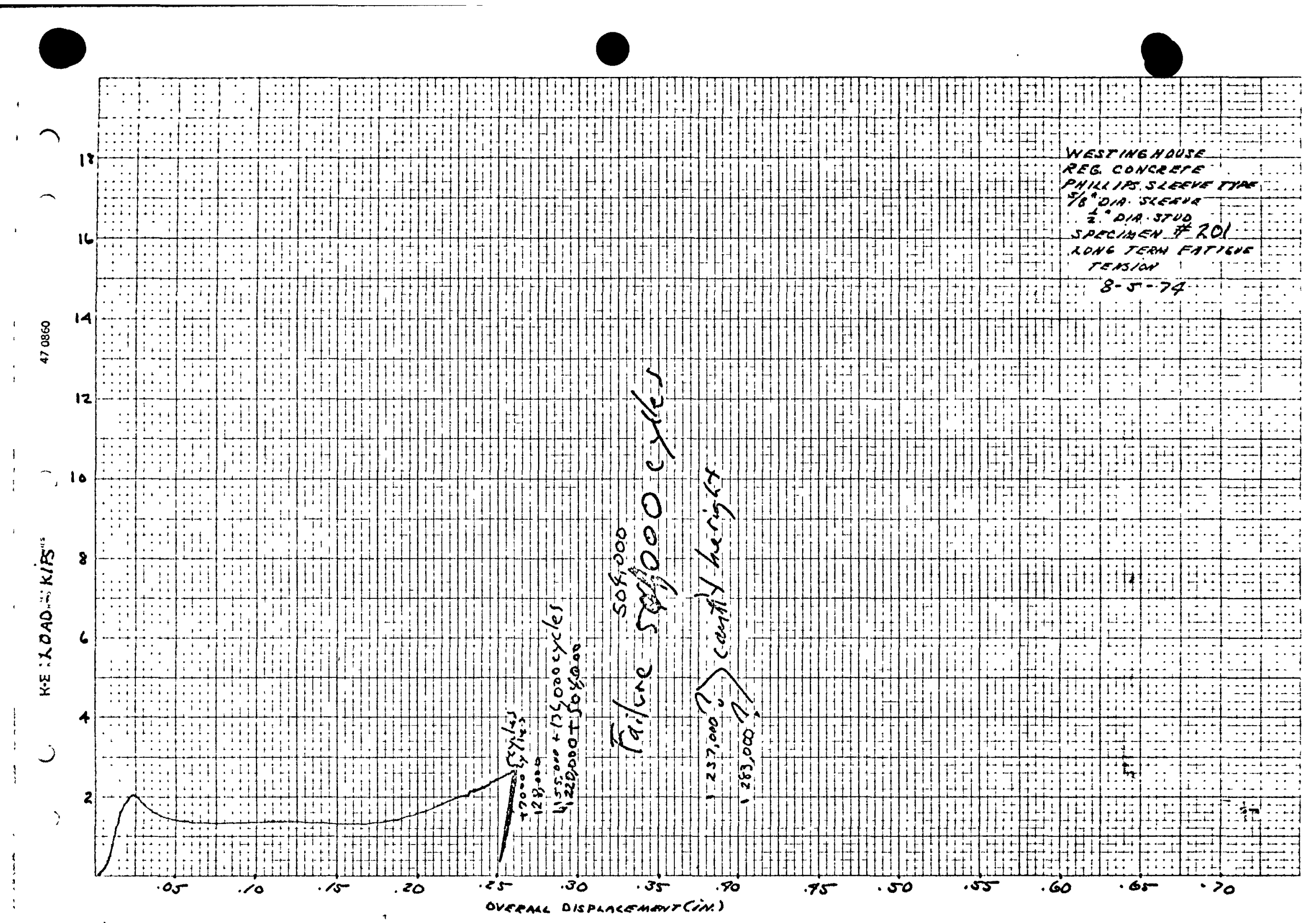




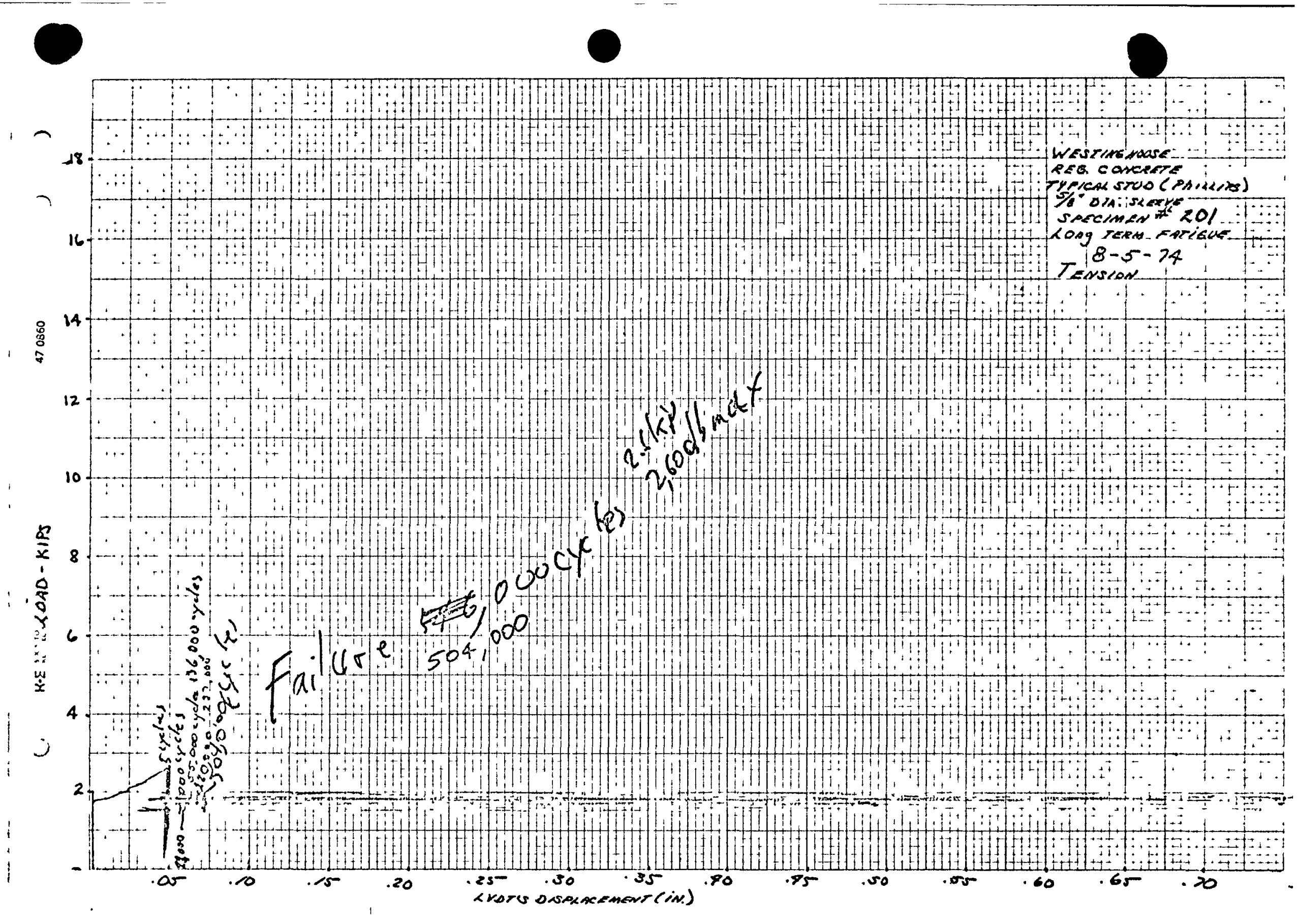




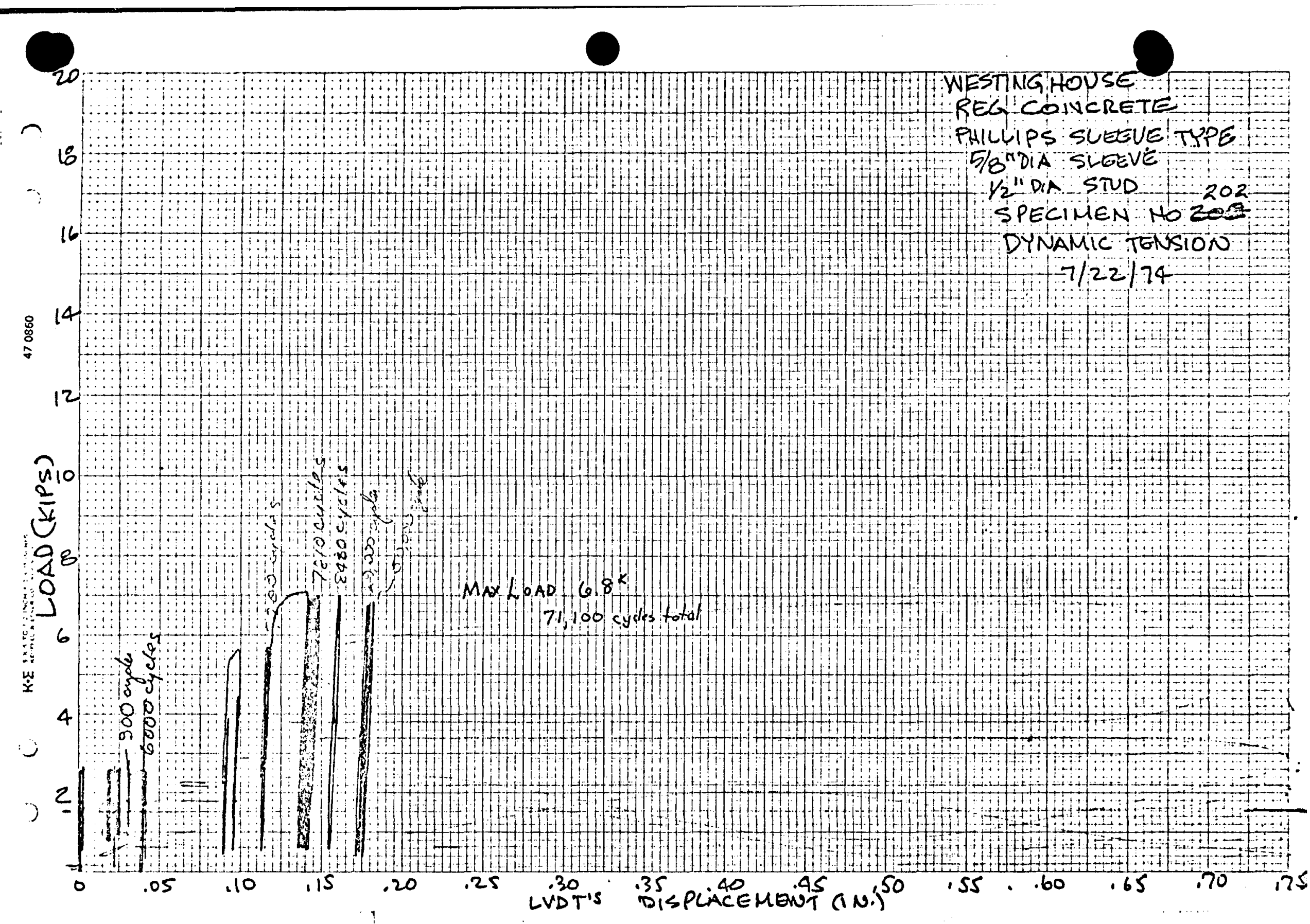




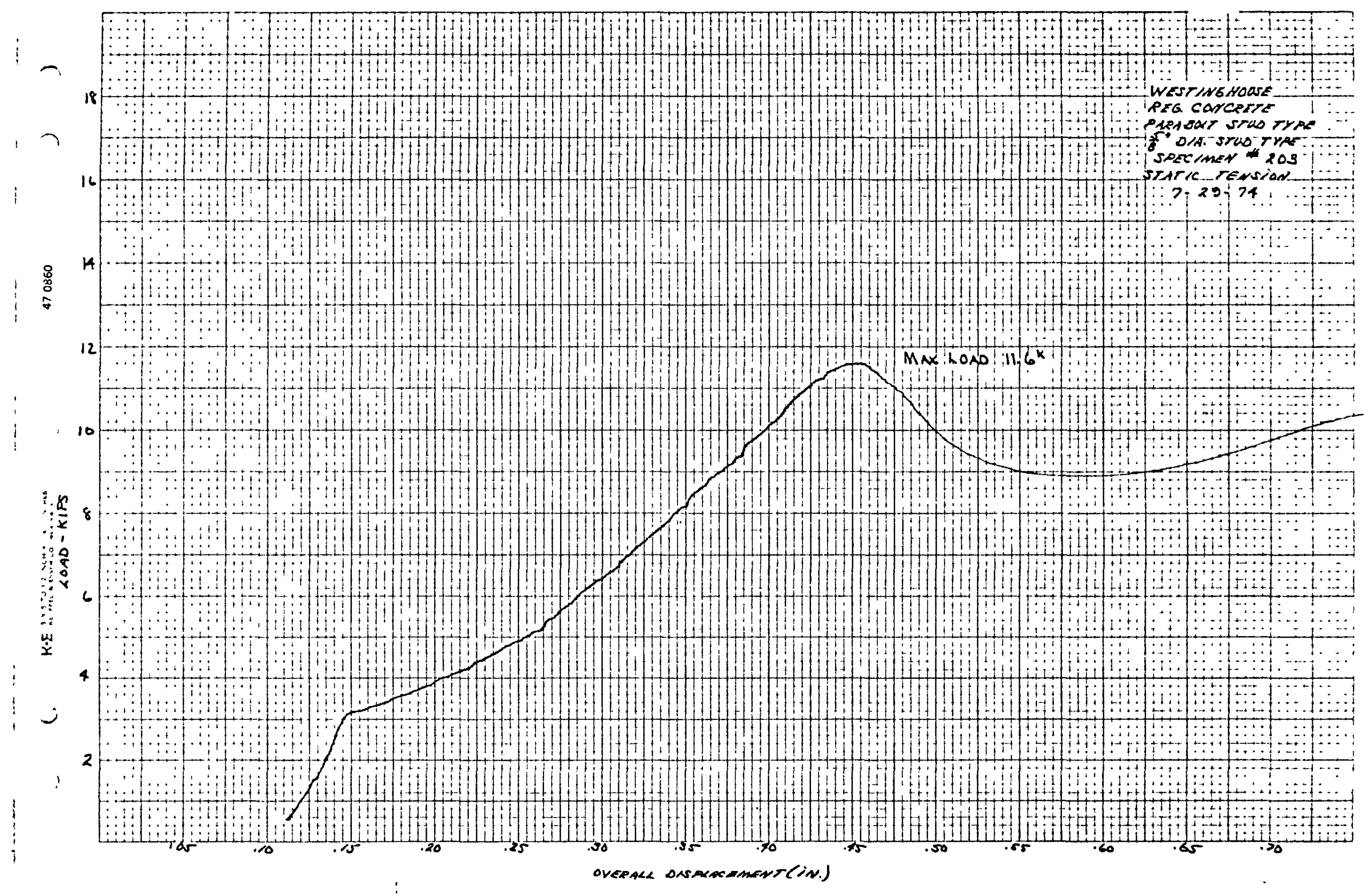




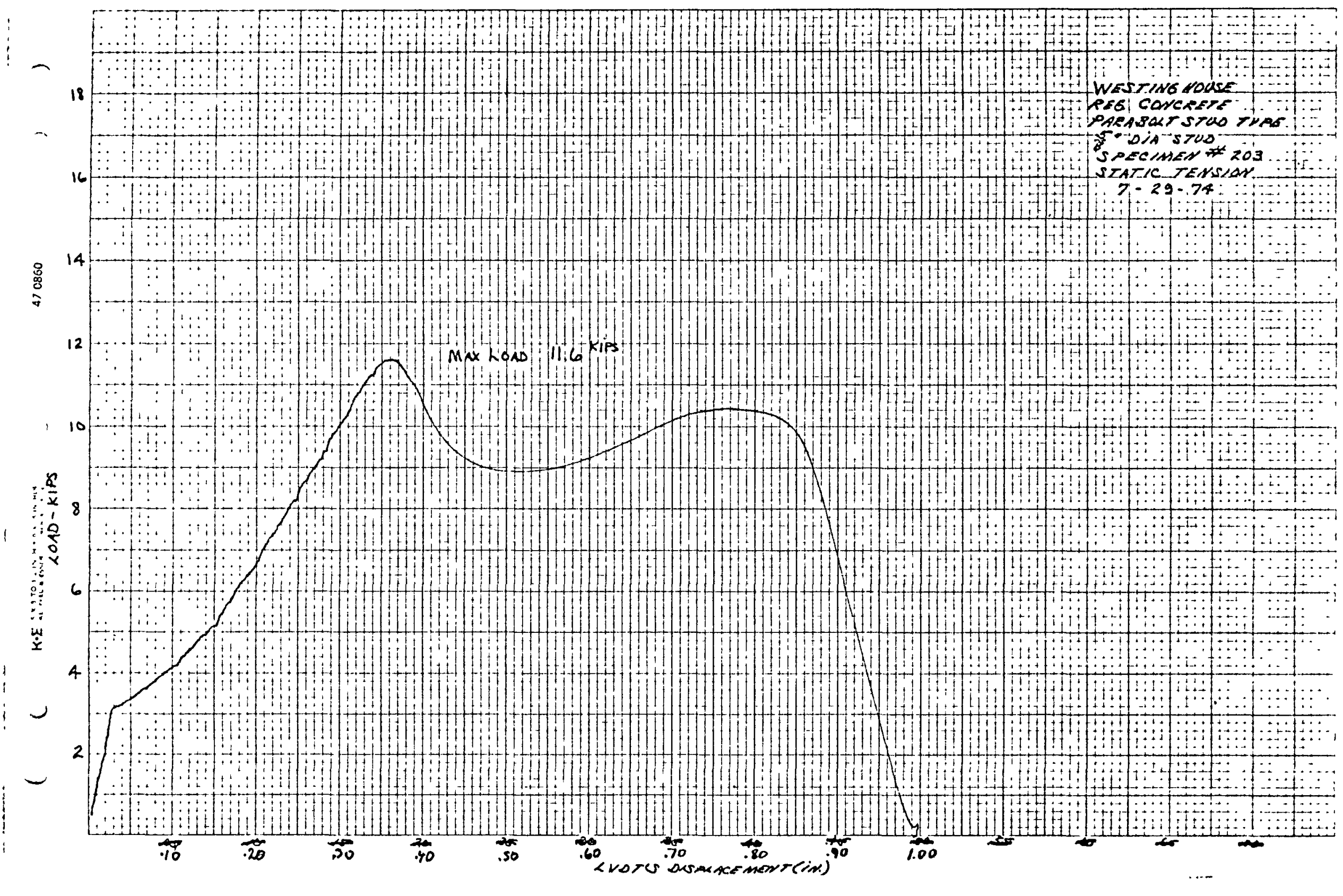




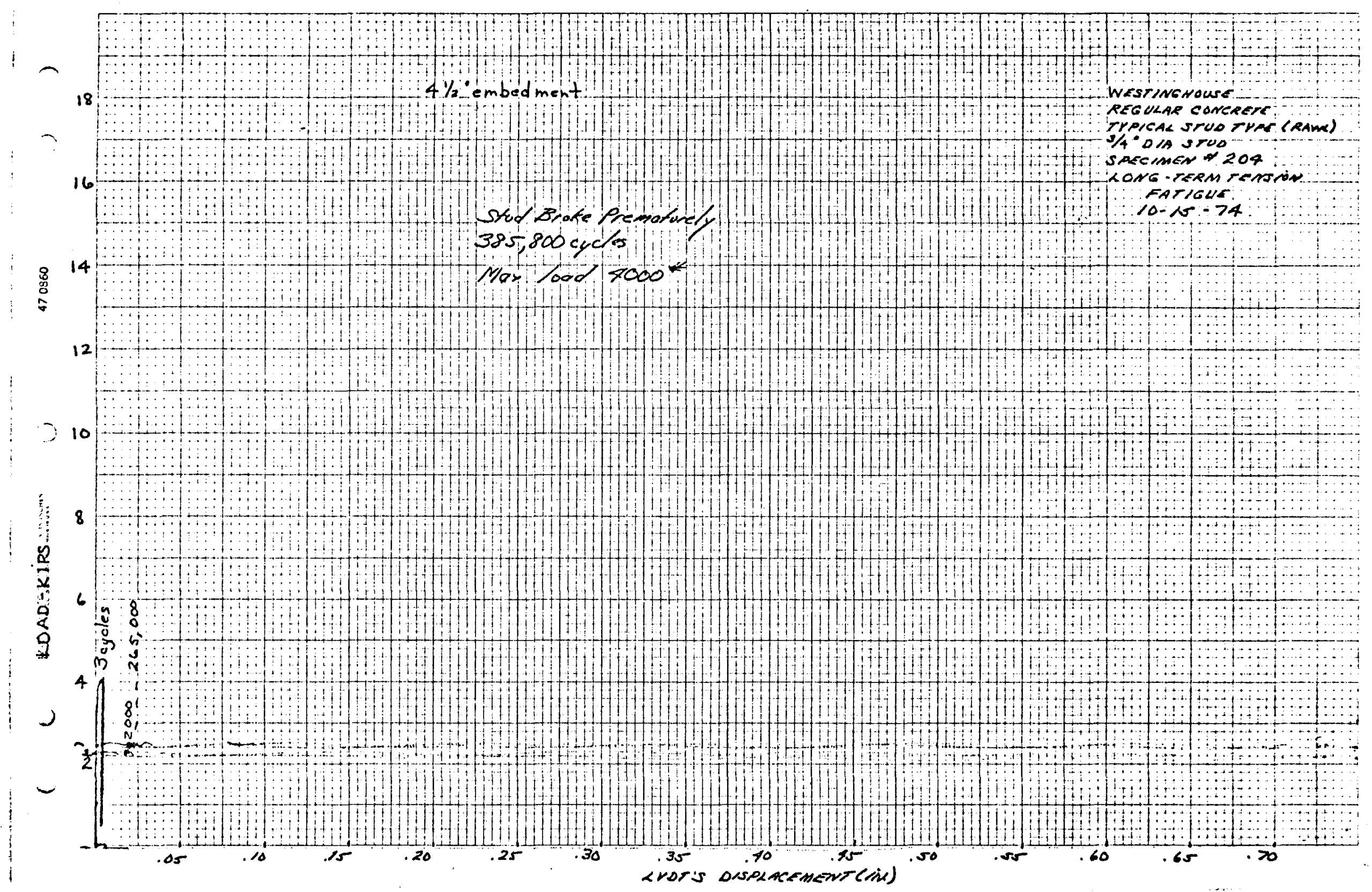




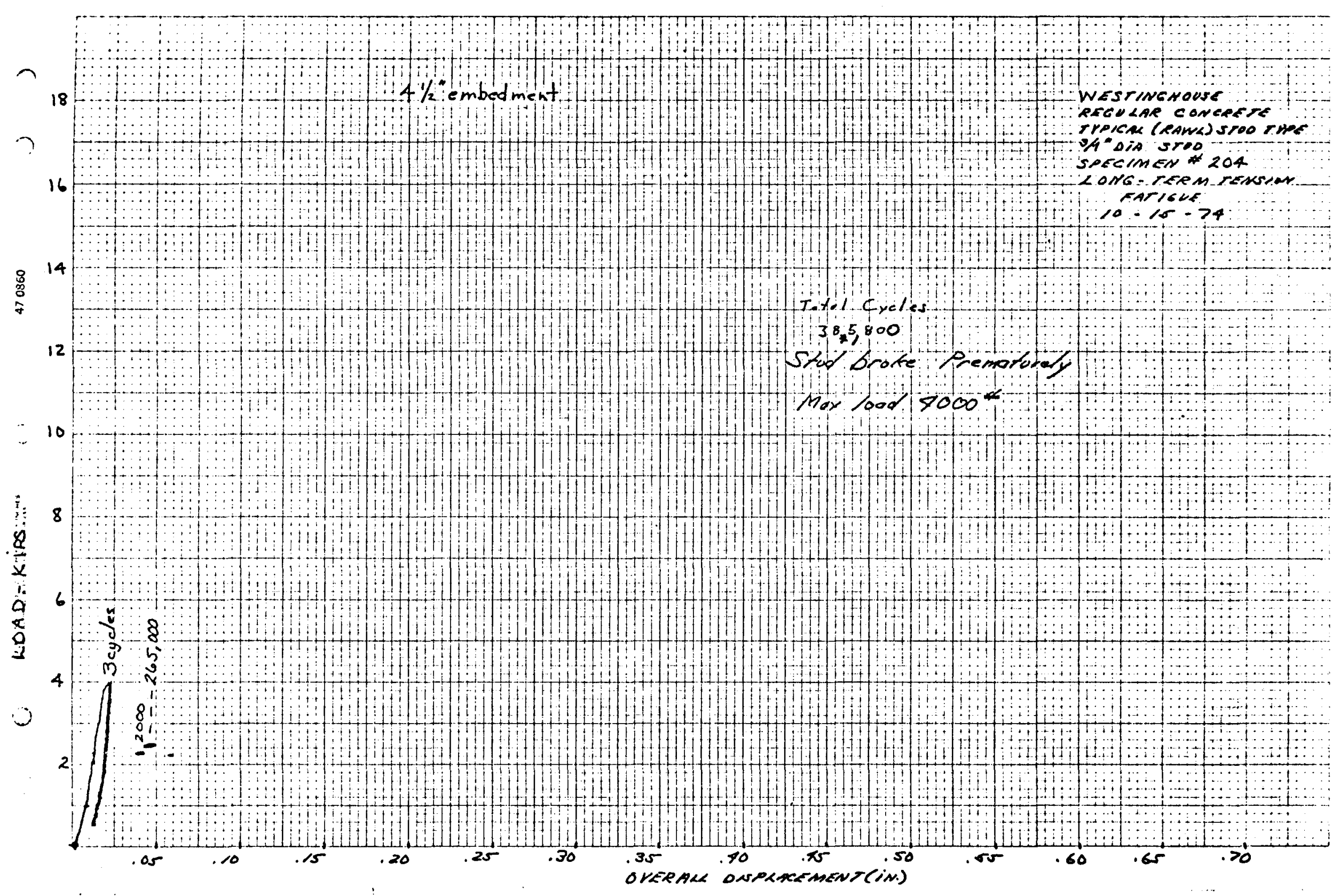




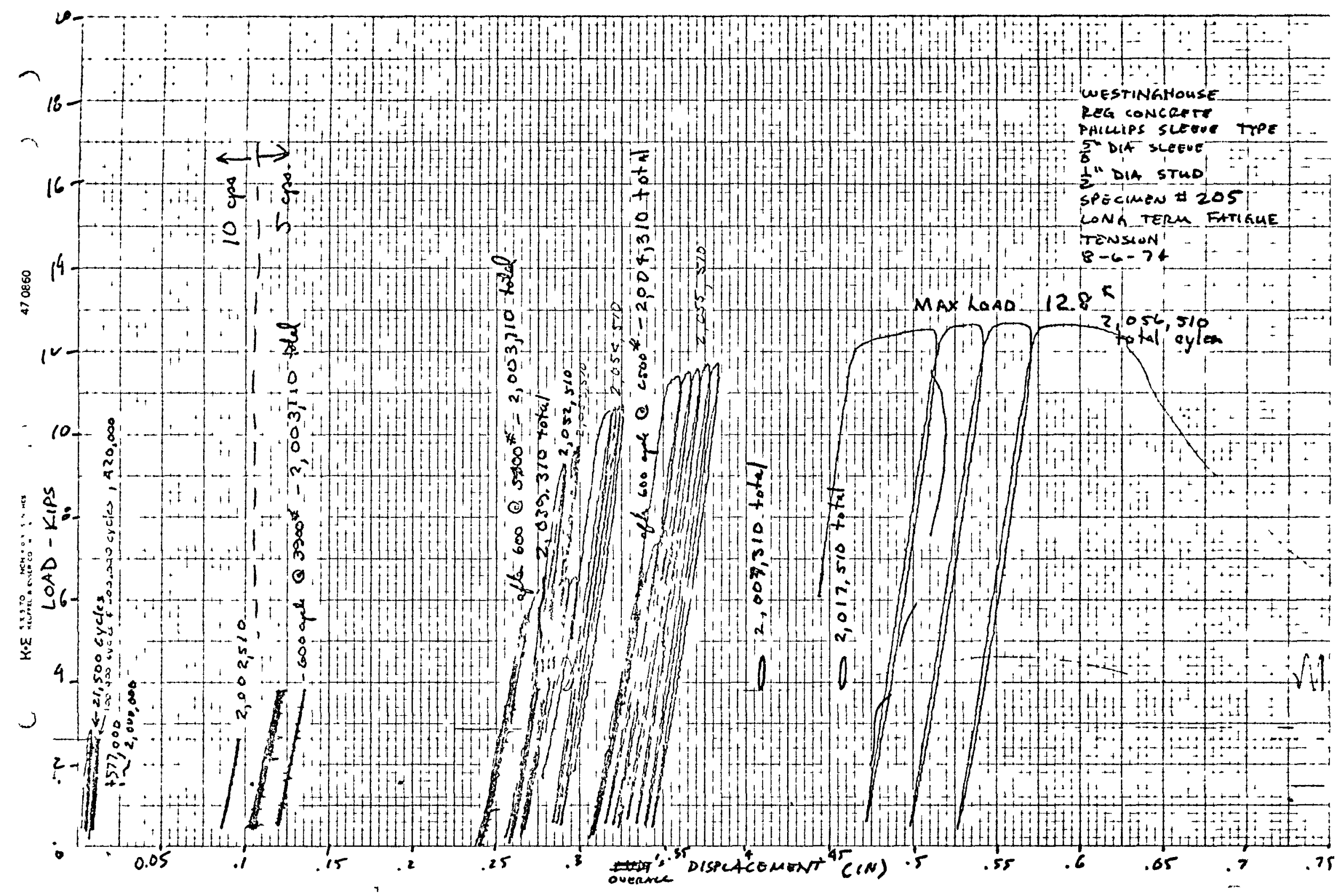




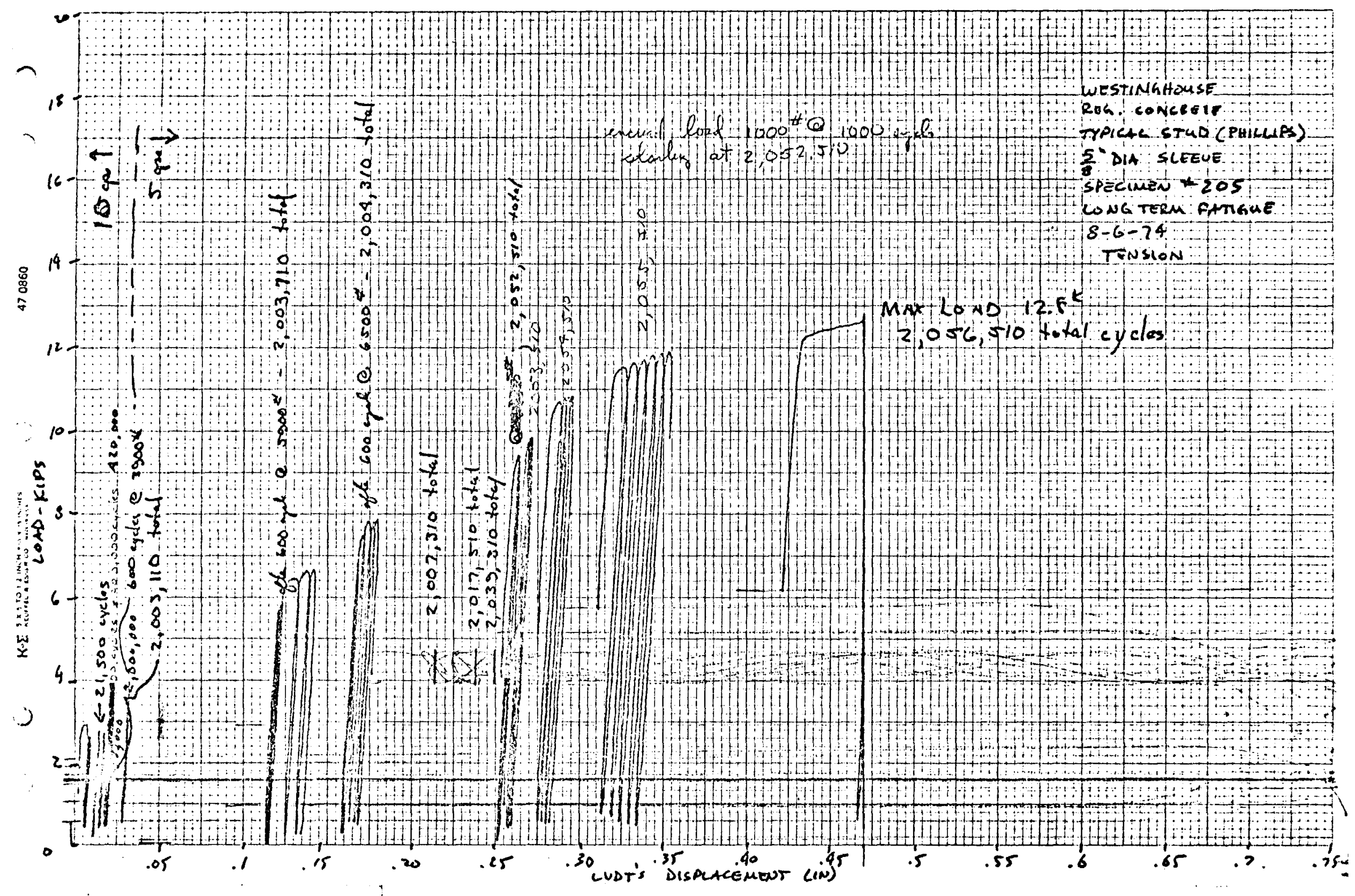




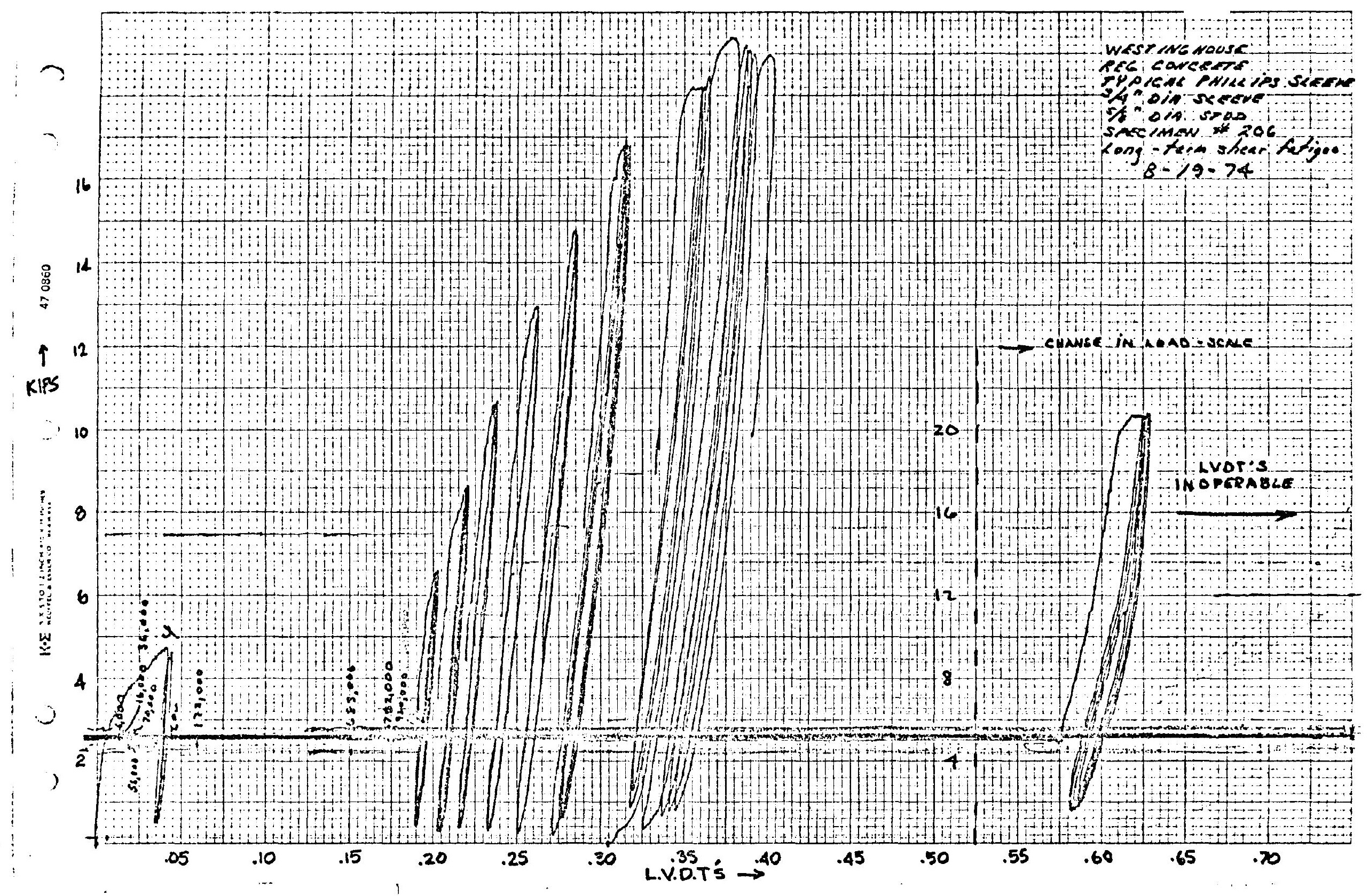




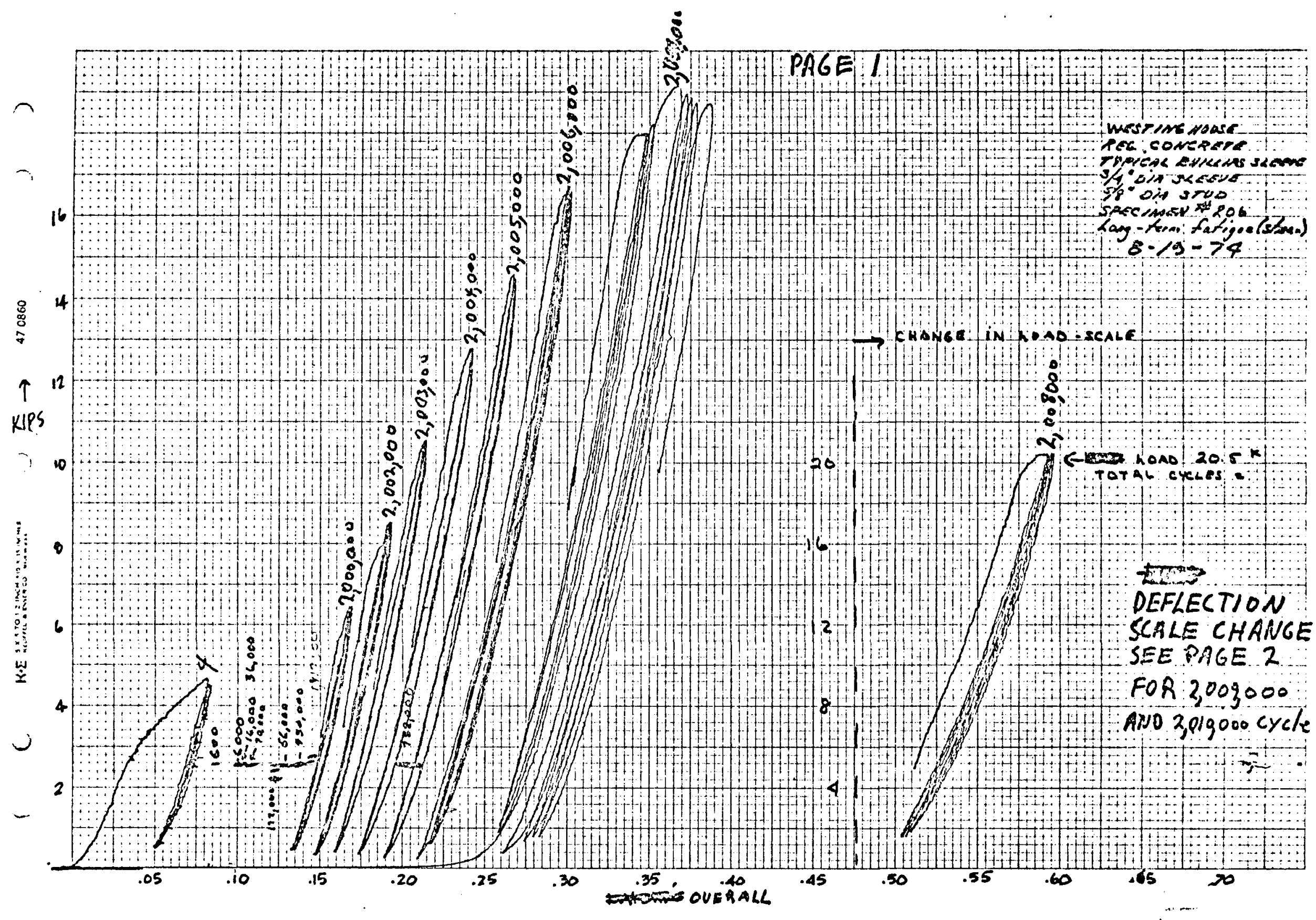




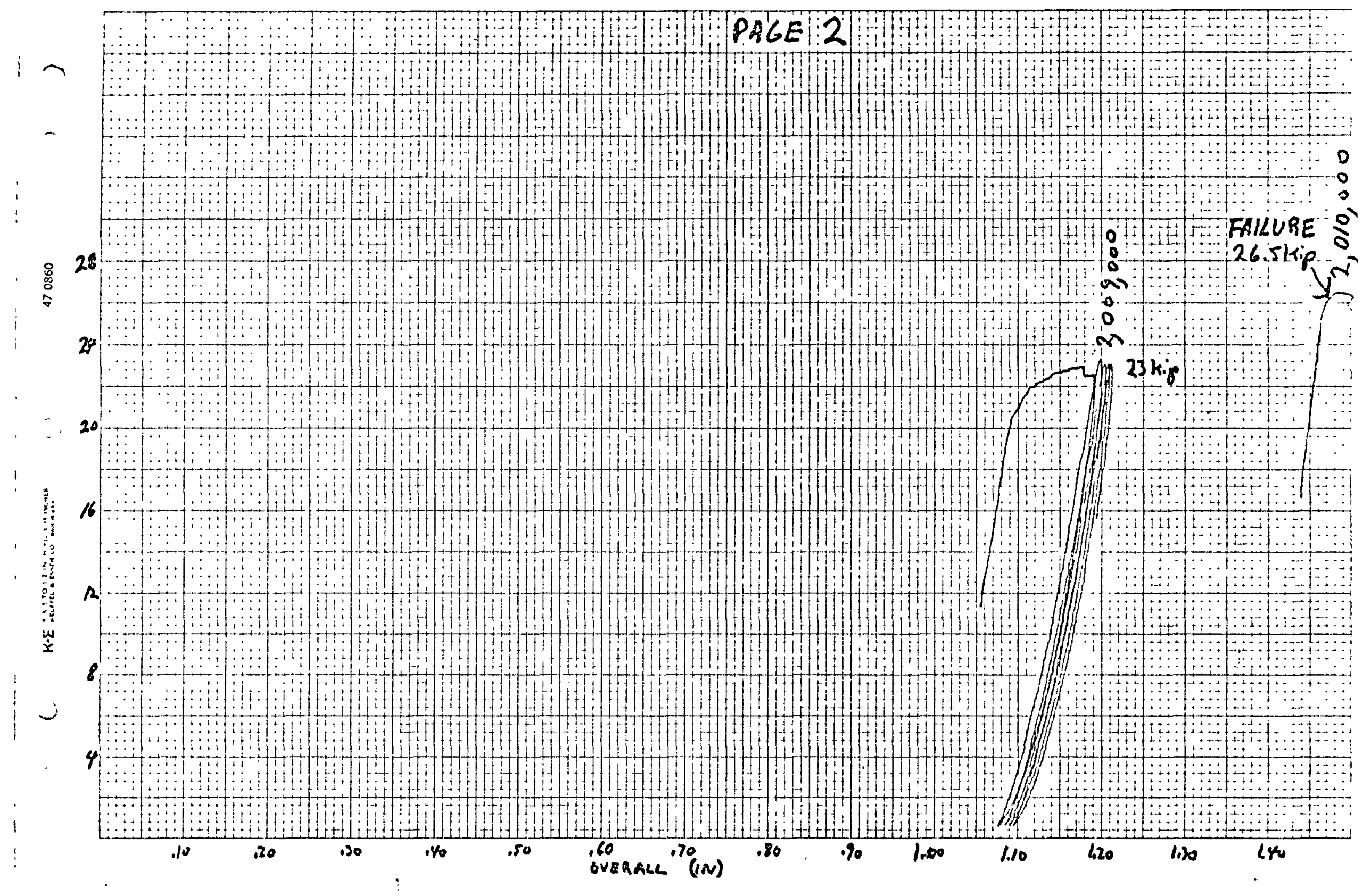




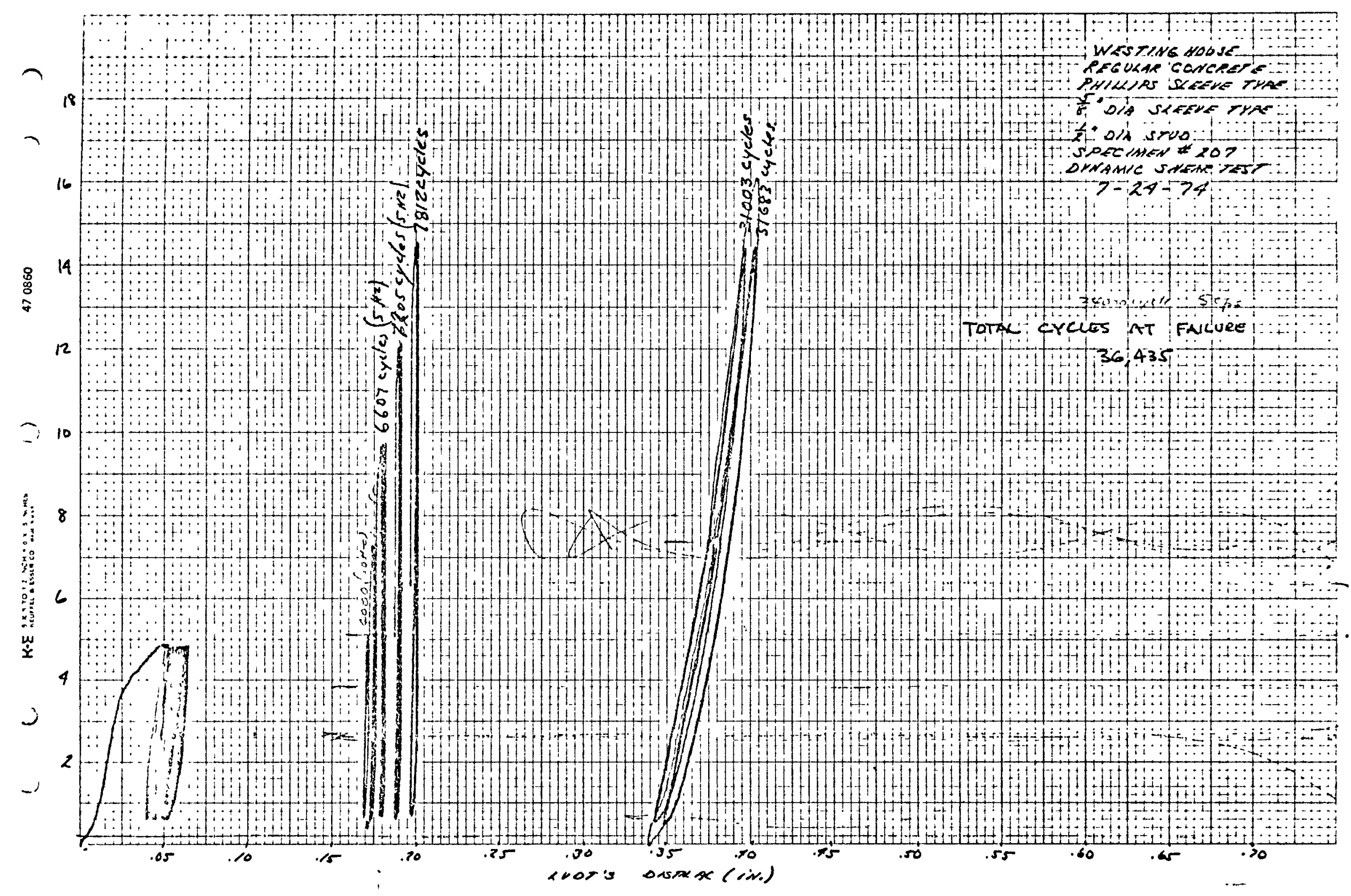




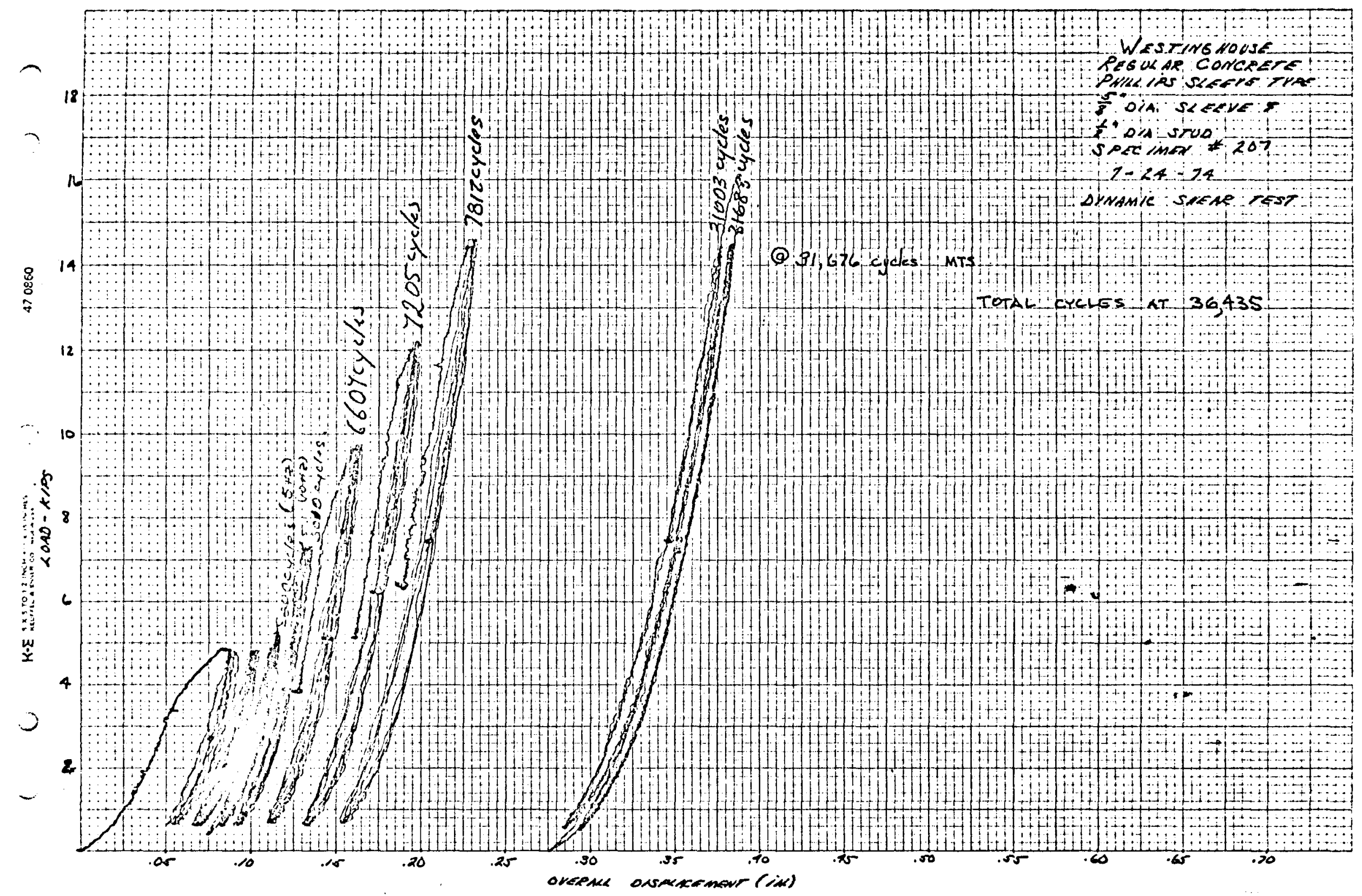




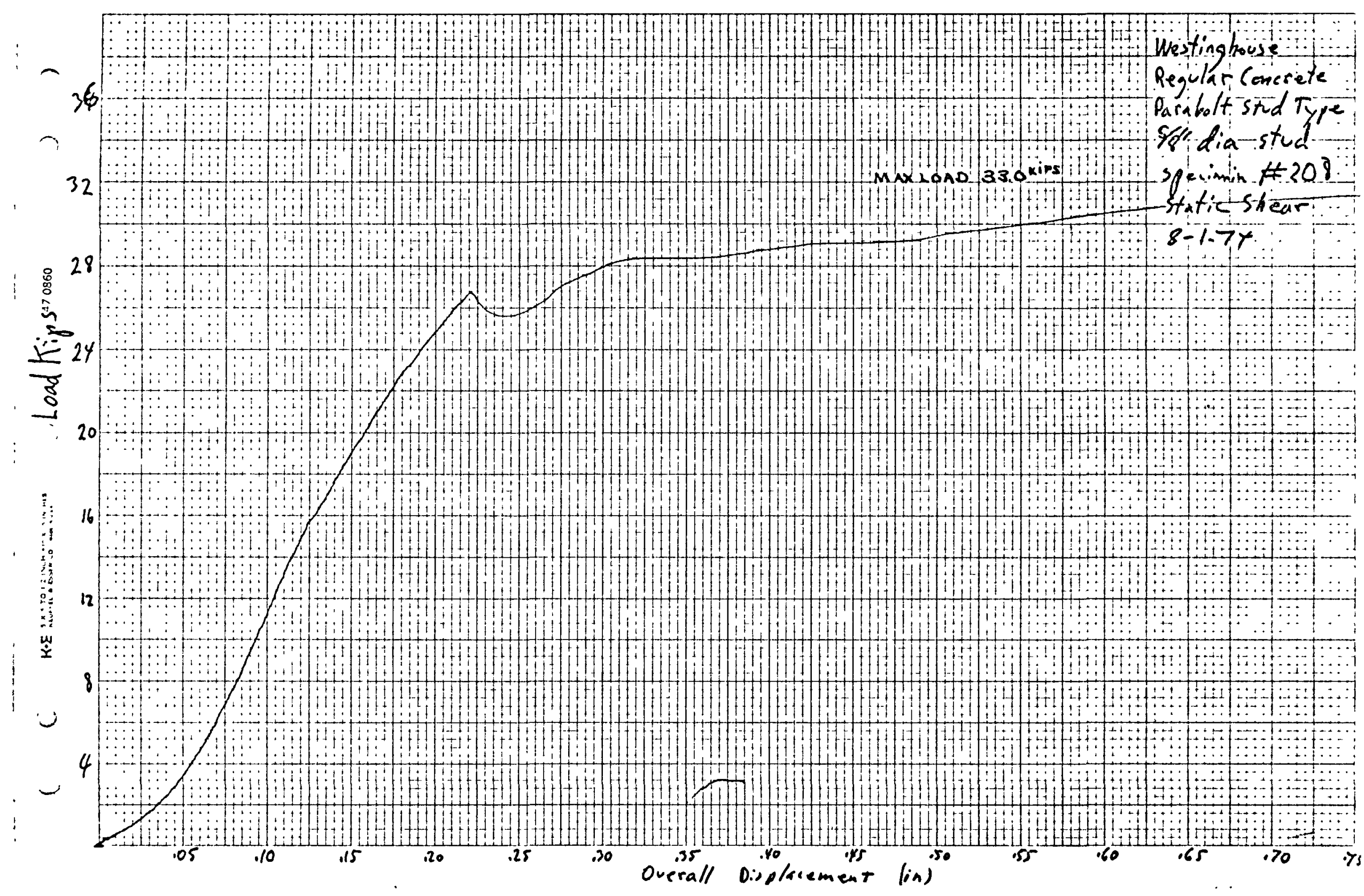




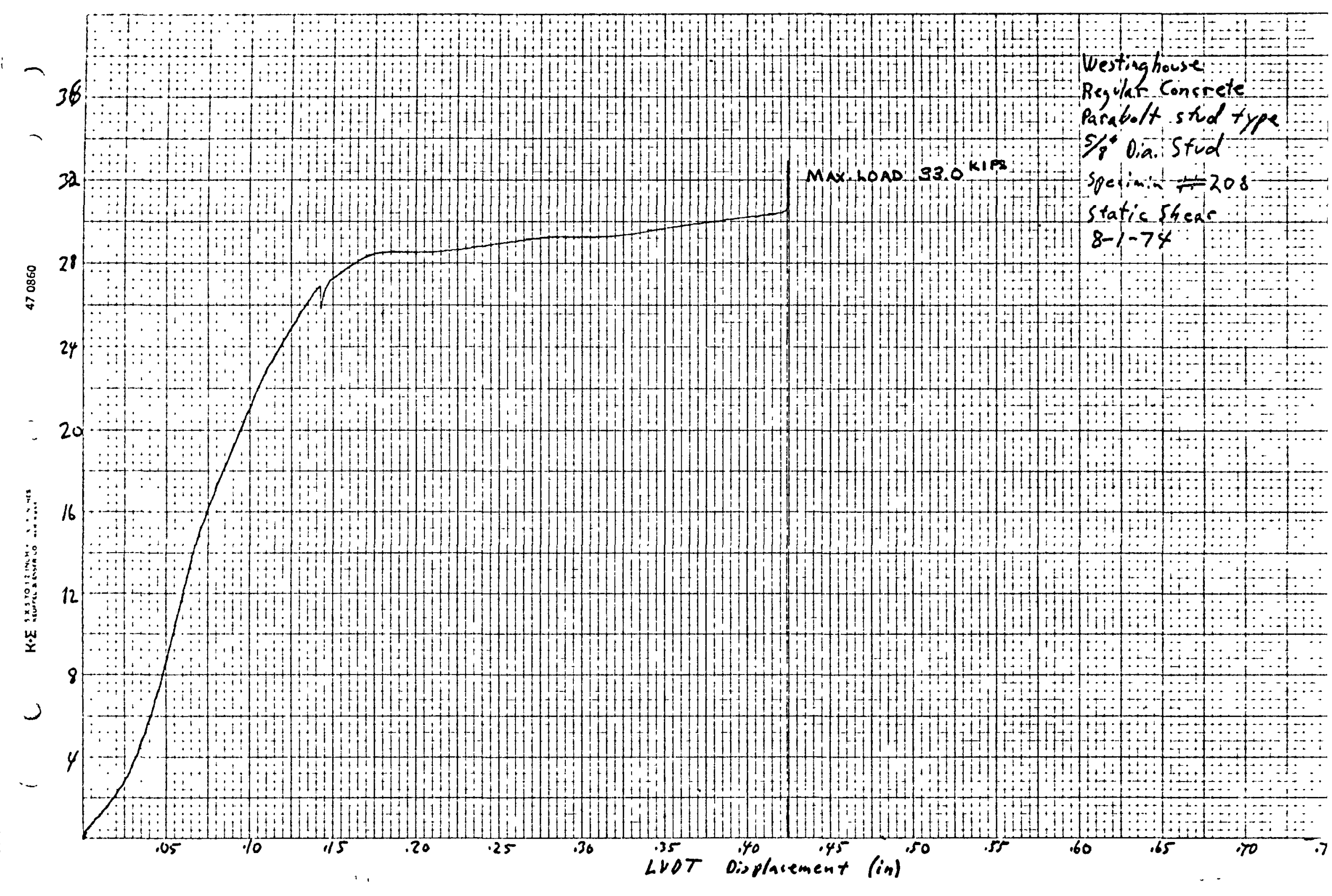




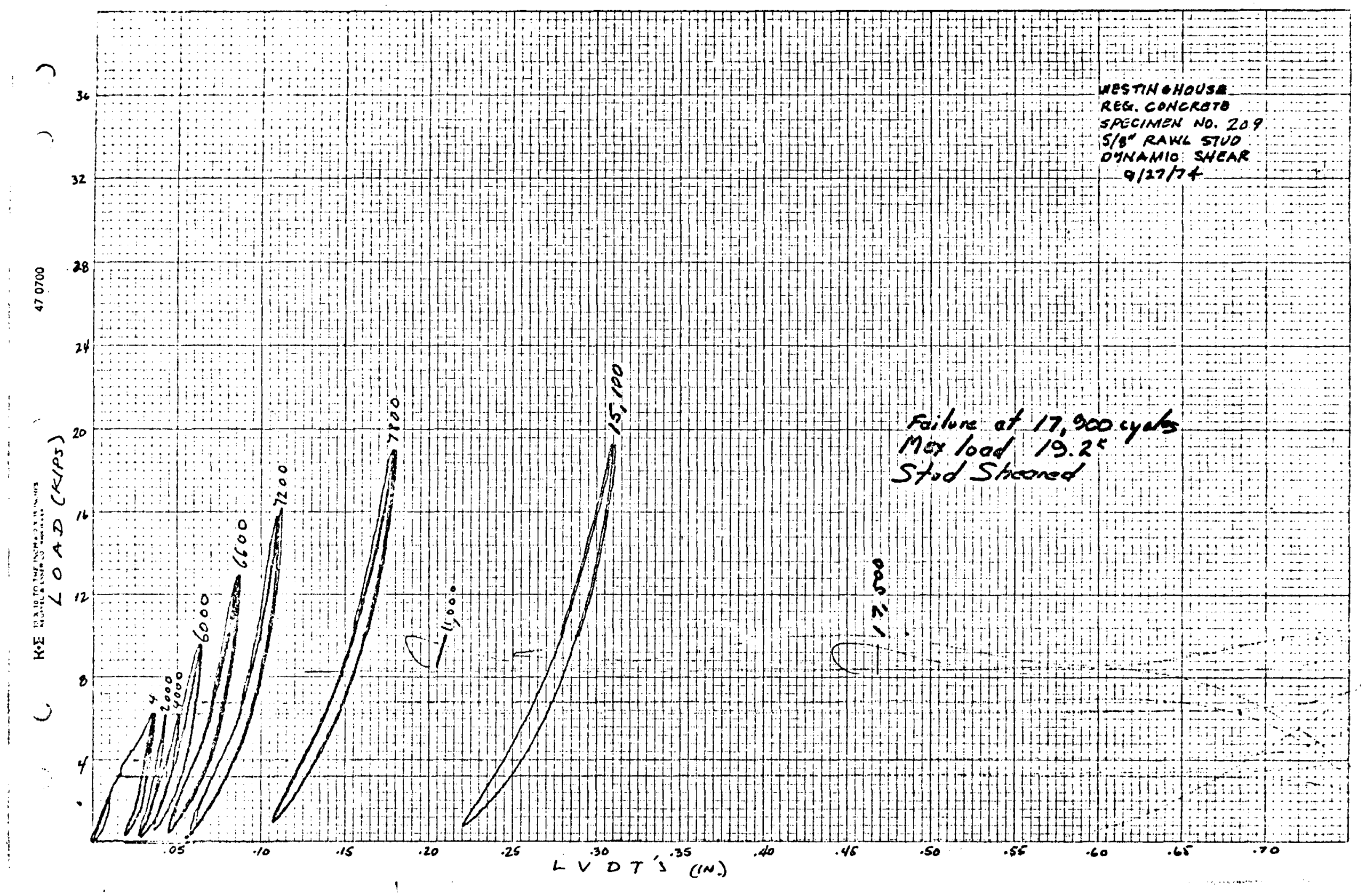




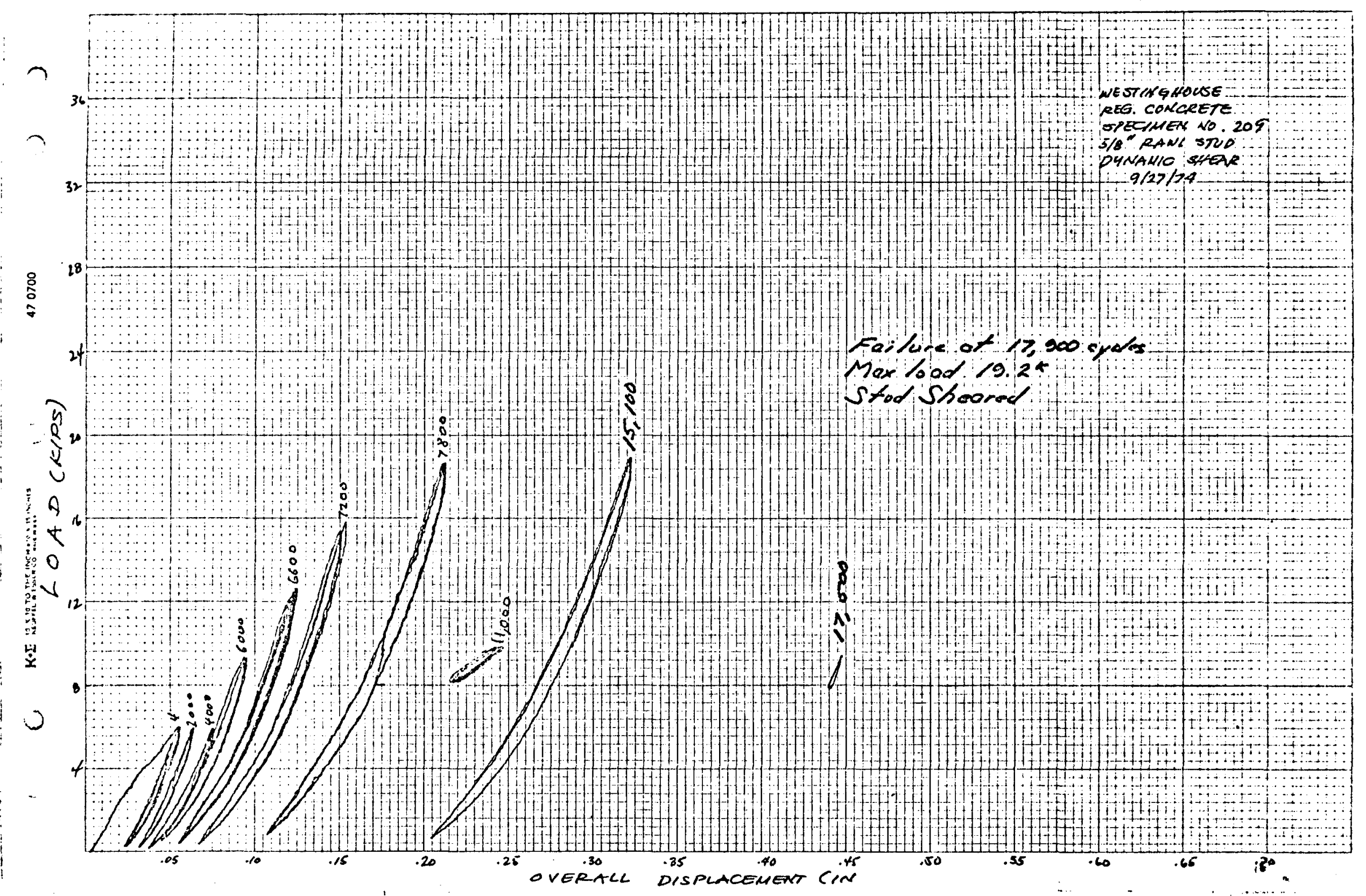




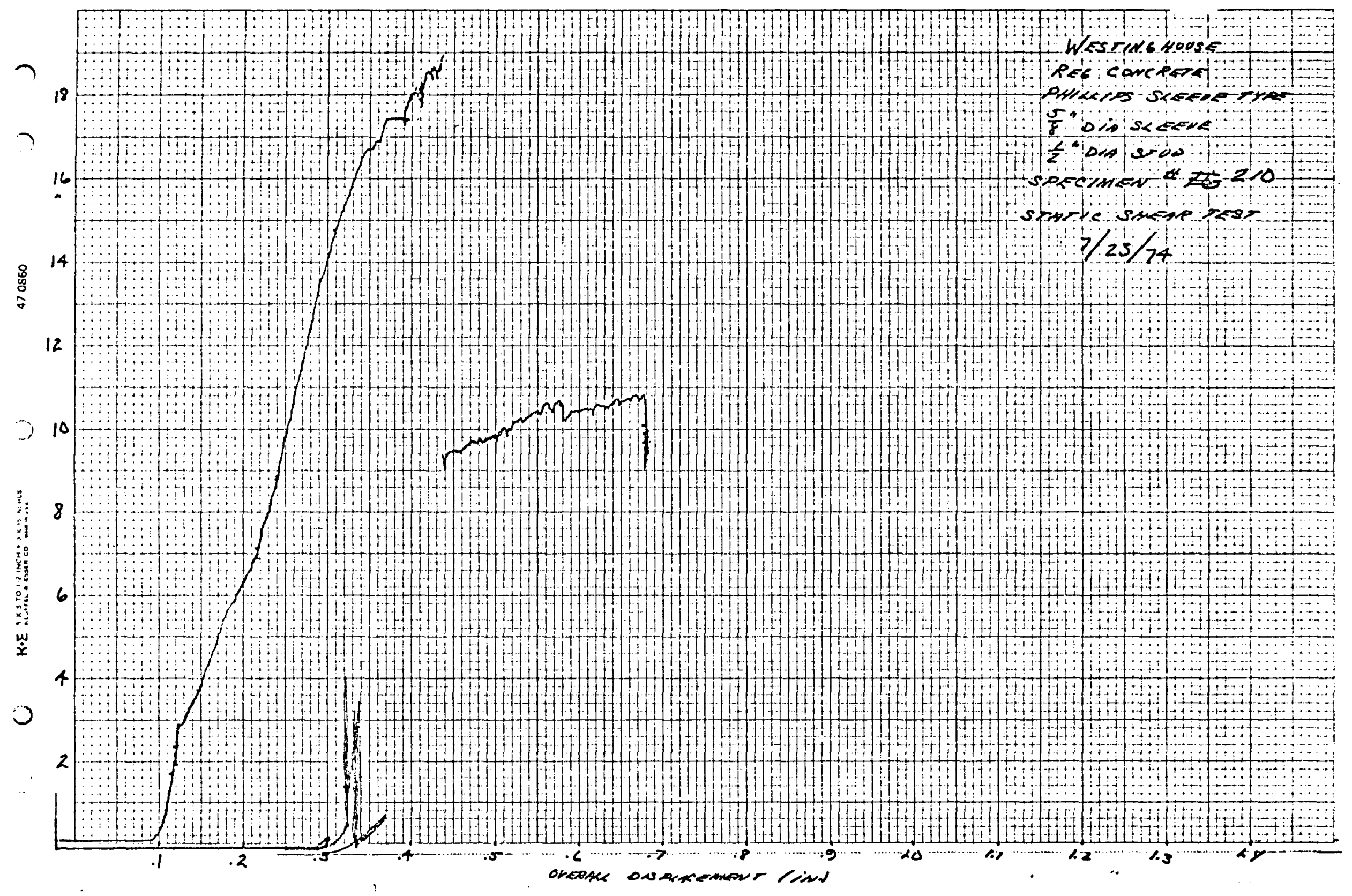




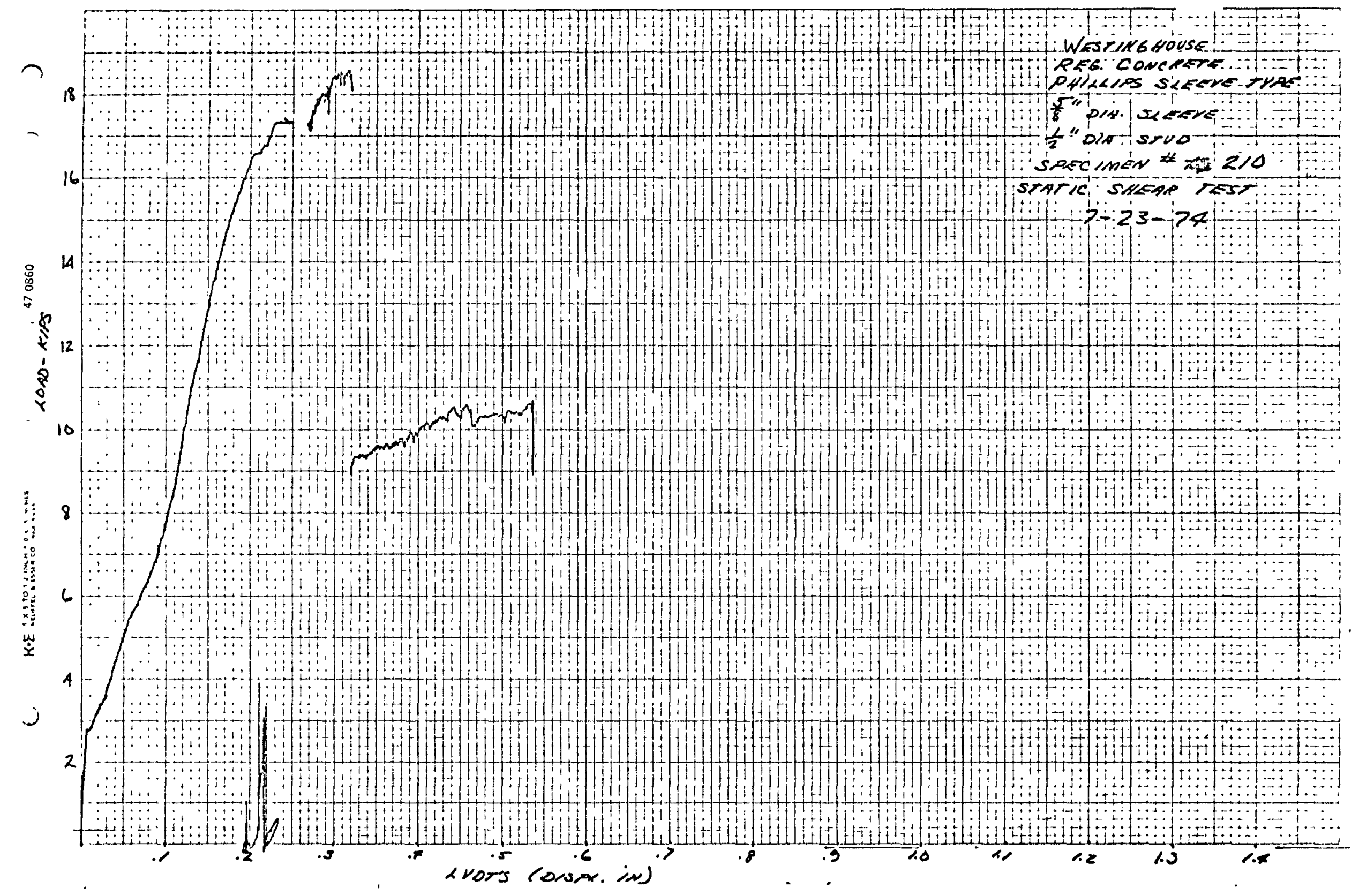




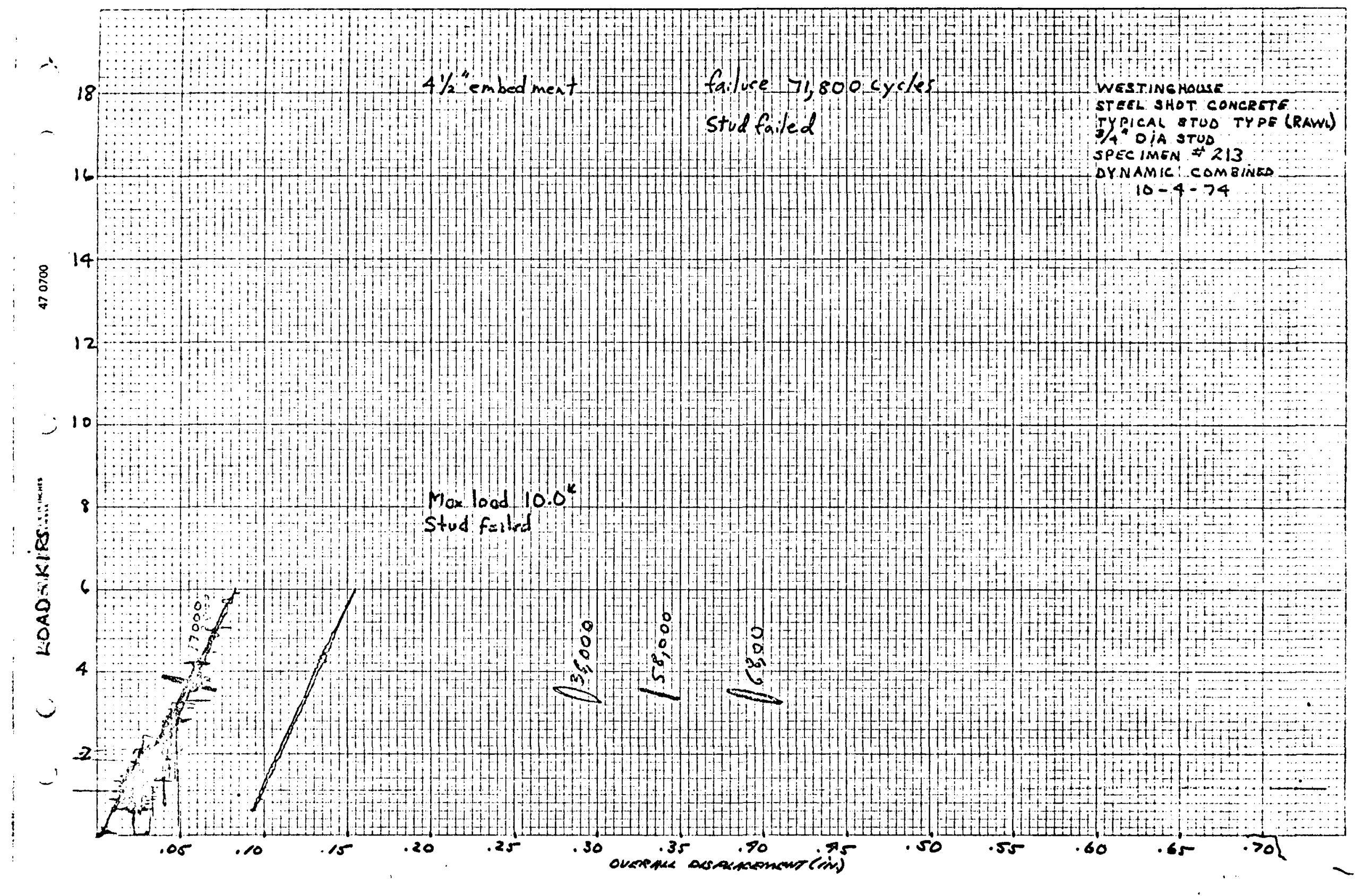




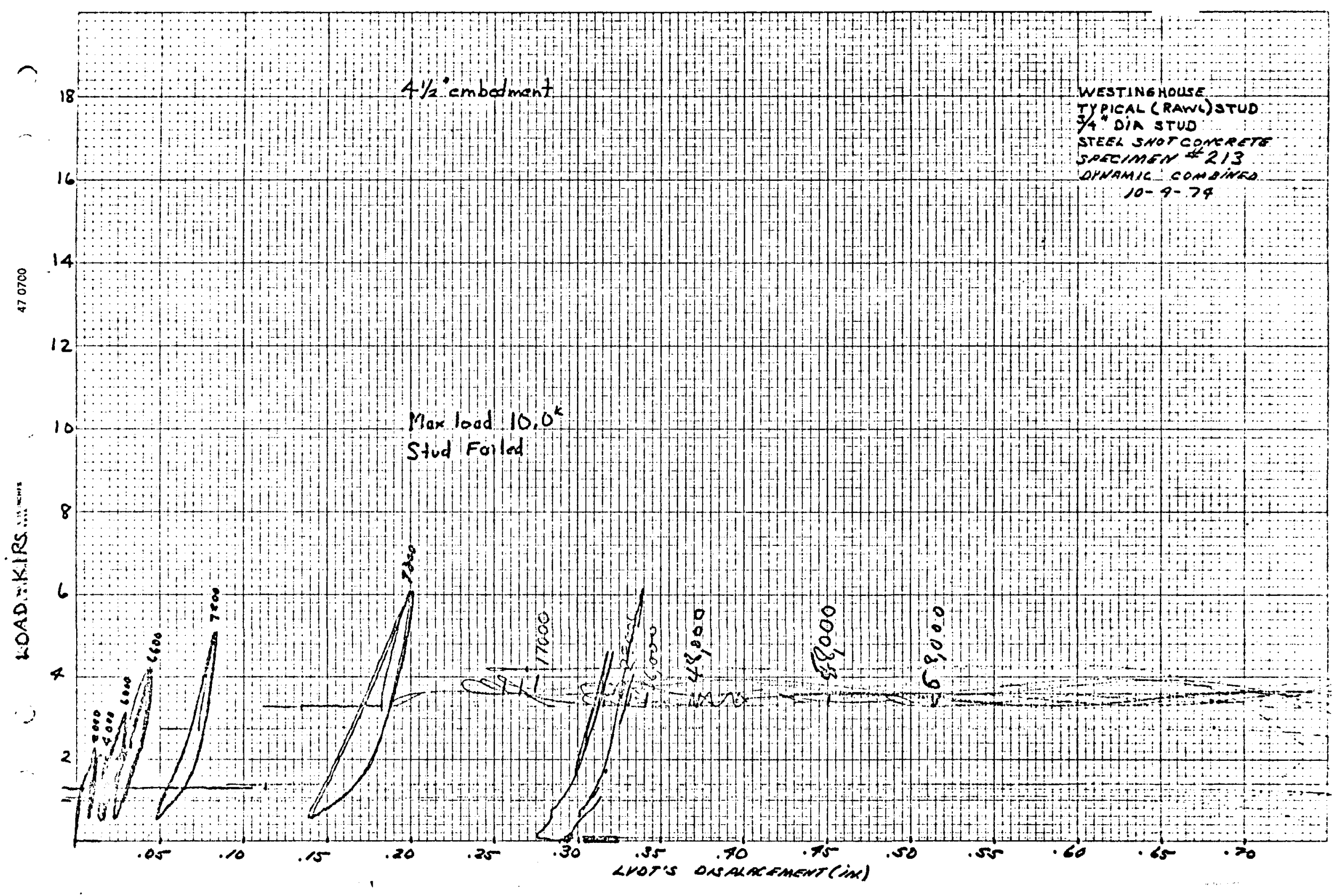




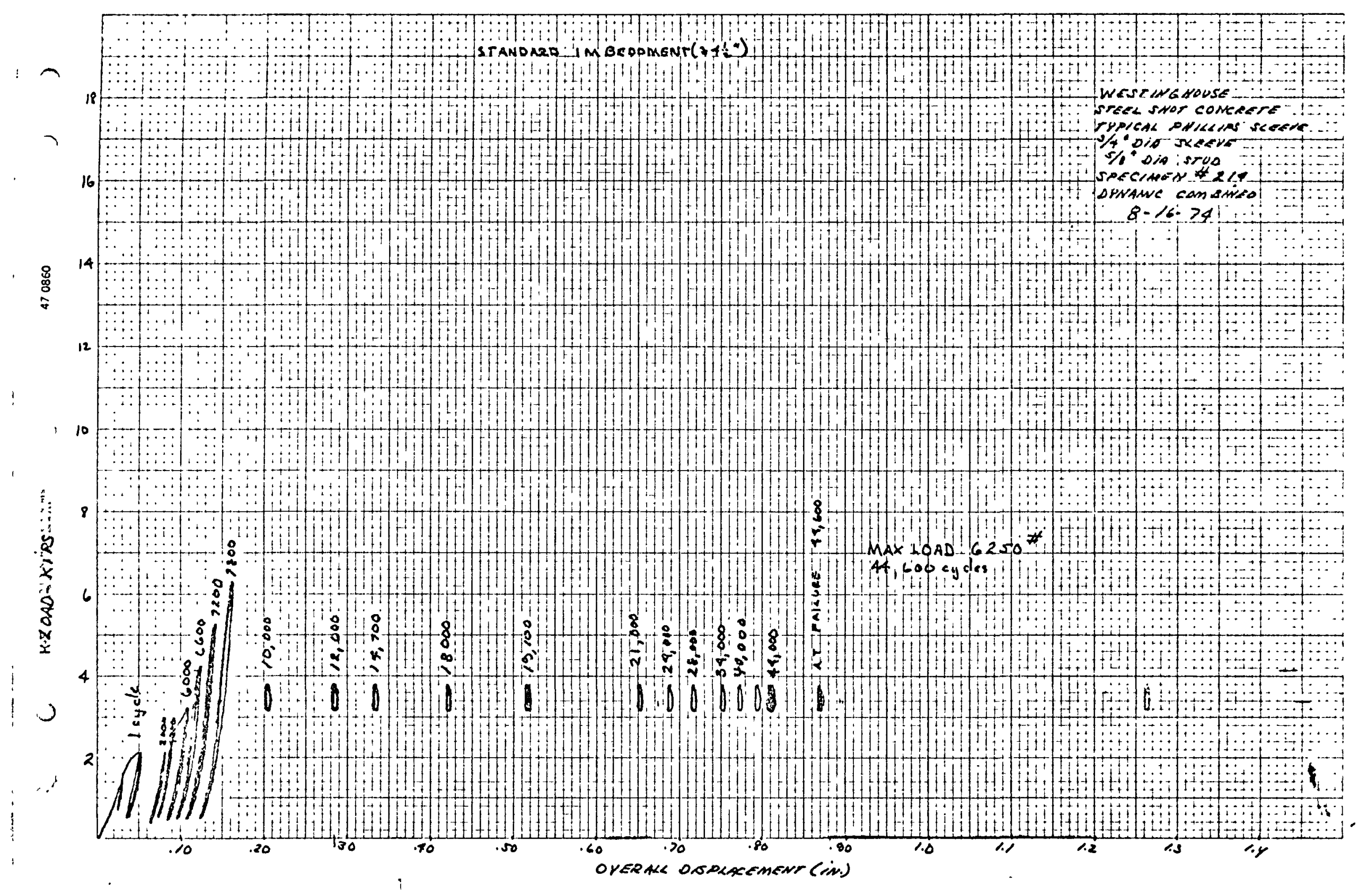




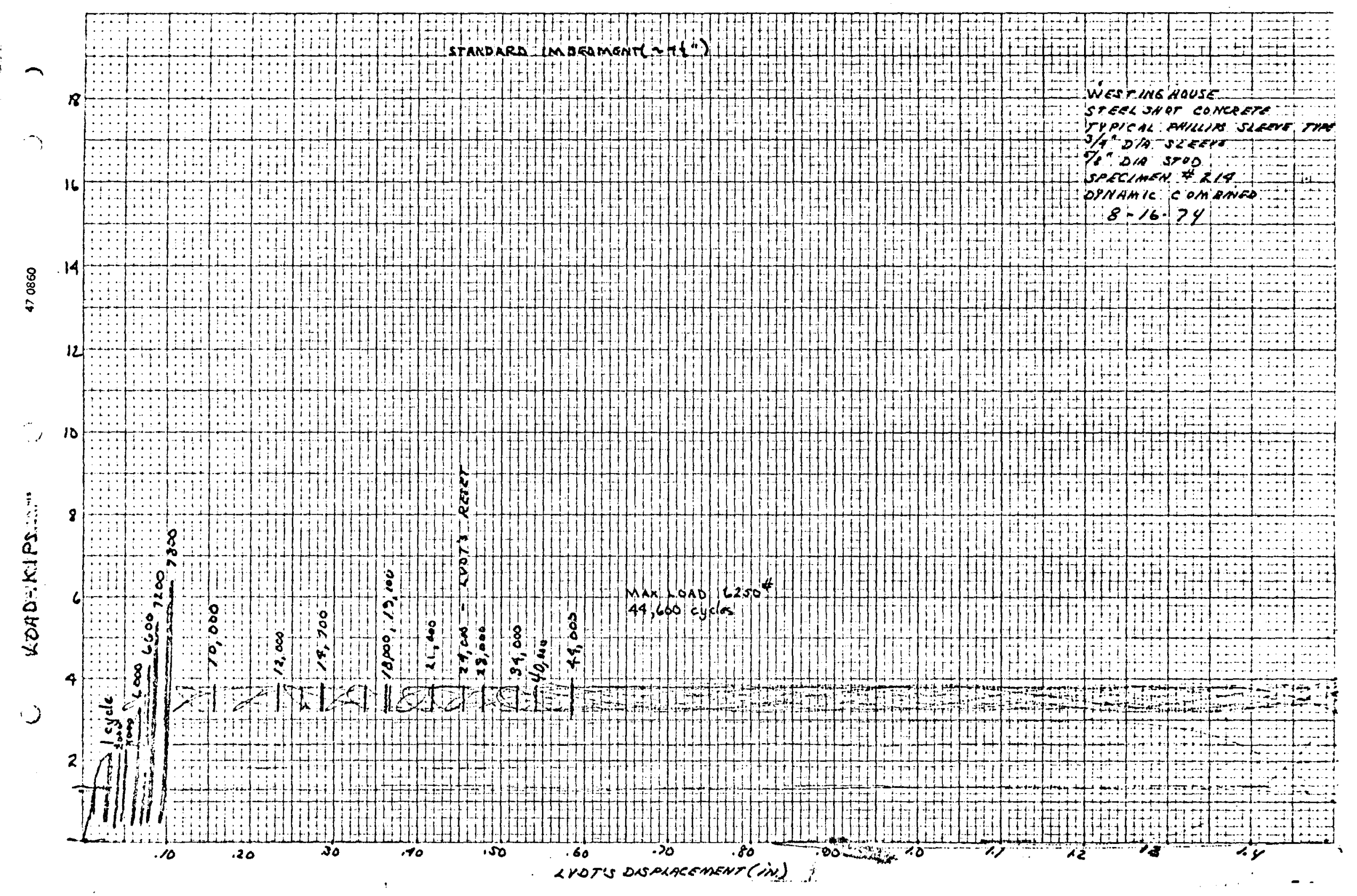




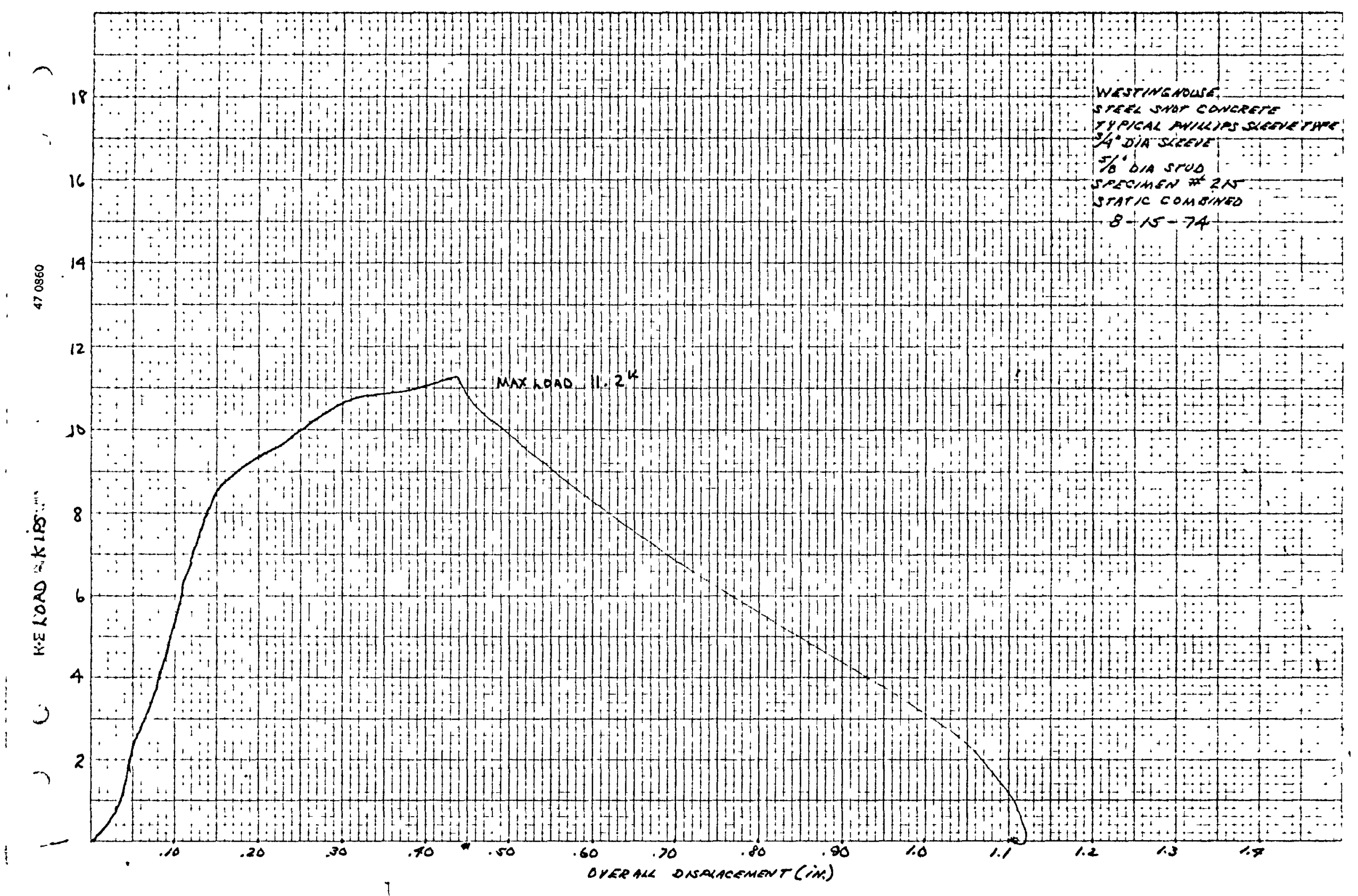




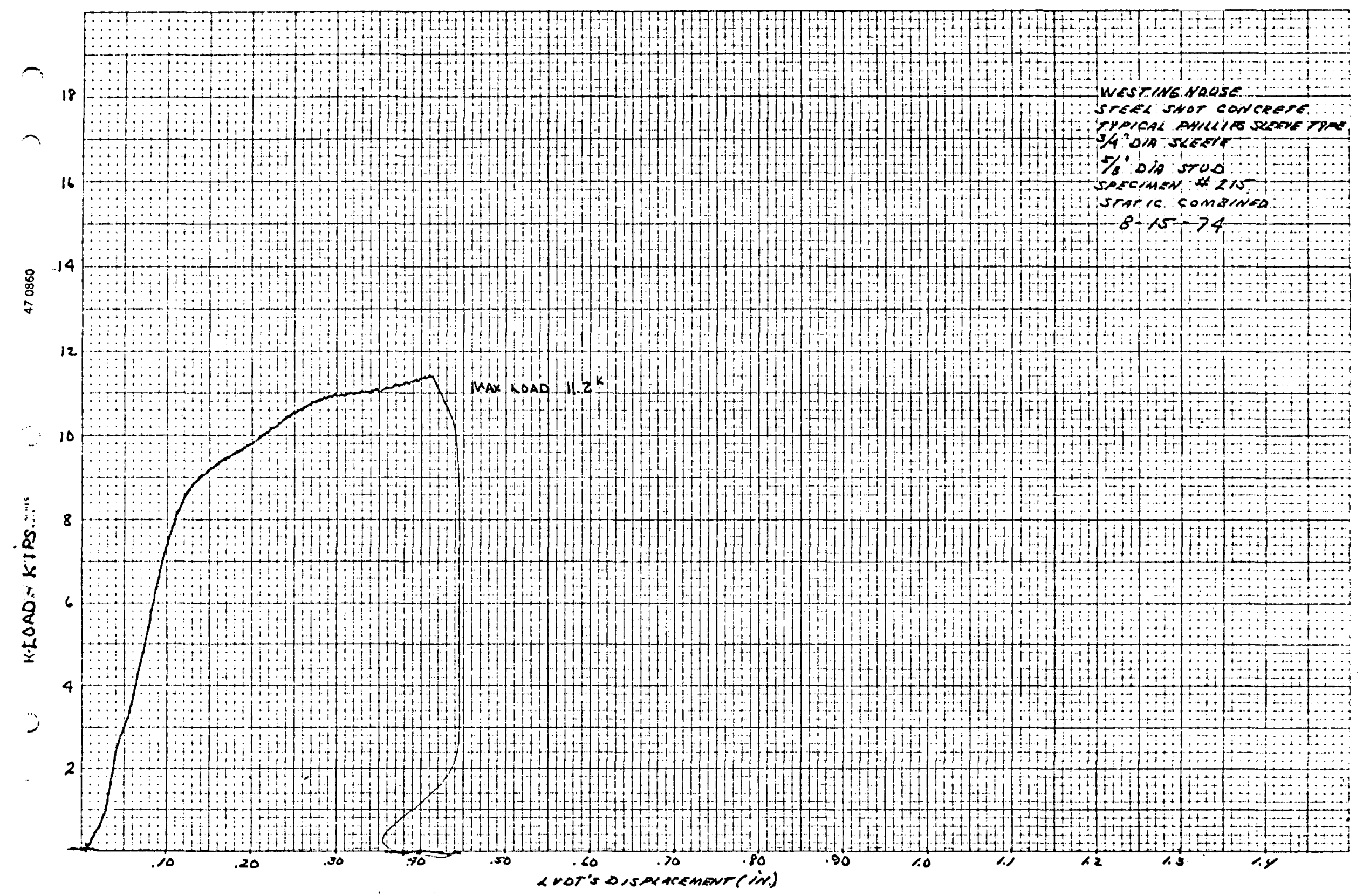




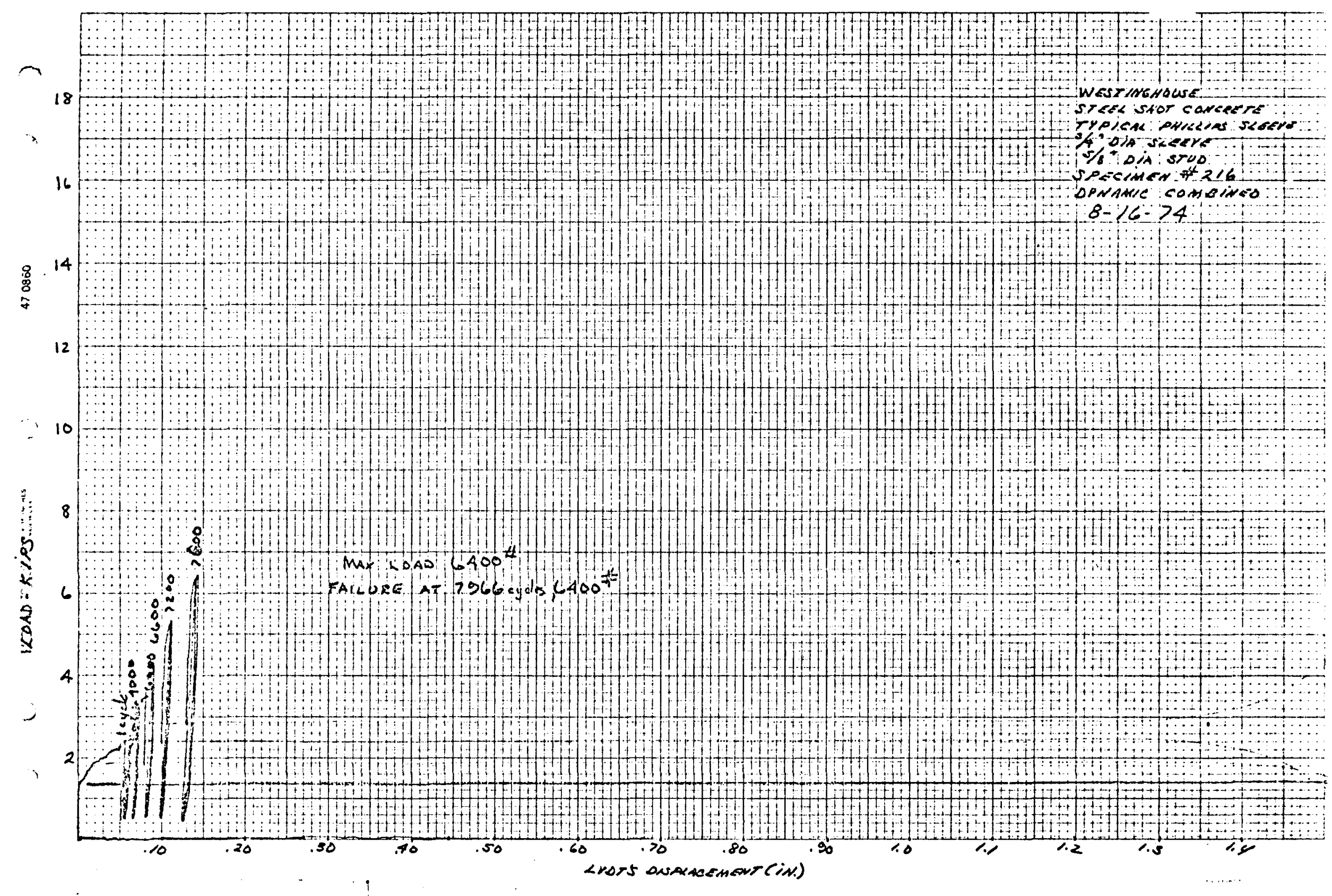




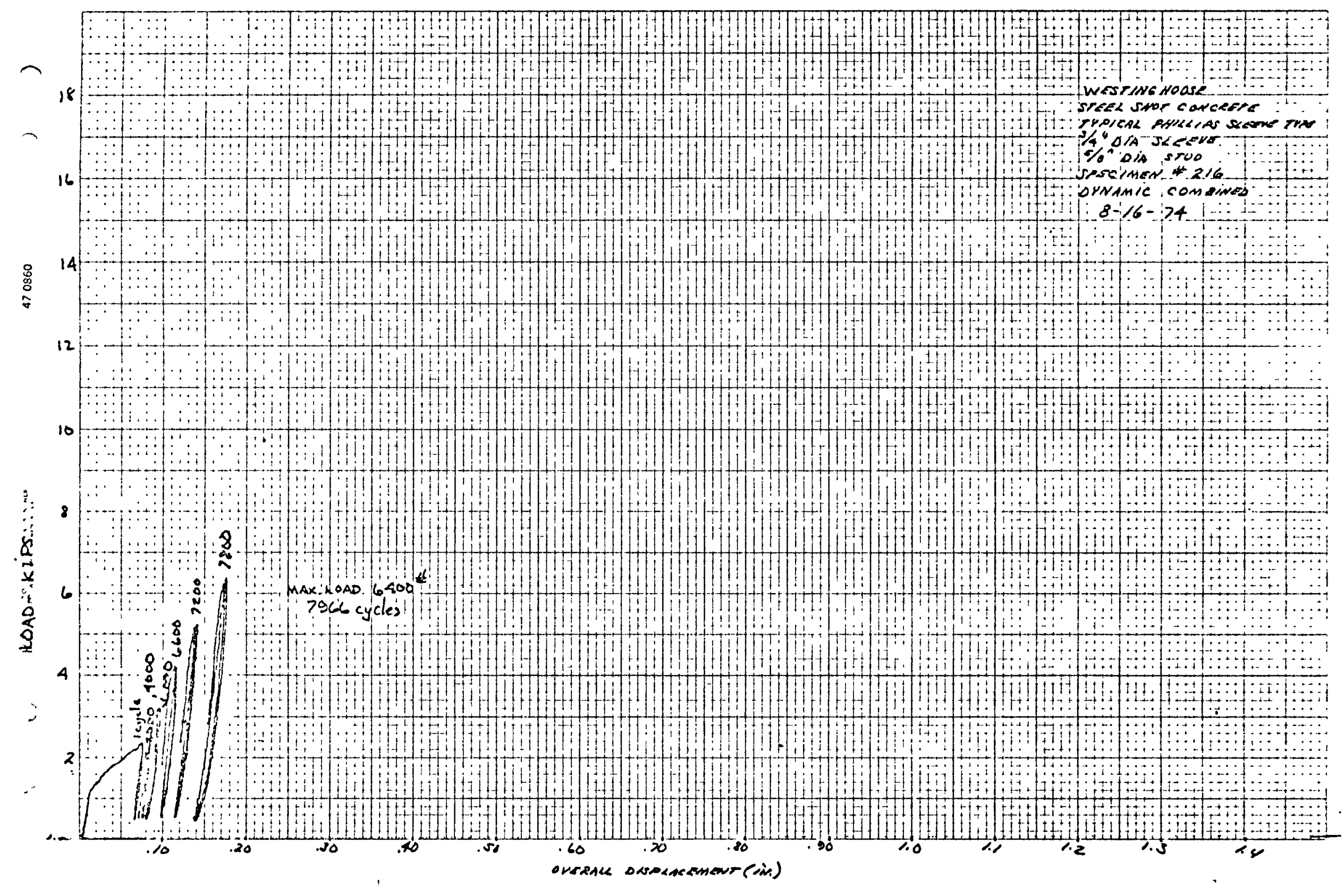




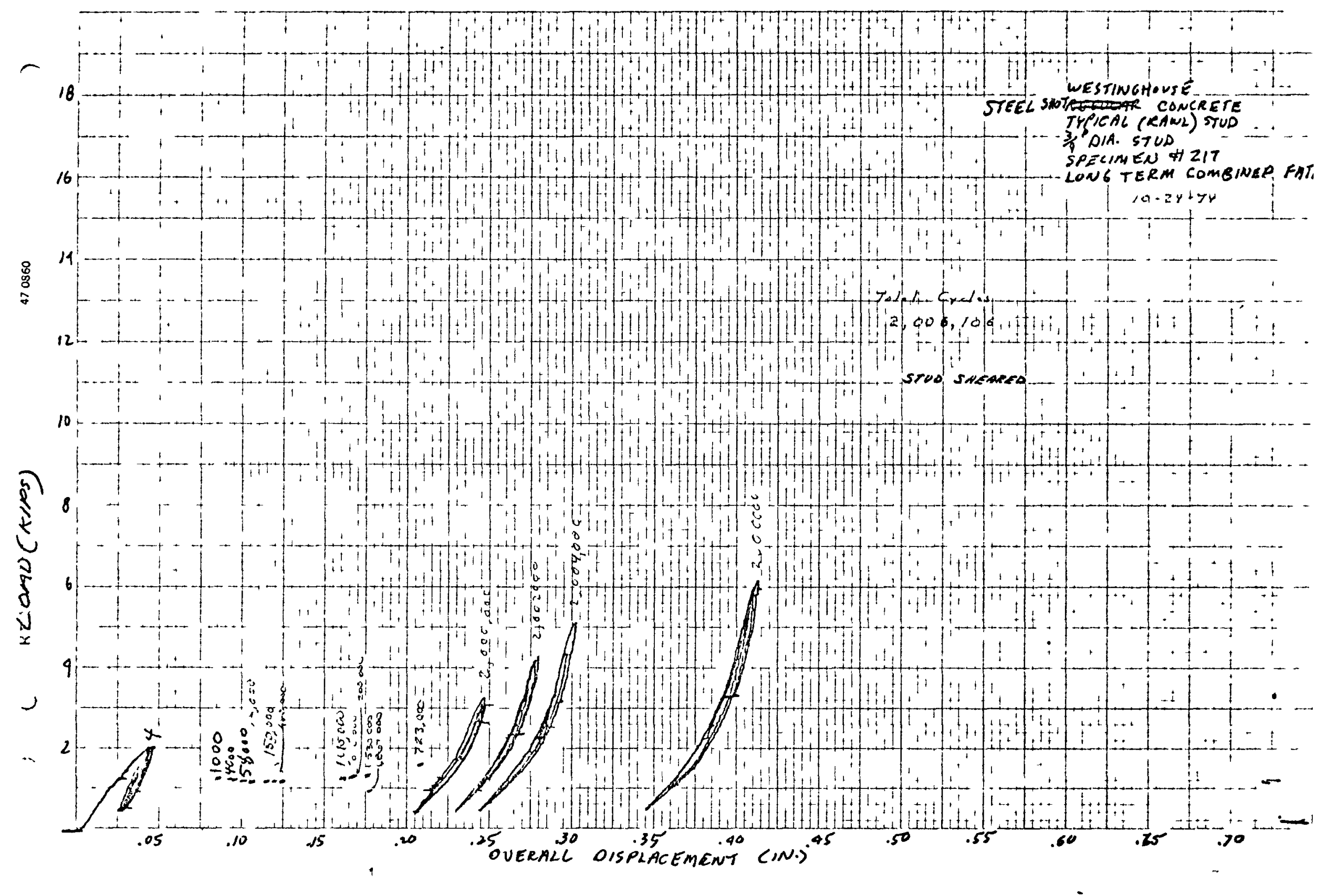




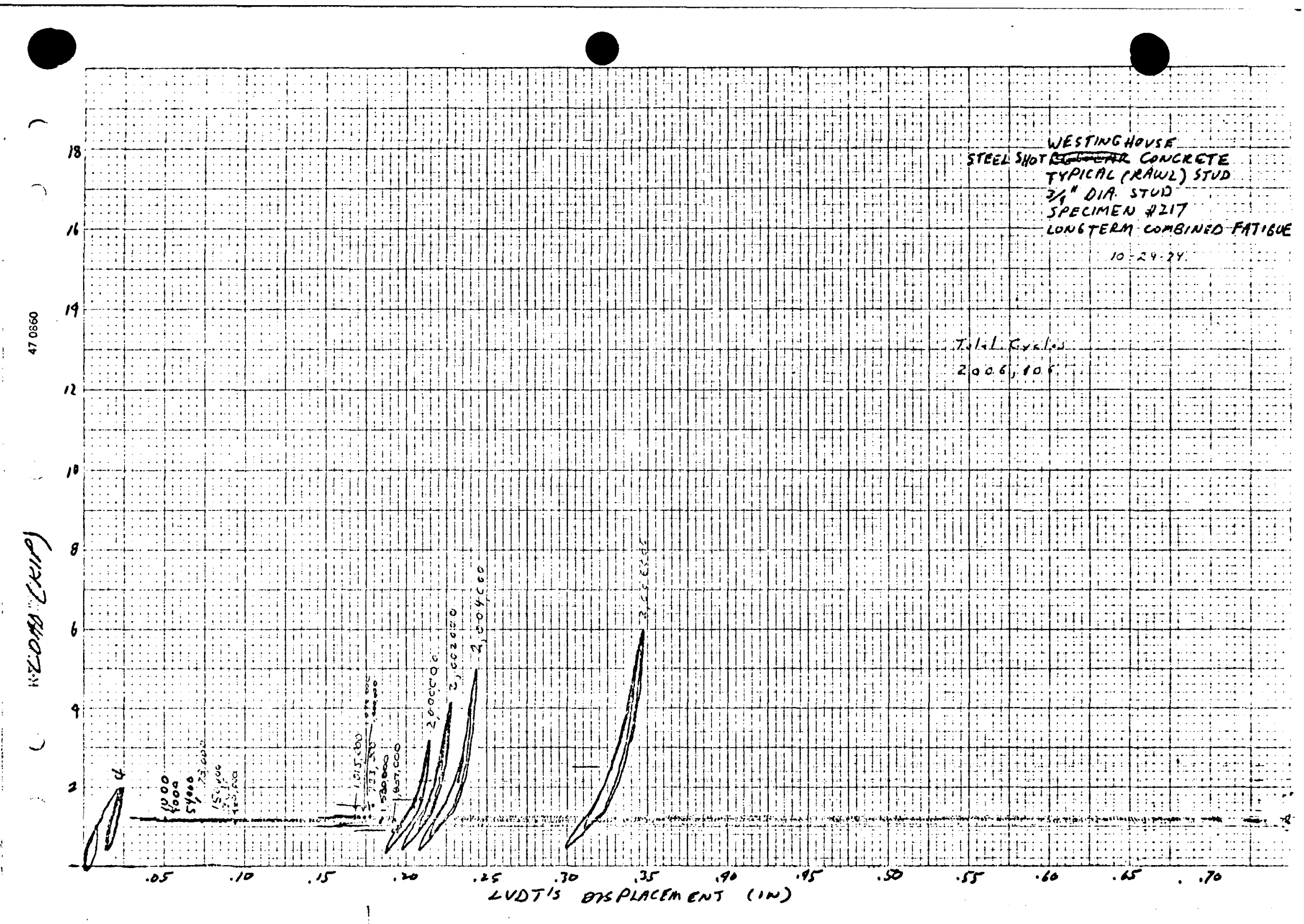




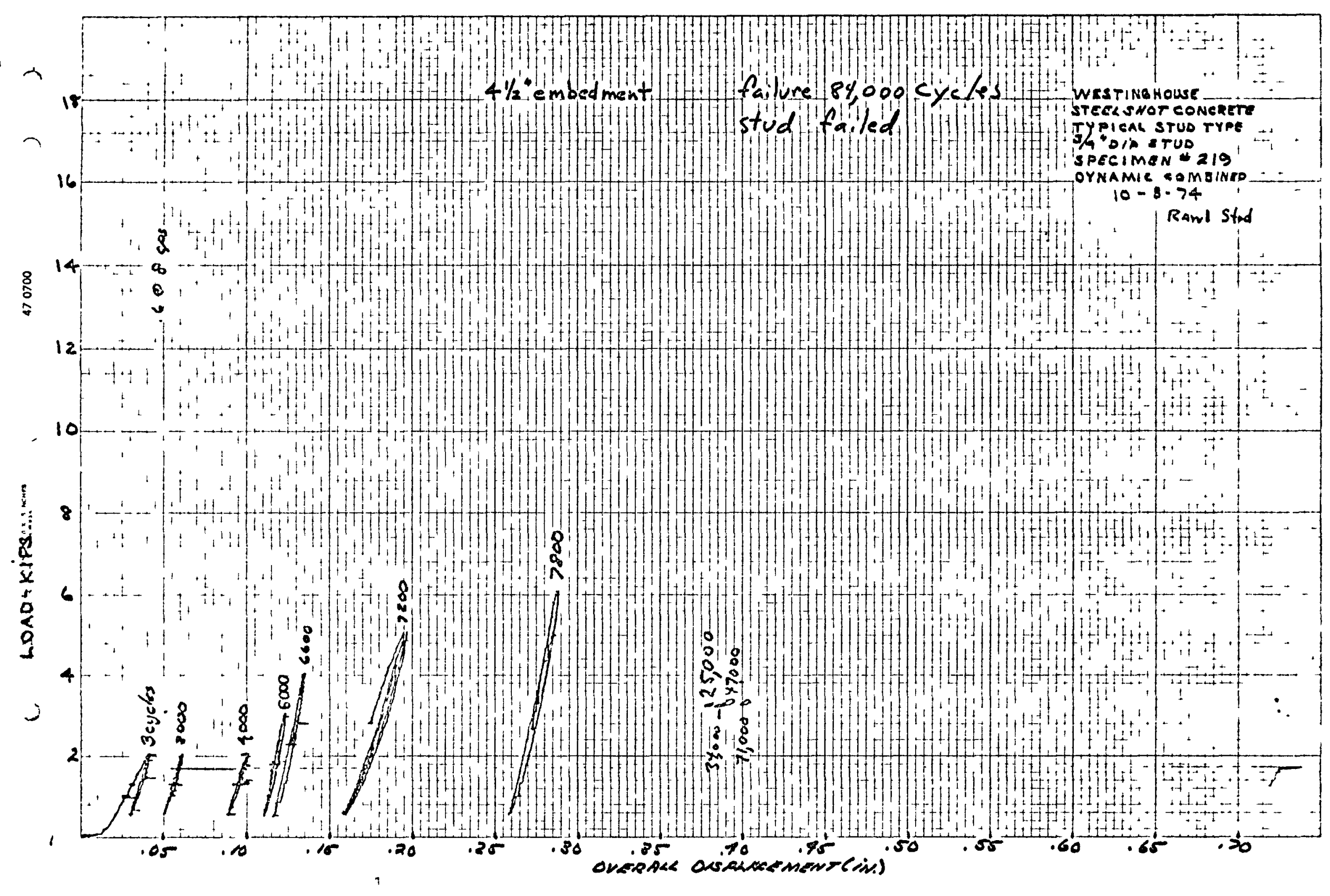




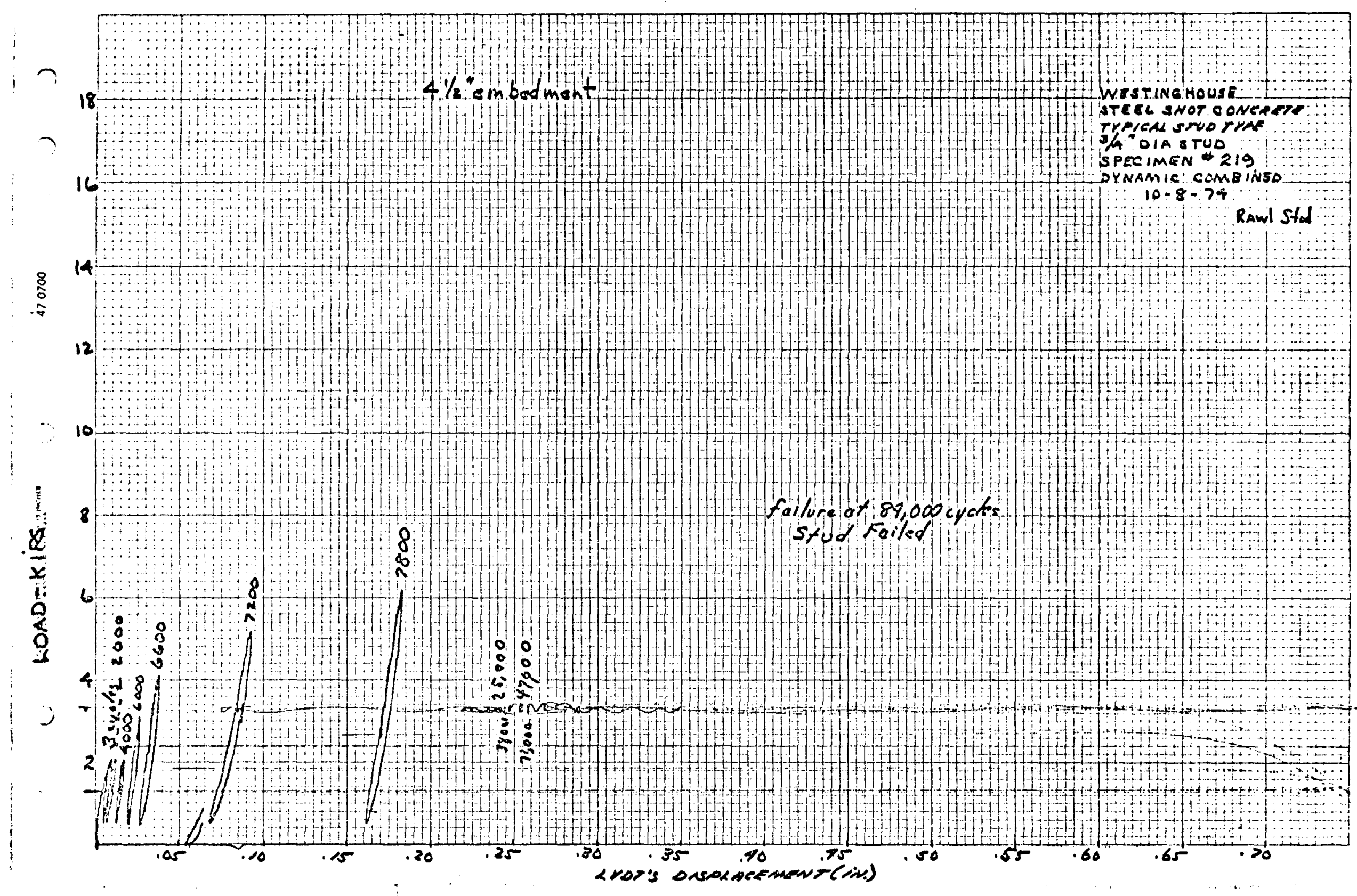




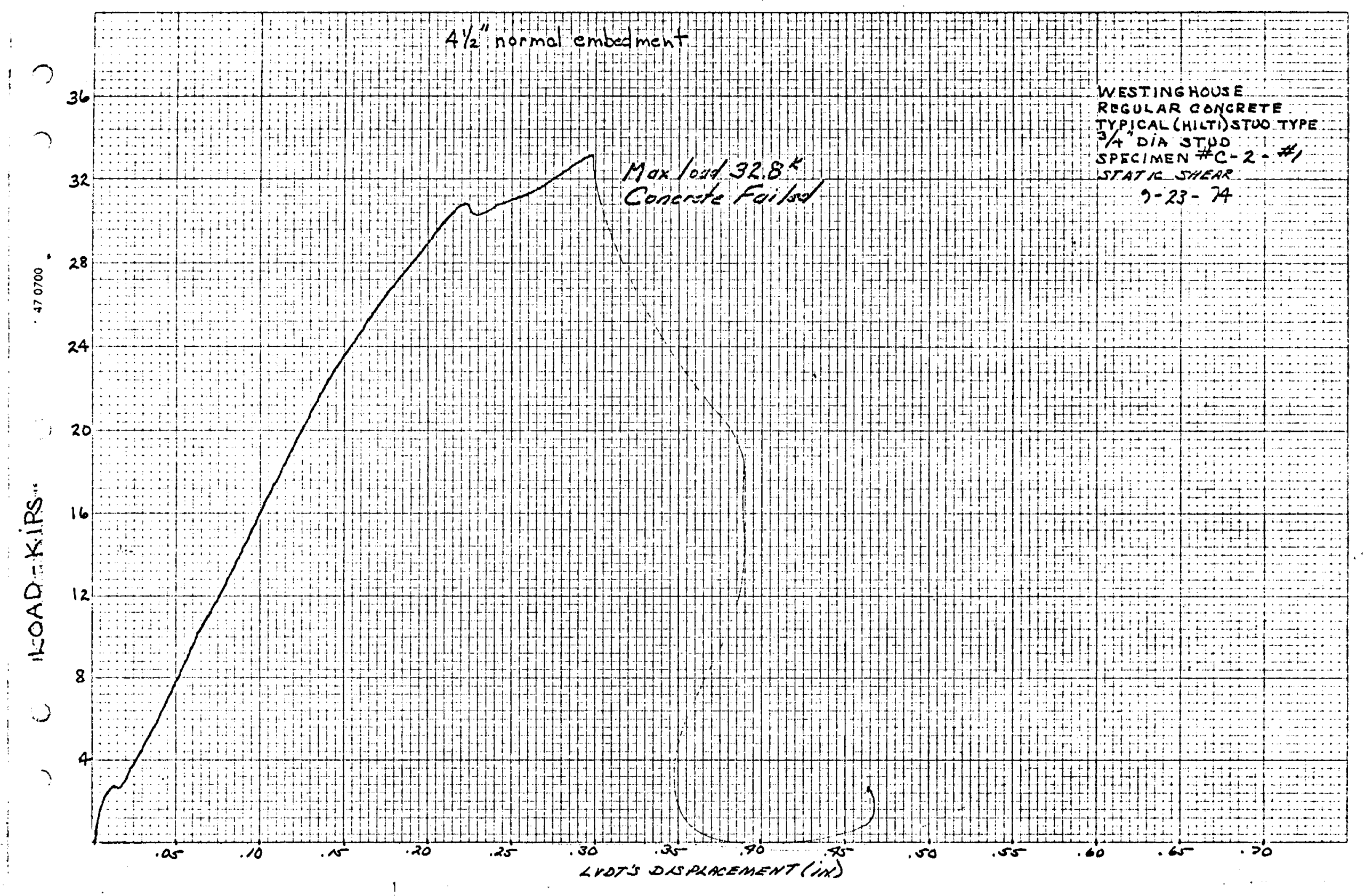




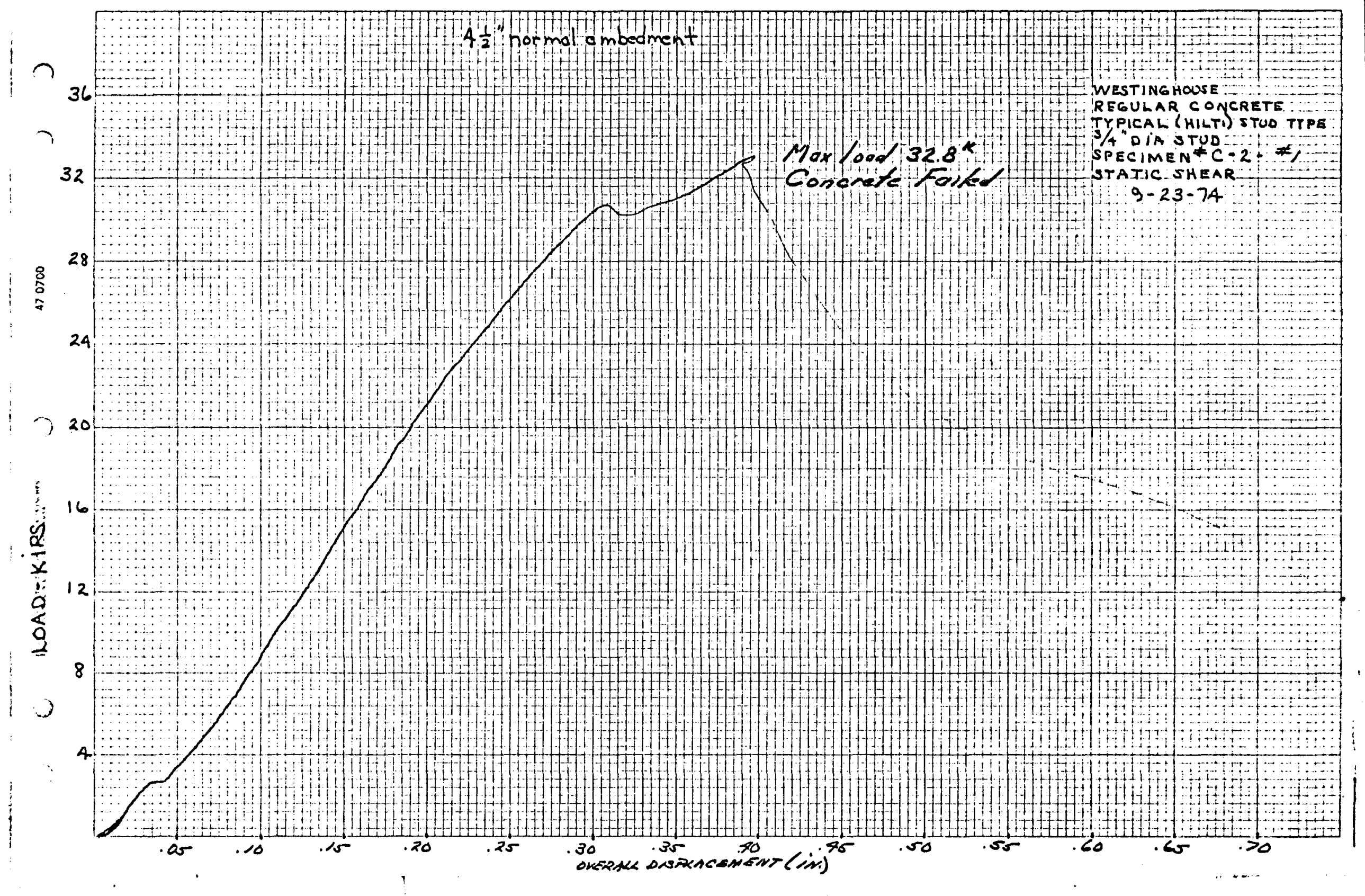




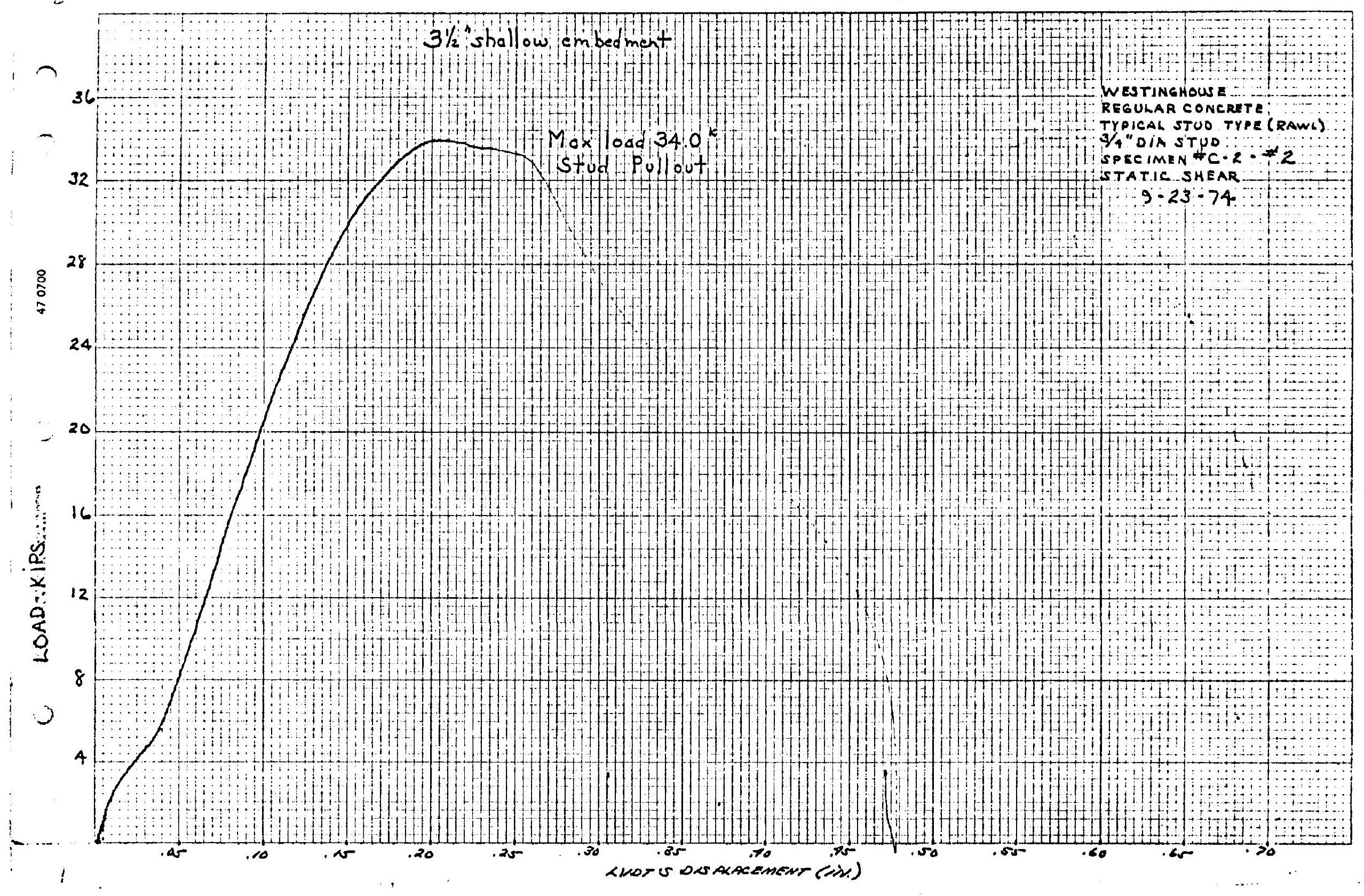




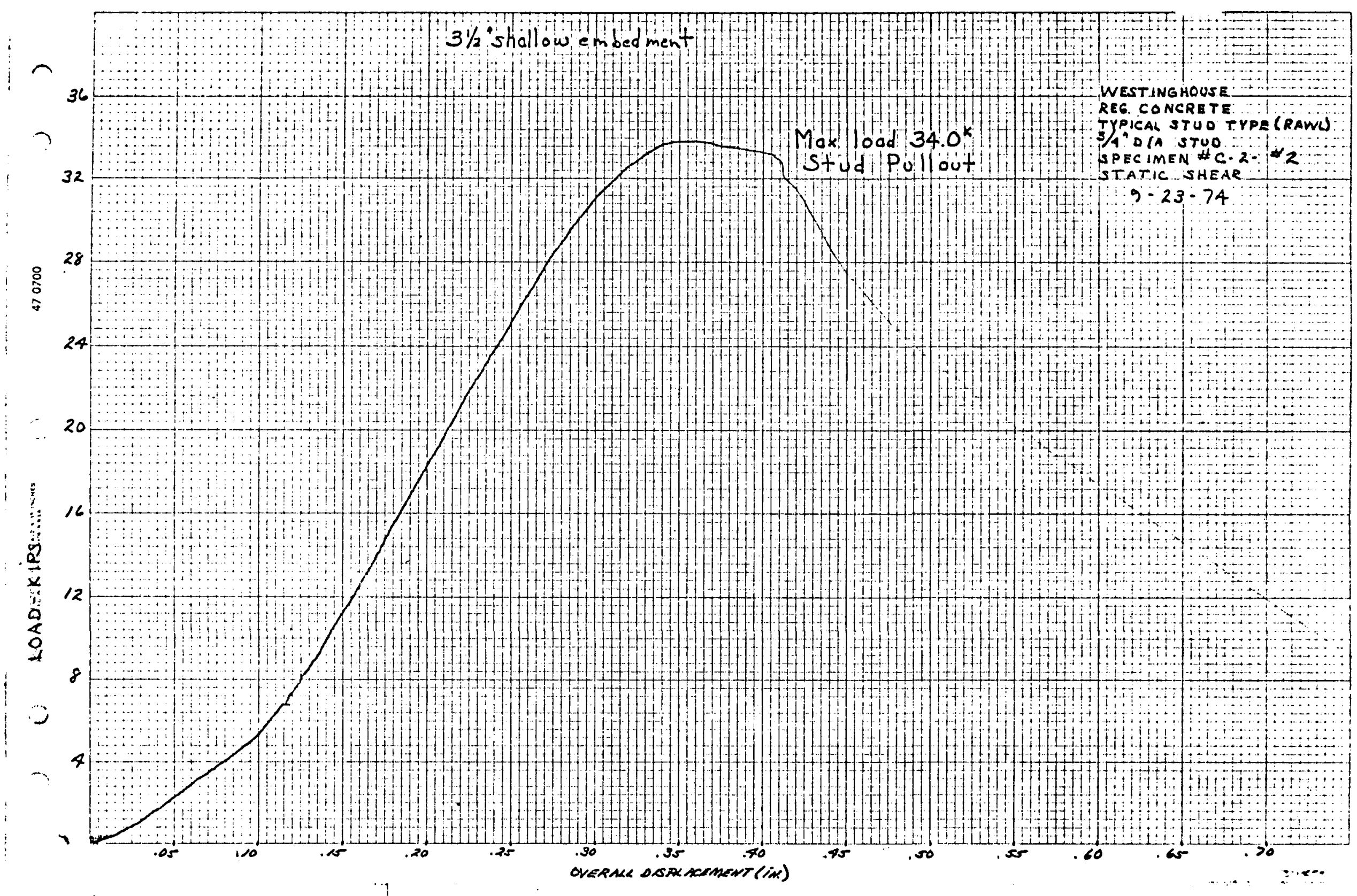




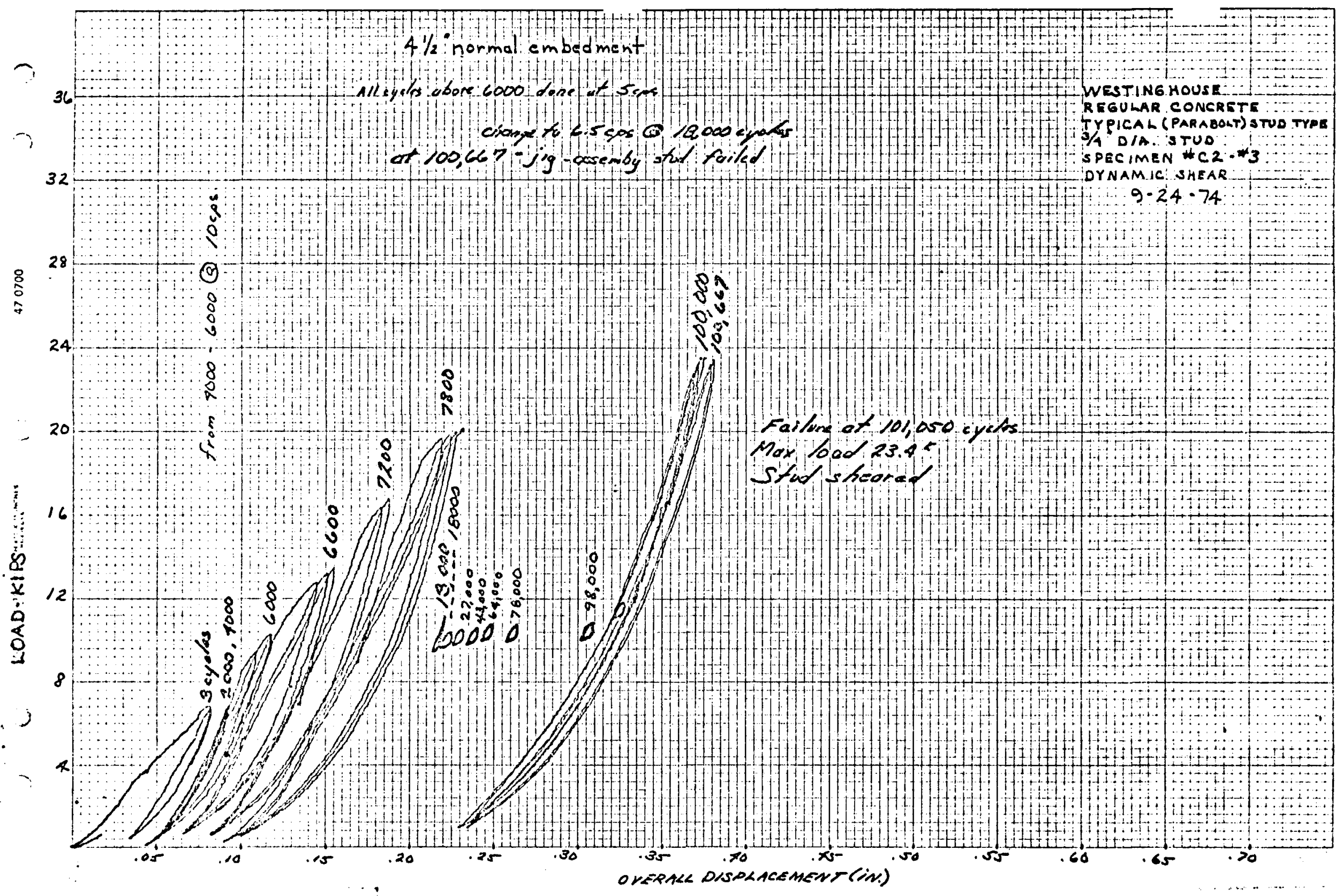




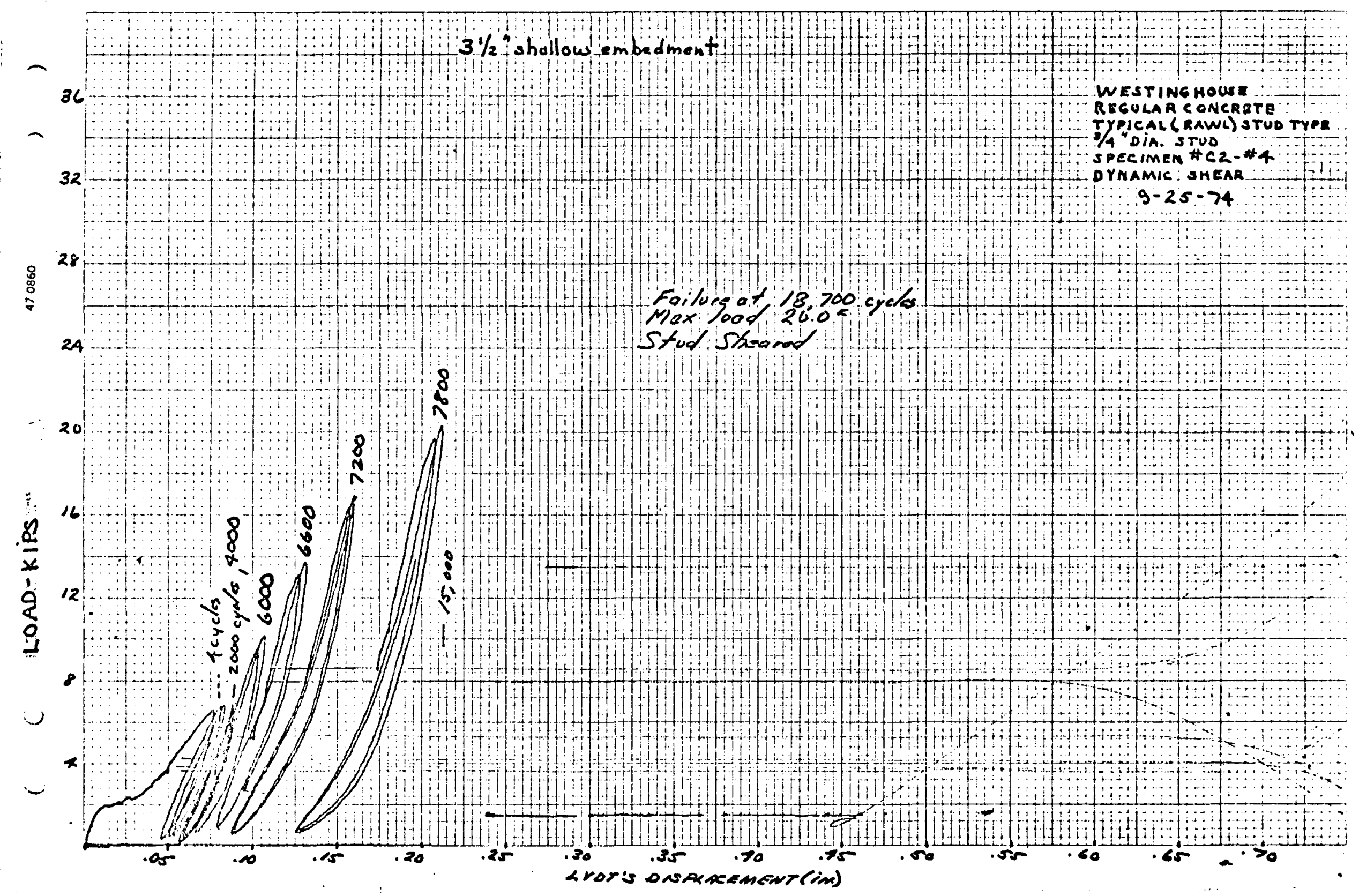




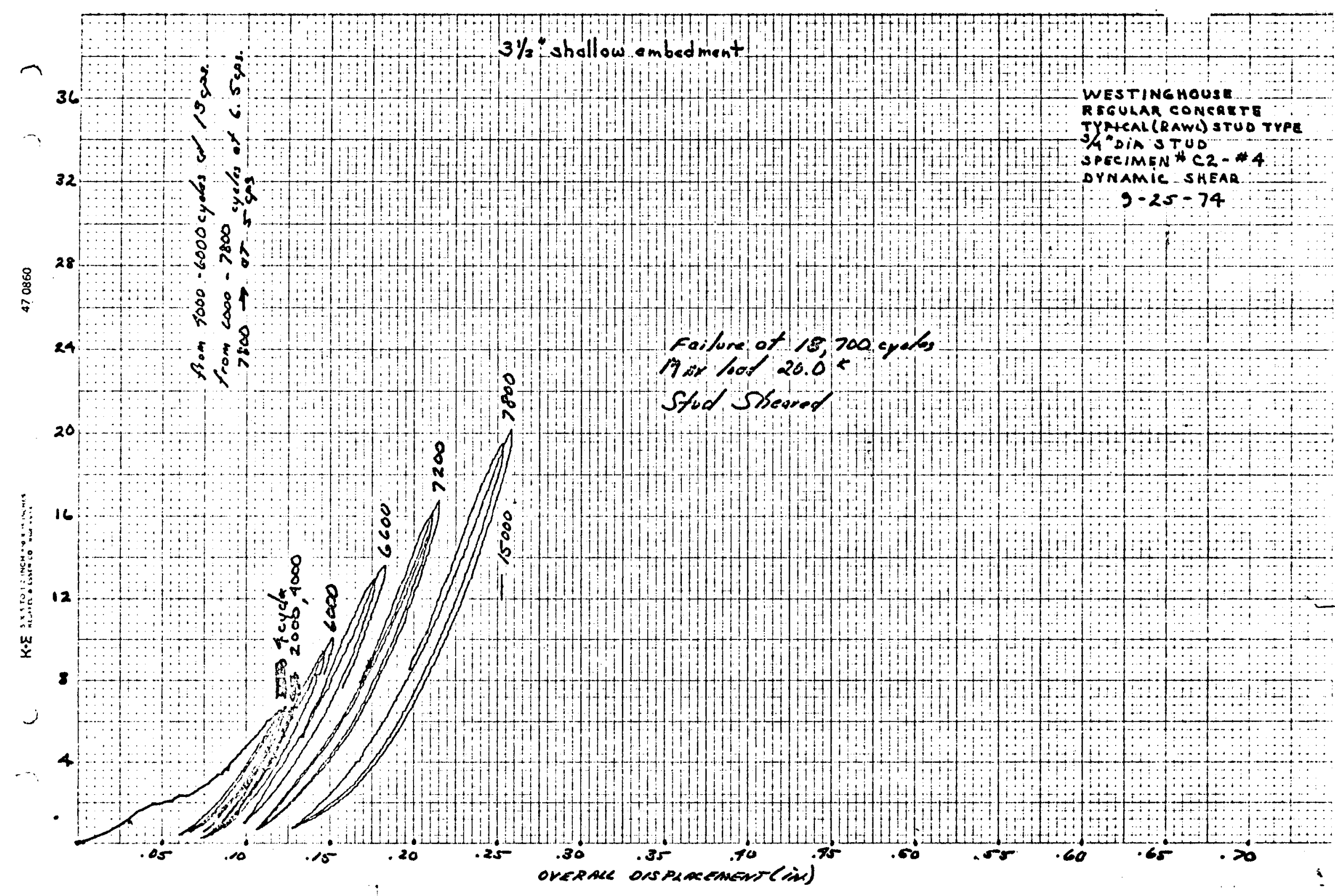




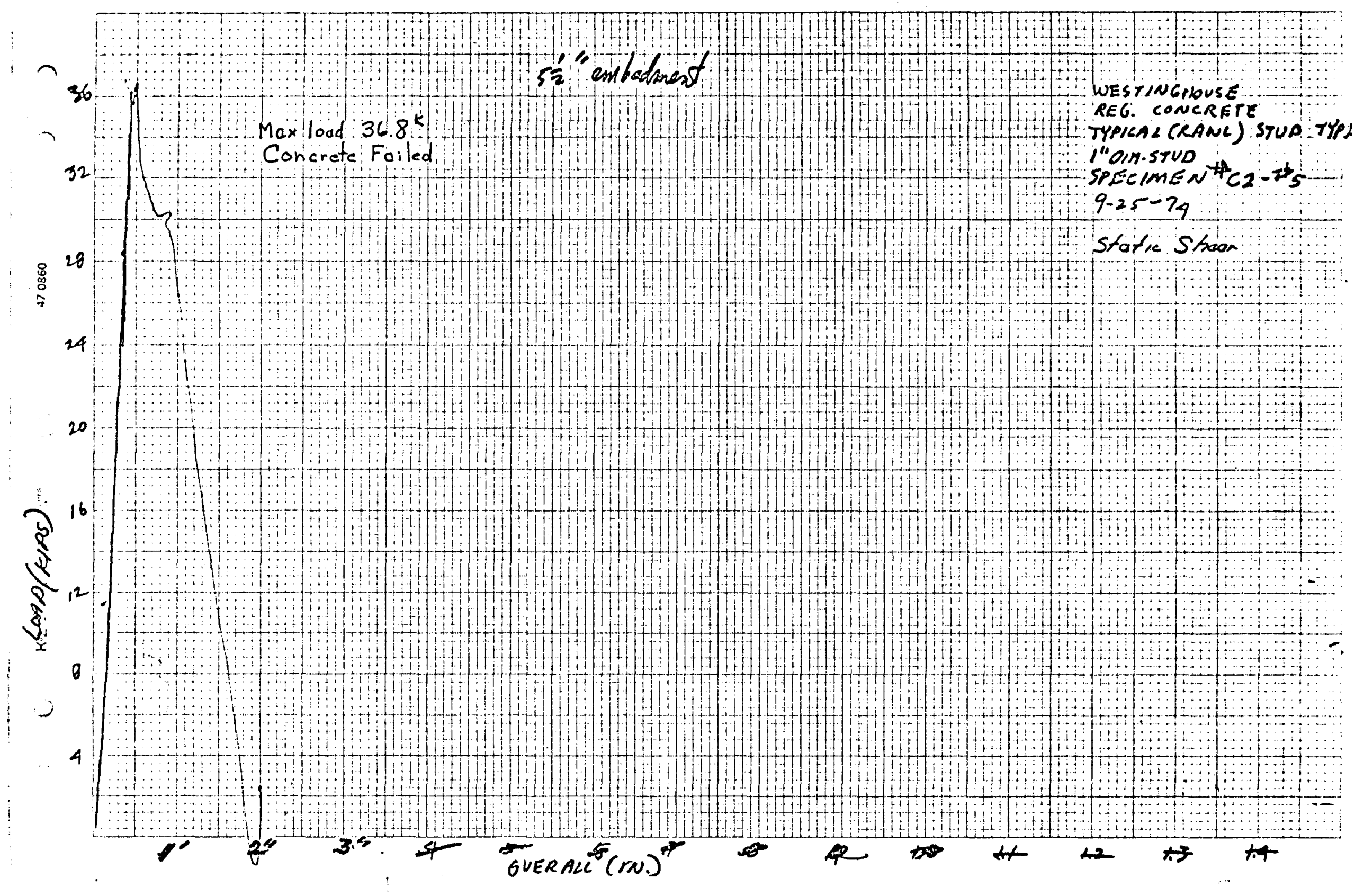




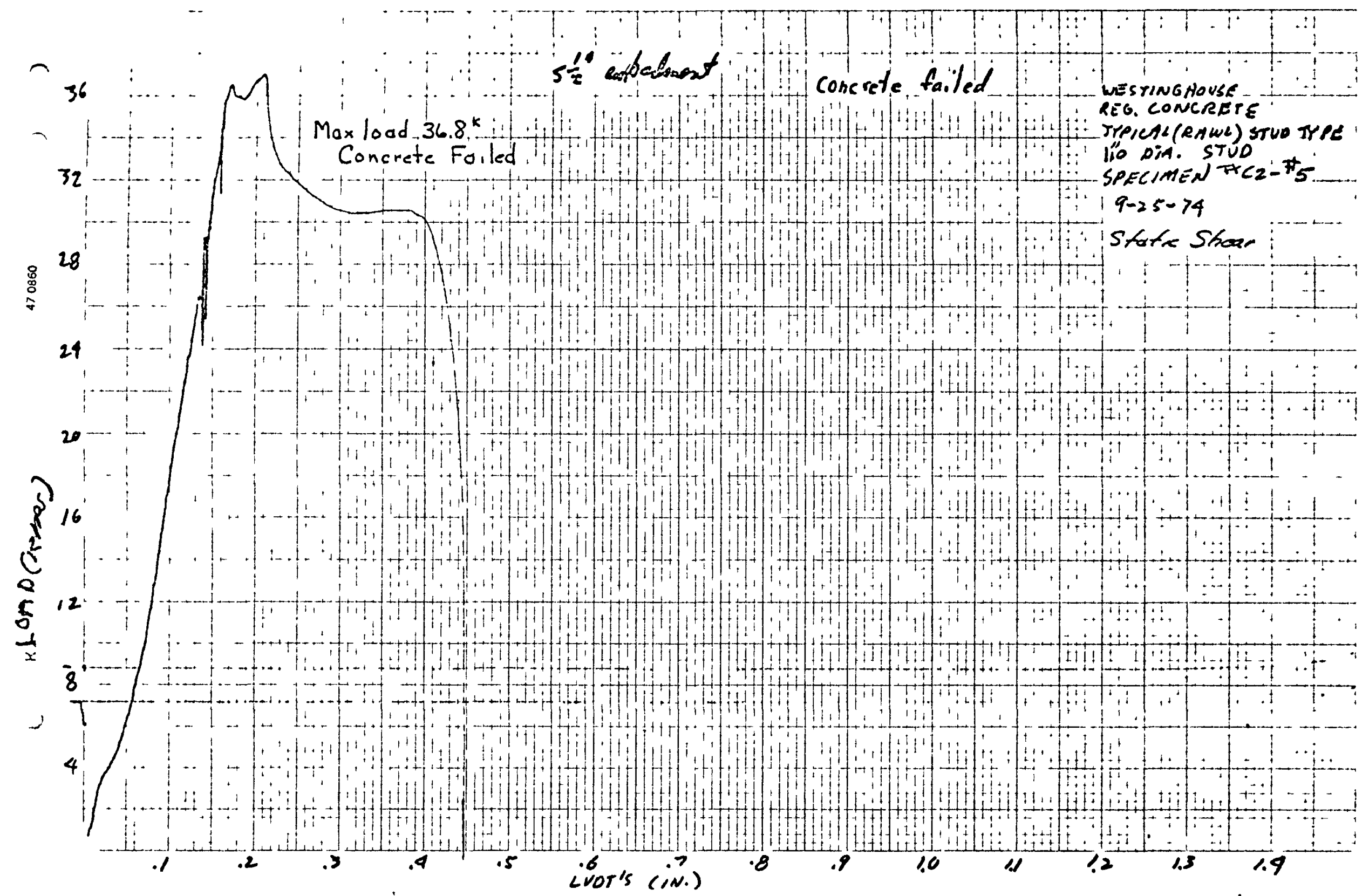




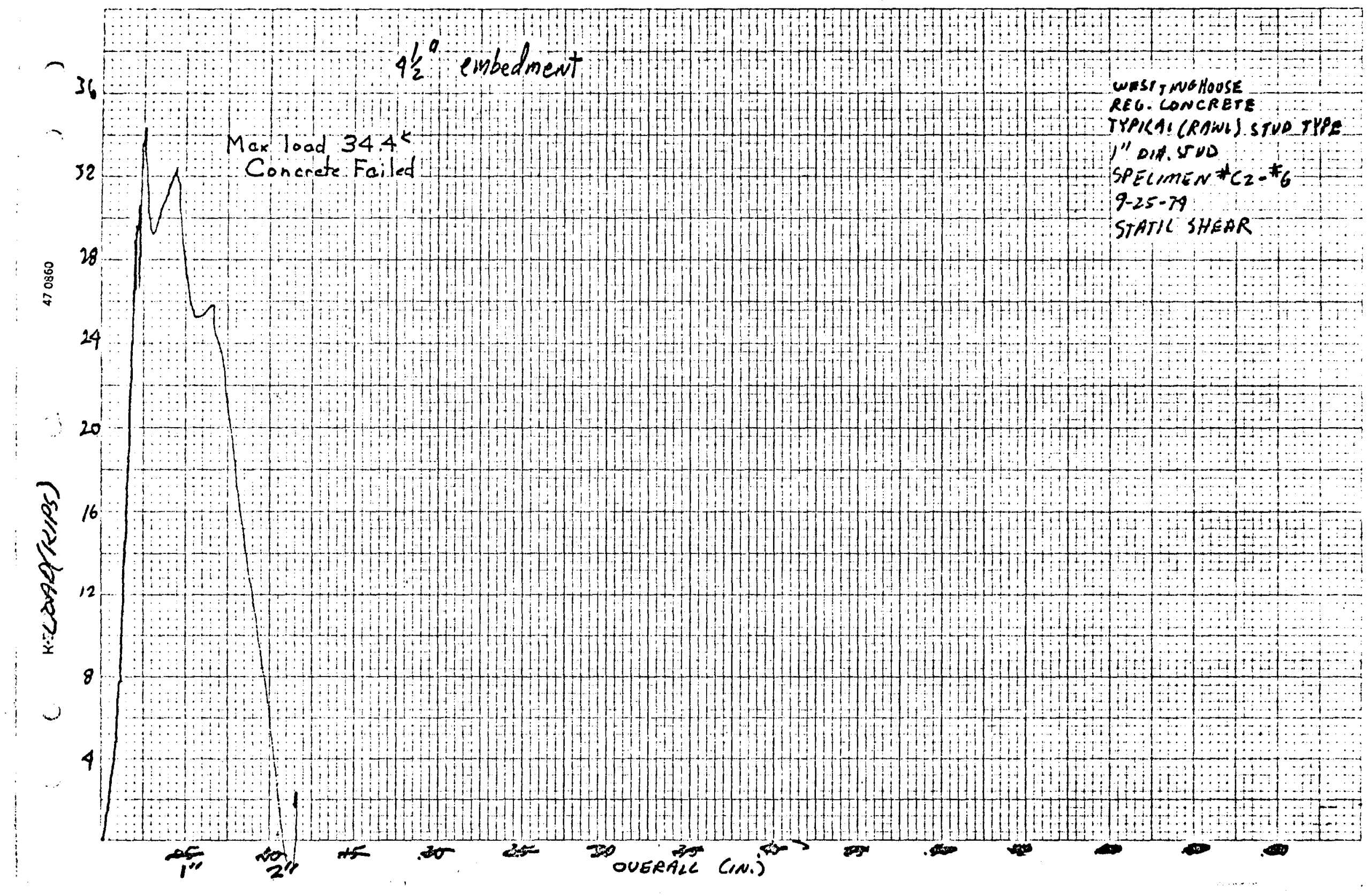




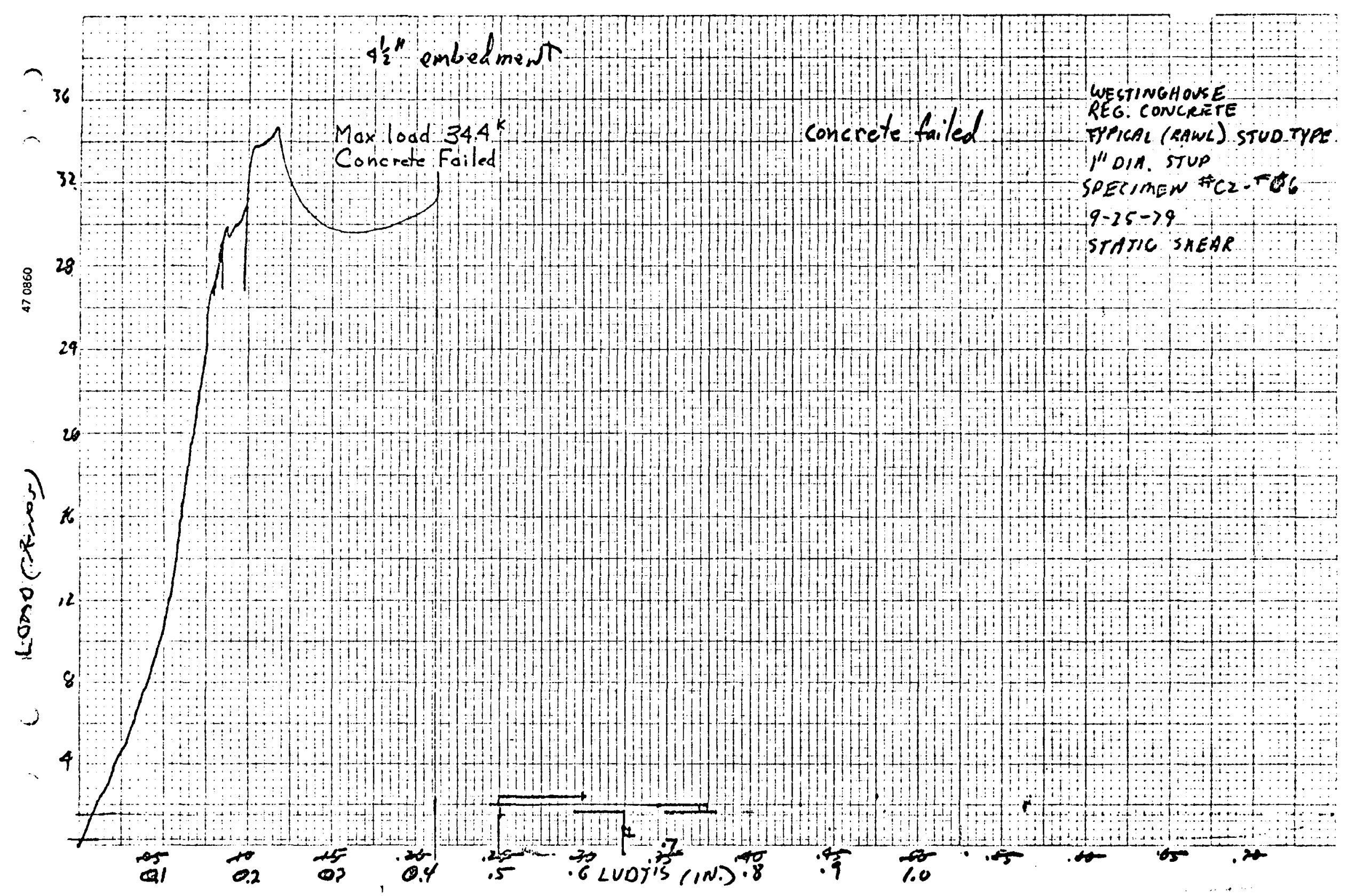




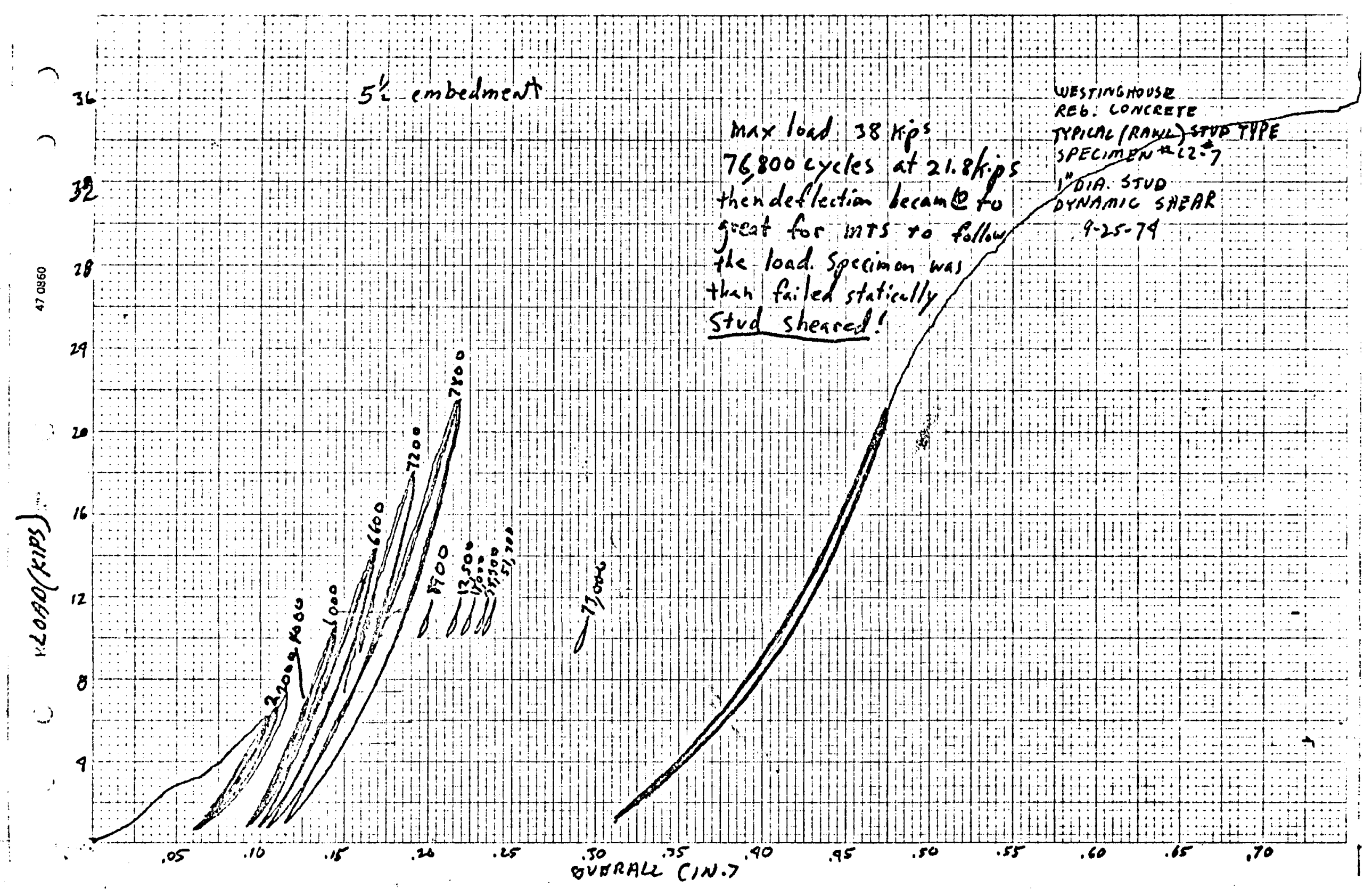




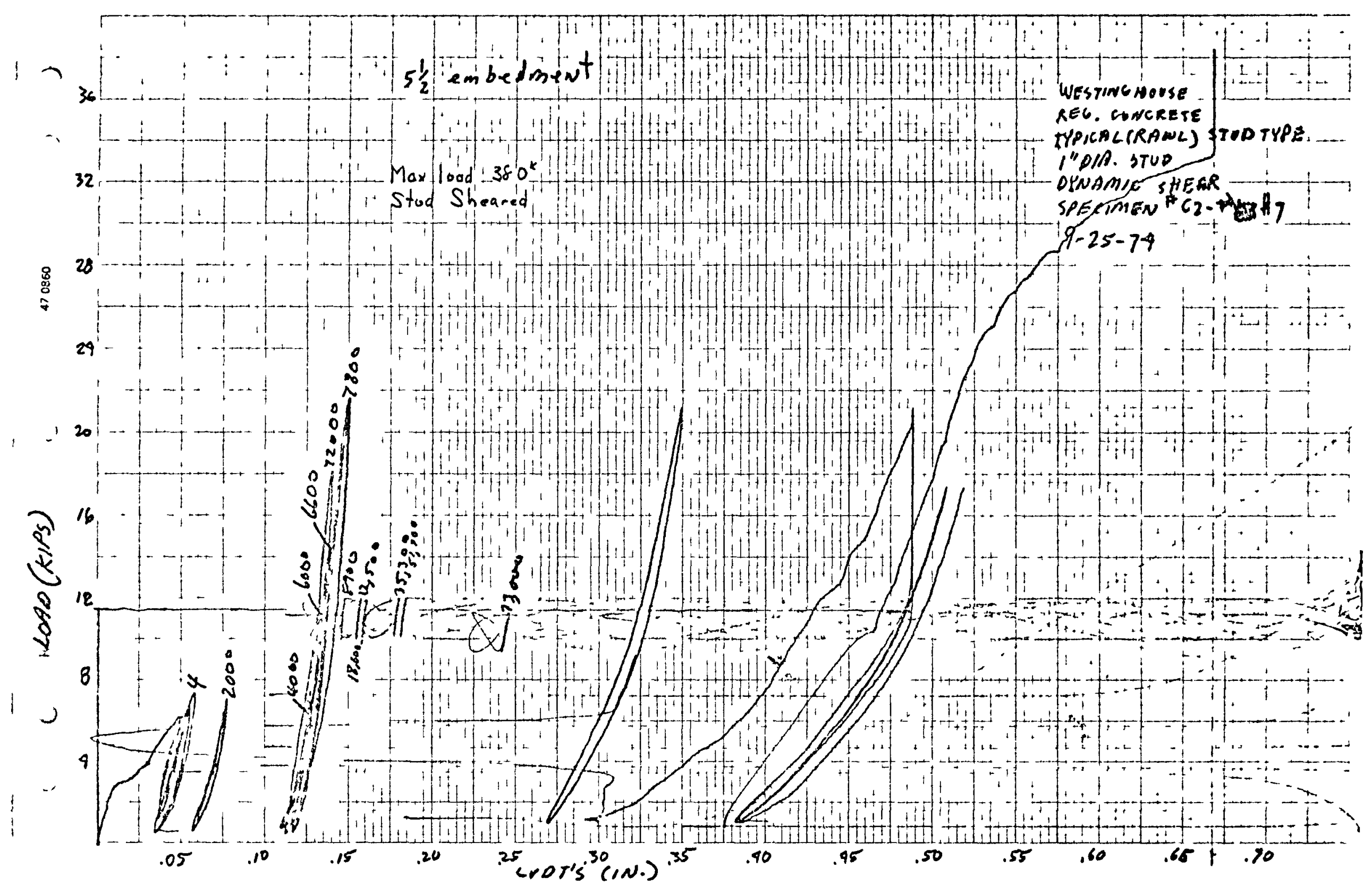




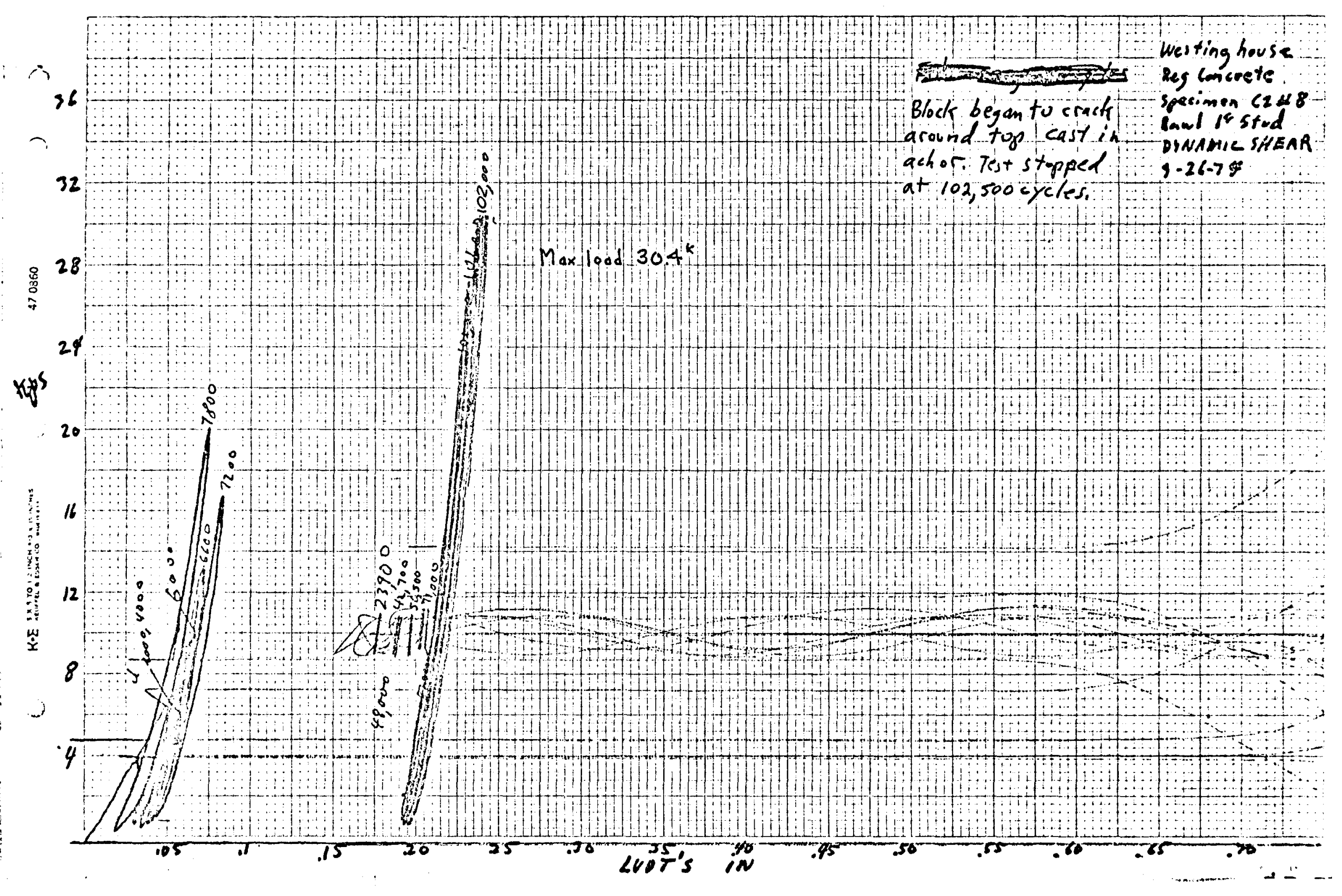




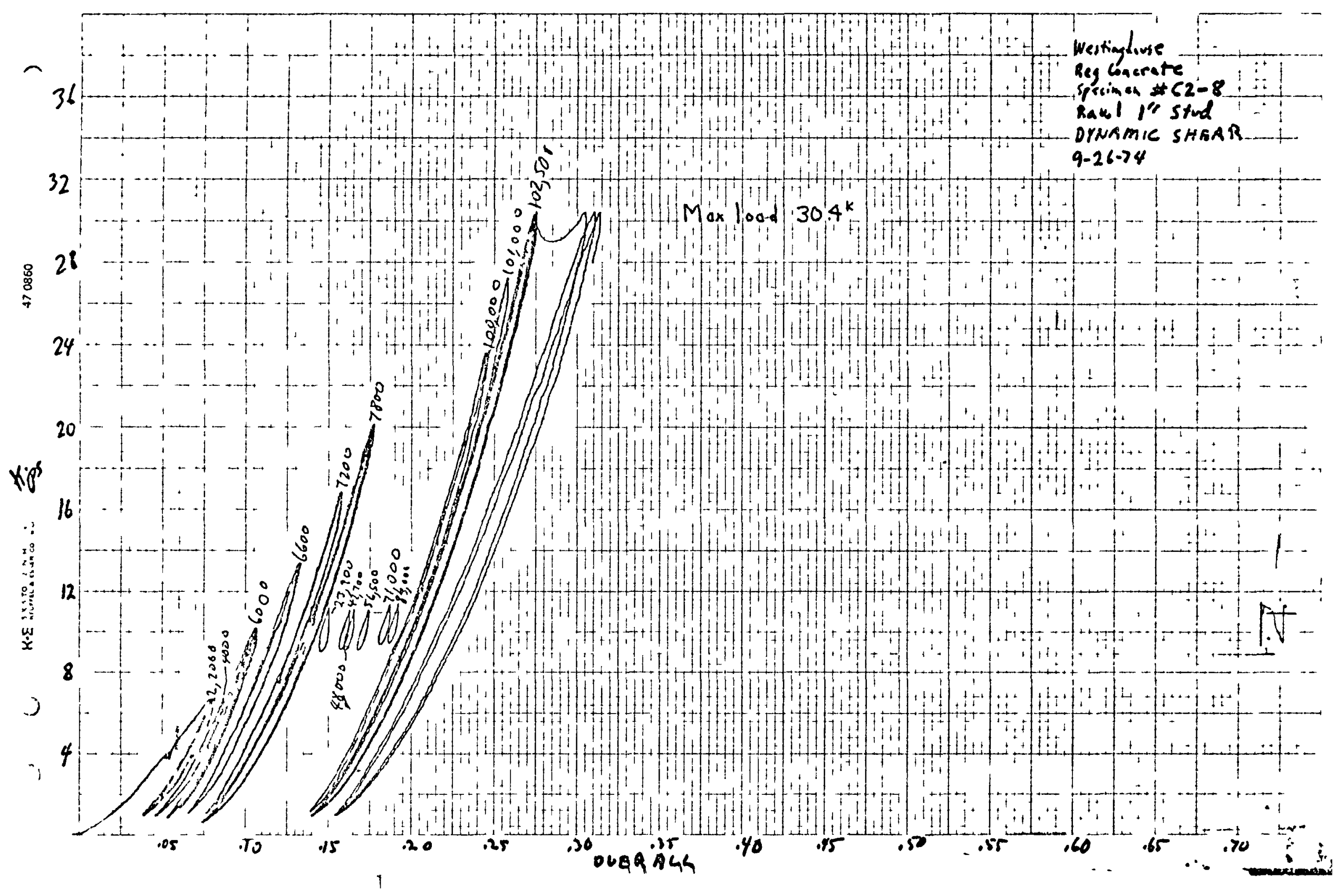




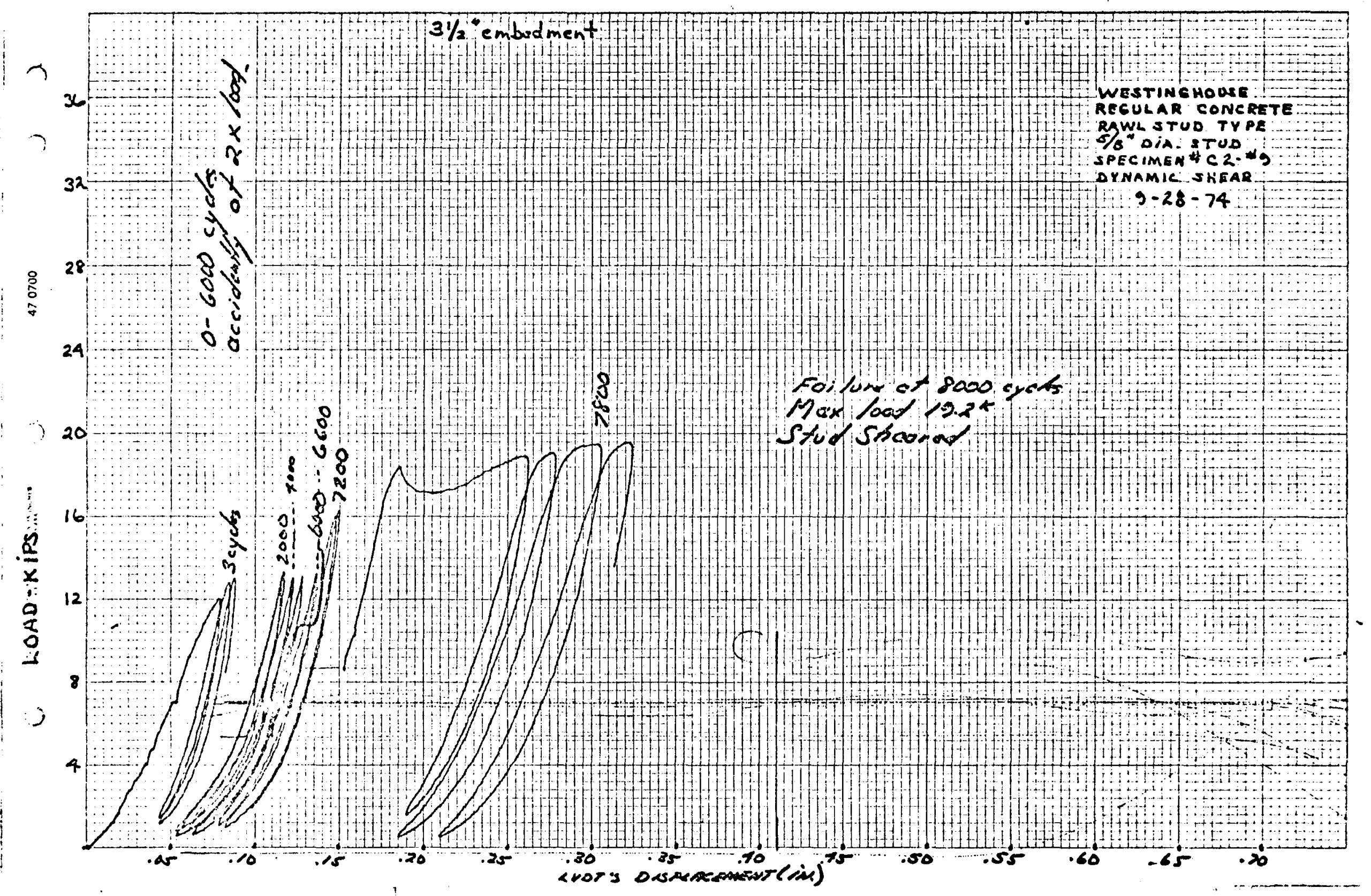




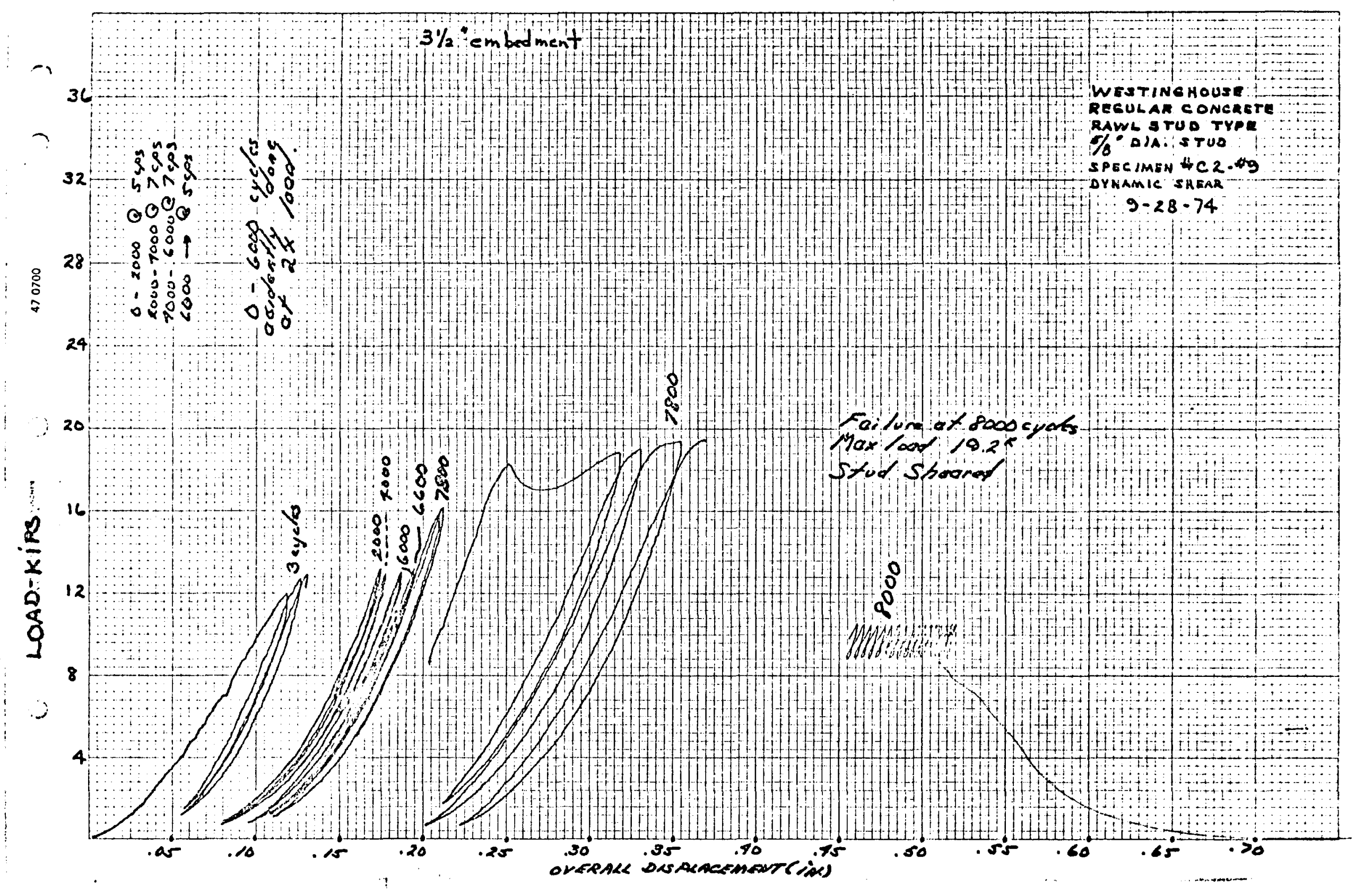




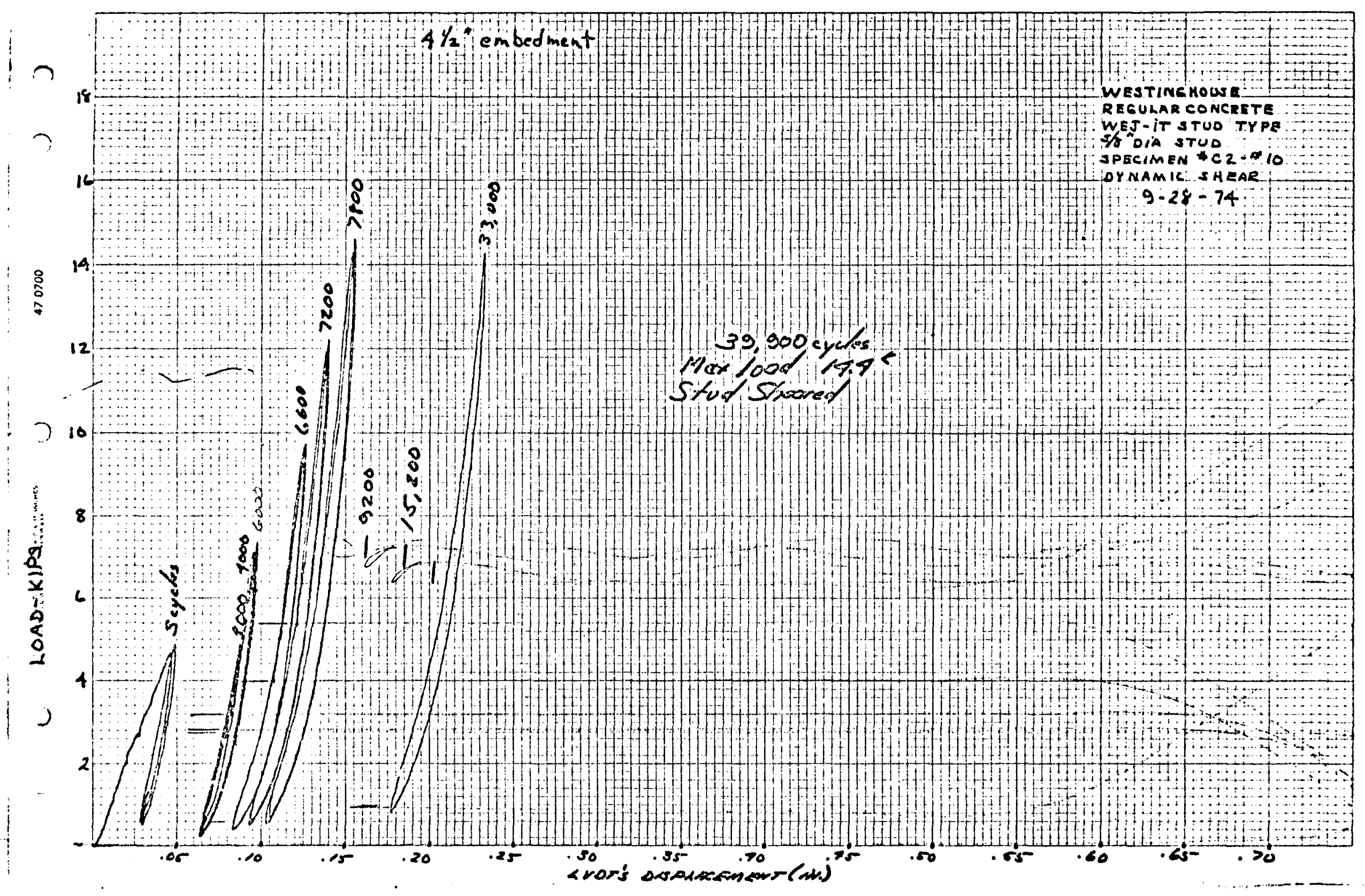




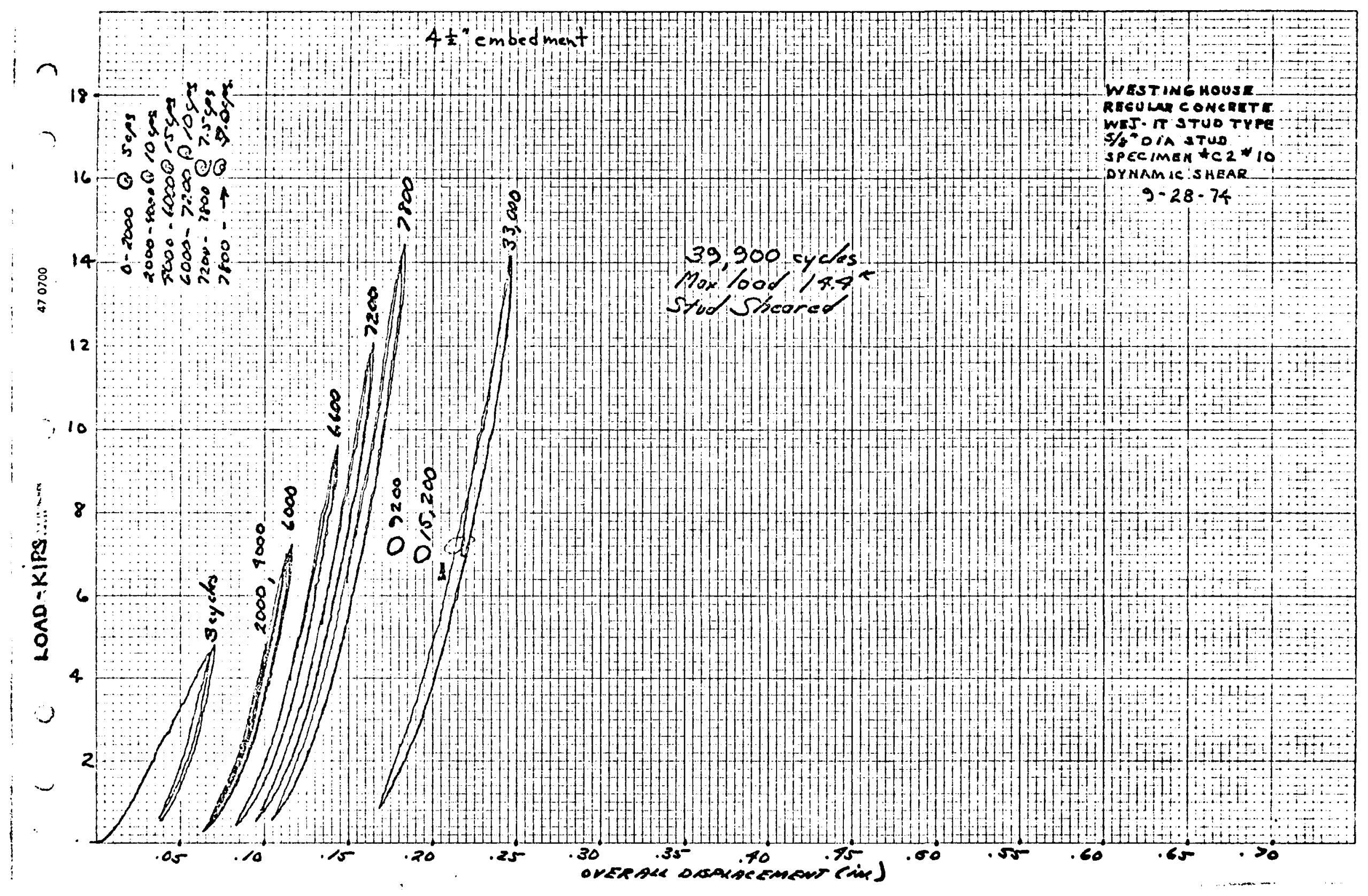




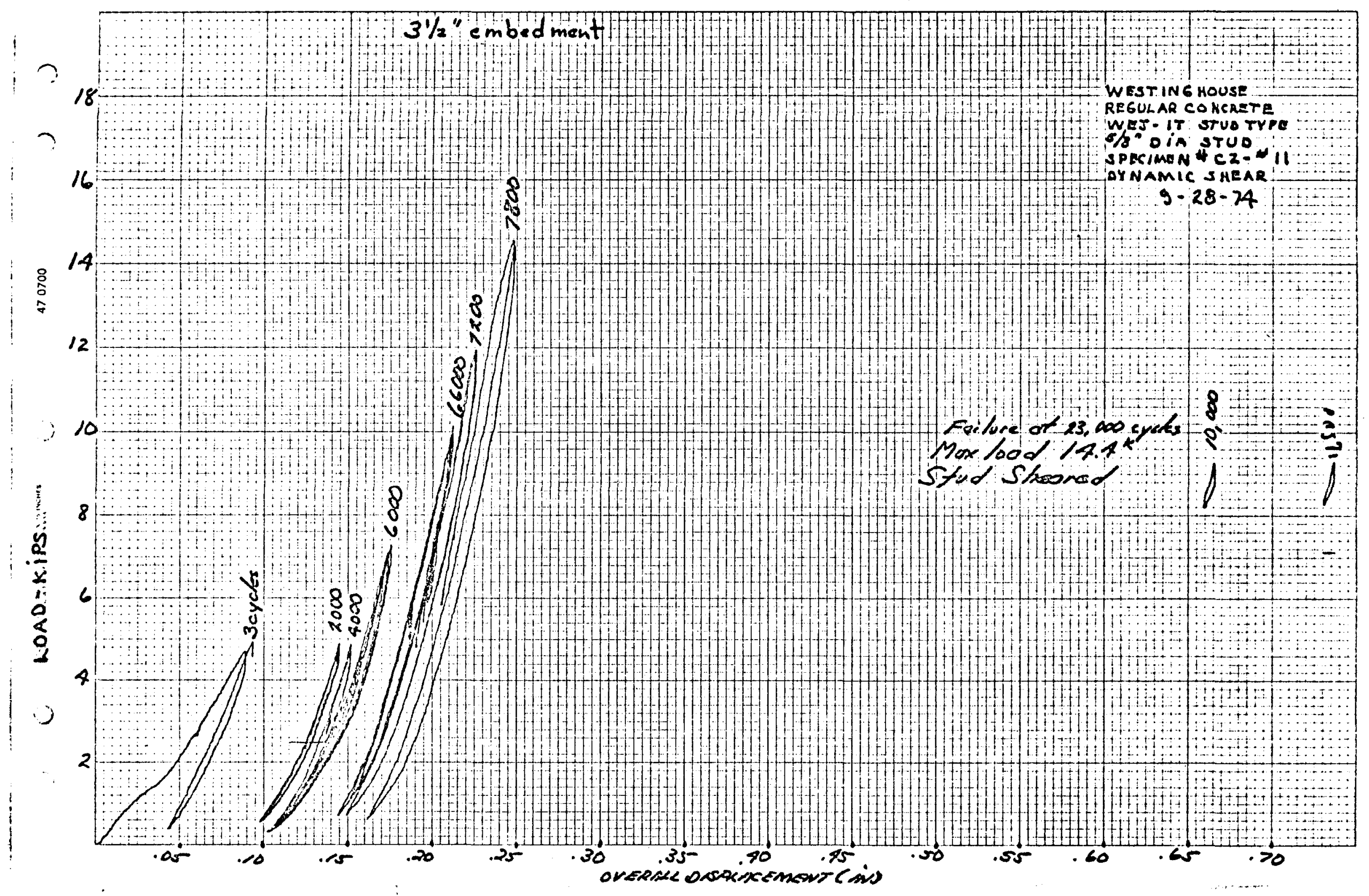




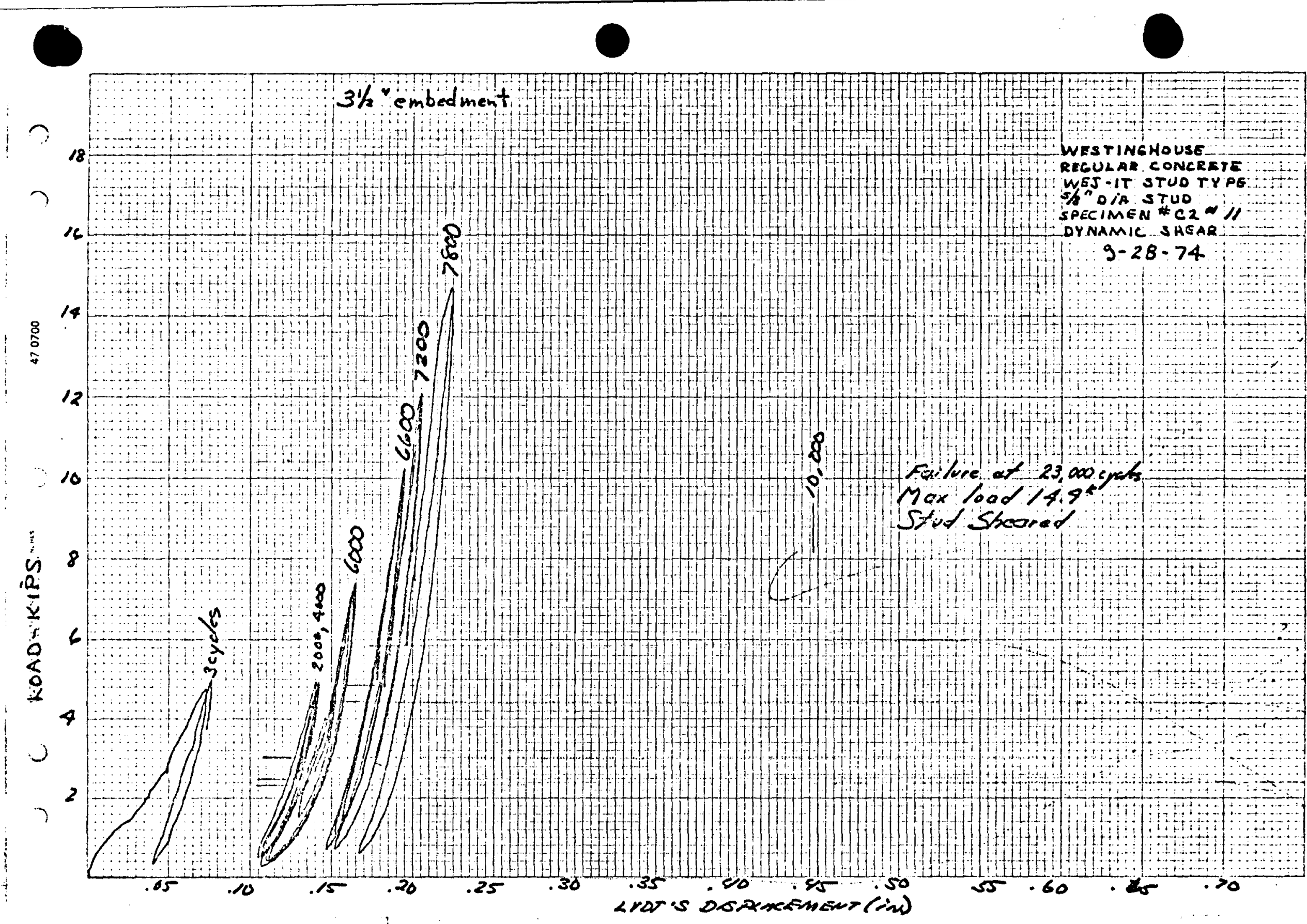




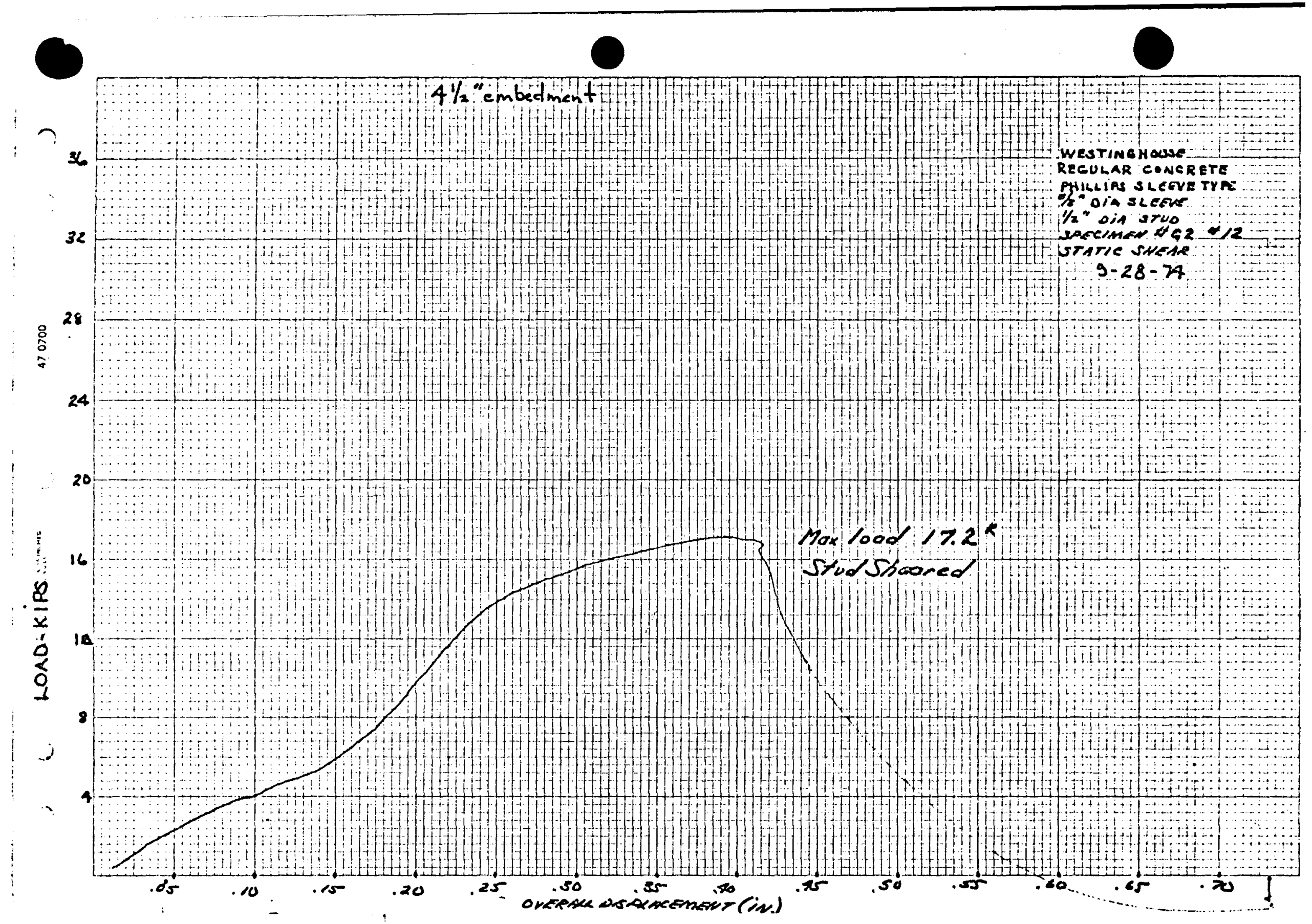




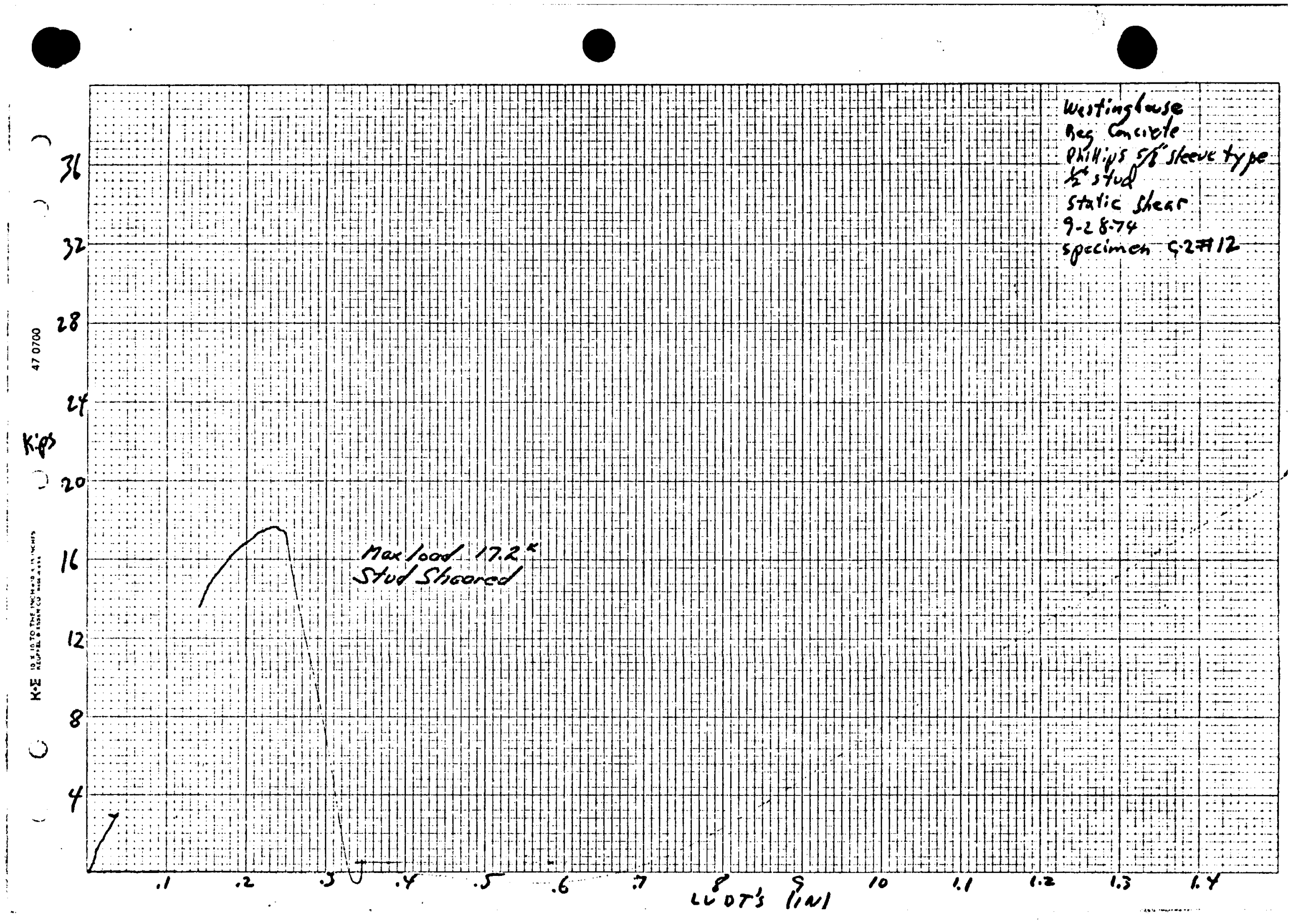




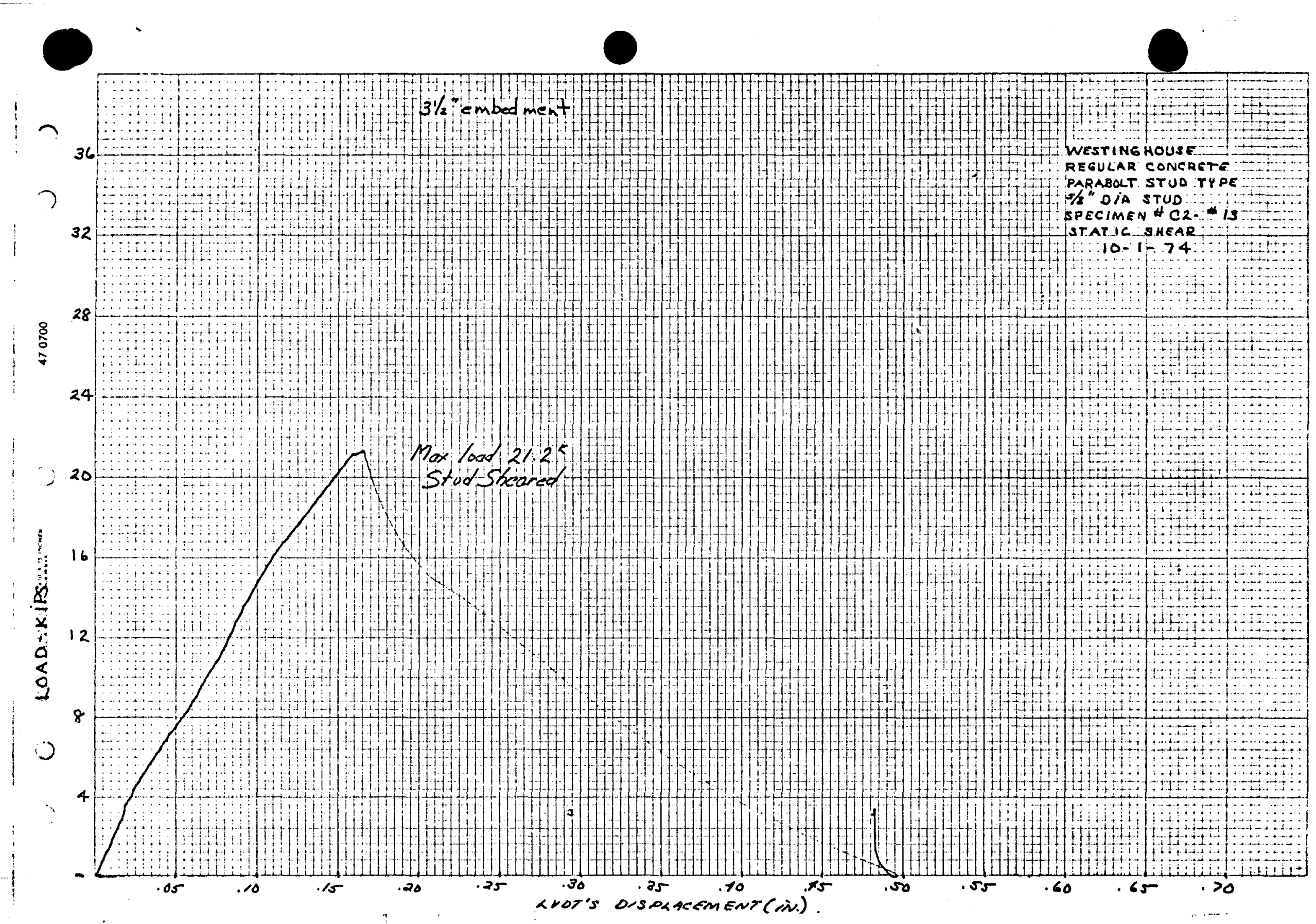




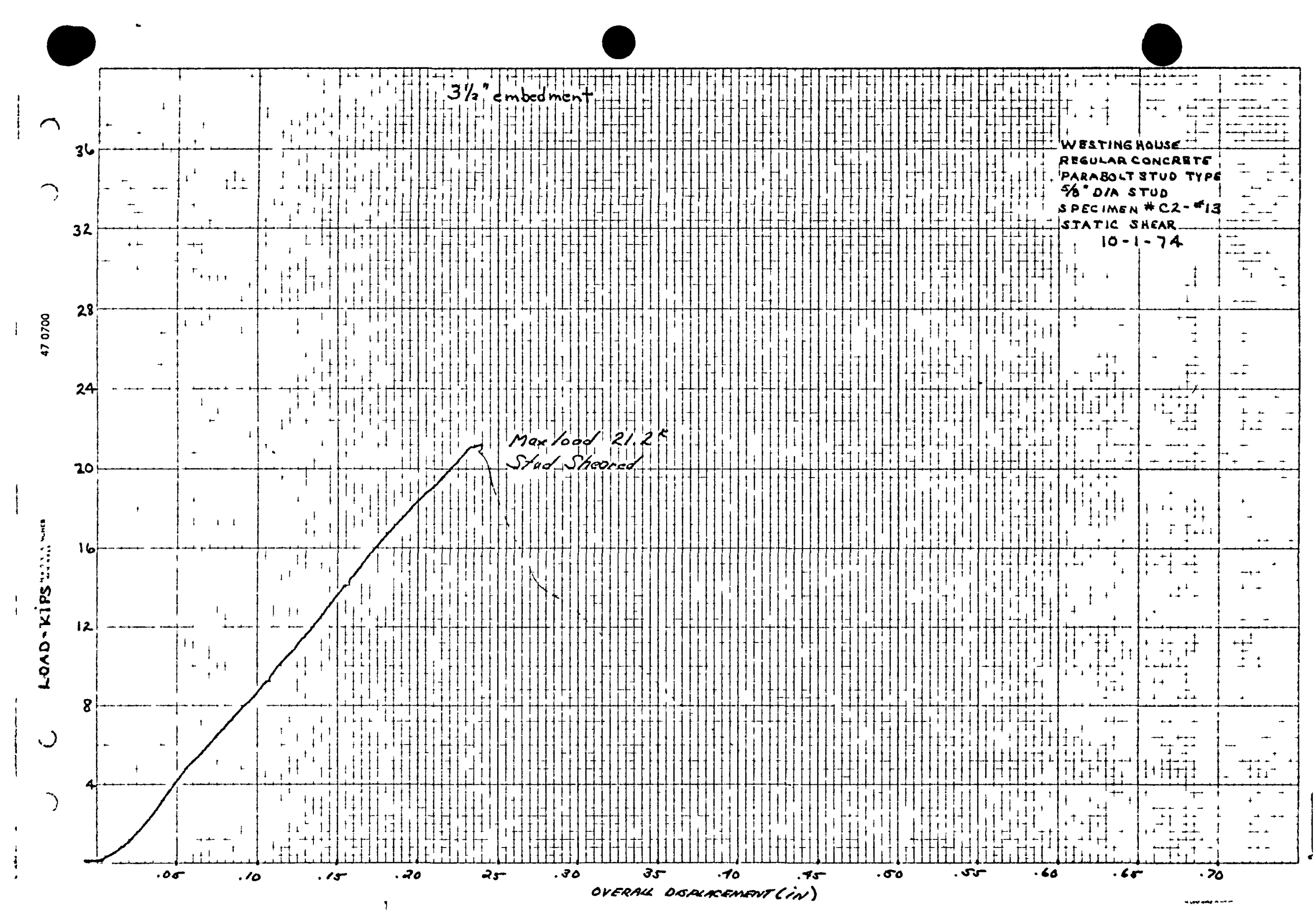




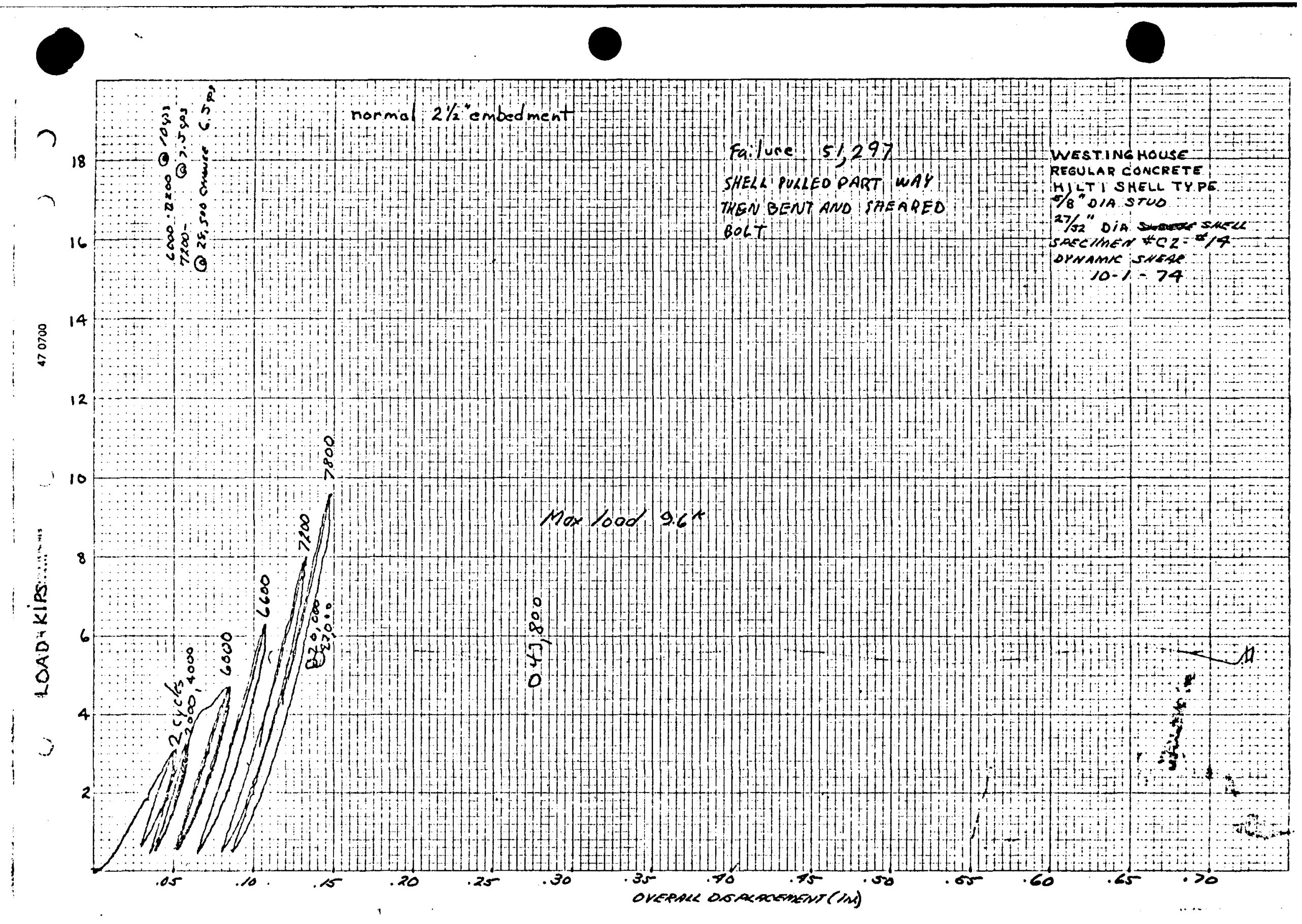




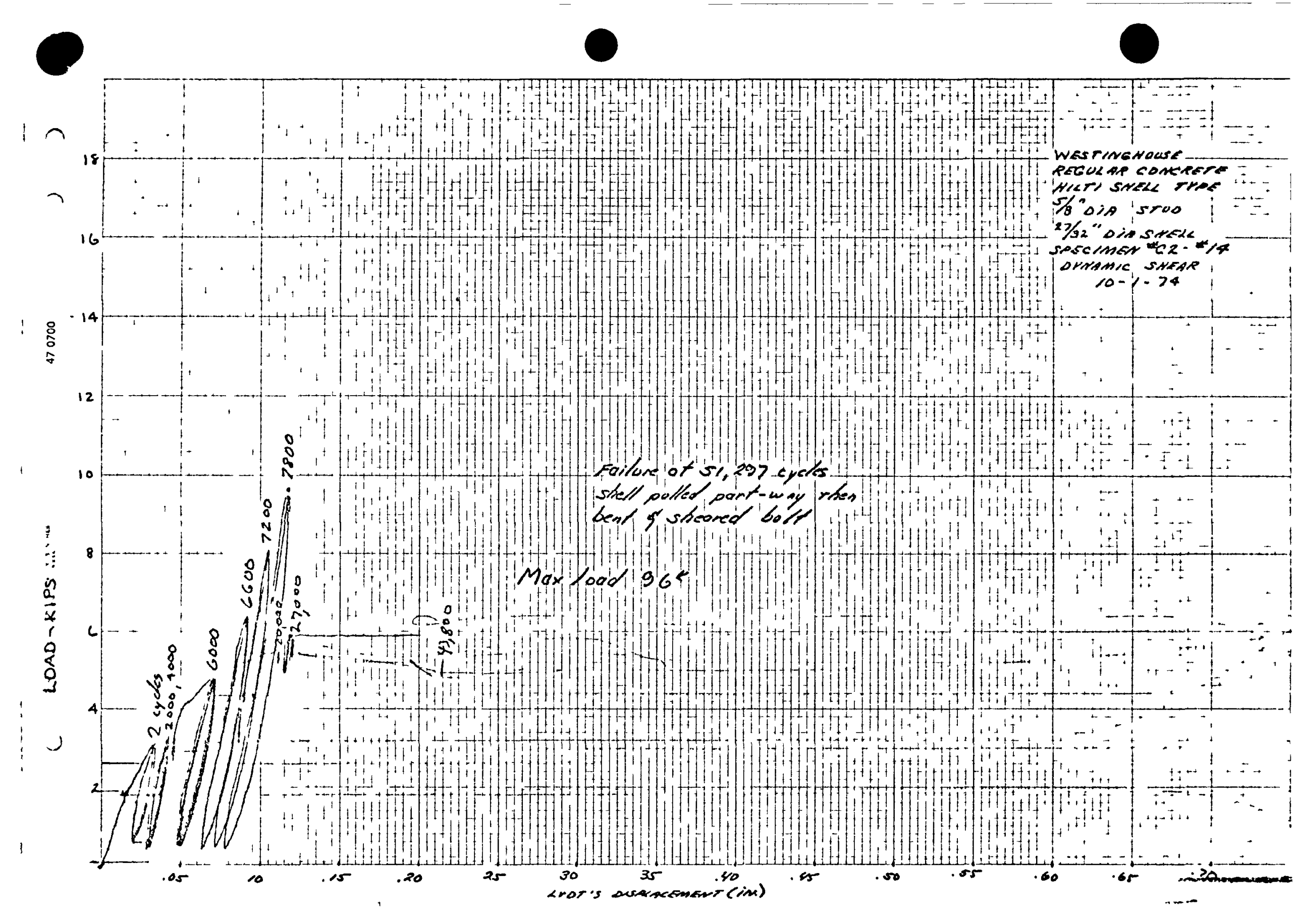




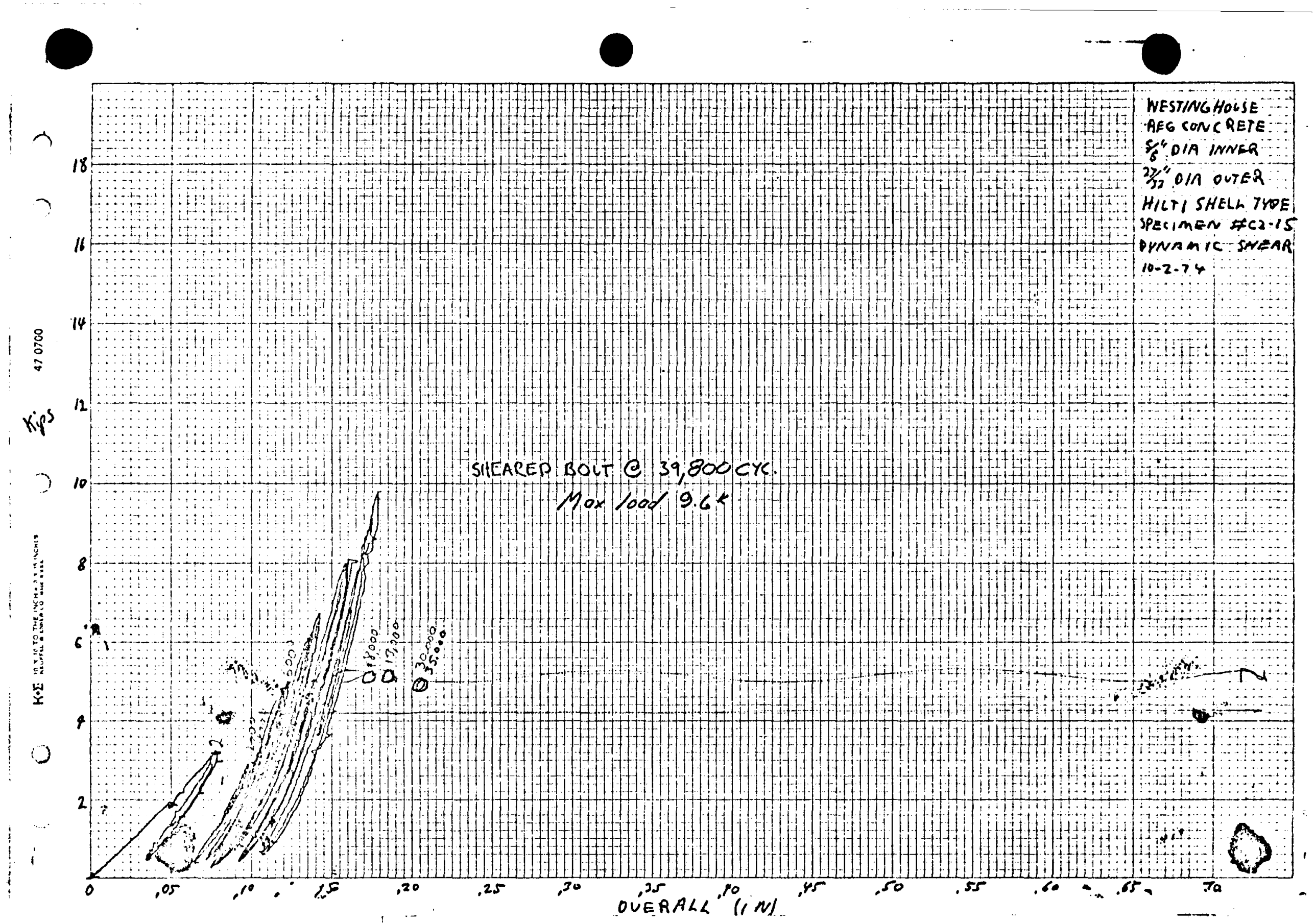




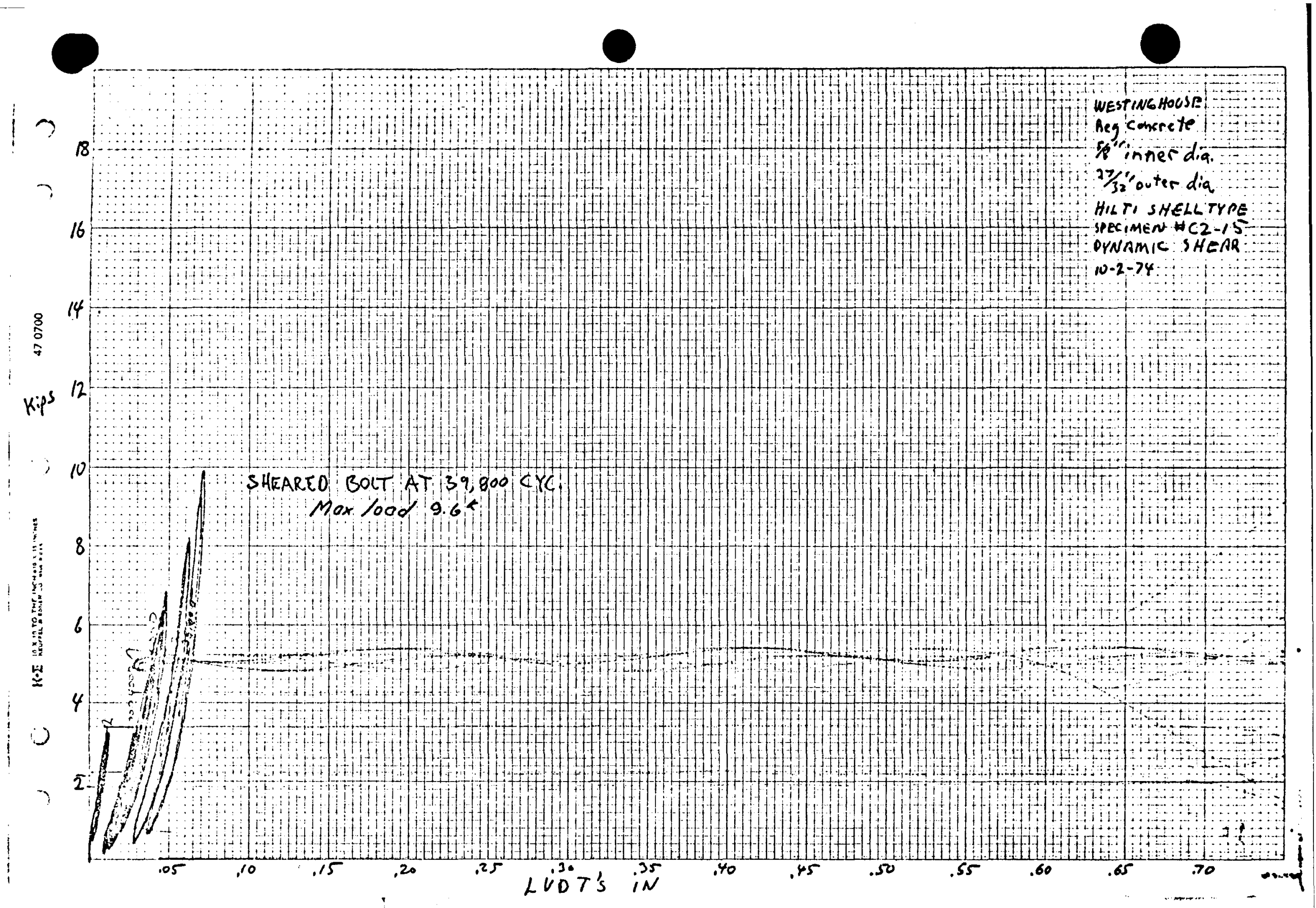




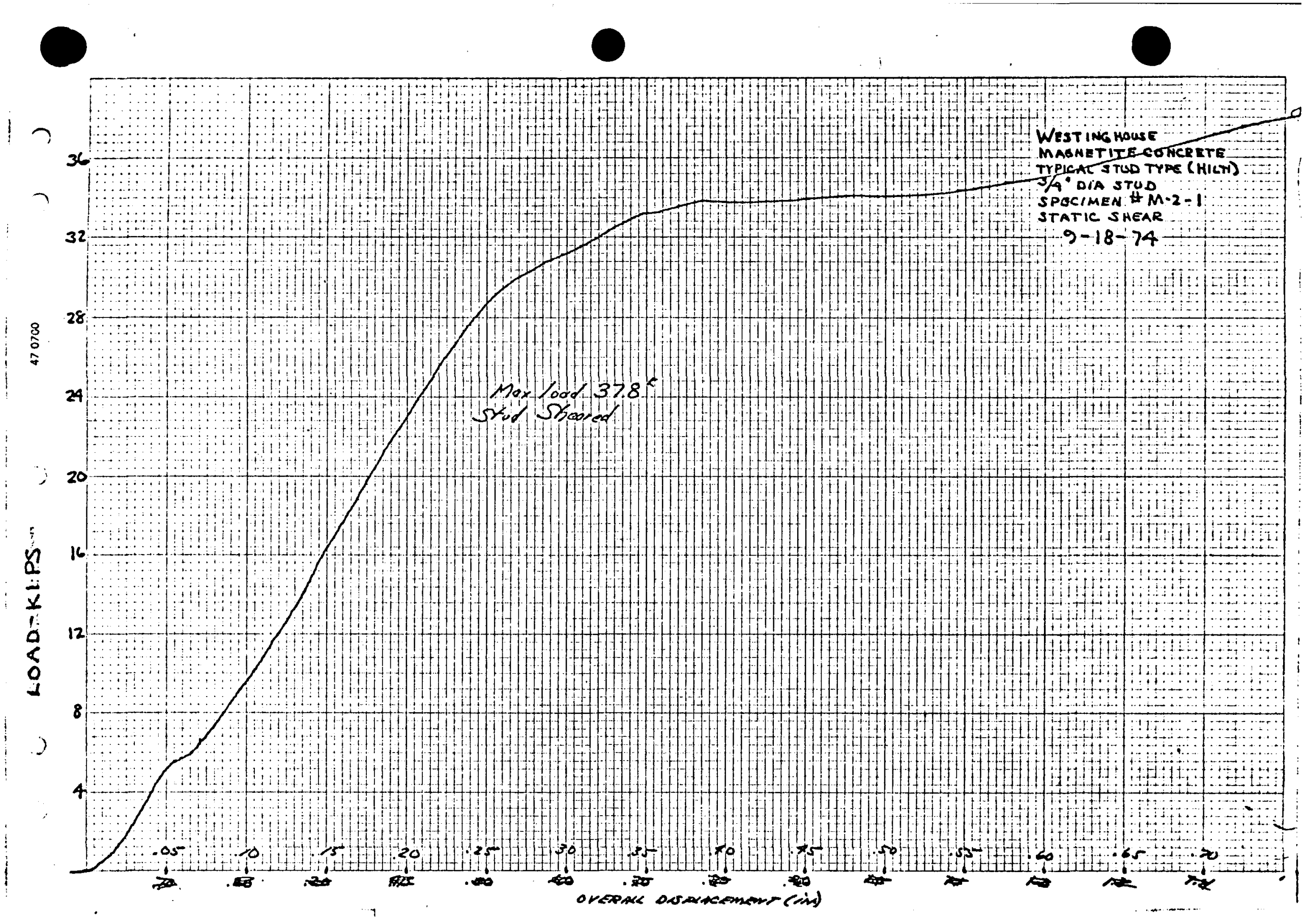




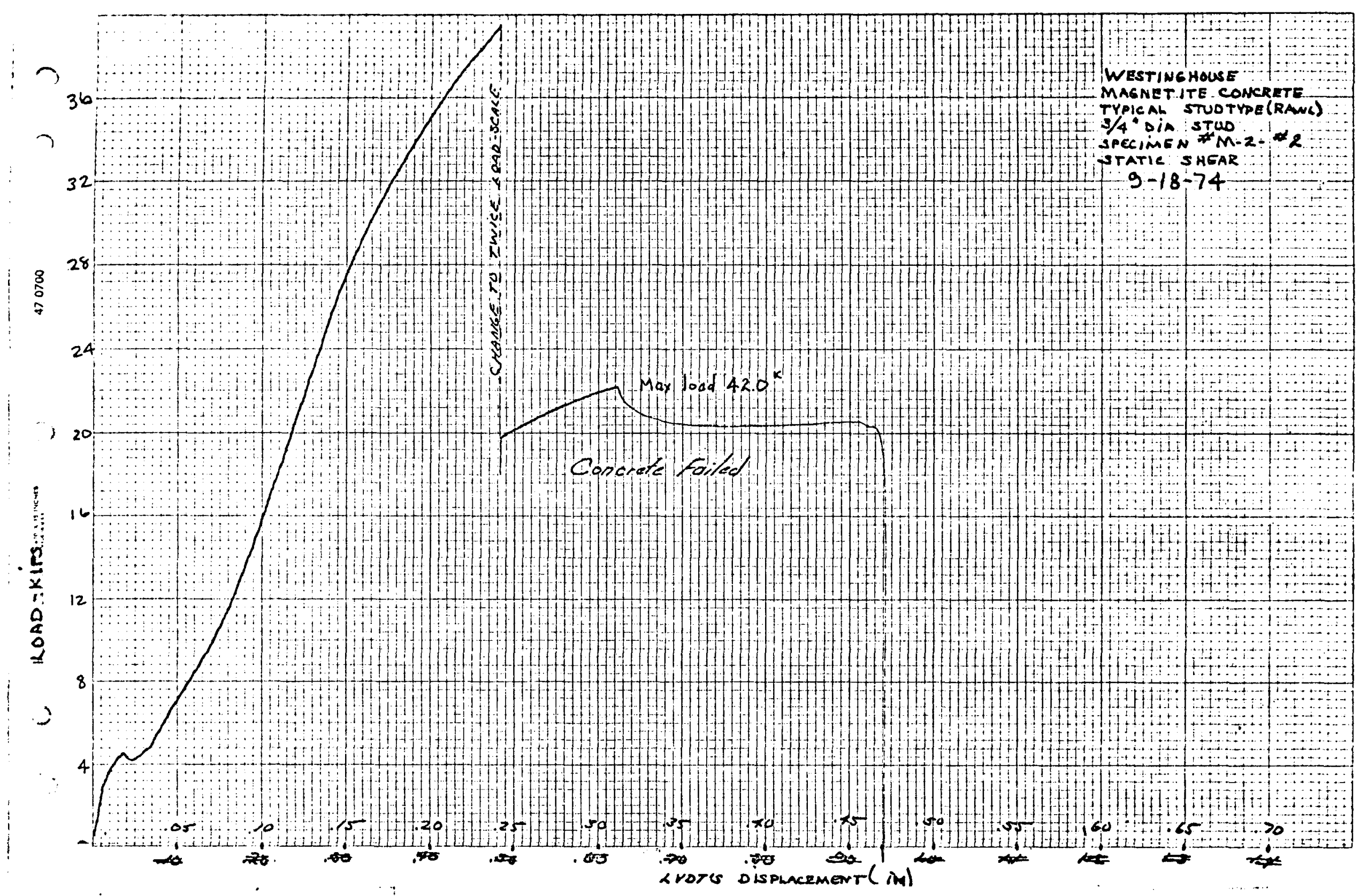




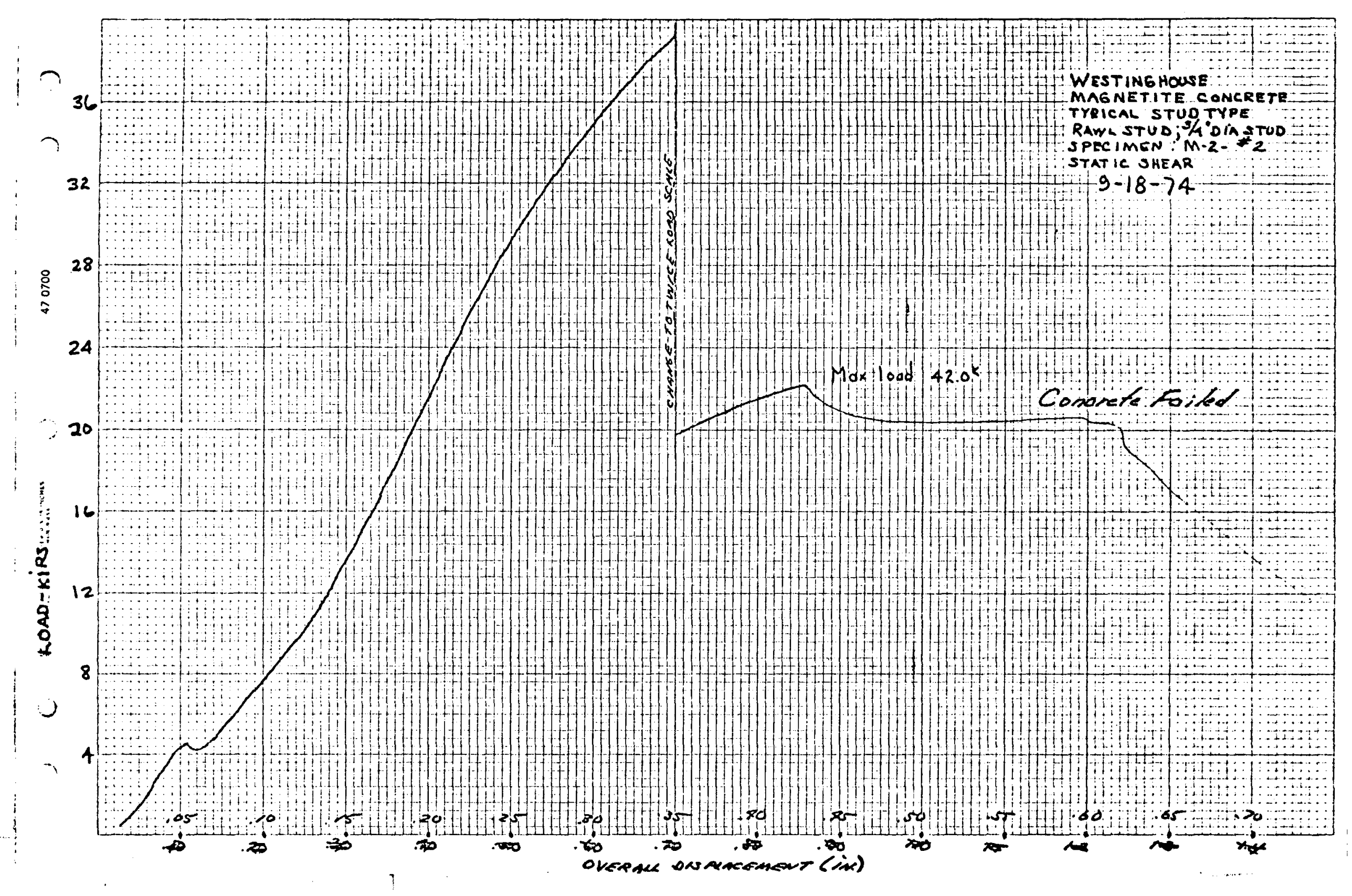




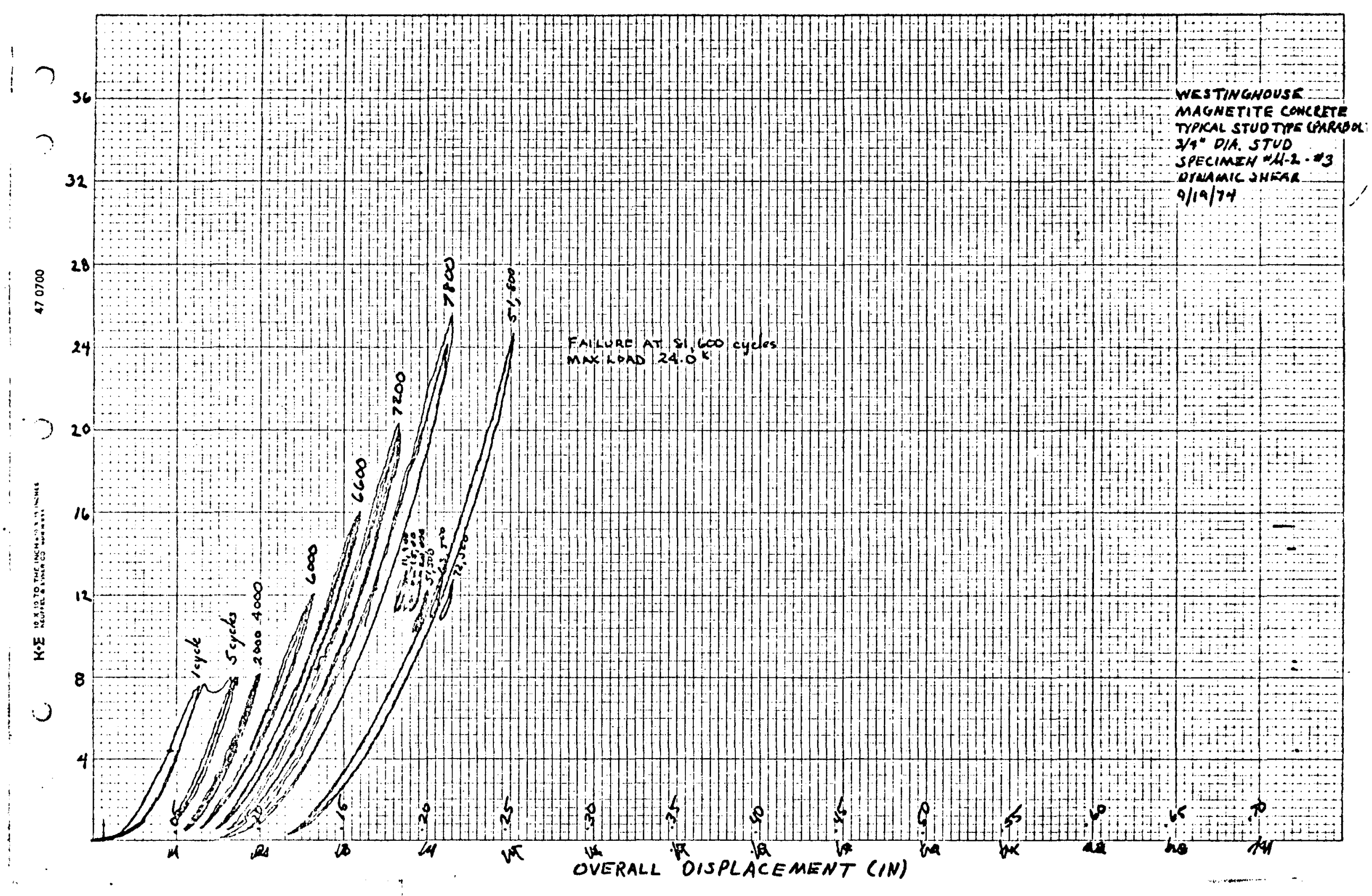




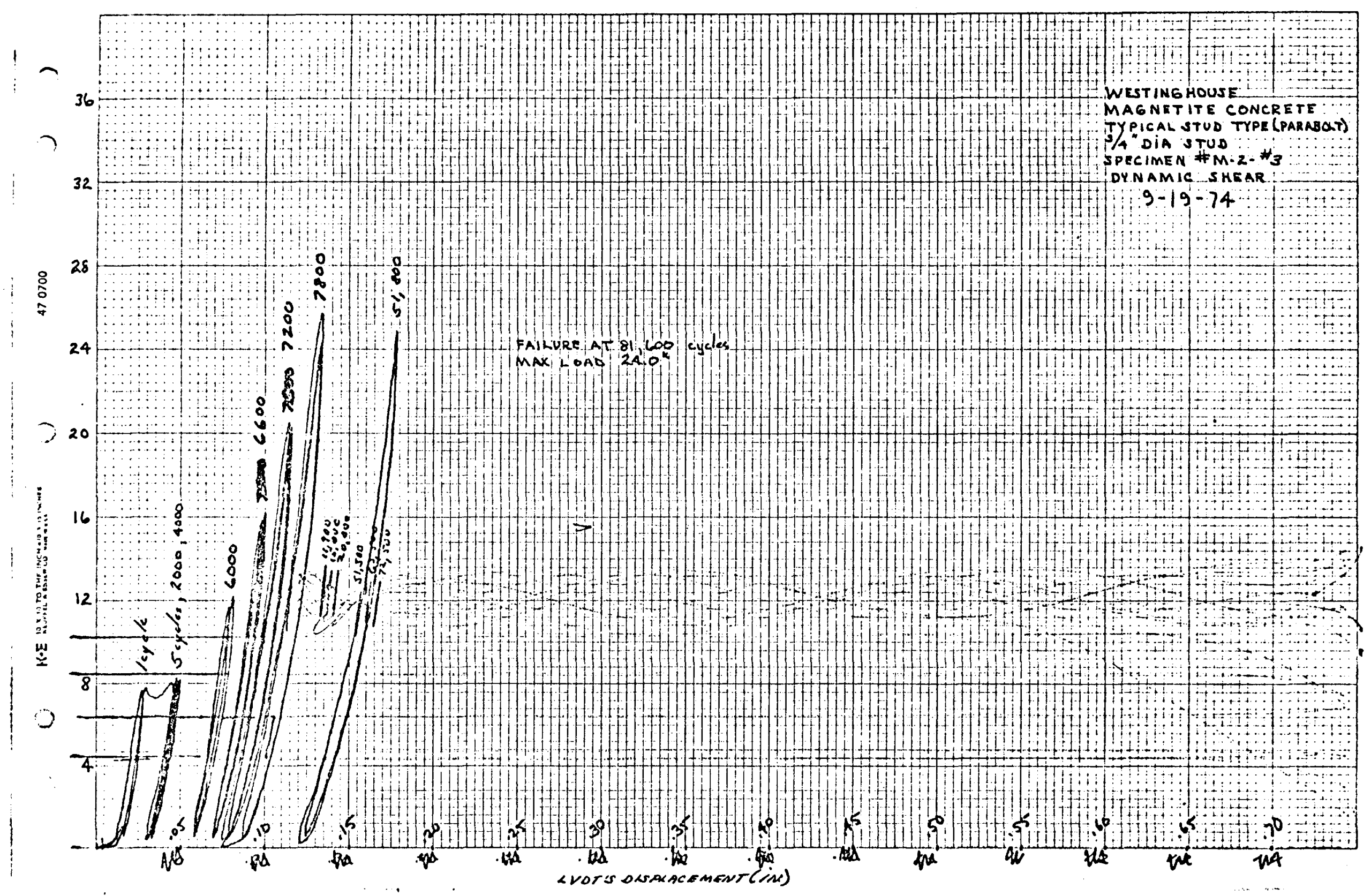




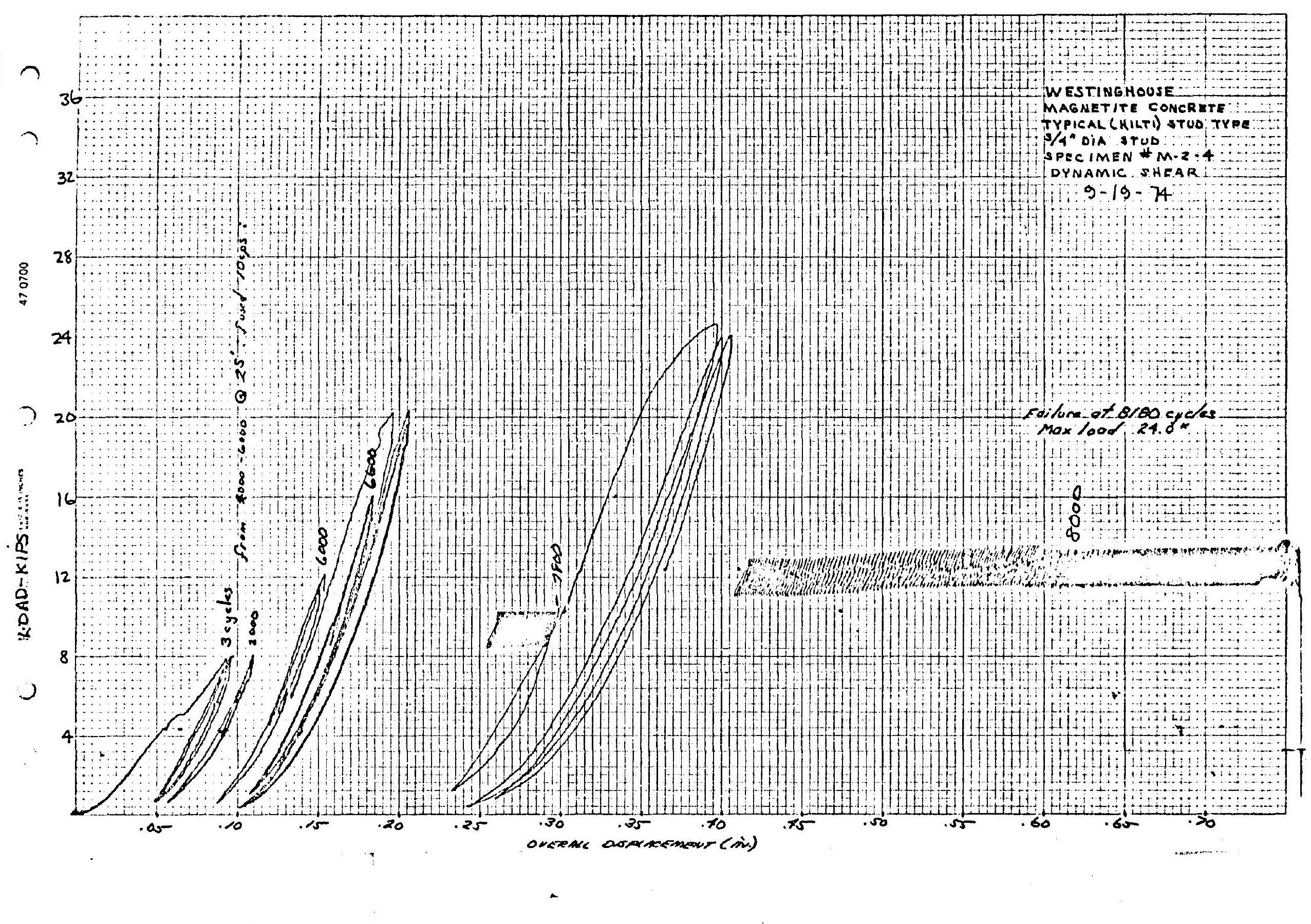




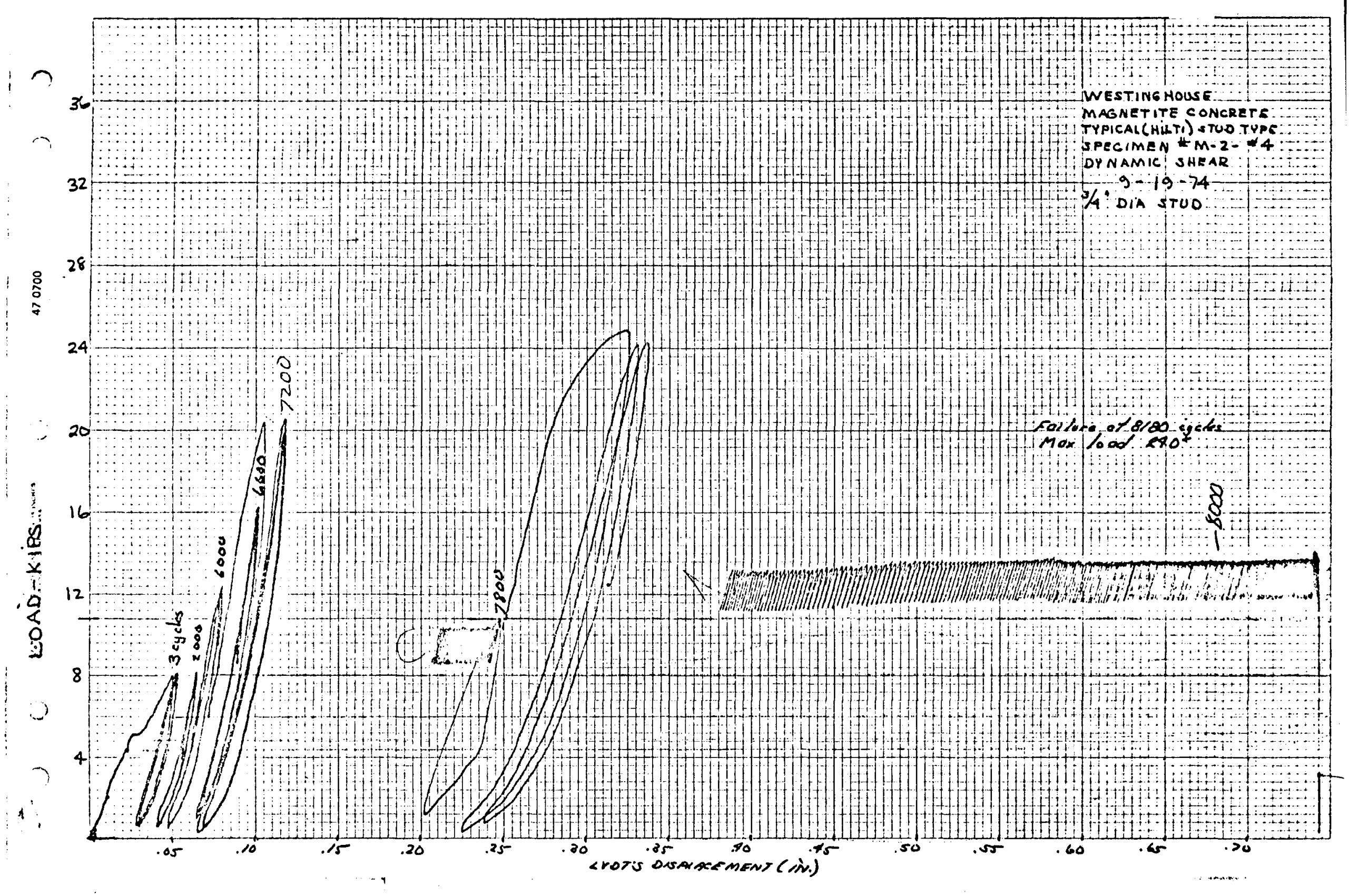




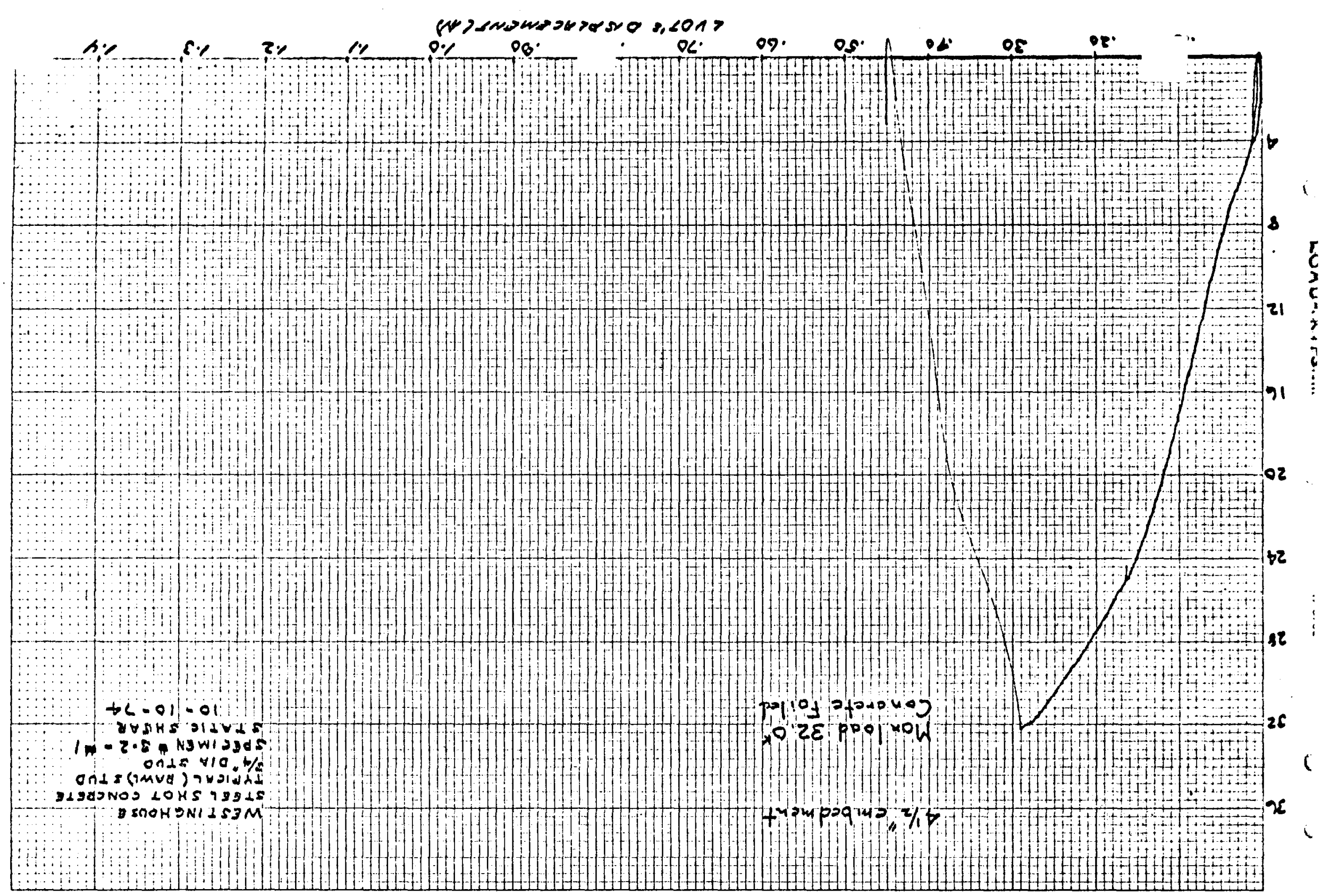




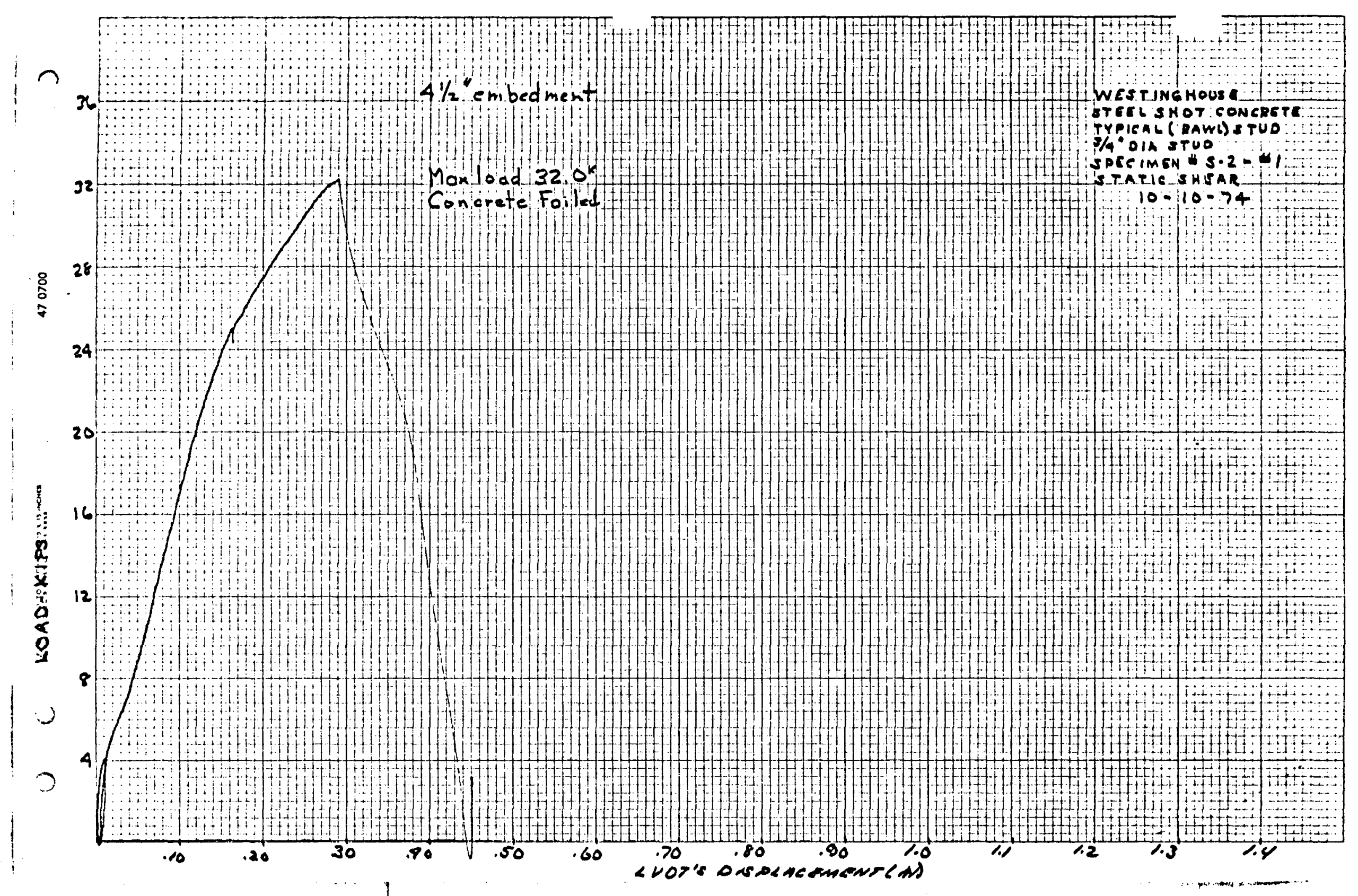




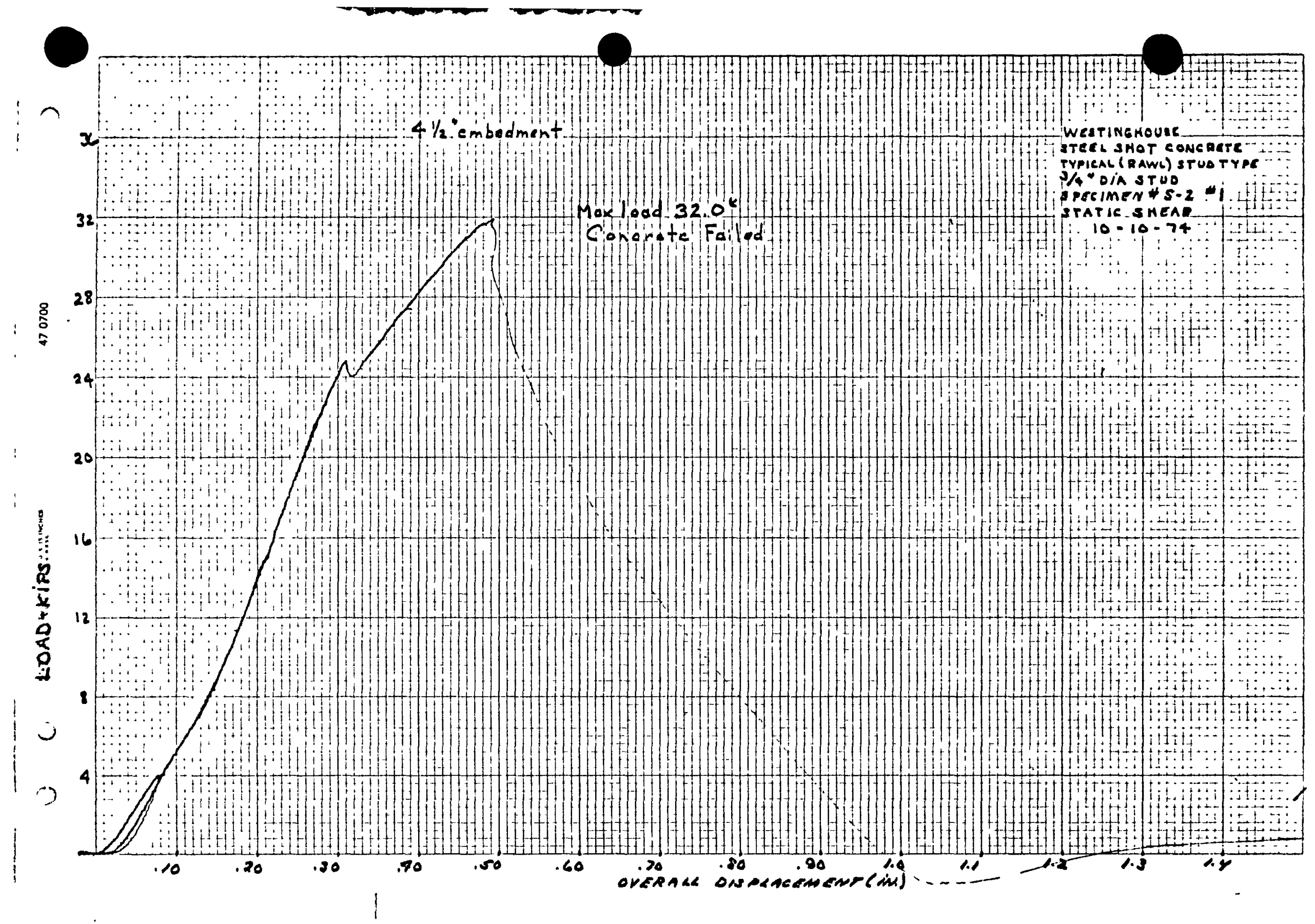




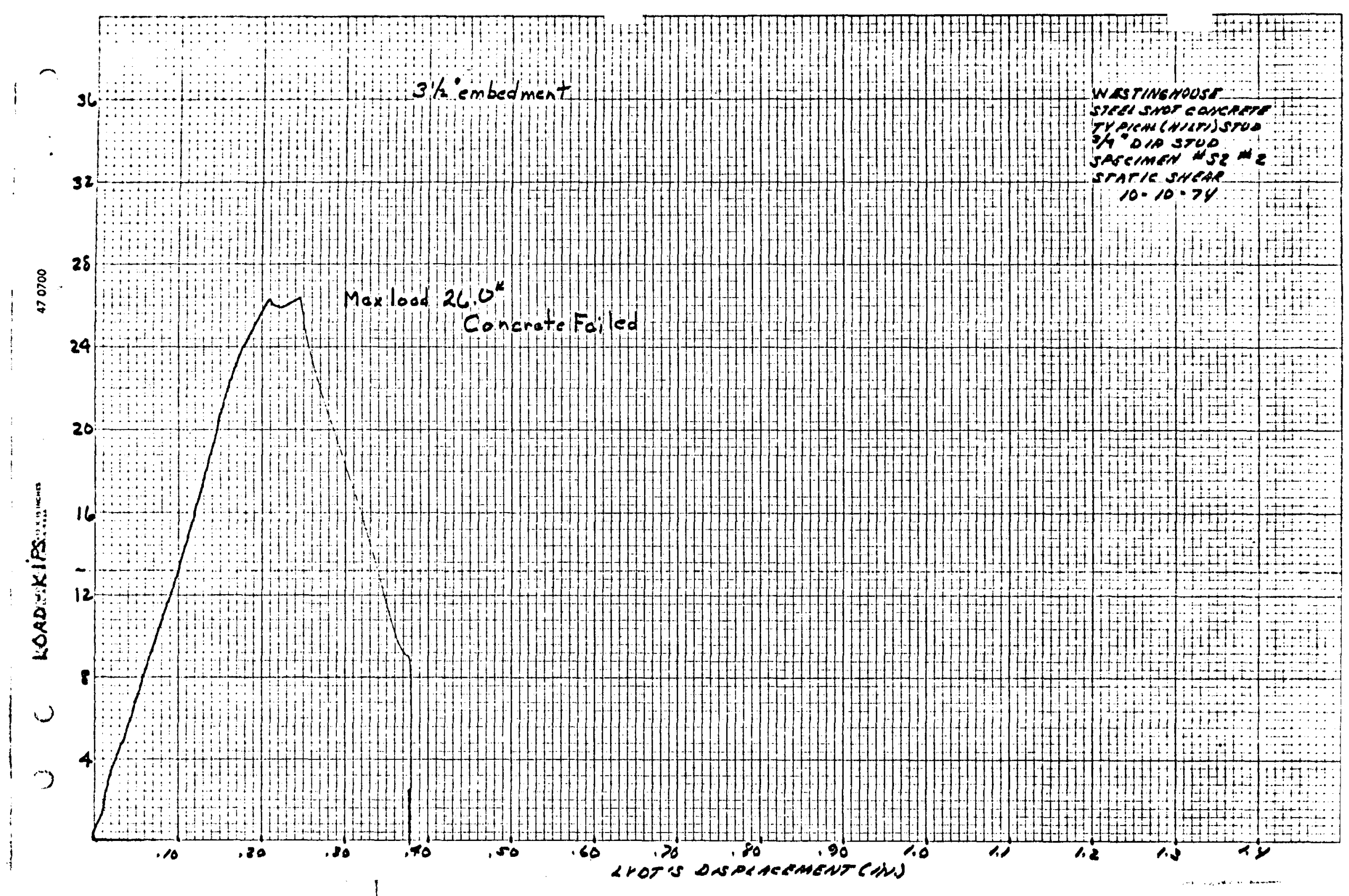




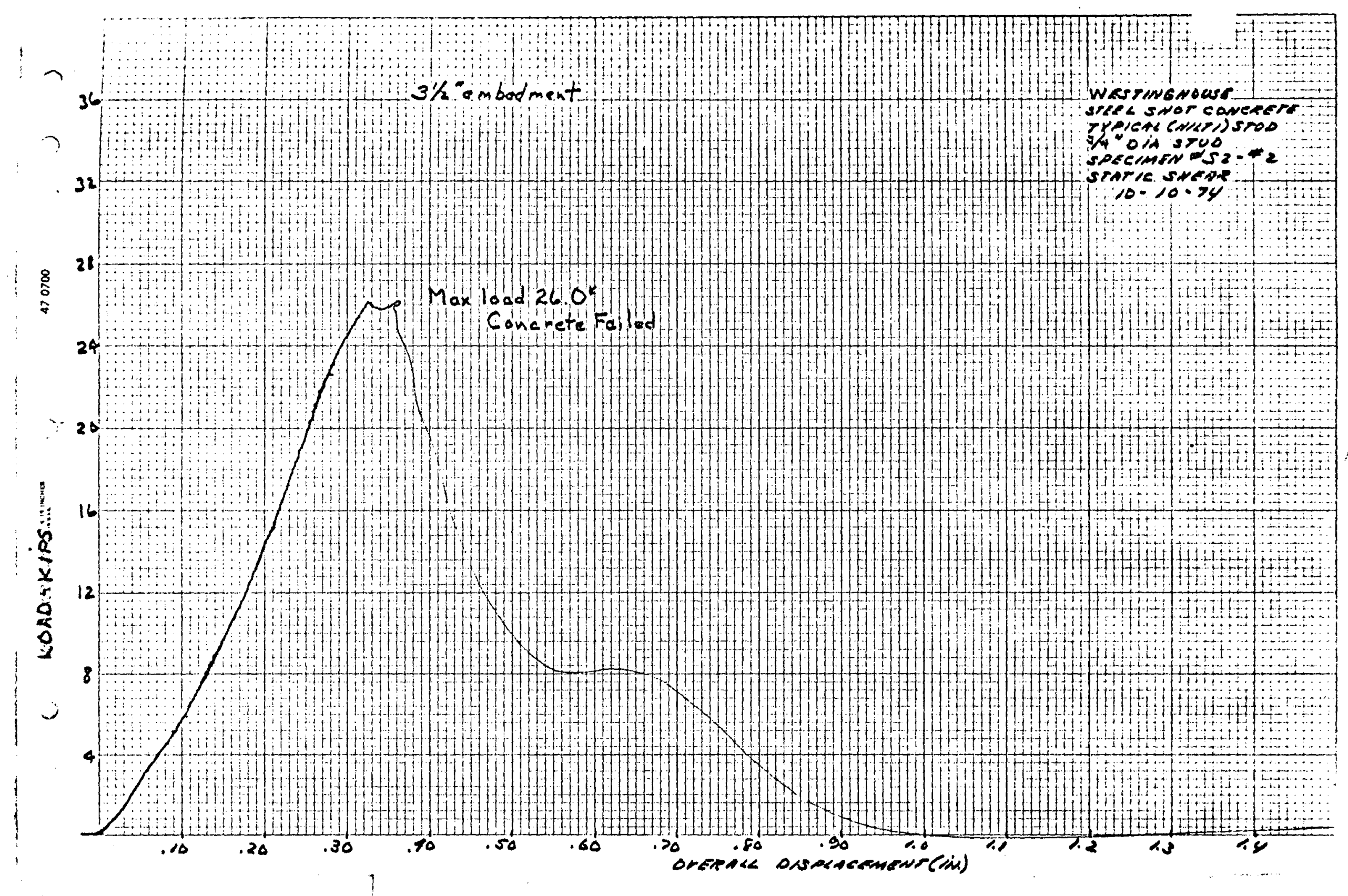




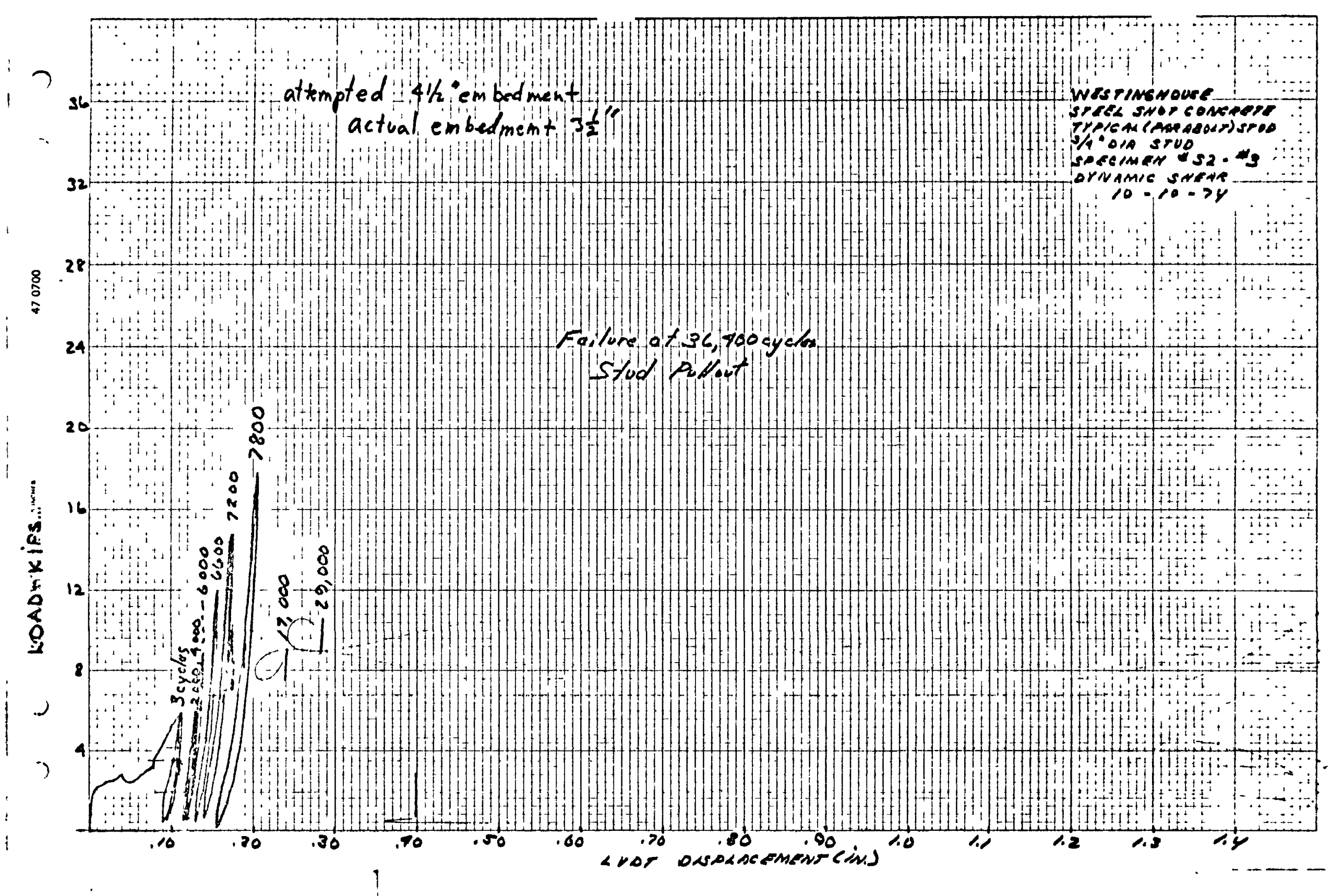




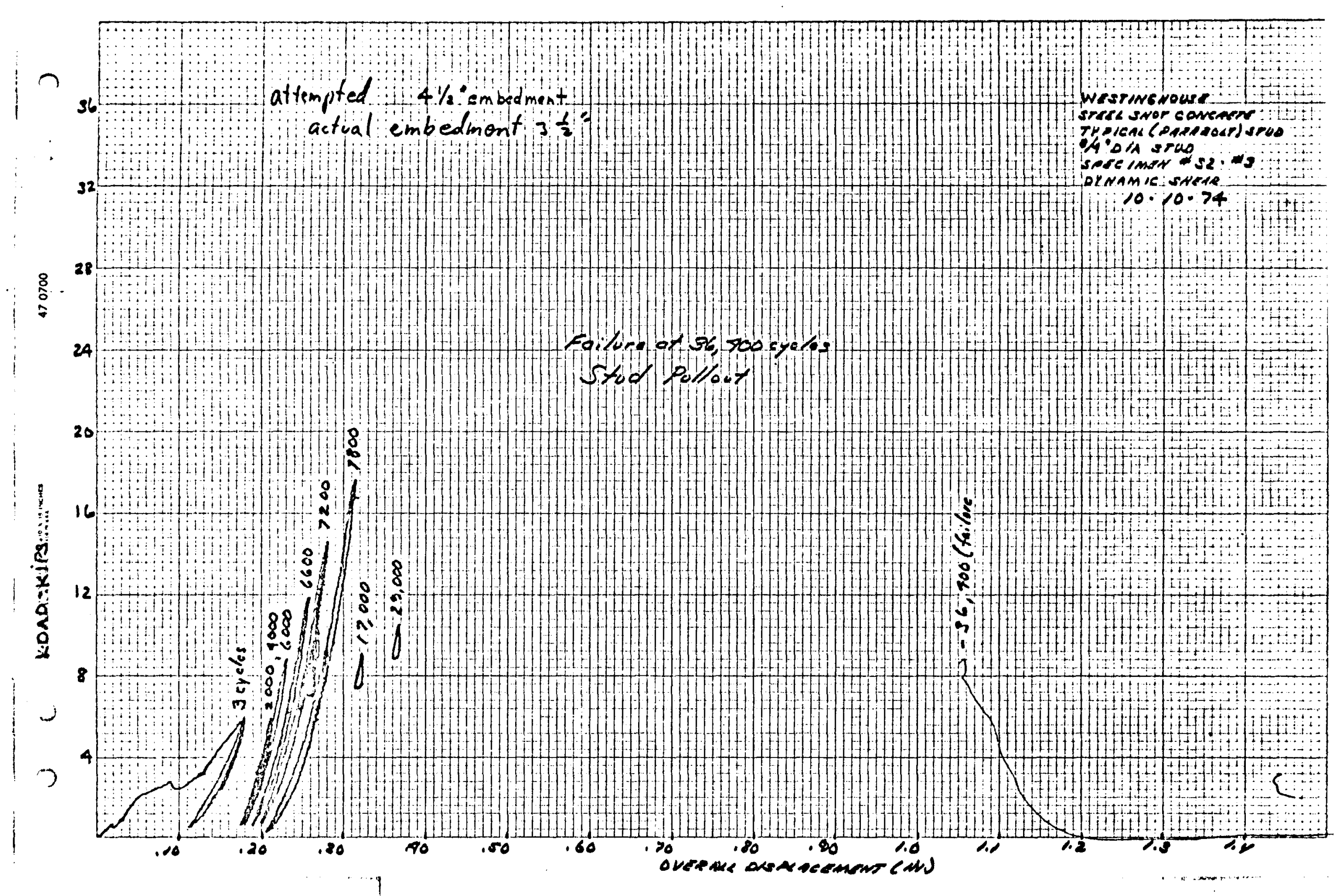




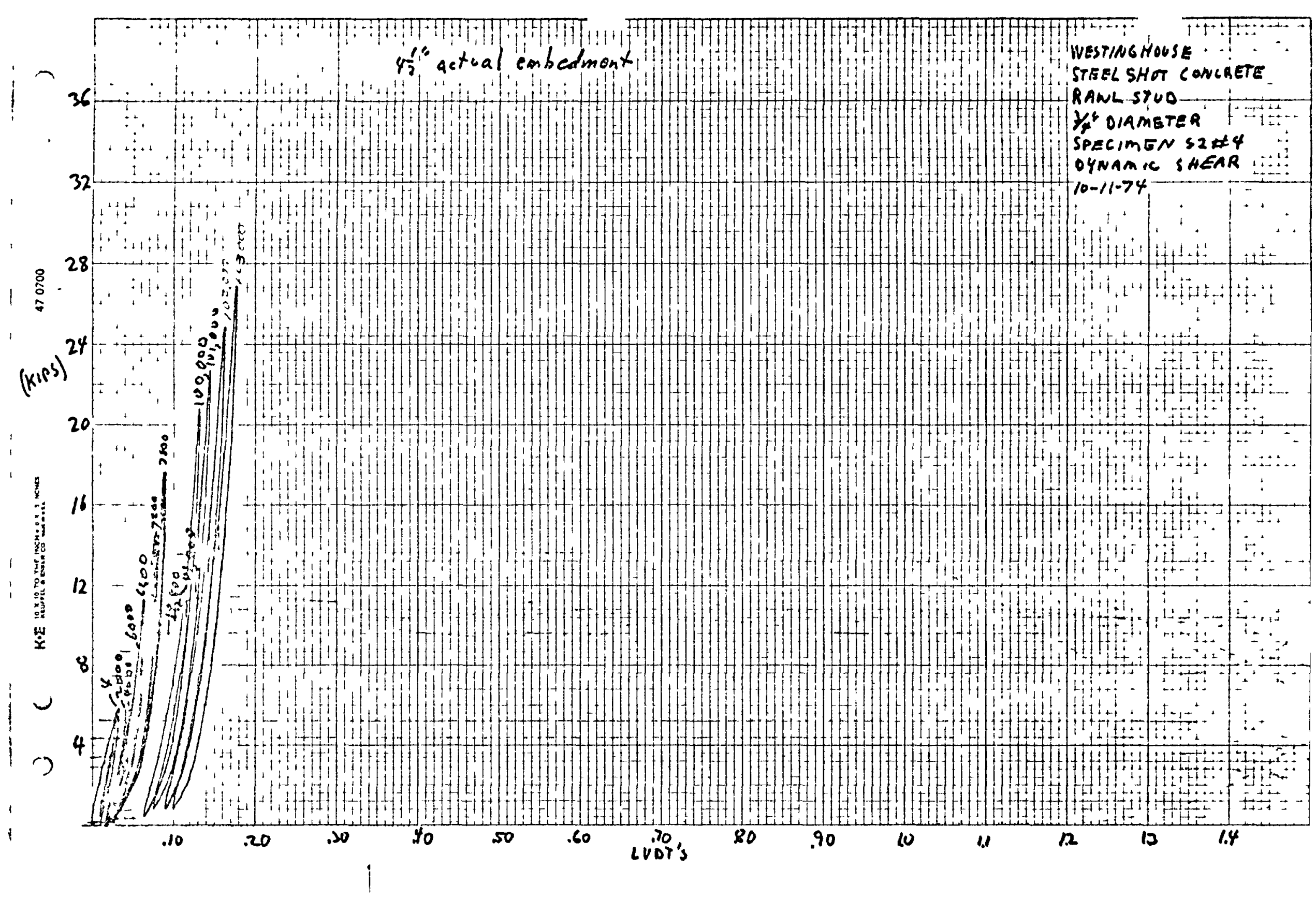




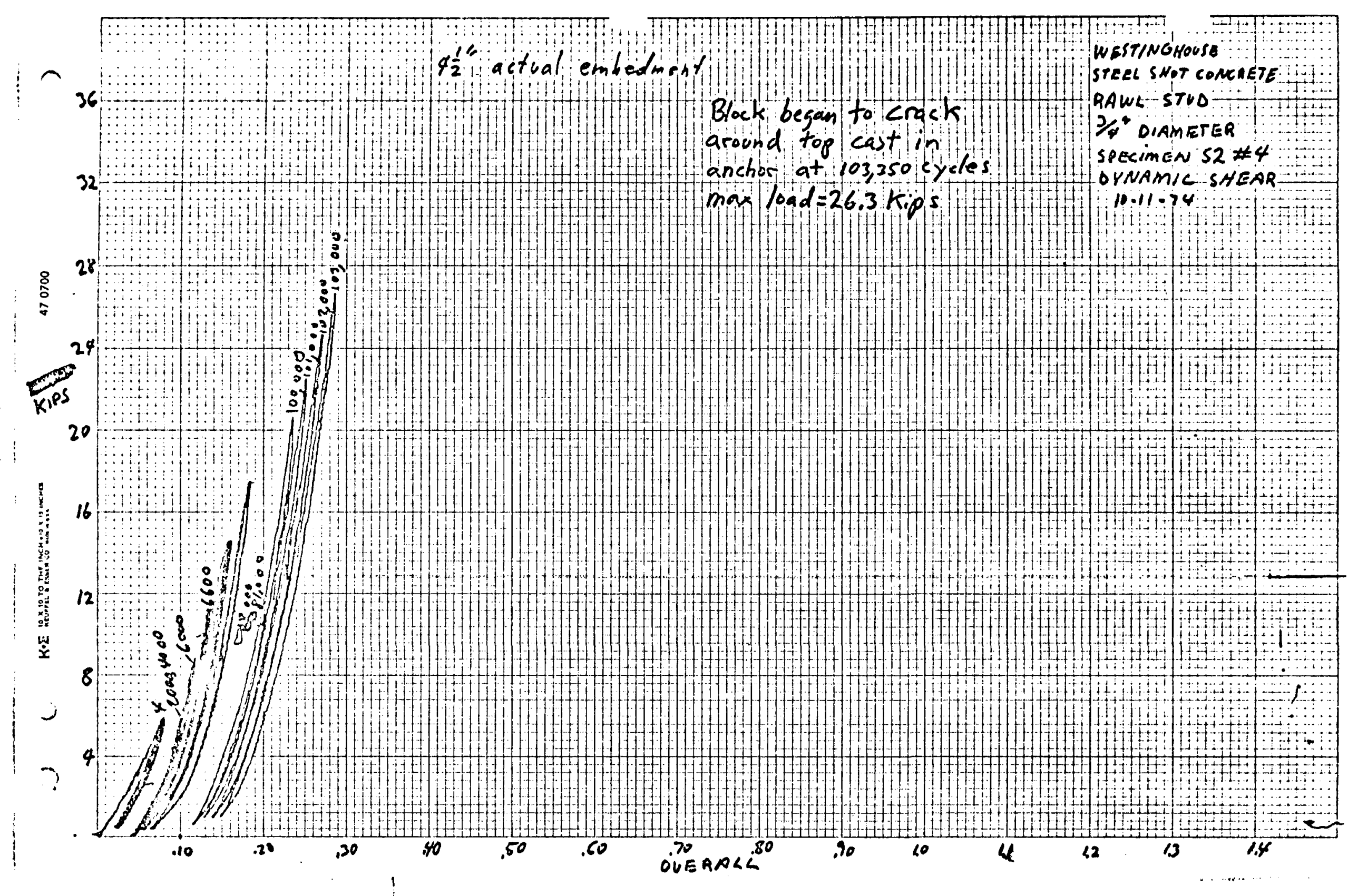




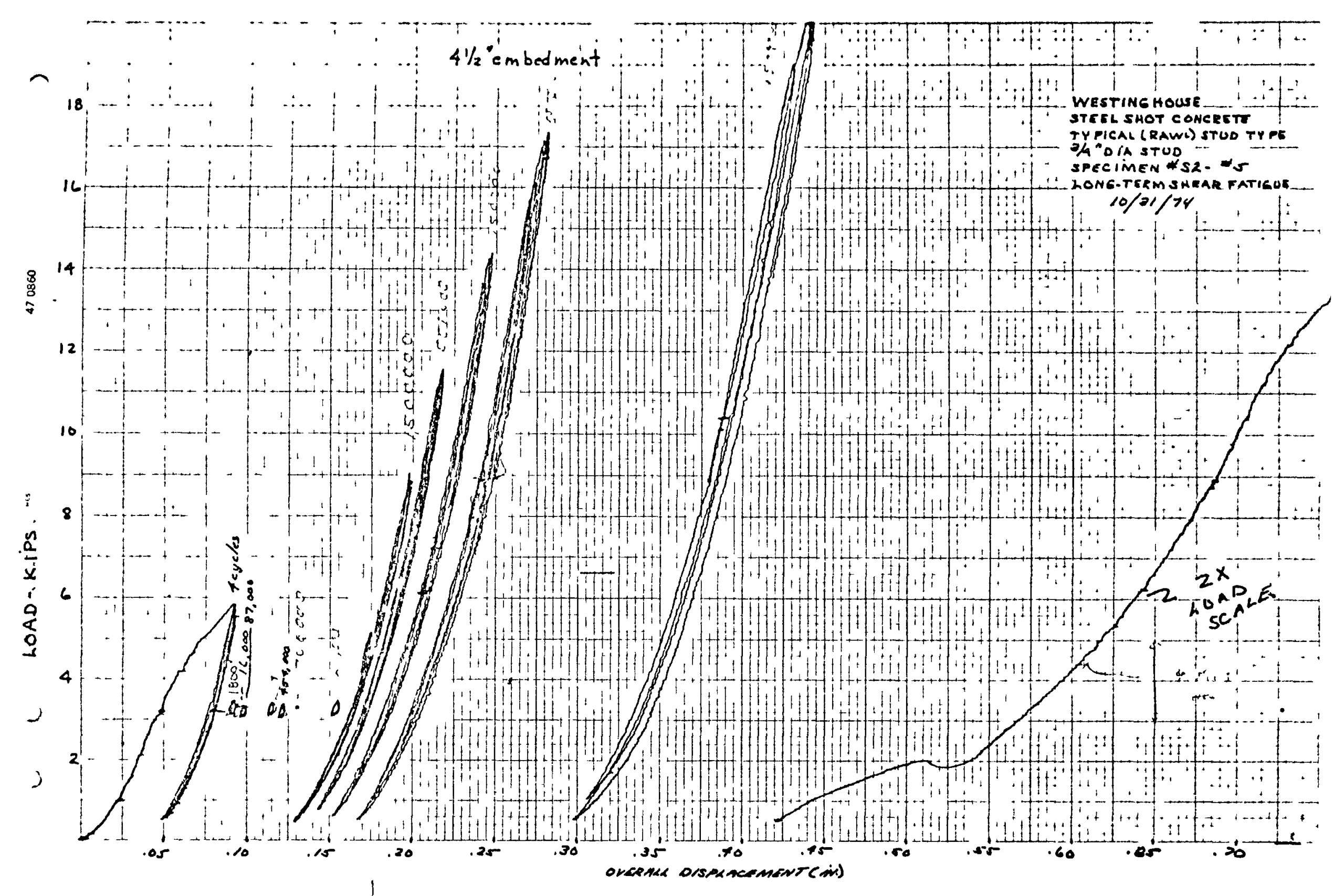




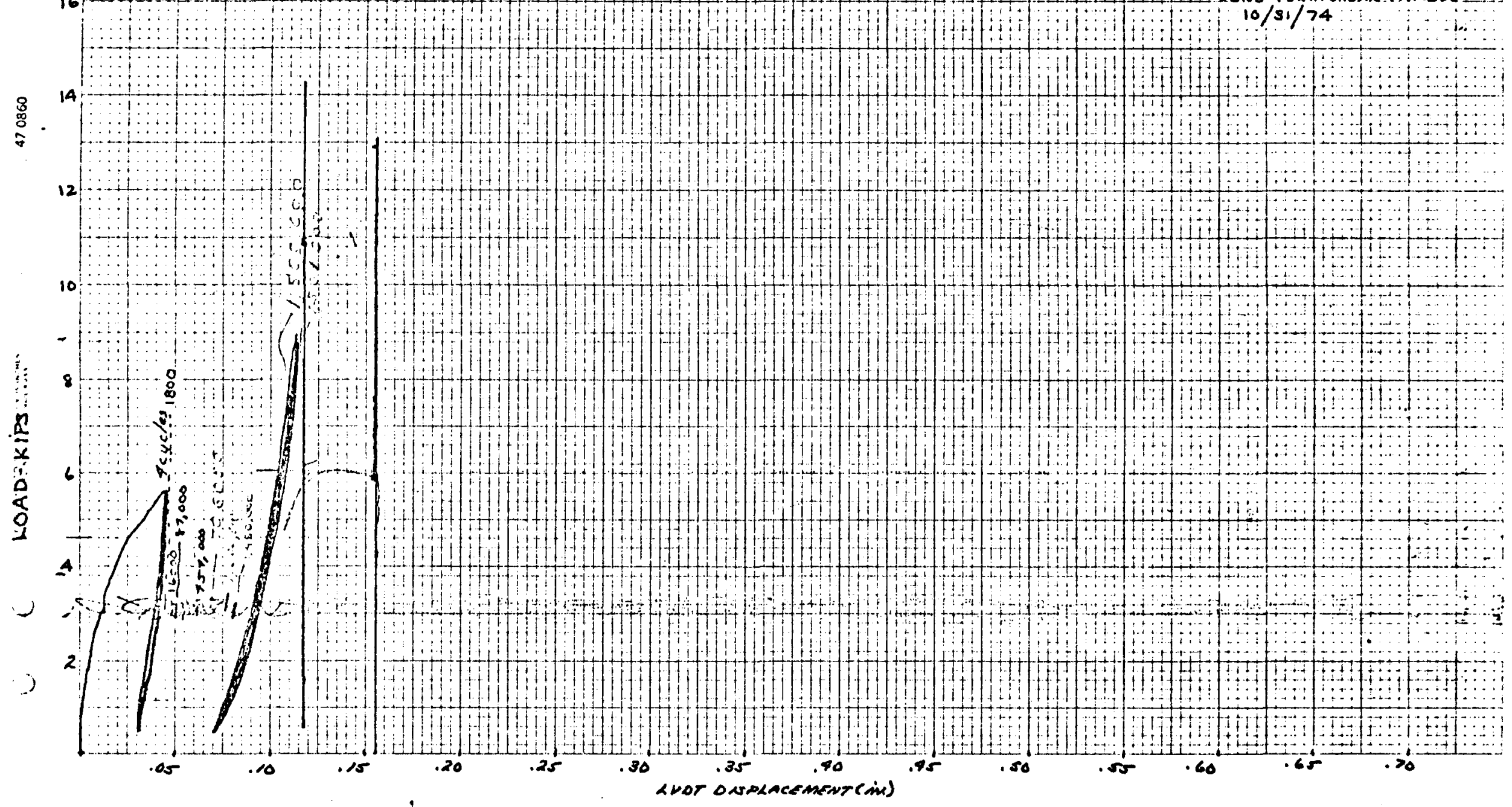



TABLE UF UATA SORTEU OY EOLT TYPE, GOLT DIA,ETER, aNU TYPE TEST, IN THAT OWDE: DO INCH SLEEVE TYNE HULTS

SHeC LUNC Fi DULT TEST. PI UI UELTA PJ UU DELTA FAILUKE NUMB TYHE KSI TYHE TYHE KIH IN KC KIF IN KC MUDE

\begin{tabular}{|c|c|c|c|c|c|c|c|c|c|c|c|c|c|}
\hline 11 & REG & 0.60 כ & レபーD & $S T A T$ & $\mathrm{~T}$ & $1<\cdot 0$ & .25 & -- & 13.0 & .40 & -- & HOLT & =AILED \\
\hline 14 & $K \in G$ & $4 \cdot 50$ & LUーS & UYN & $T$ & $11 \cdot 0$ & $\cdot 20$ & $10 \cdot 2$ & $11 \cdot 0$ & .45 & $11 \cdot 7$ & $\triangle O L T$ & FAILED \\
\hline 202 & $R \in G$ & 4.40 & Lேーb & DYN & $T$ & 6.8 & .15 & 22.0 & $6 \cdot \dot{8}$ & .20 & 71 & BOLT & FAILED \\
\hline 201 & $R \in G$ & 6.10 & LuーD & FAT & $T$ & $2 \cdot 6$ & .25 & 3.0 & $2 \cdot 6$ & . 35 & 504 & $B 3 O L T$ & FAI LED \\
\hline ع & PEG & סט: & LேーD & FAT & $T$ & $2 \cdot 0$ & .02 & 2000 & $2 \cdot 6$ & $.0 \bar{z}$ & 2000 & EOL 1 & $=A I L E D$ \\
\hline 210 & RES & 4.90 & LローD & STAT & $v$ & 19.0 & .45 & -- & 20.0 & .70 & -- & BOLT & $\triangle H E A R E U$ \\
\hline 10 & REG & 5.00 & $L B-5$ & STAT & v & 17.0 & $\cdot 20$ & -- & 24.0 & .70 & -- & BOLT & SHE $\triangle R E[$ \\
\hline$C z-12$ & $R \subset G$ & 0.87 & LE-D & STAT & v & 16.0 & .30 & -- & 17.2 & .40 & -- & EOLT & $S H E A R E=$ \\
\hline 207 & $R \subset G$ & $4 \cdot 00$ & LB-D & UYN & v & 14.4 & .20 & $7 \cdot 8$ & 14.4 & .40 & 31.6 & DOLT & SHE ARE J \\
\hline $1<$ & REG & 5.50 & Lヒ-S & DYiN & v & 14.4 & כد • & 7.0 & 14.4 & .60 & 32.0 & $=3 \cup L T$ & OMEARE- \\
\hline 200 & REG & 5.80 & $L E-5$ & FAT & $v$ & 4.6 & .07 & .60 & $26 \cdot 5$ & & 2000 & BOLT & SHEARED \\
\hline 72 & $R=0$ & $7 \cdot 17$ & レニーD & FAT & $v$ & $4 \cdot 6$ & .05 & $7 \cdot 8$ & $26 \cdot 6$ & 1.0 & 2000 & BOLT & $د H=A R E L$ \\
\hline$<1$ & RLG & כ & LE-5 & STAT & c & 6.4 & .15 & -- & 11.8 & 1.0 & - & PULLO & נUT \\
\hline 15 & $R E G$ & 5.30 & LB-5 & STAT & c & 8.0 & $\cdot 30$ & -- & $11 \cdot 4$ & $1 \cdot 1$ & -- & PULLC & $C, T$ \\
\hline 3 & $R \in G$. & 5.70 & LUーb & DYN & c & 7.0 & .05 & $7 \cdot a$ & 7.0 & .40 & 41 & FULLO & Dur \\
\hline 13 & REG & 5.00 & Lr3-D & UYN & C & $7 \cdot 2$ & .15 & $7 \cdot 0$ & $7 \cdot 2$ & .40 & 16.5 & EULT & SHEARED \\
\hline
\end{tabular}


TAELE OF UATA sURTEL OY OULT TYPE. GOLT UIA., AND TYPE TEST. CONT.

SO INCH SLEEVE TYFE DOLTS

SPLC CUNË PIL DULT TEST. PI DI UELTA FU UU UELTA FAILURE NUIND TYHE KOI TYHE TYHE KIP IN KC KIH IN KC VIUDE

\begin{tabular}{|c|c|c|c|c|c|c|c|c|c|c|c|c|}
\hline 106 & $R \in G$ & 7.15 & LBSO & STAT & $T$ & $14 \cdot 8$ & .78 & -- & $15 \cdot 4$ & - 99 & -- & PULLLOUT \\
\hline 99 & REG & 0.91 & $L B-6$ & STAT & $T$ & -- & -- & & 9.4 & $\div-$ & -- & PULLOUT \\
\hline 87 & $R \in G$ & 5.51 & LE-6 & DYN & $T$ & $7 \cdot 8$ & $\cdot 15$ & 7.8 & 15.6 & 1.01 & 103 & PULLOUT \\
\hline 86 & $R \in G$ & $7 \cdot 24$ & LUーO & UYN & $T$ & $7 \cdot 8$ & .80 & $7 \cdot 8$ & $9 \cdot 7$ & $\cdot 96$ & $9 \cdot 7$ & PULLOUT \\
\hline $1 \leftleftarrows$ & RÉ̈ & 6.08 & LUー6 & FAT & $\mathrm{T}$ & $2 \cdot 6$ & .02 & $1 \cdot 0$ & $4 \cdot 2$ & .03 & 2000 & DOLT FAILED \\
\hline 94 & REG & $0 \cdot 38$ & LேーO & FAT & $T$ & $2 \cdot 6$ & .05 & $1 \cdot 0$ & $2 \cdot 6$ & -15 & 535 & BULT FAILFD \\
\hline 121 & MIAG & 0.17 & LESO & STAT & $\mathrm{T}$ & -- & -- & & 16.6 & -- & -- & PULLOUT \\
\hline $1 \approx \overline{3}$ & NARG & 6.20 & $L \boxminus-6$ & STAT & $T$ & $3 \cdot 7$ & .01 & -- & $5 \cdot 6$ & -- & -- & PULLOUT \\
\hline $1<7$ & $M A G$ & 6.17 & LD-O & UYN & $\mathrm{T}$ & $7 \cdot 0$ & $1 \cdot 0$ & $7 \cdot 8$ & 13.0 & $1 \cdot 20$ & 107.3 & CONL FAILED \\
\hline 100 & MAG & 0.10 & レロー0 & DYN & $\mathrm{T}$ & $7 \cdot 0$ & .40 & 7.0 & $12 \bullet=$ & $\cdot 76$ & 102 & CONE FAILED \\
\hline 132 & ১১ & $6 \cdot 27$ & LE & STAT & $\mathrm{T}$ & $2 \cdot 8$ & .02 & -- & $4 \cdot 0$ & -- & -- & DULLOUT \\
\hline 134 & $\doteq$ & $6 \cdot<y$ & LE & STAT & $T$ & $4 \cdot 8$ & $\cdot 38$ & -- & $7 \cdot 2$ & 1.05 & -- & PULLOUTT \\
\hline 137 & ss & 0.20 & LB & DYN & $T$ & $3 \cdot 4$ & $\cdot 38$ & 7.8 & $5 \cdot 4$ & $\bullet 97$ & 110 & PULLOUT \\
\hline 136 & ss & $6 \cdot 24$ & L $B-6$ & DYN & $T$ & -- & -- & - & $2 \cdot 2$ & .83 & $6 \cdot 8$ & OULLOONT . \\
\hline 7 & REG & 6.78 & $L=3-0$ & $\triangle T A T$ & $v$ & $21 \cdot 3$ & .30 & -- & $29 \cdot 2$ & • 55 & -- & BUL I $\vdash A I L E D$ \\
\hline 76 & $R=G$ & 6.67 & LE-O & STAT & v & 28.0 & .40 & -- & 31.6 & .65 & -- & BOLT FAILEO \\
\hline ذ & $R=G$ & לد.7 & LUSO & UYN & $v$ & 17.3 & $\cdot 27$ & $7 \cdot 8$ & 17.8 & $\cdot 30$ & $27 \cdot 5$ & SOLT FAILES \\
\hline 70 & $R \in G$ & $6 \cdot 54$ & $L \Delta-6$ & DYN & v & $17 \cdot 8$ & 1.10 & 7.8 & $17 \cdot 8$ & -- & 19.8 & BOLT FAILEE \\
\hline 03 & iVIAG & 6.54 & Lப் 6 & STAT & v & 23.0 & .34 & -- & 32.4 & -- & -- & EOLT FAILFD \\
\hline 65 & MAG & 6.57 & L $b j-6$ & STAT & v & 25.0 & .40 & -- & $28 \cdot 2$ & - 90 & -- & FBOL-T FAILEO \\
\hline 60 & MAG & 0.48 & LB -0 & DYN & v & 17.4 & .25 & $7 . \ddot{8}$ & 17.4 & .55 & 39.6 & BOLT FAILED \\
\hline 64 & MAAG & $\epsilon .70$ & レபー6 & JYNV & v & $17 \cdot 4$ & $\cdot 27$ & $7 \cdot 5$ & 17.4 & $1 \cdot 25$ & $26 \cdot 5$ & EOLT FLILEO \\
\hline 69 & SS & 6.40 & LU-6 & STAT & $v$ & $18 \cdot 8$ & $\cdot 17$ & -- & 22.0 & $1 \cdot 20$ & -- & PULLOUT \\
\hline 65 & S3 & 0.90 & LE-O & STAT & $\mathrm{v}$ & 26.0 & .40 & -- & $28 \cdot 0$ & .60 & -- & PULLOUT \\
\hline
\end{tabular}


TAQLE OF LATA SORTEU OY OOLT TYPE, BOLT DPIA. AND TYPE TEST. CONT.

- 4 INCH SLEEVE TYPE DULTS CUNT.

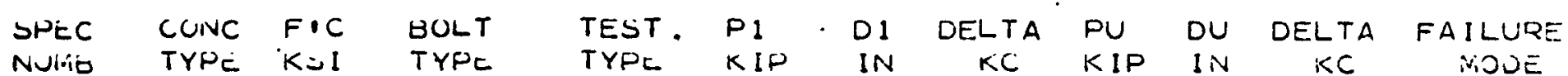

\begin{tabular}{|c|c|c|c|c|c|c|c|c|c|c|c|c|}
\hline 70 & ss & 5.16 & LB-U & UYN & $v$ & 10.8 & .22 & 7.8 & 10.8 & -- & 34 & PULLOUT \\
\hline 71 & SS & 0.43 & $L_{\square}-\theta$ & DYN & v & 16.8 & $1 \cdot 10$ & 7.8 & 10.8 & - & 7.8 & PULLCUT \\
\hline 20 & $R E G$ & -- & LB-G & STAT & c & 6.0 & .15 & -- & 10.4 & -- & -- & CONC FAILED \\
\hline 30 & REG & 5.85 & LB-O & STAT & $c$ & $8 \cdot 4$ & .19 & -- & $14 \cdot 4$ & $1 \cdot 35$ & -- & CONC FAILEO \\
\hline 20 & $R E G$ & 6.43 & LE-G & OYN & C & $7 \cdot 2$ & $\cdot 54$ & $7 \cdot 8$ & $7 \cdot 2$ & -- & $39 \cdot 5$ & EOLT SHEAREC \\
\hline 32 & $R \in G$ & 7.01 & LB-O & DYN & c & $7 \cdot 2$ & .32 & $7 \cdot 8$ & $7 \cdot 2$ & -- & $63 \cdot 3$ & BOLT SHEAREU \\
\hline 54 & MAG & $8 \cdot 14$ & LB-O & STAT & c & 5.8 & .08 & -- & $15 \cdot 4$ & -- & -- & CONC FAILED \\
\hline 1 & MAG & 8.07 & LS-6 & STAT & c & $7 \cdot 2$ & .09 & -- & $14 \cdot 2$ & .93 & - & CONC FAILED \\
\hline 56 & MIAG & 8.07 . & LB-G & DYN & c & 6.4 & .07 & $7 \cdot 8$ & $6 \cdot 4$ & -- & 67 & PULLOUTT \\
\hline دَص & IVAG & $7 \cdot 70$ & Lப-0 & OYN & c & 0.4 & .04 & $7 \cdot 8$ & $6 \cdot 2$ & -- & ב.ל & BOLT SHIEAREL \\
\hline 61 & Ss & (7.6 & Lター0 & STAT & c & 9.0 & .11 & -- & $11 \cdot 2$ & .94 & -- & CONC FAILED \\
\hline 210 & s & 6.38 & LS-O & STAT & $c$ & 8.0 & .12 & -- & 11.2 & .41 & -- & CONC FAILED \\
\hline 214 & si & 8.77 & $L B-6$ & DYN & c & 6.3 & .11 & $7 \cdot 8$ & $6 \cdot 25$ & -- & 44.6 & STUD SHEARED \\
\hline$? .16$ & SS & 6.86 & L৮-6 & DYN & $c$ & 6.4 & .14 & 7.8 & $6 \cdot 40$ & -- & $7 \cdot 5$ & PULLOUT' \\
\hline
\end{tabular}


TAÖLE UF UATA SURTLU EY BULT TYPE, BOLT DIA. AND TYPE TEST. CONT.

PE INCH STUU TYHE LULTS

SPEC CUNC FIC DULT TEST, PI DI DELTA PU DU DELTA FAILURE NUE TYPE KUI TYPE TYFE KIP IN KC KIP IN KC MODE

\begin{tabular}{|c|c|c|c|c|c|c|c|c|c|c|c|c|}
\hline 105 & $R \in G$ & $6 \cdot 54$ & So-j & STAT & T & $8 \cdot 0$ & $\cdot 20$ & -- & $14 \cdot 4$ & .40 & -- & PULLOUT \\
\hline 203 & $R=G$ & 0.20 & S: & STAT & $T$ & 11.6 & .35 & -- & 11.6 & .80 & -- & PULLOUT \\
\hline i7 & RLG & $5 \cdot 50$ & $S A-S$ & DYN & T & $8 \cdot 6$ & $\cdot 22$ & 7.8 & 18.6 & .60 & 110 & PULLOUT \\
\hline 114 & $R \subset G$ & 0.06 & $S A \dot{D}$ & DYN & $T$ & 8.6 & .50 & 7.8 & $12 \cdot 6$ & 1.0 & 102 & CONC FAILED \\
\hline 3 & REG & 0.30 & $5 j-5$ & UYN & $\mathrm{T}$ & 7.0 & .45 & $7 \cdot 8$ & 16.0 & .68 & 112 & PULLJUT \\
\hline 1 & REEG & 6.60 & SD-D & L'YN & $T$ & $7 \cdot 0$ & .07 & $7 \cdot 8$ & $12 \cdot 0$ & .45 & 102 & PULLOUT \\
\hline 89 & RLG & 6.06 & $s c-5$ & EYN & T & $5 \cdot 5$ & .07 & $7 \cdot 8$ & 10.7 & .20 & 102 & PULLOUT \\
\hline בע8 & $R E G$ & ن••• & $5 c-5$ & DYN & $T$ & כ.5. & .05 & $7 \cdot 8$ & 12.6 & .20 & 101 & PULLOUT \\
\hline 205 & RÉ́ & 0.20 & כ-נד & STAT & v & 28.0 & .15 & -- & 33.0 & .45 & $-二$ & BOLT SHEAREO \\
\hline$(-\bar{c}-13$ & REG & \pm 0 & $S t:-5$ & STAT & V & $16 \cdot 0$ & $\cdot 10$ & -- & 21.0 & $\cdot 17$ & -- & BOLT SHEAREZ \\
\hline 204 & $R=G$ & $7 \cdot 16$ & $S A-D$ & DYN & v & 19.2 & $\cdot 17$ & 7.0 & $1 \% \cdot 2$ & .40 & $17 \cdot y$ & BULT SHEAKED \\
\hline$C \ddot{-}-\dot{y}$ & $R=G$ & 0.77 & SADD & DYN & $v$ & $19 \cdot 2$, & $\cdot 30$ & $7 \cdot 8$ & $19 \cdot ?$ & $\cdot 35$ & 8.0 & BOLT SHEARED \\
\hline 7 & REG & $6 \cdot 40$ & $D E-5$ & DYN & $v$ & $20 \cdot 0$ & $\cdot 10$ & $7 \cdot 8$ & 20.0 & $\cdot 20$ & $9 \cdot 2$ & BOLT SHEARES \\
\hline 0 & $R \in G$ & -- & $5 t-5$ & DYN & V & 18.0 & - 22 & $7 \cdot 8$ & 18.0 & -- & $10 \cdot 2$ & SOLT SHEAPED \\
\hline$c-10$ & $R \in G$. & 0.40 & $=C-\sum$ & UY: & $v$ & $14 \cdot 4$ & - is & 7.0 & $14 \cdot 4$ & .23 & $33 \cdot 9$ & BULT SHEAREO \\
\hline$C \ddot{c}-11$ & $R \subset G$ & 0.64 & $\dot{S C}-=$ & UYN & v & 14.4 & . 23 & $7 \cdot 0$ & $14 \cdot 4$ & כ57 & 23.0 & EOLT $\triangle M H F A D E O$ \\
\hline 30 & $R \in G$ & 4.90 & $S B-S$ & STAT & C & $11 \cdot 6$ & .07 & -- & $11 \cdot 6$ & $\cdot 10$ & -- & CONC $r A I L E O$ \\
\hline 25 & $R=G$ & -- & $S+3-5$ & $S T . A T$ & C & $6 \cdot 8$ & - 12 & -- & 10.5 & .43 & -- & CONC FAILED \\
\hline 24 & $R \in G$ & $6 \cdot 50$ & $5 A-5$ & DYN & C & $6 \cdot 6$ & .09 & $7 \cdot 8$ & $6 \cdot 6$ & $\cdot 10$ & $22 \cdot 0$ & PULLOUT \\
\hline 36 & $R \in G$ & 0.30 & $S A-5$ & DYN & C & 6.6 & $\cdot 15$ & $7 \cdot 8$ & $6 \cdot 6$ & .45 & 37.0 & PULLOOJT \\
\hline 10 & $R \in G$ & $5 \cdot 80$ & $S E-5$ & DYN & C & $6 \cdot 6$ & .07 & $7 \cdot 8$ & $6 \cdot 6$ & -- & $17 \cdot 9$ & SHE.ARED \\
\hline 40 & $R \in G$ & 0.10 & ذே-த & OYN & C & 6.6 & $\cdot 10$ & $7 \cdot 8$ & $y \cdot 6$ & 0.45 & 102 & DULT THEAREL \\
\hline 18 & RLG & $6 \cdot 70$ & $s c-5$ & DYN & $c$ & $6 \cdot 6$ & $\cdot 27$ & $7 \cdot 8$ & $6 \cdot 6$ & -- & $28 \cdot 0$ & PULLOUT \\
\hline 4 & REG & $0 \cdot<0$ & sc-D & DYN & $c$ & 0.6 & .05 & 7.8 & 6.0 & .07 & $1 \supset .0$ & DULLOUT \\
\hline
\end{tabular}


TAGLE UF UATA SURTE'S OY DCLT TYPE, HOLT DIA., AND TYPE TEST. CONT.

- INCH STUD TYPE BCLTJ

SPEC CUINC FIC BOLT TEST. PI DI UELTA PU DU UELTA FAILURE

NUIA TYPE KOI TYPE TYPE KIP IN KC KIF IN KC WOTE

\begin{tabular}{|c|c|c|c|c|c|c|c|c|c|c|c|c|}
\hline 110 & $R E G$ & $7 \cdot 4 y$ & $5 u-0$ & STAT & $T$ & $=0$ & .10 & -- & $17 \cdot 3$ & . 5u & $-\infty$ & PULLOU \\
\hline 109 & $R \in G$ & 7.44 & $S A-O$ & DTAT & $\mathrm{T}$ & 6.0 & $\cdot 20$ & -- & $22 \cdot 0$ & .50 & -- & PULLOUT \\
\hline 110 & KESG & 0.00 & $D A-O$ & DY,V & $T$ & 10.0 & $\cdot 25$ & $7 \cdot 8$ & $16 \cdot 2$ & .45 & lub & PULLOUT \\
\hline$<0$ & $R=G$ & $7 \cdot 12$ & $=A-0$ & UYN & $T$ & 10.0 & .18 & $7 \cdot 0$ & $21 \cdot 8$ & .45 & 108 & CONC FAILED \\
\hline 04 & $R=G$ & -- & $S A-O$ & FAT & $T$ & $4 \cdot 0$ & .01 & -- & $4 \cdot 0$ & .03 & 386 & BOLT SHEARED \\
\hline 73 & PEG & -- & $5 A-6$ & FAT & $T$ & & & & & & & \\
\hline 123 & MAAG & 0.93 & $s+-6$ & STAT & $T$ & 8.0 & .04 & $\ldots$ & $14 \cdot 2$ & .15 & -- & CONC FAILED \\
\hline $1<0$ & NAG & 7.00 & $S A-O$ & STAT & $T$ & 8.0 & 5 & -- & $25 \cdot 5$ & .42 & -- & CONC FAILEO \\
\hline $1<y$ & $\vec{v}_{i} \dot{A G}$ & 0.70 & $2 E-0$ & DYN & $T$ & 13.6 & .10 & 7.8 & $1 \sqcup .7$ & .42 & 100 & BOLT FAILEO \\
\hline $1<2$ & $\therefore A G$ & 7.07 & $\triangle A-O$ & UYN & $T$ & $13 \cdot 6$ & .18 & $7 \cdot 8$ & ق. 19 & - Ẽ & 94 & EOLI $\because$ LILED \\
\hline 134 & so & 6.50 & $S A-6$ & $S T A T$ & $T$ & $12 \cdot 0$ & $\cdot 30$ & -- & $15 \cdot 6$ &.$\leqslant 0$ & $-\dot{-}$ & DULLOIT \\
\hline 133 & SS & 7.38 & $S B-S$ & STAT & $T$ & 6.0 & .40 & -- & $7 \cdot 6$ & .60 & -- & PULLCUT \\
\hline 131 & SS & 7.33 & $\triangle A-O$ & UYN & $T$ & 9.6 & .21 & 7.8 & 13.6 & .50 & 101 & PULLOUT \\
\hline 140 & S১ & 7.58 & $>A-6$ & LYN & $T$ & 8.4 & $\cdot 27$ & $7 \cdot 3$ & 14.4 & .40 & 102 & PULLCUT \\
\hline 33 & Ss & 7.61 & $s c-6$ & FAT & $T$ & $3 \cdot 2$ & - & - & $3 \cdot 2$ & . 70 & 1440 & BULT FAIL \\
\hline$\ddot{c}-\dot{1}$ & REG & 5.87 & $S H-O$ & STAT & v & 30.1 & $\cdot 23$ & -- & $3 \dot{2} \cdot 8$ & .30 & -- & HOLT SHACAREU \\
\hline$c<-2$ & REG & 6.10 & $S A-S$ & STAT & $v$ & $30 \cdot 3$ & $\cdot 15$ & -- & 34.0 & .20 & -- & SOLT :L:AOEO \\
\hline$c 2-3$ & $R=\dot{U}$ & $6 \cdot 39$ & $5 t-6$ & DYN & v & $20 \cdot 1$ & $\cdot 13$ & $7 \cdot 8$ & $23 \cdot 4$ & $\cdot 25$ & 101 & SLEAOEL \\
\hline$C<-4$ & REG & 0.68 & $S A-6$ & DYN & v & $20 \cdot 1$ & $\cdot 20$ & 7.0 & $20 \cdot 1$ & .25 & 18.7 & EOLT SHEARED \\
\hline$M 2-1$ & MAG & 6.08 & $\mathrm{SH}-6$ & STAT & v & $34 \cdot 0$ & $\cdot 30$ & $\cdots$ & $37 \cdot 8$ & $\cdot 70$ & -- & EOLT FAILES \\
\hline$M 2-2$ & MIAG & 5.81 & $3 A-6$ & STAT & v & 38.0 & $\cdot 25$ & -- & 42.0 & .33 & -- & CONC FAILED \\
\hline$\therefore i c-3$ & "AG & 5.49 & دะー6 & UYN & v & $24 \cdot 0$ & $\cdot 20$ & $7 \cdot 8$ & $24 \cdot 0$ & .25 & $8 \cdot 1$ & $E J L T F A I L \equiv 0$ \\
\hline$i n<-4$ & MAG & 5.87 & SHIOO & UYN & $v$ & $24 \cdot 0$ & - 30 & $7 \cdot 8$ & $24 \cdot 0$ & .80 & $8 \cdot 1$ & BOLT FAILFD \\
\hline$S 2-1$ & ss & $7 \cdot 30$ & $S A-O$ & STAT & v & $24 \cdot 0$ & $\cdot 20$ & -- & 32.0 & .30 & -- & CONC FAILED \\
\hline
\end{tabular}


TAOLE UF LATA SURTEU OY UOLT TYPE, DOLT DIA., AND TYPE TEST. CONT.

- 4 INCH STUD TYPE BOLTS CONT

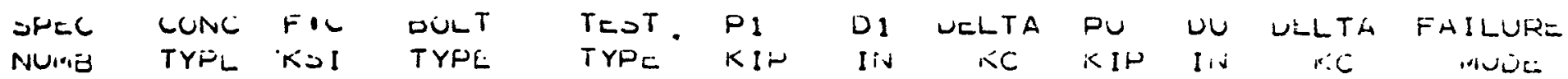

\begin{tabular}{|c|c|c|c|c|c|c|c|c|c|c|c|c|}
\hline $52-2$ & S' & 6.34 & SH-O & $S T A T$ & $v$ & 20.0 & $\cdot 15$ & -- & 26.0 & - $\Sigma 2$ & -- & CONC FAILFI \\
\hline $32-3$ & SS & $7 \cdot 44$ & Sedo & UYN & $v$ & $18 \cdot 4$ & $\cdot 20$ & $7 \cdot 8$ & $18 \cdot 4$ & .40 & $36 \cdot 4$ & PULLOUT \\
\hline 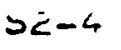 & SS & $7 \cdot 10$ & $5 A-6$ & DYN & $v$ & 18.0 & .10 & $7 \cdot 8$ & $26 \cdot 3$ & 10 & 103 & CONC FAILF= \\
\hline$b \ddot{L}$ & SS & $7 \cdot 23$ & $\triangle A-6$ & FAT & v & $5 \cdot 8$ & .05 & -- & $5 \cdot 8$ & .12 & 1500 & FAILED STAT \\
\hline 80 & $R E G$ & $7 \cdot 17$ & & $\triangle T A T$ & c & 9.0 & $\cdot 20$ & -- & 10.0 & .30 & -- & PULLOUT \\
\hline 83 & $R=G$ & 0.80 & $3 \dot{A}-6$ & UYN & c & $6 \cdot 5$ & - 25 & 7.8 & $13 \cdot 2$ & $\cdot 20$ & 107 & PULLOUT \\
\hline 31 & REG & $6 \cdot 71$ & $50-6$ & DYN & c & $6 \cdot 5$ & $\cdot 50$ & $7 \cdot 8$ & $11 \cdot 8$ & $1 \cdot 0$ & 102 & PULLCUT \\
\hline$\equiv 0$ & REEG & $0 .<2$ & $S h-0$ & FAT & c & $2 \cdot 6$ & .01 & -- & 10.3 & $\cdot 29$ & 2005 & CONC F゙AILEO \\
\hline ל ל & MAGG & -- & $5 A-6$ & STAT & c & 8.0 & .15 & -- & 11.4 & .20 & - & CONC FAILED \\
\hline 52 & $M A C$ & -- & $5 E-6$ & STAT & c & 12.0 & .02 & -- & 15.4 & .23 & -- & COVC FILE: \\
\hline בל & MAS & -- & $5 t-6$ & DYN & c & $8 \cdot 0$ & $\cdot 20$ & $7 \cdot 3$ & $14 \cdot 6$ & $\cdot 24$ & 104 & CONG FAILED \\
\hline 59 & MACG & $3 \cdot 0 َ \mathrm{c}$ & SA-O & UYN & $c$ & $8 \cdot 2$ & .04 & $7 \cdot 0$ & 12.0 & .08 & $10=$ & CONC FAILED \\
\hline 02 & ss & $7 \cdot 90$ & $\triangle E-O$ & $S T A T$ & c & 6.0 & .08 & -- & 10.4 & .17 & -- & SOLT FAILED \\
\hline 213 & ss & $8 \cdot 14$ & $S A-O$ & UYN & c & $6 \cdot 0$ & $\cdot 20$ & 7.8 & $10 \cdot 0$ & .43 & $71 \cdot 8$ & BOLT FAILEO \\
\hline 19 & SS & $0 \cdot 53$ & $s--0$ & DYN & c & $6 \cdot 1$ & $\cdot 17$ & $7 \cdot 8$ & $6 \cdot 1$ & .20 & 34.0 & DULT - A ILEJ \\
\hline$c 17$ & SS & 8.09 & $S A-6$ & FAT & c & $2 \cdot 0$ & .02 & -- & 6.0 & .35 & 2006 & ZOLT SHEAFED \\
\hline
\end{tabular}


TAELE UF LATA SURTED UY OULT TYHE, EULT DIA., AINO TYPE TEST. CONT.

1 IIVCH STUD TYHE BULTS

\begin{tabular}{|c|c|c|c|c|c|c|c|c|c|c|c|c|c|}
\hline SNEC & CUNC & $F \cdot C$ & GOLT & \multirow{2}{*}{\multicolumn{2}{|c|}{$\begin{array}{l}\text { TEST } \\
\text { TYNE }\end{array}$}} & $P_{1}$ & 01 & DELTA & $P \cup$ & DU & DFLTA & \multirow{2}{*}{\multicolumn{2}{|c|}{ FAILURE }} \\
\hline Nuiva & TYPO & $K S I$ & TYPE & & & $K I \mapsto$ & $I \dot{N}$ & $K C$ & $K \llbracket P$ & $I N$ & $k C$ & & \\
\hline Bio & $K=G$ & 6.56 & $\triangle \dot{A}-\dot{U}$ & STAT & $\mathbf{T}$ & 24.0 & - & - & $26 \cdot 4$ & .60 & - & CONC & FAIL \\
\hline 113 & $R \in G$ & $6 \cdot 13$ & $5 \sqsupset-8$ & STAT & $T$ & $16 \cdot 0$ & $1 \cdot 0$ & -- & $22 \cdot 5$ & $1 \cdot 20$ & -- & CONE & FAILED \\
\hline 91 & KES & 5.69 & $S \dot{A}-\varepsilon$ & UYN & $T$ & $16 \cdot u$ & .10 & $7 \cdot 8$ & $26 \cdot 0$ & $.3 \overline{3}$ & 103 & conc & $=\Delta I L F D$ \\
\hline 107 & $F: C G$ & 7.00 & $S t-8$ & DYN & $T$ & $15 \cdot 8$ & .65 & $7 \cdot 3$ & $20 \cdot 8$ & - 85 & 101 & CONC & FAILFR \\
\hline$C 2-5$ & $R R E$ & $6 \cdot 66$ & $S A-B$ & STAT & V & 28.0 & $\cdot 1$ & & $34 \cdot 5$ & -50 & -- & CONC & $F \triangle I L E D$ \\
\hline $\bar{c} \bar{c}-0$ & $R=O$ & $7 \cdot 14$ & $S A-O$ & STAT & V & $E \theta .0$ & $\cdot 1$ & & 36.4 & .50 & -- & & \\
\hline$c_{c-7}$ & REG & $6 \cdot 30$ & $\triangle A-B$ & DYN & V & $21 \cdot \overline{0}$ & .25 & $7 \cdot 8$ & 38.0 & $\bullet=0$ & 76 & DOLT & FAILED \\
\hline$c<-0$ & KCE & 0.10 & $\triangle A-\triangle$ & DYN & $v$ & $21 \cdot 8$ & 10 & $7 \cdot 8$ & $31 \cdot 0$ & .25 & 106 & CONC & FAILED \\
\hline 22 & $R=G$ & $7 \cdot 14$ & $S A-B$ & STAT & $C$ & 10.0 & .20 & -- & $12 \cdot 6$ & .25 & -- & CONC & FAILEN \\
\hline 17 & P.CG & 0.02 & $د B-3$ & STAT & C & 10.0 & .20 & -- & 13.0 & .25 & -- & corlo & FAILED \\
\hline $2 y$ & $R \in G$ & 0.52 & $S A-b$ & DYN & C & $8 \cdot 0$ & .14 & $7 \cdot 8$ & 8.0 & .34 & 74 & covi= & FAILED \\
\hline 20 & $R \in G$ & 0.77 & $\triangle A-B$ & OYN & C & 8.0 & .36 & $7 \cdot 8$ & $11 \cdot 6$ & .57 & 102 & Conc & FAILED \\
\hline
\end{tabular}


T E OF DATA SORTED EY GOLT TYPE, BOLT DIA.. AND TYPE TEST. CONT.

$5 / 8$ INCH SHELL ANCHORS

$\begin{array}{lllllllllllll}112 & \text { REG } & 7.49 & \text { LA-5 } & \text { DYN } & T & 6.6 & .08 & 7.8 & 10.0 & .22 & 102 & \text { CONC FAILED } \\ 100 & \text { REG } & 6.20 & \text { LA-5 } & \text { DYN } & T & 7.8 & .06 & 7.8 & 10.6 & .14 & 101 & \text { CONC FAILFD } \\ C 2-14 & \text { REG } & 6.22 & \text { LA-5 } & \text { DYN } & V & 9.6 & .15 & 7.8 & 9.6 & .30 & 51.0 & \text { BOLT SHEARED } \\ C 2-15 & \text { REG } & 6.52 & \text { LA-5 } & \text { DYN } & V & 9.6 & .15 & 7.8 & 9.6 & .25 & 39.0 & \text { BOLT SHEARED } \\ 39 & \text { REG } & 6.11 & \text { LA-5 } & \text { STAT } & C & 4.0 & .10 & -1- & 11.2 & .95 & -1 & \text { BOLT FAILED } \\ 44 & \text { REG } & 6.61 & \text { LA-5 } & \text { DYN } & C & 6.6 & .07 & 7.8 & 6.6 & .07 & 7.8 & \text { PULLOUT } \\ 34 & \text { REG } & 5.92 & \text { LA-5 } & \text { DYN } & C & 6.6 & .07 & 7.8 & 6.6 & .07 & 7.8 & \text { PULLOUT }\end{array}$

$3 / 4$ INCH SHELL ANCHORS

$\begin{array}{cclllllllllll}\text { SPEC } & \text { CONC } & F I C & \text { BOLT } & \text { TEST } & \text { PI } & \text { DI } & \text { DELTA } & \text { PU } & \text { DU } & \text { DELTA } & \text { FAILURE } \\ \text { NUMB } & \text { TYPE } & \text { KSI } & \text { TYPE } & \text { TYPE } & \text { KIP } & \text { IN } & \text { KC } & \text { KIP } & \text { IN } & \text { KC } & \text { MODE } \\ 27 & \text { REG } & 7.12 & \text { LA-6 } & \text { STAT } & C & 11.0 & .14 & -- & 12.0 & .16 & -- & \text { CONCFAILED } \\ 81 & \text { REG } & 7.10 & \text { LA-6 } & \text { STAT } & C & 9.0 & .40 & -- & 13.3 & .55 & .- & \text { PULLOUT }\end{array}$



TAUL= UF UATA JURTED OY TEST TYPE AND ULTIMATE LOAD (PU). IN THAT OZJIEL - LiVSIUIV TESTS

SPEE CUNC FIL DOLT TEST. PI DI DELTA PU DU DELTA FAILURE iVU. TYPE KJI TYP= TYPE KID IN KC KIP IN KC IISE

\begin{tabular}{|c|c|c|c|c|c|c|c|c|c|c|c|c|}
\hline Bö & REG & 5.90 & $S A-\ddot{B}$ & STAT & $T$ & $24 \cdot 0$ & $\cdot 50$ & $\div-$ & $2 \leqslant .4$ & .50 & -- & CONC FI:ILED \\
\hline $\begin{array}{l}41 \\
120\end{array}$ & $\begin{array}{l}R=G \\
R A G G\end{array}$ & $\begin{array}{l}5 \cdot 65 \\
7 \cdot 05\end{array}$ & $\begin{array}{l}S A-0 \\
S A-0\end{array}$ & $\begin{array}{l}\text { DYN } \\
\text { STAT }\end{array}$ & $\begin{array}{l}T \\
T\end{array}$ & $\begin{array}{l}16 \cdot 0 \\
8 \cdot 0\end{array}$ & $\begin{array}{l}.10 \\
.05\end{array}$ & $\begin{array}{l}7 \cdot 8 \\
--\end{array}$ & $\begin{array}{l}26 \cdot 0 \\
25 \cdot 5\end{array}$ & $\begin{array}{r}.33 \\
.42\end{array}$ & 103 & $\begin{array}{l}\text { CONC FAILED } \\
\text { CONC FAILEO }\end{array}$ \\
\hline 113 & $R=G$ & 0.13 & ذ๐ーb & $S T A T T$ & $\mathbf{T}$ & $16 \cdot 0$ & 1.0 & -- & $22 \cdot 5$ & $1 \cdot 20$ & $-\infty$ & CONC FAILED \\
\hline $10 \%$ & $\gamma=0$ & $7 \cdot 44$ & $S A-6$ & STAT & $T$ & $6 \cdot 0$ & .20 & -- & $22 \cdot 0$ & .50 & -- & PULLOUT \\
\hline 120 & REG & $7 \cdot 12$ & $S A-O$ & DYN & $T$ & 10.0 & .15 & 7.8 & $21 \cdot \varepsilon$ & .45 & 100 & CONS FAILEO \\
\hline 37 & REG & $5 \cdot 50$ & SA-D & DYN & $T$ & $8 \cdot 6$ & .22 & $7 \cdot 8$ & 18.6 & .60 & 110 & PULI_OIT \\
\hline 112 & $\dot{r}=G$ & 0.56 & $3 A-6$ & JYi & $\mathrm{T}$ & $10 \cdot 0$ & .25 & $7 \cdot 0$ & $1 \overline{5} \cdot 2$ & .45 & 105 & PULLOUT \\
\hline \pm 10 & REG & $7 \cdot 49$ & $50-6$ & STAT & $T$ & $5 \cdot 0$ & .10 & -- & $17 \cdot 3$ & .50 & -- & PULLכUT \\
\hline$i<1$ & VथिG & 6.17 & $i s-0$ & STÄT & $T$ & -- & -- & & $15 \cdot 6$ & -- & -- & DULLOLT \\
\hline はこシ & $\therefore A B$ & 0.78 & $5=-6$ & JYN & $T$ & $13 \cdot 6$ & $\cdot 10$ & $7 \cdot 8$ & $15 \cdot 7$ & .42 & 100 & EOLLT FAILES \\
\hline 07 & रㄷ & $5 \cdot 51$ & Lø-6 & UYN & $T$ & $7 \cdot 8$ & - 15 & $7 \cdot 8$ & $15: 6$ & 1.01 & 103 & PULLOUT \\
\hline 137 & SS & $6 \cdot 50$ & $5 A-6$ & STAT & $T$ & $12 \cdot 0$ & .30 & - & 15.5 & .60 & - & DULLOUT \\
\hline 100 & RヒG & $7 \cdot 16$ & LB-0 & STAT & $T$ & 14.3 & .78 & -- & 15.4 & -93 & -- & $0 ! j L 1.0: T$ \\
\hline+0 & $\dot{s}=$ & $7 \cdot ذ 8$ & $S A-6$ & DYN & $T$ & 5.4 & $\cdot \approx 7$ & $7 \cdot 0$ & $i 4.4$ & .40 & 102 & PULLOUT' \\
\hline 105 & REG & 6.54 & $5 E-S$ & STAT & $T$ & 8.0 & .20 & -- & 14.4 & .40 & -- & PULLOUT \\
\hline 123 & $M A G$ & 6.93 & $5 \theta-6$ & STAT & $T$ & $8 \cdot 0$ & .04 & -- & $14 \cdot 2$ & .15 & -- & CONC FAILE \\
\hline 131 & ১১ & ja 7 & $S A-0$ & $D Y N$ & $\mathrm{~T}$ & 9.6 & .21 & 7.5 & 13.6 & .30 & 101 & PULLOL'T \\
\hline 122 & $M A G$ & 7.07 & $5 A-6$ & DYN & $T$ & $13 \cdot 6$ & - 18 & $7 \cdot 8$ & $13 \cdot 6$ & .25 & 94 & BOLT FAILED \\
\hline 127 & MAG & 6.17 & LB-6 & DYN & $T$ & $7 \cdot 0$ & $1 \cdot 0$ & $7 \cdot 8$ & $13 \cdot 0$ & $1 \cdot 25$ & $107 \cdot 3$ & CONS $=A ! L E D$ \\
\hline $\begin{array}{l}11 \\
114\end{array}$ & $\begin{array}{l}\text { REG } \\
\text { REG }\end{array}$ & $\begin{array}{l}5 \cdot 60 \\
6.86\end{array}$ & $\begin{array}{l}L 3-5 \\
S A-S\end{array}$ & $\begin{array}{l}\text { STAT } \\
\text { UYN }\end{array}$ & $\begin{array}{l}T \\
T\end{array}$ & $\begin{array}{l}12.0 \\
8 \cdot 6\end{array}$ & $\begin{array}{l}\cdot 25 \\
.50\end{array}$ & $7 \cdot 8$ & $\begin{array}{l}13 \cdot 0 \\
12 \cdot 6\end{array}$ & $\begin{array}{l}.40 \\
1.0\end{array}$ & 102 & $\begin{array}{l}\text { BOLT FAILEO } \\
\text { CONC FAILES }\end{array}$ \\
\hline 03 & RंटG & 6.55 & $S C-5$ & DYN & $\mathrm{T}$ & $5 \cdot 5$ & .05 & $7 \cdot 8$ & $12 \cdot 6$ & .20 & 101 & PULLOUT \\
\hline 130 & نifín & $6 \cdot 10$ & LUーல & UYN & $T$ & $7 \cdot 0$ & .46 & $7 \cdot 8$ & $12 \cdot 5$ & .76 & 102 & CONE FAILES \\
\hline
\end{tabular}




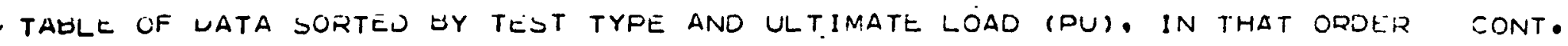
. . ISICN TESTS CONT.

SPEC CUNC FIC DOLT TEST. P1 DI UELTA PU DU DELTA FAILURE NUMB TYPE KDI TYPE TYPE KIP IN KC KIP IN KC MODE

\begin{tabular}{|c|c|c|c|c|c|c|c|c|c|c|c|c|}
\hline 1 & $R \subset G$ & 6.60 & $s=-5$ & UYN & $T$ & $7 \cdot 0$ & .07 & $7 \cdot 8$ & $12 \cdot 0$ & .45 & 102 & PULLDUT \\
\hline 203 & $R E G$ & 6.20 & St -5 & STAT & $T$ & $11 \cdot 6$ & $\cdot 35$ & -- & $11 \cdot 6$ & - 80 & -- & PULLOUT \\
\hline 14 & HEG & $4 \cdot 50$ & டேーذ & DYN & T & 11.0 & $\cdot 20$ & $10 \cdot 2$ & $11 \cdot 0$ & .45 & $11 \cdot 7$ & LHCLT FAILED \\
\hline 5 & $R \subset G$ & $6 \cdot 30$ & $\$ E-5$ & DYN & $T$ & $7 \cdot 0$ & .45 & $7 \cdot 8$ & 16.0 & .68 & 112 & DULLOUT \\
\hline 100 & $P E G$ & $0 \cdot<0$ & LA-S & DYN & $T$ & $7 \cdot 8$ & .06 & $7 \cdot 8$ & 10.0 & .14 & 101 & CONC FAILED \\
\hline 112 & $R E G$ & $7.4 y$ & LATS & DYN & $T$ & $6 \cdot 6$ & .08 & $7 \cdot 8$ & $10 \cdot 0$ & $\cdot 22$ & $10 \ddot{z}$ & CONC FAILED \\
\hline 86 & REG & 7.24 & Ly-O & DYN & $\mathrm{T}$ & 7.8 & .80 & 7.8 & 9.7 & . 90 & S. 7 & DUILLOUT \\
\hline 79 & $R=G$ & 6.91 & 1ラーの & STAT & $T$ & -- & -- & * & 9.4 & -- & -- & DULLOUT \\
\hline 89 & $\ddot{R} \in G$ & 5.05. & $S c-3$ & DYN & $T$ & $5 \cdot 5$ & .07 & $7 \cdot 3$ & 10.7 & $\cdot 20$ & 102 & PULLOUTT \\
\hline 138 & ss & $7 \cdot .18$ & $S=-6$ & STAT & $T$ & 6.0 & .40 & -- & $7 \cdot 6$ & - so & -- & PULLOL \\
\hline 134 & Sذ & $0 \cdot \dot{<9}$ & LローO & DTAT & $T$ & $4 \cdot 8$ & $\cdot 33$ & -- & $7 \cdot 2$ & 1.05 & -- & DULLOUT \\
\hline 202 & REC & 4.40 & LBS- & DYN & $T$ & 6.8 & $\cdot 15$ & $22 \cdot 0$ & $6 \cdot 8$ & .20 & 71 & BOLT FAILED \\
\hline 137. & So & $0 \cdot<0$ & Lனーல & DYN & $T$ & $3 \cdot 4$ & - 38 & $7 \cdot 5$ & 6.4 & .97 & 110 & PULLOUT \\
\hline 123 & MAE & 6.20 & LB-6 & STAT & $T$ & $3 \cdot 7$ & .01 & -- & $5 \cdot 5$ & -- & -- & DULLOUT' \\
\hline 3 & $\mathrm{REG}$ & 0.00 & $-\Xi-5$ & FAT & $T$ & $2 \cdot 6$ & .02 & $1 \cdot 0$ & $4 \cdot 2$ & .03 & 2000 & EOLT FAILED \\
\hline 204 & REG & -- & $S A-S$ & FAT & $T$ & $4 \cdot 0$ & .01 & -- & $4 \cdot 0$ & .03 & 386 & EOLT SHE \\
\hline 132 & SS & 6.27 & LE-S & $S T \Delta T$ & T & $2 \cdot s$ & .02 & -- & $\Delta \cdot C$ & -- & -- & PULLOUT \\
\hline 133 & ss & $7 \cdot 61$ & $5 i-6$ & FAT & $T$ & $3 \cdot 2$ & .05 & -- & $3 \cdot 2$ & .70 & 1440 & BOLT FAILES \\
\hline 107 & REG & 7.00 & $\mathrm{Sb}-8$ & DYN & $T$ & $15 \cdot 8$ & .65 & $7 \cdot 8$ & $20 \cdot 8$ & - 85 & 101 & CONC FAILED \\
\hline 205 & $R E G$ & $5 \cdot 50$ & LÖ-5 & FAT & $T$ & $2 \cdot 6$ & .02 & 2000 & $2 \cdot 6$ & .02 & 2000 & GOLT FATLED \\
\hline 94 & $R \in G$ & $6 \cdot 38$ & LB-G & FAT & $T$ & 2.6 & .05 & $1 \cdot 0$ & $2 \cdot 6$ & .15 & 535 & BOLT FAILED \\
\hline $20 !$ & REE & $0 \cdot 10$ & Lつー= & FAT & $T$ & $2 \cdot 6$ & • & $3 \cdot 0$ & $2 \cdot 0$ & כذ & $=04$ & כOLT FAILEO \\
\hline 136 & SS & 6.24 & $L B-S$ & DYN & $T$ & -- & -- & - & $2 \cdot 2$ & .83 & 6.8 & DULLOUT \\
\hline 73 & $R L G$ & -- & $S A-6$ & FAT & $T$ & & & & & & & \\
\hline
\end{tabular}




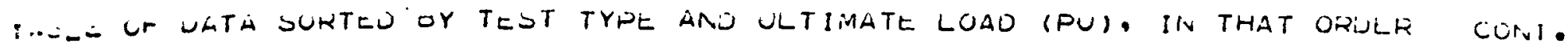
TCSTS

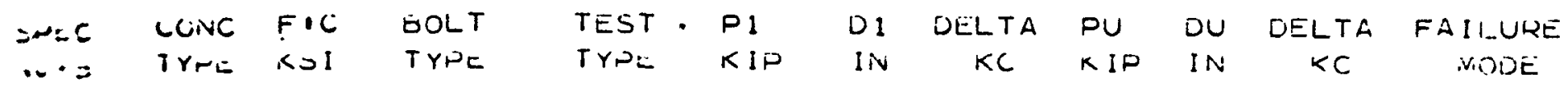

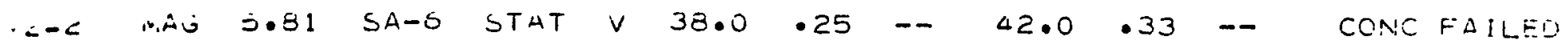

$\begin{array}{llllllllllll}C<-7 & \text { REG } & 6.36 & \text { SA-8 } & \text { UYN } & V & 21.8 & .25 & 7.8 & 38.0 & .50 & 76 \\ \cdots=-1 & \text { HAG } & 6.03 & \text { SH-G } & \text { STAT } & V & 34.0 & .30 & -- & 37.8 & .70 & -1\end{array}$

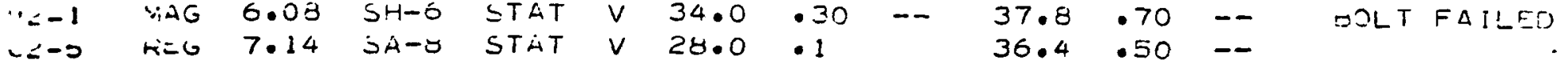

BOLT FAILED

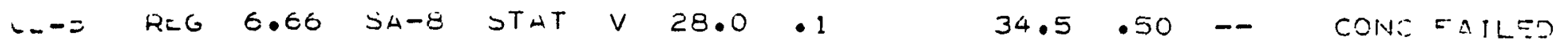

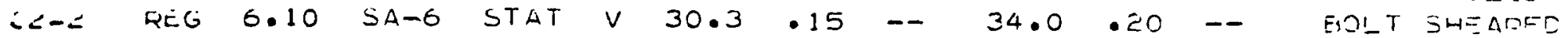

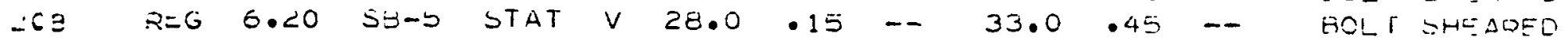

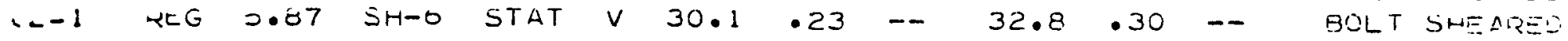

C.3 MAG 0.54 LB-6 STAT V 23.0 .34 -

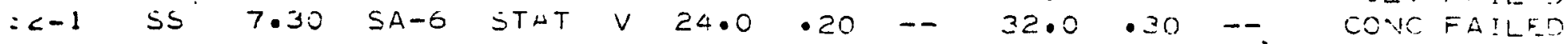

$7=$ PEG 6.67 LJ-6 STAT V 28.C .40 - - 31.6 .55 --

-. REG G.10 SA-E JYN V 21.3 .10 7.8 31.0.25 1OG CONEFAILEO

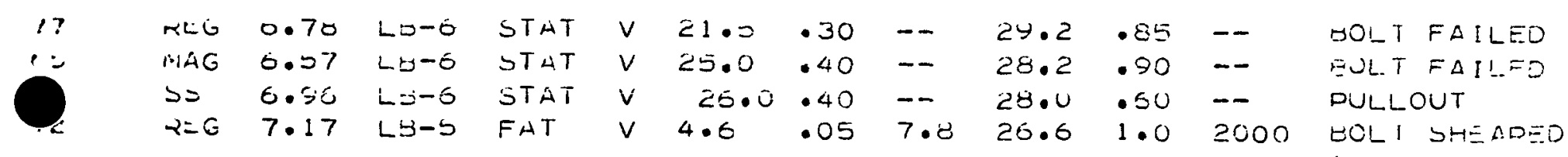

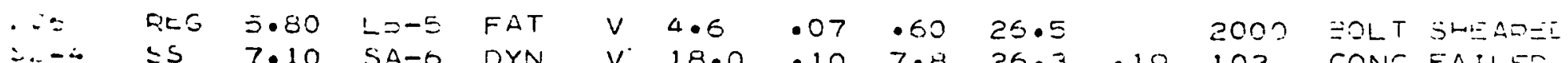

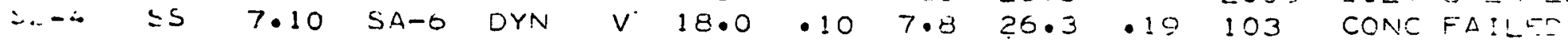

...- DS 0.84 SHIS STAT V 20.0 . 15 - $\quad 26.0$.22 -

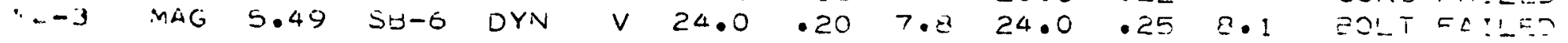

$\because-\because$ MAG 5.87 SH-G DYN V 24.0 .30 7.8 24.0 .8O B.1 BOLT FAILED

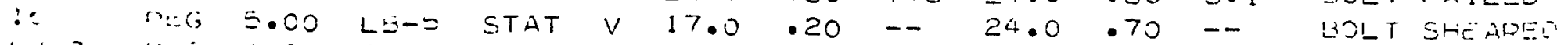

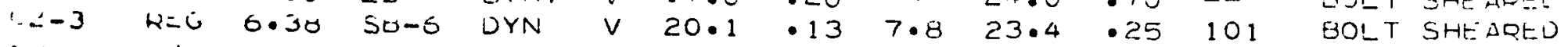

C. $=5$ 0.40 LE-O STAT V 18.8 .17 - $\quad 22.0$ 1.20 -

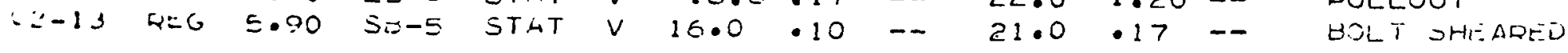


TASLE UH UATA SORTLU OY TEST TYHE AND ULTIMATE LOAD (DU), IN THAT ORDER CONT. SMEAR TESTS CONT.

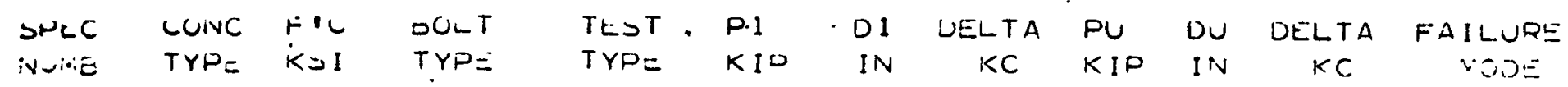

\begin{tabular}{|c|c|c|c|c|c|c|c|c|c|c|c|c|c|}
\hline$l<-4$ & $R \in G$ & 0.68 & $S A-0$ & LYN & $v$ & $20 \cdot 1$ & $\cdot 20$ & 7.8 & $20 \cdot 1$ & .25 & $1 \varepsilon \cdot 7$ & $=O L T$ & 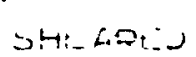 \\
\hline 7 & $R \in G$ & 6.40 & $S B-S$ & DYN & v & $20 \cdot 0$ & .10 & $7 \cdot e$ & 20.0 & .20 & $9 \cdot 2$ & BOLT & SHEAPID \\
\hline $2 C y$ & RES & $7 \cdot 10$ & $S A-D$ & OYN & $v$ & $19 \cdot 2$ & .17 & $7 \cdot 8$ & 19.2 & .45 & 17.9 & DOLT & $S H:: A R \equiv U$ \\
\hline$-2-9$ & $R \angle G$ & 0.77 & $5 A-5$ & JYiN & V & 19.2 & .30 & 7.0 & 19.2 & לد . & $8 \cdot 0$ & $\triangle O L I$ & $S H E A D E D$ \\
\hline$=--3$ & $=5$ & 7.44 & $S E-G$ & OYN & V & 18.4 & $\cdot 20$ & $7 \cdot 8$ & 18.4 & .40 & $36 \cdot 4$ & PULLS & JuT \\
\hline 1 & $R \equiv G$ & --- & si -5 & DYN & v & 18.0 & $\cdot 22$ & 7.0 & 18.0 & -- & 10.2 & UULT & ヨルAREJ \\
\hline "5 & $R=G$ & $6 \cdot 54$ & $L \bar{B}-6$ & OYN & v & 17.8 & $1 \cdot 10$ & 7.8 & 17.8 & -- & 19.8 & JOLT & $F \triangle I L E D$ \\
\hline 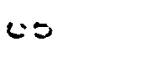 & VAG & 0.48 & LO-O & JYN & v & 17.4 & .25 & 7.8 & 17.4 & כ5 & 59.6 & $a x r$ & FAILFD \\
\hline 04 & $\therefore A B$ & 0.70 & $L=-6$ & DYNV & v & 17.4 & $\cdot \hat{2} 7$ & 7.0 & 17.4 & 1.25 & 26.5 & EOL & FAIIED \\
\hline$\therefore-1=$ & $R=3$ & 6.07 & LEーS & STAT & v & 16.0 & .30 & -- & 17.2 & .42 & -- & SOL'T & $S \omega 5 \Delta=\equiv$ \\
\hline$L \leftarrow-1 u$ & $\forall \leftarrow G$ & 6.40 & $s c-5$ & DYIV & v & 14.4 & .15 & 7.8 & 14.4 & .23 & 33.0 & BOLT & SHEARED \\
\hline $1<$ & ن́丶万 & 0.00 & LESD & UYN & v & $14 \cdot 4$ & .35 & 7.8 & $14 \cdot 4$ & .55 & 32.0 & SOLT & $S H=A R E C$ \\
\hline r. 7 & KCG & $4 \cdot=0$ & LO-S & DYN & V & 14.4 & .20 & 7.8 & $14 \cdot 4$ & .40 & $31 \cdot 6$ & SOL $T$ & SHEADEO \\
\hline$\{-! 1$ & REC & 6.64 & sc -5 & DYN & V & $14 \cdot 4$ & .25 & 7.3 & 14.4 & .75 & 23.0 & EOLT & GHF $s=5^{\circ}$, \\
\hline$\cdot-1$ & $2=0$ & 0.22 & $L^{--5}$ & DYY & V & 9.5 & .15 & $7 \cdot 8$ & 9.0 & - 30 & $\equiv \mathrm{i} \cdot 0$ & SOLT & SHEムDEU \\
\hline$c<-12$ & $R E G$ & 6.52 & $L A-5$ & DYN & v & 9.6 & .15 & 7.8 & 4.6 & מב & 35.0 & $=0 L T$ & $S H \equiv \angle R E-$ \\
\hline د 7 & KES & 7.35 & $L B-S$ & DYN & v & 17.3 & .27 & 7.8 & 17.8 & .80 & 27.5 & OLT & CAILED \\
\hline 71 & $১ ১$ & 0.45 & LB-G & OYN & v & 16.8 & 1.10 & 7.8 & 10.8 & -- & $7 \cdot 8$ & PULLC & DUT \\
\hline$=c-0$ & $\supset>$ & $7 \cdot 23$ & $\triangle A-6$ & FAT & v & 2.5 & .05 & -- & $b \cdot 8$ & .12 & 1500 & FAILE & $=\circlearrowright$ STAT \\
\hline 210 & REG & $4 \cdot 90$ & LS -5 & STAT & v & 19.0 & .45 & -- & 20.0 & .70 & -- & $E O L T$ & $\Sigma L=\triangle S E n$ \\
\hline $7 c$ & S & 5.16 & L5-S & DYN & v & 10.8 & .22 & 7.8 & 10.8 & -- & 34 & PULLO & $D U T$ \\
\hline
\end{tabular}


OF UATA SORTEJ OY TEST TYPE AND ULTIMATE LOAD (PU.) IN THAT ORDER CONT.

COMBINED SHEAR AND TENSION TESTS

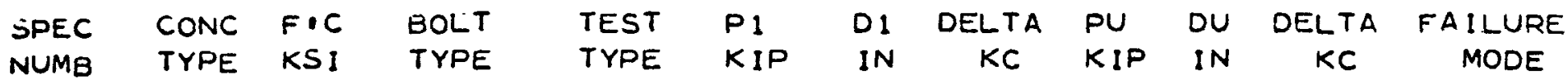

\begin{tabular}{|c|c|c|c|c|c|c|c|c|c|c|c|c|c|}
\hline 52 & MAG & -- & $S B-6$ & STAT & C & $12 \cdot 0$ & .02 & - & $15 \cdot 4$ & .23 & - & CONC & FAILED \\
\hline 54 & MAG & $8 \cdot 14$ & LB- 6 & STAT & c & $5 \cdot 8$ & .08 & -- & $15 \cdot 4$ & -- & -- & CONC & FAILED \\
\hline 58 & MAG & -- & $58-6$ & DYN & c & 8.0 & .20 & $7 \cdot 8$ & 14.6 & .24 & 104 & CONC & FAILED \\
\hline 30 & REG & $5 \cdot 85$ & $L B-6$ & STAT & C & $8 \cdot 4$ & - 19 & -- & $14 \cdot 4$ & 1.35 & -- & CONC & FAILED \\
\hline & MAG & 8.07 & LB-6 & STAT & c & $7 \cdot 2$ & .09 & -- & $14 \cdot 2$ & .93 & -- & CONC & FAILED \\
\hline 33 & REG & $7 \cdot 10$ & LA- 6 & STAT & $c$ & 9.0 & .40 & -- & $13 \cdot 3$ & .55 & $\cdot--$ & PULLO & UUT \\
\hline 33 & REG & $6 \cdot 86$ & $5 A-6$ & DYN & C & $6 \cdot 5$ & .25 & $7 \cdot 8$ & $13 \cdot 2$ & .50 & 107 & PULLO & DUT \\
\hline 7 & REG & 6.02 & $S B-8$ & STAT & c & $10 \cdot 0$ & .20 & -- & $13 \cdot 0$ & $\cdot 25$ & -- & CONC & FAILED \\
\hline & REG. & $7 \cdot 17$ & $58-6$ & STAT & c & $9 \cdot 0$ & $\cdot 20$ & -- & 13.0 & $\cdot 30$ & -- & PULLO & UT \\
\hline 23 & REG & $7 \cdot 14$ & $S A-B$ & STAT & c & $10 \cdot 0$ & $\cdot 20$ & - & $12 \cdot 6$ & .25 & -- & CONC & FAILED \\
\hline 9 & MAG & $8 \cdot 62$ & $S A-6$ & DYN & C & $8 \cdot 2$ & .04 & $7 \cdot 8$ & $12 \cdot 0$ & .08 & 102 & CONC & FAILED \\
\hline 27 & REG & $7 \cdot 12$ & LA-6 & STAT & $C$ & $11 \cdot 0$ & - 14 & - & $12 \cdot 0$ & .16 & -- & CONC & FAILED \\
\hline & REG & $6 \cdot 7 !$ & $58-6$ & DYN & C. & 6.5 & .50 & 7.8 & $11 \cdot 8$ & 1.0 & 102 & PULLO & UT \\
\hline & REG & 5.80 & LB-5 & STAT & $c$ & 6.4 & $\cdot 15$ & -- & $11 \cdot 8$ & 1.0 & -- & PULLO & UT \\
\hline 20 & REG & 6.77 & $5 A-8$ & DYN & $C$ & $8 \cdot 0$ & $\cdot 36$ & $7 \cdot 8$ & $11 \cdot 6$ & .57 & 102 & CONC & FAILED \\
\hline 35 & REG & $4 \cdot 90$ & $S B-5$ & STAT & $c$ & $11 \cdot 6$ & .07 & -- & $11 \cdot 6$ & .10 & -- & CONC & FAILED \\
\hline 46 & REG & $5 \cdot 30$ & LB-5 & STAT & $c$ & 8.0 & $\cdot 30$ & -- & 11.4 & $1 \cdot 1$ & -- & PULLO & DUT \\
\hline & MAG & $-\infty$ & $S A-6$ & STAT & $c$ & 8.0 & .15 & -- & $11 \cdot 4$ & .20 & -- & CONC & FAILED \\
\hline 215 & $\begin{array}{l}\text { REG } \\
\text { SS }\end{array}$ & $\begin{array}{l}0.11 \\
6.38\end{array}$ & $\begin{array}{l}L A-5 \\
L B-6\end{array}$ & STAT & $c$ & $\begin{array}{l}4.0 \\
8.6\end{array}$ & $\begin{array}{l}.10 \\
.12\end{array}$ & - & $\begin{array}{l}11 \cdot 2 \\
11 \cdot 2\end{array}$ & $\begin{array}{r}\because 95 \\
\because 41\end{array}$ & -- & $\begin{array}{l}\text { BOL } \\
\text { CONC }\end{array}$ & $\begin{array}{l}\text { FAILED } \\
\text { FAILED }\end{array}$ \\
\hline & SS & $7 \cdot 65$ & LB-6 & STAT & c & 9.6 & 11 & -- & $11 \cdot 2$ & . 94 & -- & CONC & FAILED \\
\hline & SS & $7 \cdot 90$ & $53-6$ & STAT & C & 6.0 & .08 & -- & 10.4 & .17 & -- & BOLT & FAILED \\
\hline & REG & 6.22 & $S A-6$ & FAT & $c$ & $2 \cdot 6$ & .01 & -- & $10 \cdot 3$ & .29 & 2005 & CONC & FAILED \\
\hline 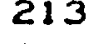 & SS & $8 \cdot 14$ & $5 A-6$ & DYN & C & 6.0 & $\cdot 20$ & $7 \cdot 8$ & $10 \cdot 0$ & .43 & $71 \cdot 8$ & BOLT & FAILED \\
\hline & REG & $6 \cdot 10$ & $S B-5$ & DYN & C & 6.6 & $\cdot 10$ & $7 \cdot 8$ & 9.6 & .35 & 102 & BOLT & SHEARED \\
\hline
\end{tabular}


TABLE OF UATA SURTED EY TEST TYHE AND ULTIMATE LOAD (PU), IN THAT ORUER CONT.

LUMBIVIED SHEAR AND TENSION TESTS

SPEC CUNC FIL DULT

Nivise

TYHE KOI TYPE
TEST, PI

TYPE
CONT.

DI UELTA FU DU JELTA FAILUKE KIP IN. KC KIP IN KC INUDE

$.177 .06 .1 \quad .25 \quad 84.0$ 3CLT FAILES .02 - -6.0 . 65 2005 EOLT SHEAREO

.12 -- $10.6 \quad .43$ - CONC FAILED

.15 -- 10.4 - -1 CONC FAIL:-D

\begin{tabular}{|c|c|c|c|c|c|c|c|c|c|c|c|c|}
\hline 29 & $R=G$ & $6 \cdot 22$ & $=A-\bar{B}$ & JYN & c & $8 \cdot 0$ & $\cdot 14$ & $7 \cdot 8$ & $8 \cdot 0$ & $\cdot 34$ & 74 & CONC FAILED \\
\hline 32 & REG & 7.01 & $L B-6$ & DYN & c & $7 \cdot 2$ & $\cdot 32$ & $7 \cdot 8$ & $7 \cdot 2$ & -- & 63.3 & BOLT SHEARED \\
\hline 29 & REG & 6.43 & $L y-0$ & DYN & c & $7 \cdot 2$ & $\cdot 54$ & $7 \cdot 8$ & $7 \cdot 2$ & -- & 29.5 & OOLT SHE \\
\hline $3 \Xi$ & $R \in G$ & 5.00 & $L \omega-5$ & DYN & $c$ & $7 \cdot 2$ & $\cdot 15$ & $7 \cdot 3$ & $7 \cdot 2$ & .40 & $16 \cdot 5$ & $B O L T$ SH $\triangle A D E D$ \\
\hline 43 & $R=G$ & $3 \cdot 7 c$ & LローD & JYN & c & $7 \cdot 0$ & .05 & $7 \cdot 2$ & $7 \cdot 0$ & .46 & 41 & PU'_L_OIJT \\
\hline 30 & REG & 6.30 & $S A-D$ & DYN & $c$ & $\epsilon \cdot 6$ & .15 & 7.8 & 6.6 & .45 & $37 \cdot c$ & PULLUUT \\
\hline 18 & $R \subset G$ & 6.70 & $s c-5$ & DYN & $c$ & $6 \cdot 6$ & .27 & 7.8 & $6 \cdot 6$ & -- & 28.0 & PULLOUT \\
\hline 24 & RES & $5 \cdot 50$ & $5 A-5$ & DYN & $c$ & $6 \cdot 6$ & .09 & $7 \cdot 3$ & $6 \cdot 5$ & .10 & $22 \cdot 0$ & \\
\hline 10 & REG & $5 \cdot 80$ & $\Delta-5$ & DYN & $c$ & $6 \cdot 6$ & .07 & $7 \cdot 8$ & $6 \cdot 6$ & -- & 17.9 & BOLT SHEARED \\
\hline 4 & REG & $0 .<0$ & $s c-j$ & DYN & $c$ & $6 \cdot 6$ & .05 & $7 \cdot 8$ & $5 \cdot 6$ & .07 & $15 \cdot 5$ & DU'LULT \\
\hline 34 & REG & $5 \cdot 4 \geq$ & LA-S & DYN & $c$ & $6 \cdot \epsilon$ & .07 & 7.8 & $6 \cdot 6$ & .07 & 7.9 & PULLOUT \\
\hline 44 & REG & 6.61 & LA-S & DYN & C & 6.6 & .07 & $7 \cdot 8$ & $5 \cdot 6$ & .07 & $7 \cdot 8$ & PJLLUUT \\
\hline & NIAG & 8.07 & $2 B-6$ & OYN & $c$ & $6 \cdot 4$ & .07 & $7 \cdot 8$ & $6 \cdot 4$ & -- & 67 & PULLOUT \\
\hline & ss & 0.80 & $1 B-\theta$ & DYN & $c$ & 6.4 & .14 & 7.8 & $\epsilon \cdot 40$ & -- & 7.9 & PUILLUT \\
\hline & ss & $8 \cdot 77$ & Lo-O & OYN & $c$ & 6.3 & .11 & $7 \cdot 8$ & $6 \cdot 25$ & -- & $44 \cdot 6$ & Sivo sr'=4 \\
\hline 53 & NAG & 7.90 & LE-S & DYiv & $c$ & 6.4 & .09 & $7 \cdot 8$ & $6 \cdot 2$ & -- & $95 \cdot 5$ & $B O L T \quad S H=A D$ \\
\hline 219 & ss & $8 \cdot 03$ & $\triangle A-0$ & DYN & $c$ & $5 \cdot 1$ & .17 & $7 \cdot 0$ & 6.1 & .25 & 84.0 & SCLT FAILES \\
\hline 217 & SS & 8.09 & $\triangle A-6$ & FAT & $c$ & $2 \cdot 0$ & .02 & -- & 6.0 & $\cdot 35$ & 2005 & EOLT SHEAREO \\
\hline 25 & REG & -- & $S B-5$ & STAT & $c$ & $6 \cdot 8$ & .12 & -- & 10.6 & .43 & -- & CONC FAILED \\
\hline 26 & REG & -- & LB-G & STAT & C & $6 \cdot 0$ & .15 & -- & 10.4 & -- & -- & CONC FAIL $=D$ \\
\hline
\end{tabular}

